List of Currently Classified Documents Relative to Hanford Production Facilities Operations Originated on the Hanford Site Between 1961-1972

\title{
PREFACE
}

The United States Department of Energy (DOE) has declared that all Hanford plutonium production- and operationsrelated information generated between 1944 and 1972 is declassified. Any documents found and deemed useful for meeting Hanford Environmental Dose Reconstruction (HEDR) objectives may be declassified with or without deletions in accordance with DOE guidance by Authorized Derivative Declassifiers.

The September 1992, letter report, Declassifications Requested by the Technical Steering Panel of Hanford Documents Produced 1944-1960, (PNWD-2024 HEDR UC-707), provides an important milestone toward achieving a complete listing of documents that may be useful to the HEDR Project. The 1429 entries listed in the report identify the documents as prioritized for eventual declassification (completed in FY 1992) by the Technical Steering Panel (TSP) and the public.

The attached listing of approximately 7,000 currently classified Hanford-originated documents relative to Hanford Production Facilities Operations between 1961 and 1972 fulfills TSP Directive 89-3.

It should be noted that this list does not include such titles as the Irradiation Processing Departrnent, Chemical Processing Department, and Hanford Laboratory Operations monthly reports generated after 1960 which have been previously declassified with minor deletions and made publicly avallable. Also note that Kaiser Engineers Hanford (KEH) Document Control determined that no KEH documents generated between January 1, 1961 and December 31, 1972 are currently classified. Additionally, titles which address work for others have not been included because Hanford Site contractors currently having custodial responsibility for these documents do not have the authority to determine whether other than their own staff have on file an appropriate need-to-know. Furthermore, these documents do not normally contain information relative to Hanford Site operations.

\section{Elemental Symbols}

When Douglas-United Nuclear (DUN) staff entered titles into its data base, those elemental symbols which consisted of upper- and lower-case letters (e.g., Plutonium $=\mathrm{Pu}$, Promethium $=\mathrm{Pm}$, Polonium $=\mathrm{Po}$, Neptunium $=\mathrm{Np}, \mathrm{Nickel}=\mathrm{Ni}, \mathrm{Niobium}$ $=\mathrm{Nb}$, etc.) did not retain the lower-case letter and upon printing recorded only a spiace.

For example, it is not immediately clear what "P." refers to in the document listing DUN-2336 "P Formation, Uranium Credits Burnout and Burnout Costs." It potentially could refer to Pu, Pm, Po, etc. However, DUN-1852

"P -238 Production Demonstration", most likely would denote "Pu" since only Pu has an isotopic of 238.

It was determined that an effort to identify the titles in question, pull and review the documents, and correct the symbol for each entry was not cost effective and outside the scope of this directive.

It should also be noted that "PT" does not normally refer to Platinum. References to "PT" in document titles and text more often refers to "Production Test".

\section{Numbering System}

This title listing was designed to consider only classified documents beginning January 1, 1961. There are some exceptions, however. Some titles may be dated in 1960 or earlier. The collection of "HW" documents for which Pacific Northwest Laboratory has custodial responsibility was not available in machine readable form when the initial listing of documents covering the years 1944-1960 was prepared. At that time, HW-68000 was chosen as the cut-off document. Most "HW" numbers greater than 68000 are dated in 1961 and later. However some exceptions were observed when preparing this listing (e.9., HW-69074RD "Control of Radio-lodine Emissions from the Separations Plants" is dated December 19, 1960.) Note that this document was once classified, but has been publicly available for a number of years. 
This list includes some of the more frequently used document prefixes during the 1961 through 1972 period. The prefixes are followed by their general descriptions, dates of origination and usape.

1. ARH

Atlantic Pichfield Hanford - Prime contractor for fusl reprocessing and waste management. Approximately 1968 to June, 1977.

2. $B N$

Battelle Northwest - 1965 to present.

3. BNWL

Battelle Northwest Laboratory - This series began in 1965 and was continuous to about document number 2260 , when in late 1977 the prefix "PNL" was aseigned for 1830 contract roports.

4. DUN

Douglas-United Nuclear - Prime contractor for operation of 100 Area production reactors. This prefix was changed to UNI (United Nuclear [inc. or Industries]) when McDonnell-Douglas relinquished its portion of the contract in 1973. United Nuclear's contract was terminated June 28, 1987.

5. FTS

Formerly Top Secret - FTS documents were later downgraded to Secret in the 1960 and 1970.

6. HAN

This accession number series was used by the Atomic Energy Commiseion, Richland Office (aleo know as HOO, Hanford Operations Office) on all documents which wore incoming to them and those which they originated.

7. $H W$

This series began with HW-7000 in July, 1947 and continued through the Coneral electric regime. H ceased in 1964 with HWs in the 89000s. All correspondenoe, memos and formal reports iseued by the General Electric Company while at Hanford were assigned an HW document number whether claseified or unctassified. Some of the very early numbers had the HEW prefix (Hanford Energy Works).

8. 150

bochem - This sories began in late 1965 and was terminated in 1867. bochem was a marrlage between U.S. Rubber and Martin Marietta and was the prime contractor for fuel reproceseing and wasto management. They were succeeded by Atlantic Richfield Hunford Company. bochem was the first operator of the 200 Areas after the General Electric departure.

9. $\mathrm{RL}$

This apha prefix followed by the initials of an oporations componont e.g. SAS (Safoguards and Socurity) has been used by DOE-RL since 1868 or 1969 . It follows the HAN series and is an accossion number used for both incoming and RL originated documents, primarily cleseified.

10. RL-GEN

The GENs were "general" to site operations and wore used in 1964 to 1965.

11. RL-NRD

The NRDs were used for documents that related to the N Pactor from 1864 until Goneral Electric turned the reactor over to Douglas-United Nuclear in 1867.

12. RL-REA

The REAs were used for documents relating to the reactors other than N Reactor that were originated in 1964 to 1965 . The REAs were followed by DUNs.

13. RL-SEP

The SEPs were used for documents relating to the separations activities which were originated in 1964 to 1965. The SEPB were followed by ISOs. 
Page No. 1

$04 / 28 / 93$

LIST OF HANFORD-GENERATED CLASSIFIED

DOCUMENTS DATED BETUEEN 1/1/61 AND 12/31/T2

DOC. NO.

TITLE

AUTHOR

LEVE

PGS

DATE

ARH-00001APR ARH-00001 FEB

ARH-00001 JAN ARH-00001 JUN ARH-00001MAR ARH-OCDOTMAY ARH-00003

$A R H-00003 A P R$

ARH-00003DEC

ARH-00003FEB

ARH-00003JAN

ARH-00003JUN

ARH-00003MAR

ARH-00003MAY

ARH-00003NOV

ARH-000030CT

ARH-00003SEP

ARH-00004APR

ARH-00004DEC

ARH-00004FEB

ARH-00004JAN

ARH-00004JUN

ARH-00004MAR

ARH-00004AMAY

ARH-00004NOV

ARH-000040CT

ARH-00004SEP

MRH -00007

ARH-00011RD
PUREX SOURCE DATA - FY 1968 - APR

PUREX SOURCE DATA - FY 1968 - FEB

PUREX SOURCE DATA - FY 1968 - JAN

PUREX SOURCE DATA - FY 1968 - JUM

PUREX SOURCE DATA - FY 1968 - MAR

PUREX SOURCE DATA - FY 1968 - MAY

PLUTONIUM PROCESSING SS MATERIALS

GENERAL LEDGER-FY 1968

PLUTONIUM PROCESSING - SOURCE DATA -

FY-1968, APRIL 1968

PLUTONIUM PROCESSING - SOURCE DATA - FY

1968 DECEMBER, 19678

PLUTONIUM PROCESSING - SOURCE DATA - FY

1968 FEBRUARY, 1968

PLUTONIUM PROCESSING - SOURCE DATA - FY

1968 JANUARY, 1968

PLUTONIUN PROCESSING - SOURCE DATA - FY

1968 JUNE, 1968

PLUTONIUA PROCESSING - SOURCE DATA - FY

1968 MARCH, 1968

PLUTONIUM PROCESSING - SOURCE DATA - FY

1968 MAY, 19688

PLUTONIUM PROCESSING - SOURCE DATA - FY

1968 NOVEMBER, 1967

PLUTONIUM PROCESSING - SOURCE DATA - FY

1968 - OCTOBER 1968

PLUTONILM PROCESSING - SOURCE DATA - FY

1968, SEPTEMBER 1967

PLUTONIUA RECLAMATION - SOURCE DATA - FY

1968 APRIL, 19688

PLUTONIUH RECL.AMATION - SOURCE DATA - FY

1968 DECEMBER, 1967

PLUTONIUN RECLAMATION - SOURCE DATA - FY

1968 FEBRUARY, 1968

PLUTONIUN RECLAMATION - SOURCE DATA - FY

1968 JaMUAARY, 1968

PLUTONIUN RECLAMATION - SOURCE DATA -

FY-1968 JUWE, 1968

PLUTONILA RECLAMATION - SOURCE DATA - FY

1968 MARCH, 1968

PLUTONIUN RECLAMATION - SOURCE DATA - FY

1968 MAY, 19688

PLUTONIUM RECLAMATION - SOURCE DATA - FY

1968 NOVEMBER, 1967

PLUTONIUA RECLAMATION - SOURCE DATA - FY

1968 - 10/01/67

PLUTONILN RECLAMATION - SOURCE DATA - FY

1968 SEPTEMBER, 19678

AEC-101 SHIPPING AND RECEIVING LOGBOOK

2 PLANT REPORT TASK I-II, RECOVERY

OPERATIONS SEPTEMBER 5, 1967 THRU

DECEMBER 31, 1967

\begin{tabular}{|c|c|c|}
\hline ARHCO & SECR & $01 / 08 / 70$ \\
\hline ARHCO & SECR & $01 / 08 / 70$ \\
\hline ARHCO & SECR & $01 / 08 / 70$ \\
\hline ARKCO & SECR & $01 / 08 / 70$ \\
\hline ARHCO & SECR & $01 / 08 / 70$ \\
\hline ARHCO & SECR & $01 / 08 / 70$ \\
\hline JG MURPHY & SECR & $09 / 04 / 67$ \\
\hline JG MURPHY & SECR & $04 / 01 / 68$ \\
\hline JG MURPHY & SRD & $12 / 01 / 67$ \\
\hline JG MURPHY & SRD & $02 / 01 / 68$ \\
\hline JG MURPHY & SRD & $01 / 01 / 68$ \\
\hline JG MURPHY & SRD & $06 / 01 / 68$ \\
\hline JG IMRPHY & SRD & $03 / 01 / 68$ \\
\hline JG MURPHY & SECR & $05 / 01 / 68$ \\
\hline JG IMUPHY & SRD & $11 / 01 / 67$ \\
\hline JG MURPHY & SECR & $10 / 01 / 67$ \\
\hline JG MURPHY & SECR & $09 / 04 / 67$ \\
\hline JG MURPHY & SECR & $04 / 01 / 68$ \\
\hline JG MURPHY & SRD & $12 / 01 / 67$ \\
\hline JG MURPHY & SRD & $02 / 01 / 67$ \\
\hline JG MURPHY & SRD & $01 / 01 / 68$ \\
\hline JG MURPHY & SRD & $06 / 01 / 68$ \\
\hline JG MURPHY & SRD & $03 / 01 / 68$ \\
\hline JG MURPHY & SECR & $05 / 01 / 68$ \\
\hline JG IMURHY & SRD & $11 / 01 / 67$ \\
\hline JG MURPHY & SECR & $10 / 01 / 67$ \\
\hline JG MURPHY & SECR & $09 / 04 / 67$ \\
\hline JG MURPHY & SRD & $09 / 04 / 67$ \\
\hline LM KNIGHTS & SRD & $09 / 05 / 67$ \\
\hline
\end{tabular}


Proe No. 2

$04 / 28 / 93$

LIST OF HANFORD-GEHERATED CLASSIFIED

DOCUMENTS DATED BETWEEN 1/1/61 AND 12/31/72

DOC. NO.

TITLE

AUTHOR

LEVE

PGS

DATE

ARH-00014

SPECTROANALYSIS OF RMA-RMC LINE

IN TAYLOR

SRD

09/05/67

PROOUCTS.

ARH-00017RD

ARCHO PRCDUCTION GRAPHS - SEPTEMBER 4 ,

D mCDOMald

SECR

$09 / 06 / 67$

ARH-00018RD

CPD CHARGING INFORMTION SEPTEMBER 5 , 1967 THRU*

ARH-00022

FY 1968 REVISION OF CS-137 IMDEX FOR MUCLEAR MATERIALS CONTENT OF COATING WASTE

ARH-00058

OPERATING DIVISION DAILY PROOUCTION

REPORTS OCTOBER, 1967 THROUGH DECEMBER, 1967

ARH-00064

ATLANTIC RICHFIELD HANFORD COMPANY, UNIT COST COMPARISONS, BUDGET FOR FY 1969 AND

D mCDONALD

SECR

$09 / 05 / 67$

WII ZIMAER

CONF

$09 / 15 / 67$

HH IMRRAY

SECR

$10 / 04 / 67$

JL GEORGE

SECR

$10 / 04 / 67$

ARH-00069

ARH -00080

ARH-00099RD

REVISION OF BUDGET FOR FY 1968.*

PRCDUCTION AND UNIT COST CHARTS."

ATLANTIC RICHLAND HANFORD PRCOUCTION

SCHEDULES OCTOBER 1967*

MUCLEAR MATERIAL TRANSFER RECORDS AND

ANALYTICAL DATA : HAF BLEND WORKSHEETS

ARH-00100RD

ARH -00109

ARH-00111RD

ARH-00116RD

DiSSOLVER OPERATION AND METAL TRANSFER

ESSENTIAL MATERIAL CONSUMPTION AND COST

REPORTS

SEPARATIONS PROCESS ENGINEERING -

WORKING PAPERS

SEPARATIONS PROCESS ENGINEERING DATA

WORKING PAPERS

WORKING PAPERS

WORKING PAPERS

QUARTERLY PROOUCTION FORECAST, 10/1/67

THROUGH 6/30/69

ARH -00127

ARH-00131

ARH-00156

PUREX OPERATIONS MEASUREMENTS QUALITY

CONTROL PROGRESS REPORT."

PRCOUCTION INVENTORY AND MANUFACTURING

STATEMENT SEPTEMBER 30, 1967.*

WORKING PAPERS - MUCLEAR MATLS.

MEASUREMENTS DATA EVALUATION-PLUTONIUM

FINISHING OPERATION JULY-DECEMBER, 1967.

ARH-00167

NONDEFENSE PLUTONIUN INVENTORY AS OF

OCTOBER 1, 1967

ARH-00173

ARH-00174RD

ARH-00185

ARH-00186

ARH-00187RD

NONDEFENSE PLUTONIUM INVENTORY AS OF

NOVEMBER 9, 1967*

MFC STUDY - KRYPTON AND XENON RECOVERY.*

NUCLEAR MATERIALS PRODUCTION REPORT

OCTOBER, 1967.*

PROOUCTION INVENTORY AND MANUFACTURING

STATEMENT OCTOBER 31, 1967.*

PLUTONIUN REDUCTION HOOD IV PROCESS

DATA.:

PLUTONIUM REDUCTION TASK III PROCESS

DATA.*

\begin{tabular}{|c|c|c|}
\hline $\begin{array}{l}\text { BA DOBBS } \\
\text { D MCDONALD }\end{array}$ & $\begin{array}{l}\text { SECR } \\
\text { SECR }\end{array}$ & $\begin{array}{l}10 / 20 / 67 \\
10 / 04 / 67\end{array}$ \\
\hline OF BENULIEU & SRD & $10 / 12 / 67$ \\
\hline OF BEAULIEU & SRD & $10 / 12 / 67$ \\
\hline EL KELLEY & SECR & $10 / 13 / 67$ \\
\hline WA BLYCKERT & SECR & $10 / 13 / 67$ \\
\hline HC RATHVON & SECR & $10 / 16 / 67$ \\
\hline DG HARLOH & SECR & $10 / 17 / 67$ \\
\hline GN WARD & SECR & $10 / 17 / 67$ \\
\hline ARCHO/DUH-RL/AEC & SECR & $10 / 31 / 67$ \\
\hline EL. KELLEY, SR. & SRD & $11 / 02 / 67$ \\
\hline GC PICKARD & SECR & $09 / 25 / 67$ \\
\hline CC JONES & SRD & $10 / 26 / 67$ \\
\hline MK HARMON & SECR & $10 / 05 / 67$ \\
\hline MK HARION & SECR & $11 / 08 / 67$ \\
\hline HP SHAK & SECR & $11 / 08 / 67$ \\
\hline JG MURPHY & SECR & $11 / 14 / 67$ \\
\hline GC PICKARD & SECR & $10 / 15 / 67$ \\
\hline PB FISK & SRD & $11 / 14 / 67$ \\
\hline PB FISK & SRD & $11 / 14 / 67$ \\
\hline
\end{tabular}


Page No. 3

$04 / 28 / 93$

LIST OF HANFORD-GENERATED CLASSIFIED

DOCUMENTS DATED BETUEEN 1/1/61 AMD 12/31/72

DOC. NO

ARH -00196

ARH-00213RD

ARH-00214

ARH-00243

ARH-00256

ARH-00258

ARH-00260

ARH-00262

ARH-00263RD

ARH-00268

ARH-00277

ARH-002771

ARH-00283

ARH-00294RD

ARH-00294RD

ARH-00294RD

ARH-00317

ARH-00318

ARH-00330

ARH-00331

ARH-00338

ARH-00341

ARH-00344RD

ARH-00375

ARH-00376

ARH-00400
TITLE

AUTHOR

GB KUKLIMSKI

END INFORMATION*

MISCELLANEOUS DATA PLUTONILA PROCESSIHG AMD PLUTONIUN RECLAMATION.*

PUREX CHEMICAL FLOUSHEET PROCESSIMG OF ALUMINUAA-CLAD URANIUN FUELS

DETERMINING ACCEPTABLE STACK

CONCENTRATIONS FOR GASEOUS MASTES

RELEASED TO THE ATMOSPHERE.*

NUCLEAR MATERIALS PRCOUCYION REPORT

NOVEMBER 1967\%

CHENICAL PROCESSING DIVISION DAILY

PROOUCTION REPORTS JAMUARY, 1968 THROUGH MARCH, 1968.*

STORAGE OF PLUTONIUN

PRCOUCTION IAVENTORY AND MANUFACTURING

STATEMENT MOVEMBER 30, 1967.*

WASTE MAMAGEMENT PERSONAL NOTES.*

OUARTERLY PRCOUCTION FORECAST, JANURAY

1, 1968 THROUGH JUNE 30, 1973.*

DESIGN CRITERIA: PUREX 3RD PLUTONIUA

SOLVENT EXTRACTION CYCLE

DESIGN CRITERIA: PUREX 3RD PLUTONIUM

SOLVENT EXTRACTION CYCLE

MONDEFENSE PLUTONIUM INVENTORY AS OF

DECEMBER 1, 1967.*

2 PLANT REPORT TASK I-II \& RECOVERY

OPERATIONS 1/1/68 THRU 12/31/68

2 PLANT REPORT TASK I-II \& RECOVERY

OPERATIONS 1/1/68 THRU 12/31/68

2 PLANT REPORT TASK I-II \& RECOVERY

OPERATIONS 1/1/68 THRU 12/31/68

NUCLEAR MATERIALS PROOUCTION REPORT

DECEMBER, 1967.*

ZPAL SAMPLE LOG

PUREX MONTH-EMD HORK SHEETS - JAMUARY,

1968 THROUGH DECEMBER 31, 1968.*

PRCOUCTION STATISTICS PUREX

PRCOUCTION INVENTORY AND MANUFACTURING

STATEMENT - DECEMBER 31, 1967.*

MONDEFENSE PLUTONIUM INVENTORY AS OF

JAMUARY 1, 1968.*

WORK PAPERS FOR PREPARING SIX WASTE

MANAGEMENT CHARTS. *

MONDEFENSE PLUTONIUN INVENTORY AS OF FEBRUARY 1, 1968*

U-236 MANAGEMENT COMMITTEE CLASSIFIED NOTES

PROOUCTION INVENTORY AND MANUFACTURIHG STATEMENT JANUARY 31, 1968.*

JP TURPING
WE MATHEISON

DJ BROM

JG MRPHY

HW MURRAY

MK HARION

GC PICKARD

QL RITTER

ARHCO-DUW-RL-AEC

RC, ROAL

RC ROAL

MK HARION

LA KNIGHTS

LH KNIGHTS

LM KNIGHTS

JG MURPHY

UR CORITY

JD MCINTOSH

EL KELLEY

GC PICKARD

MK HARAON

DL UEBELACKER

MK HARMON

VR CHAPAMN

GC PICKARD
LEVEL PGS

DATE

SECR

06/01/67

$11 / 22 / 67$

$02 / 15 / 68$

11/07/67

$12 / 14 / 67$

$01 / 01 / 68$

$12 / 19 / 67$

$12 / 22 / 67$

$12 / 19 / 67$

$02 / 29 / 68$

02/01/68

02/01/68

$01 / 04 / 68$

$01 / 01 / 68$

$01 / 01 / 68$

$01 / 01 / 68$

$01 / 15 / 68$

$01 / 16 / 68$

$01 / 17 / 68$

$01 / 17 / 68$

01/22/68

$01 / 23 / 68$

$01 / 24 / 68$

$02 / 13 / 68$

$02 / 08 / 68$

$02 / 22 / 68$ 
Page No. 4

$04 / 28 / 93$

LIST OF HANFORD-GENERATED CLASSIFIED

DOCLNENTS DATED BETWEEN 1/1/61 AMD 12/31/72

DOC. NO.

ARH-00423RD

ARH-00433

ARH -00440

ARH-00442

ARH -00450

ARH-00457

ARH-00458

ARH -00462

ARH -00463

ARH -00469

ARH -00506

ARH-00508

ARH -00515

ARH-00524

ARH-00530RO

ARH-00531RD

ARH-00532RD

ARH-00540RD

ARH-00542

ARH-00572

ARH-00589

ARH-005\%

ARH-00599RD

ARH-00611

ARH-00612
TITLE

MONTHLY REPORTS 1968 PLUTONILM PROCESS ENGINEERING SECTION.*

MUCLEAR MATERIALS REVIEW CPD ENRICHED \& DEPLETED URANILA SYSTEM FIRST \& SECOND CUARTERS FISCAL YEAR 1968 MONDEFENSE PLUTONIUN INVENTORY AS OF MARCH 1,1968*

MUCLEAR MTERIALS REVIEH - PLUTOWIUM FINISHIMG SECTION - FIRST AND SECOND CUARTERS FISCAL YEAR 1968*

MUCLEAR MATERIALS PRCOUCTION REPORT FEBRUARY, 1968*

REVIEU OF NTH POWER EVALUATION -

PRODUCTION OF PLUTONIUN

CHEMICAL PROCESSING DIVISION DAILY

PROOUCTION REPORTS APRIL, 1968 THROUGH JUME, 1968.*

PROOUCTION INVENTORY AMD MANUFACTURIMG STATEMENT FEBRUARY 29, 1968.*

GAS PURIFICATION EXPERIENCE

MONTHLY REPORT - PUREX OPERATIONS*

QUARTERLY PROOUCTION FORECAST, APRIL 1, 1968 THROUGH JUNE 30, 1970."

NONDEFENSE PLUTONIUN INVENTORY AS OF

APRIL 1, 1968*

MUCLEAR MATERIALS PROOUCTION REPORT MARCH, 1968*

PLUTONIUM RELCRK IN PUREX

ZPPR MELT PREPARATION SHEETS - LOT 1

THROUGH LOT 9

ZPPR MELT PREPARATION SHEETS - LOT 10

THROUGH LOT 17

ZPPR MELT PREPARATION SHEETS - LOT 18

THROUGH LOT -..-

COMPARISON OF PUREX L-10 AND BUTTON

PU240 VALUES.*

PROOUCTION INVENTORY AND MANUFACTURING

STATEMENT MARCH 31, 1968.*

TABLE C - KEY PROOUCTS - ARHCO

LONG-RANGE PLAN.*

MUCLEAR MATERIALS PRODUCTION REPORT

APRIL, 1968*

NONDEFENSE PLUTONIUM INVENTORY AS OF MAY 1. 1968.*

ATLANTIC RICHFIELD HANFORD COMPANY

MISSION 1 - BASIS PRCOUCTION - FY- 1968

PROOUCTION INVENTORY AND MANUFACTURING

STATEMENT APRIL 30, 1968.*

WORKING PAPERS - UNCERTAIKTY

CALCULATIONS PLUTONIUA RECLAMATIOI

IMVENTORY JAMUARY - DECEMBER, 1968.*

$$
\text { AUTHOR }
$$

LH TAYLOR

LH TAYLOR

MK HARMON

HW MURRAY

GC PICKARD

RE TOHLINSON

EL KELLEY, SR.

CD HARRIMGTOW/LM

RICHARDS/DG WILLIAMS

MK HARMON

JG IMRPHY

LL ZAHN

RN THOMPSON

RN THOHPSON

RN THOMPSON

PB FISK

GC PICKARD

VR CHAPMAN

JG MURPHY

MK HARMON

RE TONLINSON

GC PICKARD

CC JOMES
SRD

SRD

SRD

CONF

SRD

SRD

LEVEL PGS

DATE

SRD

$02 / 01 / 68$

$03 / 11 / 68$

03/08/68

$03 / 12 / 68$

$03 / 14 / 68$

$03 / 18 / 68$

$04 / 01 / 68$

$03 / 22 / 68$

$03 / 20 / 68$

$03 / 21 / 68$

$05 / 15 / 68$

$04 / 10 / 68$

$04 / 11 / 68$

$04 / 12 / 68$

$04 / 16 / 68$

$04 / 16 / 68$

$04 / 16 / 68$

$04 / 16 / 68$

$04 / 24 / 68$

$05 / 17 / 68$

$05 / 13 / 68$

$04 / 16 / 68$

$05 / 14 / 68$

$05 / 24 / 68$

$05 / 24 / 68$ 
DOC. 10.

ARH-00614

ARH-00616RD

ARH -00617

ARH-00633

ARH-00639

ARH-00647

ARH-00651RD

ARH-00654

ARH-00656

ARH-00662

ARH-00663

ARH-00664

ARH-00672

ARH -00679

ARH-00691

ARH-00691APR

ARH-00691AUG

ARH-006910EC

ARH-00691FEB

ARH-00691 JAN

ARH-00691 JUL

ARH-00691 JUN

ARH-00691MAR

ARH-00691MAY
TITLE

AUTHOR

CC JONES

WORKING PAPERS - MUCLEAR MATLS. MEASUREMENTS DATA EVALUATIOW-PLUTONIUM FINISHING OPERATION JANUARY-JULY, 1968. WORKING PAPERS

WORKING PAPERS - UMCERTAINTY

CALCULATIONS PLUTONIUN REDUCTION INVENTORY JAMUARY - DECEABER, 1968* MONDEFENSE PLUTONIUM INVENTORY AS OF JUNE 1, 1968*

MUCLEAR MATERIAL PRODUCTION REPORT MAY 1986

CHEMICAL PROCESSIMG DIVISION DAILY

PRCDUCTION REPORTS JULY, 1968 THROUGH SEPTEMBER, 1968*

PROCESS CONTROL DATA

PRODUCTION INVENTORY AND MANUFACTURING STATEMENT MAY 31, 1968.*

200 AREA OPERATION ESSENTIAL MATERIALS INVENTORY O CONSUAPTION REPORTS FYTD (BY MONTH) - 11/67 THRU 6/68 CYTD 1967."

PUREX - MUCLEAR MATERIALS DATA TO

OPERATIONS - FY 1969

MATERIAL BALANCE REPORTS - JULY THROUGH DECEMBER, 1968*

CHEMICAL PROCESSING DIVISION - SHIPPING AND RECEIVING DATA - FY 196\%

SAFECUARD SS MATERIAL PLUTONIUM

PROCESSING SOURCE DATA -FY 1969*

ARHCO LONG-RANGE PLANINING TABLES B-1

THROUGH 8-6*

PLUTONIUM PROCESSING SS MATERIALS

GEMERAL LEDGER FY 1969*

PLUTONIUM PROCESSING SOURCE DATA -

FY-1969, APRIL 1969

PLUTONIUM PROCESSING SOURCE DATA - FY

1969 - AUCUST, 19688

PLUTONIUA PROCESSING SOURCE DATA - FY

1969 - DECEMBER 1968

PLUTONIUN PROCESSING SOURCE DATA - FY

1969 - FEB 1969

PLUTONILH PROCESSING SOURCE DATA - FY

1969 JAMUARY 1969.8

PLUTONIUM PROCESSING SOURCE DATA -

FY-1969 JULY, 1968

PLUTONIUN PROCESSING SOURCE DATA - FY

1969 - JUWE, 1969

PLUTONIUN PROCESSING SOURCE DATA - FY

1969 MARCH, 1969

PLUTONILN PROCESSING SOURCE DATA - FY

1969 - MAY 1969

\begin{tabular}{|c|c|c|c|}
\hline AUTHOR & LEVEL & PGS & DATE \\
\hline CC JONES & SRD & & $05 / 24 / 68$ \\
\hline $\begin{array}{l}\text { PF KISOW } \\
\text { CC JOWES }\end{array}$ & $\begin{array}{l}\text { SRD } \\
\text { SRD }\end{array}$ & & $\begin{array}{l}05 / 24 / 68 \\
05 / 24 / 68\end{array}$ \\
\hline MK HARHOW & SRD & & $06 / 12 / 68$ \\
\hline JG MURPHY & SRD & & $06 / 12 / 68$ \\
\hline HW IURRAY & SRD & & $07 / 01 / 68$ \\
\hline $\begin{array}{l}\text { AJ LOW } \\
\text { CR BLEGEN }\end{array}$ & $\begin{array}{l}\text { SRD } \\
\text { SRD }\end{array}$ & & $\begin{array}{l}06 / 17 / 68 \\
06 / 24 / 68\end{array}$ \\
\hline LM KMAPSTAD & SRD & & $06 / 20 / 68$ \\
\hline JG MURPHY & SRD & & $07 / 01 / 68$ \\
\hline JG MURPHY & SRD & & 07/01/68 \\
\hline JG MURPHY & SRD & & 07/01/68 \\
\hline JG MURPHY & SRD & & $07 / 01 / 68$ \\
\hline MK HARMON & SRD & & $05 / 15 / 68$ \\
\hline JG MURPHY & SRD & & $07 / 01 / 68$ \\
\hline JG MURPHY & SRD & & $04 / 01 / 69$ \\
\hline JG MURPHY & SECR & & $08 / 01 / 68$ \\
\hline JG MURPHY & SECR & & $12 / 01 / 68$ \\
\hline JG MURPHY & SRD & & $02 / 01 / 69$ \\
\hline JG MURPHY & SECR & & $01 / 01 / 69$ \\
\hline JG MURPHY & SRD & & $07 / 01 / 68$ \\
\hline JG MURPHY & SRD & & $06 / 01 / 69$ \\
\hline JG MURPHY & SRD & & 03/01/69 \\
\hline JG MURPHY & SRD & & $05 / 01 / 69$ \\
\hline
\end{tabular}


Page No. 6

$04 / 28 / 93$

LIST OF MANFORD-GENERATED CLASSIFIED

DOCUNENTS DATED BETWEEN 1/1/61 AND 12/31/72

DOC. NO.

TITLE

AUTHOR

LEVEL

PGS

DATE

ARH-00691MOV

ARH-006910CT

ARH-00691SEP

ARH-00692APR

ARH-CO692AUG

ARH-00692DEC

ARH-00692FEB

ARH-00692JAK

ARH-00692JUL

ARH-00692JUN

ARH-00692MAR

ARH-00692MAY

ARH-00692MOV

ARH-006920CT

ARH-00692SEP

ARH $-006 \%$

ARH-00699

ARH -00700

ARH -00702

ARH-00704RD

ARH-00706RD

ARH-00728

ARH-00731

ARH -00743

ARH-00744

ARH-00747
PLUTONIUA PROCESSING SOURCE DATA - FY 1969 - NOVEMBER 1968

PLUTONIUN PROCESSING SOURCE DATA -

FY-1969 - OCTOBER, 1968

PLUTONIUM PROCESSING SOURCE DATA - FY 1969 - SEPTEMBER, 1968

PLUTONIUN RECLAMATION SOURCE DATA FY 1969 - APRIL 1969

PLUTONIUN RECLAMATION SOURCE DATA FY 1969 - NUESST 1968

PLUTONIUN RECLAMATION SOURCE DATA FY 1969 - DECEMBER 1968

PLUTONIUA RECLAMATION SOURCE DATA FY 1969 - FEBRUARY 1969

PLUTONIUM RECLAMATION SOURCE DATA FY 1969 - JAMUARY 1969

PLUTONIUN RECLAMATION SOURCE DATA FY 1969 - JULY 1968

PLUTONILN RECLAMATION SOURCE DATA FY 1969 - JUNE 1969

PLUTONIUN RECLAMATION SOURCE DATA FY 1969 - MARCH 1969

PLUTONIUN RECLAMATION SOURCE DATA FY 1969 - MAY 1969

PLUTOWIUM RECLAMATION SOURCE DATA FY

1969 - NOVEMBER 1968

PLUTCNIUN RECLAMATION SOURCE DATA FY 1969 - OCTOBER 1968

PLUTONIUN RECLAMATION SOURCE DATA FY

1969 - SEPTEMBER 1968

MONDEFENSE PLUTONIUM INVENTORY AS OF

JULY 1, 1968."

MBR - JUW 1968

COEI - JUNE 1968

MUCLEAR MATERIALS PROOUCTION REPORT JUNE, 1968*

ARHCO PROOUCTION GRAPHS - JULY 1, 1968

THROUGH*

SURVEY 332 - FACILITY HVA

ASSUAED PLUTONIUM REQUIREMENTS - CHART

11-A.*

PROOUCTION INVENTORY AND MANUFACTURING STATEMENT - JUNE 30, 1968.*

QUARTERLY PRODUCTION FORECAST JULY 1 , 1968 THRU JUNE 30, 1973*

NONDEFENSE PLUTONIUN INVENTORY AS OF JULY 31, 1968*

ATLANTIC RICHFIELD HANFORD COMPANY HISTORICAL DATA - CPO COST, PRODUCTION AND UNIT COST DATA

\section{JG IURPHY}

JG MURPHY

JG MURPHY

JG MUPPHY

JG MURPHY

JG MURPHY

JG MURPHY

JG MURPHY

JG IURPHY

JG MURPHY

JG MURPHY

JG MURPY

JG MURPHY

JG MURPHY

JG MURPHY

IM MARHOW

ERDA-RL

DOE-RL

JG IURPHY

D MCDONALD

RJ SLOAT

BB FIELD

CR BLEGEN

CD HARRINGTON/LM RICHARDS/OG WILLIAMS MK HARHOW

GC PICKARO
SECR

SRD

SRD

SRD

SECR

SECR

SRD

SECR

SRD

SRD

SRD

SRD

SECR

SRD

SRD

SRD

SECR

SECR

SRD

SECR

SRD

SRD

SRD

SRD

SRD

SRO
$11 / 01 / 68$

$10 / 01 / 68$

$09 / 01 / 68$

$04 / 01 / 69$

$08 / 01 / 68$

$12 / 01 / 68$

02/01/69

$01 / 01 / 69$

$07 / 01 / 68$

06/01/69

03/01/69

05/01/69

$11 / 01 / 68$

10/01/68

$09 / 01 / 68$

$07 / 10 / 68$

$07 / 92 / 68$

$07 / 12 / 68$

$07 / 15 / 68$

$07 / 01 / 68$

$07 / 09 / 68$

$06 / 14 / 68$

$07 / 25 / 68$

08/21/68

08/09/68

08/09/68 
DOC. NO.

ARH-00754

ARH-00756RD

ARH-00760

ARH-00766

ARH-0077

ARH-00789

ARH-00791RD

ARH-00797

ARH -00804

ARH-00809

ARH -00812

ARH-00814

ARH-00817

ARH-00818

ARH-00836

ARH-00841

ARH-00858

ARH -00867

ARH-00872VOL 1

ARH-00873VOL 1

ARH-00876VOL 1

ARH-00893RD

ARH-00B94RD

ARH-00895
TITLE

AT RICHFIELD MANFORD CO - 200 A

OPERATION ESSENTIAL MAT INV AND

CONSUMPTION REPORTS FYTD 1969 (BY MONTH) AND CYTD 1968*

PROOUCTION INVENTORY AHD MAMUFACTURING

STATEAENT JULY 31, 1968.*

CESIUM-137 MUCLEAR MATERIALS CONTENTS OF ALUM CLADDING UASTE AS DETERMINED FROM GRAMS PLUTONIUN PER RECAUATT DAY

AMALYTICAL DATA

PUREX CHARGIMg WORK PAPERS SEPTEMBER 6, 1968 THRU -.-*

NONDEFENSE PLUTONIUN INVENTORY AS OF AUEUST 31, 1968*

MUCLEAR MATERIALS PRODUCTION REPORT NUCUST 1968*

NUCLEAR MATERIALS REVIEW FOR PUREX AND URANILI OXIDE PLANTS, THIRD AMD FOURTH OUARTERS FISCAL YEAR 1966."

MUCLEAR MTEERIALS REVIEH - PLUTONIUM FINISHING SECTION, THIRD AND FOURTH QUARTERS FISCAL YEAR 1968.*

CURRENT AMALYTICAL LOG - FOR PERIOO

1/12/70 THRU 6/6/70

PROOUCTION INVENTORY AND MANUFACTURING STATEMENT AUGUST 31, 1968.*

CHEMICAL PROCESSING DIVISION DAILY PROOUCTION REPORTS - OCTOBER, 1968 THROUGH DECEMBER, 1968*

QUARTERLY PRODUCTION FORECAST.OCTOBER 1, 1968 THRU JUNE 30, 1970.*

NONDEFENSE PLUTONIUN INVENTORY AS OF SEPTEMBER 30, 1968.*

NUCLEAR MATERIALS PROOUCTION REPORT SEPTEMBER 1968*

PRCOUCTION IMVENTORY AND MAMUFACTURING STATEMENT SEPTEMBER 30, 1968*

UNH PRCOUCTION AMD ANALYTICAL DATA* PLUTONIUN PROOUCTION AND ANALYTICAL DATA*

TK-D5 AMALYTICAL DATA SHEETS

PRODUCTION JWVENTORY AMD MANUFACTURING

STATEMENT WORK PAPERS FY-1969.*

PRODUCTION INVENTORY AMD MANUFACTURING

STATEMENT WORK PAPERS FY-1968.*

M.l.S. COST BLDGET REPORT FY-1968.*
AUTHOR

JG MURPHY

JD AMDERSON

LM KNAPSTAD

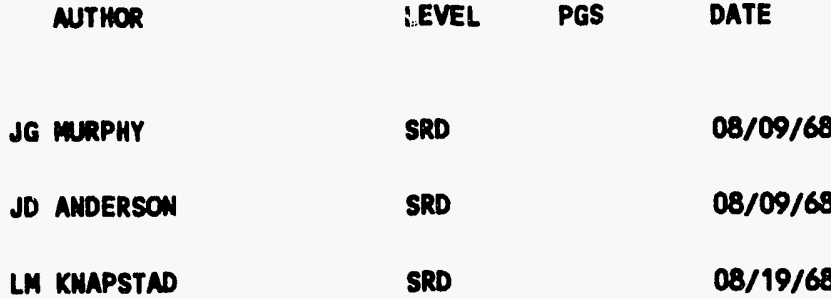

CR BLECEN

SRD

$08 / 23 / 68$

WH ZIMMER

CONF

$08 / 28 / 68$

Ad Low

SRD

$09 / 05 / 68$

$09 / 06 / 68$

MK HARMON

SRO

$09 / 17 / 68$

JG MURPHY

SRD

$09 / 16 / 68$

LH TAYLOR

SRD

$08 / 30 / 68$

RU DOERR

SRD

$08 / 30 / 68$

OV SHISET

CONF

$09 / 19 / 68$

CR BLECEM

SRD

$09 / 25 / 68$

HH MURRAY

SRD

$10 / 01 / 68$

CD HARRINGTON/LM

SRD

$10 / 31 / 68$

RICHADDS/DG WILLIAMS

I.K HABaron

$10 / 10 / 68$

JG MURPHY

$10 / 15 / 68$

RE SAITH

$10 / 22 / 68$

CN MARD

EN MARD

CN MARD

RE SAITH

RE SMITH

SRD

$10 / 21 / 68$

$10 / 24 / 68$

$10 / 25 / 68$

$10 / 25 / 68$

$10 / 25 / 68$

RE SMITH

SRD 
Page No. 8

$04 / 28 / 93$

LIST OF HANFORD-GENERATED CLASSIFIED

DOCUMENTS DATED BETLEEN 1/1/61 AMD 12/31/72

DOC. NO.

ARH-00899RD

ARH-00909RD

ARH-00914RD

ARH-00918

ARH-00934

ARH-00936

ARH-00946RD

ARH-00948RD

ARH-00950

ARH-00963RD

ARH-00965RD

ARH-00966RD

ARH-009OTRD

ARH-00981

ARH-00987RD

ARH-00988RD

ARH-00990RD

ARH-00993

ARH-00994RD

ARH-00995RD

ARH-00995RD BK2

ARH-009\%RD

ARH-01000PT2

ARH-01005RD

ARH-01006RD

ARH-01007RD

ARH-01013RD

ARH-01019

ARH-01023

ARH-010232

ARH-010233
TITLE

PROOUCTION INVENTORY AND MAMUFACTURIMG

STATEMENT WORKING PAPERS SECOND OUARTER

FY-1969 - OCTOBER THROUGH DECEMBER 1968

ROUGH DRAFT - WORKING PAPERS MASTE

status SUMARIES

D5 COMPOSITE DATA

MONDEFENSE PLUTONIUN STATUS REPORT AS OF OCTOBER 31, 1968."

WUCLEAR MAERIALS PROOUCTION REPORT OCTOBER, 1968*

PUREX L-10 IN-LINE SAMPLER TEST.*

PUREX LAB VS $Z$ PLANT LAB PLUTONIUM

WORKING PAPERS OF MUC MAT CONTROL PROG

ARHCO PRCDUCTION INVEMTORY AMD

MANUFACTURING STATEMENT OCTOBER 31, 1968.*

MASS SPECTROMETER DATA ON PUREX PU

MITRATE BATCHES.*

WORKING PAPERS

PLUTONIUN PROOUCTION

PLUTONIUN PRCOUCTION AND ANALYTICAL

DATA.*

NUCLEAR MATERIALS PRODUCTION REPORT -

MOVEMBER 1968*

MONTHLY HOOO 4 MAKE-UP

MONTHLY HOOD 7 DATA

MONTHLY BUTTON TRAMSFER RECORD."

MONDEFENSE PLUTONIUN STATUS REPORT AS OF NOVEMBER 30, 1968*

MONTHLY BUTTON MEUTRON COUWT

MATERIAL TRANSFER REQUESTS FROM PUREX TO PU PROCESSING SUBSECTION.*

MATERIAL TRAMSFER REQUESTS FROM PUREX TO PLUTONIUN PROCESSING SUBSECTION - BOOK 2

DAILY PERFORMANCE REPORT

B PLANT AMALYTICAL RESULTS

MUCLEAR MATERIALS FOR CHEMICAL

SEPARATIONS BUSIMESS (WORKING DRAFT)*

UWIT COST OF RELORKING WAR RESERVE PU METAL*

THE EFFECT OF PU-241 MM-241 REACTION TO VEAPONS QUALITY*

HOOO \& DATA \& MONTHLY TOTALS

PRODUCTION INVENTORY AND MANUFACTURING

STATEMENT - NOVEMBER 30, 1968."

CPD DAILY PRCOUCTION REPORTS JAN, 1969

THROUGK MARCH, 1969*

CPD DAILY PROOUFTION REPORTS APRIL, 1969

THROUGH JUNE, 1969*

CPD DAILY PROOUCTION REPORTS JULY, 1969

THROUGH SEPT, 1969.*
AUTHOR

RE SUITH

DL UEBELACKER

EN MARD

IMK MARanow

JG MURPHY

EL KELLEY

LF JAMIN

FR LOHDEFINCK

RE SAITH

LL THons

RC FORENAN

LL THouAs

LL THows

JG IURPHY

AH HIMKSON

AH HIMKSON

AH HINKSON

MK MARHON

DH UNDERLDOO

LF JANIM

LF JAMIN

AH HIAKSON

ML MADEEN

AE SNITH

TR MCKENZIE

TR MCKENZIE

LL THOAnS

RE SAITH

HU MURRAY

HH MURRAY

HW MURRAY
LEVEL PGS

DATE

$10 / 30 / 68$

$11 / 05 / 68$

$11 / 04 / 68$

$11 / 12 / 68$

$11 / 15 / 68$

$11 / 08 / 68$

$11 / 14 / 68$

10/01/68

$11 / 22 / 68$

$11 / 12 / 68$

$11 / 26 / 68$

10/31/68

10/31/68

$12 / 12 / 68$

$12 / 03 / 68$

$12 / 03 / 68$

$12 / 03 / 68$

$12 / 10 / 68$

$12 / 04 / 68$

$12 / 04 / 68$

$10 / 04 / 71$

$12 / 04 / 68$

$01 / 01 / 71$

$12 / 10 / 68$

$12 / 10 / 68$

$12 / 10 / 68$

$01 / 01 / 68$

$12 / 26 / 68$

$01 / 01 / 69$

$04 / 01 / 69$

07/01/69 
Page No. 9

$04 / 28 / 93$

LIST OF HANFORD-GENERATED CLASSIFIED

DOCIMENTS DATED BETUEEN 1/1/61 AND 12/31/72

DOC. NO.

ARH-010234

ARH-01031

ARH-01039

ARH-01045RD

ARH-01048RD

ARH-01048RD

ARH-01057

ARH-01060RD

ARH-01060RD

ARH-01065

ARH-01075

ARH-01083RD

ARH-01090RD

ARH-0109401

ARH -0109402

ARH -0109403

ARH-0109404

ARH-0109405

ARH-0109406

ARH -0109407

ARH-0109408

ARH -0109409

ARH -0109410

ARH-0109411

ARH-0109412

ARH-0109908

ARH-0109909
TITLE

CPD DAILY PRCDUCTION REPORTS OCT, 1969 THROUGH DEC, 1969.*

QUARTERLY PRCOUCTION FORECAST - 1/1/69

THROUEH 6/30/74

NUCLEAR MATERIALS PROOUCTION REPORT

DECEMBER, 1968."

MEUTRON CONTER MONOGRAPHS

2 PLANT REPORT TASK I\&II, RECOVERY OPERATIONS 1969

2 PLANT REPORT TASK IRII, RECOVERY OPERATIONS 1969

NONDEFENSE PLUTONIUN STATUS REPORT AS OF DEC 31, 1968.*

BUTTON LIME DAILY REPORT JAMUARY 5, 1967 THRU OEC 3, 1968."

BUTTON LIME DAILY REPORT JANUARY 5, 1967 THRU DEC 3, 1968.*

ARHCO, CPD, PRCDUCTION FORECAST, JAN 1, 1969 THROUEH JUNE 30, 1974."

PROOUCTION INVENTORY ANO MAMUFACTURING

STATEMENT DEC 31, 1968.*

PUREX MONTH ENO WORK SHEETS, BEGIMNING

AUG 1966*

HANFORD PRCDUCTION CUT-BACK STUDIES."

MONDEFENSE PLUTONIUN STATUS REPORT AS OF

JAN 31, 1969.*

NONDEFENSE PLUTONIUN STATUS REPORT AS OF FEB 28, 1969.*

FUEL GRADE PLUTONILN STATUS REPORT AS OF

MARCH 31, 1969.*

FUEL-GRADE PLUTONIUM STATUS REPORT AS OF APRIL 30, 1969.*

FUEL-GRADE PLUTONIUN STATUS REPORT AS OF MYY 31, 1969*

FUEL-CRADE PLUTONIUN STATUS REPORT AS OF JUNE 30, 1969.*

FUEL-GRADE PLUTONIUM STATUS REPORT AS OF JULY 31, 1969.*

FUEL-GRADE PLUTONIUN STATUS REPORT AS OF NUEUST 31, 1969.*

FUEL-CRADE PLUTONIUN STATUS REPORT AS OF SEPTEMBER 30, 1969*

FUEL-GRADE PLUTONIUN STATUS REPORT AS OF OCTOBER 31, 1969.*

FUEL-GRADE PLUTONIUN STATUS REPORT AS OF NOVEABER 30, 1969.*

FUEL-GRADE PLUTONIUN STATUS REPORT AS OF DECEMBER 31, 1969.*

MBR - NUG 1969

MBR - SEP 1969
NUTHOR

CD MARRINGTCW/LM

RICMARDS/DG HILLIAMS

JG MRPAY

RN THOWPSON

LM KHIGHTS

LM KNIGHTS

MK HAREON

WN KIMNEY

W KIMNEY

D mCDOMALD

RE SAITH

EL KELLEY

VR CHAPMAN

MK HARMOY

MK MARMON

MK MARnow

MK MARMOM

m. HARMO

MK MARArOM

MK HARArOY

IXK HAREON

MK HARMON

MK MARMON

AK HARMON

MK HARMON

ERDA-RL

ERDA-RL
LEVEL PGS DATE

SRD

$10 / 01 / 69$

$0: / 17 / 69$

$01 / 15 / 69$

$01 / 08 / 69$

01/02/69

$01 / 02 / 69$

$01 / 15 / 69$

$01 / 16 / 69$

$01 / 16 / 69$

$01 / 21 / 69$

$01 / 24 / 39$

01/29/69

02/03/69

02/10/69

$03 / 14 / 69$

$04 / 17 / 69$

$05 / 15 / 69$

$06 / 11 / 69$

$06 / 15 / 69$

$08 / 14 / 69$

$09 / 15 / 69$

$10 / 09 / 69$

$11 / 07 / 69$

$12 / 11 / 69$

$01 / 09 / 70$

$09 / 10 / 69$

$10 / 14 / 69$ 
DOC. NO.

ARH-0109910

ARH-0109911

ARH-0109912

ARH-0111212

ARH-01113

ARH-0111310

ARH-0111311

ARH-0191312

ARH -011132

ARH-011133

ARH-011134

ARH -011135

ARH-099,36

ARH-011137

ARH-011138

ARH-011139

ARH-01114

ARH 0111410

ARH-0111411

ARH-0111412

ARH-011142

ARH-011143

ARH-011144

ARH-011145

ARH-011146

ARH-011147

ARH-011148
TITLE

HVA MBR - OCT 1969

HVA MBR - NOV 1969

HVA MBR - DEC 1969

COEI - DEC 1969

material Balance AND MET PRCOUCTION REPORT-JAN, 196\%*

material balamce and net PRCOUCTION REPORT, OCT, 1969.*

MATERIAL. BALANCE AND MET PRCOUCTION REPORT - NOV 1969*

MATERIAL BALANCE AND MET PRODUCTION REPORT, DEC, 1969.*

MATERIAL BALANCE AMD MET PROOUCTION REPORT - FEB, 1969.*

MATERIAL BALANCE AMD MET PROOUCTION REPORT - MARCH 1969*

MATERIAL BALANCE AND MET PROOUCTION REPORT APRIL, 1969.*

material BALAMCE AMD MET PRCOUCTION REPORT, MAY, 1969.*

MATERIAL BALANCE AMD MET PROOUCTION REPORT JUNE, 1969.*

matriRial balance and MET PROOUCTION REPIRT, JULY, 1969.*

material balance ano met production REPORT - AUG 196\%

MATERIAL BALANCE AND MET PROOUCTION REPORT, SEPT, 1969.*

MUCLEAR MATERIALS PROOUCTION REPORT JAN, 1969*

MUCLEAR MATERIALS PROOUCTION REPORT -

OCT 1969*

NUCLEAR MATERIALS PRODUCTION REPORT -

NOV 1969*

NUCLEAR MATERIALS PRODUCTION REPORT DEC, 9969.*

NUCLEAR MATERIALS PROOUCTION REPORT FEB, 1969.*

NUCLEAR MATERIALS PRODUCTION REPORT

MARCH 1969*

MUCLEAR MATERIALS PRODUCTION REPORT

APRIL, 1969.*

MUCLEAR MATERIALS PROOUCTION REPORT MAY, 1969.*

MUCLEAR MATERIALLS PROOUCTION REPORT JUNE, 1969.*

MLCLEAR MATERIALS PRODUCTION REPORT JULY, 1969."

MUCLEAR MATERIALS PRCOUCTION REPORT AUG 1969*
AUTHOR

LEVEL

PGS

DATE

ERDA-RL

SECR

$11 / 10 / 69$

ERDA-RL

SECR

SECR

ARHCO

JG MURPHY

SECR

SECR

$12 / 09 / 69$

$01 / 12 / 70$

$01 / 12 / 70$

$02 / 12 / 69$

JG MURPHY

SECR

$11 / 11 / 69$

JG MURPHY

SECR

$12 / 11 / 69$

JG IMUPHYY

SECR

$01 / 13 / 70$

JG MURPHY

SECR

$03 / 13 / 69$

JG MURPHY

SECR

$04 / 14 / 69$

JG MURPHY

SECR

$05 / 15 / 69$

JG IURPHY

SECR

$06 / 16 / 69$

JG MURPHY

SECR

$07 / 11 / 69$

JG MURPHY

SECR

$08 / 11 / 69$

JG MURPHY

SECR

$09 / 12 / 69$

JG MURPHY

SECR

$10 / 13 / 69$

JG MURPHY

SECR

$02 / 14 / 69$

JG MURPHY

SECR

$11 / 11 / 69$

JG IMRPHY

SECR

12/11/69

JG MURPHY

SECR

$01 / 12 / 70$

JG MURPHY

SECR

03/13/69

JG MURPHY

SECR

$04 / 11 / 69$

JG MURPHY

SECR

$05 / 12 / 69$

JG MURPHY

SECR

$06 / 13 / 69$

JG MURPHY

SECR

$07 / 10 / 69$

JG MURPHY

SECR

$08 / 11 / 69$

JG MURPHY

SECR 
Page No. 11

$04 / 28 / 93$

LIST OF MANFORD-GENERATED CLASSIFIED

DOCUMENTS DATED BETUEEN 1/1/61 ANO 12/31/72

DOC. NO.

ARH-011149

ARH-0111510

ARH-0111511

ARH-0111512

ARH-01115:3

ARH-01115i

$A R H-01115$

ARH-011156

ARH-011157

$A R H-01115 B$

ARH-011159

ARH-01127

ARH-011441

ARH-011442

ARH-011443

ARH-011444

ARH -011445

ARH-011446

ARH -019447

ARH-01156RD

ARH-01160

ARH-01164RD

ARH-01167

ARH-01168

ARH-01179

ARH-01181
TITLE

MUCLEAR MUTERIALS PRCOUCTION REPORT SEPT, 1969:

RESEARCH AND DEVELOPMENT MUCLEAR

MATERIALS PROJECT EEËURT - OCT 1969

RESEARCH AND DEVELOPMENT MUCLEAR

MATERIALS PROJECT REPORT - MOV 1969

RESEARCH AND DEVELOPHENT MUCLEAR

MMTERIALS YROJECT REPORT - DEC 1989

RESEARCK AMD DEVELOPMENT MUCLEAR

MATERIALS PROJECT REPORT - MAR 1969

RESEARCH \& DEVELOPNENT MUCLEAR MATERIALS

PROJECT REPORT - APRIL 1969

RESEARCH \& DEVELOPMENT MUCLEAR MATERIALS

PROJECT REPORT, MAY 1969

RESEARCH \& DEVELOPNENT MUCLEAR MATERIALS

PROJECT REPORT, JUWE 1969

RESEARCH \& DEVELOPMENT MUCLEAR MATERIALS

PROJECT REPORT, JULY 1969

RESEARCH \& DEVELOPMENT MUCLEAR MATERIALS

PROJECT REPORT, AUCUST 1969

RESEARCH \& DEVELOPHENT MUCLEAR MATERIALS

PROJECT REPORT, SEPPTEMBER 1969

2PAL SAMPLE LOG

PRODUCTION INVENTORY AND MANUFACTURING

STATEMENT JAN 31, 1969.*

PRCOUCTION INVENTORY AND MANUFACTURING

STATEMENT FEB 28, 1969.*

PRODUCTION INVENTORY AND MANUFACTURIMG

STATEMENT MARCH 31, 1969.*

PRODUCTION INVENTORY AND MANUFACTURING

STATEMENT APRIL 30, 1969.*

PRODUCTION IAVENTORY AND MANUFACTURING

STATEMENT MAY 31, 1969.*

PROOUCTION INVENTORY ANO MANUFACTURING

STATEMEUT JUME 30, 1969.*

PROOUCTION INVENTORY AND MANUFACTURING

STATEMENT JULY 31, 1969."

ISOTOPIC COMPOSITION OF PLUTONIUN FEED."

LAB MEASUREMENT TEST PROGRAM - PHASE I

AND IA PUREX LIO SAMPLES.*

26- PU-240 PRCD, FEB 27, 1969

MUCLEAR MATERIALS REVIEH FOR PUREX AMO

URANIUM OXIDE PLAKTS, FIRST O SECOND

CUARTERS FISCAL YEAR 1969.*

MUCLEAR MATERIALS REVIEW - PLUTONIUM

FINISHIMG SECTION - FIRST 0 SECOND

QUARTERS FY 196\%

FIVE YEAR PROOUCTION STUDY FORECAST

4/1/69 THROUGH 6/30/74

WORKIHG PAPERS - MUCLEAR MATERIALS

MEASUREMENTS DATA EVALUATION SYSTEM

PLUTONIUA FINISHẼNG OPERATION AULY-DEC, 1968.

\begin{abstract}
AUTHOR
\end{abstract}
JG MURPHY

JG MURPHY

JG MUPPHY

JG MURPHY

JG MURPHY

JG MURPHY

JG MURPY

JG MURPHY

JG MRPHY

JG MURPHY

JG MURPHY

UR CORITY

RE SWITH

RE SMITH

RE SMITH

RE SMITH

CR BLECEN

CR BLECEN

CR BLEGEM

MK MARMON

CP SUTtER

LL THOHS

LH TAYLOR

RW DOERR

SECR

ARHCO/OUN-RL/AEC

SECR

CC JONES

SECR
03/27/69

$03 / 28 / 69$

$10 / 10 / 69$

$11 / 11 / 69$

$12 / 11 / 69$

$01 / 12 / 70$

$04 / 08 / 69$

05/08/69

$06 / 13 / 69$

$07 / 91 / 69$

08/07/69

$09 / 10 / 69$

$10 / 10 / 69$

$02 / 10 / 69$

02/25/69

$03 / 24 / 69$

$04 / 21 / 69$

05/21/69

$06 / 23 / 69$

$07 / 23 / 69$

$08 / 22 / 69$

$04 / 22 / 69$

$02 / 26 / 69$

$02 / 27 / 69$

$03 / 20 / 69$

$09 / 13 / 68$ 
DOC. NO.

ARH-01182

ARH-01194

ARH-01195

ARH-011953

ARH-011954

ARH-01205RD

ARH-012288K1

ARH-01248RD

ARH-01249

ARH-01251

ARH-01255

ARH-01283

ARH-01292RD

ARH-01293RD

ARH-01294RD

ARH-01295RD

ARH-01302

ARH-01303

ARH-01307RD

ARH-01310SUP

ARH-01314RD

ARH-01315

ARH-01315APR

ARH-01315AUG

ARH-01315DEC

ARH-01315FEB

ARH-01315JAN

ARH-01315JUL
TITLE

ARHCO ACCUWLATIVE MANUFACTURING COSTS PER UNIT PUREX AND PLUTONIUN FINISHING.* TAMK FARM DATA

ARHCO-CPD - PRODUCTION STUDY FORECAST

APRIL 1, 1969 THROUGH JUHE 30, 1974."

ARHCO-CPD - PRODUCTION FORECAST - JULY

1, 9969 THROUGH JUNE 30, 1974*

ARHCO-CPD - PRCOUCTION FORECAST, 10/01/60 THROUGH 06/30/71

MANUFACTURING DEPARTMENT MO MAMAGEAENT RPT JAN THROUEK DEC, 1969*

METAL LOG - MAY 1, 1969

PUREX OPERATIMG PLANT APRIL, 1969

THROUGH MARCH, 1971*

ARHCO BUDEET FOR FY-1971, REV OF BUDGET

FOR FY 1970 PROD COST SUMMARY.*

ARHCO UNIT COST COAPARISONS QUDGET - FY

1971 AND REV OF BUDEET - FY 1970.*

RESIMPTION OF OS ACTIVITY

AMALYSIS OF PLUTONIUA STORAGE.*

FUEL GRADE PLUTONIUN INVENTORY BALANCE

SUMMARY WORK SHEETS

FUEL GRADE PLUTONIUA STATUS REPORT WORK

SHEETS FY 1969*

NONDEFENSE PLUTONILM INV RPT WORK SHEETS

FY 1968*

FUEL GRADE PLUTONILM INVENTORY -

HONTH-END COMPOSITION

CUARTERLY PRCOUCTION FORECAST 7/1/69

THROUGH 6/30/74

MUCLEAR MRTERIALS MEASUREMENT

STATISTICS. *

ARHCO PRCOUCTION GRAPHS JULY 1, 1969

THRU JULY 1, 1970.*

VISUAL AIDS PRESENTED AT BUDGET REVIEW MEETING.*

FFTF OXIDE PRCOUCTION DATA JUNE, 1969.* PLUTONIUN PROCESSING SS MATERIALS

GENERAL LEDGER FY 1970.*

PLUTONIUM PROCESSING SOURCE DATA - FY

1970-APRIL 1970

PLUTONIUM PROCESSING SOURCE DATA - FY

1970 AUCUST 1969.8

PLUTONIUM PROCESSING SOURCE DATA - FY

1970 DECEMBER, 19698

PLUTONIUN PROCESSING SOURCE DATA - FY

1970 FEBRUARY 1970.8

PLUTONIUN PROCESSING SOURCE DATA - FY

1970 JaNUARY 1970

PLUTONIUN PROCESSING SOURCE DATA - FY

1970 JULY 1969
NUTHOR

EH BARTLETT, JR

JG MURPHY

D medomald

D mCDOMALd

D mCDOMLAD

M MARTY

HO SUYDER

D mcoculald

BH IMRICK

EW BARTLETT

LJ BRECKE

LI BRECKE

ML MDEEN

ML. MDEEEN

ML MADEEN

ML MADEEN

ARHCO/DUN-RL/AEC

CC JONES

D mcoomalo

RW HARVEY

PB FISK

JG MURPHY

JG IMRPAY

JG MURPHY

JG MURPHY

JG MURPHY

JG MURPHY

JG MURPHY
LEVEL PGS

DATE

$03 / 20 / 69$

07/07/69

$04 / 07 / 69$

07/29/69

$11 / 04 / 69$

$01 / 29 / 69$

05/01/69

$04 / 18 / 69$

05/23/69

05/23/69

05/23/69

$06 / 09 / 69$

$06 / 17 / 69$

$06 / 17 / 69$

$06 / 16 / 69$

$06 / 16 / 69$

07/28/69

$09 / 29 / 69$

07/01/69

$07 / 10 / 69$

07/01/69

07/01/69

$04 / 01 / 70$

$08 / 01 / 69$

$12 / 01 / 69$

$02 / 01 / 70$

01/01/70

07/01/69 
DOC. NO.

ARH-01315JUH

ARH-01315MAR

ARH-01315MAY

ARH-01315MON

ARH-013150CT

ARH-01315SEP

ARH-01316APR

ARH-01316AUG

ARH-013160EC

ARH-01316FEB

ARH-01316JAN

ARH-01316JUL

ARH-01316JUN

ARH-01316JUN BK2

ARH-01316JUN BK3

ARH-01316MAR

ARH-01316MAY

ARH-01316NOV

ARH-013160CT

ARH-01316SEP

ARH-01319S

ARH-01322RD

ARH-01327

ARH-01331

ARH-013312
TITLE

PLUTONIUM PROCESSING SUURCE DATA - FY 1970 - JUNE 1970

PLUTONIUA PROCESSIIIG SOURCE DATA - FY

1970 MRCH-1970.8

PLUTONIUN PROCESSIMG SOURCE DATA - FY

1970-MAY 1970.8

PLUTONIUN PROCESSING SOURCE DATA - FY

1970 NOVEMBER 19698

PLUTONIUM PROCESSING SOURCE DATA - FY

\$970 OCTOBER 1969

PLUTONIUA PROCESSIHG SOURCE DATA - FY

1970 SEPTEMBER 1969

PLUTONILN RECLAMATION SOURCE DATA FY 1970 - APRIL 1970

PLUTONIUN RECLAMATION SOURCE DATA FY

1970 - AUEUST 1969

PLUTONIUM RECLAMATION SOURCE DATA FY

1970 - DECEABER 1969

PLUTONIUN RECLAHATION SOURCE DATA FY 1970 - FEBRLARY 1970

PLUTONILM RECLAMTION SOURCE DATA FY

1970 - JAMUARY 1970

PLUTONIUM RECLAMATION SOURCE DATA FY 1970 - JULY 1969

PLUTONIUN RECLAMATION SOURCE DATA FY 1970. JUWE, 1970

PLUTONIUN RECLAMATION SOURCE DATA FY 1970

PLUTONIUM RECLAMATION SOURCE DATA FY

1970, JUME, 1970

PLUTONIUN RECLAMATION SOURCE DATA FY

1970 - MARCH 1970

PLUTONIUM RECLAMATION SOURCE DATA FY 1970 - MAY 1970

PLUTONIUA RECLAMTION SOURCE DATA FY 1970 - NOVEIMBER 1969

PLUTONIUM RECLAMATION SOURCE DATA FY 1970- OCTO BER 1969

PLUTONIUN RECLAMATION SOURCE DATA FY 1970 - SEPTEMBER 1969

SAFEGUARD SS MATERIAL PLUTONIUM

PROCESSING SOURCE DATA - FY 1970.*

PUREX PRODUCTION STATISTICS

SHIPPIMG-RECEIVING REPORT FISCAL YEAR

1970.*

WORKING PAPERS MUCLEAR MATLS.

MEASUREMENTS DATA EVALUATION-PLUTONIUM

FINISHING OPERATION JAMUARY-JUNE, 1969.

WORKING PAPERS - IIUCLEAR MATIS.

MEASUREMENTS DATA EVALUATION-PLUTOWIUA

FINISHING OPERATION JULY-DECEMBER, 1969.
NTHOR

JG MURPHY

JG MURPHY

JG MURPHY

JG MURPIYY

JG MURPHY

JG IMRPHY

JG MURPHY

JG MURPHY

JG MURFHY

JG MURPHY

JG MURPHY

JG MURPHY

JG MURPHY

JG MURPHY

JG MURPHY

Ja MURPHY

JG MURPHY

JG MURPHY

JG MURPAY

JG MURPHY

JG MURPHY

EL KELLEY

JG MURPHY

CC JOAES

CC JONES
LEVEL PGS

DATE

06/01/70

03/01/70

$04 / 01 / 70$

$11 / 01 / 69$

10/01/69

$09 / 01 / 69$

$04 / 01 / 70$

$08 / 01 / 69$

$12 / 01 / 69$

$02 / 01 / 70$

$01 / 01 / 70$

07/01/69

06/01/70

$06 / 01 / 70$

06/01/70

03/01/70

05/01/70

$11 / 01 / 69$

$10 / 01 / 69$

$09 / 01 / 69$

07/01/69

07/15/69

07/22/69

07/25/69

03/25/70 
Page No. 14

$04 / 28 / 93$

LIST OF HANFORD-GENERATED CLASSIFIED

DOCUMENTS DATED BETHEEN 1/1/61 AMD 12/31/72

DOC. NO.

ARH -01342

ARH-01345

ARH -01349

ARH-01350APR

ARH-01350AUG

ARH-1113500EC

ARH-ง 1350 FEB

ARY,-01350JAN

MRH-01350JUN

ARH-01350MAR ARH-01350MMY

ARH-01350NOV

ARH-013500CT

ARH-01350SEP

ARh'01351RD

ARH-01363

ARH-01364

ARH-01370

ARH-01371

ARH-01372

ARH -01378

ARH-01389

ARH-01394

ARH-01400RD
TITLE

ARHCO - 200 AREAS OPERATION ESSENTIAL MATERIALS INVENTORY AND CONSUMPTION REPORT FYTD 1970 AND CYTD 1969* AEC - 101 SHIPPING AND RECEIVING LOGBOOK ARHCO ACCUMLATIVE MANUFACTURING COSTS PER UNIT PUREX AND PLUTOMIUM FINISHING." PRODUCTION INVENTORY AND MANUFACTURING STATEMENT-APRIL 30, 1970 PRODUCTION INVENTORY AND MANUFACTURING STATEMENT AUGUST 31, 1969.* PRODUCTION INVENTORY AND MAMUFACTURING STATEMENT DECEMBER 31, 1969.* PRODUCTION INVENTORY AND MANUFACTURING STATEMENT FEBRUARY 28, 1970.*

PROOUCTION INVENTORY AND MANUFACTURING STATEMENT JANUARY 31, 1970. PROOUCTION INVENTORY AND MANUFACTURING STATEMENT JUNE, 1970.

PRODUCTION INV AND MFG STATERENT." PROOUCTION INV AND MFG STATEMENT MAY, 1970

PRODUCTION INVENTORY AMD MANUFACTURIMG

STATEMENT NOVEAMER 30, 1969.*

PRCDUCTION INVENTORY AND MANUFACTURING

STATEMENT OCTOBER 31, 1969.*

PRODUCTION INVENTORY AND MANUFACTURING STATEMENT - SEPTEMBER 30, 196\%*

ARHCO PRCDUCTION COSTS, TOTAL COSTS, O UNIT COSTS - JULY 1969 THRU JUNE 1970.* MUCLEAR MATERIALS REVIEH FOR PUREX AND URANIUM OXIDE PLANTS - THIRD AND FOURTH QUARTERS FISCAL YEAR 196\%

NUCLEAR MATERIALS REVIEY - PLUTONIUM

FINISHING SECTION THIRD AND FOURTH

QUARTERS FISCAL YEAR 1969."

MEPTUNIUM ANALYTICAL DATA DOE C-1 TAMK UNH TRANSFERSO AUEUST 1 THROUGH AUCUST 31, 1969*

PRODUCTION AND UNIT COST CHARTS.*

ATLANTIC RICHFIELD MANFORD COMPANY PRCOUCTION INVENTORY ACCOUNTING.

NEPTUNIUM AHALYTICAL DATA FOR C-1 TARK UNH TRANSFERSO JUNE 1 THRU JULY 31 , 1969*

QUARTERLY PRODUCTION FORECAST OCTOBER 1. 1969 THROUGH JUNE 30, 1971.*

MUCLEAR \& CHEMICAL SAFETY AMALYSIS PUREX PLANT 1970 THORIUM CAMPAIGN

CHEMICAL PROCESSING CONSIDERATIONS IN

TOLL IRRADIATION OF MEPTUNIUN

PRESENTATION ON SEP 26, 1969.*
AUTHOR

HI SHEPARD

JG MRPHY

EW BARTLETT, JR

CR BLEGEN

CR BLEGEN

CR BLECEN

CR BLECEN

CR BLEGEN

CR BLEGEN

CR BLECEN

CR BLEGEN

CR BLEGEN

CR BLECEN

CR BLEEEN

CR BLEGEN

LH TAYLOR

RU DOERR

JD MCINTOSH

HF PATTERSON

CR BLEGEN

JD MCINTOSH

ARHCO-DUN-RL-AEC

GC OBERG

LE BRUNS
LEVEL

PGS

DATE

SECR

07/31/69

$08 / 01 / 69$

SRD

$08 / 11 / 69$

$05 / 21 / 70$

$09 / 23 / 69$

$01 / 22 / 70$

$03 / 25 / 70$

$02 / 25 / 70$

$07 / 23 / 70$

$04 / 23 / 70$

$06 / 23 / 70$

$12 / 23 / 69$

$11 / 25 / 69$

$10 / 22 / 69$

$08 / 15 / 69$

$09 / 02 / 69$

$08 / 25 / 69$

09/02/69

$10 / 27 / 69$

$07 / 20 / 70$

$08 / 04 / 69$

$10 / 29 / 69$

05/22/70

$10 / 06 / 69$ 
DOC. MO.

ARH-01405RD

ARH-01407RD

ARH-01420

ARH-01421

ARH-01423RD

ARH-01424RD

ARH-01425RD

ARH-01425RD1

ARH-01425RD2

ARH-01425RD3

ARH-01425RD4

ARH-01426

ARH-01429

ARH-01429

ARH-01443RD

ARH-01444RD

ARH-01445

ARH-01447RD

ARH-01448RD

ARH-01452

ARH-01463

ARH-01489RD

ARH-01490

ARH-01491

ARH-01498RD
TITLE

PRODUCTION INVENTORY AMD MANUFACTURING

STATEMENT - WORKING PAPERS - FOURTH

QUARTER FY-1969 - APRIL, MAY, AMD JUME, 1969*

PROOUCTION INVENTORY AND MANUFACTURING

STATEMENT - WORKIMG PAPERS - FISCAL YEAR

1970*

PRODUCTION INVENTORY AND MAMUFACTURIMG

STATEMENT - WORKING PAPERS - FIRST

QUARTER FY-1970 - JULY-SEPT. 1969.

PRODUCTION INVENTORY AND MANUFACTURING

STATEMENT - WORKING PAPERS - SECOND

QUARTER FY-1970 - OCTOBER-DECEMBER,

1969.

PRODUCTION INV AND MANUFACTURING

STATEMENT JAN, FEB, MAR, 1970.*

PRODUCTION INV AND MANUFACTURING

STATEMENT APRIL, MAY, JUNE, 1970.*

MEMT INFO SYS FY-1969

HISTORICAL PLUTONILM MUF TREMD ANALYSIS

PUREX. JAMUARY, 1965 TO JULY, 1969.*

HISTORICAL PU MFF TREND AMALYSIS JAMUARY

1965 TO JULY 1969

PUREX PLUTONIU: MATERIAL UNACCOUNTED

FOR.*

MUF BY CAMPAIGN FOR FY 1969 - FISCAL

YEAR END MUF 1966 THROUGH 1969, PUREX OPERATIONS.

PLUTONIUN UNACCOUNTED FOR (MUF) AT

PUREX.*

PUREX CALCULATIONS

DISSOLVING 210 CO-PRCDUCT

NEPTUNIUN ANALYTICAL DATA FOR C-1 TANKK

UNH TRANSFERSA OCTOBER 1 THROUGH OCTOBER

31, 1969.*

PROGRESS REPORT - 1970 PUREX THORIA

CAMPAICN

STAMDARD BUTTON CHECKS

CPD-MAJOR PROCRAM ISSUE-STORAGE OF

PLUTONILA PRODUCTS AND SCRAP\#

STUDY FOR SOLIDIFICATION OF PUREX UASTES

TEN-YEAR PROOUCTION STUDIES
AUTHOR

JD ANDERSON

EL KELLEY

HQ SHYDER

HO SWYOER

CR BLECEN

CR BLECEN

SRD

$10 / 22 / 69$

CR BLECEN

SECR

$10 / 22 / 69$

LEVEL

PGS

DATE

$10 / 07 / 69$

10/07/69

10/01/69

10/01/69

10/22/69

CR BLECEN

SECR

$10 / 22 / 69$

CR BLECEN

SECR

$10 / 22 / 69$

CR BLEGEN

SECR

$05 / 18 / 70$

CR BLEGEN

SECR

$05 / 18 / 70$

CR BLECEN

SECR

SECR

$10 / 22 / 69$

$10 / 01 / 69$

ROCKMELL HANFORD

SECR

$10 / 01 / 69$

EL KELLEY,SR

SECR

$03 / 14 / 69$

EL KELLEY SR

SECR

$07 / 11 / 69$

RP CORLEH

SECR

$11 / 05 / 69$

RA PEASE

RA PEASE

JD MCINTOSH

SRD

SRD

SECR

$10 / 31 / 69$

$10 / 31 / 69$

$11 / 05 / 69$

RW MCCULLLGG

SECR

$11 / 17 / 69$

RN THOMPSON

CA LYMERS

SRD

12/05/69

$12 / 10 / 69$

AEC-RL

SECR

SECR 
Page No. 16

$04 / 28 / 93$

LIST OF HANFORD-GENERATED CLASSIFIED

DOCUMENTS DATED BETUEEN 1/1/61 AMD 12/31/72

DOC. NO.

TITLE

AUTHOR

LEVE

PGS

DATE

ARH-01515

ARHCO SEMIANMUAL REPORT PROCESS

AEC-RL

SECR

$12 / 31 / 69$

ARH-015261

DEVELOPMENT 5/1/69 THRU 10/31/69

HH MURRAY

SECR

$01 / 02 / 70$

THROUGH MAR, 1970.*

ARH-015262

CPO DAILY PROOUCTION RPT APR 1970

HW MURRAY

SECR

$04 / 01 / 70$

ARH -015263

THROUGH JUNE 1970.*

HW MURRAY

SECR

07/01/70

ARH-015264 SEPT 1970.

CPD DAILY PRODUCTION REPORTS

HW MURRAY

SECR

$10 / 01 / 70$

ARH-01527RD

OCTOBEK -DECEMBER 1970.

PUREX DAILY PERFORMANCE RPT - JAN 1.

OF BENLLIEU

SECR

$01 / 01 / 70$

ARH-0154001

ARH-0154002

ARH-0154003

ARH-0154004

ARH-0154005

ARH-0154006

ARH-0154007

ARH -0154008

ARH-0154009

ARH-0154010

ARH -0154011

ARH-0154012

ARH-0154112

ARH-015416

ARH-015441

ARH-0154410

1970 THROUGH DEC 31, 1970.*

MBR - JAN 19T0

MBR - FEB 1970

MBR - MAR 1970

HVA MBR - APR 1970

HVA MBR - MAY 1970

HVA MBR - JUN 1970

HVA MBR - JUL 1970

HVA MBR - AUG 1970

HVA MBR - SEP 1970

HVA MBR - OCT 1970

HVA MBR - NOV 1970

HVA MBR - DEC 1970

COEI - DEC 1970

COEI FY 1970 - JUME

RESEARCH AHD DEVELOPMENT NUCLEAR

MATERIALS PROJ RPT JAN, 1970."

RESEARCH AMD DEVELOPMENT MUCLEAR

MATERIALS PROJECT REPORT OCTOBER 1970

RESEARCH AND DEVELOPMENT MUCLEAR

MATERIALS PROJECT REPORT MOVEMBER 1970

RESEARCH AND DEVELOPMENT MUCLEAR

MATERIALS PROJECT REPORT - DECEMBER 1970

RESEARCH AND DEVELOPMENT MUCLEAR

MMTERIALS PROJECT RPT FEB, 1970.*

RESEARCH AND DEVELOPNENT MUCLEAR

ARH -015443

MUERIALS PROJECT RPT MAR, 1970.*

RESEARCH AMD DEVELOPMENT MUCLEAR

MATERIALS PROJECT REPORT APRIL, 1970.*

RESEARCH AND DEVELOPNEAT MUCLEAR

MATERIALS PROJECT REPORT: MAY 1970

ARH -015446

RESEARCH AND DEVELOPMENT MUCLEAR

MATERIALS PROJECT REPORT JUNE, 1970.

RESEARCH AND DEVELOPMENT MUCLEAR

MATERIALS PROJECT REPORT JULY, 1970.

ARH-015448

RESEARCH AND DEVELOPMENT NUCLEAR

MATERIALS PRONEECT REPORT AUCUST, 1970.

ARH -015449

RESEARCH AMD DEVELOPNENT MUCLEAR

MATERIALS PROJECT REPORT SEPTEMBER,

ERDA-RL

ERDA-RL

ERDA-RL

ERDA-RL

ERDA-RL

ERDA-RL

ERDA-RL

ERDA-RL

ERDA-RL

ERDA-RL

ERDA-RL

ERDA-RL

DOE-RL

DOE-RL

JG MURPHY

$02 / 11 / 70$

$03 / 91 / 70$

$04 / 10 / 70$

05/12/70

$06 / 10 / 70$

$07 / 13 / 70$

$08 / 12 / 70$

$09 / 10 / 70$

$10 / 12 / 70$

$11 / 11 / 70$

$12 / 10 / 70$

$01 / 11 / 71$

$01 / 11 / 71$

$07 / 13 / 70$

$02 / 11 / 70$

JG MURPHY

$11 / 11 / 70$

JG MURPHY

$12 / 11 / 70$

JG MURPHY

$01 / 11 / 71$

JG MURPHY SRD

$03 / 11 / 70$

JG MURPHY

SRD

$04 / 10 / 70$

JG MURPHY

SRD

$05 / 12 / 70$

JG MURPHY

SRD

$06 / 11 / 70$

JG MURPHY

SRD

$07 / 13 / 70$

JG MURPHY

SRD

08/12/70

JG MURPHY

SRD

$09 / 11 / 70$

JG MURPHY

SRD

$10 / 09 / 70$ 
Page No. 17

$04 / 28 / 93$

LIST OF HANFORD-GENERATED CLASSIFIED

DOCUMENTS DATED BETWEEN 1/1/61 AND 12/31/72

DOC. NO.

ARH-01546

ARH-015462

ARH-015463

ARH-015464

ARH-01553

ARH-01554

ARH-01561

ARH-01563RD

ARH-015671

ARH-0156710

ARH-0156711

ARH-0156712

ARH-015672

ARH-015673

$A R H-015674$

ARH -015675

ARH -015676

ARH-015677

ARH-015678

ARH-015679

ARH-015711

ARH-015712

ARH-015713

ARH-015714

ARH-01572

ARH-01577

ARH-015T7
TITLE

AUTHOR

ARHCO-DUN-AEC-RL

ARHCO-DUN-AEC-RL

ARHCO, DUN, AEC-RL

ARHCO DUN AEC-RLOO

RP CORLEH

JD MCINTOSH

UR FORITY

EL KELLEY, SR

MK HARMON

MK HARMON

MK MARHON

MK HARMON

MK HARMOY

MK HARMON

MK MARMON

MK MARHON

MK HARMON

MK HARMOM

MK MARMON

MK HARMON

D medomald

D mCDOMAld

D MCDOMALD

D mCDOMALO

BH UYRICK

RW DOERR

RW DOERR
LEVEL PGS DATE

SEC

$01 / 27 / 70$

SECR

$04 / 21 / 70$

SECR

$07 / 27 / 70$

SECR

$10 / 26 / 70$

CUSI

$01 / 09 / 70$

01/07/70

$02 / 12 / 70$

$01 / 20 / 70$

$02 / 11 / 70$

$11 / 02 / 70$

$12 / 15 / 70$

01/08/71

$03 / 10 / 70$

04/09/70

$05 / 11 / 70$

$06 / 09 / 70$

07/06/70

$08 / 04 / 70$

$09 / 03 / 70$

$10 / 06 / 70$

$01 / 30 / 70$

$04 / 23 / 70$

07/29/70

$10 / 27 / 70$

$01 / 29 / 70$

$02 / 02 / 70$

MISTORICAL MUF TREND AHALYSIS PU

FINISHIMG.*

HISTORICAL MUF TREND ANALYSIS PU

FINISHING 
Page No. 18

04/28/93

LIST OF HANFORD-GENERATED CLASSIFIED

DOCUMENTS DATED BETWEEN 1/1/61 AMD 12/31/72

DOC. NO.

TITLE

AUTHOR

LEVEL

PGS

DATE

ARH-015808K1

ARH-01580BK2

ARH -015856

ARH-01586

ARH-015862

ARH-015863

ARH-01590RD

ARH-01594RD

ARH-01606RD

ARH-01606RD

ARH-01614RD

ARH-01616RD

ARH-01618RD

ARH-01622

ARH-01624RD

ARH-01629

ARH -01630

ARH-01637

ARH-01642

ARH -01653

ARH-01668

ARH-01672

ARH-01672REV

ARH-01685RD

ARH-01685RD VG

ARH-01710

ARH-01720

ARH-01720APR

ARH-0172OAUG
BUTTON DATA

HO SUYDER

BUTTON DATA

HQ SNYDER

LF JANIN

MEPTUNIUM AMALYTICA DATA UNH TRAMSFERS

- JAN 1970

MEPTUWIUM AMALYTICAL DATA UNH TRANSFERSO FEB 9970.*

MEPTUWIUNA AMALYTICAL DATA FOR C- 9 TAMK

LWH TRAMS APRIL 1 THRU MAY 2, 1970

NOTES ON PU STORACE AND PU SCRAP.

DATA AND CALCULATIONS RELATING TO

CLASSIFIED INFORIMTION*

PLUTONIUN PROCESS ENG SECT PROCESS

CONTROL RPT 1970.*

PLUTONIUM PROCESS ENG SECT PROCESS

CONTROL RPT 1970.*

FUEL-ERADE PU STATUS RPT WORK SHEETS

JULY THROUGH DEC, 1969*

MO PRCDUCTION STATISTICS PUREX OPER.*

SS MATERIALS BALANCE \& ESSENTIAL

MATERIAL CONSUAPTION PUREX OPERATIONS

STUDY MOTES - PLUTONIUN INVENTORY

INFORMATION,*

VAULT STORAGE INVENTORY

MMA REVIEW FOR PUREX AND URANIUN OXIDE

PLANTS FT AND SEC QTS F Y 1970.*

NMA REVIEW - PU FIN SECT FT AND SEC OTS FY 1970.*

IMPURITIES IN PUREX FEED SOLUTIONS (U)

CY 1970 DNR THORIZ - U-233 DATA

STUOY INFO ON HANFORD SUPPORT OF DMA OS WORK.*

PLUTONIUN SUPPLY FOR FFTF

ARHCO BLDGET FOR FY 1972 AND REV OF

BUDGET FOR FY 1971 PRCOUCTION COST

SUMURY.*

ARHCO BUDGET FOR FY 1972 AMD REVISION OF BUDGET FOR FY 1971 PROOUCTION COST

SUMUARY.

WU-GRAPHS PRESENTED DURING DMA-ALO VISIT

APR 28, 1970.*

ORIGINAL W GRAPHS, DMA-ALO VISIT APRIL

28, 1970

ARHCO ANNUAL REPORT - PROCESS

DEVELOPMENT 11/1/69-4/30/70

URANIUA OXIDE OPERATION SS MATERIALS

GENERAL LEDGER-FY 1971

URANIU OXIDE - SOURCE DATA - FY 1971 -

APRIL 1971

URANIUM OXIDE - SOURCE DATA - FY 1971 -

ANEUST 1970
JD MCINTOSH

JD MCInTOSH

JD MCINTOSH

LI BRECKE

AE BARBER

LH KNIGHTS

LM KNIGHTS

ML MADEEN

EL KELLLEY,SR

EL. KELLEY SR

LI BRECKE

HQ SWYOER

LH TAYLOR

RU DOERR

ra pease

ARHCO

RP CORLEH

RP CORLEH

BH WYRICK

BW WYRICK

RW MARVEY

RU HARVEY

ARHCO

JG MURPHY

JG MURPHY

JG MURPHY
CONF

SECR

SECR

SECR

SECR

SECR

SECR

SECR

SECR

SECR

SECR

SECR

SECR

SECR

SECR

SECR

SECR

SECR

SECR

CRD

SECR

SECR

CoNf

CONF

SECR

SECR

SRD

SRD

SRD
01/01/70

01/01/70

07/01/70

02/05/70

03/12/70

$06 / 15 / 70$

$02 / 11 / 70$

$02 / 16 / 70$

01/01/70

01/01/70

$12 / 31 / 69$

$03 / 13 / 70$

$03 / 13 / 70$

03/09/70

03/01/70

$02 / 25 / 70$

$02 / 25 / 70$

$03 / 18 / 70$

$03 / 25 / 70$

$04 / 15 / 70$

$04 / 24 / 70$

$04 / 30 / 70$

$06 / 30 / 70$

05/08/70

05/08/70

$06 / 12 / 70$

$07 / 01 / 70$

$04 / 04 / 71$

08/01/70 
Page No. 19

$04 / 28 / 93$

LIST OF HANFORD-GENERATED CLASSIFIED

DOCLMENTS DATED BETUEEN 1/1/61 AND 12/31/72

DOC. NO.

ARH-017200EC

ARH-01720FEB

ARH-01720JAN

ARH-01720JUL

ARH-01720JUN

ARH-01720N1

ARH-0172ON2

ARH-0172013

ARH-0172014

ARH-0172OMAR

ARH-01720maY

ARH-0172ONOV

ARH-017200CT

ARH-01720SEP

ARH-01721

ARH-01721APR

ARH-01721AUG

ARH-01721DEC

ARH-01721FEB

ARH-01721JAK

ARH-01721JUL

ARH-01721 JUN

ARH-01721M1

ARH-01721M2

ARH-01721MB
TITLE

URANIUM OXIDE - SOURCE DATA - FY 1971 december 1, 1970.

URANIUM OXIDE - SOURCE DATA - FY

1971-FEBRUARY 1971

URANIUM OXIDE - SOURCE DATA - FY

1971-JANUARY, 1971

URAMIUN OXIDE - SOURCE DATA - FY 1971 JULY, 1970

URANIUN OXIDE - SOURCE DATA - FY 1971 JUNE 1971

U OXIDE OPERATION MONTH-END REPORT - FY 1971 - JUL-SEP 1970

U OXIDE CPERATION MONTH-END REPORT - FY

1971 - OCT-DEC 1970

U OXIDE OPERATION MOHTH-END REPORT - FY 1971 - JAN-MAR 1971

U OXIDE OPERATION MONTH-END REPORT - FY 1971 - APR-JUN 1971

URANIUN OXIDE - SOURCE DATA - FY 1971

MARCH 1971

URANIUN OXIDE - SOURCE DATA - FY 1971 -

maY 1971

URANIUM OXIDE - SOURCE DATA - FY-1971 -

MOVEMBER 1, 1970.

URANIUN OXIDE - SOURCE DATA - FY 1971, OCTOBER, 1970.

U OXIDE - SOURCE DATA - FY 1971 -

SEPTEMBER, 1970

PUREX SS MATERIALS GENERAL LEDGER

FY-1971

PUREX - SOURCE DATA - FY 1971 - APRIL

1971

PUREX - SOURCE DATA - FY 1971 - AUG,

19708

FUREX - SOURCE DATA - FY 1971 - DECEMBER

1. 1970

PUREX - SOURCE DATA - FY 1971 FEBRUARY 1

1979

PUREX - SOURCE DATA - FY 1971 JANUARY 1

1971

PUREX - SOURCE DATA - FY 1971 - JULY,

19708

PUREX - SOURCE DATA - FY 1971 - JUAE

1971

PUREX - MONTH-END REPORT - FY 1971 - JUL THRU SEPT 1970

PUREX - MONTH END - FY1971 - JULY

THROUGH SEPTEMBER 1970

PUREX - MONTH-END REPORT - FY 1971 - JAN THRU MAR 1971
NUTHOR

JG MURPHY

JG IURPHY

JG MURPHY

JG MURPHY

JG MURPHY

JG MURPHY

JG MURPHY

JG MURPHY

JG IMRPAY

JG IURPHY

IG IURPHY

JG MURPHY

JG MURPHY

JG MURPHY

JG MURPHY

JG MURPYY

JG MURPHY

JG MURPHY

JG MURPHY

JG IURPHY

JG MURPHY

JG MURPHY

JG MURPHY

JG MURPHY

JG MURPHY
LEVEL.

PGS

DATE

SRD

$12 / 01 / 70$

SRD

02/01/71

SRD

$01 / 01 / 71$

SRD

07/01/70

SRD

06/01/71

SRD

07/01/70

SRD

$10 / 01 / 70$

SRD

01/01/71

SRD

$04 / 01 / 71$

SRD

SRD

SRD

SRO

SRD

SECR

$03 / 01 / 71$

05/01/71

$11 / 01 / 70$

$10 / 01 / 70$

$09 / 01 / 70$

07/01/70

SECR

$04 / 01 / 79$

SECR

08/01/70

SECR

$12 / 01 / 70$

SECR

02/02/71

SECR

$01 / 01 / 79$

SECR

07/01/70

SECR

$06 / 01 / 71$

SECR

07/01/70

SECR

$09 / 16 / 70$

SECR

$01 / 01 / 71$ 
Page No. 20

$04 / 28 / 93$

LIST OF HANFORD-GENERATED CLASSIFIED

DOCUMENTS DATED BETHEEN 1/1/61 AND 12/31/72

DOC. NO.

ARH-01721N4

ARH-01T21MAR

ARH-01721MAY

ARH-01721MOV

ARH-017210CT

ARH-01721SEP

ARH-01722

ARH-01722APR

ARH-01722AUG

ARH-01722DEC

ARH-01722FEB

ARH-01722JAN

ARH-01722JUL

ARH-01722JUN

ARH-01722MAR

ARH-01722MAY

ARH-01722NOV

ARH-017220CT

ARH-01722SEP

ARH-01723

ARH-01723APR

ARH-01723AUG

ARH-017230EC

ARH-01723FEB

ARH-01723JAN

ARH-01723JUL.
TITLE

PUREX - MONTH-END REPOR' - FY 1971 - APR THRU JUN 1971

PUREX - SOURCE DATA - FY 1971 MARCH 1 1971

PUREX - SOURCE DATA - FY 1971 - MAY 1971

PUREX - SOURCE DATA - FY 1971 - MOVEMBER 1. 1970

PUREX - SOURCE DATA - FY 1971 - OCTOBER

1. 1970.

PUREX - SOURCE DATA - FY 1971 - SEPT

19708

PU PROC SS MATERIALS GEMERAL LEDGER - FY 1979*

PLUTONIUN PROCESSING SOURCE DATA - FY

1971 APRIL 1979

PU PROC - SOURCE DATA - FY 1971 - AUEUST 1970

PLUTONIUN PROCESSING SOURCE DATA - FY

1971 - DECEMBER, 1970.

PLUTONIUN PROCESSING SOURCE DATA - FY

1971 FEBRUARY 1971

PLUTONIUN PROCESSING SOURCE DATA - FY

1971 - JAMUARY 1971

PLUTONIUM PROCESSING - SOURCE DATA - FY

1971 - JULY 1970

PLUTONIUM PROCESSING SOURCE DATA - FY

1971 - JUNE 1971

PLUTONIUN PROCESSING SOURCE DATA - FY

1971 MARCH 1971

PLUTONIUM PROCESSING SOURCE DATA - FY

1971 - MAY 1971

PLUTONIUN PROCESSING SOURCE DATA - FY

1971 - NOVEMBER, 1970.

PLUTONILY PROCESSING SOURCE DATA - FY

1971 - OCTOBER, 1970.

PU PROC - SOURCE DATA - FY 1979 -

SEPTEMBER 1970

PU RECLAMATION SS MATERIALS GENERAL

LEDGER - FY 1971*

PLUTTONIUN RECLAMATION SOURCE DATA FY

1971 APRIL 1971

PU RECLAMATION - SOURCE DATA - FY-1971 NUG 1970

PLUTONIUN RECLAMATION SOURCE DATA FY 1971, DECEMBER, 1970.

PLUTONIUN RECLAMATION SOURCE DATA FY 1971 FEBRUARY 1971

PLUTONIUN RECLAMATION SOURCE DATA FY

1971 JAMUARY 1971

PLUTONILA RECLAMATION SOURCE DATA FY

1971 JULY 1971

$$
\text { AUTHOR }
$$

LEVEL.

PGS

DATE

JG IURPHY

SECR

04/01/79

JG IMRPHY

SECR

$03 / 01 / 71$

JG MURPHY

SECR

SECR

05/01/71

$11 / 01 / 70$

JG MURPHY

SECR

$10 / 01 / 70$

JG MURPHY

SECR

$09 / 01 / 70$

JG MURPHY

SECR

07/01/70

JG MURPHY

SRD

JG MURPHY

SRD

SECR

SECR

SRD

SRD

SRD

SRD

SECR

SRD

SRD

SRD

SECR

SRD

SRD

SECR

SECR

SRD

JG MURPHY

SECR
$01 / 01 / 71$

$04 / 01 / 79$

08/01/70

$12 / 01 / 70$

02/01/71

$01 / 01 / 71$

07/01/70

$06 / 01 / 71$

03/01/79

05/01/71

$11 / 01 / 70$

$10 / 01 / 70$

$09 / 01 / 70$

07/01/70

$04 / 01 / 79$

08/01/70

$12 / 01 / 70$

$02 / 01 / 71$

07/01/70 
DoC. NO.

ARH-01723JUW

ARH-01723MAR

ARH-01723mAY

ARH-01723MON

ARH-017230CT

ARH-01723SEP

ARH-01745

ARH-01745SUP

ARH-01754RD

ARH-01755RD

ARH-01758RD

ARH-01760

ARH-01784RD

ARH-01786

ARH-01789RD

ARH-01793

ARH-01795

ARH-01796PT2

ARH-01797RD

ARH-01798RD

ARH-01817

ARH-01823

ARH-01824

ARH-01832RD
TITLE

AUTHOR

PLUTONIUM RECLAMATION SOURCE DATA FY 1971 JUNE, 1971

PLUTONIUN RECLAMATION SORCE DATA FY 1971 MARCH 1971

PLUTONIUN RECLAMTION SOURCE DATA FY 1971 MY 1979

PLUTONIUM RECLAMATION SOURCE DATA FY 1971, NOVEMBER, 1970.

PLUTONIUN RECLAMATION SOURCE DATA FY 1971 OCTOBER 1970

PLUTONIUN RECLAMATION SOURCE DATA FY 1971 SEPTEMBER 1970

WU-GRAPHS PRESENTED AT MEADQUARTERS

PROOUCTION DIVISION FY 1979-72 BUDGET REVIEH

W-GRAPHS PRESENTED AT MEADQUARTERS PROOUCTION DIVISION FY 1971-7R BUDET REVIEH

PUREX PROOUCTION STATISTICS

MUCLEAR MATERIAL TRAMSFERS

ARHCO PRCOUCTION GRAPHS JULY 1, 1970

THRU JULY 1, 1971.

ARHCO UNIT COST COMPARISONS BUDGET FOR FY 1972 AND REVISION OF BUDGET FOR FY

1971.

DATA FROA PROCESS TEST BL-70-2 EVALUATION OF THE DOUBLE CONTAIMER STORAGE SYSTEM FOR PLUTONIUA METAL. COAPUTER OUTPUT THORIA CHARGING SCHEDULE 1970.

ARCHO FINANCIAL STATENENT WORK PAPS FOR FY-1970

SHIPPIMG AND RECEIVING REPORT FY-1971. PUREX - MUCLEAR MATERIALS DATA TO OPERATIONS FY 1971.

CHEMICAL FLOUSHEET FOR THE PUREX PLANT SECOND (2B COLUNA) AND THIRD PLUTONIUM SOLVENT EXTRACTION CYCLES.

PU REDUCTION-HOOO IV PROCESS DATA-FY

1971.

PU REDUCTION-TASK III PROCESS

DATA-FY-1971.

WORKIMG PAPERS - PUREX SYSTEM.

MUCLEAR MATERIALS REVIEU PUREX AND URANIUN OXIDE PLANTS THIRD AND FOURTH CUARTERS FISCAL YEAR 1970.

NUCLEAR MATERIALS REVIEU - PLUTONILM FINISHING SECTION THIRD AND FOURTH QUARTERS FISCAL YEAR 1970. PROCESS CONTROL INFORMATION FISCAL YEAR 1971.

\begin{tabular}{|c|c|c|}
\hline JG MURPHY & SRD & $06 / 01 / 71$ \\
\hline JG MURPHY & SRD & 03/01/71 \\
\hline JG MURPHY & SECR & $05 / 01 / 71$ \\
\hline JG MURPHY & SRD & $11 / 01 / 70$ \\
\hline JG MURPHY & SRD & $10 / 01 / 70$ \\
\hline JG IURPHY & SRD & $09 / 01 / 70$ \\
\hline RH MARVEY & SRD & $06 / 17 / 70$ \\
\hline RU MARVEY & SECR & $06 / 29 / 70$ \\
\hline EL KELLEY & SECR & $06 / 25 / 70$ \\
\hline EL KELLEY & SECR & $06 / 25 / 70$ \\
\hline D MCOONALD & SECR & $07 / 01 / 70$ \\
\hline EW BARTLETT & SECR & $07 / 01 / 70$ \\
\hline LL THOHS & SECR & $07 / 14 / 70$ \\
\hline RA MATROUS & SRD & $07 / 24 / 70$ \\
\hline CR BLECEN & SRD & $07 / 29 / 70$ \\
\hline JG MURPHY & SECR & $07 / 01 / 70$ \\
\hline JG MRPAY & SECR & $08 / 04 / 70$ \\
\hline RL MALSER & SECR & $08 / 12 / 70$ \\
\hline RN THOMPSON & SRD & $08 / 06 / 70$ \\
\hline RN THOMPSON & SRD & $08 / 06 / 70$ \\
\hline RD SHIMER & SECR & $08 / 26 / 70$ \\
\hline W MALL & SECR & $08 / 31 / 70$ \\
\hline RW DOERR & SECR & $08 / 18 / 70$ \\
\hline AJ LOW & CONF & $09 / 14 / 70$ \\
\hline
\end{tabular}


Page No. 22

$04 / 28 / 93$

LIST OF MAMFORD-GENERATED CLASSIFIED

DOCUNENTS DATED BETWEEN 1/1/61 AND 12/31/72

Doc. NO.

TITLE

NUTHOR

LEVEL

PGS

DATE

ARH-01841APR

ARH-01841AUG

ARH-018410EC

ARH-01841FEB

ARH-01841JAN

ARH-01841 JUW

ARH-01841MAR

ARH-01841MYY

ARH-01841MOV

ARH-018410CT

ARH-01841SEP

ARH-01855

ARH-01864RD

ARH-01869RD

ARH-01869RD1

ARH-01869RD2

ARH-01869RD3

ARH-01869RD4

ARH-0187612

ARH-01882

ARH-018951

ARH-018952

ARH-018953

ARH-018954

ARH-01896RD
PROOUCTION INVENTORY AND MANUFACTURING STATEMENT - APRIL 1971

PROOUCTION INVENTORY AND MANUFACTURING STATEMENT AUCUST, 1970.

PROOUCTION INVENTORY AND MANUFACTURING STATEMENT DECEMBER 1970

PRODUCTION INVENTORY AND MAMUFACTURING

STATEMENT - FEBP' IAPY 1971

PROOUCTION INVEMIORY AND MANUFACTURING STATEMENT JAMUARY 1971

PROOUCTION INVENTORY ANO MANUFACTURING

STATEMENT - JUNE 1971

PRCOUCTION INVENTORY AND MANUFACTURING

STATEMENT - MARCH 1971

PROOUCTION INVENTORY AND MAMUFACTURING

STATEMENT - MAY 1971

PRCOUCTION INVENTORY AND MANUFACTURING

STATEMENT MOVEMBER, 1970

PROOUCTION INVENTORY AND MANUFACTURIMG

STATEMENT OCTOSER 1970

PROOUCTIOA INVENTORY AND MANUFACTURING

STATEMENT SEPTEMBER, 1970.

WEAPONS PLUTONIUN BUTTON DATA.

ARHCO PRCOUCTION COSTS AND UNIT COSTS

JULY 1970 THRU JUWE 1971.

PROOUCTION INVENTORY AND MAMUFACTURIMG

STATEAENT WORKING PAPERS FY 1971.

PROO INVEN + MFGT STAT WORKING PAPERS

FY-1971 10

PROO INVEN + MFGT STAT WORKING PAPERS

FY-1971 20

PROD INVEN + MFGT STAT WORKING PAPERS

FY-1971 30

PROD INVEN + MFGT STAT WORKING PAPERS

FY-1971 40

WA - COEI - DEC 1970

SPECTROAMALYSIS OF RMA-RMC LINE PROOUCTS

CHENICAL PROCESSING DIVISION DAILY

PRCOUCTION REPORTS JANUARY 1971 THROUGH

MARCH 1971

CHEMICAL PROCESSING DIVISION DAILY

PRODUCTION REPORTS - APRIL 1971 THRU

JUNE 1979

CHEMICAL PROCESSING DIVISION DAILY

PROOUCTION REPORTS JULY 1971 THROUGH

SEPTEMBER 1971

CHEMICAL PROCESSING DIVISION DAILY

PRCOUCTION REPORTS - SEPTEMBER 1971 THRU DECEMBER 1971

PRCOUCTION GRAPHS CY 1966 - FY 1971
Cr blecen

CR BLECEN

CR BLECEN

CR BLECEN

CR BLEGEN

CR BLEeEN

CR BLECEN

CR BLEGEN

CR BLECEN

CR BLECEN

CR BLECEN

MH CURTIS

CR BLECEN

CR BLECEM

CR BLECEN

CR BLECEN

CR BLECEN

CR BLECEN

ARHCO

AJ LOW

MU MURRAY

WW MURRAY

HU MURRAY

MH IURRAY

HW MURRAY
SECR

SECR

SECR

$05 / 25 / 71$

SECR

$09 / 30 / 70$

$01 / 25 / 71$

03/25/71

02/22/71

$07 / 23 / 71$

$04 / 22 / 71$

$06 / 23 / 79$

$12 / 23 / 70$

$11 / 23 / 70$

$10 / 26 / 70$

$10 / 16 / 70$

$10 / 26 / 70$

$11 / 05 / 70$

$11 / 05 / 70$

$12 / 16 / 70$

$05 / 21 / 71$

$05 / 21 / 71$

$01 / 11 / 71$

10/01/70

$01 / 01 / 71$

$04 / 01 / 71$

07/01/71

$09 / 01 / 71$

SECR

$12 / 10 / 70$ 
Page No. 23
$04 / 28 / 93$

Doc. No.

ARH-018991

ARH-018993

ARH-018994

ARH-019001

ARH-019003

ARH-019004

ARH-019004RD

ARH-019132

ARH-019133

ARH-019134

ARH-01914

ARK-01922RD

ARH-019251

ARH-0192510

ARH-0192511

ARH-0192512

ARH-019259

ARH-01926RD

ARH-0192801

ARH-0192802

ARH-0192803

ARH-0192804

ARH-0192805

ARH-0192806
TITLE

ARHCO CHEMICAL PROCESSING DIVISION PROOUCT:ON FORECAST - JAMUARY 1, 1971 THRU JUNE 30, 1976

ARH SO CPD PRCOUCTION FORECAST - JULY 1 , 1971 THRU JUNE 30, 1976

ARHCO - CPD - PRCOUCTION FORECAST OCtOser 1, 1971 tMRU JUNE 30, 1976

QUARTERLY PRCOUCTION FORECAST - JAMUARY 1. 1971 THRU JUNE 30, 1976

CUARTERLY PRCOUCTION FORECAST - JULY 1, 1971 - JUNE 30, 1976

OUARTERLY PRCOUCTION FORECAST - OCTOBER

1, 1971 THRU JUNE 30, 1976

OUARTERLY PRCOUCTIOW FORECAST - OCTOBER

1. 1979 THRU JUNE 30, 1976

BURIAL CARDENS RECORDS - WA - FY 1971 MOUTH EWD AND SOURCE DATA OCTOBER THRU DECEMBER 1970

QURIAL GARDENS RECORDS - WA - FY 1971 MONTH END AND SOURCE DATA JAMUARY THRU MARCH 1971

BURIAL GARDENS RECORDS - WA - FY 1971

MONTH END AND SOURCE DATA - JUNE 1971

BURIAL GARDEN INVENTORY FY 1971 -

OCTOBER 1970 THRU JUWE 1979

PUREX PROCESSING IORKIMG PAPERS

ATLANTIC RICHFIELD MANFORD PROOUCTION

SCHEDULES JAMUARY 1971

ATLANTIC RICHFIELD MANFORD PRCOUCTION SCHEDULES OCTOBER 1971

ATLANTIC RICHFIELD HANFORD PRCOUCTION

SCHEDULES - NOVEMBER 1971

ATLANTIC RICHFIELD MANFORO PRODUCTION

SCHEDULES - DECEMBER 1971

ATLANTIC RICHFIELD MANFORD PRCDUCTION

SCHEDULES - SEPTEMBER 1971

PUREX DALLY PERFORMAMCE REPORT - JAMUARY

1, 1971 THRU DECEMBER 31, 1971

AMALYSIS OF MEPTUWIUA BATCH PUREX NO.

1-71

AMALYSIS OF MEPTUNILA BATCH PUREX NO.

2-71

AMALYSIS OF MEPTUNIUA BATCH PUREX NO.

3-71

AMALYSIS OF MEPTUNIUH BATCH PUREX NO.

6-71

AMALYSIS OF MEPTUNIUN BATCH PUREX NO.

8-71

ANALYSIS OF MEPTUNIUN BATCH PUREX NO.

9. 71 AND 10-71
AUTHOR

D MCDOMAL.D

D mooonalo

D mCDOMALd

ARCHO, DUW, AEC-RL

ARCHO DUN AEC-RL

ARCHO DUN AEC-RL

DUN, ARCHO, AEC-RL

JG MURPYY

JG MURPHY

Ja MRPHY

JG MURPHY

GJ RNA

D medomald

- mcoomald

D monounalo

D medomald

D MCDOMALD

OF BENULIEU

AEC-RL

AEC-RL

AEC-RL

AEC-RL

AEC-RL

AEC-RL
SECR

$01 / 01 / 71$

SECR

LEVEL

PGS

DATE

SECR

$01 / 28 / 71$

$06 / 29 / 71$

02/18/72

$01 / 25 / 71$

$06 / 26 / 71$

$10 / 26 / 79$

10/18/71

$10 / 01 / 70$

SECR

04/01/71

$10 / 01 / 70$

$01 / 04 / 71$

$01 / 01 / 71$

10/01/71

$11 / 01 / 71$

12/01/71

$09 / 01 / 71$

$01 / 04 / 71$

$05 / 13 / 71$

08/27/71

09/20/71

10/13/79

$11 / 05 / 71$

$11 / 23 / 71$ 
Page No. 24

$04 / 28 / 93$

LIST OF HANFORD-GENERATED CLASSIFIED

DOCUMENTS DATED BETWEEN 1/1/61 AND 12/31/72

DOC. NO.

TITLE

NTHOR

LEVEL

PGS

DATE

ARH-0192807

ARH-01932

ARH-01933RD

ARH-01943

ARH-0195510

ARH-0195511 REV

ARH-0195512

ARH-019556

ARH-019557

ARH-019558

ARH-019559

ARH-0195601

ARH-0195602

ARH-0195603

ARH-0195604

ARH-0195605

ARH-0195606

ARH -0195607

ARH-0195608

ARH-0195609

ARH-0195610

ARH-0195611 REV

ARH-0195612

ARH-0195613

ARH-0195710

ARH-0195711 REV

ARH-0195712

ARH-0195713

ARH-019576

ARH-019577

ARH-019578

ARH-019579

ARH-019601

ARH-0196010

ARH-0196011

ARH -0196012

ARH-019602

ARH -019603

ARH-019604
ANALYSIS OF MEPTUNIUM BATCH PUREX NO.

10-71 AMD 11-71

PFAL SAMPLE LOG

MAMUFACTURING DEPARTMENT MONTHLY

MANACEMENT REPORTS - JAMUARY THRU

DECEMBER 1971

INTERIM STORAGE OF RADIOACTIVE XENON AND KRYPTON CAS

HVA - COEI - OST 1971

HVA - COEI - NOV 1971

HVA - COEI - DEC 1971

HVA - COEI - JUW 1971

HVA - COEI - JUL 1971

HVA - COEI - NUG 1971

HVA - COEI - SEP 1971

HVA MSR - JAN 1971

HVA MSR - FEB 1971

HVA MSR - MAR 1971

HVA MSR - APR 1971

HVA MSR - MAY 1971

HVA MSR - JUN 1971

HVA MSR - JUL 1971

HVA MSR - AUG 1971

HVA MSR - SEP 1971

HVA MSR - OCT 1971

HVA MSR - NOV 1971

HVA MSR - DEC 1971

HVA MSR - JUL 1971 (SUPPLEMENT)

WA - COEI - OCT 1971

WA - COEI - NOV 1971

WA - COEI - DEC 1971

WA - COEI - JUN 1971 (SUPPLENENT)

WA - COEI - JUN 1971

WA - COEI - JUL 1971

WA - COEI - AUG 1971

WA - COE! - SEP 1971

RESEARCH AND DEVELOPMENT MUCLEAR

MATERIALS PROJECT REPORT - JAMUARY 1971

RESEARCH AMD DEVELOPMENT MUCLEAR

MATERIALS PROJECT REPORT - OCTOBER 1971

RESEARCH AND DEVELOPNENT MUCLEAR

MATERIALS PROJECT REPORT - MOVEMBER 1971

RESEARCH AND DEVELOPMENT MUCLEAR

MATERIALS PROJECT REPORT: DECEMBER 1971

RESEARCH AND DEVELOPMENT MUCLEAR

MATERIALS PROJECT REPORT - FEBRUARY 1971

RESEARCH AND DEVELOPMENT MUCLEAR

MATERIALS PRDJECT REPORT - MARCH 1971

RESEARCH AND DEVELOPMENT MUCLEAR

MATERIALS PROJECT REPORT - APRIL 1971
AEC-RL

SECR

$12 / 20 / 71$

WR GORITY

CRD

$01 / 04 / 71$

IN MARTY

SECR

$01 / 04 / 71$

RP CORLEW

SECR

$01 / 14 / 79$

DOE-RL

DOE-RLL

DOE-RL

DOE-RL

DOE-RL

DOE-RL

DOE-RL

JG MURPHY

JG MURPHY

JG MURPHY

JG MURPHY

JG MURPHY

JG MURPHY

JG MURPHY

JG MURPHY

JG MURPHY

JG MURPHY

JG MURPHY

JE MURPHY

JW JORDAN

DOE-RL

DOE-RL

DOE-RL

DOE-RL

DOE-RL

DOE-RL

DOE-RL

DOE-RL

JG MURPHY

SECR

$11 / 12 / 71$

12/17/79

$01 / 11 / 72$

$07 / 08 / 71$

$08 / 12 / 71$

09/09/71

10/12/71

$02 / 19 / 79$

03/12/71

04/09/71

$05 / 11 / 71$

05/11/71

07/09/71

$08 / 11 / 71$

09/09/71

10/12/71

$11 / 12 / 71$

$12 / 17 / 71$

$01 / 11 / 72$

$08 / 26 / 71$

$11 / 08 / 71$

$12 / 14 / 71$

$01 / 11 / 72$

$11 / 11 / 71$

07/09/71

$08 / 11 / 71$

$09 / 09 / 71$

$10 / 11 / 71$

$02 / 11 / 71$

JG MURPHY

$11 / 10 / 71$

JG MURPHY

$12 / 08 / 71$

JG MURPHY

$01 / 12 / 72$

JG MURPHY

03/05/71

JG MMRPHY

$04 / 09 / 71$

JG MURPHY

$05 / 11 / 71$ 
Page No. 25

$04 / 28 / 93$

LIST OF HAMFORD-GEMERATED CLASSIFIED

DOCLNENTS DATED BETUEEN 1/1/61 AND 12/31/72

DOC. NO.

ARH-0196C5

ARH-019606

ARH-019607

ARH -019608

ARH-019609

ARH- C1965RD

ARH -019741

ARH -0197410

ARH -0197411

ARH-0197412

ARH-019742

ARH-019743

ARH-019744

ARH-019745

ARH-019746

AKH-019747

ARH-019748

ARH-019749

ARH-01975

ARH-01976

ARH-01977

ARH-01989RD

ARH-01989RD

ARH -01996

ARH-02011
TITLE

RESEARCH AND DEVELOPMENT NUCLEAR MATERIALS PROJECT REPORT - MAY 1971 RESEARCH AND DEVELOPMENT NUCLEAR MTERIALS PROJECT REPORT JUNE 1971 RESEARCH AND DEVELOPMENT MUCLEAR MATERIALS PROJECT REPORT JULY 1971 RESEARCH AMD DEVELOPMENT MUCLEAR MMTERIALS PROJECT REPORT - NUGUST 1971 RESEARCH AND DEVELOPHENT MUCLEAR MATERIALS PROJECT REPORT - SEPTEMBER 1971 STUOY NOIES - CY 1971

FUEL-GRADE PLUTONIUM STATUS REPORT AS OF JANUARY 31, 1971

FUEL-GRADE PLUTONIUN STATUS REPORT AS OF OCTUBER 31, 1971

FUEL-GRADE PLUTONIUN STATUS REPORT AS OF NOVEMBER 30, 1979

FUEL-CRADE PLUTONIUA STATUS REPORT AS OF DECENEER 31, 1971

FLEL-GRADE PLUTONIUM STIATUS REPORT AS OF FEBRUARY 28, 1971

FUEL-GRADE PLUTOWILM STATUS REPORT AS OF MARCH 31, 1971

FUEL-GRADE PLUTONIU STATUE REPORT AS OF APRIL 30, 1979

FUEL-GRADE PLUTONIUN STATUS REPORT AS OF MAY 31, 1971

FUEL-GRANE PLUTONIUM STATUS REPORT AS OF JUNE 30, 1971

FUEL-GRADE PLUTOWIUM STATUS REPORT -

JULY 31, 1971

FUEL-GRADE PLUTONIIY STATUS REPORT AUEUST 1979

FUEL-GRADE PLUTONIUN STATUS REPORT AS OF SEPTEMBER 30, 1979

LOA PRESSURE REACTOR DISCMARGE DATA DEFLETED 72 METAL RATIO PU-240 VS U-235

N REACTOR TRAMSFER PROOUCTION DATA -

CASE 1 AND CASE 2 - APRIL

ONE YEAR FORECAST - B PLAYT CONTROLLING

PLUTONIUM PROCESS ENGINEERING SECTION -

PROCESS CONTROL REPORT .

JANUARY-DECEMBER 1971

PLUTONIUM PROCESS EMGINEERING SECTION

PROCESS CONTROL REPORT JANUANY -

DECEMBER 1971

STAMDARD FEED RATES FOR FFTF PLUTONIUM

DIOXIDE PRCDUCTION

BILLIMGS OF FAST FLUR TEST FACILITY OXIDE

\section{AUTHOR}

LEVEL

PGS

DATE

JG IURPHY

SRD

$06 / 11 / 79$

JG MURPHY

SRD

07/09/71

JG MURPHY

SRD

$08 / 11 / 71$

JG MURPHY

SRD

$09 / 09 / 71$

JG MURPHY

SRD

$10 / 11 / 71$

UR CHAPWAN

MK HARMOH

SECR

$01 / 01 / 71$

02/05/71

MK HARHOW

SECR

$11 / 16 / 71$

MK HARMON

SECR

$12 / 16 / 71$

MK HARHON

SECR

$01 / 14 / 72$

MK HARHOW

SECR

03/02/79

MK MARHON

SECR

$04 / 01 / 79$

MK MARETON

SECR

05/05/71

MK HARHON

SECR

$06 / 02 / 71$

MK MARHOW

SECR

07/08/71

MK HARMON

SECR

$08 / 04 / 71$

MK HARMON

SECR

$09 / 03 / 71$

MK HARMON

SECR

$10 / 11 / 71$

ML MADEEN

SECR

$11 / 07 / 68$

D medonald

SECR

$04 / 24 / 70$

RG ZUNHOFF

SECR

SECR

$09 / 17 / 70$

02/17/71

Ad LoN

SECR

$02 / 17 / 71$

LM KNIGHTS

CONF

$02 / 24 / 71$

RE SNITH

SECR

03/08/79 
Page No. 26

$04 / 28 / 93$

LIST OF HANFORD-GEMERATED CLASSIFIED

DOCLMENTS DATED BETUEEN 1/1/61 AND 12/31/72

DOC. NO.

ARH-02011REV2

ARH-02016RD

ARH-02028

ARH-02033

ARH-02039RD

ARH -02042

ARH-02054

ARH-02055RD

ARH-02079

ARH-02081

ARH-02081SUP

ARH-02096

ARH-02097RD

ARH-ก2097RD VOL2

ARH-02099RD

ARH-02105RD

ARH-02107RD

ARH-02114RD

ARH-02128RD

ARH-02132

ARH-02132APR

ARH-02132AUG

ARH-02132DEC

ARH-02132FEB

ARH-02132JAN

ARH-02132JUL
TITLE

BILLINGS OF FAST FLUX TEST FACILITY OXIDE

ARHCO PRODUCTION FORECAST WORKING PAPERS

- jamuarr 1971

MIDYEAR REVIEW OF SELECTED YEAR-END

STATEMENTS

MUCLEAR MATERIALS REVIEH - PLUTONIUM

FINISHING SECTION - FIRST AND SECOND

QUARTERS FISCAL YEAR 1971

MISCELLLANEOUS WORKING PAPERS

RECYCLE PU PROCESSING AT PUREX PROCESS

FLONSHEET, CAPITAL COST AND OPERATING COSTS

QUANTITY AND CUALITY OF PU PRODUCED FROM CURRENTLY PLANHED PUREX OPERATIONS MANPONER AMD OPERATING BASES FOR COSTING PU RECYCLE PROCRNM AT PUREX

PLUTONIUN METAL PURIFICATION AT Z PLANT W-GRAPHS PRESENTED AT HEADQUARTERS PRODUCTION DIVISION - FY 1972-73 BUSGET REVIEW

W-GRAPHS PRESENTED AT HEADQUARTERS PRCDUCTION DIVISION FY 1972-73 BLDGET REVIEW

PLUTONILM BLENDIMG PLANS

PUREX D-5 IN-LINE SAMPLE STATION INVENTORY RESULTS

PUREX D-5 IN-LINE SAMPLE STATION

IMVENTORY RESULTS

PLUTONIUA FINISHING METAL LOG

ION EXCHANGE DATA SHEETS AND SAMPLE SUMUARIES

STUDY MOTES - $Z$ PLANT STAFFING

PLUTONILN SCRAP STANDARD VALUATION WORKSHEETS FOR IUVENTORY JAMUARY 1, 1971

ALLOCATIONS OF FUELS GRADE PLUTONILM

FEED TO THE FFTF AND WEAPONS PROGRAMS

PUREX SS MATERIALS GENERAL LEDGER FY

1972

PUREX - SOURCE DATA - FY 1972 - APRIL

1972

PUREX - SOURCE DATA - FY 1972 AUGUST

1971

PUREX - SOURCE DATA - FY 1972 DECEMBER

1971

PUREX - SOURCE DATA - FY 1972 - FEBRUARY

1972

PUREX - SOURCE DATA - FY 1972 JAMUARY

1972

PUREX - SOURCE DATA - FY 1972 JULY 1971
AUTHOR

ET MCINTYRE

D mCDONALD

BM DOBBS

RU DOERR

GU UPINGTON

RA uAtrous

MK HARMON

RC FORSman

LE BRUNS

EF CURREM

EF CURREM

RP CORLEW

JD AMDERSON

JD AMDERSON

AJ LCW

KH TAMAKa

LI BRECKE

CR BLECEN

PB FISK

JG MURPHY

JG MURPHY

JG MURPHY

JG MURPHY

JG MURPHY

JG MURPHY

JG MURPHY
SECR

SECR

SRD

SECR

LEVEL.

PGS

DATE

SECR

$06 / 30 / 79$

$03 / 105 / 71$

03/18/71

$03 / 24 / 71$

03/31/71

$07 / 28 / 71$

$04 / 14 / 71$

$04 / 14 / 71$

$06 / 04 / 71$

05/04/71

$05 / 04 / 71$

$05 / 14 / 71$

05/21/71

07/09/71

05/12/71

05/18/71

05/20/71

06/02/71

$06 / 21 / 71$

$07 / 01 / 71$

$04 / 01 / 72$

08/01/71

$12 / 01 / 71$

02/01/72

$01 / 01 / 72$

07/01/71 
Page No. 27

$04 / 28 / 93$

LIST OF HAMFORD-GENERATED CLASSIFIED

DOCUMENTS DATED BETHEEN 1/1/61 AND 12/31/72

DOC. NO.

TITLE

WTHoR

LEVEL

PGS

DATE

ARH-02132JUN

PUREX - SOURCE DATA - FY 1972 - JUME

JG MURPHY

SRD

06/01/72

ARH-02132M1

1972

JG MURPHY

SECR

07/01/71

ARH-02132N2

THRU SEPT 1971

PUREX HONTH-END REPORTS - FY 1972 - OCT

JG MURPHY

SECR

$10 / 01 / 71$

ARH-0213243

ARH-02132144

PUREX MONTH-END REPORTS - FY 1972 - JAN

JG MURPHY

SECR

$01 / 01 / 72$

THRU MAR 1972

PUREX MONTH-END REPORTS - FY 1972 - APR

JG MURPHY

SECR

$04 / 01 / 72$

ARH-02132MAR

THRU JUW 1972

PUREX - SOURCE DATA - FY 1972 - MARCH

JG MURPHY

SECR

03/01/72

ARH-02132MAY

ARH-02132NOV

PUREX - SOURCE DATA - FY 1972 - MAY 1972

JG MURPHY

PUREX - SOURCE DATA - FY 1972 NOVEMBER

JG MURPAY

SECR

05/01/72

1971

ARH-021320CT

PUREX - SOURCE DATA - FY 1972 OCTOBER

JG MURPHY

SECR

$11 / 01 / 71$

1971

ARH-02132SEP

PUREX - SOURCE DATA - FY 1972 SEPTEMBER

1971

ARH-02133

URANIUM OXIDE OPERATION SS MATERIALS

GENERAL LEDGER FY 1972

ARH-02133APR

URANIUM OXIDE - SOURCE DATA - FY 1972 APRIL 1972

URANIUM OXIDE - SOURCE DATA - FY 1972

AUCUST 1, 1971

ARH-02133DEC

URANIUM OXIDE - SOURCE DATA - FY 1972

DECEMBER 1, 1971

URANIUN OXIDE - SOURCE DATA - FY 1972 -

FEBRUARY 11972

URANIUY OXIDE - SOURCE DATA - FY 1972 -

Ja mippuY

SECR

$10 / 01 / 71$

SECR

09/01/71

JG MURPHY

SRD

07/01/71

JG MURPHY

SRD

$04 / 01 / 72$

JG MURPHY

SRD

08/01/71

JG MURPHY

SRD

$12 / 01 / 71$

JG MURPHY

SRD

02/01/72

JG MURPHY

SRD

01/01/72

JAMUARY 1, 1972

URANIUN OXIDE - SOURCE DATA - FY 1972

JG MURPHY

SECR

$07 / 01 / 79$

ARH-02133JUN

JULY 1, 1971

URANIUM OXIDE - SCURCE DATA - FY 1972 -

JG MURPHY

SRD

06/01/72

JUNE 1972

URANIUN OXIDE OPERATION MONTH-END JG MURPHY

SRD

07/01/71

REPORTS FY 1972 JUL-SEPT 1971

URANIUA OXIDE OPERATION MONTH-END

SRD

$10 / 01 / 71$

REPORTS FY 1972 OCT-DEC 1971

URANIUN OXIDE OPEARTION MONTH-END

JG MURPHY

$\operatorname{sRD}$

$01 / 01 / 72$

REPORTS FY 1972 JAK-MAR 1972

URANIUM OXIDE OPERATION MONTH-END

JG MURPHY

SRD

$04 / 01 / 72$

REPORTS FY 1972 APR-JUN 1972

ARH-02133MAR

ARH-02133MAY

ARH-02133NOV

URANIUM OXIDE - SCURCE DATA - FY 1972 -

JG MURPHY

SRD

03/01/72

MARCH 1, 1972

JG MURPHY

SRD

05/01/72

MAY 1972

URANIUM OXIDE - SOURCE DATA - FY 1972

JG MURPHY

SRD

$11 / 01 / 71$

MOVEMBER 1, 1971

URANIUN OXIDE - SOURCE DATA - FY 1972

OCTOBER 1. 1971

JG MURPHir

SRD

$10 / 01 / 71$ 
Page No. 28

$04 / 28 / 93$

LIST OF HAMFORD-GEMERATED CLASSIFIED

DOCUMENTS DATED BETUEEN 1/1/61 AND 12/31/72

Doc. Mo.

TITLE

AUTHOR

LEVEL

PGS

DATE

ARH-02133SEP

ARH-02134APR

ARH-02134ANG

ARH-02134DEC

ARH-02134FEB

ARH-02134JAM

ARH-02134JUL

ARH-02134JUW

ARH-02134HAR

ARH-02134MAY

ARH-02134MOV

ARH-021340CT

ARH-02134SEP

ARH-02135APR

ARH-02135AUG

ARH-02135DEC

ARH-02135FEB

ARH-02135JAN

ARH-02135JUL

ARH-02135JUN

ARH-02135MAR

ARH-02135MAY

ARH-02135NOV

ARH-021350CT

ARH-02135SEP
URANIUN OXIOE - SOURCE DATA - FY 1972 SEPTEMBER 1, 1971

PLUTONIUN PROCESSING SOURCE DATA - FY 1972 APRIL 1972

PLUTONIUN PROCESSING SOURCE DATA - FY 1972 AUCUST 1971

PLUTONIUN PROCESSING SOURCE DATA - FY 1972 DECEMBER 1971

PLUTONIUM PROCESSING SOURCE DATA - FY 1972 FEBRUARY 1972

PLUTONIUM PROCESSING SOURCE DATA - FY 1972 JANULARY 1972

PLUTONIUN PROCESSING SOURCE DATA - FY 1972. JULY 1972

PLUTONIUN PROCESSING SOURCE DATA - FY 1972 JUNE 1972

PLUTONIUN PROCESSING SOURCE DATA - FY

1972 MARCH 1972

PLUTONIUM PROCESSING SOURCE DATA - FY 1972 MAY 1972

PLUTONIUN PROCESSING SOURCE DATA - FY

1972 NOVEMBER 1971

PLUTONIUN PROCESSING SOURCE DATA - FY 1972 OCTOBER 1979

PLUTONIUM PROCESSING SOURCE DATA - FY 1972 SEPTEMBER 1971

PLUTONIUM RECLAMATION SOURCE DATA - FY 1972 - APRIL 1972

PLUTONIUA RECLAMATION SOURCE DATA FY 1972 AUGUST 11971

PLUTONIUM RECLAMATION SOURCE DATA - FY

1972 - DECEMBER 1971

PLUTONIUA RECLAMATION SOURCE DATA - FY

1972 - FEBRUARY 1972

PLUTONIUN RECLAMATION SOURCE DATA - FY

1972 - JANUARY 1972

PLUTONIUM RECLAMATION SOURCE DATA FY 1972 JULY 1971

PLUTONIUA RECLAMATION SOURCE DATA - FY

1972 - JUME 1972

PLUTONILA RECLAMATION SOURCE DATA - FY 1972 - MARCH 9972

PLUTONIUM RECLAMATION SOURCE DATA - FY

1972 - MAY 1972

PLUTONIUM RECLAMATION SOURCE DATA

FY-1972

PLUTONIUA RECLAMATION SOURCE DATA - FY

1972 - OCTOBER 1971

PLUTONIUA RECLAMATION SOURCE DATA FY

1972 SEPTEMBER, 1971

\begin{tabular}{|c|c|c|}
\hline JG MURPHY & SECR & $09 / 01 / 71$ \\
\hline JG MURPHY & SECR & $04 / 01 / 72$ \\
\hline JG MURPHY & SECR & 08/01/71 \\
\hline JG MURPHY & SRD & $12 / 01 / 71$ \\
\hline JG MURPHY & SRD & $02 / 01 / 72$ \\
\hline JG MURPHY & SRD & $01 / 01 / 72$ \\
\hline JG MURPHY & SRD & 07/01/71 \\
\hline JG MURPHY & SRD & $06 / 01 / 72$ \\
\hline JG MURPHY & SRD & 03/01/72 \\
\hline JG MURPHY & SRD & 05/01/72 \\
\hline JG MURPHY & SRD & $11 / 01 / 71$ \\
\hline JG MURPHY & SRD & 10/01/71 \\
\hline JG MURPHY & SRD & 09/01/71 \\
\hline JG RURPHY & SECR & $04 / 01 / 72$ \\
\hline JG MURPHY & SECR & 08/01/71 \\
\hline JG MURPHY & SRD & $12 / 01 / 71$ \\
\hline JG MURPHY & SRD & $02 / 01 / 72$ \\
\hline JG MURPY & SRD & $01 / 01 / 72$ \\
\hline JG MURPHY & SRD & 07/01/71 \\
\hline JG MURPHY & SRD & $06 / 01 / 72$ \\
\hline JG MURPHY & SRD & 03/01/72 \\
\hline JG MURPHY & SRD & 05/01/72 \\
\hline JG MURPHY & SRD & $11 / 01 / 71$ \\
\hline JG MURPHY & SRD & $10 / 01 / 71$ \\
\hline JG NURPHY & SRD & 09/01/71 \\
\hline
\end{tabular}


DOC. NO

ARH -02140

ARH-021401

ARH-021402

ARH-021403

ARH-021406

ARH-02148

ARH-02149RD

ARH-02150RD

ARH-02151RD

ARH-02157SUP

ARH-02157SUP-V

ARH-02157SUP-VG

ARH-02160RD

ARH-02172RD

ARH-02192

ARH-02194

ARH-02196APR

ARH-02196AUG

ARH-021960EC

ARH-02196FEB

ARH-02196JAN

ARH-02196JUL

ARH-02196JUN

ARH-02196MAR
TITLE

GEMERAL LEDGER - BURIAL GARDENS RECORDS

- WA - FY 1972

BURIAL GARDENS RECORDS - WA - FY 1972 MONTH END AND SOURCE DATA - JULY THRU SEPTEABER 1971

BURIAL GARDENS RECORDS - WA - FY 1972 MONTH END AAD SOURCE DATA OCTOBER THRU DECEMBER 1971

BURIAL GARDENS RECORDS - WA - FY 1972 MONTH END AND SOURCE DATA - JANUARY THRU MARCH 1972

BURIAL GARDENS RECORDS - WA - FY 1972 MONTH-END AMD SOURCE DATA - APRIL-JUNE 1972

FUEL GRADE PLUTONIUM TRANSACTIOAS

MUCLEAR MATERIAL TRANSFERS FY 1972

PUREX PROOUCTION STATISTICS FY 1972

ARHCO PROOUCTION GRAPHS JULY 1, 1971

THRU JULY 1, 1972

WU-GRAPHS PRESENTED AT RL O2 PROGRAM

BUDGET REVIEH

VELLUMS USED TO MAKE COLOR

TRANSPARENCIES PRESENTED AT RL 02 PROGRAM BUDGET REVIEW

COLOR TRANSPARENCIES PRESENTED AT RL 02 PROGRAM BUDGET REVIEH

CAH HEAD-END BATCH DATA SHEETS RUNS 71-3 THROUGH 71-4

ARHCO WASTE MANAGEMENT MODEL DESCRIPTION

OF THE SEPARATIONS MODULE

IMPACT OF PROPOSED SPECIFICATIONS FOR PLUTONIUM METAL

MUCLEAR MATERIALS REVIEW - PLUTONIUM

FINISHING SECTION - THIRD AMD FOURTH QUARTERS FY 1971

APR 1972 PRODUCTION INVENTORY \&

MANUFACTURING STATMENT

AUG 1971 PRODUCTION \& MANUFACTURING

STATEMENT

DEC 1971 PRODUCTION \& MANUFACTURING

STATEMENT

FEB 1972 PROOUCTION INVENTORY \&

MANUFACTURIMG STATMENT

JAN 1972 PRODUCTION IAVENTORY \&

MANUFACTURING STATMENT

JUL 1971 PRODUCTION \& MANUFACTURING

STATEMENT

PROOUCTION INVENTORY AND MAMUFACTURING

STATEMENT - JUNE 1972

MR 1972 PRODUCTION INVENTORY \&

MANUFACTURING STATMEAT

\begin{tabular}{|c|c|c|c|}
\hline AUTHOR & LEVEL & PGS & DATE \\
\hline JG MURPHY & SECR & & $07 / 01 / 71$ \\
\hline JG MURPHY & SECR & & $07 / 01 / 71$ \\
\hline JG MRPHY & SECR & & $10 / 01 / 71$ \\
\hline JG MURPHY & SECR & & $01 / 01 / 72$ \\
\hline JG MURPYY & SECR & & $04 / 01 / 72$ \\
\hline RP CORLEH & SECR & & $07 / 02 / 71$ \\
\hline EL KELLEY & SRD & & $07 / 01 / 71$ \\
\hline EL KELLEY & SECR & & $07 / 01 / 71$ \\
\hline D MCDONALD & SECR & & $07 / 01 / 71$ \\
\hline RU HARVEY & SECR & & $07 / 08 / 71$ \\
\hline RU HARVEY & SECR & & 07/08/71 \\
\hline AW MARVEY & SECR & & $07 / 08 / 71$ \\
\hline PR MCIURRAY & SECR & & $07 / 09 / 71$ \\
\hline RW SHERMAN & SECR & & $07 / 21 / 71$ \\
\hline RP CORLEH & CONF & & $08 / 26 / 71$ \\
\hline RU DOERR & SECR & & $08 / 30 / 71$ \\
\hline CR BLEGEN & SECR & & $05 / 25 / 72$ \\
\hline CR BLEGEN & SECR & & $09 / 30 / 71$ \\
\hline CR BLECEN & SECR & & $01 / 20 / 72$ \\
\hline CR BLECEN & SECR & & $03 / 23 / 72$ \\
\hline CR BLEGEN & SECR & & $02 / 23 / 72$ \\
\hline CR BLEGEN & SECR & & $08 / 26 / 71$ \\
\hline CR BLEGEN & SECR & & $07 / 31 / 72$ \\
\hline CR BLEGEN & SECR & & $04 / 25 / 72$ \\
\hline
\end{tabular}


Page No. 30

$04 / 28 / 93$

LIST OF HANFORD-GENERATED CLASSIFIED

DOCUMENTS DATED BETWEEN 1/1/61 ANO 12/31/72

DOC. NO.

ARH-02196MAY

ARH-02196NOV

ARH-021960CT

ARH-02196SEP

ARH-02198

ARH-02201

ARH-02202RD

ARH-02208

ARH-02212RD

ARH-02217RD

ARH-02227

ARH-02229

ARH-02230

ARH-02231

ARH-02245

ARH-02249

ARH-02256RD

ARH-02256RD 1

ARH-02256RD2

ARH-02256RD 3

ARH-02256RD4

ARH-02257RD

ARH-02264RD

ARH-02266RD
TITLE

MAY 1972 PROOUCTION INVENTORY \& MANUFACTURING STATMENT NOV 1971 PRCOUCTION \& MANUFACTURING STATEMENT

OCT 1979 PRCOUCTION \& MANUFACTURING STATEMENT

SEP 1971 PROOUCTION \& MANUFACTURIHG STATEMENT

MUCLEAR MATERIALS REVIEH - PUREX AMD URANIUN OXIDE PLANTS - THIRD AND FOURTH CUARTERS FY 1971

RICHLAND MON-WEAPON PLUTONIUN PROOUCTION DATA

INFORMAL PRESENTATION TO DR MCKIM

PLANNIMG INFORMATION FOR FFTF

ARHCO PROOUCTION FORECAST WORKING PAPERS

- JULY 1971

LABORATORY PROJECTION STUDY WORKING

PAPERS

ARHCO ACTIVE AND IN PROCESS IMVENTORIES PLUTONIUN - 02 PROGRAM AS OF JUNE 30, 1971

ARHCO PROJECTION OF MATERIAL USAGE

PLUTONIUN - 02 PROGRAM FOR FY 1972

ARHCO PROJECTION OF MATERIAL USAGE ENRICHED URANIUM - 02 PROGRAM FOR FISCAL YEAR 1972

ARHCO PROJECTION OF MATERIAL USAGE

PLUTONIUM - 02 PROGRAM FOR FY 1973

ARHCO - CPD - PLUTONIUM FINISHING

TWELVE-MONTH OPERATING PLAN - OCTOBER 1, 1971 THRU SEPTEMBER 30, 1972

WU-GRAPHS PRESENTED TO HEADQUARTERS

WASTE MAMAGEMENT AMO TRANSPORTATION DIV

HASTE MANAGEMENT PROGRAM REVIEW

PROO INVEN + MFGT STAT WORKING PAPERS FY-1972

PROD INVEN + MFGT STAT WORKING PAPERS

FY-1972 10

PROD INVEN + MFGT STAT WORKING PAPERS

FY-1972 20

PROO INVEN + MFGT STAT WORKING PAPERS

FY- 197230

PRCO INVEN + MFGT STAT WORKING PAPERS

FY- 197240

CAH HEAD END BATCH DATA SHEETS - RUNS

79-5 THRU -....-

PU RECYCLE STUDY WORKING PAPERS FOR

ARH-2042

D-5 COMPOSITE ANALYTICAL RESULTS
NTHOR

CR BLEGEN

CR BLEGEN

CR BLECEM

CR BLECEN

VN MALL

RP CORLEM

HP SHAY

RJ KOFOED

D mCDONALD

DL UEBELACKER

GB RUKLINSKI

GB RUKLIMSKI

GB RUKLINSKI

GB KUKLINSKI

D mCDONald

WC SCHMIDT

CR BLEGEN

CR BLEgEN

CR BLEgEN

CR BLEGEN

CR BLEgen

PR MCINURRAY

RA WATROUS

ah case
LEVEL

PGS

DATE

$06 / 23 / 72$

$12 / 29 / 71$

$12 / 01 / 71$

$10 / 27 / 71$

$08 / 20 / 71$

$08 / 26 / 71$

$08 / 24 / 71$

$09 / 15 / 71$

$09 / 16 / 71$

$09 / 22 / 71$

$09 / 30 / 71$

$09 / 30 / 71$

$09 / 30 / 71$

09/30/71

$10 / 14 / 71$

$10 / 19 / 71$

$10 / 28 / 71$

$10 / 28 / 79$

$10 / 28 / 71$

$10 / 28 / 71$

$10 / 28 / 79$

$10 / 28 / 71$

$11 / 16 / 71$

$11 / 17 / 71$ 
DOC. NO.

ARH-02294RD

ARH-02316RD

ARH-02321BK1 RD

ARH-023221

ARH-023222

ARH-023223

ARH-023224

ARH-02324RD

ARH-0232501

ARH-0232502

ARH -0232503

ARH -0232504

ARH-0232505

ARH-0232506

ARH -0232507

ARH-02326BK1

ARH-023268K2

ARH-02327BK1

ARH -02329

ARH-02332RD BK1

ARH-02332RD BK2

ARH-023371

ARH-023372

ARH-023373

ARH-023374

ARH-023375

ARH-02348RD

ARH-02356

ARH-023581

ARH-023582

ARH -023583
TITLE

AUTHOR

D medonald

ARHCO PROOUCT
OCTOBER 1971

PUREX DAILY PERFORMANCE REPORT - JAMUARY

OF BENULIEU

1 THRU DECEMBER 1972

B PLANT ACCOUWTABILITY WORKING PAPERS

CPD - DAILY PRODUCTION REPORTS - JAMLARY

THRU MARCH 1972

CPD - DAILY PRCOUCTION REPORTS - APRIL

THRU JUME 1972

CPO DAILY PROOUCTION REPORTS JULY 1972

THRU SEPT 1972

CPD DAILY PRCOUCTION REPORTS - OCT 1972

THRU DEC 1972

PLUTONIUN REDUCTION - HOOD IV PROCESS

DATA

ARHCO JAN 1972 PRCOUCTION SCHEDULES

ARHCO FEB 1972 PRODUCTION SCHEDULES

ARHCO MAR 1972 PRCOUCTION SCHEDULES

ARHCO APR 1972 PRCDUCTION SCHEDULES

ARHCO MAY 1972 PRODUCTION SCHEDULES

ARHCO JUN 1972 PRCOUCTION SCHEDULES

ARHCO JUL 1972 PRODUCTION SCHEDULES

HISTORICAL DATA FOR 741 RECEIPTS

HISTORICAL DATA FOR 741 RECEIPTS

HISTORICAL DATA FOR 741 SHIPMENTS

PFAL SAMPLE LOG - CY 1972

ION EXCHANGE - DATA SHEETS AMD SAMPLE

SUMMARIES

ION EXCHANGE - DATA SHEETS AND SAMPLE

SUMMARIES

FUEL-GRADE P STATUS REPORT AS OF

$1 / 31 / 72$

FUEL-GRADE P STATUS REPORT AS OF

2/29/72

FUEL-GRADE $P$ STATUS REPORT AS OF

$3 / 31 / 72$

FUEL-GRADE P STATUS REPORT AS OF

$4 / 30 / 72$

FUEL-GRADE $P$ STATUS REPORT AS OF

$5 / 31 / 72$

MANUFACTURING DEPT MONTHLY MANAGEMENT

REPORTS - JAMUARY THRU DECEMBER 1972

MUCLEAR MATERIALS REVIEH - PUREX AMD

URANIUM OXIDE PLANTS - FIRST AMD SECOND

QUARTERS FY 1972

QUARTERLY PRCOUCTION FORECAST - JAMUARY

1. 1972 THRU JUNE 30, 1976

QUARTERLY PRCOUCTION FORECAST - APRIL 1, 1972 THRU JUNE 30, 1976

QUARTERLY PROOUCTION FORECAST JULY 1 , 1972 THRU JUNE 30, 1974
DA DOOD

HH MURRAY

HY MURRAY

HH MRRAY

HH MURRAY

GH UPIHGTON

- mCDOMALD

D modonalo

mCDONald

mcDomald

mcoonald

mcoonald

D mCDOMald

JG MURPHY

JG MURPHY

JG MURPHY

CP SUTTER

KH TAMAKA

KH TAMAKA

MK HARMON

MK MARMON

MK MARMON

MK MARHON

MK HARMON

HP SHAW

VW HALL

DUN ARCHO AEC-RL

RJ KOFOED

AEC RL ARCHO DUN
LEVEL

PGS

DATE

$06 / 29 / 71$

$01 / 03 / 72$

$01 / 01 / 72$

01/01/72

$04 / 01 / 72$

07/01/72

$10 / 01 / 72$

01/01/72

$01 / 03 / 72$

02/01/72

03/01/72

$04 / 04 / 72$

05/02/72

ns/06/72

07/03/72

$01 / 03 / 72$

03/09/72

$01 / 03 / 72$

01/03/72

01/01/72

07/01/72

$02 / 16 / 72$

03/14/72

$04 / 18 / 72$

05/08/72

07/20/72

$01 / 21 / 72$

$01 / 27 / 72$

$02 / 24 / 72$

$04 / 24 / 72$

$10 / 11 / 72$ 
DOC. NO.

ARH-023591

ARH-0236001

ARH-0236002

ARH-0236003

ARH-0236004

ARH -0236005

ARH-0236006

ARH-02364SUP

ARH-023671

ARH-0236710

ARH-0236711

ARH-023672

ARH-023673

ARH-023674

ARH-023675

ARH-023676

ARH -023677

ARH-023678

ARH-023679

ARH -023681

ARH -0236810

ARH -0236811

ARH -023682

ARH-023683

ARH-023684

ARH-023685

ARH 023686

ARH- 023687

ARH -023688

ARH-023689

ARH-0236911
TITLE

NUTHOR

D MCDONALD

RD ALLAN

JAMUARY 1, 1972 THRU JUME 30, 1976

ARHCO 200 AREAS OPERATION ESSENTIAL

MATERIAL INVENTORY \& CONSUMPTION REPORT

FOR THE MONTH OF JAMUARY 1972

ARHCO 200 AREAS OPERATION ESSENTIAL

MATERIAL INVENTORY \& CONSUMPTION REPORT

FOR THE MONTH OF FEBRUARY 1972

ARHCO 200 AREAS OPERATION ESSENTIAL

MATERIAL INVENTORY \& CONSUMPTION REPORT FOR THE MONTH OF MARCH 1972

ARHCO 200 AREAS OPERATION ESSENTIAL

MATERIAL INVENTORY \& CONSUMPTION REPORT FOR THE MONTH OF APRIL 1972

ARHCO 200 AREAS OPERATION ESSENTIAL MATERIAL INVENTORY \& CONSUMPTION REPORT FOR THE MONTH OF MAY 1972

ARHCO 200 AREAS OPERATION ESSENTIAL. MATERIAL INVENTORY \& CONSUMPTION REPORT FOR THE MONTH OF JUWE 1972

W-GRAPHS PRESENTED AT RL-02 PROGRAM MID-YEAR BUDGET REVIEW

HVA - COEI - JAN 1972

HVA - COEI - OCT 1972

HVA - COEI - NOV 1972

COEI - FEB 1972

HVA - COEI - MAR 1972

COEI - MAR 1972

HVA - COEI - MAY 1972

HVA - COEI - JUN 1972

HVA - COEI - JUL 1972

HVA - COEI - AUG 1972

HVA - COEI - SEP 1972

MATERIAL STATUS REPORT - JANUARY 1972

HVA - MATERIAL STATUS REPORT - OCTOBER 1972

HVA - MATERIAL STATUS REPORT - NOVEMBER 1972

MATERIAL STATUS REPORT - FEBRUARY 1972

MATERIAL STATUS REPORT - MARCH 1972

MATERIAL STATUS REPORT - APRIL 1972

HVA - MATERIAL STATUS REPORT - MAY 1972

HVA - MATERIAL STATUS REPORT - JUNE 1972

HVA - MATERIAL STATUS REPORT - JULY 1972

HVA - MATERIAL STATUS REPORT - AUGUST

1972

HVA - MATERIAL STATUS REPORT - SEPTEMBER 1972

WA - COMPOSITION OF ENDING INVENTORIES

- NOVEMBER 1972

\begin{tabular}{|c|c|c|}
\hline AUTHOR & LEVEL PGS & DATE \\
\hline D MCDONALD & SECR & $02 / 24 / 72$ \\
\hline RD ALLAN & SECR & $02 / 07 / 72$ \\
\hline RD ALLAN & SECR & 03/07/72 \\
\hline RD ALLAN & SECR & $04 / 10 / 72$ \\
\hline RD ALLAM & SECR & 05/11/72 \\
\hline RD ALLAN & SECR & $06 / 06 / 72$ \\
\hline RD ALLAN & SECR & 07/12/72 \\
\hline WC SCHAIDT & SECR & 02/09/72 \\
\hline DOE-RL & SECR & $02 / 11 / 72$ \\
\hline DUE-KL & SECR & $11 / 13 / 72$ \\
\hline$D C E-R L$ & SECR & $12 / 11 / 72$ \\
\hline ARHCO & SECR & $04 / 11 / 72$ \\
\hline DOE-RL & SECR & $05 / 10 / 72$ \\
\hline DOE-RL & SECR & $06 / 09 / 72$ \\
\hline DOE-RL & SECR & $07 / 12 / 72$ \\
\hline DOE-RL & SECR & 08/09/72 \\
\hline DOE-RL & SECR & 09/08/72 \\
\hline DOE-RL & SECR & $10 / 10 / 72$ \\
\hline JH JORDAN & SECR & $02 / 10 / 72$ \\
\hline JH JORDAN & SECR & $11 / 09 / 72$ \\
\hline JH JORDAN & SECR & $12 / 11 / 72$ \\
\hline JW JORDAN & SECR & 03/10/72 \\
\hline JW JORDAN & SECR & $0,4 / 11 / 72$ \\
\hline JW JORiban & SECR & 05/10/72 \\
\hline JH JORDAN & SECR & $06 / 09 / 72$ \\
\hline JW JORDAN & SECR & $07 / 12 / 72$ \\
\hline JW JORDAN & SECR & 08/09/72 \\
\hline & & \\
\hline JW JORDAN & SECR & 10/11/72 \\
\hline JG MURPHY & SRD & $12 / 11 / 72$ \\
\hline
\end{tabular}


Doc. No.

ARH-023700

ARH-0237002

ARH-0237002

ARH-0237003

ARH-0237003

ARH-0237004

ARH-0237004

ARH-0237005

ARH-0237006

ARH-0237007

ARH -0237008

ARH -0237009

ARH -0237010

ARH-0237011

ARH-023711

ARH-0237110

ARH-0237111

ARH-023712

ARH-023713

ARH-023714

ARH-023715

ARH-023716

ARH -023717

ARH-023718

ARH -023719
TITLE

material balance and MET proOUCtion REPORT JANUARY 1972

MATERIAL BALANCE AND MET PRCOUCTION REPORT FEBRUAR 1972

mTERIAL BaLANCE AND MET PRCOUCTION REPORT FEBRUAR 1972

MATERIAL BALANCE AND net PROOUCTION REPORT MARCH 1972

MATERIAL BALANCE AND NET PRODUCTION REPORT MARCH 1972

MATERIAL BALANCE AND NET PRODUCTION REPORT APRIL 1972

material BalaNCE AND MET PRODUCTION REPORT APRIL 1972

material BaLANCE AND MET PRODUCTION REPORT MAY 1972

material baLANCE AMd MET PRCOUCTION REPORT JUWE 1972

MATERIAL BALANCE AMD MET PRCOUCTION REPORT JULY 1972

MATERIAL BALANCE AND NET PROOUCTION REPORT AUEUST 1972

MATERIAL BALANCE AND MET PRODUCTION REPORT SEPTEMBER 1972

MATERIAL BALANCE AND NETT PRCOUCTION REPORT OCTOBE 1972

MATERIAL BALANCE AND HET PRCOUCTION REPORT NOVEMER 1972

NUCLEAR MATERIALS PRODUCTION REPORT JANUARY 1972

NUCLEAR MATERIALS PROOUCTION REPORT OCTOBER 9972

NUCLEAR MATERIALS PROOUCTION REPORT NOVEMBER 1972

NUCLEAR MATERIALS PRCOUCTION REPORT FEBRUARY 1972

MUCLEAR MATERIALS PRCOUCTION REPORT MARCH 1972

NUCLEAR MATERIALS PRCOUCTION REPORT APRIL 1972

MUCLEAR MATERIALS PRCOUCTION REPORT MAY 1972

NUCLEAR MATERIALS PRCOUCTION REPORT JUNE 1972

NUCLEAR MATERIALS PROOUCTION REPORT JULY 1972

MUCLEAR MATERIALS PRODUCTION REPORT AUCUST 1972

NUCLEAR MATERIALS PROOUCTION REPORT SEPTEMBER 1972
NTHOR

JG MURPHY

JG MURPHY

JG IMRPHY

JG MURPHY

JG MURPHY

JG MRPHY

JG MURPHY

JG MURPHY

JG MURPHY

JG MURPHY

JG MURPHY

JG MURPHY

JG MURPHY

JG IURPHY

JG MURPHY

JG MURPHY

JG MURPHY

JG MURPHY

JG MURPHY

JG IURPHY

JG MURPHY

JG MURPHY

JG MURPHY

JG MURPHY

JG IURPHY
LEVEL

PGS

DATE

SECR

$02 / 09 / 72$

03/09/72

03/09/72

$04 / 12 / 72$

$04 / 12 / 72$

$05 / 11 / 72$

$05 / 11 / 72$

$06 / 08 / 72$

$07 / 13 / 72$

$08 / 10 / 72$

$09 / 12 / 72$

10/11/72

$11 / 09 / 72$

$12 / 08 / 72$

$02 / 09 / 72$

$11 / 09 / 72$

$12 / 08 / 72$

$03 / 10 / 72$

$04 / 11 / 72$

$05 / 09 / 72$

06/08/72

$07 / 11 / 72$

$08 / 09 / 72$

09/08/72

10/09/72 
Page No. 34

$04 / 28 / 93$

LIST OF MANFORD-GENERATED CLASSIFIED

DOCUMENTS DATED BETWEEN 1/1/61 AND 12/31/72

DOC. NO.

ARH-02372 07

ARH-02372 08

ARH-02372 09

ARH-02372 10

ARH-02372 11

ARH -023724

ARH-023725

ARH-023726

ARH-02375RD

ARH-02385

ARH-02391RD

ARH-02391RD

ARH-02393

ARH-02400SUP1

$A R H-02409$

ARH-02416RD

ARH-02423

$A R H-02440$

ARH-02441RD

ARH-02448

$A R H-02457$

ARH-02475RD

ARH-02498

ARH-02498AUG

ARH-024980EC

ARH-02498JUL
TITLE

MUCLEAR MATERIALS PROJECT REPORT - JULY

1972

MUCLEAR MATERIALS PROJECT REPORT -

MUGUST 1972

MUCLEAR MATERIALS PROJECT REPORT -

SEPTEMBER 1972

MUCLEAR MATERIALS PROJECT REPORT -

OCTOBER 1972

MUCLEAR materials PROJECt REPORT -

MOVEMBER 1972

RESEARCH AND DEVELOPMENT MUCLEAR

MATERIALS PROJECT REPORT - APRIL 1972

MUCLEAR MATERIALS PROJECT REPORT - MAY 1972

MUCLEAR MATERIALS PROJECT REPORT - JUNE

1972

HD-98 HOURLY LOG

MUCLEAR MATERIALS REVIEH - PLUTONIUN

FINISHING SECTION - FIRST ANO SECOND

CUARTERS - FISCAL YEAR 1972

PLUTONIUN PROCESS ENGINEERING SECTION

PROCESS CONTROL REPORT - JAMUARY -

DECEMBER 1972

PLUTONIUN PROCESS ENGINEERIMG SECTION

PROCESS CONTROL REPORT - JAMUARY -

DECEMBER 1972

QUARTERLY PROOUCTION FORECAST

ALTERWATIVE

ARHCO HIGH-LEVEL LIOUID RADIOACTIVE

UASTE MAMAGEMENT PROCRAM - SUPPLENENT

PLUTONIUN BLENOING SCHEDULE - JAMUARY 1 , 1972 THRU JUNE 30, 1976

CHEMICAL PROCESSING DIVISION MONTHLY

REPORT SUMUARIES JAN 1972 THRU DEC 1973

WUCLEAR MATERIALS MAMAGEMENT PLANS

FIMAL LOW-PRESSURE REACTOR FUEL

PROCESSING CAMPAIEN - PUREX PLANT

WASTE PROCESSING SECTION MONTHLY REPORTS

- jamuary 1 thru december 31, 1972

COOED BUTTOW MUMBER LISTING

ALTERNATIVE PLUTONIUM BLENDING PLANS

FISCAL YEARS 1973-1976

STUDY NOTES - CY 1972

PLUTONIUM PROCESSING SS MATERIAL GEMERAL LEDGER

PLUTONIUM PROCESSING SOURCE DATA - FY

1973 AUEUST 1972

PLUTONIUA PROCESSING SOURCE DATA - FY

1973 DECEMBER 1972

PLUTONIUN PROCESSING SOURCE DATA - FY

1973 - JULY 1972
AUTHOR

JG MURPHY

SRD

SRD

SRD

$\operatorname{sen}$

SECR

SECR

SECR

SECR

SRD

Ad LoN

RW DOERR

SECR

SRO

SECR

LM RICHARDS

SECR

DE LARSON

SRD

LM RICHARDS

SECR

SECR

SECR

SECR

CONF

RE SMITH

PB FISK

LM RICHARDS

VR CHAPMAN

JG MURPHY

JG MURPHY

JG MURPHY

JG MURPHY
$02 / 18 / 72$

$02 / 25 / 72$

08/09/72

$09 / 11 / 72$

10/09/72

$11 / 09 / 72$

$02 / 09 / 72$

$05 / 10 / 72$

$06 / 09 / 72$

$07 / 13 / 72$

$05 / 05 / 71$

02/23/72

$02 / 18 / 72$

$04 / 14 / 72$

$03 / 30 / 72$

$01 / 01 / 72$

03/28/72

$04 / 07 / 72$

$09 / 01 / 72$

$04 / 11 / 72$

$04 / 25 / 72$

01/01/72

07/01/72

08/01/72

12/01/72

07/01/72 
DoC. NO.

ARH-02498NOV

ARH-024980CT

ARH-02498SEP

ARH-02499

ARH-02499AUG

ARH-024990EC

ARH-02499JUL

ARH-02499NON

ARH-024990CT

ARH-02499SEP

ARH-02504

ARH-025041

ARH-025042

ARH-02505RD

ARH-02507SUP

ARH-02520RD

ARH-02544

ARH-02553

ARH-02559RD

ARH-02564

ARH-02566

ARH-02572

ARH-02582

ARH-02583

ARH-02584
TITLE

PLUTONIUN PROCESSING SOURCE DATA - FY
1973 MOVEMBER 1972
PLUTONIUN PROCESSING SOURCE DATA - FY
1973 OCTOBER 1972
PLUTONIUM PROCESSING SOURCE DATA - FY
1973 SEPTEMBER 1972
PLUTOWIUN RECLAMATION SS MATERIALS

PLUTONIUN RECLAMA

PLUTONIUN RECLAMATION SOURCE DATA FY

1973 - AUEUST 1972

PLUTONIUN RECLAMATION SOURCE DATA FY

1973 - DECEMERR 1972

PLUTOAIUN RECLAMATION SOURCE DATA FY

1973 - JULY 1972

PLUTONILN RECLAMATION SOURCE DATA FY

1973 - NOVEMER 1972

PLUTONIUN RECLAMATION SOURCE DATA FY

1973 - OCTOBER 1972

PLUTONIUN RECLAMTION SOURCE DATA FY

1973 - SEPTEMBER 1972

GENERAL LEDGER - BURIAL GARDENS RECORDS

- WA - FY 1973

BURIAL GARDEN RECORDS - WV - FY 1973 -

MONTH-END AND SOURCE DATA JULY THRU

SEPTEMBER 1972

BURIAL GARDEN RECORDS - WA - FY 1973 -

MONTH END AND SOURCE DATA - OCTOBER THRU DECEMBER 1972

PROCESS CONTROL INFORMATION - CALENDAR

YEAR 1972

WU-GRAPHS PRESENTED AT RL O2 PROCRAM

FY-74 BUDCET REVIEW

BLENDING PLAN WORKING PAPERS

ATOHIC ENERGY COMISSION PLOUSHARE TASK

FORCE GROUP MEETIMG

REVISED MUCLEAR MATERIALS MAMACEMENT

PLAN

ARHCO PRCOUCTION CRAPHS - JULY 1, 1972

THRU JUNE 30, 1973

STLOY III - UTILIZATION OF 2 PLANT FOR

PLOUSHARE PROCRAM

ATLANTIC RICHFIELD HANFORD COMPANY

BUDGET FOR FY 1974 AND REVISION OF

BUDGET FOR FY 1973 JULY 11, 1972 EDITION

OFFSITE RADIOACTIVE SHIPMENT RECORD

MAMAGEMENT IMPROVEMENT PROGRAM - SCRAP

MUCLEAR MATERIALS

TRIP REPORT PLOUSHARE TASK FORCE GROUP

MEETING

MUCLEAR MATERIALS REVIEH - PLUTONIUM

FINISHING SECTION THIRD AND FOURTH

OUARTERS FY 1972

\begin{tabular}{|c|c|c|c|}
\hline AUTHOR & LEVEL & PGS & DATE \\
\hline 6 MURPHY & SECR & & $11 / 01 / 72$ \\
\hline MURPHY & SECR & & $10 / 01 / 72$ \\
\hline 6 MURPAY & SRD & & $09 / 01 / 72$ \\
\hline 16 MURPUY & SECR & & $07 / 01 / 72$ \\
\hline 16 MUPPHY & $\operatorname{sed}$ & & $08 / 01 / 72$ \\
\hline 16 MURPHY & SECR & & $12 / 01 / 72$ \\
\hline 16 MURPHY & SRD & & $07 / 01 / 72$ \\
\hline IG MURPHY & SECR & & $11 / 01 / 72$ \\
\hline 16 MURPHY & SECR & & $10 / 01 / 72$ \\
\hline 16 IURPHY & SRD & & $09 / 01 / 72$ \\
\hline 16 IMRPHY & SECR & & $07 / 01 / 72$ \\
\hline 16 MURPHY & SECR & & $07 / 01 / 72$ \\
\hline JG MURPHY & SECR & & $10 / 01 / 72$ \\
\hline As LOA & SECR & & $05 / 18 / 72$ \\
\hline NC SCHMIDT & SECR & & $06 / 12 / 72$ \\
\hline $\begin{array}{l}\text { MCOOMALD } \\
\text { KNIGHTS }\end{array}$ & $\begin{array}{l}\text { SECR } \\
\text { SECR }\end{array}$ & & $\begin{array}{l}05 / 30 / 72 \\
06 / 19 / 72\end{array}$ \\
\hline RP CORLEY & SECR & & $07 / 05 / 72$ \\
\hline MCDOMALD & SECR & & $07 / 01 / 72$ \\
\hline LE BRUWS & SRD & & $07 / 20 / 72$ \\
\hline BN DoBas & SECR & & $07 / 11 / 72$ \\
\hline HO SUYDER & SECR & & $07 / 21 / 72$ \\
\hline LM RICHARDS & SECR & & $07 / 21 / 72$ \\
\hline LE BRUWS & SECR & & $08 / 02 / 72$ \\
\hline RW DOERR & SECR & & $08 / 30 / 72$ \\
\hline
\end{tabular}


DOC. NO.

ARH-02585

ARH-02587

ARH-02593

ARH-02595AUE

ARH-02595JUL

ARH-02595NOV

ARH-025950CT

ARH-02595SEP

ARH-02603

ARH-02606

ARH -02607

ARH -02618

ARH-02638

ARH-02645

ARH-02646

ARH-02658

ARH-02668RD

ARH-C-00001

ARH $-N-00003$

ARH-N-00004

ARH $-\mathrm{N}-00008$

ARH-N-00009

ARH-N-00011

ARH $-\mathrm{N}-00017$

ARH-N-00023

ARH-N-00024

ARH $-N-00038$

ARH-N-00039

ARH-N-00045

ARH $-\mathrm{N}-00052$
TITLE

STLOY IV - UTILIZATION OF 2 PLANT FOR PLOUSHARE PROGRAM

ARHCO 200 AREAS OPERATION ESSENTIAL MATERIALS INVENTORY AND CONEMAPTION FYTD 1973 -BY MONTH

ISOTOPIC ASEAY THIRD AND FOURTH CORE

FAST FLUX TEST FACILITY

PRCOUCTION IMVENTORY AND MANUFACTURING

STATENENT - NUCUST 1972

PROOUCTION INVENTORY AND MANUFACTURING

STATEMENT - JULY 1972

PRCOUCTION INVENTORY AMD MAMUFACTURING

STATEMENT - NOV 1972

PROOUCTION INVENTORY AMD MANUFACTURING

STATENENT - OCTOBER 1972

PROOUCTION IMVENTORY AMD MANUFACTURING

STATEMENT BEPTEMBER 1972

ISOTOPIC ASSAY THIRD AND FOURTH CORE

FAST FLUX TEST FACILITY - DECAY

CORRECTIONS

SPECIAL MUCLEAR MATERIAL PROJECT

IUVENTORY AS OF JUWE 30, 1972

PLUTONIUN REDUCTION NOOD IV PROCESS DATA

PLUTONIUN SCRAP MAMAGEMENT PLAN

REPORT REVIEH - APPLICATIONE OF THE

CONCEPTUAL DESIGN FOR SAFECUARDS AT

ARHCO - DRAFT

EFFLUENT DATA - CALEMDAR YEAR 1971

FORM AEC 343C MONTHLY DISTRIBUTION OF

OPERATING COSTS IMCURRED ANO MONTHLY

MARRATIVE COMAENTS AT 45-1 - 2130

ISOTOPIC ASSAY THIRD AND FOURTH CORES

FFTF

PLUTONIUM BLENOING MODEL TEST CASES -

WORKING PAPERS

REPORT TO THE ATLANTIC RICHFIELD HAMFORD

COMPANY AN INDEPEMDENT OPINION OF

ISOCHEM INVENTORY AS AT AUEUST 31, 1967

SS MTERIAL REVIEH

PERSONAL NOTES

PERSONAL MOTES.

PROOUCTION DATA.

PERSONAL MOTES.

URANIUN REDUCTION LOGBOOK

WASTE STORAGE DATA

PERSONAL MOTES

PUREX PLANT CAPACITY FACTOR

PUREX PLANT PLUTONILW INVENTORY

PERSONAL MOTES.

PERSONAL MOTES.

$$
\text { AUTHoR }
$$

LE BRUNS

RE SMITH

RP COALEW

SECR

$08 / 25 / 72$

CR BLECEN

SECR

$09 / 25 / 72$

CR BLECEN

SECR

$08 / 31 / 72$

CR BLECEN

SECR

$12 / 27 / 72$

CR blecen

SECR

$11 / 25 / 72$

CR BLECEM

SECR

$10 / 25 / 72$

RP CORLEW

SECR

$09 / 19 / 72$

CB RUKLIMSKI

SECR

$09 / 12 / 72$

CW UPIMETON

RE FELT

LM RICHARDS

SeD

SECR

CONF

LA RICHARDS

LI RICHARDS

SRD

CONF

RP CORLEY

cowf

DL SPRENGER

SRD

RF LUM

SECR

LH TAYLOR

VO DONIHEE

VR CMAPUAM

VR CHAPUAN

AE SMITH

ML MNDEEN

DL UEBELACKER

WN SMITH

GH MARD

CPD - PUREX

RE VANDER COOK

RD CARTER
SECR

SECR

SECR

SECR

SRD

SECR

SRD

SECR

SECR

SECR

SRD

SECR
$10 / 31 / 72$

$11 / 06 / 72$

$12 / 01 / 72$

$12 / 11 / 72$

$10 / 09 / 67$

$09 / 01 / 72$

10/09/72

$10 / 20 / 72$

$09 / 05 / 67$

09/05/67

$09 / 05 / 67$

09/05/67

$09 / 05 / 67$

09/05/67

09/05/67

$09 / 05 / 67$

$09 / 05 / 67$

$09 / 05 / 67$

$09 / 05 / 67$

$09 / 14 / 67$ 
Page No.

DOC. NO.

ARH-N-00054

ARH-N-N00055

ARH-N-00064

ARH-N- -00065

ARH-N-00068

ARH-N-00080

ARH-N-00082

ARH $-N-00091$

ARH-N-00094

ARH-N-00095

ARH-N-00114

ARH-N-00121

ARH-N-00124

ARH-N-00125

ARH-N-00127

ARH-N-00128

ARH-N-00142

ARH-N-00144

ARH-N-00167

ARH-N-00154

ARH-N-00155

ARH-N-00156

ARH-N-00170

ARH-N-00190

ARH-N-00204

ARH-N-00221

ARH-PH-00369REV

ARH-R-00042

ARH-R-00049

ARH-R-00051

ARH-R-00052

ARH-R-00053

ARH-R-00054

ARH-R-00055DR

ARH-R-00055DR2

ARH-R-00057

ARH-R-00058

$A R H-R-00060$

ARH-R-00063

ARH-R-00064
TITLE

PERSONAL MOTES.

PERSONAL MOTES

PERSOHAL nOTES.

PERSOMAL MOTES.

PERSOMAL MOTES

TECHMICAL MOTES.

FISSIOW PROCESS PRCOUCTS SU

PERSOMAL MOTES

PERSOUAL MOTES.

SHIPPER - RECEIVER INFORMATION

PUREX MEASUREMENTS.

PERSONAL NOTES

PERSOUAL NOTES.

PU-240 RESULTS.

ENRICHED METAL DISCHARGES.

CHEMICAL PROCESSING STATISTICS.

PERSONAL MOTES - AMALYTICAL STLDIES

PERSONAL MOTES - MASTE MAMACEMENT

MOOELS, BOOK I

MUCLEAR MATERIAL PROC IMFORMATION.

HISTORIC TREWDS.

WASTE MAMAGEMENT MODEL SEPARATION

MASTE MAMACEMENT MODEL

TRITIUN CALCULATIONS

FISSION PROCESS PRCOUCT SUMARY - LOG

Mo.2

MASTE DISPOSAL STREAMS

PUREX MEASUREMENTS

HANFORD WASTE MAMAGEMENT BRIEFIMG

USAEC TRAMSFER

VOUCHER-56-MMA-HVA-6-69-1.

USAEC TRANSFER

VOUCHER-6- $M A-H V A-10-70-1$.

STRONTIUM ISOTOPE RATIOS

USAEC TRANT.EER

VOUCHER-14-MAA-HVA-12-70-1.

USAEC TRAMSFER VOUCHER 26-MMA-HVA-1-70-1

USAEC TRAMSFER VOUCHER-1-MAA-HVA-4-70-1.

TASK GROUP-2 DR RPT, PU STORAEE AMD PU

SCRAP.

TASK GROUP-2 DR RPT PU STORAGE AMD PU

SCRAP.

USAEC TRAMSFER VOUCHER-2-MAA-HVA-4-70-2.

USAEC TRANSFER

VOUCHER-30-MMA-HVA-4-70-3.

USAEC TRANSFER VOUCHER

46-MMA-HVA-5-70-1.

USAEC TRANSFER VOUCHER -

48-MMA-HVA-6-70-1.

USAEC TRAMSFER VOUCHER - 069-MMA-HVA-002

FIMAL.

\begin{tabular}{|c|c|c|c|}
\hline $\begin{array}{l}\text { Md } \\
\text { RL }\end{array}$ & $\begin{array}{l}\text { SZULIMSKI } \\
\text { HALSER }\end{array}$ & $\begin{array}{l}\text { SECR } \\
\text { SECR }\end{array}$ & $\begin{array}{l}09 / 18 / 67 \\
09 / 18 / 67\end{array}$ \\
\hline GL & manson & SECR & $10 / 26 / 67$ \\
\hline JS & DUCXINGHAY & SECR & $10 / 26 / 67$ \\
\hline JH & IARREN & SECR & $10 / 26 / 67$ \\
\hline HA & MOULTHROP & SECR & $01 / 04 / 68$ \\
\hline MC & JAcoss & SECR & $01 / 23 / 68$ \\
\hline eN & MARD & SECR & $05 / 02 / 68$ \\
\hline RW & THOWPSON & SECR & $05 / 23 / 68$ \\
\hline LH & TAYLOR & SECR & $06 / 03 / 68$ \\
\hline LH & TAYLOR & SECR & $11 / 06 / 68$ \\
\hline TR & MCKENZIE & SECR & $12 / 10 / 68$ \\
\hline RM & DOERR & SECR & $02 / 03 / 69$ \\
\hline D & MCDOMALD & SECR & $02 / 04 / 69$ \\
\hline D & MCDOUALD & SECR & $04 / 07 / 69$ \\
\hline D & MCDOMLLD & SECR & $04 / 07 / 60$ \\
\hline of & HILL & SECR & $08 / 21 / 69$ \\
\hline$D P$ & KOWICHEK & SECR & 09/09/69 \\
\hline JG & IMRPHY & SECR & $10 / 27 / 69$ \\
\hline LH & TAYLOR & SECR & $01 / 08 / 70$ \\
\hline sYs: & S DEPT & SECR & $01 / 13 / 70$ \\
\hline SY: & $S$ DEPT & SECR & $01 / 15 / 70$ \\
\hline J & AKAMIME & SECR & $06 / 23 / 70$ \\
\hline MC & JACoss & SECR & $01 / 01 / 71$ \\
\hline Bd & ERAOY & SECR & $04 / 15 / 71$ \\
\hline w & MALL & SECR & $10 / 13 / 71$ \\
\hline SJ & BEARD & SECR & $04 / 01 / 68$ \\
\hline$L$ & BRYAN & SECR & $06 / 30 / 69$ \\
\hline$L$ & BRYAM & SECR & $10 / 15 / 69$ \\
\hline HH & I VAN TUYL & SECR & $12 / 01 / 69$ \\
\hline L & BRYAN & SECR & $12 / 17 / 69$ \\
\hline $\mathbf{L}$ & BRYAM & SECR & $01 / 26 / 70$ \\
\hline$L$ & BRYAM & SECR & $03 / 27 / 70$ \\
\hline GB & PLEAT & SECR & $03 / 27 / 70$ \\
\hline CB & PLEAT & SRD & $04 / 22 / 70$ \\
\hline L. & BRYAM & SECR & $04 / 01 / 70$ \\
\hline $\mathbf{L}$ & BRYAN & 'SECR & $04 / 18 / 70$ \\
\hline L & ERYAN & SECR & $05 / 25 / 70$ \\
\hline L & BRYAM & SECR & $06 / 26 / 70$ \\
\hline L & BRYAM & SECR & $06 / 30 / 70$ \\
\hline
\end{tabular}


DOC. NO.

ARH-R-00067DR

ARH-R-00068

ARH-R-00118

ARH-R-00121

ARH-RL-00006

ARH-RL-00007

ARH-RL-00008

ARH-RL-00009

$A R H-R L=00011$

ARH-RL-00014

ARH-RL-00017

ARH $-V-00124$

ARH $-V-00144$

ARH-V -00145

ARH-V-00146

BNCC-0001

BNCC-0009

BNCC-0009 2

BNCC-0009 3

BNCC- 00094

BNCC-0009 5

BNCC- 0010

BNCC-0014 1

BNCC-0014 2

BNCC-0014 3

BNCC-0014 4

BNCC-0014 5

BMCC-0015 5

BNCC-0016

BNCC-0034

BNCC-0037 APR

BNCC-0037 MAR
PLUTONIUN IN-PROCESS INVENTORY FIMAL DRAFT REPORT.

TASK EROUP (II) REPORT - PLUTONIUY STORAGE AND PLUTONIUN SCRAP.

USAEC TRAMSFER VOUCHER O64-FAA-HVA-002

USAEC TRAMSFER VOUCHER 311-NUA-HVA-1

UO3 STANDARD VALUES

LOW PRESSURE REACTORS - JULY REACTOR

PRODUCT ION

COMHISSION ASSUMPTION PREPARATION OF

21-MO CUARTERLY PRCD FORECAST OCT 1 , 1968 THRU JUNE 30, 1970.

NUEUST REACTOR PROOUCTION

LO-3 PLANT APPRAISAL

USAEC TRAMSFER VOUCHER -

16-MMA-HVA-6-69-1.

APPRAISAL REPORT

USAEC TRANSFER VOUCHER 021-HVA-DZA-001F

USAEC TRAMSFER VOUCHER 311-HVA-DZA-001

USAEC TRANSFER VOUCHER 311-HVA-AUA-006

USAEC TRANSFER VOUCHER 311-HVA-FYA-004

LETTER TO G MATSON MASA-LEHIS RESEARCH

CENTER

MATERIAL BALANCE REPORT JAMUARY 1965

MATERIAL BALANCE REPORT FEBRLARY 1965

MATERIAL BALANCE REPORT

MATERIAL BALANCE REPORT APRIL 1965

MATERIAL BALAMCE REPORT MAY 1965

INLET AND OUTLET SAMPLES - 100-KM

REACTOR

MONTHLY INVENTORY REPORT SAN-32500

JAMUARY 1965

MONTHLY INVENTORY REPORT SAN-32500

FEBRUARY 1965

MONTHLY INVENTORY REPORT SAN-32500 MARCH

1965

MONTHLY INVENTORY REPORT SAN-32500 APRIL 1965

MONTHLY INVENTORY REPORT SAN-32500 MAY

1965

MEAPONS RESEARCH AND DEVELOPMENT SS

MATERIAL PROJECTS MAY 1965

PRESSURE DROP CHARACTERISTIICS OF SOLID

ALUMINUM DUMAY PATTERNS IN $K$ ZIRCALOY-2 PROCESS TUBE

MRD TARGET SUPPORT STUDIES IEEKLY REPORT

3

COMPOSITION OF ENDING INVENTORY APRIL 1965

COMPOSITION OF ENDING INVENTORY MARCH 1965

\author{
AUTHOR
}

T MALranick

CB PLEAT

UH HENDERSOW

BH MEST

MH HENDERSON

AEC

OJ ELCERT

AEC

OJ ELCERT

WR POHLCO

OJ ELGERT

CR BLECEN

CR BLEGEN

CR BLECEN

CR BLEGEN

SHAW CH

BUDD RO

BUDD RO

BUDD RO

BUDD RO

BUDD RO

SKEIE DS

BUDD RO

BUDD RO

BUDD RO

BUDD RO

BUDO RO

OUDO RO

ANGLE CW

MEBER JW

BUDD RO

BUDD RO
LEVEL

PGS

DATE

$07 / 21 / 70$

$06 / 16 / 70$

$06 / 23 / 72$

$11 / 01 / 72$

$07 / 10 / 68$

$08 / 13 / 68$

$09 / 10 / 68$

$09 / 13 / 68$

10/03/68

$06 / 26 / 69$

$08 / 19 / 69$

$01 / 31 / 72$

$11 / 07 / 72$

$11 / 07 / 72$

$11 / 07 / 72$

01/26/65

CRD

02/12/65

03/10/65

$04 / 12 / 65$

05/13/65

05/11/65

02/05/65

$02 / 15 / 65$

03/11/65

$04 / 13 / 65$

05/13/65

$06 / 11 / 65$

$06 / 11 / 65$

$02 / 16 / 65$

03/11/65

$05 / 13 / 65$

SRD

12

$04 / 12 / 65$ 
Page No. 39

$04 / 28 / 93$

LIST OF HANFORD-GENERATED CLASSIFIED

DOCUMENTS DATED BETUEEN 1/1/61 AND 12/3:/72

DOC. NO.

BNCC-0037 MAY

BNCC-0051

BNCC-0054

BNCC-0055

BNCC-0085

BMCC-0089

BNCC-0091

BNCC-0098

BNCC-0100

BNCC-0101

BNWC-0006

BNWC-0008

BNWC-0008 11

BNWC-0008 11A

BNWC-0008 IA

BNWC-0008 2

BNWC-0008 2A

BNWC-0008 3

BNHC-0008 3A

BNWC-0008 4

BNWC-0008 4A

BNUC-0008 5

BNHC-0008 5A

BNHC-0008 6

BNWC-0008 6A

BNWC-0072
TI:LE

COMPOSITION OF ENDING INVENTORY MAY 1965 CLASSIFICATION REVIEW OF PHOTOGRAPHS

TELEPHONE CONVERSATION WITH CP

BLAMKENSHIP MASA-LEHIS - 3-30-65

PRELIMIMARY AMALYSIS OF K-REACTOR

DONMCOAER APPROACH SECTION PRESSURES

DURING TEMPERATURE TRANSIENTS

ISOT "C ANALYSES IN WEIGHT PERCENT

LETTER: CLASSIFICATION OF MEUTRON FLUX

IN HANFORD IRRADIATION TEST FACILITIES

LETTER TO MR FRANK ROM, LEUIS RESEARCH

CENTER

LOW-TEMPERATURE HYDRIDING OF ZIRCALOY-2

LETTER TO MT SAUNDERS MASA-LEUIS

RESEARCH CENTER

SNPO AMD SNAP BIOLOGICAL STLDIES FOR

USHRDL

PROGNESS REPORT - PROGRAM DESCRIBED IN HW-83921

BATTELLE-NORTHUEST MONTHLY ACTIVITIES REPORT, JAMUARY 1965

FACIFIC MORTHLEST LABORATORY MONTHLY

ACTIVITIES REPORT - MOVEMBER 1965

REACTOR \& MATERIALS TECHNOLOGY MOWTHLY

REPONT D2 PROGRAK - NOVEMBER 1965

REACTOR \& MATERIALS TECHNOLOGY MONTHLY

REPORT - JAMUARY 1965

BATTELLE-NORTHLEST MONTHLY ACTIVITIES

REPORT - FEBRUARY 1965

REACTOR AND MATERIALS TECHNOLOGY MONTHLY

REPORT - FEBRUARY 9965

BATTELLE-NORTHUEST MONTHLY ACTIVITIES

REPORT - MARCH 9965

REACTOR AND MATERIALS TECHNOLOGY MONTKLY

REPORT - MARCH 1965

BATTELLE-NORTHUEST MONTHLY ACTIVITIES

REPORT - APRIL 1965

REACTOR AND MATERIALS TECHNOLOGY MONTHLY REPORT - APRIL 1965

BATTELLE-NORTHUEST MONTHLY ACTIVITIES

REPORT - MAY 1965

REACTOR \& MATERIALS TECHNOLOGY MONTHLY

REPORT - MAY 1965

BATTELLE-NORTHUEST MONTHLY ACTIVITIES

REPORT - JUWE 1965

R:ACTOR \& MATERIALS TECHMOLOGY MOHTHLY

REPORT - JUNE 1965

HANFORD CONTRIBUTION FOR THE TWENTIETH

HIGH TEMPERATURE FUELS COMIITTEE

MEETIHG, MAY 18, 19, 20, 1965

$$
\text { AUTHOR }
$$

BUDD RO

SWYDER WA

DRUAHELLIER $K$

ROWE DS

LAGERGREN CR

SUYDER MA

ALBAUEH FH

HINEGARDNER WK

FAUCETT SL

BUDD RO

SWANSOW $d L$, MISSEN DA

CRD

ALBAUEH FM

ALBAUGH FH

ALraugh FH

ALBAUEH FW

SRD

ALBAUTH FH

SRD

ALBNuGH FY

ALBAUGh FW

LAST GA
SRD

CRD

SRD

SRD

SRD

SRD

68

07/15/65

LEVEL PGS

DATE

$06 / 14 / 65$

$04 / 02 / 65$

$04 / 01 / 65$

03/26/65

05/12/65

$05 / 17 / 65$

05/20/65

$06 / 01 / 65$

06/28/65

05/25/65

$01 / 19 / 65$

$02 / 15 / 65$

12/01/65

$12 / 15 / 65$

$02 / 15 / 65$

$03 / 15 / 65$

$03 / 15 / 65$

$04 / 15 / 65$

$04 / 95 / 65$

$05 / 14 / 65$

$05 / 14 / 65$

$06 / 15 / 65$

$06 / 15 / 65$

07/01/65

CRD $30 \quad 04 / 26 / 65$ 
Page No. 40

$04 / 28 / 93$

LIST OF HANFORD-GENERATED CLASSIFIED

DOCUNENTS DATED BETWEEN 1/1/61 AND 12/31/72

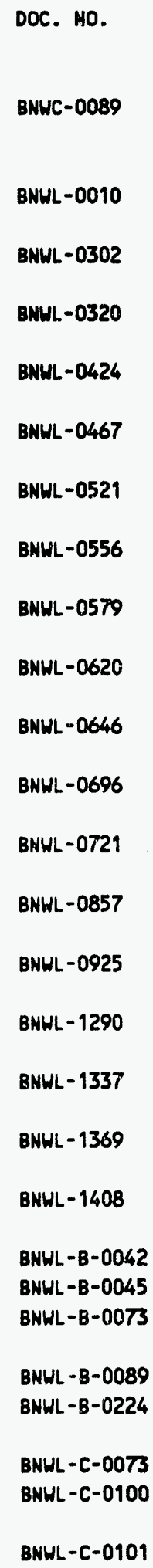

TITLE

AUTHOR

LEVEL PGS

DATE

HUNTER DO, BROWN RL

SRD

45

$05 / 19 / 65$

PLUTONIUH PLATING THICKNESS MITK

ULTRASONIC AND EDDY CURRENT TECHNIOUES

PLUTONIUM FUELING STUDIES FOR FAST

FLETCHER JF

CRD

85

$01 / 01 / 65$

COMPACT REACTORS

PACIFIC NORTHLEST LABORATORY MONTHLY

ACTIVITIES REPCRT FOR JULY 1966

PACIFIC MORTHLEST LABORATORY MONTHLY

ACTIVITIES REPORT FOR AUGUST 1966

PACIFIC MORTHLEST LABORATORY MONTHLY

ACTIVITIES REPORT FOR MARCH 1967

PACIFIC NORTHMEST LABORATORY MONTHLY

ACTIVITIES REPORT FOR MAY 1967

PACIFIC MORTMLEST LABORATORY MONTHLY ACTIVITIES REPORT FOR JULY 1967

PACIFIC NORTHWEST LABORATORY MONTHLY ACTIVITIES REPORT FOR AUEUST 1967

PACIFIC MORTHUEST LABORATORY MONTHLY ACTIVITIES REPORT FOR SEPTEMBER 1967 PACIFIC MORTHUEST LABORATORY MONTHLY ACTIVITIES REPORT FOR OCTOBER 1967 PACIFIC NORTHUEST LABORATORY MONTHLY ACTIVITIES REPORT FOR MOVEMBER 1967

PACIFIC NORTHWEST LABORATORY MONTHLY ACTIVITIES REPORT FOR JAMUARY 1968

PACIFIC NORTHLEST LABORATORY HONTHLY ACTIVITIES REPORT FOR FEBRUARY 1968

PACIFIC MORTHLEST LABORATORY MONTHLY ACTIVITIES REPORT JUNE, 1968

PACIFIC NORTHUEST LABORATORY MONYHLY ACTIVITIES REPORT OCTOBER 1968

PACIFIC NORTHUEST LABORATORY MONTHLY ACTIVITIES REPORT DECEMBER 1969

PACIFIC NORTHLEST LABORATORY MONTHLY

ACTIVITIES REPORT - FEBRUARY 1970

PACIFIC NORTHUEST LABORATORY MONTHLY

ACTIVITIES REPORT MARCH 1970

PACIFIC MORTHLEST LABORATORY MONTHLY

ACTIVITIES REPORT APRIL 1970

CPTF RUN 4 FLUX MONITORING

CPTF RUN 5 FLUX MONITORING

AMALIYSIS OF PROMETHIUM-147 DEMAMD AND

COSTS OF SUPPLY

CPTF RUN 6 FLUX MONITORING

AEC RECULATORY SAMPLES AND A WORK

PROFILE OF THE NEH BRUNSHICK LABORATORY

TRITIUM TESTUELL DATA - 1964-65

MANAGEMENT IMPROVEMENT PROGRAM SCRAP

MUCLEAR MATERIALS

LETTER: CLASSIFICATION OF AMS PAPER BNUL-4237-S USE OF NUCLEAR EXPLOSIVE ARRATS TO FRACTURE HOT ROCK

\begin{tabular}{|c|c|c|c|}
\hline \multirow{3}{*}{ 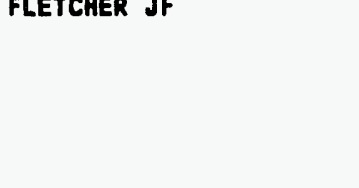 } & CNo & s & תם \\
\hline & SRD & 58 & 08/01/66 \\
\hline & SRD & 59 & 09/01/66 \\
\hline FAMCETT SL & CRD & 84 & $04 / 01 / 67$ \\
\hline FAMCETT SL & CRD & 92 & $06 / 01 / 67$ \\
\hline FALCETT SL & SRD & 82 & $08 / 01 / 67$ \\
\hline FALCETT SL & CSRD & 69 & $08 / 01 / 67$ \\
\hline FAWCETT SL & CRD & 69 & $10 / 01 / 67$ \\
\hline ALBAUGH FW & SRD & 69 & $11 / 01 / 67$ \\
\hline ALBAUGH FW & CRD & 84 & 12/01/67 \\
\hline \multirow[t]{2}{*}{ ALBAUGH FW } & CRD & 89 & 02/01/68 \\
\hline & CRD & 83 & $03 / 01 / 68$ \\
\hline ALBAUGH, FH & CRD & 89 & $07 / 01 / 68$ \\
\hline ALBAUGH, FU & CRD & 87 & $11 / 01 / 68$ \\
\hline ALBAUGH FH & CRD & 60 & $01 / 01 / 70$ \\
\hline ALBAUGH FH & CRD & 62 & $03 / 01 / 70$ \\
\hline ALBAUGH FH & CRD & 70 & $04 / 01 / 70$ \\
\hline ALBAUGH FU & SRD & 66 & $05 / 01 / 70$ \\
\hline DIVINE JR & CRD & 25 & $12 / 18 / 70$ \\
\hline DIVINE JR & CRD & 19 & $02 / 22 / 71$ \\
\hline $\begin{array}{l}\text { HAMSEK JE, HAYMOND VL, } \\
\text { VANTUYL HH }\end{array}$ & SRD & 90 & $03 / 19 / 71$ \\
\hline DIVIME JR & CRD & 27 & $05 / 07 / 71$ \\
\hline BROUNS RJ, ROBERTS FP & CRD & 52 & $10 / 01 / 72$ \\
\hline KELLOGG LS & CRD & 6 & $05 / 18 / 71$ \\
\hline RICHMOND WD & SRD & 2 & $07 / 14 / 72$ \\
\hline BURNHAM JB & SRD & 3 & $07 / 21 / 72$ \\
\hline
\end{tabular}


Page No. 41

$04 / 28 / 93$

LIST OF HANFORD-GENERATED CLASSIFIED

DOCUMENTS DATED BETUEEN 1/1/61 AMD 12/31/72

DOC. NO.

BNHL-C-0102

BNWL-CC-0128 10

BNHL-CC-0128 11

BNUL-CC-0128 12

BNHL-CC-0128 6

BNUL-CC-0128 7

BNHL-CC-0128 8

BNWL-CC-0128 9

BNWL-CC-0129 10

BNWL-CC-0129 11

BNHL-CC-0129 12

BNWL-CC-0129 6

BNHL-CC-0129 7

BNHL-CC-0129 8

BNLL-CC-0129?

BNHL-CC-0130 12

BNWL-CC-0130 6

BNHL-CC-01309

BNHL-CC-0131 AUGUST

BNHL-CC-0131

DECEMBER

BNWL-CC-0131 JULY

BNWL-CC-0131 JUNE

BNLL-CC-0131

NOVEMBER

BNHL-CC-0131 OCTOBEK COMPOSITION OF ENDING INVENTORY OCTOBER

BNHL-CC-0131

SEPTEMBER

BNHL-CC-0133 21

BNHL-CC-013322

BNWL-CC-0133 23

BNWL-CC-013324

\section{5}

SCRAP STATUS REPORTS FOR PLUTONIUN-239 AND ENRICHED URANIUN MATERIAL BALANCE REPORT, OCTOBER 1965 MATERIAL BALANCE REPORT, NOVEMBER 1965 MATERIAL BALANCE REPORT, DECEMBER 1965 MATERIAL BALANCE REPORT JUNE 1965 MATERIAL BALAMCE REPORT, JULY 1965 MATERIAL BALAHCE REPORT, AUCUST 1965 MATERIAL BALANCE REPORT, SEPTEMBER 1965 WEAPONS RESEARCH AND DEVELOPMENT SS MATERIAL PROJECTS OCTOSER 1965 WEAPONS RESEARCH AND DEVELOPMENT SS MATERIAL PROJECTS NOVENRER 1965 MEAPONS RESEARCH AMD DEVELOPMENT SS MATERIAL PROJECTS DECEMBER 1965 WEAPONS RESEARCH AND DEVELOPMENT SS MATERIAL PROJECTS JUNE 1965

WEAPONS RESEARCH AND DEVELOPMENT SS MATERIAL PROJECTS, JULY 1965 WEAPONS RESEARCH AND DEVELOPMENT SS MATERIAL PROJECTS AUEUST 1965 WEAPONS RESEARCH AMD DEVELOPMENT SS MATERIAL PROJECTS SEPTEMBER 1965 QUARTERLY INVENTORY REPORT, SAN-32500, DECEMBER 1965

MONTHLY INVENTORY REPORT SAN-32500 JUNE 1963

QUARTERLY INVENTORY REPORT, SAN-32500, SEPTEMBER 1965

COMPOSITION OF ENDING INVENTORY AUGUST 1965

COMPOSITION OF ENDING INVENTORY DECEMBER 1965

COMPOSITION OF ENDING INVENTORY JULY 1965

COMPOSITION OF ENDING INVENTORY JUNE 1965

COMPOSITION OF ENDING INVENTORY NOVEMBER 1965

COMPOSITION OF ENDING INVENTORY

SEPTEMBER 1965

USAEC TRANSFER VOUCHER

4-HYA-FZA-10-66-1F

USAEC TRANSFER VOUCHER

2-HYA-JZ8-10-66-1F

USAEC TRANSFER VOUCHER

3-HYA-AUA-10-66-1F

USAEC TRANSFER VOUCHER

5-HYA-HZA-10-66-1F
AUTHOR

MENRY HL

LEVEL

PGS

Date

\section{BUDD RO \\ BUDD RO \\ BUDD RO \\ BUDD RO \\ BUDD RO \\ BUDD RO \\ BUDD RO \\ BUDD RO}

BUDD RO

QUDD RO

BUDD RO

BUDD RO

BUDD RO

BUDD RO

BUDD RO

BUDD RO

BUDD RO

BUDD RO

BUDD RO

BUDD RO

BUDD RO

BLDD RO

BUDD RO

BUDD RO

CLOUSE HE

CLOUSE HE

CLOUSE HE

CLOUSE HE
SRL

CRD

CRD

CRD

SRD

CRD

CRO

CRD

CRD

CRD

CRD

SRD

CRD

CRD

CRD

CRD

SRD

CRD

CRD

CRD

CRD

SRD

CRD

CRD

CRD

13

SRD

SRD

SRD

SRD
08/01/72

$11 / 11 / 65$

$12 / 13 / 65$

$01 / 13 / 66$

07/14/65

08/11/65

09/13/65

10/13/65

$11 / 10 / 65$

$12 / 15 / 65$

$01 / 13 / 65$

$07 / 13 / 65$

08/11/65

$09 / 14 / 65$

$10 / 14 / 65$

$01 / 14 / 66$

$07 / 13 / 65$

$10 / 14 / 65$

$09 / 13 / 65$

$01 / 13 / 66$

$08 / 11 / 65$

$07 / 14 / 65$

$12 / 14 / 65$

$11 / 10 / 65$

$10 / 13 / 65$

$11 / 01 / 65$

$11 / 01 / 65$

$11 / 01 / 65$

$11 / 03 / 65$ 
Page No. 42

$04 / 28 / 93$

LIST OF MANFORD-GENERATED CLASSIFIED

DOCLMENTS DATED BETHEEN 1/1/61 AMD 12/31/72

TITLE

AUTHOR

PGS

DATE

BNWL-CC-0133 25

BNWL-CC-013326

BNHL-CC-0133 27

BNWL-CC-0133 28

BNUL-CC-013329

BNWL-CC-0133 30

BNHL-CC-0133 31

BNUL-CC-0133 32

BNHL-CC-0133 33

BNHL-CC-0133 34

BNWL-CC-0133 35

BNHL-CC-0133 36

BNWL-CC-0133 37

BNHL-CC-0133 38

BNHL-CC-0133 39

BNHL-CC-0133 40

BNUL-CC-0133 41

BNWL-CC-0133 42

BNWL-CC-0133 43

BNHL-CC-0133 44

BNWL-CC-0133 45 BNWL-CC-0133 46 BNHL-CC-0133 47 BNHL-CC-0133 48 BNWL-CC-0133 49 BNWL-CC-0133 50 BNHL-CC-0133 51 BNHL-CC-0133 52 BNHL-CC-0133 53 BNHL-CC-0133 54 BNHL-CC-0133 55 BNHL-CC-0133 56 BNWL-CC-0133 57 BNLL-CC-0133 58 BNWL-CC-0133 59 BNWL-CC-0190 BNHL-CC-0222
USAEC TRANSFER VOUCHER

1-HYA-CZA-11-66-1F

USAEC TRAASFER VOUCHER

2-HYA-JZA-19-66-1F

USAEC TRANSFER VOUCHER

4-HYA-HXA-11-66-1F

USAEC TRANSFER VOUCHER

5-HYA-HZA-11-66-1F

USAEC TRANSFER VOUCHER

6-HYA-HAA-11-66-1F

USAEC TRAMSFER VOUCHER

1-HYA-JZA-12-66-1F

USAEC TRANSFER VOUCHER

2-HYA-CZA-12-66-1F

USAEC TRANSFER VOUCHER

2-HYA-FZA-12-66-1F

USAEC TRANSFER VOUCHER

4-HYA-HXA-12-66-1F

USAEC TRANSFER VOUCHER

5-HYA-HZA-12-66-1F

USAEC TRANSFER VOUCHER 1-HYA-LZA-1-66-1F USAEC TRANSFER VOUCHER 2-HYA-LAA-1-66-1F USAEC TRANSFER VOUCHER 3-HYA-ALA-1-66-1F USAEC TRANSFER VOUCHER 4-HYA-PZA-1-66-1F USAEC TRANSFER VOUCHER 5-HYA-ASA-1-66-1F USAEC TRANSFER VOUCHER 7-HYA-FXA-1-66-1F USAEC TRANSFER VOUCHER 8-HYA-HHA-1-66-1F LUSAEC TRANSFER VOUCHER 9-HYA-HXA-1-66-1F USAEC TRAMSFER VOUCHER

10-HYA-HZA-1-66-1F

USAEC TRANSFER VOUCHER

11-HYA-HAA-1-66-1F

USAEC TRAMSFER VOUCHER 1-HYA-ASA-2-66-1F USAEC TRAMSFER VOUCHER 2-HYA-J2A-2-66-1F USAEC TRANSFER VOUCHER 3-HYA-LM-2-66-1F USAEC TRANSFER VOUCHER 5-HYA-HZA-2-66-1F USAEC TRANSFER VOUCHER 6-HYA-HWA-2-66-1F USAEC TRAHSFER VOUCHER 7-HYA-HXA-2-66-1F USAEC TRANSFER VOUCHER 8-HYA-ALA-2-66-1F USAEC TRANSFER VOUCHER 1-HYA-CZA-3-66-1F USAEC TRANSFER VOUCHER 2-HYA-LZA-3-66-1F USAEC TRAMSFER VOUCHER 3-HYA-ASA-3-66-1F USAEC TRANSFER VOUCHER 5-HYA-JZA-3-66-1F USAEC TRANSFER VOUCHER 6-HYA-FAA-3-66-1F USAEC TRANSFER VOUCHER 7-HYA-HZA-3-66-1F USAEC TRANSFER VOUCHER 8-HYA-HHA-3-66-1F USAEC TRANSFER VOUCHER 9-HYA-HXA-3-66-1F K-AREA HOT TEST HOLE TEMPERATURES MONTHLY PROGRESS REPORT ON WORK ORDERS C-55995 AND C84887

$\begin{array}{lccc}\text { CLOUSE HE } & \text { SRD } & 1 & 12 / 01 / 65 \\ \text { CLOUSE HE } & \text { SRD } & 1 & 12 / 01 / 65 \\ \text { CLOUSE HE } & \text { SRD } & 1 & 12 / 03 / 65 \\ \text { CLOUSE HE } & \text { SRD } & 1 & 12 / 03 / 65 \\ \text { CLOUSE HE } & \text { SRD } & 1 & 12 / 03 / 65 \\ \text { CLOUSE HE } & \text { SRD } & 1 & 01 / 03 / 66 \\ \text { CLOUSE HE } & \text { SRD } & 1 & 01 / 03 / 66 \\ \text { CLOUSE HE } & \text { SRD } & 1 & 01 / 03 / 66 \\ \text { CLCUSE HE } & \text { SRD } & 1 & 01 / 04 / 66 \\ \text { CLOUSE HE } & \text { SRD } & 1 & 01 / 04 / 66\end{array}$

CLOUSE HE CLOUSE HE CLOUSE HE CLOUSE HE CLOUSE HE CLOUSE HE CLOUSE HE CLOUSE HE CLOUSE HE

CLOUSE HE

CLOUSE HE CLOUSE ME CLOUSE ME CLOUSE HE CLOUSE HE CLOUSE HE CLOUSE HE CLOUSE HE CLOUSE HE CLOUSE HE CLOUSE HE CLOUSE HE CLOUSE HE CLOUSE HE CLOUSE HE COX JH HARMON KM
02/01/66

$02 / 01 / 66$

$02 / 01 / 66$

$02 / 01 / 66$

$02 / 01 / 66$

$02 / 01 / 66$

02/02/66

$02 / 02 / 66$

$02 / 02 / 66$

$02 / 01 / 66$

$02 / 25 / 66$

$02 / 25 / 66$

$02 / 25 / 66$

$02 / 28 / 66$

$02 / 28 / 66$

$02 / 28 / 66$

$02 / 25 / 66$

$03 / 31 / 66$

$03 / 31 / 66$

$03 / 31 / 66$

$03 / 31 / 66$

$03 / 31 / 66$

$03 / 31 / 66$

03/31/66

$03 / 31 / 66$

08/04/65

$08 / 27 / 65$ 
Page No. 43

$04 / 28 / 93$

LIST OF HANFORD-GENERATED CLASSIFIED

DOCUAAETS DATED BETHEEN 1/1/61 AND 12/31/72

DOC. NO.

BNHL-CC-0309

BNHL-CC-0315

BNHL-CC-0333

BNUL-CC-0337

BNWL-CC-0382

BNWL-CC-0410

BNHL-CC-0418

BNAL-CC- 0437

BNHL-CC-0442

BNUL-CC-0459

BNHL-CC- 0460

BNHL-CC-0473 9 BNHL-CC-0473 10 BNWL-CC-0473 11 BNHL-CC-0473 12 BNHL-CC-0473 2 BNHL-CC-0473 3 BAWL-CC-0473 4 BNWL-CC-0473 5 BNHL-CC-0473 6 BNUL-CC-0473 7 BNHL-CC-0473 8 BNHL-CC-04739 BNLL-CC-0474 1

BNHL-CC-0474 10

BNHL-CC-0474 11

BNHL-CC-0474 12

BNHL-CC-0474 2

BNHL-CC-0474 3

BNWL-CC-0474 4
TITLE

JOWOG-22 AGENDA ITEM NO. 5C PRELIMIMARY STUDIES OW TIVE STABILITY OF BETA PHASE PLUTONIUN ALLOYS

TRAMSFER MEASUREMENT STATISTICS DERIVED FROM PUREX PROCESSING MEASUREMENTS' QUALITY CONTROL PROGRAM

ESTIMATED COSTS FOR CLASSIFIED PROJECT TRITIUM RELEASE FROM LITHIUM SILICATE AND LITHIUM ALUMIMAE, IN-REACTOR AND OUT-OF-REACTOR

TECHAICAL SERVICES - TESTING OF MASA FUEL ELEMENTS FOR BATTELLE-COLUMBUS PROGRESS REPORT ON WORK ORDER C-84887 SEPTEMBER - DECEMBER, 1965

LETTER TO PG HOLSTED, RE: RADIOBARIUM FEASIBILITY STUOY FOR PERIMETER INSPECTION OF SHUTDOWN PRCOUCTION REACTORS

LETTER TO PG HOLSTED, RE: BNWL-118, IN AMSWER TO BNWI-160

TRITIUN RECOVERY FROM CERAMIC TARGETS IMTERIM REPORT

LETTER TO GEN. DL CRONSON, DIRECTOR OF MILITARY APPLICATION, USAEC, HASHINGTON DC

MATERIAL BALANCE REPORT - JAMUARY 1966

MATERIAL BALANCE REPORT - OCTOBER 1966

MATERIAL BALANCE REPORT - NOVEMBER 1966

MATERIAL BALAHCE REPORT - DECEMBER 1966

MATERIAL BALANCE REPORT - FEBRUARY 1966

MATERIAL BALANCE REPORT - MARCH 1966

MATERIAL BALANCE REPORT - APRIL 1966

MATERIAL BALANCE REPORT - MAY 1966

MATERIAL BALANCE REPORT - JUNE 1966

MATERIAL. BALANCE REPORT - JULY 1966

MATERIAL BALANCE REPORT - AUGUST 1966

MATERIAL BALANCE REPORT - SEPTEMBER 1966

COMPOSITION OF ENDING INVENTORY JANUARY

1966

COMPOSITION OF ENDING INVENTORY OCTOBER

1966

COMPOSITION OF ENDING INVENTORY MOVEMBER 1966

COMPOSITION OF ENDING INVENTORY DECEMBER

1966

COMPOSITION OF ENDING INVEHTORY FEBRUARY

1966

COMPOSITION OF ENDING INVENTORY MARCH

1966

COMPOSITION OF ENDING INVENTORY APRIL 1966

\begin{tabular}{|c|c|c|c|}
\hline AUTHOR & LEVEL & PGS & DATE \\
\hline TAYLOR JAA & SRD & 11 & $10 / 20 / 65$ \\
\hline IOUGH CG & SRD & 32 & $11 / 23 / 65$ \\
\hline $\begin{array}{l}\text { IANSLYKE WJ } \\
\text { JOHNSON AB }\end{array}$ & $\begin{array}{l}\text { SRD } \\
\text { SRD }\end{array}$ & $\begin{array}{l}4 \\
57\end{array}$ & $\begin{array}{l}11 / 01 / 65 \\
11 / 03 / 65\end{array}$ \\
\hline DRLTOA DC & CRD & 6 & $12 / 03 / 65$ \\
\hline LARAON KM & SRD & 14 & $12 / 27 / 65$ \\
\hline $\begin{array}{l}\text { GRANOUIST DP, BROUNS RJ } \\
\text { FULLAER GL', GREEN DR, } \\
\text { GRANOUIST DP }\end{array}$ & $\begin{array}{l}\text { CRD } \\
\text { SRD }\end{array}$ & $\begin{array}{l}1 \\
63\end{array}$ & $\begin{array}{l}01 / 04 / 66 \\
02 / 01 / 66\end{array}$ \\
\hline IIPTON CR & CRD & 8 & $01 / 18 / 66$ \\
\hline HARMON KM & SRD & 25 & $01 / 25 / 66$ \\
\hline ROESCH WC & SRD & 2 & $01 / 25 / 68$ \\
\hline SUDD RO & CRD & 41 & $02 / 14 / 66$ \\
\hline UDD RO & CRD & 38 & $11 / 11 / 66$ \\
\hline UDD RO & CRD & 63 & $12 / 13 / 66$ \\
\hline 300 RO & CRO & 64 & $01 / 13 / 67$ \\
\hline SUDD RO & CRD & 41 & $03 / 10 / 66$ \\
\hline UDD RO & CRO & 40 & $04 / 12 / 66$ \\
\hline ULD RO & CRD & 41 & $05 / 13 / 66$ \\
\hline UDD RO & CRD & 43 & $06 / 14 / 66$ \\
\hline SUDO RO & CRD & 42 & $07 / 15 / 66$ \\
\hline SUDO RO & CRD & 34 & $08 / 11 / 66$ \\
\hline BUD RO & CRD & 36 & $09 / 15 / 66$ \\
\hline BUDD RO & CRD & 37 & $10 / 14 / 66$ \\
\hline BUDD RO & CRD & 13 & $02 / 15 / 66$ \\
\hline BUDD RO & CRD & 11 & $11 / 14 / 66$ \\
\hline BUDD RO & CRD & 11 & $12 / 14 / 66$ \\
\hline BLDD RO & CRD & 17 & $01 / 16 / 67$ \\
\hline BUDO RO & CRD & 13 & $03 / 11 / 66$ \\
\hline BUDD RO & CRD & 12 & $04 / 14 / 66$ \\
\hline BLDD RO & CRD & 13 & $05 / 13 / 66$ \\
\hline
\end{tabular}


Page No. 44

$04 / 28 / 93$

LIST OF HANFORD-GENERATED CLASSIFIED

DOCUMENTS DATED BETUEEN 1/1/61 AND 12/31/72

DOC. NO.

BNHL-CC-0474 5

BNWL-CC-0474 6

BNUL-CC-04747

BNHL-CC-04748

BNWL-CC-04749

BAHL-CC-0473 1

BNWL-CC-0475 10

BNWL-CC-0475 11

BNUL-CC-0475 12

BNHL-CC-0475 2

BNUL-CC-0475 3

BNHL-CC-0475 \&

BAWL-CC-0475 5

BNUL-CC-0475 6

BNWL-CC-0475 7

BNWL-CC-04758

BNWL-CC-0475 9

BNWL-CC-0492

BNWL-CC-0492 RD

BNWL-CC-0507

BNWL-CC-0572

BNHL-CC-0583 1

BNHL-CC-0583 2

BNHL-CC-0583 3

BNHL-CC-0583 4
TITLE

COMPOSITION OF ENDING INVENTORY MAY 1966 COMPOSITION OF ENDING INVENTORY JUNE 1966

COMPOSITION OF ENDING INVENTORY JULY 1966

COMPOSITION OF ENDING INVENTORY AUGUST

1966

COMPOSITION OF ENDING INVENTORY

SEPTEMBER 1966

WEAPONS RESEARCH AND DEVELOPWENT SS

MATERIAL PROJECTS, JANUARY 1966

MEAPONS RESEARCH AMD DEVELOPMENT SS

MATERIAL PROJECTS, OCTOBER 1966

WEAPONS RESEARCH AND DEVELOPMENT SS

MATERIAL PROJECTS, NOVEMBER 1966

MEAPONS RESEARCH AMD DEVELOPMENT SS MATERIAL PROJECTS, DECEMBER 1966

WEAPONS RESEARCH AMD DEVELOPMENT SS MATERIAL PROJECTS, FEBRUARY 1966

WEAPONS RESEARCH AMD DEVELOPMENT SS

MATERIAL PROJECTS, MARCH 1966

WEAPONS RESEARCH AND DEVELOPMENT SS

MATERIAL PROJECTS, APRIL 1966

WEAPONS RESEARCH AND DEVELOPMENT SS

MATERIAL PROJECTS, MAY 1966

WEAPONS RESEARCH AND DEVELOPMENT SS

MATERIAL PROJECTS, JUNE 1966

WEAPONS RESEARCH AND DEVELOPMENT SS

MATERIAL PROJECTS, JULY 1966

VEAPONS RESEARCH AND DEVELOPMENT SS

MATERIAL PROJECTS, AUCUST 1966

WEAPONS RESEARCH AND DEVELOPNENT SS

MATERIAL PROJECTS, SEPTEMBER 1966

COMPARISON OF UPSTREAM AMD DOWSTREAM

RIVER TEMPERATURES - 1964-1965

COMPARISON OF UPSTREAM AND DOMNSTREAM

RIVER TEMPERATURES - 1963-64 AND

1964-1965 HATER YEARS

FORECAST OF SPECIAL NUCLEAR MATERIAL

REQUIREMENTS JULY 1, 1966 TMRU JUNE 30, 1967

LETTER - W. A. SHYDER TO CAPT. R. HAUVER QUARTERLY INVENTORY REPORT, L-32500 AS OF MARCH 1966

QUARTERLY INVENTORY REPORT, L-32500 AS

OF JUNE 1966

QUARTERLY INVENTORY REPORT, L-32500 AS OF SEPTEMBER 1966

QUARTERLY INVENTORY REPORT, L-32500 AS OF DECEMBER 1966

\begin{tabular}{|c|c|c|c|}
\hline AUTHOR & LEVEL & PGS & DATE \\
\hline $\begin{array}{l}\text { BLDD RO } \\
\text { BUDD RO }\end{array}$ & CRD & 13 & $\begin{array}{l}06 / 14 / 66 \\
07 / 15 / 66\end{array}$ \\
\hline DOOD KO & CRD & 15 & 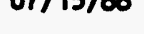 \\
\hline BUDD RO & CRD & 11 & $08 / 10 / 66$ \\
\hline BUDD RO & CRD & 11 & $09 / 16 / 66$ \\
\hline BUDD RO & CRD & 11 & $10 / 14 / 66$ \\
\hline BUDD RO & CRD & 4 & $02 / 15 / 66$ \\
\hline BUDD RO & CRD & 3 & $11 / 14 / 66$ \\
\hline BLDD RO & CRD & 4 & $12 / 13 / 66$ \\
\hline BUDD RO & CRD & 3 & $01 / 13 / 67$ \\
\hline BUDD RO & CRD & 4 & $03 / 11 / 66$ \\
\hline BUDD RO & CRD & 4 & $04 / 12 / 66$ \\
\hline BUDD RO & CRD & 4 & $05 / 13 / 66$ \\
\hline BUDD RO & CRD & 4 & $06 / 15 / 66$ \\
\hline BUDD RO & CRD & 4 & $07 / 15 / 66$ \\
\hline BUDD RO & CRD & 3 & $08 / 11 / 66$ \\
\hline BUDD RO & CRD & 3 & $09 / 16 / 66$ \\
\hline BUDD RO & CRD & 2 & $10 / 14 / 66$ \\
\hline CORLEY JP & CRD & 10 & $04 / 08 / 66$ \\
\hline CORLEY JP & CRD & 4 & $02 / 10 / 66$ \\
\hline BUDD RO & CRD & 2 & $02 / 08 / 66$ \\
\hline SHYDER WA & CRD & 2 & $03 / 29 / 66$ \\
\hline BUDD RO & CRD & 2 & $04 / 12 / 66$ \\
\hline BUDD RO & CRD & 2 & $07 / 18 / 66$ \\
\hline BLDD RO & CRD & 2 & $10 / 13 / 66$ \\
\hline BUDD RO & CRD & 2 & $01 / 12 / 67$ \\
\hline
\end{tabular}


Page No. 45

$04 / 28 / 93$

LIST OF MANFORD-GENERATED CLASSIFIED

DOCUMENTS DATED BETWEEN 1/1/61 AND 12/31/72

DOC. NO

BNWL-CC-0592

BNWL-CC-0687

BNWL-CC-0780 RPT1

BNWL-CC-0780 RPT10

BNHL-CC-0780 RPT11

BNHL-CC-0807

BNHL-CC-0829 DRAFT

BNUL-CC-0839

BNHL-CC-0872

BNWL $-C C-1010$

BNHL-CC-1026 1

BNWL-CC- 10262

BNHL-CC-1027 6

BNWL-CC- 1121

BNWL-CC- 1161

BNHL-CC-1184

BNHL-CC- 12591

BNWL-CC- 125910

BNWL-CC-1259 11

BNWL-CC-1259 12

BNWL-CC-1259 2

BNWL-CC-1259 3

BNWL-CC- 12594

BNWL-CC-1259 5

BNWL-CC- 12596

BNHL-CC- 12597

BNWL-CC- 12598
TITLE

TRAMSFER MEASUREMENT STATISTIC DERIVED FROM PUREX PROCESSING MEASUREMENTS'

QUALITY CONTROL PROGRAM SECOND HALF OF 1965

INVENTION REPORT PROCESS FOR SEPARATION OF URANIUM ISOTOPES

PRCOUCTION INVENTORY AND MANUFACTURING

STATEMENT JULY 31, 1966

PRODUCTION INVENTORY AND MANUFACTURING

STATEMENT APRIL 30, 1967

PRODUCTION INVENTORY AND MANUFACTURING

STATEMENT MAY 31, 1967

INTER-REACTOR RIVER TEMPERATURE DATA

MOTES TAKEN AT meETING in aLBUaUERQUe, MM, SEPTEMBER 9, 1966

STATUS REPORT ON ATTENUATOR STUDIES

LETTER TO DR. LARRY QUINH

DRAHING H-2-64052 FOR MELDMENT

COAPOSITE OF ENDING INVENTORY - JANUARY 1967

COMPOSITE OF ENDING INVENTORY - FEBRUARY

1967

WEAPONS RESEARCH AND DEVELOPMENT - SS

MATERIAL PROJECTS - JUNE 1967

LETTER TO M JOHNSON, DOW-ROCKY FLATS

DRAHINGS FOR PILOT PLANT DEMONSTRATION

LETTER: CLASSIFICATION CUIDANCE

LETTER, BATTELLE-NORTHWEST REPORT

BNHL- 118

PROOUCTION INVENTORY AND MARUFACTURING

STATEMENT JUAE 30, 1967

PROOUCTION INVENTORY AND MANUFACTURING STATEMENT MARCH 31, 1968

PROOUCTION INVENTORY AND MANUFACTURING

STATEMENT APRIL 30, 1968

PROOUCTION INVENTORY AND MANUFACTURING

STATEMENT MAY 31, 1968

PRCOUCTION INVENTORY AND MANUFACTURING

STATEMEAT JULY 31, 1967

PRODUCTION INVENTORY AND MANUFACTURING STATEMENT AUGUST 31, 1967

PRCDUCTION INVENTORY AMD MANUFACTURIHG STATEMENT SEPTEMBER 30, 1967

PROOUCTION INVENTORY AND MANUFACTURING STATEMENT OCTOBER 31, 1967

PROOUCTION INVENTORY AND MANUFACTURING STATEMENT NOVEMBER 30, 1967

PROOUCTION INVENTORY AND MANUFACTURING

STATEMENT DECEMBER 31, 1967

PROOUCTION INVENTORY AND MANUFACTURING

STATEMENT JANUARY 31, 1968
AUTHOR

HOUGH CG

LEVEL PGS DATE

SRO

34

$03 / 31 / 66$

BRONELL LE

CRD

5

06/07/66

GRAY RE

SRD

15

$08 / 23 / 66$

GRAY RE

SRD

18

05/22/67

GRAY RE

SRD

16

$06 / 20 / 67$

CORLEY JP

SRD

SRD

$09 / 09 / 66$

ESSIG TH

O'KEEFE DP

TIMGEY GL

KING RR

BUDD RO

BUDD RO

BUDD RO

KING RR

CRD

SRD

CRD

CRD

68

$09 / 09 / 66$

$10 / 04 / 66$

$10 / 13 / 66$

$01 / 24 / 67$

$02 / 15 / 67$

$03 / 14 / 67$

$07 / 18 / 67$

03/22/67

SORENSON RJ

TIPTON CR

$04 / 14 / 67$

04/28/67

GRAY RE

$07 / 24 / 67$

GRAY RE

$04 / 22 / 68$

GRAY RE

GRAY RE

GRAY RE

GRAY RE

GRAY RE

GRAY RE

GRAY RE

GRAY RE

GRAY RE

$05 / 21 / 68$

$06 / 20 / 68$

$08 / 22 / 67$

$09 / 21 / 67$

$10 / 23 / 67$

$11 / 17 / 67$

$12 / 20 / 67$

$01 / 23 / 68$

SRD

$02 / 21 / 68$ 
Page No. 46

$04 / 28 / 93$

LIST OF HANFORD-GENERATED CLASSIFIED

DOCUMENTS DATED BETWEEN 1/1/61 AND 12/31/72

DOC. MO.

BNHL-CC-12599

BNWL-CC- 1260

BNUL-CC-1269

BNHL-CC- 1314

BMUL-CC-1328

BNUL-CC-1358

BNWL-CC-1408

BNHL-CC-1426

BNHL-CC- 1437

BNHL-CC- 1472

BNWL-CC-1483 AOPG

BNWL-CC-1512

BNWL-CC- 1579

BNHL-CC- 9580 AOPG

BWHL-CC-1600 AOPG

BNLL-CC-1634 AOPG

BNWL-CC- 1655

BNWL-CC-1684 AOPG

BNWL-CC-1738 AOPG

BNHL-CC-2019 AOPG

BNHL-CC-2027-5

BNHL-CC-2091

BNUL-CC-2139 RD

BNWL-CC-2161

BNUL-CC-2221 RD

BNHL-CC-2376

BNHL-CC-2407 RD
PRCOUCTION INVENTORY AND MANUFACTURING

STATEMENT FEBRUARY 29, 1968

DATA REPORT

GENERAL VEAPONS DEVELOPNENT - PLUTONIUM

METALLURGY SECTION MONTHLY REPORT - MAY, 1967

AN AMALYSIS OF HOT DIE SIZE FUEL BONDING LABORATORY STUDIES OF AL-NI-U BONDING INTERIM REPORT

SUMUARY OF THE AOPG BRAINSTORMING

SESSION OF JULY 25, 1967

MFC-12 STUDIES

MFC-12 STUDIES

FUNDING OF THE MFC- 12 PROJECT FOR THE REMAINDER OF FY 1968

LIMITED MARFARE

REPORT OF SPECIAL ACTIVITIES UNDER

MFC-12

MFC-12 PROGRN

CONTINUATION OF MFC-12 STLDIES

SPECIAL ACTIVITIES UNDER MFC-12 REVIEW

AND PROGRAM

EFFECTS OF RADAR BLACKOUT PLASTIS ON

OPTICAL BEAM PENETRATION

SPECIAL ACTIVITIES UNDER MFC-12 MONTHLY

REPORT MARCH 1968

DECLASSIFICATION REVIEN OF HH-72402

SPECIAL ACTIVITIES UNDER MFC- 12 MONTHLY

REPORT APRIL 1968

TRIP REPORT: VISIT TO LALRENCE RADIATION

LABORATORY, LIVERMORE, CALIFORMIA, ON JULY 9, 1968

FIMAL REPORT OF SPECIAL ACTIVITIES UNDER MFC- 12

KPR AS IS, ALTERNATE TUBE - 237-NP-AL

TARGET /IBH FORM COMPUTER OUTPUT/

CLASSIFICATION BULLETINS

SS MATERIAL AUDIT 68-1

5-69 COPE STUDIES

ENERGY BUDGET

PROPOSED RESEARCH PROGRAM: FLUORESCENCE SPECTRA AND LIFETIME MEASUREMENTS OF

RARE EARCH ELEMENTS IN SOLUTIONS

CONTAINIMG URANIUM OXIDE ION FOR IDENTIFICATION OF POSSIBLE LASSIMG SOLUTIONS

MAVAL REACTOR STUDY - MISCELLANEOUS DATA
AUTHOR

LEVEL PGS

DATE
GRAY RE

SCHNEIDER RA

STEWART, RH

GURMELL ME

GURLELL WE

SHIPPERT RE

NICHOLS LL, VALI $H$, KEDER SRD

IM. WILSON AS

VALI H

PAUL, RS

BENDER JH

REARDOW UA

MEELEY VI

WORLTON D, CORDON RL,

VALI $H$

REARDON UA

LESSOR D

REARDON UA

TIPTON CR

REARDON

REARDOW UA

REARDON WA

SHAVER RE

SORENSON R.

WOODRUFF HJ

RICHMOND WD

CORLEY JP

GORDON RL

SHAVER RE

$\begin{array}{lll}\text { SRD } & 20 & 03 / 20 / 68 \\ \text { CRD } & 3 & 06 / 20 / 67 \\ \text { SRD } & 9 & 05 / 31 / 67\end{array}$

CRD

CRD

CRD

22

08/01/67

$11 / 02 / 67$

07/01/67

$10 / 30 / 67$

$11 / 16 / 67$

$11 / 30 / 67$

$12 / 13 / 67$

$01 / 12 / 68$

$01 / 12 / 68$

$03 / 06 / 68$

$03 / 25 / 68$

$03 / 22 / 68$

$04 / 18 / 68$

05/10/68

$05 / 31 / 68$

$07 / 17 / 68$

02/01/70

01/03/69

$04 / 14 / 69$

$08 / 04 / 67$

06/05/69

$09 / 10 / 65$

CON $T$

24

$09 / 06 / 70$
CON

$01 / 20 / 70$ 
Page No. 47

$04 / 28 / 93$

LIST OF HANFORD-GENERATED CLASSIFIED

DOCUMENTS DATED BETWEEN 1/1/61 AND 12/31/72

Doc. No

BNHL-CC-2408 RD

BNML-CC-2409 RD

BNHL-CC-2497

BNHL-CC-2447 RD

BNUL-CC-2488 RD

BNWL-CC-2526

BNWL-CC-2663

BNHL-D-004

BNHL-D-023 RD

BNWL-D-087

BNHL-D-137

BNHL-D-140

C0-858

CON-65-00001

CON-70-00503

CON-72-00012

CON-SEC-69-00001 CON-SEC-71-00004

CPD-00069

CPD-00076

CPD-00109

CPD-00142

CPD-00144

CPD-00179

CPD -00187

CPD -00213

CPD-00218

CPD-00220ADD

CPD-00221

CPD-00232
TITLE

NUTHOR

SHAVER RE

SHAVER RE

MONTHLY REPORT FOR AOPG /SEP-68/ SR PRELIMINARY STUDY

LETTER TO JH DOYLE - DOU ROCKY FLATS

FLUX DATA - HANFORD REACTORS /IBM CARDS/

REACTOR OUTPUT - PRCOUCTION INFORMATION OCEAN NUCLEAR REACTOR PONER - PRESENT AND FUTURE

MUCLEAR MATERIALS MAMAGEMENT PLUTONIUM SHIPNENT DATA

MONDESTRUCTIVE MEASUREMENTS OF EXTRATERRESTRIAL MATERIAL

LETTER TO MR FP JOHANSEN - LRL REFERENCE SANL-815-007

RELATED SERVICES FEASIBILITY STUOY 300A00621

LETTER TO HE RAWSOM - AEC-RL INFORMATION PRESENTED DURING 10-4-72 VISIT TO 231-2

LETTER TO PG RHOADES, REF: TWX - MB BILES TO TA MEMZEK, OCTOBER 18, 1972 ANALYSIS OF DISSOLVER SOLUTIONS RECOVERY OF PLUTONIUN AND URANIUN FROM REACTOR FUEL METALLURGICAL SCRAP -ROUGH DRAFT-

N REACTOR SHUTDON AND COMAITTEE INVESTIGATION HILLIAMS/BARAMONSKI AMD BILES

RECOVERY OF PLUTONIUN SCRAP FROM PACIFIC MORTHLEST LABORATORY

INCIDENTS OF PHYSICAL SECURITY INTEREST

AEC MANUAL CHAPTER APPENDIX 2701 SECTION $G$

POSSIBLE SAVINGS FOR THORIA PROGRAM

RICHLAND UO3 SHIPMENTS

SHIPMENT OF U03 TO PADUCAH

PUREX PLANT APPRAISAL

SCHEDULED U03 SHIPMENTS TO FERNALD

APPRAISAL OF PU FINISHING OPERATIONS

PUREX NEPTUNIUN RECOVERY

UO3 SHIPMENTS TO PADUCAH

STATUS OF ORANGE OXIDE DELIVERIES -

DECEMBER 31, 1968

HANFORD RADIOACTIVE WASTE MANAGEMENT

PLANS.

TABLE I - MORMAL DEPLETED U IN STORAGE

-TABLE II - FORECAST UO-3 PROOUCTION \&

ESTIMATED U-235 \& U-230 CONTENT

LO3 RETURNS TO FERHALD

\begin{tabular}{|c|c|c|c|}
\hline AUTHOR & LEVEL & PGS & DATE \\
\hline SHAVER RE & SRD & $\begin{array}{l}\operatorname{con} \\
T\end{array}$ & $01 / 20 / 70$ \\
\hline SHAVER RE & SRD & $\begin{array}{l}\text { CON } \\
T\end{array}$ & $01 / 20 / 70$ \\
\hline $\begin{array}{l}\text { MAYS DO } \\
\text { KULCINSKI GL. }\end{array}$ & $\begin{array}{l}\text { SRD A } \\
\text { SRD }\end{array}$ & $\begin{array}{l}10 \\
\text { con } \\
T\end{array}$ & $\begin{array}{l}01 / 19 / 70 \\
02 / 01 / 70\end{array}$ \\
\hline SYMOGROUND IN & SRD & 19 & $04 / 01 / 70$ \\
\hline CONN PD & CRD & 20 & 11 \\
\hline FISHBAUGHER JR & SRO & 3 & $08 / 03 / 70$ \\
\hline Hociun MA & SWSI & 11 & $09 / 25 / 70$ \\
\hline KING RR & CRD & 7 & $12 / 08 / 70$ \\
\hline HAMSEN JE & SRD & 11 & $08 / 11 / 71$ \\
\hline KING RR & CNSI & 3 & $10 / 05 / 72$ \\
\hline DAVIDSON JM & SRD & 1 & $10 / 26 / 72$ \\
\hline $\begin{array}{l}\text { CHRISTY JT } \\
\text { WH SCHULZ }\end{array}$ & $\begin{array}{l}\text { CRD } \\
\text { CONF }\end{array}$ & 1 & $\begin{array}{l}07 / 05 / 66 \\
02 / 01 / 65\end{array}$ \\
\hline RO BRANCH & CONF & & $10 / 06 / 70$ \\
\hline RP CORLEY & CONF & & $10 / 13 / 72$ \\
\hline ERDA-RL & CNSI & & $05 / 21 / 69$ \\
\hline$A E C-R L$ & CHSI & & $11 / 04 / 71$ \\
\hline$A E C-R L$ & SECR & & $08 / 30 / 68$ \\
\hline$A E C-R L$ & SECR & & $09 / 06 / 68$ \\
\hline AEC-RL & SECR & & $10 / 09 / 68$ \\
\hline AEC-RL & SECR & & $11 / 04 / 68$ \\
\hline AEC-RL & SECR & & $11 / 08 / 68$ \\
\hline$A E C-R L$ & SECR & & $12 / 09 / 68$ \\
\hline AEC-RL & SECR & & $12 / 11 / 68$ \\
\hline AEC-RL & SECR & & $01 / 03 / 69$ \\
\hline AEC-RL & SECR & & $01 / 06 / 69$ \\
\hline AEC-RL-CPD & SECR & & $01 / 15 / 69$ \\
\hline AEC-RL & SECR & & $01 / 10 / 69$ \\
\hline A SCHWANKOFF & SRD & & $01 / 22 / 69$ \\
\hline
\end{tabular}

$01 / 22 / 69$ 
Page No. 48

$04 / 28 / 93$

LIST OF HANFORD-GEMERATED CLASSIFIED

DOCUMENTS DATED BETWEEN 1/1/61 AND 12/31/72

DOC. No.

CPD-00250

CPD-0025301

CPD-0025302

CPO-0025303

CPD-0025304

CPD-0025305

CPD -0025306

CPD-0025307

CPD-0025308

CPD-0025309

CPD-0025310

CPD-0025311

CPD-0025312

CPD-00261

CPD-00279

CPD -00300

CPD-00301

CPD-00312

CPD-003121

CPD -00329

CPD -00336

CPD-00339

CPD -00340

CPD -00344

CPD -0035001

CPD-0035002

CPD -0035003

CPD -0035004

CPD-0035005

CPD-0035006

CPD -0035007

CPD-0035008

CPD-0035009

CPD -0035010

CPD-0035011

CPD-0035012

CPD -00375

CPD -00378

CPD -00381

CPD -00390

CPD-00396

CPD-00398

CPD-05321
TITLE

AUTHOR

STATUS OF ORANGE OXIDE DELIVERIES -

JAMUARY 31, 1969

COB MONTHLY REPORT - JAN 1969

COB MONTHLY REPORT - FEB 1969

COB MONTHLY REPORT - MAR 1969

COB MONTHLY REPORT - APR 1969

COB MONTHLY REPORT - MAY 1969

COB MONTHLY REPORT - JUW 1969

COB MONTHLY REPORT - JUL 1969

COB MONTHLY REPORT - AUG 1969

COB MONTHLY REPORT - SEP 1969

COB MONTHLY REPORT - OCT 1969

COB MONTHLY REPORT - NOV 1969

COB MONTHLY REPORT - DEC 1969

SHIPMENT OF PLUTOWIUM TO SAVANMAH RIVER

STATUS OF ORANEE OXIDE DELIVERIES -

FEBRUARY 28, 1969

SHIPMENT OF UO-3 TO PADUCAH

SHIPMENT OF WO-3 TO FERMALP

ORAMGE OXIDE DELIVERY

ATTACHMENT RE ORANGE OXIDE DELIVERY REPORTS

UO-3 HOPPER SUPPLY \& CONTROL

PLUTONIUN PRCOUCT AND SCRAP STCRAGE

REVISED UO-3 SHIPMENTS TO FERMALD

RL APPRAISAL REPORT OF ATLANTIC

RICHFIELD HANFORD COMPANY CHEMICAL

OPERATIONS

DEPLETEO WO-3 SHIPMENT TO FERMALD

COB MONTHLY REPORT - JAN 1970

COB MONTHLY REPORT - FEB 1970

COB MONTHLY REPORT - MAR 1970

COB MONTHLY REPORT - APR 1970

COB MONTHLY REPORT - MAY 1970

COB MONTHLY REPORT - JUN 1970

COB MONTHLY REPORT - JUL 1970

COB MONTHLY REPORT - NUG 1970

COB MONTHLY REPORT - SEP 1970

COB MONTHLY REPORT - OCT 1970

COB MONTHLY REPORT - NOV 1970

COB MONTHLY REPORT - DEC 1970

FFTF BLEND PLAN

FFTF SUPPLY SPECIFICATION

WU GRAPH, PUREX U-233 PROOUCTION \&

SHIPPING SCHEDULE

PLUTONIUN PRODUCT STORAGE - FY-1971

APPRAISAL OF ARHCO - CHEMICAL OPERATIONS

W0-3 SHIPPING

EXTRACTION OF PLUTONIUN AND MEPTUNIUN

FROM AQUEOUS SOLUTIONS
AEC-RL

JT CHRISTY

JT CHRISTY

JT CHRISTY

JT CHRISTY

JT CHRISTY

JT CHRISTY

JT CHRISTY

JT CHRISTY

JT CHRISTY

JT CHRISTY

JT CHRISTY

JT CHRISTY

EP BARAMOUSKI

AEC-RL

AEC-RL

AEC-RL

AEC-RL

AEC-RL

AEC-RL

DG HILLIAMS

AEC-RL

OJ ELGERT

AEC-RL

JT CHRISTY

JT CHRISTY

JT CHRISTY

JT CHRISTY

JT CHRISTY

JT CHRISTY

JT CHRISTY

JT CHRISTY

JT CHRISTY

JT CHRISTY

JT CHRISTY

JT CHRISTY

AEC-RL

OJ ELGERT

AEC-RL

AEC-RL

OJ ELGERT

AEC-RL

WW SCHULZ
LEVEL PGS DATE

SECR 02/04/69

SECR

$02 / 11 / 69$

SECR

$03 / 14 / 69$

$04 / 15 / 69$

$05 / 09 / 69$

$06 / 12 / 69$

$07 / 11 / 69$

$08 / 11 / 69$

$09 / 12 / 69$

$10 / 14 / 69$

$11 / 14 / 69$

$12 / 10 / 69$

$01 / 13 / 70$

$02 / 14 / 69$

03/03/69

03/03/69

$04 / 03 / 69$

$05 / 29 / 69$

05/29/69

$08 / 20 / 69$

$10 / 10 / 69$

$10 / 28 / 69$

10/29/69

$12 / 11 / 69$

$02 / 11 / 70$

$03 / 13 / 70$

$04 / 13 / 70$

05/12/70

$06 / 15 / 70$

$07 / 13 / 70$

$08 / 17 / 70$

$09 / 15 / 70$

$10 / 19 / 70$

$11 / 23 / 70$

$12 / 16 / 70$

$01 / 14 / 71$

05/25/70

$06 / 16 / 70$

$08 / 31 / 70$

$08 / 31 / 70$

$11 / 03 / 70$

$11 / 20 / 70$

03/19/65 
Page No. 49

$04 / 28 / 93$

LIST OF HANFORD-GENERATED CLASSIFIED

DOCUNENTS DATED BETWEEN 1/1/61 AND 12/31/72

Doc. No.

CPD- 05331

CPD-05335

CPD-220 ADD

DUW-00013

DUN-00033 RD BK4

DUN-00038 RD

DUW-00040 RD DUN-00041RD

DUN-00060

DUW-00123

DUN-00130

DUN-00162RD

DUN -00180

DUN-00189RD

DUN-00200

DUN-00201

DUN-00203RD

DUN-00211

DUN-00217

DUN-00233

DUN-00234

DUN -00247

DUN-00264F

DUN-00264G

DUN-00271
TITLE

NUTHOR

PATENT AEC CASE S-35, 174 - MHIR 1874 LETTER, GEORCE H. LEE TO RICHARD C. ENMIS, SUBJ: AEC CASE S-35,174 MUIR- 1874

hANFORD RADIOACTIVE MMAGEMENT PLAMS

B-C REACTOR MUNAGEMENT REPORTS FROM

11/1/65 THRU JANUARY 1969

"KE" REACTOR OUTACE TIME ACCOUNTABILITY

11/1/65 THROUBH -.....

REACTOR OPERATING STATISTICS CUMULATIVE CALEMDAR EFISCAL YEAR 11-1-65 THRU

MISCELLAMEOUS BOOK 11/1/65 THRU -..REACTOR OPERATING BOOK 11/1/65 THRU ...REACTOR DATA

PRCOUCTION REPORT - DUN PROOUCTION

OPERATION 11-1-65 THRU 6-30-66

WATER PLANT CAPACITIES 100-KE AND 100-KM

LETTER, CE BOWERS TO TW MAUFF SECURITY

CLASSIFICATION - HW-84219 RD

ENGINEERING SPECIFICATIONS HOT DIE

SIZING FUELS FABRICATION PROCESS

KW OUTAGE TIME ACCOUNTABILITY REPORT

STATUS REPORT OF CONFINEMENT STUDIES DUN OPERATED REACTORS

MAXIMUN U-235 CONCENTRATION FOR

COPROOUCTS

MONTHLY MANAGEMENT REPORTS PRODUCTION

PLANNING \& SCHEDULING UNIT NOVEMBER

1965 THRU APRIL 1979

PRESSURE MONITOR SYSTEM AMALYSIS \&

PERFORMANCE CRITERIA B, C, D, KE \& KH REACTORS

MANUFACTURING PROCESS SPECIFICATIONS

METALLIC URANIUM FUEL ELEMENTS

FABRICATED BY THE HOT DIE SIZING

PROCESS

N PRCOUCTION DATA NOTES

U-236 CONCENTRATIONS AVAILABLE FOR THE

HANFORD SHALL REACTOR \& $K$ REACTORS

SEMIANMUAL RESEARCH AMO DEVELOPMENT

REPORT DOUGLAS UNITED MUCLEAR,

INC.

CHARGE-DISCHARGE SUMUARY 105-KE-FY 1970, 7/1/69 THRU 6/30/70

CHARGE-DISCHARGE SUMARY, 105-KE, FY 1971, 7/1/70 - 6/30/71

REACTOR UNIT COST SUMMARY FY 1969 THRU 4 MONTHS OF FY 1966
GH LEE

AEC-RL

SECR

SECR

$05 / 22 / 67$

01/16/68

ELGERT DJ, RUHLMN CW, VOILAND EE, MARREN JH AR MAUIRE

SRD

8

$01 / 15 / 69$

SRD

$11 / 01 / 65$

DL DEENEAL

SECR

$11 / 01 / 65$

DL DEMEAL

SRD

$11 / 01 / 65$

DL DENEAL

CRD

$11 / 01 / 65$

$11 / 01 / 65$

05/25/79

11/01/65

DUN-RL

RW Baw

SECR

SRD

RM SCOTT

CE BOWERS

CRD

$11 / 05 / 65$

$11 / 22 / 65$

ADVAMCED PROCESS

SECR

01/04/66

ENGINEERING UNIT

CRO

$04 / 08 / 71$

$08 / 19 / 71$

OC SCHROEDER

SRD

$11 / 18 / 65$

T PRUDICH

SRD

11/01/65

TM CLEMENT, PA CROSETTI CRD

$02 / 28 / 66$

PROOUCTION FUELS SECTION CRD

$12 / 01 / 65$

RP RONE

SRD

SECR

$03 / 08 / 68$

$08 / 30 / 72$

TH MHBRose

CRO

$11 / 30 / 65$

SECR

$11 / 04 / 71$

RP ROWE

SRD

$07 / 01 / 70$

SECR

$06 / 09 / 71$ 
DOC. NO.

DUW-00271REV1

DUN-00276

DUN-00315RD

DUN-00317

DUN-00353

DUN-00355RD

DUN-00403RD30

DUN-00403RD31

DUN-00403RD32

DUN-00403RD33

DUN-00403RD34

DUN-00403RD35

DUN-00403RD36

DUN-00403RD37

DUN-00405

DUN-004065

DUN-00407

DUN-00413

DUN-00416

DUW-00416

DUN-00416RD

DUN-004 18

DUN-00419

DUN-00430

DUN-00432

DUN-00446

DUN-00446SUP1

DUN-00447

DUN-00447

DUN-00455

DUN-00456

DUN-00476 RD5
TITLE

AUTHOR

REACTOR UNIT COST SUMARY FY 1960 ESTIMATED FY 1967

MOTES ON FORECASTING SCHEDULING \&

MUCLEAR MATERIALS

IRRAD AMALY PT IP-691 SUP C IRRAD OF

W-U02 OR W-P 02, PLATE CAPSULES IN THE

KE OR KW SWOUT FACILITIES

MUCLEAR MATERIALS PROOUCTION REPORT

MOVEMBER 1965

CLASSIFICATION - PONER LEVEL CAPABILITY

COMIENT ISSUE RUPTURE COST AMALYSIS

105-KW OUTACE DATA BK 10, 11 \& 12-68

105-KH OUTACE DATA BK 1, 2-69

OUTACE DATA BK 3-1-69/4-30-69

CUTACE DATA BK 5-69/6-30-69

OUTACE DATA BK 7-69/8-69

OUTAGE DATA BK 9-69/10-69

OUTACE DATA BK 11-69/12-69

OUTACE DATA BOOK 1/70 - 2/70

PRESENTATION TO THE ADVISORY COMAITTEE

ON REACTOR SAFECUARDS 9/10/65 AT MANFORD FMPC SHIPPIMG LIST

FUEL MELTING TIME FOLLONING A LOSS OF

COOLANT

RESULTS OF 8/2/65, 190-8 DRALDOWN TEST

FUEL AND TUBE PERFORMANCE MEMORAMDUM

NOVEMBER 1965

PROPOSAL FOR MGT OF FACILITY CAPABILITY PROJECT-SAFETY OF HANFCRD PRODUCTION REACTORS UTILIZING HIGHLY ENRICHED FUEL PROPOSAL FOR MGT OF FACILITY CAPABILITY PROJECT-SAFETY OF HANFORD PROOUCTION REACTORS UTILIZING HIGHLY ENRICHED FUEL E-N CORE CONVERSION DATA

APPROX. OPERATIMG-RAMGE DATA FOR APRIL STUDIES

BUDGET STLOY HELIUM PURIFICATION UNIT MONTHLY RPT 12/28/65

APPROX. OPERATING-RAMGE DATA FOR APRIL STUDIES

SUPPLEMENTARY OPERATING-RANGE DATA FOR

SPECIAL PRCOUCTS - APRIL STUDIES

SAFETY TRIP LIMITS FOR THE HIGH SPEED SCANNER - K REACTORS

SAFETY TRIP LIMITS FOR THE HIGH SPEED SCANMER $K$ REACTORS

DUN, INC. RPT TO THE WORKING COMITTEE OF THE FEDC

IMCENTIVES ZIRCONIUM FUEL JACKETS

MATER TREATMENT DAILY LOG 900-KM

\begin{tabular}{|c|c|c|}
\hline \multirow[t]{2}{*}{ AUTHOR } & LEVELL POS & DATE \\
\hline & SECR & $06 / 10 / 71$ \\
\hline \multirow[t]{2}{*}{ T PRLDICH } & SECR & 12/01/65 \\
\hline & SECR & $08 / 19 / 71$ \\
\hline \multirow[t]{9}{*}{ T PRUDICH } & SRD & $12 / 09 / 65$ \\
\hline & SECR & 12/13/65 \\
\hline & SECR & $12 / 13 / 65$ \\
\hline & SECR & $04 / 09 / 71$ \\
\hline & $\begin{array}{l}\text { SECR } \\
\text { SECR }\end{array}$ & $\begin{array}{l}04 / 09 / 71 \\
04 / 09 / 71\end{array}$ \\
\hline & SECR & $04 / 09 / 71$ \\
\hline & SECR & $04 / 09 / 71$ \\
\hline & SECR & $04 / 09 / 71$ \\
\hline & SECR & $04 / 09 / 71$ \\
\hline \multirow{2}{*}{ JR SPINK } & CRD & $\begin{array}{l}04 / 00 / 71 \\
12 / 31 / 65\end{array}$ \\
\hline & SECR & $03 / 02 / 72$ \\
\hline \multirow[t]{2}{*}{ DE BUSH } & CRD & $01 / 03 / 66$ \\
\hline & SECR & $12 / 21 / 65$ \\
\hline \multirow{10}{*}{ RD JENSEN } & & \\
\hline & SECR & $12 / 29 / 65$ \\
\hline & SECR & $12 / 21 / 65$ \\
\hline & SECR & 12/22/65 \\
\hline & SECR & $12 / 22 / 65$ \\
\hline & SECR & $12 / 28 / 66$ \\
\hline & SECR & $12 / 28 / 65$ \\
\hline & & \\
\hline & SECR & $02 / 18 / 66$ \\
\hline & SECR & $02 / 15 / 66$ \\
\hline \multirow[t]{3}{*}{ JP HAMRIC } & CRD & $02 / 15 / 66$ \\
\hline & SECR & $01 / 04 / 66$ \\
\hline & SECR & $06 / 11 / 71$ \\
\hline JW MCLAUGHLIH & SECR & $08 / 01 / 68$ \\
\hline
\end{tabular}


Page No.

$04 / 28 / 93$

LIST OF HANFORD-GENERATED CLASSIFIED

DOCUNENTS DATED BETWEEN 1/1/61 AND 12/31/72

Doc.

DUN-00492

DUN-00500

DUN-00504

DUN-0052454

DUW-00529RD

DUN-00538RD

DUN-00546

DUN-00580

DUN-00584

DUN-00603RD

DUN-00604

DUN-00605

DUW-00605NDD 1

DUW-00612

DUN-00616

DUN-00619

DUN-00620

DUN-00628

DUN-00630

DUN-00635SUP 1

DUN-00643

DUN-00645

DUN-00647

DUN-00648RD

DUN-00650

DUN-00651

DUN-00660

DUN-00665
TITLE

NUTHOR

PRODUCTION STATISTICS VS FORECAST JAN

THRU JUW 1966

PRCOUCTION - CY 1965

NEUTRON BENM FACILITY TECHMICAL REPORT

STATUS OF IRRADIATIONS PERFORMED BY

REACTOR PHYSICS \& IRRADIATION TECHNOLOCY

AS of 12/31/70

K PLANT DATA

P ENRICHMENT CONCEPT

MUCLEAR MATERIALS PROOUCTION REPORT

DECEMBER 1965

DUN PIEM STATENENT 12/31/65 \& FY TO DATE

MIXED BUMPER \& MONBUMPER FUEL CHARGES B,

C, D, KE \& KU REACTORS

FUEL PERFORMANCE INFORMATION

VISIT MITH AEC IN GERMANTOW

D DOANCOMER DATA

D REACTOR DOMMCONER DATA

ECONOMIC CONSEQUENCES OF ELEVATED

GRAPHITE TEMPERATURES B \& D REACTORS

A REVIEN OF THE COOLANT FLON DECAY

ASSUMPTION USED IN CONTROL SYSTEM

ANALYSES

JUSTIFICATION FOR DISCHARGE CHUTE

CLEARING EQUIPMENT - B \& D REACTORS

JUSTIFICATION FOR DISCHARGE CHUTE

CLEARING EOUIPHENT - $K$ REACTORS

CARBON DIOXIDE FOR PH NDJUSTMENT, 100-KE

8 KW

BUDGET STUDY PROCESS MATER TREATMENT FOR REACTOR EFFLUENT ACTIVITY CONTROL

FIZIKR - INPUT DATA PACK \& SAMPLE

CALCULATION

MUCLEAR MATERIALS PRCOUCTION REPORT JAMUARY 1 THRU "JUNE 30, 1966

MECHANICAL PROPERTIES IRRADIATED

ALUNINUM REACTOR PROCESS TUBING

PERICOIC COMPETITOR \& MARKET DEVELOPMENT

RPT

UNUSUAL OPERATION - KE REACTOR JAN 16 TO JAN 20, 1966

BUDGET STUDY COMMENTS CHARGE-DISCHARGE

DURING OPERATION 100-K

FY 68 \& REVISED FY 67 PACE BUDGET -

DISCHARGE CHUTE CLEARIMG ECUIPMENT, 105-B, D, KE \& KW REACTORS

OPTIMIZATION OF COBALT-6O PRCOUCTION IN

THE HANFORD TESTING FACILITIES

TRIP RPT - JAN 18 \& 19, 1966 - CASE

GEMERATOR MEETIMG IN OAK RIDGE

\begin{tabular}{|c|c|c|}
\hline \multirow[t]{5}{*}{ AUTHOR } & LEVEL PGS & DATE \\
\hline & SECR & $04 / 09 / 71$ \\
\hline & SECR & $01 / 10 / 66$ \\
\hline & SECR & $11 / 29 / 65$ \\
\hline & SECR & $06 / 10 / 71$ \\
\hline \multirow[t]{2}{*}{ RE DUWN } & SECR & $01 / 12 / 66$ \\
\hline & SECR & $01 / 13 / 66$ \\
\hline \multirow[t]{13}{*}{ T PRLDICH } & SRD & $01 / 14 / 66$ \\
\hline & SECR & $07 / 06 / 71$ \\
\hline & & \\
\hline & SECR & $07 / 06 / 71$ \\
\hline & SECR & $01 / 21 / 66$ \\
\hline & SECR & $01 / 26 / 66$ \\
\hline & SECR & $02 / 18 / 66$ \\
\hline & & $02 / 02 / 00$ \\
\hline & SECR & $01 / 27 / 66$ \\
\hline & SECR & $01 / 28 / 66$ \\
\hline & SECR & $01 / 28 / 66$ \\
\hline & SECR & $01 / 29 / 66$ \\
\hline & SECR & $06 / 11 / 71$ \\
\hline PD GROSS & SRD & $03 / 03 / 66$ \\
\hline \multirow[t]{3}{*}{ T PRUDICH } & SRD & $02 / 03 / 66$ \\
\hline & SECR & $02 / 10 / 66$ \\
\hline & SECR & $02 / 14 / 66$ \\
\hline DUW-RL & SECR & $02 / 03 / 66$ \\
\hline \multirow[t]{2}{*}{ KL. HLADEK } & SECR & $02 / 04 / 66$ \\
\hline & SECR & $02 / 01 / 66$ \\
\hline \multirow[t]{2}{*}{ MH HONTCOMERY } & SECR & $02 / 02 / 66$ \\
\hline & SECR & $02 / 10 / 66$ \\
\hline
\end{tabular}


DOC. NO.

TITLE

DESICN OF THE 7/8 INCH REAR FACE

FLEXIBLE CONECTOR FOR B \& D REACTORS MODIFICATION OF ORIFICE PATTERN \& DESIGN D-REACTOR

CASE GENERATOR EVALUATOR SYSTEM PROOUCTION ESTIMATE FOQ USE IN APRIL COST STUDIES

DUN-00693

DUN-00697

DUW-00700

OUw-00704

DUN-0070606

DUN-00714

DUN-00715

DUN-00727

DUN-00728

DUN-00729

DUN-00740

DUN-00741

DUN-00747

DUN-00750

DUN-00751

DUN-00753

DUN-00756

DUN-00767

DUN-007T7

DUN-00782

DUN-00809
FUEL AMD TUBE PERFORMANCE MEMORAMDUM JAMUARY 1966

QUDGET FACILITY DESCRIPTION FY 1968 REV OF FY 1967 PACE BUDGET TARGET ELEMEAT CHARGE DURING OPERATION 100-K REACTORS

CARBOW DIOXIDE VERSUS NITROGEN AT $K$ REACTOR

B, $C$ \& D DOWNCONER CCOLANT PRESSURE \& FLON LIMITATIONS

DUN PIEM STATEMENT 06/30/66

STUCK FUEL ELEMENT FAILURE FREQUENCY

DURING THE PERIOD OF IMPROVED EAMM

MONITOR FACILITIES AT KE \& KW REACTORS

FUEL ELEMENT FLUSHING POTENTIAL BY

DIESEL PUMP STARTS - B \& C REACTORS

KLE FUEL - PHYSICS AND ENGIMEERIMG

PARAMETERS FROMMOFDA COMPUTER CODE RUNS

K4N FUEL - PHYSICS AND ENGIMEERIMG

PARAMETERS FROMHOFDA COMPUTER COOE RUNS

FUEL FAILURE DATA 9/1/65 - 12/31/65

TOTAL CONTROL. TEMPERATURE EFFECT CURVES

IRRADIATION OF TARGET MATERIAL IN

K-REACTOR SPLINE CAP TUBES

BUDGET STUDY TARGET ELEMENT CHARGIMG

DURIMG OPERATIONS, 100-K REACTORS, FY-1968 \& REV OF FY-1067 PACE BUDEET FUEL FAILURE LOT KY-928-Q - D REACTOR PRELIMINARY ENGR RPT \& JUSTIFICATION COOLANT BACKUP 100-D AREA

TEMP \& FLON DATA EBUR FUEL ROO BUNDLES, C REACTOR 2/6/65 TO 1/1/66

THE EFFECTS OF INLET MATER TEMP

FLUCTUATION AT D REACTOR

REACTOR STARTUP PHYSICS

INTERLABORATORY MEUTRON FLUX SPECIAL.

MEASUREMENT PROGRAM

FEASIBILITY OF PRCOUCING RADIOISOTOPES

IN TEST FACILITIES 0074 \& 0065 IN A K REACTOR

K5E FUEL - PHYSICS AMD ENGINEERING PARAMETERS FROM MOFOA COMPUTER COOE RUNS

\begin{tabular}{|c|c|c|c|}
\hline AUTHOR & LEVEL & PGS & DATE \\
\hline \multirow{4}{*}{ DK CLEM } & SECR & & $02 / 08 / 66$ \\
\hline & SECR & & $02 / 17 / 66$ \\
\hline & SECR & & $02 / 15 / 65$ \\
\hline & SECR & & $02 / 15 / 66$ \\
\hline \multirow[t]{2}{*}{ AK HARDIN } & CRD & & $02 / 16 / 66$ \\
\hline & SECR & & $02 / 16 / 66$ \\
\hline \multirow[t]{5}{*}{ JP COOKE } & CRD & & $02 / 17 / 66$ \\
\hline & SECR & & $02 / 17 / 66$ \\
\hline & SECR & & $07 / 06 / 71$ \\
\hline & SECR & & $02 / 18 / 66$ \\
\hline & SECR & & $02 / 18 / 66$ \\
\hline ER PERL & SRD & & $02 / 21 / 66$ \\
\hline \multirow[t]{9}{*}{ ER PERL } & DID & & $02 / 21 / 66$ \\
\hline & SECR & & $02 / 23 / 66$ \\
\hline & SECR & & $02 / 23 / 66$ \\
\hline & SECR & & $02 / 23 / 66$ \\
\hline & SECR & & $01 / 27 / 66$ \\
\hline & SECR & & $02 / 25 / 66$ \\
\hline & SECR & & $03 / 15 / 66$ \\
\hline & SECR & & $02 / 25 / 66$ \\
\hline & SECR & & $02 / 28 / 66$ \\
\hline \multirow[t]{3}{*}{ GF BAILEY } & CONF & & $03 / 01 / 66$ \\
\hline & SECR & & $03 / 07 / 66$ \\
\hline & SECR & & $03 / 01 / 66$ \\
\hline ER PERL & SRD & & $03 / 11 / 66$ \\
\hline
\end{tabular}


Page No. 53

$04 / 28 / 93$

LIST OF HANFORD-GENERATED CLASSIFIEO

DOCUMENTS DATED BETWEEN 1/1/61 AND 12/31/72

DOC. NO.

DUN-00818

DUH -00822

DUN-00823RD

DUN-00825

DUN-00832

DUN-00833

DUN-00838

DUN-00840

DUN-00847

DUN-00849

DUN-00849REV

DUN-0085001

DUN-0085002

DUN-0085003

DUN-0085004

DUN-0085005

DUN-00855RD

DUN-00856

DUN-00857RD

DUN-00899

DUN-00909

DUN-00903

DUN-00910

DUN-00912

DUN-00921

DUN-00922

DUN-00933
TITLE

SHIELD PROTECTION LONDINGS

CLASSIFIED NOTES ON ACCOUNTABILITY

COMPARISON OF VARIOUS $K$ REACTOR

DISCHARGE SCHEMES

EFFECT OF REAR HARDWARE MODS ON B

REACTOR OPERATION

HARDWARE MOO FLOW IMCREASES D-REACTOR

AMALYSIS \& CONTROL OF THE "PIMHOLE" MELD

DEFECT SITUATION

FUEL \& TUBE PERFORMANCE MEMORANDUN

FEBRUARY 1966

CHARTS USED IN MARCH 3 MEETIMG MITH $F$.

P. BARAMOUSKI \& E. E. FOWLER

JUSTIFICATION FOR INCREASED PUMPING

CAPACITY 181 B/C RIVER PUMPHOUSE

STUDY OF APPLICATIONS FOR HANFORD

PRCOUCED COLBALT-60

STUDY OF APPLICATIONS FOR HANFORD

PRCDUCED COLBALT-60

MANUFACTURING SECTION SEMIANHUAL SUMARAY

RPT - PERIOD ENDING JUNE 30, 1966)

MANUFACTURING SECTION SEMIANMUAL SUMMARY

RPT - PERIOD ENDING DEC 31, 1966)

MANUFACTURING SECTION SEMIANMUAL SUMAARY RPT - PERIOD ENDING JUN 30, 1967)

MANUFACTURING SECTION SEMIAMNUAL SUMMARY RPT - PERIO0 ENDING DEC 31, 1967)

MANUEACTURING SECTION SEMIANMUAL SUMAARY RPT - PERIOD ENDING JUN 30, 1968)

DESIGN CRITERIA FOR COOLANT BACKUP 100-D AREA

FACTORS INFLUENCING REACTOR LIFE

PREDICITED COSTS OF ZIRCONIUNA FUEL

CLADDING FOR DUN OPERATED HANFORD

REACTORS (PRESENT LEVELS OF OPERATION)

BRIEF REVIEW OF POTENTIALITIES FOR GAS

PURIFICATION AT $K$ REACTORS

SPECIAL IRRADIATIONS HANDBOOK

FUEL USACE AUTHORIZATIOH - 01

IRRADIATION OF SUBSTANDARD FUEL ELEMENTS

CASE CASES SUBMITTED MARCH 30, 1966

PREVIEW \& RESPONSIBILITIES FOR THE

"HONITOR COLUNAS" PT

ABSTRACT A DESCRIPTION OF IIN

TEMPERATURE HYDRIDING OF ZIRCALOY AT

HANFORD

INTERIM RPT - EXPLORATORY COBALT

IRRADIATIONS

DIFFERENCE BETWEEN PRCOUCTION FORELAST \& BASE CASE STUDY
AUTHOR

ER PERL

SECR

SRD

SECR

SECR

SECR

SECR

PD GROSS

CRD

$03 / 17 / 66$

SECR

$03 / 30 / 66$

SECR

$03 / 24 / 66$

SECR

$04 / 26 / 66$

SECR

$04 / 26 / 66$

SECR

09/01/66

SECR

$01 / 31 / 67$

SECR

$09 / 15 / 67$

SECR

$03 / 15 / 68$

SECR

$09 / 01 / 68$

LB BRINKMAN

CRD

$04 / 07 / 66$

SECR

SECR

$03 / 25 / 66$

03/25/66

SECR

$03 / 23 / 66$

SECR

$06 / 15 / 66$

SECR

$04 / 27 / 66$

HG SPENCER

SRD

$04 / 08 / 66$

03/30/66

SECR

$03 / 31 / 66$

SECR

$04 / 01 / 66$

SECR 
Page No. 54

$04 / 28 / 93$

LIST OF HANFORD-GENERATED CLASSIFIED

DOCUMENTS DATED BETWEEN 1/1/61 AND 12/31/72

Doc. NO.

DUN-00934

DUN-00939RD

DUN-00942

DUN-00943

DUN-00961

DUN-00968

DUH -00970

DUN-00976 04

DUN-00980

DUN-00985

DUN-01013

DUW-01016

DUN-01016

DUN-01017

DUN-01017 RD

DUN-01023

DUN-01032

DUN-01033

DUN-01050

DUN-01056

DUN-01060

DUN-01061

DUN-01062RD

DUN-01065

DUN-01069

DUN-01069EX

DUN-01070

DUN-01082

DUN-01085RD

DUN-01086RD

DUN -01090
TITLE

HOT DIE SIZING DEVELOPMENT PROGRAM

SCHEDULE

OUTER SIX VSR CHANNELS AT K-REACTORS

FUTURE COURSES OF ACTION

REACTOR WATER LEAKS CY-1964 \& 1965

ANALYSIS OF PROVEN TECHMOLOGY CASES

ALTERNATE CO-60 PRCOUCTION METHODS

SOME REACTOR PHYSICS ASPECTS OF BALL 3X

FLOW RATES - C AND D REACTORS

GAS EVOLUTION IN REACTOR COOLAAT

FUEL ELEMENT LOCALIZED CORROSION

\section{EXAMINATION D REACTOR}

PRODUCTION - MITH \& HITHOUT PROJECTIONS

4/1/66 THRU 12/31/67

INTERIM PERFORMANCE REVIEW, FY 1966

PROPOSED SYS FOR OPERATION OF THE

VSR-HCR CONTROL SYS \& POWER MONITORING

INSTRUNATS B, C, \& D REACTORS

PCCF FLUSH - B \& D REACTORS, SAFETY

ASPECTS

PCCF FLUSH B \& D REACTORS SAFETY ASPECTS

MEETING MINUTES ON UO2 RUPTURE

INVESTIGATION 2/2/66

MEETING MINUTES ON 102 REPTURE

INVESTIGATION FEBRUARY 28, 1966

HIGH EXPOSURE P FOR ZPPR

CHARTS USED IN APRIL 6 MEETING WITH F.

P. BARANOUSKI

PROJECT PROPOSAL DISCHARGE CMUTE

CLEARING EQUIPMENT, K REACTORS

UNATTENDED COOLING FOR HANFORD

PROOUCTION REACTORS

CLASSIFICATION - REACTOR POUER LEVELS

DISCUSSIONS WITH F.P. BARAMOUSKI - MAY

5, 1966

PROOUCTION TEST AUTHORIZATION -031, IRR

OF $M-L 02 \& N-L O 2$ FUELS PINS IN KE \& KW REACTORS TISR-701

IRR AMALY PRCDUCTION TEST AUTHORIZATION

-032, ETC.

COST OF RUPTURES

RICHLAND ISOTOPE PRODUCTION MATRIX

TABLE 5 - REACTOR PROOUCTION CAPABILITY

REACTOR MOOERNIZATION TEST FACILITY

FUEL \& TUBE PERFORHANCE MEMORANDUM APRIL 1966

WORK SHEETS

WORK SHEETS

REACTIVITY TESTING OF PROOUCTION REACTOR

FUELS IN THE HTR
AUTHOR

LEVEL

PGS

DATE

$04 / 04 / 66$

$07 / 11 / 67$

$04 / 05 / 66$

LE RUSLER

SECR

SRD

SECR

$04 / 06 / 66$

$04 / 13 / 66$

$04 / 13 / 66$

SECR

CRD

$04 / 21 / 66$

JF QUIRK

SECR

$04 / 15 / 66$

VB SCHUINBERG

SECR

SECR

$04 / 19 / 66$

$04 / 29 / 66$

SECR

05/02/66

JP HAMRIC

CRD

SECR

$05 / 02 / 66$

$04 / 28 / 66$

JR SPINK

CRD

$04 / 28 / 66$

SECR

$05 / 03 / 66$

SECR

05/06/66

TH LYOWS

SECR

$02 / 20 / 67$

SECR

$06 / 10 / 66$

SECR

SECR

$05 / 10 / 66$

$05 / 11 / 66$

DUN-RL

SECR

$06 / 11 / 71$

SECR

$06 / 11 / 71$

LE KUSLER

SECR

$05 / 13 / 66$

05/12/66

$06 / 14 / 66$

$05 / 17 / 66$

$05 / 17 / 66$

CRD

SECR

SECR

$05 / 20 / 66$

05/20/66

05/23/66 
Doc. No.

DUN-01099

DUN-01109

DUN-01125

DUN-01130

DUN-01143

DUN-01144

DUN-01150

DUN-01153REV

DUN-01155

DUN-01156

DUN-01186RD

DUN-01216

DUN-01217

DUN-01224

DUN-01229

DUN-01244

DUN-01249

DUN-01253

DUN-01254

DUN-01255

DUN-01256RD

DUN-01263

DUN-01266

DUN-01268

DUN-01269

DUN-01277RD

DUN-01279

DUN-01290

DUN-01292

DUN-01296

DUN-01302
TITLE

MEUTRON FLUX PROFILES FOR K REACTOR SIDE-TO-SIDE-TEST HOLES

COST COAPARISON FOR TUBES VERSUS IMPACTS

FOR HOT DIE SIZE FUEL MANUFACTURE

PERTINENT CLASSIFIED ROUGH DRAFTS

K4N MODEL BUMPER FUEL ELEMENT USAGE -

KE-KW REACTORS

ENGR RPT - FRONT FACE FOG SPRAY SYSTEM

FOR B, C, D, KE \& KW REACTORS

$B$ \& D DOWICONER FLOH LIMIT

CALCULATION OF LAST-DITCH BACKUP

ADEQUACY AT THE SWALL REACTORS

FY-1967 COALS RESEARCH \& ENGINEERING SECTIOM

2.1 E N DEWONSTRATION

NOTES ON IRRADIATION OF MECOYMIUN

PRCO SUB-SECT CLASSIFIED HORKING PAPERS FY 1967

CONSTRUCTION PROJECT DATA SHEET REACTOR IMPROVEMENT FOR POLONIUN-210 PROOUCTION RICHLAND, UA

FUEL \& TUBE PERFORMANCE MEMORAMDUM MAY 1966

CLASSIFICATION - PANELLIT RECORDS

BULK TEMPERATURE SURGES

ECONOMICS OF CHARGING K4 FUEL WITH $K 5$

CHARGING MACHINES

MANUFACTURING GOALS FY-1967 PART II -

CLASSIFIED INFORMATION

P ASSAY VARIATIOH CASES

DESIGK CHANGE 1099 - PRESSURE MONITOR

COLUMN CIRCUIT TIME DELAY - 105-KE-KU

C5NS FUEL EXPOSURE STUDY

BPA PONER INTERRUPTIONS - JUNE $7 \& 10$, 1966

K-REACTOR SPLINE TUBE COOLANT FLOW

RECYCLE OF NORMAL URANIUM AT RICHLAMD

PRODUCTION RECORDS PROOUCYION SCHEDULING 6/67

PIECE RECORDS PRODUCTION SCHEDULING 6/67

MAXIMIZING P -238 PROOUCTION FRON N -237

IRRADIATIONS

DUN DISCHARGE GOAL EXPOSURE PLAN

(REVISED $7 / 1 / 66$ )

WELD PINEHOLES

REVISION OF CASE IIA MEPTUMIUN SCHEDULE

MUCLEAR MATERIALS PRODUCTION REPORT JULY 1, 1966 THRU JUNE 30, 1967

EMERGEMCY GUIDE KE-KW PROCESSING UNITS

EXPELLED IRRADIATED FUEL HANDLING
AUTHOR

LEVEL

PQS

DATE

LR HALLIS

SECR

05/25/66

SECR

05/02/66

CRD

$05 / 31 / 66$

SECR

$06 / 03 / 66$

SECR

07/27/66

SECR

$06 / 06 / 66$

$04 / 28 / 67$

TH AMBrose

SECR

$07 / 26 / 66$

SECR

03/09/66

UK woods

SRD

$07 / 07 / 66$

$04 / 09 / 71$

SECR

$06 / 27 / 66$

PD GROSS

$06 / 21 / 66$

$06 / 23 / 66$

$06 / 24 / 66$

$06 / 28 / 66$

RS BELL

$06 / 20 / 66$

$06 / 30 / 66$

SECR

$07 / 08 / 66$

SECR

$07 / 06 / 66$

SECR

07/06/66

H TOFFER

SECR

SRD

$07 / 01 / 66$

07/05/66

$04 / 08 / 71$

SECR

$04 / 08 / 71$

SECR

07/05/66

SECR

$07 / 06 / 66$

SECR

07/07/66

$06 / 11 / 71$

T PRUDICH

SECR

SRD

07/01/66

SECR

$07 / 12 / 66$ 
DOC. NO.

DUN-01328

DUN-01333

DUM-01337

DUN-01346

DUN-01360

DUN-01371

DUN-01371

DUN-01380

DUN-01382

DUN-01384

DUN-01387

DUN-01389

DUN-0140206

DUN-0140207

DUN-0140208

DUN-0140209

DUN-0140210

DUN-0140211

DUN-0140212

DUN-01403

DUN-01403ADD 1

DUN-01416

DUN-01428

DUN-01429

DUN -01450

DUN-01456RD

DUN-01462

DUN-01497
TITLE

ACTION PLAN FOR POLONILAN-210 PROGRAM IN SMALLER RICHLAND REACTORS

DOWNCONER PRESSURE READINGS - C REACTOR PRODUCTION TEST IP-766, SUPA EXTEN COAL EXPOSURE FOR L P -A FUEL ELEMENT COLUNA FUEL \& TUBE PERForMANCE MEMORANDUM JUNE 1966

REDUCED FUEL CHARGE LENGTH K REACTOR SPLINE TUBES

ECONONIC EVALUATION OF OPTIMUM GRAPHITE MOOERATOR TEMPERATURES - C REACTOR

ECONOMIC EVALUATION OF OPTIMUM GRAPHITE MOOERATOR TEMPERATURES C REACTOR MANAGEMENT REPORT - JULY, 1966 ADVANCES CONCEPTS PLANHING SECTION REVISED HIGH TANK BACKUP ADEQUACY LIMITS C REACTOR

GRAMS GENERATED, UNIT COST, 4TH QUARTER

\& FY 1966

A PROGRAM FOR PRODUCTION OF CERTAIN NONDEFENSE PLUTONIUN NEEDS IN DUN REACTORS

HIGH TANK COOLANT BACKUP ADEQUACY LIMITS B REACTOR

PRCOUCTION INVENTORY \& MANUFACTURING

STATEMENT

PROOUCTION INVENTORY \& MANUFACTURING

STATEMENT

PROOUCTION INVENTORY \& MANUFACTURING

STATEMENT

PRODUCTION INVENTORY \& MANUFACTURING

STATEMENT

PRODUCTION INVENTORY \& MANUFACTURING

STATEMENT

PROOUCTION INVENTORY \& MANUFACTURING

\section{STATEMENT}

DUN PIBN STATEMENT O6/30/67

SHIELD STATUS - DUN OPERATED REACTORS

SHIELD STATUS - DUN OPERATED REACTORS ADDENDUM 1

COMPANY MEASUREMENTS, FY 1966

REVISED HIGH TANK COOLANT BACKUP

ADEQUACY LIMITS D REACTOR

PLUTONIUM ASSAY IH VARIATION STUDY

FUEL FAILURE PREDICTION 130 PERCENT HIGH PONER DENSITY PROGRAM

-G- REPORT - 11/65 THRU -..

MAMAGEMENT REPORT - AUG 1966 ADVANCED CONCEPTS AND PLANHING SECTION

U03 STAMDARD VALUES
AUTHOR

LEVEL

PGS

DATE

SECR

$08 / 15 / 66$

SECR

$07 / 19 / 66$

SECR

$06 / 11 / 71$

PD GROSS

CRD

$07 / 25 / 66$

SECR

08/01/66

LJ MEWELL

CRD

08/08/66

LJ MELELL

CRD

08/08/66

R NILSON

SECR

08/01/66

AN KUGLER

CRD

08/05/66

SECR

$05 / 25 / 71$

DH BAMGERTER/RH BOWN

SRD

08/05/66

GL SMITH

CRD

$08 / 10 / 66$

SECR

05/26/71

SECR

$05 / 26 / 71$

SECR

05/26/71

SECR

$05 / 26 / 71$

SECR

05/26/71

SECR

$05 / 26 / 71$

SECR

07/06/71

SECR

SECR

$09 / 28 / 79$

$09 / 28 / 71$

W FAGER

SECR

$08 / 16 / 66$

RV SKINNER

CRD

$08 / 22 / 66$

RH BOWN

SRD

SECR

$09 / 01 / 66$

$08 / 25 / 66$

DL DENEAL

SRD

SECR

$09 / 06 / 66$

$08 / 31 / 66$

SECR 
DOC. NO.

DUH-01503

DUN-01504

DUN-01508

DUN-01527

DUN-01536

DUN-01538

DUN-01545

DUN-01557

DUN-01565

DUN-01581

DUN-01593REV1

DUN-01594

DUN-01594REV

DUN-01604

DUN-01607

DUN-01608

DUN-01621

DUN-01629

DUN-01635

DUN-01644

DUN-01651

DUN-01653

DUN-01656

DUN-01673

DUN-01683

DUN-01718

DUN-01722
TITLE

CHARTS FOR DECREMENTAL COST STUDY AMALYSIS

JUSTIFICATION FOR DISCHARGE CHUTE

CLEARIMG ECUIPMENT - 210 ENRICHMENT

LOADIMG $K$ REACTORS

NOTES ON THE SIDE HOT SPOT FUEL FAILURE MODEL

FUEL PERFORMANCE IMPROVEMENTS

HOT DIE SIZING CASE STUDIES

EMS 207-C, REACTOR SHIELD COOLANT SYSTEM PROOUCTION TEST AUTHORIZATION -044, ETC.

IMPACT OF DELAY IN HOT DIE SIZING

PROJECT FUNDS

LETTER TO GF OUSLEY FROM RS PETERSON, SUBJECT: BALL 3X SYSTEM

HIGHLY ENRICHED LONDING ANTICIPATED

REACTOR MATERIALS HANDLING PROBLEMS

UNIT COST PER GRAM COMPARISON BY REACTOR

FY 1963 THRU FY 1966

REPORT OF THE IN-REACTOR TEMPERATURE AMD CREEP TEST OF AN UNCOOLED HORIZONTAL CONTROL ROO SECMENT

REPORT OF THE IN-REACTOR TEMPERATURE AND CREEP TEST OF AN UNCOOLED HORIZONTAL CONTROL ROO SECMENT

ORALLOY COST FOR PROOUCTION REACTOR FUEL USE

K REACTOR OPERATION AT REDUCED FLOUS

REPORT OF THE WORKING COWIITEE OF THE

FUEL ELEMENT DEVELOPMENT COMMITTEE

EFFECT OF AXIAL FLUX SHAPING ON GREEN-END FUEL

MAINTENANCE STUDIES

IRR ANALYSIS PTA-044, MASA FUEL PIN,

ETC.

SUPPLEMENTARY REACTIVITY CONTROL SYSTEMS B, C, \& D REACTORS

EVALUATION OF MASS JACKBOLT FOAMING "D" REACTOR

TECHNICAL EVALUATION OF E-D LOADINGS $K$ REACTORS

THE ROLE OF DECREMENTAL \& ALLOCATED

COSTS IN SYSTEMS AHALYSIS

PROCESS ENGINEERING SUPPLEMENTARY AUDIT

PROOUCTION TEST AUTHORIZATION -049

NOZZLE CAP MOLDED O-RING GASKETS FOR C REACTOR

FLOW CAPABILITIES B, C \& D WATER PLANTS HANFORD REACTOR \& FUELS STRENGTHS \& VEAKMESSES
AUTHOR

LEVEL

PGS

DATE

SECR

$09 / 01 / 66$

SECR

$08 / 22 / 66$

RG LAUER

SECR

$08 / 31 / 66$

SECR

08/10/66

SECR

$09 / 12 / 66$

09/07/66

06/11/71

$11 / 01 / 66$

SECR

SECR

$09 / 29 / 66$

SECR

$09 / 29 / 66$

SECR

$06 / 09 / 71$

WN ADACHI

CRD

$10 / 19 / 66$

WN ADACHI

CRD

$09 / 29 / 67$

SECR

$10 / 05 / 66$

STAFF

$10 / 07 / 66$

$10 / 01 / 66$

$10 / 07 / 66$

03/01/66

SECR

$06 / 11 / 71$

IL HUFFMAN

$10 / 14 / 66$

$10 / 18 / 66$

$11 / 15 / 66$

GR VANCE

SRD

$10 / 19 / 66$

DG MEIS

SECR

$10 / 10 / 66$

$11 / 14 / 66$

SECR

$12 / 01 / 66$

SECR 
Page No. 58

$04 / 28 / 93$
LIST OF HANFORD-GENERATED CLASSIFIED

DOCUMENTS DATED BETUEEN 1/1/61 AND 12/31/72
DOC. NO.

DUN-01730

DUN-01745

DUN-01747

DUN-01753

DUN-01771

DUN-01771REV2

DUN-01821

DUN-01833

DUN-01836

DUN-01837

DUN-01838RD

DUN-01844

DUN-01846

DUN-01849

DUN-01849 RD

DUN-01849RD

DUN-01852

DUN-01864

DUN-01865REV1

DUN-01866

DUN -01867

DUN -01890

DUN-01900

DUN-01904

DUN-01928

DUN-01929

DUN-01934RD

DUN-01935RD

DUN-01936RD

DUN-01943
TITLE

MUCLEAR PARAMETERS \& FACTORS FOR MASA CAPSULES

POTENTIAL FOR VSR DROP TIME IMPROVEMENT

V-72 VALVE CLOSURE DURING K REACTOR SHUTDOWN

ELEMENTS BASIC TO A LONG-RANGE PLAN CHARTS USED INPRESENTATION BY ADVANCED CONCEPTS AND PLANNING

ROL CHARGE CARD LOND COOE DEFINITIONS

ROL CHARGE CARD LOAD COOE DEFINITIONS REVISION NO. 2

THE HOT-DIE-SIZING PROCESS

COOLANT BACKUP ADEQUACY CONSIDERATIOAS

DURING THE E-D LOADING PROGRAM - $K$

REACTORS

FLUX MONITOR ANALYTICAL RESULTS $2 B$

KE-REACTOR

DOWNCOMER DATA - D REACTOR

SAMPLE CALCULATIONS GVR VALUES FOR

DETERMINING TRITIUM SEPARATIONS COSTS

DAP-507 WORK AREA FOG SPRAY SYSTEM

CLASSIFICATION - POWER LEVEL \& FLUX

RELAXATION

PRCOUCTION FORECAST DOUGLAS UNITED

NUCLEAR, IMC. JANUARY 1, 1967 THROUGH

JUNE 30, 1972

PROOUCTION FORECAST DOUGLAS UNITED

NUCLEAR INC., JAMUARY 1, 1967 THRU JUNE 30, 1972

PRCOUCTION FORECAST - DOUGLAS UNITED MUCLEAR, INC. - JAMUARY 1, 1967 THROUGH JUNE 30. 1972

P -238 PROOUCTION DEMONSTRATION

SEMIANMUAL RED REPORT DUN, INC

FUEL IRRADIATIONS AT HANFORD

PERFORMANCE OF T-SECTION BUMPERS

THORIUM OXIDE SPECIFICATIOAS

DEC 1966 REACTIVITY BALANCE \& ASSOCIATED

REACTOR PHYSICS DATA

CLASSIFIED WORKING PAPERS

LOCALIZED CORROSION COMPARISON BETWEEN

HDS \& ALSI FUEL

PROOUCTION FUELS SECTION PRODUCTION COST

REPORT DEC 1966

REPORT TO WORKING COMN FRON DUN

MISCELLANEOUS CORRESPONDENCE

TRITIUN PROOUCTION MOOES

MISCELLANEOUS RECYCLE INFORMATION

FUELS COSTS, PRODUCTION, \& UNIT COSTS

DEC 1966
AUTHOR

LEVEL

PGS

DATE

HM BURTON

SECR

$11 / 22 / 66$

SECR

$11 / 30 / 66$

$11 / 30 / 66$

R NILSON

SRD

$\$ 1 / 30 / 66$

DL HIRSCHEL

CRD

CRD

$12 / 16 / 66$

DL HIRSCHEL

SECR

SECR

$12 / 08 / 66$

$08 / 30 / 72$

SECR

$08 / 30 / 72$

SECR

RH BOWN

SRD

$08 / 30 / 72$

$12 / 12 / 66$

SECR

SECR

08/30/72

$08 / 30 / 72$

AP VINTHER

SRD

$01 / 12 / 67$

AP VINTHER

SRD

$12 / 29 / 66$

AP VINTHER

SECR

$12 / 30 / 66$

SECR

SECR

TW AMBROSE

TW AMBROSE

DE CLARK

CRD

SECR

CRD

CRO

ER TINNEY

SRD

SECR

SECR

SECR

SECR

SECR

SECR

SECR
$06 / 14 / 71$

07/06/72

03/03/67

$08 / 30 / 72$

$12 / 28 / 66$

01/05/67

01/05/67

$08 / 30 / 72$

06/09/71

$06 / 19 / 71$

07/06/71

07/06/71

$08 / 30 / 72$

$06 / 09 / 71$ 
Page No. 59

$04 / 28 / 93$

LIST OF MANFORD-GENERATED CLASSIFIED

DOCUMENTS DATED BETHEEN 1/1/61 AND 12/31/72

DOC. NO.

TITLE

AUTHOR

LEVE

PGS

DATE

DUN-01950

MONTHLY UNIT COST REPORT DEC 31, 1966

INTERIM REVIEW OF COALS FY 1967

SECR

06/09/71

DUN-01974

DUH -01975

SUPPLEMENTARY INFORMATION ON MATURAL

RECYCLE

DUW-01984

DUN-01999

DUN-02000

DEC 1966 MONTHLY MEASUREMENTS REPORT

REACTOR DAILY OPERATION REPORT 1967

PROCESS SPECIFICATIONS FUEL AND TAREET

DUN-02026

ELEMENT MANUFACTURING PROCESSES

ELECTRICAL NOISE GENERATOR TESTS ON

REACTOR PLANT INSTRLMENTATION

DUN-02031

PRESENTATION CHARTS

THORIA SHIELD PROTECTION COSTS

DUN-02033

PRODUCT COST, GRAMS GENERATED 2ND

QUARTER 12/31/66 \& FY TO

DUN-02059

OPERATIONAL CHARGE-DISCHARGE FOR TARGET

MATERIAL

DUN-02063

DUN GOALS INTERIM REVIEW - FY 67

MANUFACTURING SECTION

DUN-02064

DUN GOALS INTERIM REVIEW - FY 67 R\&E SECTION

DUN-02065

DUN COALS FY 67 INTERIM REVIEW OF

COMPANY GOALS

DUW-02066

DUN GOALS INTERM REVIEW - FY 67

PRODUCTION FUELS SECTION

DUN-0206881

DUN-0206882

DUN-02073

DUN-02074

REACTOR OUtAGE SCHEDULE (TENTATIVE)

REACTOR OUTAGE SCHEDULE (TENTATIVE)

FUEL RUPTURE MONITORING IMPROVEMENTS

BUDGET STUOY, FISCAL YEARS $68 \& 69$

PRODUCTION FUELS SECTION PROOUCTION COST

REPORT JAN 1967

DUN-02078

DUN-02085

DUN-02092

DUN-02101RD

DUN-02125

DUN-02133

DUN-02167RD

DUN-02168 RD

BACKUP NDEQUACY LIMITS

FUELS COSTS, PROOUCTION \& UNIT COSTS JAN

1967

MONTHLY UNIT COST REPORT 01/31/67

WORKING PAPERS FOR PRODUCTION \& UNIT

COST STUDY - FISCAL \& CALENDAR YEARS

1963 - 1971

JAN 67 MONTHLY MEASUREMENTS REPORT

OPERATING TIME RECAP - TISP-307 3B TEST

HOLE - 105-KW

WORKING PAPERS FOR LONG-RAMGE PLAN (1

\& 2 (TERATIONS)

LONG RANGE PLAN WORKIMG PAPERS (3RD

INTERATION)

DUN-02181REV

UNIT COST REACTOR \& FUELS ACTUAL FY-65,

FY-66 ESTIMATED FY-67 \& FY-68

DUN -02192

PRODUCTION FUELS SECTION PROOUCTION COST

FUELS ENGINEERING

SECR

$06 / 09 / 71$

SECR

08/30/72

SUBSECTION

SECR

$01 / 19 / 67$

$04 / 08 / 71$

$05 / 16 / 66$

RG GEIER

CRD

$08 / 30 / 72$

SECR

$01 / 27 / 67$

SRD

$06 / 14 / 71$

$06 / 09 / 71$

SECR

08/30/72

IN FAGER

SECR

$02 / 06 / 67$

WA FAGER

SECR

$02 / 06 / 67$

IM FAGER

SECR

02/06/67

IN FAGER

CONF

$02 / 06 / 67$

RP ROWE

CONF

01/01/71

RP ROWE

GL ERICKSOW

CRD

CRD

$01 / 95 / 71$

$02 / 14 / 67$

SECR

06/09/71

SECR

08/30/72

SECR

$06 / 09 / 71$

PC MCMAHON

SECR

06/09/71

$02 / 14 / 67$

DH BAMGERTER

$02 / 22 / 67$

$08 / 30 / 72$

SECR

$08 / 30 / 72$

$R$ NILSON

SECR

$02 / 28 / 67$

SECR

$06 / 09 / 71$

SECR

$06 / 09 / 71$

REPORT FEB 1967

FUELS COSTS, PRODUCTION \& UNIT COSTS FEB 1967

SECR

06/09/71 
Page No. 60

$04 / 28 / 93$

LIST OF HANFORD-GENERATED CLASSIFIED

DOCUMENTS DATED BETUEEN 1/1/61 AND 12/31/72

DoC. NO.

TITLE

AUTHOR

LEVEL

PGS

DATE

DUH-02207RD

DUN-02215

DUN-02224

DUN-02224ADD 1

DUN-02226

DUN-02236

DUN-02241

DUN-02268

DUN -02269

DUN-02271

DUN-02272 B

DUN-02272 B

DUN-02324

DUN-02335

DUN-02336

DUN-02350

DUN-02354

DUN-02358

DUN-02367

DUN-02375

DUN-02377

DUN-02386

DUN-02395

DUN-02397

DUN-02410

DUN-02434

DUN-02441

DUN-02449

DUN-02450RD
REACTOR OUTAGE SCHEDULE (TENTATIVE)

MONTHLY UNIT COST REPORT 02/28/67

SPEED-OF-CONTROL PONER LEVEL LIMITS FOR

E-D LOADING - $K$ REACTORS

SPEED-OF-CONTROL PONER LEVEL LIMITS FOR

E-D LONDING - $K$ REACTORS, ADDENDUM

1

HOT DIE SIZE 313 BLDG VS hOT HIE SIZE CAGE

FEB 67 MONTHLY MEASUREMENTS REPORT

ISOTOPE INFORMATION SERIES PRESENTED TO PROOUCTION FUELS SECTION

DONCONER DATA - 105-C REACTOR

LAST DITCH COOLANT BACXUP ADEQUACY

LIMITS D REACTOR

PRESENTATION MATERIAL DUN MUCLEAR SAFETY ADVISORY COMIITTEE MEETING FEBRUARY 15, $16 \& 17,1967$

FLOW \& PONER CALCULATIONS (POP PROGRAM)

FOR PTA-063 OUICK TURNAROUNO MEPTUNIUM

FLOW \& POWER CALCULATIONS (POP PROGRAM)

FOR PTA-063 QUICK TURMAROUND MEPTUNIUN

PRCO FUELS SECT FY-69 \& REV OF FY-68

PROO COSTS \& UNIT COSTS

DECREMENTAL COST - D REACTOR

P FORHATION, URANIUN CREDITS BURNOUT \& BURNOUT COSTS

PROOUCTION FUELS SECTION PROOUCTION COST REPORT MAR 1967

MAR 67 MONTHLY MEASUREMENTS REPORT

FUELS COSTS, PRODUCTION \& UNIT COSTS MAR 1967

FUEL \& TUBE REDESICN FOR B OR D REACTOR

LTR, "ELECTRICAL NOISE GEMERATOR" DATED

11/10/66, HU HEACOCK FROM J KAVECKIS

MONTHLY UNIT COST REPORT 03/31/67

FUEL DESIGNS FOR HIGH POWER DENSITY FUEL

TESTS

REVIEW TESTS

BUDGET FY-69, REV FY-68 SUMHARY OF 02

SPECIAL MUCLEAR MATERIALS BY AEC

CLASSIFICATION

INTERIM REPORT HALF-PLANT ZETA POTENTIAL

TEST

PTA-054 DATA SHEETS

PTA-069 NEPTUNIUN-237 PRODUCTION FROM

MATURAL -- UITH A HIGH U-236 CONTENT

PROD COST-GRAMS GENERATED 3RD QUARTER

3/31/67 \& FY-TD

WORKING PAPERS FY-69, BUDGET \& REV TO

FY-68 BUDGET PROD FUELS SECTION

\begin{tabular}{|c|c|c|}
\hline & SECR & $06 / 10 / 71$ \\
\hline & SECR & $06 / 09 / 71$ \\
\hline IR LANGTON/ER PERL & SRD & $06 / 29 / 67$ \\
\hline F MENEC & SRD & $09 / 29 / 67$ \\
\hline & SECR & $08 / 30 / 72$ \\
\hline H BANGERTER & $\begin{array}{l}\text { SECR } \\
\text { SECR }\end{array}$ & $\begin{array}{l}03 / 21 / 67 \\
08 / 30 / 72\end{array}$ \\
\hline $\begin{array}{l}\text { IN KUGLER } \\
\text { 2V SKIMMER }\end{array}$ & SECR & $\begin{array}{l}04 / 25 / 67 \\
03 / 31 / 67\end{array}$ \\
\hline & SECR & $08 / 30 / 72$ \\
\hline
\end{tabular}

RE BAMRS

SECR

$01 / 08 / 68$

RE BAARS

SECR

$01 / 08 / 68$

SECR

06/09/71

SECR

SECR

$08 / 30 / 72$

$07 / 06 / 71$

SECR

06/09/71

DH BANGERTER

SECR

$04 / 24 / 67$

06/09/71

SECR

SECR

08/30/72

08/30/72

SECR

SECR

SECR

SECR

06/09/71

$08 / 30 / 72$

08/30/72

$06 / 09 / 71$

JR LANGTON

SECR

08/30/72

DUN-RL

CRD

05/01/67

$06 / 14 / 71$

SECR

06/09/71

SECR

06/09/71 
DOC. NO.

DUN-02454

DUN-02464

DUN-02465

DUN-02.482

DUN-02494

DUN-02495

DUN-02501

DUN-02508RD

DUN-02509

DUN -02513

DUN-02533

DUN-02537RD

DUN-02538RD

DUN-02542RD DUN-02564

DUN-02576

DUN-02584

DUN-02585

DUN-025851

DUN-02590

DUN-02594

DUN -02597

DUN-02598

DUN-02602

DUN-02608

DUN-02612RD

DUN-02624

DUN-02624RD DUN-02625
TITLE

FINAL RPT HALF PLANT ACTIVATED ALUMIMA TEST

DEVELOPMENT TEST O72 IRRADIATION OF SERPENTINE CONCRETE 222 CAPSULE, TISR 372

IRRADIATION ANALYSIS DEVELOPNENT TEST OT2 IRRADIATION OF SERPENTIME CONCRETE 222 CAPSULE, TISR 372 PRODUCTION FUELS SECTION PRCDUCTION COST REPORT APR 1967

MONTHLY UNIT COST REPORT 04/30/67

FUELS COSTS, PRODUCTION \& UNIT COSTS APR 1967

ECONOMICS OF REDUCING FUEL COLUNA

LENGTHS IN CENTRAL ZONE ALUMINUM TUBES $K$ REACTORS

RESEARCH \& DEVELOPMENT NOTES

K REACTOR LOW ALUM FEED TEST

APR 67 MONTHLY MEASUREMENTS REPORT

K REACTOR ADMINISTRATIVE LIMITS

RECEIPTS REGISTER FY 1968

PRCDUCTION SHIPPING REGISTER ONSITE \& OFFSITE FY 1968

PROO COST GRAMS GENERATED REPORT FY 1968

$B$ \& D PONER \& WATER PLANT MODS AND

OPERATION AFTER DEACTIVATION OF D

REACTOR

CLASSIFICATION GUIDANCE FOR CONTRACT

DDR-104

PTA-080, PHYSICS TESTING AT D REACTOR

DEACTIVATION

MONTHLY UNIT COST REPORT 05/31/67

REACTOR UNIT COST COMPARISONS 5/31/67

DUN REACTORS REPORT TO COST FY 1968

MAY 67 MONTHLY MEASUREMENTS REPORT

PRESSURE MONITOR CUAGE MOOEL 157 \& MODEL

158 RESPONSE TIME CHARACTERISTICS TEST

AIR ACCUMULATION \& PRESSURE STEP CHANGES

2.1\% ENRICHED URANIUA-LITHIUM LONDING MEMORAMDUM

BACKUP ADEQUACY LIMITS, B REACTOR 6/1/67

TO $6 / 1 / 68$

PTA-031, SUP A, IRRADIATION AMALYSIS,

ETC.

LONG-RANGe PLAN MORKING PAPERS (4 ITERATION)

DUN PRODUCTION FORECAST 7/1/67 THRU 6/30/72

DUN PRODUCTION FORECAST THRU 6/72

REACTOR PROCESSING ISOTOPE PRCDUCTION

AMD FUEL CONVERSION TABLES

\begin{abstract}
AUTHOR
\end{abstract}
LEVEL.

PGS

DATE

SECR

$08 / 30 / 72$

PJ ZIMMERMAN

CRD

07/03/67

PJ ZIMMERMAN

CRD

05/31/67

SECR

$06 / 09 / 71$

SECR

06/09/71

SECR

$06 / 09 / 71$

SECR

$08 / 30 / 72$

DH BAMGERTER

SECR

SECR

SECR

SECR

SECR

SECR

SECR

SECR

$04 / 01 / 71$

08/30/72

TW MAUFF

CRD

$06 / 06 / 67$

GF BAILEY

conf

$06 / 14 / 71$

SECR

SECR

SECR

OH BAMGERTER

SECR

SECR

06/09/71

06/09/71

$04 / 08 / 71$

$06 / 20 / 67$

08/30/72

SECR

$06 / 14 / 71$

SECR

$08 / 30 / 72$

SECR

$06 / 14 / 71$

SECR

$08 / 30 / 72$

SECR

07/08/71

ER PERL

SECR

07/08/71

CRD 
Page No. 62

$04 / 28 / 93$

LIST OF HANFORD-GENERATED CLASSIFIED

DOCLMENTS DATED BETMEEN 1/1/61 AND 12/31/72

DOC. NO.

TITLE

AUTHOR

LEVEL PGS

DATE

DUN-02626

RECOMNENDATIONS FOR PROCESS STANDARD ON

SECR

$08 / 30 / 72$

DUN-02631

HOT STARTUPS

FUELS COSTS, PROOUCTION \& UNIT COSTS MAY

sech

$06 / 09 / 71$

DUN-02674

1967

IRON-MASONITE SHIELD STATUS INTERIM RPT

SECR

08/30/72

DUN-02680

II6

SECR

$08 / 30 / 72$

DUN-02712

PARAMETRIC CURVES FOR TOTAL CONTROL FOR

SECR

$04 / 08 / 79$

DUN-02713

PROOUCTION RECORDS, PRCOUCTION SCHEDULTE

SECR

$06 / 27 / 67$

PIECE RECORDS PRODUCTION SC HEDULIMG ALL

RP MALTON

SRD

$08 / 30 / 72$

TEST RESULTS OF PROOUCTION TEST IP-05I

SECR

$08 / 30 / 72$

DUN-02758

TRIPOUT TEST - $K$ REACTORS

SCHEDULE FOR TEST FUEL IRRADIATIONS

$X$-RAY TESTING OF OFF-ChaRged PROCESS

SECR

$08 / 30 / 72$

DUN-0280001

TUBES

PROOUCTION INVENTORY \& MANUFACTURING

SECR

$05 / 26 / 71$

DUN-0280002

STATEMENT

SECR

$05 / 26 / 71$

DUN-0280003

PROOUCTION INVENTORY \& MANUFACTURING

SECR

$05 / 26 / 71$

PROOUCTION INVENTORY \& MANUFACTURING

SECR

$05 / 26 / 71$

PRCOUCTION INVENTORY \& MAHUFACTURING

SECR

05/26/71

PRODUCTION INVENTORY \& MANUFACTURING

SECR

$06 / 10 / 71$

DUN PROOUCTION INVENTORY \& MANUFACTURING

SECR

$06 / 10 / 71$

DUN-0280007

STATEMENT 12/31/67 \& FYTD

DUN PRODUCTION INVENTORY \& MANUFACTURING

SECR

$06 / 10 / 71$

DUN PRCDUCTION INVENTORY M MANUFACTURING

SECR

$06 / 10 / 71$

DUN PROOUCTION INVENTORY \& MANUFACTURING

SECR

$06 / 10 / 71$

DUN PRODUCTION INVENTORY \& MANUFACTURING

SECR

$06 / 10 / 71$

DUN PRODUCTION INVENTORY \& MANUFACTURING

SECR

07/06/71

DUN PIEN STATEMENT 06/30/68 \& FYTD

CHARTS FOR RL PRESENTATION FOR E.P.

SECR

$07 / 06 / 71$

BARAMONSKI, 7/11/67

PLANNINg ESTIMATE CASES

ECONOMIC ANALYYSIS SEGMENTAL DISCHARGE OF

$08 / 30 / 72$

DUN-02809
DUN-02810RD

REACTOR FUEL NO BENEFIT FROM LESS FUEL

$08 / 30 / 72$

DUN -02823

DUN-02824

FAILURES

NMS REPORT FY 1968

MONTHLY UNIT COST REPORT 06/30/67

REACTCR UNIT COST COAPARISONS 6/30/67

FUELS COSTS, PRODUCTION \& UNIT COSTS JUN 1967

SECR

SECR

$12 / 05 / 72$

SECR

06/09/71

DUN-02828 
Page No. 63

$04 / 28 / 93$

LIST OF HAMFORD-GENERATED CLASSIFIED

DOCUNENTS DATED BETWEEN 1/1/61 AND 12/31/72

DOC. NO.

TITLE

AUTHOR

LEVEL

PGS

DATE

DUN-02898

HEAT LOND ON CONFIHENENT SYSTEM FILTERS

SECR

$08 / 30 / 72$

DUW-02899

$K$ REACTORS

FIMAL RPT PILOT TEST PROCRAM CO2

SECR

$08 / 30 / 72$

DUN-02903RD

H CONTROL

DRAFT OF MISSION W MUCLEAR SAFETY

PROCRAM

DUN-02920

PROD COST-CRAMS GENERATED 4TH OUARTER

6/30/67 \& FY-TO

DUN-02958

DUN-02960

DUN-02961

DUN-02971

DUN-02972

DUN-02976

DUW-02979

DUN-02981

DUN-02983RD

NUCLEAR MATERIALS PRCOUCTION REPORT JULY 1, 1967 THRU JUNE 30, 1968

ADVANCED PLANMING ENGIMEERING MOTES

CRITICALITY CONTROL 2.1 E-N LONDING PROOUCTION FUELS

LIA102 TARGET DESIEN

K REACTOR OPERATION WITH REDUCED FLON

CONDITIONS

FUELS COSTS, PRODUCTION \& UNIT COSTS JUL

1967

MONTHLY UNIT COST REPORT 07/31/67

P -238 PRODUCTION TARGET FABRICAITON

PROPOSAL

KEY NLMBERS FOR FUEL DISCHARGED FROM DUN REACTORS

ADVANCED TECHNOLOGY INCENTIVES

$K$ REACTOR GRAPHITE TEMPERATURE ECONOHICS RELATIVE TO HELIUH LOSS RATES

DUN-02993RD

DUN-03002

DUN-03017

TECHNICAL EVALUATION OF 210 E-N LOADINGS

$K$ REACTORS

SUMHARY PROCESS WORK REQUEST \#69-7 \&

SUPPLEMENT "A" (CNIE ROD)

UTILIZATION OF HIGH U-236 MATERIAL

PREDICTED FLATTENING OF THE OVERBORE

ZIRCALOY PROCESS TUBES IN THE "CM

REACTOR FUEL TEST BED

DUN-0303101

DUN-030311

DUH-03040

DUN-03055

DUN-03061

DUN-03076

DUN-03101

DUN-03112RD

DUN-03121

ADP EQUIPMENT SYSTEM STLDY W6801 PART II AUTOMATIC DATA PROCESSING EQUIPMENT STUOY \#6801, PT II

SUMMARY REPORT PROCESS MATER FLCMETER

STUDY

REVISED DATA FOR USE IN TOTAL CONTROL

CALCULATIONS

DESIGN STUDY - TRITILM EXTRACTION

FACILITY - 100-N INCREASED CAPABILITIES

REVIEU OF ACCOUNTABILITY \& CONTROL OF

SOURCE \& SPECIAL MUCLEAR MATERIALS

MULTIPLE FUEL MOOEL STUOY

N-REACTOR PONER LEVEL CAPABILITY

SCHEDULE OF SPECIAL ACTIVITIES UNDER

MFC-12

$T$ PRUDICH

CR STRAND

LH RICE

RP MALTON

BECR

$08 / 30 / 72$

SECR

$06 / 09 / 71$

SRO

SECR

SECR

SECR

SECR

SECR

SECR

SECR

SRD

RD SHIMER

LJ MEVELL

SECR

SRD

SECR

CM SCHLEIKHARDT

CONF

JW MICKOLAUS

WEB LANDER

SECR

CRD

RL BENCINI, GE GREGER

SRD

SECR

SECR

SECR

SECR

SECR

SECR

SECR

SECR
07/01/67

$06 / 14 / 71$

07/28/67

$08 / 11 / 67$

08/30/72

$06 / 09 / 71$

$06 / 09 / 71$

$08 / 30 / 72$

$08 / 15 / 67$

08/17/67

08/20/67

$07 / 06 / 71$

$08 / 23 / 67$

08/25/67

$08 / 30 / 72$

$02 / 01 / 68$

02/01/68

$08 / 30 / 72$

$08 / 30 / 72$

$10 / 05 / 67$

$09 / 12 / 67$

$09 / 15 / 67$

$09 / 15 / 67$

08/30/72 
Page No. 64

$04 / 28 / 93$

LIST OF MANFORD-GENERATED CLASSIFIED

DOCLNENTS DATED BETMEEN 1/1/61 AND 12/31/72

DoC. NO.

DUN-03123

DUN-03124

DUN-03125

DUN-03137

DUN-031371

DUN-0313710

DUW-031372

DUN-031373

DUN-031374

DUN-031375

DUN-031376

DUW-031377

DUN-031378

DUN-031379

DUN-03137RD

DUN-03153RD

DUN-03154RD

DUN-03159

DUN-03163

DUN-03169

DUN-03179

DUN-03176

DUN-03190RD

DUN-03194RD

DUN-03204

DUN-03214C RD

DUN-03220

DUN-03235

DUN-03236RD

DUN-03245

DUN-03247

DUN-03249

DUN-03260

DUN-03262

DUN-03266

DUN-03270
TITLE

FUELS COSTS, PRCOUCTION \& UNIT COSTS AUG

1967

SPECIALIZED FUEL MODELS

INVENTION DESCRIPTION - CARBON-14

EXTRACTION FRON REACTOR CAS

MONTHLY UWIT COST REPORT 08/31/67

MONTHLY UNIT COST REPORT 09/30/67

MONTHLY UNIT COST REPORT JUN 1968

MONTHLY UNIT COST REPORT 10/31/67

MONTHLY UNIT COST REPORT 11/30/67

MONTHLY UNIT COST REPORT DEC 1967

MONTHLY UNIT COST REPORT JAN 1968

MONTHLY UNIT COST REPORT FEB 1968

MONTHLY UNIT COST REPORT MAR 1968

MOUTHLY UNIT COST REPORT APR 1968

MONTHLY UNIT COST REPORT MAY 1968

PROOUCTION COST \& UNIT COST INFORMATION FY 1968

LETTERS CONCERNING MEPTUNIUN PRODUCTION REACTOR PARAMETERS

NUG 67 MONTHLY MEASURENENTS REPORT

DUN FY67 COMPANY MEASUREMENTS

210 E-N DEMONSTRATION LONDING IN $K$

REACTORS

HDS - STUDY TEAM REPORT

DESCRIPTION OF REACTOR OPERATING LIMITS

\& REACTOR MASTER FILE REPORTS K-REACTORS

HDS PROGRAM

MANUFACTURING ENG MOTES - 7/67 - 7/68

MEAR LAPSE OF TOTAL CONTROL, 105-KW

REACTOR $9 / 15 / 67$

POP CALCULATIONS FOR PTA-103 ON INCH

OVERBORE

IMCREASING FAST MEUTRON FLUX IN TEST

FACILITIES

LETTER: .95 ENRICHED FUEL CORES SS

MATERIAL REQUEST \#27 AND 28

DUAL-CYCLE DICHROMATE NUTOCLAVE FILMS OW 8001-F ALLOY FUEL CLADDING

PRCOUCTION FUELS SECTION PROOUCTION COST REPORT SEP 1967

K-REACTOR - LOSS OF COOLANT TEMPERATURE STUDY OF THE BIOLOGICAL SKIN

FUELS COSTS, PRODUCTION \& UNIT COSTS SEP 1967

COPROOUCT TARGET SHIPMENTS

SUPPORT CORROSION PROBLEM

TISR-309 SAMPLE DATA

ECONOMIC AMALYSIS SEGMENTAL DISCHARGE OF $K$ REATOR FUEL.
NTHOR

LEVEL Pas

DATE

JP COOKE

SECR

$06 / 09 / 71$

SECR

$08 / 30 / 72$

$09 / 20 / 67$

SECR

06/09/71

SECR

SECR

SECR

SECR

SECR

SECR

SECR

SECR

SECR

SECR

SECR

AF RUPINSKI

SRD

AF KUPIMSKI

DH BAMEERTER

KL ROBERTSON

CRD

SECR

SECR

SECR

SECR

JO SKOLRLD

SECR

SECR

SECR

SECR

SECR

SECR

SECR

SECR

SECR

SECR

WJ FERCUSON

SECR

RK MARKFELDER

JR YOUNG
$08 / 30 / 72$

06/09/71

$10 / 24 / 67$

$10 / 19 / 67$

$10 / 20 / 67$

06/09/71

$06 / 09 / 71$

$06 / 09 / 71$

$06 / 10 / 71$

06/10/71

$06 / 10 / 71$

$06 / 10 / 71$

$06 / 10 / 71$

04/08/71

$09 / 25 / 67$

$09 / 25 / 67$

$09 / 28 / 67$

$09 / 29 / 67$

$08 / 30 / 72$

$10 / 04 / 67$

$08 / 30 / 72$

$06 / 09 / 71$

$08 / 30 / 72$

$11 / 04 / 71$

$08 / 30 / 72$

08/30/72

$08 / 30 / 72$

$10 / 25 / 67$ 
Page No. 65

$04 / 28 / 93$

LIST OF MANFORD-GENERATED CLASSIFIED

DOCIMENTS DATED BETWEEN 1/1/61 AND 12/31/72

Doc. No.

DUN-03283

OUN-03287RD

DUN-03287RD SUP1

DUN-03305 SUP1

DUN-03310RD

DUN-03311RD

DUN-03330

OUN-03332

DUN-03333

DUN-03336RD VOL2

DUN-03342RD

DUN- 03353

DUN-03360

DUN-03363RD

DUN-03379

DUN-03380

DUN-03387

DUN -03400

DUA -03409

DUN-03416

DUN-03418

DUN-03419

DUN-03424RD

DUN-03425RD

DUN-03431RD

DUN-03432RD

DUN-03442

DUN-03479

DUN-03482

DUN-03493

DUN-03498RD

DUN-03499
TITLE

REACTOR OPERATING LIMITS EQUATIONS \& DEFIMITIONS

MINIMCDEL FINAMCIAL DATA

ADDEEDUM TO DUN-3287 RD MIMIMODEL

FIMANCIAL MODEL

PRCOUCTION TEST CHANGE AUTHORIZATION

NO.1 TO PT-NR-95, IRRADIATION OF HICH U-236 MARK I-C FUEL

MONTHLY REPORTS \& MISC CORRESPONDENCE

CLASSIFIED DRAFTS \& WORKING PAPERS

PRCO COST-GRAMS GENERATED IST CUARTER

FY 68

PRCDUCTION FUELS SECTION PRODUCTION COST

REPORT OCT 1967

LITHIUM-ALUMIMUN SEPARATIONS REQUEST

WORKING PAPERS

THORIA IRRADIATIONS - MISCELLAMEOUS DATA

VARYING ENRICHMENT OF V-R TAILS

FUELS COSTS, PRODUCTION \& UNIT COSTS OCT

1967

COST REDUCTION STUDY

105-KE CONTROL ROON LOG FRON 12/1/67

THRU 2/29/68

LO5-KW CONTROL ROOM LOG FROM 12/1/67

THRU 2/29/68

H REDUCTION AT C REACTOR

COST OF DISCONYINUING THE UPSTREAM

PROGRAM

WATER FLOUS - 183 - 190 BUILDINGS FY 1959

THRU FY 1968

FIMAL RPT HALF-PLANT ZETA POTENTIAL TEST

FLUX MONITOR TRIP SETTINGS

U-233 PROOUCTION CAPABILITY

REACTOR MODERATOR DISTORTION STUOY

OVERBORE REWOVAL W4T5

IMFORMATION FOR FUELS REQUIREMENT

MEETING IN GERMANTOM

PRCOUCTION FUEL INVENTORIES 11/30/67

ROL PROGRAM CHANGE "IT2

PRCOUCTION FUELS SECTION PROOUCTION COST REPORT NON 1967

STARTUP COST SAVINGS REALIZED DUE TO HIGH SPEED SCANMER

FUELS COSTS, PROOUCTION \& UNIT COSTS NOV 1967

MONTHLY MANAGENENT REPORT JAMUARY 1, 1968 THROUGH DECEMBER 31, 1968 PHYSICS SUBSECTION

PTA-031, SUPPLEMENT B, IRRADIATION OF MO-U02 \& N -UO2 FUEL PINS IN THE KE \& KW REACTORS
AUTMOR

LEVEL

PGS

Date

SECR

$06 / 09 / 71$

OH BANGERTER

SRD

$10 / 31 / 67$

DH BAMGERTER

SRD

03/05/68

HE JACKEL

SECR

$02 / 26 / 68$

RL MILLER

EC BENMETT

HF TEW

RE CLOUGH

SRD

11/03/67

$11 / 03 / 67$

06/09/71

SECR

SECR

06/09/71

SECR

SRD

SECR

SECR

SECR

SECR

CONF

CONF

SECR

SECR

SECR

SECR

SECR

SECR

SECR

AN RUGLER

SECR

SECR

SECR

SECR

SECR

SECR

SECR

SRD

SECR

$06 / 14 / 71$

$09 / 25 / 70$

$07 / 06 / 72$

$08 / 30 / 72$

06/09/71

08/30/72

12/01/67

$12 / 01 / 67$

$08 / 30 / 72$

$08 / 30 / 72$

$08 / 30 / 72$

$08 / 30 / 72$

$08 / 30 / 72$

$08 / 30 / 72$

$08 / 30 / 72$

$04 / 05 / 67$

08/30/72

$08 / 30 / 72$

$08 / 30 / 72$

$06 / 09 / 79$

$08 / 30 / 72$

$06 / 09 / 71$

GC FULLMER

$07 / 06 / 71$ 
Page No. 66

$04 / 28 / 93$

LIST OF HAMFORD-GENERATED CLASSIFIED

DOCUMENTS DATED BETMEEN 1/1/61 AMD 12/31/72

DoC. NO.

TITLE

DUN -03500

DUN-03501

DUN-03502

DUN-03503 RD REV1

DUN-03505

DUN-03523

DUN-03527RD

DUN-03530

DUN-03531

DUN-03533RD

DUN-03548

DUN-03555

DUN-03558RD

DUN-03572

DUN-03595

DUN-03596

DUN-03597

DUN-03604RD

DUN-03605

DUN-03612RD

DUN-03617

DUN-0361701

DUN-0361702

DUN-0361703

DUN-0361704

DUN-0361705

DUN-0361706

DUN-0361707

DUN-0361708

DUN-0361709

DUN-0361710

DUN-0361711

DUN-0361712

DUN-0361713 CAI-170 FUEL SYSTEMS
IRRAD AMALYSIS FOR PTA-031 SUP B, IRRAD OF MO-UO2 \& $N$-U02 FUEL PINS IN THE KE \& KW REACTORS, TISR 701

PTA-112 IRRADIATION OF UN FUEL PINS IN THE FLOUING HELIUN ATMOSPHERE, TISR 702 IRRAD AMALYSIS PROD TEST AUTHORIZATION 112, IRRAD OF UN FUEL PINS IN A FLONIMG HELIUM ATMOSPHERE TISR 702

PROJECT PROPOSAL REPLACERENT FUEL

PRODUCTION PROCESS (HOT DIE SIZING)

P FORHATION, U CREDITS, BURNOUT \& BURNOUT COSTS

PREPARATION \& CALORIMETRIC ASSAY OF 170

$T$ SOURCES

WORKING NOTES

TRITIUN PRCOUCTION USING HIGHLY ENRICHED

K REACTOR PROCESS TUBE RUPTURE

MISCELLANEOUS REACTOR STATISTICS

PTA-107 - IRRADIATION OF HIGH U-236

CONTENT 95 METAL IN KE REACTOR

ALSI PROCESS DIRECT LABOR STAMDARDS

DUN, INC. PRODUCTION FUEL SECTION

SELECTED COST CONPARISONS-SEP, OCT \& NOV

1967 SEP, OCT \& NOV 1966

DESIGN BASIS FOR CALCULATIONS FOR

LONG-TERM P -AL. IRRADIATIONS

DUN MONTHLY REPORT JAN 1968

DUN MONTHLY REPORT FEB 1968

DUN MONTHLY REPORT MAR 1968

WORKING NOTES

TECHNICAL NOTE ON INCREASING THE NDEQUACY OF THE B/C LAST-DITCH COOLING

WORKING PAPERS - PTA-069

DEC 67 MONTHLY MEASUREMENTS REPORT

JAN 68 MONTHLY MEASUREMENTS REPORT

FEB 68 MONTHLY MEASUREMENTS REPORT

MAR 68 MONTHLY MEASUREMENTS REPORT

APR 68 MONTHLY MEASUREMENTS REPORT

MAY 68 MONTHLY MEASUREMENTS REPORT

JUN 68 MONTHLY MEASUREMENTS REPORT

JUL 68 MONTHLY MEASUREMENTS REPORT AUG 68 MONTHLY MEASUREMENTS REPORT SEP 68 MONTHLY MEASURENENTS REPORT OCT 68 MOWTHLY MEASUREMENTS REPORT NOV 68 MONTHLY MEASUREMENTS REPORT DEC 68 MONTHLY MEASUREMENTS REPORT JAN 69 MONTHLY MEASUREMENTS REPORT
AUTHOR

LEVEL

PGS

DATE

SECR

$07 / 06 / 71$

SECR

07/06/71

SECR

07/06/71

RE DALING

SRD

$02 / 08 / 68$

SECR

$08 / 30 / 72$

SECR

$09 / 20 / 72$

JP HAMRIC

CRD

$12 / 15 / 67$

SECR

$09 / 20 / 72$

SECR

$09 / 20 / 72$

AF KUPINSKI

JF CAMP III

JF CAMP III

JF CAMP III

JF CAMP III

JF CAMP III

JF CAMP III

JF CAMP III

JF CAMP III

JF CAMP III

JF CAMP III

JF CAMP III

JF CAMP III

JF CANP III

JF CAMP III
SECR

SECR

SECR

SECR

$09 / 20 / 72$

$09 / 20 / 72$

SECR

$09 / 20 / 72$

SECR

SECR

SECR

CRO

SECR

$09 / 20 / 72$

$02 / 01 / 68$

03/01/68

$04 / 01 / 68$

01/03/68

09/20/72

CRD

SECR

SECR

SECR

SECR

SECR

SECR

SECR

SECR

SECR

SECR

SECR

SECR

SECR

SECR
$01 / 08 / 68$

$01 / 26 / 68$

$02 / 20 / 68$

$03 / 20 / 68$

$04 / 29 / 68$

$05 / 27 / 68$

$06 / 28 / 68$

$07 / 29 / 68$

$08 / 27 / 68$

10/03/68

$10 / 25 / 68$

$11 / 22 / 68$

$12 / 30 / 68$

$01 / 22 / 69$

$03 / 30 / 69$
$12 / 28 / 71$ 
Page No. 67

$04 / 28 / 93$

LIST OF HANFORD-GENERATED CLASSIFIED

DOCUMENTS DATED BETWEEN 1/1/61 AND 12/31/72

DOC. NO.

DUN-0361715

DUN-0361716

DUN -0361717

DUN-0361718

DUN-0361719

DUN-0361720

DUN-0361721

DUN-03620

DUN-03637

DUN-03639

DUN-03644

DUN-03671

DUN-03672

DUN-03678

DUN-03685

DUN-03688

DUN-03695

DUN-03707

DUN-03711

DUN-03728

DUN-03732

JUN-03752RD

DUN-03756

DUN-03758RC

DUN-03759

DUN-03775
TITLE

QUARTER FY69

QUARTERLY MEASUREMENTS REPORT 4TH

QUARTER FY69

QUARTERLY MEASUREMENTS REPORT 1ST

QUARTER FYTO

QUARTERLY MEASUREAENTS REPORT 2ND

QUARTER FYTO

QUARTERLY MEASUREMENTS RPT 3 QUARTER

FY-1970

QUARTERLY MEASUREMENTS RPT 4 QUARTER FY-1970

QUARTERLY MEASUREMENTS RPT 1 QUARTER

FY-1971

IRRADIATED FUEL SHIPMENTS FROM THE B, C

\& K REACTORS

KE REACTOR FACTOR DATA 1/1/68 - 12/31/68

REACTOR OPERATIONS DAILY REPORTS FOR 1968

REPORT OF URANILM ACCOUNTABILITY IN PRODUCTION FUELS OPERATION PROCESS FOR PERIOO ENDNG 12/29/67

B - C BACK-UP ADEQUACY

PROOUCTION FUELS SECTION PRCOUCTION COST REPORT DEC 1967

PROGRAM O2 PLANNING, FY-1970 - 1974

CAPITAL BUDGET ITEMS \& SIGNIFICANT

PPERATING EFFECTS

PROOUCTION FUELS SECTION - CAPITAL

BUDGET ITEMS INCLUDED IN PROGRAM 02

PLANNING FY-1970 - 1973

STATUS OF UPSTREAM FUEL PROGRAM FY-1967

DUN FUEL COSTS, PRODUCTION \& UNIT COSTS

DEC 1967

DUN N REACTOR SEMIANMUAL REPORT OF

RESEARCH \& DEVELOPMENT

SLIYARY OF LITHIUM ALUMINATE TARGET

ELEMENT PERFRRMANCE FROM PTA-054 210 E-N DEMONSTRATION LOADING

LONG RANGE 02 RED PROGRNM

FABRICATION OF IRE BARE CORES BY

EXTRUSION

MONTHLY MANAGEMENT REPORTS - CY 1968

REACTOR PROCESS DEVELOPNENT UNIT

REPORT OF THE HORKING COMMITTEE OF THE

FEDC

WORKING PAPERS

GRAPHITE STACK LIFE

RADIOMETALLURGY EXAMINATION OF B REACTOR

WATERAiXX ELEHENT FÁLLURE
AUTHOR

JF CAMP III

JF CAMP III

LEVEL PGS DATE

DG OLAMDER

SECR

DG OLANDER

SECR

$03 / 04 / 70$

SECR

$09 / 20 / 72$

SECR

$09 / 20 / 72$

SECR

$09 / 20 / 72$

SECR

$09 / 20 / 72$

RM FULSMAS

SRD

$.01 / 02 / 68$

RM FULSMS

SECR

$01 / 02 / 68$

JW REMFRO

CONF

$01 / 08 / 68$

SECR

$09 / 20 / 72$

SECR

$06 / 10 / 71$

SECR

$09 / 20 / 72$

SECR

$09 / 20 / 72$

SECR

$09 / 20 / 72$

$06 / 10 / 71$

$01 / 18 / 68$

SECR

$09 / 20 / 72$

RH SCAMION

$09 / 20 / 72$

$01 / 31 / 68$

PA CARLSON

SRD

$02 / 05 / 68$

SECR

$04 / 08 / 71$

RL MILLER

SRD

SECR

SECR

$02 / 01 / 68$

$09 / 20 / 72$

$09 / 20 / 72$ 
Page No. 68

$04 / 28 / 93$

LIST OF HANFORD-GENERATED CLASSIFIED

DOCUMENTS DATED BETUEEN 1/1/61 AND 12/31/72

DOC. NO.

TITLE

DUN-03783

DUN-03786REV2

DUN-03788

DUN-03796

DUN-03804RD

DUN-03806

DUN-03809

DUN-03810

DUN-03812RD

DUN-03814

DUN-03815

DUN-03816

DUN-03816RD

DUN-03817RD

DUN-03819

DUN-03822

DUN-03830

DUN-03831RD REV2

DUN-03832

DUN-03835

DUN-03848

DUN-03852RD

DUN-03858

DUN-03862

DUN-03886RD

DUN-03890RD

DUN-03892RD

DUN-03894

DUN -03895

DUN-03896 REV2

REPORT

6/30/73

6/30/73

JAN 1968 $5 / 31 / 68$

$5 / 31 / 78$
FLUX MONITOR HIGH TRIP SETTINGS KE REACTOR, REACTOR STARTUP 1/11/68

ALSI FUEL PERFORMANCE HONITORING PT-011,

DUN C-K REACTORS SEMIAMMUAL RPT OF RED

KE-REACTOR FLUX MONITOR TRIP SETTINGS

N RESEARCH \& EMGIMEERIHG MAMAGEMENT

DUN OPERATED PROOUCTION REACTORS

OPERATING RELIABILITY

C2N - C5N FUEL SWELLING PROBLEM, RADIOMETALLURGY EXAMIMATION

HIGHLY ENRICHED FUEL DEVELOPMENT PROGRAM

WORKING PAPERS - PU 238

PRODUCTION FUELS SECTION PRODUCTION COST REPORT JAN 1968

EVALUATION OF SUGGESTION MR-2209

INCREASED STEPOUT TOOL TIP RADIUS

DUN, PRODUCTION FORECAST - 1/1/68 TO

DUN, PROOUCTION FORECAST - 1/1/68 TO

BY-PRCOUCT ROO

HCR COMPENSATION OF TOP \& BOTTON E-RING

$C \& K$ REACTORS

INFCRMATION ON 00-RON IRRADIATION

TESTING FACILITIES

DUN FUEL COSTS, PRCOUCTION \& UHIT COSTS

DUN LONG-RANGE PLAN (OVERVIEH)

USE OF O-TYPE FUEL INVENTORY

PROOUCT COST GRAMS GENERATED 2ND CUARTER JAN 1968 \& FYTO

DESIGN OF SPECIFICATIONS PLUTONIUN

ALUMINUM FUEL ELEMENTS

FUELS ENGR CONTRIBUTIONS TO FEDC RPT

PARAMETRIC PHYSICS DATA FOR SEGMENTAL

DISCHARGE

MAMAGEMENT REPORT FEBRUARY 1968 FUELS MANUFACTURING SUBSECTION

MONTHLY MGT RPT 1968 ADVANCED CCNCEPTS \& PLANMING SECTION

PROBLEM \#19 UP, AOPG ADVANCED TECHMOLOGY EVALUATION

WORKING PAPERS - BJ KNUDSEN

105-KE CONTROL ROON LOG FROM 3/1/68 THRU

105-KW COWTROL ROOM LOG FROM 3/1/68 THRU

METALLURGY EXAMINATION OF 125 FUEL

ELEMENT FAILURE IN KW REACTOR PTA-067
AUTHOR

LEVEL PGS

DATE

TS DODGE

SECR

$09 / 20 / 72$

SECR

09/20/72

SECR

09/20/72

SECR

SECR

$09 / 20 / 72$

$05 / 28 / 71$

EF KRAUTTER

CRD

$09 / 20 / 72$

SECR

$09 / 20 / 72$

SECR

SRD

$09 / 20 / 72$

02/07/68

SECR

$06 / 10 / 71$

SECR

$09 / 20 / 72$

SECR

$06 / 09 / 71$

SECR

06/09/71

SECR

$09 / 20 / 72$

$09 / 20 / 72$

SECR

09/20/72

SECR

$06 / 10 / 71$

SECR

SECR

$09 / 20 / 72$

$09 / 20 / 72$

$06 / 10 / 71$

GL SMITH

CRD

$02 / 15 / 68$

SECR

$09 / 20 / 72$

$09 / 20 / 72$

CO AMY

SRD

$02 / 20 / 68$

SECR

$04 / 09 / 71$

SECR

07/08/71

BJ KNUDSEN

SRD

CONF

$02 / 23 / 68$

STAFF

CONF

$02 / 26 / 68$

SECR

$09 / 20 / 72$ 
DOC. NO.

DUW-03900

DUN-03909

DUN-03910

DUN-03911

DUN-03924

DUN-03931

DUN-03939RD

DUN-03949

DUN-03953

DUN-03955

DUN-03961

DUN-03962

DUN-03963RD

DUN-03968RD

DUN-03970

DUN-03971

DUN-03982

DUN-03984

DUN-03990

DUN-03999

DUN-04000VOL 1

DUN-04000VOL2

DUN-04003

DUN-04004

DUN-04012

DUN -04017

DUN-04018

DUN-04019
TITLE

PROOUCTION FUELS ENGR PROGRAMS (SINGLE PASS REACTORS) INCLLDING ASSUMPTIONS ORGANIZATION \& FORECAST BUDGET ASSLMPTIONS

RESEARCH \& ENGIMEERING PT SCHEDULE FLUX CAPSULE IRRADIATION IN A OO-ROW SPECIALLY IRRADIATION FACILITY CLASSIFIED SUPPLEMENT TO PACE IMPROVEMENT PROPOSAL W2332

CALCULATED GAWA FLUX \& SPECTRLM IN A HANFORD REACTOR

USE OF MATURAL TAILS FOR HIGH P -240 P PRODUCTION

RPT TO THE FEDC FRON DUN, INC. FOR THE SINGLE PASS, LOW PRESSURE REACTORS PROOUCTION FUELS SECTION PROOUCTION COST REPORT FEB 1968

THORIA DELIVERY SCHEDULE

FISSION PRODUCT RELEASE RATE FROM ALLMINUM CLAD URANIUM FUEL

HEAT GENERATION IN UNCLOOLED DY203 HCR OLIVETTI PROGRAM FOR P -238 PRCOUCTION PTA-069 COMPUTER OUTPUT DATA

REACTOR OPERATIONS MONTH END RPT $11 / 30 / 67$

DUN FUEL COSTS, PRODUCTION \& UNIT COSTS FEB 1968

CAPITAL PLANT \& EQUIPMENT BUDGET FYTO REV OF FY69 DUN, IMC.

EMERGENCY STEAM LOND, 100-B AREA

STRAP-ON RTD RESPONSE MEASUREMENTS AT KW REACTOR - STATUS REPORT

OFFSITE SHIPMENT \& RECEIPT REGISTERED

BEGINNING NOV 1, 1965

U-235 \% IN REACTOR DISCHARGES \& BASIC INVENTORIES

ENGINEERING SPECIFICATIONS HANFORD SINGLE PASS REACTOR FUEL ELEMENTS ENGIMEERING SPECIFICATIONS HANFORD SIMGLE PASS REACTOR TARGET AMD OTHER CORE-RELATED ELEMENTS

44-TUBE OVERBORE - REACTOR MODERNIZATION TEST FACILITY - 105-C BLDG - OUTAGE SUMHARY REPORT

U-233 PROGRAM TECHNICAL \& SCHEDULIMG INFORMATION

FUELS MANUFACTURING UNIT COST AMALYSIS

DUN MONTHLY REPORT APR 1968

DUN MONTHLY REPORT MAY 1968

DUN MONTHLY REPORT JUM 1968
AUTHOR

JT STRIMGER

SECR

$09 / 20 / 72$

$09 / 20 / 72$

$09 / 20 / 72$

$09 / 20 / 72$

PJ ZIMMERMAN

SECR

$09 / 20 / 72$

AF KUPIMSKI

SRD

$09 / 20 / 72$

SECR

$09 / 20 / 72$

SECR

$06 / 10 / 71$

SECR

$09 / 20 / 72$

SECR

$09 / 20 / 72$

SECR

SECR

AF KUPINSKI

CED

SECR

SECR

SECR

$09 / 20 / 72$

$09 / 20 / 72$

$03 / 15 / 68$

09/20/72

$06 / 10 / 71$

$04 / 01 / 68$

T PRLDICH

SECR

SECR

$09 / 20 / 72$

$09 / 20 / 72$

$03 / 18 / 68$

$12 / 05 / 7$

FUELS ENGINEERING

CRD

$06 / 01 / 68$

SUBSECTION

CM SCHLEIKHARDT

$05 / 14 / 69$

SECR

$09 / 20 / 72$

SECR

$09 / 20 / 72$

SECR

06/10/71

05/01/68

06/01/68

$07 / 01 / 48$ 
Page No. 70

$04 / 28 / 93$

LIST OF HANFORD-GEMERATED CLASSIFIED

DOCUMENTS DATED BETUEEN 1/1/61 AND 12/31/72

Doc. NO.

DUN-04022

DUN-04023

DUN-04053

DUN-04059RD

DUN-04082RD

DUN-04083RD

DUN-04084RD

DUN-04089RD

DUN -04099

DUN-04104

DUN-04107

DUN-04117

DUN-04120

DUN-04121RD

DUN-04130

DUN-04139

DUN-04139REV1

DUN-04144

DUN-04156

DUN-04161

DUN-04201RD

DUN-04202RD

DUN-04206RD

DUN-04207

DUN-04214

DUN-04222
TITLE

AUTHOR

LEVEL

PGS

DATE

PTA 133: IRRADIATION OF W-UO2 OR W-P 02, PLATE CAPSULES IN THE KE OR KW REACTOR SNOUT FACILITIES TISR 700 IAP TEST AUTH 133: IRRADIATION OF H-UO2 OR W-P 02, PLATE CAPSULES IN THE KE OR KW REACTOR SHOUT FACILITIES TISR 700 105-KE TEMPERATURE MAPS FROM 4/1/68 THRU 6/30/68

MISCELLLANEOUS REPORTS

LONG-RANGE PLAN WORKING PAPERS 5

INTERATION

DUN LONG-RANGE PLANNING - N-B-C-K PROGRAMS

B, C, K REACTORS ISOTOPE CAPABILITIES ALTERAATE PROGRAM EMPHASIS 02 RRD REPORT OF INVENTION SEGMENTAL DISCHARGE OF REACTOR FUEL REACTOR OPERATIONAL IMPLICATIONS RESULTING FRON COLUABIA RIVER TEMPERATURE RESTRICTIONS

BUDGET FOR FY-1970 \& REVISION OF FY 1969 SIMGLE PASS AND N REACTORS DOUGLAS UNITED MUCLEAR, INC. O2 RESEARCH AMD DEVELOPMENT

FUELS SECTION PROOUCTION FUELS COST REPORT MAR 1968

INFLUENCE OF GAS ATMOSPHERE ON MONDEFENSE PLUTONIUN PRODUCTION ECONOMICS AT C REACTOR

DUN MISSION 5 OTHER ISOTOPES PROGRAM FY-1968

DUN FUEL COSTS, PRODUCTION \& UNIT COSTS MAR 1968

DUN, PRODUCTION FORECAST - 4/1/68 TO 6/30/70

DUN, PROOUCTION FORECAST - 4/1/68 THRU 6/30/70

FOUR-INCH SPIRE FAILURE B-REACTOR

FULLY ENRICHED FUEL DEVELOPNENT PROGP.MM TISR-327, REV1

INVENTORY STLOY CHARTS

PRODUCTION CAPABILITY FOR P AT 12\% P

-240 LON PRESSURE REACTORS

FUEL TYPE AND $P-241$ CONTENT

RESULTS OF C-REACTOR DIESEL SYSTEM FLUSH TEST

FUELS SECTION FY 1970 DUDGET \& REV OF FY 1969 PRODUCTION COST \& UNIT COSTS MANUFACTURING PROCESS SPECIFICATIONS METALLIC URANIUM FUEL ELEMENTS FABRICATED BY THE ALSI CANHING PROCESS

\begin{tabular}{|c|c|c|}
\hline \multirow[t]{3}{*}{ AUTHOR } & LEVEL PGS & DATE \\
\hline & SECR & $07 / 06 / 71$ \\
\hline & SECR & $07 / 06 / 71$ \\
\hline \multirow[t]{3}{*}{ STAFF } & CoNF & $03 / 26 / 68$ \\
\hline & SECR & $09 / 20 / 72$ \\
\hline & SECR & $09 / 20 / 72$ \\
\hline GC FULLLMER & SECR & $10 / 12 / 67$ \\
\hline \multirow[t]{3}{*}{ RL MILLER } & SECR & $04 / 02 / 68$ \\
\hline & SECR & $09 / 20 / 72$ \\
\hline & SECR & $11 / 21 / 68$ \\
\hline RM SCOTT & CONF & $04 / 08 / 68$ \\
\hline \multirow[t]{2}{*}{ TH AMBROSE/JW RICHES } & SECR & $04 / 08 / 68$ \\
\hline & SECR & $06 / 10 / 71$ \\
\hline \multirow[t]{6}{*}{ LJ MELELL } & SRD & $04 / 22 / 68$ \\
\hline & SECR & $09 / 20 / 72$ \\
\hline & SECR & $06 / 10 / 71$ \\
\hline & SECR & $06 / 09 / 71$ \\
\hline & SECR & $06 / 09 / 71$ \\
\hline & SECR & $09 / 20 / 72$ \\
\hline \multirow[t]{7}{*}{ EM EPPERSON } & SECR & $04 / 24 / 68$ \\
\hline & SECR & $09 / 20 / 72$ \\
\hline & SECR & $09 / 20 / 72$ \\
\hline & SECR & $09 / 20 / 72$ \\
\hline & SECR & $09 / 20 / 72$ \\
\hline & SECR & $09 / 20 / 72$ \\
\hline & SECR & $06 / 10 / 71$ \\
\hline $\begin{array}{l}313 \text { PROCESS ENGINEERING } \\
\text { UNIT }\end{array}$ & CRD & $07 / 01 / 68$ \\
\hline
\end{tabular}


Page No. 71

$04 / 28 / 93$

LIST OF HANFORD-GENERATED CLASSIFIED

DOCUMENTS DATED BETWEEN 1/1/61 AND 12/31/72

DOC. NO.

DUN-04226

DUN-04232

DUN-04234RD

DUN-04235

DUN-04248

DUN-04256

DUN-04263

DUN-04264

DUN-04277

DUN-04289

DUN-04298

DUN-04303

DUN-04308

DUN-04316

DUN-04317RD

DUN-04328RD

DUN-04334

DUN-04334SUP

DUN-04340

DUN-04341

DUN-04345

DUN-04349

DUN-04354

DUN-04355

DUN-04356

DUN-04358

DUN-04369

DUN-04376
TITLE

AUTHOR

NR MILLER

RH KOGA

DUN BUDGET FOR FYTO \& REV OF FY69

PRCOUCTION \& RED CONTRACTORS COST \&

EMPLOYEE ASSIGNMENT AMALYSIS

C-BACKUP ADEQUACY

SPLIME SAVIMGS

FUELS SECTION PRODUCTION FUELS COST

REPORT APR 1968

PROOUCT COST GRAMS GENERATED 3RD QUARTER

3/31/68 \& FYTD

DUN FUEL COSTS, PRCOUCTION \& UNIT COSTS

APR 1968

IRRADIATION ANALYSIS PROD TEST AUTH 135. IRRADIATIOWS IN URANIUN SWELLING TYPE CAPSULES IN KE \& KU REACTORS

DUN, INC. FY 1970 PROGRAM 02 BUDGET

ALTERMATES

CHARGE-DISCHARGE SEQUENCE FOR E-D/E-Q

TRANSITION

COPRODUCT FUEL DESIGNS

FUEL \& TUBE REDESIGN FOR C REACTOR

TOTAL CONTROL TEMPERATURE EFFECT - $K$

REACTORS

THCRIA CHARGING

SPECIAL STUOY

SPECIAL STLOY (SUPPLEMENT)

105-KE CONTROL ROOW LOG 6/68 - 8/68

105-KE CONTROL ROOH LOG 6/68 - 8/68

POISON BURNUP IN REACTOR HCR'S

EFFECTIVE PU-238 \& NP-237 HEUTRON

ABSORPTION CROSS SECTIONS IN N \& $K$

REACTORS

K REACTOR PU-238 DEHONSTRATIOH RESULTS

(INTERIM RPT PTA-063)

ROL PROGRAM CHANGE $\$ 74$, EMERGENCY

COOLANT NDEQUACY LIMITS, 105-B, C, KE \& KW REACTORS

SIMGLE PASS REACTORS UNIT COST

COMPARISON FY 1968 THRU FY 1970

COLD LONG TERM GAINS - C REACTOR

TONHAGE \& MDS DISCHARGED FY 1969

PROOUCTION RECORDS, PRCOUCTION SCHEDULE 100 AREAS FY 1969
LEVEL PGS DATE

SECR

$09 / 20 / 72$

SECR

$09 / 20 / 72$

SECR

$05 / 08 / 68$

05/03/68

SECR

$09 / 20 / 72$

$09 / 20 / 72$

$06 / 10 / 71$

SECR

SECR

$06 / 10 / 71$

SECR

$06 / 10 / 71$

SECR

$07 / 06 / 71$

SECR

$09 / 20 / 72$

SECR

09/20/72

SECR

SECR

SECR

$09 / 20 / 72$

$09 / 20 / 72$

09/20/72

SECR

SECR

SECR

SECR

SECR

SECR

SECR

$09 / 20 / 72$

09/20/72

09/20/72

$04 / 08 / 71$

$04 / 08 / 71$

09/20/72

$09 / 20 / 72$

SECR

$09 / 20 / 72$

SECR

$09 / 20 / 72$

SECR

$06 / 10 / 71$

SECR

$09 / 20 / 72$

SECR

$04 / 08 / 71$

$04 / 08 / 71$ 
Page No. 72

$04 / 28 / 93$

LIST OF HANFORD-GENERATED CLASSIFIED

DOCUMENTS DATED BETHEEN 1/1/61 AND 12/31/72

Doc. NO.

TITLE

NUTHOR

LEVEL

PGS

DATE

DUN-04382

FUELS SECTION PRCOUCTION FUELS COST

SECR

$06 / 10 / 71$

REPORT MAY 1968

DUN-04388

DUN-043954

DUN-043955

DUN-043956

DUN-04396

DUN-04399RD

COMPENDIUM OF REACTOR INCIDENTS

DUN-RL

$07 / 01 / 68$

DUN CONVENTIONAL REACT \& ENG DATA

DUN CONVENTIONAL REACT/ENG SOURCE DATA

SECR

$04 / 09 / 71$

DUN CONVENTIONAL REACT/ENG SOURCE DATA

DUN CONVENTIONAL REACTORS \& ENGINEERING

$04 / 08 / 71$

$04 / 08 / 79$

REPORT TO COST FY69

CP DORISS

$07 / 01 / 68$

PRESENTATION CHARTS FUELS SECTION, DUN,

SECR

SECR

$09 / 20 / 72$

DUN-04400RD

IMC.

HANFORD PRODUCTION REACTOR CONPLIANCE

WITH THE GEMERAL DESIGA CRITERIA FOR

DUN-RL

SECR

$06 / 01 / 68$

DUN-04405JRD NUCLEAR POUER REACTORS

N OPERATIONS MUCLEAR MATERIALS REPORTS

N OPERATIONS MUCLEAR MATERIALS REPORTS

N OPERATIONS MUCLEAR MATERIALS REPORTS

DUN-04405LRD

DUN-04405MRD

DUN-04406 L

N OPERATIONS MUCLEAR MATERIALS REPORTS

N OPERATIONS MUGLEAR MATERIALS MONTHLY INVENTORY REPORTS FY69

DUN-04491C

DUN-044910

DUN MUCLEAR MATERIALS MONTH AND REPORTS

FOR CONSOLIDATION OF MATERIAL

BALANCE REPORT FISCAL YEAR 1969

JANUARY - MARCH 1969

DUN MUCLEAR MATERIALS MONTH END REPORTS

FOR CONSOLIDATION OF MATERIAL

BALANCE REPORT APRIL - JUNE

1969 FISCAL YEAR 1969

DUN-04416J

DUN PRODUCTION FUELS INVENTORIES, SOURCE

DATA

DUN-04416K

DUN PROOUCTION FUELS INVENTORIES, SOURCE

DATA

DUN-0.4418

DUN-04433

DUN-04435

DUN-0.436

DUN-0.4437RD

DUN PRODUCTION FUELS COST REPORT FY 1969

TUBE 3560-KU FLOW STOPPAGE EVENT

COEI

MBR

CONCENTRATION OF P -241 IN PRODUCTION

MATRIX SLIGHTLY ENRICHED URANIUM DRIVERS

DUN-04444RD

GRAPHITE COEFFICIENT FOR E-D LOAD - $K$

REACTOR

DUN-04445

PTA-148 IRRADIATIONS, UNITED MUCLEAR

CORP. TISR 807

DUN-04446

DUN-04448

IAPTA-148 (U-P) C IRRADIATIONS, UNITED

MUCLEAR CORP. TISR-807

ARHCO \& DUN, REVISED FY-1969 \& FY- 1970

PROGRAM 02 BUDGET ALTERNATIVES

DUN MONTHLY REPORT JUL 1968

DUN MONTHLY REPORT AUG 1968

DUN MONTHLY REPORT SEP 1968

NUCLEAR MATERIALS PRCOUCTION REPORT JULY 1, 1968 THRU JUNE 30, 1969

sech

$04 / 08 / 71$

CP DORISS

T PRUDICH

T PRLDICH

SRD

07/01/68

(s)

SECR

SECR

SECR

SECR

SECR

SECR

SECR

SECR

SECR

SECR

SECR

SECR

T PRUDICH

DUN-OA 
Page No. 73

$04 / 28 / 93$

LIST OF HANFORD-GENERATED CLASSIFIED

DOCUMENTS DATED BETWEEN 1/1/61 AND 12/31/72

DoC. NO.

TITLE

AUTHOR

LEVEL PGS

DATE

DUN-04468

DUN-04469

DUN-0447301

DUN-0447302

DUN-0447303

DUN-0447304

DUN-0447305

DUN-0447312

DUN-04481

DUN-04482

DUN-04484

DUN-04485

DUN-04488

DUN-04489

DUN-04499RD REV2

DUN -04500

DUN-04501

DUN-04505RD

DUN- 04507

DUN -04513

DUN -04514

DUN -04530

DUN-04534RD

DUN-04539

DUN-04542

DUN-04544

DUN-04545

DUN-0.4547RD

DUN-04549

DUN-04550

DUN -04553
OUTAGE TIME ACCOUNT'Y C REACTOR 7/68 6/69

OUTAGE HISTORY C REACTOR 7/68-6/69

PRODUCTION INVENTORY \& MANUFACTURING

STATEAENT 7/31/68

PRODUCTION INVENTORY \& MANUFACTURING

STATEMENT 8/31/68

PROOUCTION INVENTORY \& MANUFACTURING

STATEMENT 9/30/68

PROOUCTION INVENTORY \& MANUFACTURING

STATEMENT 10/31/68

PRODUCTION INVENTORY \& MANUFACTURING

STATEMENT 11/30/68

DUN PI\&M STATEMENT 06/30/69

DUN FUEL COSTS, PRODUCTION \& UNIT COSTS

MAY 1968

FUELS SECTION PRODUCTION FUELS COST

REPORT JUN 1968

E-Q LOADING ERROR COAPENSATION IN

BUCKLED ZONE

U-233 PROGRAM LOADING PATTERN

CONSIDERATIONS

DUN FUEL COSTS, PRCOUCTION \& UNIT COSTS

JUN 1968

PRESSURE MONITOR CUAGE RESPONSE TIME

TEST TUBE 3560 - KW REACTOR

DUN LONG-RANGE PLAN (DETAILS)

RPT ON THE ANALYSIS OF DEPLETED URANILM

SASAPLES:

RADIONETALLURGY EXAM OF K5E FUEL

FAILURES

COST SAVINGS MEASURES

CYCLE CONTROL - REACTOR, AUGUST 1967

PERSCNNEL EXPOSURE REDUCTION

SUMMARY PTA-OLO - HIGH POWER DENSITY

CORE ALLOY EVALUATION

1-HXA-DMA-7-69-1 FINAL

WORKING PAPERS FOR PROD COST FY 1969

PROGRAM IRRADIATION DATA \& U-238 HISTORY

PROOUCTION FORECAST DUN, INC., 7/1/68

THRU 6/30/73

2-HXA-HYA-7-69-1

3-HXA-HVA-69-1 FIMAL

BARE CORE FABRICATION COSTS

PRCOUCT COST - GRAMS GEMERATED 4

QUARTER 6/30/68 AND FY TO DATE

UPSTREAM FUEL REPORT SECOND QUARTER - CY 1968

FUELS SECTION PROOUCTION FUELS COST RPT JUL 1968
SECR

$04 / 09 / 71$

SECR

SECR

SECR

SECR

SECR

SECR

SECR

SECR

SECR

SECR

SECR

SECR

SECR

SECR

DUN-RL

SRD

SECR

SECR

SECR

SECR

SECR

SECR

SECR

RP UALTOM

SECR

SECR

VA SCHOUVILLER

BA KENNEDY

SECR

SECR

SECR

SECR

SECR

SECR
$04 / 09 / 71$

$06 / 10 / 71$

$06 / 10 / 71$

$06 / 10 / 71$

$06 / 10 / 71$

$06 / 10 / 71$

07/06/71

$06 / 10 / 71$

$06 / 10 / 71$

$09 / 20 / 72$

09/20/72

$06 / 10 / 71$

09/20/72

$09 / 20 / 72$

09/20/72

$09 / 20 / 72$

$09 / 20 / 72$

$09 / 20 / 72$

09/20/72

08/12/69

09/20/72

$04 / 08 / 71$

08/01/68

09/20/72

07/31/68 $09 / 20 / 72$

$08 / 01 / 68$

$09 / 20 / 72$

08/05/68

$09 / 20 / 72$ 
Page No. 74

04/28/93

LIST OF HANFORD-GENERATED CLASSIFIED

DOCUMENTS DATED BETUEEN 1/1/61 AND 12/31/72

Doc. No.

TITLE

AUTHOR

LEVEL PGS DATE

DUN-04554

JULY PRODUCTION FORECAST FUELS SECTION -

SECR

$09 / 20 / 72$

DUN, INC., JUL 1968 THROUGH JUNE 1973

DUN, INC. PRODUCTION - MNO JUL FY 1968

DUN-04558

DUN- 0455802

DUN, INC. PRCOUCTION - MUD AUG FY 1969

WATER (MN GAS) \& STEAM DISTRIBUTION (M

LBS)

DUW-0456002

HATER (HM GAS) \& STEAM DISTRIBUTION (M

LBS)

DUN-0456003

WATER (MA GAS) \& STEAM DISTRIBUTION (M LBS) SEP FY 1969

DUN-0456004

HATER (MN CAS) \& STENM DISTRIQUTION (M LBS) OCT FY 1969

DUN-0456005

WATER (NM GAS) \& STEAM DISTRIBUTION (M LBS) NOV FY 1969

DUN-0456006 WATER (MN GAS) \& STEAM DISTRIBUTION (M LBS) DEC FY 1969

DUN-0456007

DUN-0456008

WATER (MN CAS) \& STEAM DISTRIBUTION (M

L.BS) JAN FY 1969

WATER (IM GAS) \& STEAM DISTRIBUTION (H LBS) FEB FY 1969

DUN-0456009

MATER (MM GAS) \& STEAM DISTRIBUTION (H

LBS) MAR FY 1969

DUN-0456010

WATER (MN GAS) \& STEAM DISTRIBUTION (M LBS) APR FY 1969

DUN -0456011

WATER (MM GAS) \& STEAM DISTRIBUTION (M LBS) MAY FY 1969

DUN-0456012

DUN-04561

DUN-04562

DUN -04563

DUN-04565

DUN-04573

WATER (WM GAS) \& STEAM DISTRIBUTION (M LBS) JUN FY 1969

CORROSIOH IMDUCED FUEL FAILURES

STATUS OF SPECIAL REACTOR PROCESS TUBE

LOADINGS 8/9/68

FUELS COSTS, PRCOUCTION \& UNIT COSTS

JULY 1968

WASHINGTON STATE MATER QUALITY

TEMPERATURE STAMDARDS AS RELATED TO

REACTOR OPERATION

PRCD ASSUMPTIONS \& SCHEDULES JULY

SECR

$09 / 20 / 72$

$09 / 20 / 72$

SECR

$09 / 20 / 72$

SECR

$09 / 20 / 72$

SECR

$09 / 20 / 72$

SECR

$09 / 20 / 72$

SECR

$09 / 20 / 72$

SECR

$09 / 20 / 72$

SECR

$09 / 20 / 72$

SECR

$09 / 20 / 72$

SECR

$09 / 20 / 72$

SECR

$09 / 20 / 72$

SECR

$09 / 20 / 72$

SECR

$09 / 20 / 72$

SECR

$09 / 20 / 72$

SECR

$09 / 20 / 72$

SECR

$09 / 20 / 72$

SECR

$09 / 20 / 72$

PROOUCTION FORECAST F. S. 7/68 THRU 6/73.

SECR

$09 / 20 / 72$

(FUEL ELEMENTS FOR SINGLE PASS \&

REACTORS)

DUN-04575

DUN-04576

DUN-04578

DUN-04581

DUN-0458201

DUN-0458202

DUN-0458203

DUN-0458204

DUN-0458205

\section{SECR}

MATERIAL BALANCE RPT JULY 1968

FUELS ACCOUNTING SUBSECTION MONTHLY COST

ANALYSIS RPT $8 / 68$

DIESEL FLUSHING INCIDENT - 105-KE

MONTHLY UNIT COST RPT 7/31/68

MONTHLY UNIT COST RPT $8 / 31 / 68$

MONTHLY UNIT COST RPT 9/30/68

MONTHLY UNIT COST RPT 10/31/68

MONTHLY UNIT COST RPT 11/30/68
SECR

SECR

DUN-RL

SECR

SECR

SECR

SECR

SECR

SECR
$09 / 20 / 72$

$09 / 20 / 72$

$09 / 20 / 72$

$09 / 20 / 72$

09/20/72

$09 / 20 / 72$

$09 / 20 / 72$

$09 / 20 / 72$

$09 / 20 / 72$ 
Page No. 75

$04 / 28 / 93$

LIST OF HANFORD-GENERATED CLASSIFIED

DOCUMENTS DATED BETHEEN 1/1/61 AND 12/31/72

DOC. NO.

DUN-0458206

DUN-0458207

DUN-0458208

DUN-0458209

DUN-0458210

DUN-0458211

DUW-0458212

DUW-0458301

DUN-0.458302

DUN- 0458303

DUN-0458304

DUN-0458305

DUN-0458306

DUN-0458307

DUN-0458308

DUN-0458309

DUW-0458310

DUN-0458311

DUN-0458312

DUN-04584

DUN-04585

DUN -04591

DUN-04602

DUN -04603

DUN-04604

DUN-04605

DUN-04618

DUN-04631

DUN-04636RD

DUN-04637

DUN-04638RD

DUN-04639

DUN -04643

DUN-04647

DUN-04650
TITLE

MONTHLY UNIT COST RPT 12/31/68

MONTHLY UNIT COST RPT 1/31/69

MONTHLY UNIT COST RPT 2/28/69

MONTHLY UNIT COST RPT 3/31/69

MONTHLY UNIT COST RPT 4/30/69

MONTHLY UNIT COST REPORT MAY 31, 1969

MONTHLY UNIT COST REPORT JUNE 30, 1969

DISTRIBUTION OF PONER COSTS JULY 1968

DISTRIBUTION OF PONER COSTS AUG 1968

DISTRIBUTION OF PONER COSTS SEPT 1968

DISTRIBUTION OF PONER COSTS DEC 1968

DISTRIBUTION OF PONER COSTS FYTD DEC

\section{8}

DISTRIBUTION OF PONER COSTS JAN 1969

DISTRIBUTION OF PONER COSTS FEB 1969

DISTRIBUTION OF PONER COSTS MAR 1969

DISTRIBUTION OF PONER COSTS APR 1969

DISTRIBUTION OF PONER COSTS MAY 1969

DISTRIBUTION OF PONER COSTS JUNE 1969

DISTRIBUTION OF PONER COSTS FYTD JUNE 1969

RESEARCH AND DEVELOPNENT ALLOCATIONS AND

PLANT ASSISTANCE SUPPORT FY-1969

RESEARCH AND DEVELOPAENT ALLOCATIONS AND

PLANT ASSISTANCE SUPPORT FY-1969

U-233 PROOUCTION PROGRAM

OPERATIONAL PHYSICS ASPECTS OF A K E-D

LOAD

DEV TEST AUTH 152 IRRAD OF BORON CARBIDE

IN KE \& KH REACTORS TISR-384

DUN FY 1964 - FY 1966 UNIT COST

ADDITIONS OF AN IMPURITY TO THE ALSI

BOND

DEVELOPMENT TEST AUTHORIZATION 146

IRRADIATION OF CORROSIOW PROOUCTS IN THE C-1 FACILITY

ESTIMATED DEFECT COST (COEXTRUSION SHOP)

EFFECTS KLE CHARGE LENGTH FOR THORIA

SUPPORT ENRICHMENT

RECOMMENDATION ON THE UTILIZATION OF K4E

FUEL FOR K REACTOR CENTRAL ZONE LOADIMGS

REACTOR PROOUCTION STUDIES

DUN REPORT TO FEDC SEP 1968

N REACTOR FUEL SYSTEMS CAPACITIES

TOTAL CONTROL AND OPERATIONAL PHYSICS

CURVES - E-Q LATTICE

RPT OF INVENTION AN OPERATING PROCESS

DETECTING BREACHED 2R-2 SHEATHED

COPROOUCT TARGETS DISCHARGE FROM REACTOR

- STRIPPED ALLWIMUM CLAD SUBASSEMBLY
AUTHOR LEVEL PGS DATE
a

$\begin{array}{cc}\text { SECR } & 09 / 20 / 72 \\ \text { SECR } & 09 / 20 / 72 \\ \text { SECR } & 09 / 20 / 72 \\ \text { SECR } & 09 / 20 / 72 \\ \text { SECR } & 09 / 20 / 72 \\ \text { SECR } & 06 / 16 / 69 \\ \text { SECR } & 07 / 22 / 69 \\ \text { SECR } & 08 / 15 / 68 \\ \text { SECR } & 09 / 24 / 68 \\ \text { SECR } & 10 / 21 / 68 \\ \text { SECR } & 01 / 21 / 69 \\ \text { SECR } & 01 / 21 / 69 \\ & \\ \text { SECR } & 02 / 19 / 69 \\ \text { SECR } & 03 / 13 / 69 \\ \text { SECR } & 04 / 23 / 69 \\ \text { SECR } & 05 / 13 / 69 \\ \text { SECR } & 06 / 19 / 69 \\ \text { SECR } & 07 / 21 / 69 \\ \text { SECR } & 07 / 21 / 69\end{array}$

CD HARRINGTON CRD

$08 / 22 / 68$

JH RICHES CRD

$08 / 15 / 68$

$\begin{array}{ll}\text { SECR } & 08 / 16 / 68 \\ \text { SECR } & 08 / 20 / 68\end{array}$

SECR

07/06/71

SECR

$06 / 10 / 71$

$08 / 13 / 68$

SECR

$10 / 18 / 68$

SECR

$08 / 22 / 68$

SECR

$08 / 26 / 68$

SECR

$08 / 27 / 68$

SECR

$05 / 20 / 71$

$09 / 01 / 68$

JM LUTTON SECR $\quad 08 / 29 / 68$

$08 / 28 / 68$

R COOPERSTEIN/HJ BELLARTS CRD

08/01/68 
DOC. NO.

DUN-04651

DUN-04652RD

DUN-04656

DUN-04657

DUN-04670

DUN-04672

DUN-04677

DUN-04678

DUN-04684RD

DUN-04690

DUN-06694

DUN-04701

DUN-04705RD

DUN-04707

DUH-04709

DUN-04710RD

DUN-04711

DUN-04718

DUN-04724

DUN-04729

DUN-04736RD

DUN-04737

DUN-04738RD

DUN-04745

DUN-04747

DUN-04747RD A DUN-04747RD B DUN-04787RD

DUN-04794

DUN-04800
TITLE

AUTHOR

R COOPERSTEIM

SRD

08/08/68 IN-REACTOR, FOR IMPROVED

PROPERTIES AMD PROLONGED REACTOR LIFE

OPERATIONS DIVISION BLDGET REDUCTION

105-KE CONTROL ROOH LOG 9/68 - 11/68

105-KE CONTROL ROON LOG $9 / 68$ - 11/68

PRODUCTION ASSUMPTIONS \& CONVERSION UNIT COST COMPARISON

C3E CORE ALLOY EVALUATION INTERIM REPORT

U-233 PROGRAM LOADING PATTERN

CONSIDERATIONS

MEMORANDUM OF PROCESS STANDARDS 8-TM

TUBE POWER ANO TORP LIMIT - $K$ REACTORS

REACTOR OPERATING STATISTICS

RECAPITULATION 7/1/68THRU

PRESSURE DROP CHARACTERISTICS: LAST

DITCH SYSTEM

U-233 PRODUCTION PROGRAM

FAST FLUX ENHANCEMENT IN A $K$ TEST

FACILITY

STATISTICAL DATA

ORALLOY PROGRAM PRIORITIES

SUMUARY REPORT K REACTOR INLET RISER

EVALUATION

REACTIVITY CHANGE DUE TO FLON REDUCTION

AT KW REACTOR

MAJOR ACCOAPLISHMENTS, N REACTOR
COPROOUCT DEMONSTRATION

FUELS SECTION PRCOUCTION FUELS COST

REPORT AUG 1968

DUN FUEL COSTS, PRCOUCTION \& UNIT COSTS

AUG 1968

MEUTRON FLUX TAILORING FOR HANFORD

IRRADIATION REQUEST NO. 811

$K$ PLANT ENGINEERING DATA

THORIA DELIVERY SCHEDULE

THORIA PROGRAMS

CARBON-14 EXTRACTION FROM REACTOR EAS

PROGRESS REPORT PRODUCTION TEST

AUTHORIZATION \#124

PTA-150, K REACTOR PLUTONIUM

IRRADIATIONS

PTA-150 DATA SHEETS

PTA-150 DATA SHEETS

THORIA PROGRAM - EFFECTS OF TORP

REDUCTION AND TUBE PONER LIMITS AT $K$

AREAS

DUN, INC. FINISHED FUEL INVENTORY CHARTS

OC SCHROEDER

SECR

SECR

SECR

SECR

SECR

SECR

SECR

DL DENEAL

SRD

SECR

SECR

SECR

Ja COWan

JM LUTTON

SRD

CONF

SECR

SECR

RG GEIER

SECR

SECR

JR LAMGTON

RL MILLER

BASED ON JULY PROOUCTION FORECAST

PRESENTATION TO AEC MEADQUARTERS

DiVISION OF PROOUCTION STAFF SEPT. 24, 1968 AT HANFORD
SECR

SECR

SECR

SECR

SECR

SECR

SECR

$06 / 14 / 71$

CRD

CRD

SECR

SECR

$09 / 26 / 68$

$08 / 12 / 68$

$04 / 08 / 71$

$04 / 08 / 71$

$06 / 09 / 71$

09/05/68

$09 / 18 / 68$

$09 / 05 / 68$

09/05/68

08/01/68

$09 / 12 / 68$

$09 / 12 / 68$

$09 / 10 / 68$

$09 / 11 / 68$

$09 / 10 / 68$

$09 / 11 / 68$

$09 / 11 / 68$

$06 / 10 / 71$

$06 / 10 / 71$

$09 / 17 / 68$

$05 / 20 / 71$

$09 / 18 / 68$

$05 / 25 / 79$

$01 / 24 / 69$

$02 / 24 / 69$

$11 / 12 / 70$

$09 / 25 / 68$

SECR 
Page No. 77

$04 / 28 / 93$

LIST OF MANFORD-GENERATED CLASSIFIED

DOCUMENTS DATED BETMEEN 1/1/61 AND 12/31/72

DOC. NO.

DUN-04801

DUN-04808RD

DUN-04811

DUN-04812

DUN-04813

DUN-04819

DUN-04825

DUN-04828

DUN-04837

DUN-04839RD

DUN-04845

DUN-04862

DUN-04863

DUN-04870RD

DUN-04885RD

DUN-04904

DUN-04911

DUN-04914

DUN-04926

DUN-04927

DUN-04933

DUN-0493302

DUN-0493303

DUN-04935RD

DUN-04943RD

DUN-04944RD

DUN-04952RD

DUN-04953

DUN-04962

DUN-04964

DUN-04969

DUN-04971 RD
TITLE

AUTHOR

CHARGING OF K4 EMRICHED FUEL IN CEHTRAL ZONE $K$ REACTOR TUBES

GRAMS GENERATED REPORTS FY 1969

DUW MONTHLY REPORT OCT 1968

DUN MONTHLY REPORT MON 1968

DUN MONTHLY REPORT DEC 1968

DEVELOPNENT TEST - 38 FRONT RISER

PRESSURE TEST - $K$ REACTCR

DUN, PROOUCTION FORECAST - 10/1/68 THRU

6/30/70

SPLINES FOR OVERBORE LATTICE

MONTHLY ECUIPMENT MAINTEMANCE STANDARDS

CHECK SHEETS

FUELS SECTION MONTHLY RECORD REPORT -

MARCH 1968 THROUGH JUNE 1969

FLUX FACTORS FOR ITU TEST FACILITIES

FUELS SECTION PRODUCTION FUELS COSY

REPORT SEP 1968

SCRAP AMD RELDRK COSTS 313 ALSI

PRCOUCTION 7-23-68 - 8-16-68 ANO 8-17-68

- 9-19-68

MONTHLY MEMT REPORTS FRON 4/66

CLASSIFIED WORKING PAPERS FUELS

MANUFACTURING

FUEL COSTS, PRCOUCTION \& UNIT COSTS SEP

1968

105-KW OUTAGE SUMMARIES

BASIN STORAGE CAPACITIES

CHARGE LENGTHS \& DOWNTREAM SPACER

PATTERNS C, KE \& KU REACTORS

C REACTOR FUEL FAILURES TUBES 2562 \&

3361

UTILIZATION OF 1.114 WTX U-235 AS PLANT

STAMDARD EMRICHMENT FOR CEK REACTORS

111 METAL - INTERIM REPORT MEAPONS GRADE PLUTONIUN PROOUCTION

111 METAL - INTERIM REPORT - 111 E-Q

REACTOR LONDINGS

PROGRAM ANALYSIS OVERBORE

MANAGEMENT \& RECORD REPORTS

N REACTOR STUDIES (CP)

WORKING PAPERS - 100 N AREA

K REACTOR PROCESS PIPING CALCULATIONS

UPSTREAM FUEL REPORT THIRD CUARTER CY

1968

OUTAGE SCHEDULES

GRAPHITE TEMPERATURE READINGS FOR SPEED OF CONTROL

PRODUCTION DATA FOR BUDGET REVIEH PURPOSES

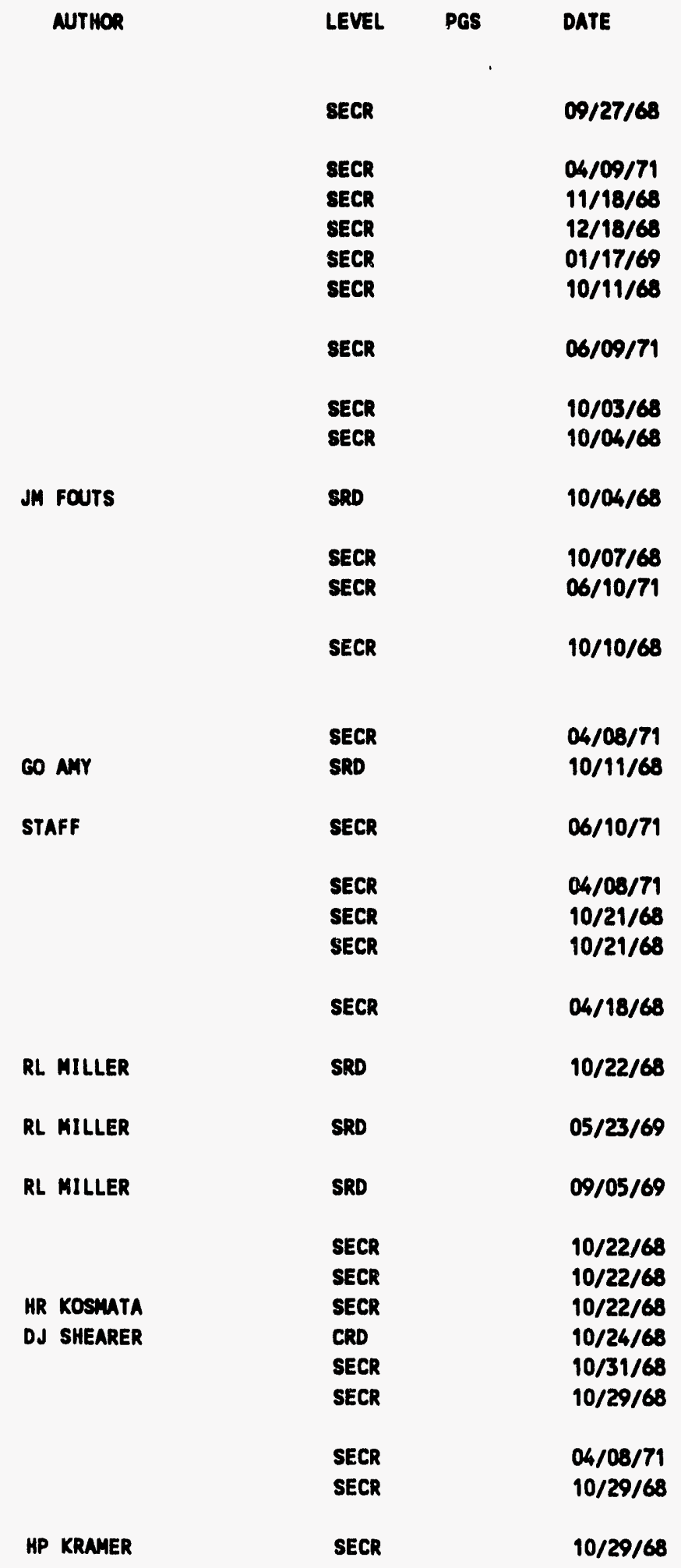


Page No. 78

O4/28/93

LIST OF MANFORD-GENERATED CLASSIFIED

DOCUMENTS DATED BETWEEN 1/1/61 AND 12/31/72

Doc. NO.

DUN-04973RD

DUN-04977

DUN-04979

DUN-04985RD

DUN-04989

DUN-04991RD

DUN-05000PT4

DUN-05000PT5

DUN-05000PT6

DUN-05000PT7

DUN-05006 RD

DUN-05006RD

DUN-05012

DUN-05014

DUN-05023RD

DUN-05026

DUN-05028

DUN-05029

DUN-0502901

DUN-05032

DUN-05046

DUN-05050

DUN-05052

DUN-05053RD

DUN-05056

DUN-05062

DUN-05064

DUN-05066

DUN-05068RD

DUN-05069RD

DUN-05070RD

DUN-05071RD

DUN-05072RD

DUN-05073RD
TITLE

OPERATIONS CHARGE- DISCHARGE

MATER TREATMENT TEST FACILITY STUDIES JULY 1964 THRU JULY 1967

MANFORD ISOTOPE PROOUCTION CAPABILITIES

FOR SIMGLE PASS REACTORS

- REVIEN OF OVERBORE UNIT COST INCENTIVES ORALLOY TIMIME

MEAT RPTS PROO PLANNING OPERATION

KW INCIDENT MISCELLANEOUS IMFORMATION

KW IMCIDEAT MISCELLAMEOUS INFORMATION

KW INCIDENT MISCELLAMEOUS IMFORMATIOW

RH INCIDENT MISCELLANEOUS INFORMATION

COMENTS ON TRITIUN PROERN AMALYSIS

COMNENTS ON TRITIUN - PROCRAM ANALYSIS

REPORT ON SHORT POISON COLLMM LENGTH FOR

TOTAL CONTROL - KW REACTOR

PRODUCT COST GRAMS GENERATED IST QUARTER FY 1969

SPECIAL MUCLEAR MATERIALS MARKET STUDY

FOR PLANNING PURPOSES

DUN FIVE YEAR ELDEET PLAN, FY 1971-1975

PROGRAM O2 CAPITAL QUDGET LIME ITENS

SECRET WORKING PAPERS

COST BUDGET REPORT

COST BUDGET REPORT

FUELS SECTION PRODUCTION FUELS COST

REPORT OCT 1968

FIHAL REPORT N REACTOR COPROOUCT

DEMONSTRATION

FUEL COSTS, PRODUCTION \& UNIT COSTS OCT 1968

EXTRACTION OF TRITIUN FROM LITHIUM -

ALUMINATE TARGETS

PLAMMIMG ESTIMATE CHARTS

STATUS OF PTA-127 SUP1, IRRADIATION OF FULL LENGTH SURPLUS B REACTOR FUEL

COLUMUS IN THE FRIMGE OF C REACTOR

GRAPHITE BAR IRRADIATION HISTORY C REACTOR

FDS FUEL FABRICATION AND USAGE

PRCOUCTION OF ALL PILES - FY 1969 - 1970

- 1971

COST STUDIES

COST CHARTS

ABCM - CIRIUM MODEL TEST CASES

CONVERSION RATIO POLLI FITS - ALL FUEL

SSA COMPUTER RUN WORKING PAPERS

GRAPHITE TEMPERATURE DATA - KW REACTOR

$(10 / 11 / 55$ - 10/12/56)(7/25/58 -
MTHOR

LEVEL

PGS

DATE

DUN-RL.

DUN-RL.

DUN-RL

TH AMBROSE

R NILSON

JP MAMRIC

SECR

SECR

10/29/68

$11 / 05 / 68$

SECR

$12 / 12 / 68$

SECR

$10 / 30 / 68$

$10 / 31 / 68$

$04 / 08 / 71$

$05 / 20 / 71$

$05 / 20 / 71$

05/20/71

$09 / 25 / 68$

$11 / 04 / 68$

$11 / 04 / 70$

$11 / 18 / 68$

$06 / 10 / 79$

$11 / 08 / 68$

STAFF

$12 / 18 / 68$

11/07/68

$06 / 10 / 71$

$06 / 10 / 71$

$06 / 10 / 71$

RG GEIER

SECR

$11 / 15 / 68$

SECR

$06 / 10 / 71$

R COOPERSTEIn

SECR

$11 / 13 / 68$

RW REID

SECR

CONF

$11 / 13 / 68$

$11 / 14 / 68$

SECR

$11 / 14 / 68$

RH KOGA

SECR

SRD

$11 / 13 / 68$

$11 / 15 / 68$

RA MELSOW

SRD

$11 / 20 / 68$

ER PERL

SECR

SRD

SECR

ER PERL.

LR MOWSON
$11 / 20 / 68$

01/01/67

$12 / 28 / 71$

$11 / 20 / 68$

$11 / 01 / 68$

$11 / 6 / 60)$ 
Page Ho. 79

$04 / 28 / 93$

LIST OF HAMFORD-GENERATED CLASSIFIED

DOCUMENTS DATED BETWEEN 1/1/61 AMD 12/31/72

DOC. NO.

DUN-05073RD SUP1

DUN-05073RD SUP2

DUN-05073RD SUP3

DUN-05075RD

DUN-05089

DUN-05090RD

DUN-05091RD

DUN-05094

DUN-05094RD1

DUN-05094RD2

DUN-05094RD3

DUN-05094RD 4

DUN-05094RD5

DUN-05094R06

DUN-05094RD7

DUN-05094RD8

DUN-05095

DUN-050951

DUN-050952 RD

DUN-05095RD3

DUN-05095RD 4

DUN-05095RDS

DUN-05103

DUN-05112RD

DUN-05119RD

DUN-05123RD

DUN-05129RD

DUN-05141RD

DUN-05142

DUN-05147RD

DUN-05149RD

DUN-05151RD

DUN-05152RD

DUN-05155RD

DUN-05165RD

DUN-05169RD

DUN-05170RD

DUN-05171RD

DUN-05172RD

DUN-05173RD

DUN-05174RD

DUN-05178
TITLE

GRAPHITE TEMPERATURE DATA - KY REACTOR

PONER DATA

FISCAL YEAR 1969 MID-YEAR BUDGET REVIEH

COLLMBIA RIVER PROCRNK

BRITTLE FRACTURE PROCRAM

105-KE CONTROL ROON LOG 2/68

105-KE CONTROL ROON LOG 3/69

105-KE CONTROL ROCN LOS 6/69

105-KE CONTROL ROCH LOG 9/69

105-KE CONTROL ROOH LOS 12/69

105-KE CONTROL ROON LOS 3/70

105-KE CONTROL ROON LOG 6/70

105-KE CONTROL ROON LOG

105-KE CONTROL ROOA LOG

105-KE CONTROL ROON LOG 2/69

105-KE CONTROL ROON LOG 3/69

105-KE CONTROL ROON LOG 6/69

105-KE CONTROL ROON LOG 9/69

105-KE CONTROL ROON LOG 12/69

105-KE CONTROL ROON LOG 3/70

STRAP-ON RTD'S TRIP LIMITS - KW REACTOR

OCTOBER 31, 1968 INVENTORY

210 E-N WORKING PAPERS

DATA SHEETS FOR PTA-127 \& SUP A

CLASSIFIEO ROUGH DRAFTS

MONTHLY MANAGEMENT REPORTS PROCESS

EVALUATION SUBSECTION

FUEL EXPOSURE C REACTOR

LOAD CODES - C REACTOR

N REACTOR COST PERFORMANCE

POTENTIAL MODES OF REACTOR OPERATION

S-RD, A.P. VINTHER \& JR YOUNG 7-7-66

EFFECT OF 2 ALTERKATE URANIUN FEED CYCLE

MODES ON URANIUM COST AND MEPTUNIUM

PRODUCTION

MAXIMIZING THE REACTOR INVENTORY OF

U-236

ADVANCED TECHMOLOGY COST STUDIES

LONG RANGE PLAN QUESTIONS

1968 PLANNING ESTIMATE CORRESPONDENCE

AND MISCELLANEOUS INFORMATION

INFORMAL SNM REQUIREMENTS

GRAPHITE GRONTH AS A REACTOR LIMITATION

PREVIOUS PLANMING ASSUMPTIONS

LONG RANGE PLAN - MANPONER

DUN PRCOUCTION FORECAST 1/1/69 THRU

$6 / 30 / 74$
AUTHOR

LR MONSON

LR MONSON

LR MONSON

LEVEL pes DATE

CRO

$11 / 01 / 68$

$11 / 01 / 68$

$11 / 01 / 68$

$11 / 19 / 68$

$11 / 22 / 68$

JW BALLOWE $\quad$ CRD $\quad 11 / 21 / 68$

DH DIEDIKER CRD $\quad 11 / 21 / 68$

SECR $\quad 04 / 08 / 71$

SECR $\quad 04 / 08 / 71$

SECR $\quad 04 / 08 / 71$

SECR $\quad 04 / 08 / 71$

SECR $\quad 04 / 08 / 71$

SECR $\quad 04 / 08 / 71$

SECR $\quad 04 / 08 / 71$

SECR $\quad 04 / 08 / 71$

SECR $\quad 04 / 08 / 71$

SECR $\quad 04 / 08 / 71$

SECR $\quad 04 / 08 / 71$

SECR $\quad 04 / 08 / 79$

SECR $\quad 04 / 08 / 71$

SECR $\quad 04 / 08 / 71$

SECR $\quad 04 / 08 / 71$

JP HAMRIC CRD $\quad 11 / 26 / 68$

SECR $\quad 11 / 27 / 68$

JR LANGTOW CONF 12/02/68

$04 / 09 / 71$

$12 / 03 / 68$

01/02/69

$12 / 10 / 68$

$12 / 04 / 68$

$08 / 18 / 66$

$10 / 01 / 68$

$08 / 15 / 68$

SECR

$06 / 17 / 68$

SECR

$04 / 14 / 66$

SECR

SECR

$04 / 30 / 68$

$12 / 05 / 68$

SECR

$02 / 20 / 67$

$02 / 13 / 68$

$01 / 23 / 68$

07/02/68

$06 / 10 / 71$ 
Page No. 80

04/28/93

LIST OF MAMFORD-GENERATED CLASSIFIED

DOCLMENTS DATED BETHEEN 1/1/61 AND 12/31/72

Doc. No.

TITLE

AUTHOR

PGS

DATE

DUN-05178RD PT1

DUN-05178RD PT2

DUN-05178RD PT3

DUN-05185RD

DUN-05195

DUN-05201

DUN-05204RD

DUN-05206

DUW-05207

DUN-05209

DUN-05211

DUN-05212

DUN-05221

DUN-05226

DUN-05227RD

DUN-05230

DUN-052371

DUN-05238

DUN-0523910

DUN-0523911

DUN-05239RD 1

DUN-05239RD 12

DUN-05239RD 15

DUN-05239RD17

DUN-05239RD 18

DUN-05239RD19

DUN-05239RD21

DUN-05239RD3

DUN-05239RD 4

DUN-05239RD5

DUN-05239RD6

DUN-05239RD7

DUN-05239RD8

DUN-05239RD9
DUN, PROOUCTION FORECAST - 01/1/69 THRU 6/30/71

QUARTERLY PRODUCTION FORECAST N REACTOR

- DUN 1-1-69 THRU 6-30-74

DUN OUARTERLY PRCOUCTION FORECAST FUELS

- 1/1/69 - 6/30/74

LIME CAPITAL ITEMS UNDER CONSIDERATION

IN DUW

COOLAMT PIPING STRESS AMALYSIS C \& $K$

AREASUN

FUELS SECTION PRCOUCTION FUELS COST

REPORT NOV 1968

HAMDLING OF EMPTY MMGAZINES $K$ REACTOR

FUEL CHARGIM

DQUGLAS UNITED MUCLEAR, IMC SEMIANMUAL

RESEARCH AMD DEVELOPNENT REPORT

SUIMURY

DOUGLAS UNITED MUCLEAR, INC SEMIANMUAL

REPORT OF RESEARCH AMD DEVELOPNENT

HI-SPEED SCAMNER TRIP LIMITS

PROCESS STANDARDS VIOLATION C-07O - RATE

OF PONER INCREASE C REACTOR - NOV. 26, 1968

DUN FUEL COSTS, PRODUCTION \& UNIT COSTS

NOV 1968

PLUTONIUM FORMATION, URANIUN CREDITS, BURNOUT, AND BURNOUT COSTS

URANIUN DELIVERY SCMEDULE FUELS SECTION

\section{- ADDENDUA}

PERSONAL NOTES

MATERIALS IRRADIATION REVIEH FOR 309

CAPSULE HITH MIXED OXIDE FUEL

MONTHLY RECORD REPORT JAN THRU DEC 1970

1.114 E-O PERFORMANCE

KE REACTOR OUTAGE DATA $11 / 69$

KE REACTOR OUTAGE DATA 12/69

KE REACTOR OUTAGE DATA 1/69

KE REACTOR OUTAGE DATA

KE REACTOR OUTAGE DATA

KE REACTOR OUTAGE DATA

KE REACTOR OUTAGE DATA 7/70

KE REACTOR OUTAGE DATA 8/70

KE REACTOR OUTAGE DATA

KE REACTOR OUTAGE DATA 4/69

KE REACTOR OUTAGE DATA 5/69

KE REACTOR OUTAGE DATA 6/69

KE REACTOR OUTAGE DATA $7 / 69$

KE REACTOR OUTAGE DATA 8/69

KE REACTOR OUTAGE DATA 9/69

KE REACTOR OUTAGE DATA $10 / 69$
SECR

$06 / 09 / 71$

W JOHNSON

SECR

$12 / 17 / 68$

SECR

$06 / 10 / 71$

SECR

$10 / 31 / 68$

SECR

$12 / 16 / 68$

SECR

$06 / 10 / 71$

SECR

$12 / 04 / 68$

TW AMBROSE

SRo

$12 / 12 / 68$

TU Ambrose

$12 / 31 / 68$

$12 / 12 / 68$

$12 / 12 / 68$

SECR

SECR

$06 / 10 / 71$

SECR

$01 / 10 / 69$

SECR

$12 / 18 / 68$

RV SKINMER

CRD

SECR

SECR

SECR

SECR

SECR

SECR

SECR

SECR

SECR

SECR

SECR

SECR

SECR

SECR

SECR

SECR

SECR

SECR

SECR
$12 / 18 / 68$

$12 / 26 / 68$

$04 / 09 / 71$

$12 / 26 / 68$

$04 / 08 / 71$

$04 / 08 / 71$

$04 / 08 / 71$

$04 / 08 / 71$

$04 / 08 / 71$

$04 / 08 / 71$

$04 / 08 / 71$

$04 / 08 / 71$

04/08/71

$04 / 08 / 71$

04/08/71

$04 / 08 / 71$

$04 / 08 / 71$

$04 / 08 / 71$

$04 / 08 / 71$

$04 / 08 / 71$ 
Page No. $\$ 1$

$04 / 28 / 93$

LIST OF HANFORD-GENERATED CLASSIFIED

DOCUMENTS DATED BETHEEN 1/1/61 AND 12/31/72

Doc. No.

DUN-0j240

DUN-05244RD

DUN-05250

DUN-05251

DUN-05254

DUW-05255

DUN-05256

DUN-05258

DUN-05258RD

DUN-05262

DUN-05270

DUN-05272

DUW-05277

DUW-05282

DUN-05293

DUN-05295RD

DUN-05296RD

DUN-05298RD

DUN-05299RD

DUN-05300RD

DUN-05301RD

DUN-05302RD

DUN-05303RD

DUN-05304RD

DUN-05309

DUN-05313

DUN-053?.1

DUN-05325RD

DUN-05329

DUN- 05336
TITLE

SOME OPERATIONAL PHYSICS ASPECTS OF AN E-N DEMONSTRATION LOADING IN A K REACTOR WORKING PAPERS FUELS SECTION FY 1969 MIDYEAR BUDGET REVIEH MATERIAL BALANCE REPORT - DECEMBER 1968 CCAPOSITION OF ENDING INVEATORY - DEC 1968

DUN MONTHLY REPORT JAMUARY 1969

DUN MONTHLY REPCRT FEBRLARY 1969

DUN MONTHLY REPORT MARCH 1969

STRESS AMALYSIS OF 100-K PRIMARY PIPING STRESS AMALYSIS OF 100-K PRIMARY PIPING CUTACE DATA BK C REACTOR $3 / 69$ COSTS OF PREPARING A REACTOR FOR IMPLEMENTATION OF MFC- 12 PROJECTS AVERAGE U-236 CONTENT ON INCOMIMG ENRICHED CORES FOR SINGLE PASS REACTORS MINUTES OF CLASSIFICATION MEETIMG 12-10-68 DIRECT COAPEMSATION METHOO FOR TOTAL CONTROL

SUMARY PROCESS WORK REQUEST HO 75-8 AND SUPPLENENTS A, B, C AND D

SECTION 1, REACTOR INCIDENT FILE GEMERAL IAFORMATION FROA 1945

SECTION 2, REACTOR INCIDENT FILE GENERAL INFORMATION FROM 1945

SECTION 4, REACTOR INCIDENT FILE GENERAL INFORMATION FROM 1945

SECTION 5, REACTOR INCIDENT FILE GEMERAL INFORMATION FROM 1945

SECTION 6, REACTOR INCIDENT FILE GENERAL INFORMATION FRON 1945

SECTION 7, REACTOR IMCIDENT FILE GENERAL INFORMATION FRON 1945

SECTION 8, REACTOR INCIDENT FILE GENERAL INFORMATION FRON 1945

SECTION 9, REACTOR IMCIDENT FILE GEMERAL INFORMATION FROM 1945

SECT 10, REACTOR INCIDENT FILE GEMERAL INFORMATION FRON 1945

SEGMENTAL CHARGE-DISCHARGE ECONOMICS $x$ REACTOS

ECONOMICS OF SEGMENTAL CHARGE-DISCHARGE

OF K REACTOR FUEL

FUELS ACCOUNTING SUBSECTION MONTHLY COST

ANALYSIS REPORT - 1/69

URANIUN IAVENTORI:S AS OF MOV 1968

URANIUN IMVENTORY

TORP LIMITS FO: $K$ REACTORS
AUTHOR

LEVEL PGS DATE

DM DIEDIKER

SECR

01/02/69

SECR

$12 / 31 / 68$

SECR

$12 / 31 / 68$

SECR

$12 / 31 / 68$

SECR

SECR

$02 / 18 / 69$

$03 / 18 / 69$

$04 / 18 / 69$

08/01/69

08/01/69

$04 / 08 / 71$

$01 / 06 / 69$

$01 / 08 / 69$

SECR

01/08/69

$01 / 16 / 69$

$01 / 10 / 69$

DUN-RL

05/20/71

DUN-RL

05/20/71

DUN-RL

$05 / 20 / 71$

DUN-RL

SECR

$05 / 20 / 71$

DUN-RL

SECR

$05 / 20 / 71$

DUN-RL

SECR

$05 / 20 / 71$

DUN-RL

SECR

05/20/71

DUN-RL

SECR

$05 / 20 / 71$

DUN-RL

SECR

$05 / 20 / 71$

SECR

$01 / 27 / 69$

SECR

$01 / 15 / 69$

SECR

$06 / 10 / 71$

SECR

$06 / 10 / 79$

SECR

06/10/79

$01 / 20 / 69$ 
Page No.

DOC. NO.

DUN-05353

DUN-05356

DUN-05357

DUN-05359

DUN-05362

DUN-05370

DUN-05376

DUN-05385

DUN-05386

DUN-05397

DUN-05398

DUN-05400

DUN-05402

DUN-05403

DUN-05412

DUN-05427

DUN-05434RD

DUN-05439

DUN-05444RD

DUN-05445

DUN-05454

DLN-05477

DUN-05487

DUN-05491

DUN-05495

DUN-05496

DUN-05498

DUN-05503

DUN-05504RD PT!
TITLE

KE REACTOR FACTOR DATA FOR 1969

KE REACTOR MON-MATURAL URANIUM LOADING STATUS FOR 1969

KH REACTOR MON-MATURAL URANIUM LOADING REPLACEMENT OF ALKALINE CLEAMER BY ETCHANT CLEAMER ON THE CAP AMD CAN MACHIME

INTERIM REPORT: DEVELOPMENT OF TUBE

PONER LIMIT FOR K REACTORS

COST BUDGET CONTRACTOR \& EDIT REPORT FY

1971

SHUTDOW MEUTRON FLUX TRAVERSE PT 4561

105 KE, 1-20-69

THE USE OF BLENDED MATURAL TAILS FOR

HIGH PU-240 PLUTONILA PROOUCTION

LOAD CODES - C REACTOR

SCRAM TRANSIENTS - SIMGLE PASS REACTORS

K REACTOR IN-CORE CHAMBER BURMOUT DATA

LONG RANGE HANFORD FUEL COST REDUCTION

RESIDENCE TIME FOR OVERBORE FUEL

STATUS OF UPSTREAM FUEL PROGRAM CY 1968

NUCLEAR SAFETY SUMMARY FOR DUH OPERATED

B, C, $K$ AND M REACTORS

RESULTS OF DEVELOPMENT \& TESTING FOR

CHARGING PU-ALUMINUM TARGET ELEMENTS

OVER SPLINES IN K REACTOR ZIRCONIUN

TUBES

CONVERSION \& DEPRECIATION FY 1969

PU BURMING - TARGET FABRICATION PROPOSAL DUN LONg-Range PLANNIng - N,B,C,K PROGRAMS

BPA FLOW LIMITS FOR NTTF TUBES

MEASURED STRESS AMALYSES OF THE ELLIPTIC AND SPHERICAL RISER DOMES 105 KE, D

RISER

STRAP-OW RTD RESPONSE MEASUREMENTS AT KW REACTOR - STATUS REORT

STATUS RE PORT NO. 5 K REACTOR BRITTLE

FRACTURE PRT $3 R A M$

KW INCIDENT

FUELS ACCOUNTING SUBSECTION MONTHLY COST

ANALYSIS REPORT FEB 1969

OVERTIME USAGE STUDY

LOAD COOES - $K$ REACTORS

MEMORANDUM OF PROCESS STANDARDS

RELAXATION 9-3M DEVIATIOHS FROM MORMAL

CHARGE CIMPOSITION - MIXED CHARGE, C

REACTOR

DUN PRODUCTIO STLY FOPECAST 4/9/69

THRU 6/30/74
AUTHOR

RM FULSMAS

RM FULSMAS

SRD

SRD

$01 / 23 / 69$

$01 / 23 / 69$

SECR

$04 / 08 / 79$

SECR

07/01/69

VA SCHOUVILLER

SECR

$01 / 13 / 69$

SRD

$01 / 24 / 69$

SECR

$01 / 27 / 69$

SECR

$01 / 31 / 69$

SECR

$01 / 29 / 69$

02/03/69

$01 / 31 / 69$

$02 / 04 / 69$

$02 / 04 / 69$

02/03/69

$01 / 31 / 69$

08/07/68

VA SCHOUVILLER

SRD

CRD

SECR

$07 / 01 / 68$

EM EPPERSON

SECR

SECR

$02 / 11 / 69$

$02 / 13 / 69$

SECR

$02 / 19 / 69$

SECR

$02 / 19 / 69$

DUN-PL

SECR

SECR

$04 / 08 / 71$

$06 / 09 / 71$

SECR

$02 / 24 / 69$

SECR

$02 / 19 / 69$

$02 / 20 / 69$

SECR 
Page No. 83

$04 / 28 / 93$

LIST OF HANFORD-GENERATED CLASSIFIED

DOCUMENTS DATED BETVEEN 1/1/61 AND 12/31/72

Doc. NO.

DUN-05506

DUN-05513

DUN-05533

DUN-05534RD

DUN-05535B RD

DUN-05535RD A

DUN-05538RD

DUN-05542

DUN-05544

DUN-05550

DUN-05550RD PT1

DUN-05350RD PT2

DUN-05552

DUN-05555RD

DUN-05557

DUN-05565

DUN-05568RD

OUN-05576

DUN-05578

DUN-05585

DUN-05588

DUN-05590

DUN-05591

DUN-05592

DUN-J5594

DUN-05607

DUN-05610

DUN-05611

DUM-05612

DUN-05614
TITLE

RADIOMETALLURGY EXAM REPORTS K 5E HOLE

FAILURE

KE PROCESS ENGINEERING LOG

LOAD CODES - C REACTOR

PTA-150 POP RUNS

PTA-171 MERICIUM IRRADIATION TEST DATA

PTA-171

PRESENTATION CHARTS FOR 3560-KW INCIDENT

CONSIDERATION OF POSSIBLE FRAGMENT

EJECTIONS FROM A BRITTLE FAILURE OF A K

RISER

FY71 BLEND CASE - $N$ \& $K$ REACTORS

REVISION - DUN PRODUCTION STUDY FORECAST

4/1/69 THRU 6/30/74

REVISION - DUN PRODUCTION STUDY FORECAST

4/1/69 THRU 6/33/74

REVISED PRODUCTION STUDY FORECAST FUELS

- DUN, INC.., APRIL 1, 1969 THRU JUNE 30, 1974

ROL CHANGES \& ADDITIONS

PRODUCTION DIVISION O2 MID-YEAR REVIEH -

FEB 17-19, 1969

LAST DITCH BACKUP DURING ALTERNATE

OPERATION OF THE K REACTORS

$K$ REACTOR TUBE PONER LIMITS

PU-238 WORKING PAPERS, BOOK II

REACTOR STACK MATERIAL PROPERTIES AND

TEMPERATURE MAP

DEVELOPMENT TEST 38 FRONT RISER PRESSURE

TEST - $K$ REACTOR RESULTS OF TESTS ON C

AND $D$ RISERS

PROPOSED DECLASSIFICATION OF ENHANCED

FAST MEUTRON FLUX IN SPECIAL TEST

FACILITIES

MEMORANDUM OF PROCESS STAMDARDS 9-5M

MAXIMUM AND MINIMMM TORP RECUIREMENTS,

KE AND KU REACTORS

MATERIALS IRRADIATION REVIEW GRAPHITE COATING STUDIES, DUN-053

INVESTIGATION OF ONE-IHCH OVERBORE TUBE RUPTURE

FUELS ACCOUNTING SUBSECTION MONTHLY COST ANALYSIS REPORT MARCH 1969

STATUS REPORT NO. $6 \mathrm{~K}$ REACTOR BRITTLE FRACTURE PROGRAM

THE DNR THORIA IRRADIATION PROGRAM

DUN MONTHLY REPORT APRIL 1969

DUN MONTHLY REPORT MAY 1969

DUN MONTHLY REPORT JUNE 1969

SM DIP AT C REACTOR ON 3-10-69
AUTHOR

LEVEL PGS DATE

SECR

$02 / 28 / 69$

TA GREEN

CONF

$02 / 24 / 69$

JR LAMGTON

SECR

$02 / 27 / 69$

03/03/69

$07 / 03 / 69$

$06 / 19 / 69$

05/20/71

AF KUPINSK

03/27/69

OC SCHROEDER

SECR

$03 / 11 / 69$

$06 / 10 / 71$

SECR

$06 / 10 / 71$

SECR

$03 / 13 / 69$

DL HIRSCHEL

CRD

$03 / 04 / 69$

SECR

$02 / 19 / 69$

SECR

$03 / 06 / 69$

TS DODGE

SECR

SRD

SECR

$03 / 12 / 69$

03/10/69

03/03/69

SECR

03/12/69

SECR

$03 / 13 / 69$

SECR

03/13/69

SECR

$03 / 17 / 69$

WK ALENXANDER

CoNf

$03 / 17 / 69$

PC MCMAHON

SRD

$06 / 18 / 69$

SECR

03/18/69

SECR

03/19/69

05/16/69

$06 / 16 / 69$

$07 / 16 / 69$

03/21/09 
Page No. 84

$04 / 28 / 93$

LIST OF HANFORD-GENERATED CLASSIFIED

DOCUMENTS DATED BETUEEN 1/1/61 AND 12/31/72

DoC. NO.

DUN-05621

DUN-05630RD

DUN-05632RD

DUN-05640

DUN-05645

DUN-05652

DUN-05653

DUN-05658

DUN-05660

DUN-05664

DUN-05679RD

DUN-05697

DUN-05700

DUN-05702

DUN-05719

DUN-05721

DUN-05723

DUN-05724

DUN-05726

DUN-05728

DUN-05735

OUN-05741

DUN-05742

DUN-05745RD

DUN-05746

DUN-05751 RD

DUN -05753

DUN-05754
TITLE

RELAXATION 9-6N DEVIATION FRON MORMAL CHARGE COMPOSITION-MIXED CHARGES, C REACTOR

WORKING PAPERS FOR PROD COST FY 1969

MANAGENENT REPORT TECHNICAL DIVISION

CALENDAR YEAR1969

PROGRAM PLAN - OTHER ISOTOPES

OUTAGE DATA BK $5 / 69$

BUDGET STLDY FY- 1970 REPLACEMENT OF K

REACTOR 36" COOLANT SUPPLY PIPING

PROCESS DEVELOPMENT FUNDING TO DOUGLAS

UNITED MUCLEAR, INC

COBALT-60 ACTIVITY OF THERMAL SHIELDS -

SPR

ENRICHED URANIUA METAL FOR THE THORIA

IRRADIATION PROGRAM

REINFORCEMENT OF BRANCH CONNECTIONS

100-K PRIMARY COOLANT PIPING

RP STUOY FORECAST CASE $V, 4 / 1 / 69$ THRU

6/30/74

DEVELOPMENT TEST AUTHORIZATION 175

Z-HOLE NEUTRON FLUX MEASUREMENT

REACTOR PHYSICS MANUAL OF TRANSIENT

CALCULATIONS

MFC-12: A FEASIBILITY STUDY FOR A

MULTIPURPOSE IRRADIATION TESTING

FACILITY

SUGGESTED STUDIES

STATUS REPORT NO. 7 K REACTOR BRITTLE

FRACTURE PROGRAM

FUELS ACCOUNTING SUBSECTION HONTILIY COST

ANALYSIS REPORT - $4 / 69$

OPERATIONAL PHYSICS ASPECTS OF A K E-Q

LOND

USE OF K REACTOR FUEL IN C REACTOR

DNR U-233 PROGRAM

TIME CONSTANT FOR COOLANT TEMPERATURE

TRANSIENT MEASUREMEMTS

EQUATION FOR U-236 BUILDUP

HOT DIE SIZE PROCESS CASE STLDIES \&

OPERATING MODES

WORKING PAPERS FY 1971

CLASSIFICATION - UNC TEST PHOTOGRAPHS

FUELS SECTION - PERSONNEL BLOGET WORKING PAPERS

URANIUN DELIVERY SCHEDULE FOR ALTERNATE $K$ REACTOR OPERATING: MODES

DESIGN TEST - 53 K REACTOR INLET PIPING

ISOLATION MATERIAL IMPACT TESTS
AUTHOR

RH REID

SRD

$03 / 21 / 69$

LEVEL PGS DATE

CU RUHLMAN

SECR

$04 / 08 / 71$

$04 / 17 / 69$

RG GEIER

EJ FILIP

LB BRINIMAAN

SRD

03/25/69

$04 / 08 / 71$

03/27/69

CD HARRIMGTON

$04 / 04 / 69$

RL MILLER

$04 / 03 / 69$

04/02/69

DM DIEDIKER

CRD

$04 / 10 / 69$

SECR

$06 / 10 / 71$

EC BENMETT

CRD

05/20/69

PROCESS PHYSICS

$04 / 01 / 69$

KJ MATTY/AJ EBENS

05/08/69

CD HARRINGTON

$04 / 11 / 69$

HU HEACOCK

CRD

SRD

$04 / 17 / 69$

$06 / 10 / 71$

ED BOYER

SECR

$04 / 21 / 69$

SA 10000

WV JOHNSON

I THIERER

$04 / 15 / 69$

$04 / 17 / 69$

$04 / 18 / 69$

AF KUPINSKI

05/05/69

RG COWAN, ETAL

SRD

$05 / 15 / 69$

TW HAUFF

$04 / 08 / 71$

04/21/69

$04 / 22 / 69$

$06 / 10 / 71$

WW ADACHI

$04 / 30 / 69$
OC SCHROEDER 
Page No. 85

$04 / 28 / 93$

LIST OF HAMFORD-GEMERATED CLASSIFIED

DOCUMENTS DATED BETMEEN 1/1/61 AND 12/31/72

DOC. NO.

DUN-05756

DUN-05757

DUN-05759

DUN-05763

DUN-05772

DUN-05774

DUN-05786SUP2

DUN-05790

DUN-05801RD

DUN-05811

DUN-05812

DUN-05817

DUN-05832RD

DUN-05833

DUN-05834

DUN-05835RD

DUN-05841

DUN-05842

DUN-05843

DUN-05850

DUN-05851

DUN-05860

DUN-05862

DUN-05866

DUN-05871

DUN-05873

DUY -05880
TITLE

PP: DCE-524 REPLACEMENT FUEL PRCOUCTION PROCESS HOT DIE SIZE DESIGN CRITERIA HOT DIE SIZE PROCESS

CLADDING FAILURE OF ONE-INCH OVERBORE ELEMENT FROAC REACTOR

PROPOSED EUIDE TOPICS INCORPORATING FAST FLUX CLASSIFICATION CUIDAMCE

FAST FLUX ENHANCEMENT STUDY FOR MFC-12 PROCEDURE FOR GRAPHITE COATING EXPERIMENT

USE OF SURPLUS SWALL REACTOR FUEL AT THE $J$ REACTOR

THE DNR THORIA IRRADIATION PROGRAM MONTHLY REPORTS REACTOR PHYSICS

FLOU LAB TESTS FOR THE PTA-157 DOUBLE ORIFICE ASSEMBLY

PRELIMINARY CRITERIA - ELECTROCHEMICAL

ZIRCONIUN HYORIDE REMOVAL PROCESS

FUELS ACCOWTING SUBSECTION MONTHLY COST

AMALYSIS REPORT - $5 / 69$

DUN FORECAST STUOY WORKING PAPERS CASE

VIII, APR 1969

PRODUCTION TEST AUTHORIZATION - 161

OPERATIONAL TEST OF 190-K HIGH LIFT

PUMPS REPORT OF RESULTS

STATUS REPORY NO. $8 \mathrm{~K}$ REACTOR BRITTLE

FRACTURE PROGRAM

RP STUDY FORECAST CASE VI, 4/1/69 THRU 6/30/74

KOT WAFER THORIA ELEMEMTS

THE I SE OF SH203 BALLS FOR IMSERTABLE POISON

DUN PROOUCTION \& RED CONTRACTORS COST \&

EMPLOYEE ASSIGNMENT AMALYSIS

OPERATING DATA FOR THORIA MAFER

DISSOLUTION SAMPLES

EVALUATION OF LAST-DITCH SYSTEM FLUSHES:

EVIDENCE OF CROSSTIE CORROSIOW

ISOLATION OF K RISERS \& RISER APPROACH

LIMES

"STRESS" A COMPUTER PROGRAM FOR

CONVERTING MEASURED STRAIN TO

STRESS IN PIPING SYSTEMS

IRRADIATED THORIA

REACTIVITY EFFECTS OF C2N \& C3E FUEL

ELEMENTS IN AK-LATTICE

TECHNICAL BASES FOR RESISTANCE

TEMPERATURE DETECTORS - $K$

REACTORS

OVERBORE RUPTURE REPORT
AUTHOR

LEVEL

PGS

DATE

RH TOIVONEN

SECR

05/07/68

SECR

05/07/69

$04 / 25 / 69$

TL DECBALD

CRD

07/03/69

PJ ZIMUERTAN

SECR

05/01/69

EC BENNETT

SRD

$04 / 29 / 69$

SECR

07/06/71

OC SCHROEDER

H TOFFER

SRD

05/05/69

06/01/63

SA 1000

WK ALEXADER

CRD

CRD

05/19/69

SECR

$06 / 10 / 71$

SECR

$06 / 10 / 71$

RJ EHLERS

CRD

06/05/69

HU HEACOCK

SRD

$05 / 16 / 69$

SECR

$06 / 10 / 71$

JP SCHMIDT

CRD

$05 / 21 / 69$

CD BOYER

SRD

05/21/69

FM KIMNEY

SECR

$05 / 20 / 69$

JP SCHMIDT

SRD

05/22/69

GA KUGLER

SRD

05/26/69

LB BRINXWAN

CRD

$06 / 09 / 69$

CW HARRISOM

CRD

$06 / 10 / 69$

T PRUDICH

SRD

05/29/69

JR BAICHTAL

SRD

$06 / 04 / 69$

RL BENCIMI

SRD

09/08/69

GC SORENSEN 
Page No. 86

$04 / 28 / 93$

LIST OF HANFORD-GENERATED CLASSIFIED

DOCUMENTS DATED BETHEEN 1/1/61 AND 12/31/72

DOC. NO.

DUN-05883

DUN-05887RD

DUN-05887RD REV1

DUN-05896

DUN-05898

DUW-05904RD

DUN-05905 05

DUN-05905 06

DUN-059054

DUN-05906

DUN-05907 03

DUN-05908

DUN -05910

DUN-05916

DUN-05921

DUN-05926

DUN-05927

DUN-05929 G

DUN-05929A

DUN-U5929B

DUN-05929C

DUN-059290

DUN-05929E

DUN-05929F
TITLE

AN ENGINEERING STLOY OF POSSIBLE PIPE WHIP IN $K$ REACTOR PROCESS PIPING REVISION OF CORPORATE COALS REVISION OF CORPORATE GOALS

N REACTOR POAER-ONLY FUEL FABRICATION COST STUDY (TUBE-IN-TUBE, CLUSTER, METAL, \& OXIDE MODELS)

OPERATIMG EXPERIENCE MITH K-REACTOR HIGH

LIFT PLMPS

DUN JUL 1969 FORECAST PAPERS

DUN SINGLE PASS REACTORS \& EMGINEERING

SOURCE DATAFYTO MARCH \& APRIL 1970

DUN SINGLE PASS REACTORS \& ENGINEERING

SOURCE DATA FYTO MAY \& JUNE 1970

DUN SINGLE PASS REACTORS \& ENGR SOURCE

DATA FY 1970

DUN SINGLE PASS RECTORS \& ENGINEERING REPORT TO COST, FYTO

SINGLE PASS RECTORS \& ENGIMEERING MONTH END COAPUTER DATA, FISCAL YEAR 1970 JAN, FEB, MAR 1970

SUMmLARY, PTA-114, IRRADIATION OF ALSI

FUEL CONTAINING

SOLID-INDUCTION-OIL-HEAT-TREATED

URANIUN CORES

K REACTOR CRASH DISCHARGE CAPABILITY PRODUCTION RECORDS, PRODUCTION SCHEDULE 100 AREA - 6/70

THOUGHTS ON K-REACTOR POWER LEVEL

ASSUAPTIOW FOR CORE

FUELS ACCOUNTING SUBSECTION MONTHLY COST

ANALYSIS REPCRT JUN 1969

DUN PRCOUCTION FORECAST 7/69 - 6/74

$N$ OPERATIONS NUCLEAR MATERALS MONTHLY

INVENTORY REPORTS FISCAL YEAR 1970

N OPERATIONS MUCLEAR MATERIAL INVENTORY

REPORTS FISCAL YEAR 1970 JULY 1969

N OPERATIONS MUCLEAR MATERIALS MONTHLY INVENTORY REPORTS FISCAL YEAR 1970 AUGUST 1970

N OPERATIONS MUCLEAR MATERIALS MONTHLY INVENTORY REPORTS FISCAL YEAR 1970 SEPTEMBER 1969

N OPERATIONS MUCLEAR MATERIALS MONTHLY INVENTORY REPORTS FISCAL YEAR 1970 OCTOBER 1969

N OPERATIONS MUCLEAR MATERIALS MONTHLY INVENTORY REPORTS FISCAL YEAR 1970 N OPERATIONS MUCLEAR MATERIALS MONTHLY INVENTORY REPORTS FISCAL YEAR 1970 DECEMBER 1969
AUTHOR

PF MERCIER

SRD

SECR

SRD

R NILSON

JP KEEMAN

SECR

RJ ENLERS

SRD

SECR

SECR

SECR

SECR

SECR

SECR

CP DORISS

JP SCHMIDT/RH TOIVONEN

SRD

$06 / 24 / 69$

JT BAKER

SRD

SECR

$06 / 13 / 69$

$04 / 08 / 71$

R NILSON

SRD

06/10/69

SECR

06/09/71

CP DORISS

SECR

SRD

SRD

SRD

$06 / 10 / 71$

07/01/69

CP DORISS

07/01/69

CP DORISS

07/01/69

CP DORISS

SRD

$07 / 01 / 69$

CP DORISS

SRD

$07 / 01 / 69$

CP DORISS

SRD

07/01/69

CP DORISS

07/01/69 
Page No. 87

$04 / 28 / 93$

LIST OF HANFORD-GENERATED CLASSIFIED

DOCLMENTS DATED BETWEEN 1/1/61 AND 12/31/72

DOC. NO.

TITLE

AUTHOR

LEVEL

PGS

DATE

DUN-05929H

N OPERATIONS MUCLEAR MATERIALS MONTHLY

CP DORISS

SRD

$07 / 01 / 69$

INVENTORY REPORTS FISCAL YEAR 1970

FEBRUARY 1970

DUN-05929J

N OPERATIONS MUCLEAR MATERIALS MONTHLY

CP DORISS

SRD

07/01/69

INVENTORY REPORTS FISCAL YEAR 1970

MARCH 1970

DUN-05929K

n OPERATIONS mUCLEAR Materials MONTHLY

CP DORISS

SRD

07/01/69

IMVEMTORY REPORTS FISCAL YEAR 1970

APRIL 1970

DUN-05929L

N OPERATIONS MUCLEAR MATERIALS MONTHLY

CP DORISS

SRD

07/01/69

DUN-05932A

INVENTORY REPORTS FISCAL YEAR 1970

NUCLEAR MATERIALS BALANCE ANO

CP DORISS

SRD

07/01/69

DUN-05932B JULY THRU SEPTEMBER

1969 FISCAL YEAR 1970

NUCLEAR MATERIALS BALAMCE AMD

CONSOLIDATIONS OCTOBER THRU

CP DORISS

SRD

07/01/69

DECEMBER 1969 FISCAL YEAR 1970

DUA-05932C

NUCLEAR MATERIALS BALANCE AND

CP DORISS

SRD

07/01/69

CONSOLIDATIONS JANUARY THRU MARCH

1970 FISCAL YEAR 1970

DUN-059320

NUCLEAR MATERIALS BALANCE AND

CP DORISS

SRD

07/01/69

FISCAL YEAR 1970

TEST FACILITY STATUS, KE REACTOR

TEST FACILITY STATUS, KE REACTOR

REACTOR OUTAGE TIME \& UTILIZATION 7/1/69

LV BARKER

CRD

CRD

DUN-059392

DUN-05948RD

- 6/30/70

DUN-059525

DUN-059526

PRCOUCTION INVENTORY \& MFG STATEMENT 1/07/70

PRCOUCTION INVENTORY \& MFG STATEMENT

LV BARKER

CRD

$04 / 27 / 70$

C O'MALLEY, JR

$07 / 01 / 69$

SECR

05/25/71

$1 / 27 / 70$

PROOUCTION INVENTORY \& MFG STATEMENT

2/26/70

DUN-05952A

PROOUCTION INVENTORY \& MFG STATEMENT

SECR

05/25/71

$1 / 31 / 70$

DUN-05958 G

DUN PROOUCTION FUELS INVENTORIES \&

SOURCE DATA FOR SINGLE PASS REACTOR FYTO JANUARY 1970

DUN-05958 K

DUN PRODUCTION FUELS INVENTORIES \&

SOURCE DATA FOR SINGLE PASS REACTOR FY7O

FEBRUARY 1970

DUN-05958 !

DUN PRCOUCTION FUELS INVENTORIES \&

$05 / 25 / 71$

SOURCE DATA FOE SINGLE PASS REACTOR FY7O

MARCH 1970

DUN-05958 J

DUN PRCDUCTION FUELS INVENTORIES \&

CP DORISS

SECR

07/08/69

SOURCE DATA FOR SINGLE PASS RECTOR FY7O MAY 1970

DUN-05958 K

DUN PRODUCTION FUELS INVENTORIES \&

CP DORISS

SECR

07/08/69 MAY 1970 
Page No. 88

$04 / 28 / 93$

LIST OF HANFORD-GENERATED CLASSIFIED

DOCLNENTS DATED BETHEEN 1/1/61 AND 12/31/72

Doc. No.

TITLE

AUTHOR

LEVEL

PGS

DATE

DUN-05958 L

DUN PRCOUCTION FUELS INVENTORIES \&

CP DORISS

SECR

07/01/69

SOURCE FOR SIMGLE PASS REACTOR FYTO JUNE 1970

DUN-05959 C

DUN PROOUCTION FUELS MUCLEAR MATERIAL

CP DORISS

SECR

07/01/69

MONTH END REPORT FOR SIMGLE PASS REACTOR

FYTO MAR THROUGH JUW 70

DUN-05\%60

DUN PRODUCTION FUELS COST REPORT FOR

SIMGLE PASS REACOTRS FY1970 JULY 1969

CP DORISS

SECR

07/01/69

THROUCH JUNE 1970

DUN-05961

DUN-05963

DEACTIVATED BASINS: STORAGE CAPACITIES

DEVELOPMENT \& TESTING OF

LITHIUM-ALUMINUM ALLOY SPLIMES

W JOHWSON

SRD

$06 / 30 / 69$

WK ALEXANDER/RL MILLER

SRD

07/02/69

DUN-05965

DUN-05966

DUN-05967

DUN-05969

DUN-0599306

DUN-0599307

DUN-0599308

DUN-0599309

DUN-0599310

(PTA-136)

DUN MONTHLY REPORT JULY 1969

DUN MONTHLY REPORT AUGUST 1969

DUN MONTHLY REPORT SEPT 1969

K-REACTOR COOLANT TEMPERATURE RESPONSE

MONTHLY UNIT COST REPORT 12/31/69

MONTHLY UNIT COST REPORT 01/31/70

MOWTHLY UNIT COST REPORT 02/28/70

MONTHLY UNIT COST REPORT 03/31/70

MONTHLY UNIT COST REPORT 04/30/70

MONTHLY UNIT COST REPORT 05/31/70

MONTHLY UNIT COST REPORT 06/30/70

REVIEW OF C-REACTOR SHUTDOW

REACTOR INVENTORY \& DISCHARGE SUMUARY FY 1970

WORK PROPOSED IN FURTHERANCE OF

PATENTABLE MATTER COVERED IN INVENTION

DESCRIPTION DUH-IR-31 (DUN-5489)

AEC PATENT CASE NO. S-37861

DUN-06005

K-REACTOR ENRICHMENT REQUIREMENTS FOR

RECYCLED FUEL

DUN-06019

OPTIMM USE OF SPLINES FOR MINIMUA

OUTAGES AT THE $K$ REACTORS

DUN-06022

DUN-06023RD

POTENTIAL BUDGET SAVINGS \& RELATED

PENALTIES FY 1970

K-REACTOR COOLANT SUPPLY SYSTEM FLOW

TRANSIENT \& WATER HAMMER FOLLOWING A

PIPE BREAK

DUN-06024

K5N SINGLE ELEMENT ISOTOPE PRODUCTION \&

ER PERL/RH MEICHLE

CRD

$08 / 18 / 69$

SECR

$09 / 18 / 69$

GL ERICKSON

SECR

SECR

$10 / 17 / 69$

VA SCHOUVILLER

SRD

$07 / 01 / 69$

VA SCHOUVILLER

SRD

SRD

$01 / 13 / 70$

VA SCHOUVILLER

$02 / 16 / 70$

03/18/70

VA SCHOUVILLER

SRD

$04 / 15 / 70$

$05 / 13 / 70$

VA SCHOUVILLER

SRD

$06 / 17 / 70$

VA SCHOUVILLER

07/21/70

DH BANGERTER

07/09/69

EF FAIRHEATHER

07/01/69

JP COOKE

$07 / 10 / 69$

CONVERSIONTABLE

STATUS REPORT \#10, K-REACTOR BRITTLE

FRACTURE PROGRAM

RL MILLER

SRD

$07 / 11 / 69$

LA NIELSEN

SRD

07/17/69

DH BAMGERTER

07/17/69

WG CONH

$07 / 21 / 69$

ANALYSIS OF THE PRESSURE RETAINING

ABILITY OF K REACTOR BIOLOGICAL

SHIELD

DUN-06033

FINAL DIGITAL GAMMA ENERGY MONITOR

DEVELOPMENT REPORT

FUELS ACCOUNTING SUBSECTION MONTHLY COST

ANALYSIS REPORT JUL 1969

HW HEACOCK

SRD

07/21/69

$07 / 17 / 69$

RL RICKS

CRD

$07 / 25 / 69$

KR I.ATTIM

CRD

09/05/69

DUN-06034 
DOC. NO.

DUN-06042

DUN-06047

DUN-06050

DUN-06058

DUN-06059

DUN-06059ADD 1

DUN-06062

DUN-06064

DUN-06070

DUN-06071

DUN-06074

DUN-06080

DUN-06082

DUN -06083

DUN-06084

DUN-06087 DUN-06096

DUN-06097

DUN-06103

DUN-06106

DUN-06109

DUN-06111

DUN-06112

DUN-06112REV

DUN-06113

DUN-06121

DUN-06152

DUN-06153
TITLE

SS MATERIAL MEASUREMENT REVIEW

PP: ISOLATION OF PROCESS COOLANT RISERS

- K REACTORS - PROJECT DCE-532

CLASSIFICATION - FUEL \& TARGET

INVENTORIES

FUEL \& TARGET PROOUCTION TEST SCHEDULES

K REACTOR OVERBORING - REACTOR PHYSICS ASPECTS

K REACTOR OVERBORING - REACTOR PHYSICS ASPECTS, ADDENDUN 1

WORKIMG PAPERS

TECHNICAL EVALUATION FOR THE IRRADIATION

OF NMERICUM (PT-171) IN A HANFORD

K REACTOR

N COALS DATA

NUCLEAR MATERIALS PRCOUCTION REPORT JULY 1, 1969 THRU JUNE 30, 1970

FACILITY MEUTRON FLUX PROFILE FOR

WM-140 TEST

RESPOUSE TO COWENTS ON K-REACTOR SAFETY ANALYSIS REPORT

REACTIVITY EFFECTS ON O3N \& O3E FIVEL

ELEMENTS IN AK-LATTICE

EXCESS REACTIVITY - KW REACTOR

INTERIM FEASIBILITY STLOY DIVERSION OF

INDIVIDUAL CROSSHEADER EFFLUENT

K-REACTOR

STARTUP AND TURMAROUAD MOTEBOOK

RADIOMETALLUURG EXAMINATION OF ONE INCH

OVERBORE FAILURES AND URANIUM

METALLOGRAPHY OF HIGH EXPOSURE

ELEMENTS

KE REACTCR UNSCHEDULED OUTAGE REPORT

SAFETY TRIP LIMITS FOR THE HIGH SPEED

SCANNING SYSTEM - $K$ REACTORS

REACTOR INVENTORIES FY1979 7-1-69 THRUN

6-30-70

K-REACTOR SUPPLY COOLANT TRANSIENTS

FOLLOUING LOSSOF TWO INLET RISERS

THEORETICAL STARTUP TOP-OF-RISER

PRESSURE FOR THE K-REACTORS

SLIDES FOR $N$ COALS MEETING

SLIDES FOR N GOALS MEETING (REVISION)

PROCESS DEVELOPMENT ACCOMPLISHMENTS

DUN-RL

PLUTONIUM BURMING CAPABILITIES OF

HANFORD $K$ REACTORS

FUELS ACCOUNTING SUBSECTION MONTHLY COST

ANALYSIS REPORT JUL 1969

PLUTONIUM FORMATION, URANIUM CREDITS, BURNOUT, AND BURMOUT COSTS

$$
\text { NUTHon }
$$

JW MANDSHUH

SRD

SECR

CRD

TH HAUFF

HF TEW

GR CORRELL

SRD

SRD

SRD

GR CORRELL

TH YOUNG

AF KUPINSKI

CRD

SRD

HG SPENCER

SECR

SRD

CRD

RV SKINWER

CD HARRINGTON

SRD

JR BAICHTAL

SRD

JP HAMRIC/LA MIELSEN

RL RICKS

SRD

SRD

JR BAICHTAL

RU TOIVONEA

CRD

SRD

$07 / 31 / 69$ 08/08/69

RG CLOUGH, JR.

SRD

SRD

$08 / 04 / 69$

JF NEAEC

SECR

07/01/69

WG CONN

SRD

08/06/69

TA GREEM

SRD

08/11/69

HG SPENCER

HG SPENCER

SECR

SECR

SECR

SRD

SECR

SRD

09/04/69

$08 / 25 / 69$

07/22/69

$07 / 31 / 69$

07/25/69

07/25/69

$07 / 24 / 69$

$09 / 01 / 70$

$08 / 04 / 69$

$04 / 01 / 69$

08/01/69

08/01/69

$07 / 31 / 69$

$07 / 31 / 69$

08/08/69

08/07/69

CW KUHLMAM

ER PERL/RH MEICHLE
06/09/71

$08 / 08 / 69$

08/08/69

08/08/69

$08 / 13 / 69$

$09 / 01 / 69$ 
Page No. 90

$04 / 28 / 93$

LIST OF HAMFORD-GENERATED CLASSIFIED

DOCUMENTS DATED BETWEEN 1/1/61 AND 12/31/72

DOC. NO.

DUN-06159

DUN-06161

DUN-06162

DUN-06170

DUN-06172

DUN-06173

DUN-06177

DUN-06182

DUN-06197

DUN-06200

OUN-06204

DUN-06210

DUN-06211

DUN-06217

DUN-06224

DUN-06224REV

DLA-06231

DUN-06241

DUN-06245

DUN-06245SUPA

DUN-06245SUPB

DUN-06251

DUN-06256

DUN-06266

DUN-06270

DUN-06271

DUN-06272

DUN-06273
TITLE

K-REACTOR PIPING \& STRAINER EVALUATION

STATUS REPORT NO. 11 K REACTOR BRITTLE

FRACTURE PROGRAM

TONMAGE \& MDS DISCHARGED FY 1970

MBR - NUG 1969

ENGIMEERNG STAFF AT RICHLANO

DECLASSIFICATION - GAS LEAK DETECTION

REPORT TO FEDC

UO3 \& THORIA RECYCLE TOWMAGE

ALSI SCRAP \& RELORK COSTS CALCULATION

PROCEDURES

DATA SUMUARY OF TAPERED BASE K5E

COMPONENTS USED IN CANNING STANDARD K5E CORES

ADDITIONAL DIVISIONAL COALS

COMPOSITION OF ENDING INVENTORY FISCAL

YEAR 1970

AEC 101 LISTIHG - FISCAL YEAR 1970

GRAPHITE TEMPERATURE COEFFICIENTS FOR

THORIA LOADED K REACTORS

DOUGLAS UNITED MUCLEAR, INC. MEW MISS

ASSET ACCOUNTS - 1611 \& 1631

DOUGLAS UNITED NUCLEAR, INC. NEH MIS

ASSET ACCOUNTS - $1611 \& 1631$

SUPPLEMENTAL NOTES TO HH-74095 SUP5

(SAFETY ANALYSIS OF COOLANT SUPPLY

PIPING, HANFORD $K$ PRCOUCTION

REACTORS)

THERMAL AND HYDRAULIC PERFORMANCE OF C-

AND O-TYPEFUEL IN THE $K$ REACTORS

PRELIMIMARY TEST DETAILS IN SUPPORT OF

INITIAL KE REACTOR SIMGLE CROSSHEADER

DECONTAMIMATION

PRELIMIMARY TEST DETAILS IN SXIPPORT OF

INITIAL KE REACTOR SINGLE CROSSHEADER

DECONTAMINATION, SUPPLEMENT A

MUMERICAL RESULTS OF DUN-6245

"PRELIMIMARY TEST DETAILS IN SUPPORT

OF INITIAL KE REACTOR SINGLE

CROSSHEADER DECONTAMINATION"

SENSITIVITY OF VSR FAILURE - $K$ PIPE

BREAK ACCIDENT

MBR - SEP 1969

FUELS ACCOUNTING UNIT MONTHLY COST

ANALYSIS REPORT AUG 1969

DUN MONTHLY REPORT OCT 1969

DUN MONTHLY REPORT NOV 1969

DUN MONTHLY REPORT DEC 1969

$X$ REACTOR UNCOOLED HORIZONTAL CONTROL

$R O O$ TEMPERATURE STUDY FOR A NEW

MAMUFACTURING PROCESS

\begin{tabular}{|c|c|c|c|}
\hline AUTHOR & LEVEL & PGS & DATE \\
\hline $\begin{array}{l}J \text { EHLERS } \\
\text { W HEACOCK }\end{array}$ & $\begin{array}{l}\text { CRD } \\
\text { SRD }\end{array}$ & & $\begin{array}{l}08 / 15 / 69 \\
08 / 15 / 69\end{array}$ \\
\hline & $\begin{array}{l}\text { SECR } \\
\text { SECR }\end{array}$ & & $\begin{array}{l}04 / 08 / 71 \\
08 / 31 / 69\end{array}$ \\
\hline H BAMCERTER & Conf & & $08 / 15 / 69$ \\
\hline W HAUFF & $\begin{array}{l}\text { CRD } \\
\text { SECR }\end{array}$ & & $\begin{array}{l}08 / 18 / 69 \\
04 / 08 / 71\end{array}$ \\
\hline STEM & SRD & & $08 / 21 / 69$ \\
\hline BAKER & CRD & & $08 / 29 / 69$ \\
\hline R PRAETORIUS & SRD & & $08 / 25 / 69$ \\
\hline MILSON & SECR & & $08 / 25 / 69$ \\
\hline P DORISS & SRD & & $07 / 01 / 69$ \\
\hline P DORISS & SRD & & $07 / 01 / 69$ \\
\hline MUNM/JR BAICHTAL & SRD & & $08 / 29 / 69$ \\
\hline A SCHOWVILLER & SRD & & $09 / 02 / 69$ \\
\hline IA SCHOUVILLER & SRD & & $08 / 12 / 70$ \\
\hline & & & \\
\hline
\end{tabular}

SA 1000

SRD

$09 / 10 / 69$

TA GREEH

SRD

$09 / 12 / 69$

TA GREEN

SRD

$09 / 30 / 69$

TA GREEN

SRD

$10 / 29 / 69$

RH MEICHLE

SRD

$09 / 12 / 69$

SECR

10/13/69

SECR

$06 / 09 / 71$

SECR

$11 / 18 / 69$

SECR

$12 / 18 / 69$

$01 / 16 / 70$

JA WHITE

$09 / 24 / 69$ 
Page No. 91

$04 / 28 / 93$

LIST OF HANFORD-GENERATED CLASSIFIED

DOCUMENTS DATED BETHEEN 1/1/61 AND 12/31/72

DOC. NO.

DUN-06274

DUN-06274

DUW-06282

DUN-06282PT 1 RD DUN-06282PT2 RD

DUN-06286

DUN-06287RD

DUN-06288RD

DUN-06289

DUN-06289REV

DUN-05289REV1 SUP2

DUN-06295

DUN-06295SUP1

DUN-06299

DUN-06312

DUN-06314

DUN-06315

DUN-06316

DUN-06325

DUN-06326RD

DUN-06331RD

DUN-06341

DUN-06342RD

DUN-06343RD

DUN-06344

DUN-06350
TITLE

FAILURE TO COMPLY MITH OPERATING LIMITS - 105-KW FLUX MONITORS

FAILURE TO COMPLY WITH OPERATING LIMITS

- 105-KH FLUX MONITORS

DUN PRODUCTION FORECAST 10/69 THRU 6/79

DUN PRODUCTION FORECAST 10/69 THRU 6/79

DUN PRODUCTION FORECAST 10/69 THRU 6/71

K REACYOR SUPPLY COOLANT TRAHSIENTS

PRELIMIMARY O2 CORPORATED COALS

PRELIMIMARY 02 CORPORATED COALS

PROPOSED INITIAL TECHNICAL BASES FOR

TUBE PONER LIMITS FOR THE $K$ REACTORS MATURAL LOADING COMNENT ISSUE

INITIAL TECHNICAL BASES FOR TUBE POWER

LIMITS FOR THE $K$ REACTORS MATURAL

LOADING

BASIS FOR EVALUATION OF EFFECTIVE VOID COEFFICIENTS REACTOR PONER

LIMITS

EXTENSION OF INITIAL TECHMICAL BASES FOR

TUBE POWER LIMITS TO LONER INLET TEMPERATURES

MEMORANDUN OF PROCESS STAMDARDS 9-13 $M$

BRITTLE FRACTURE TUBE POWER LIMITS

AND RISER PRESSURE LIMITS K REACTORS

MEMORAMDLM OF PROCESS STAMDARDS 9-13 M SUUPLEMENT 1

K REACTOR "WYE" JOINT OUTAGES

OPERATIONS APPRAISAL - FY69

DOUGLAS UNITED MUCLEAR, INC. CHARTS USED IN PRESENTATIONS TO F. $P$.

BARANOUSKI OCTOBER 1, 1969

CHARTS PREPARED FOR F.P. BARAMOUSKI

MEETING OCTOBER 1, 1969 (NOT USED)

COMNENTS ON DRL REPORT - K PIPING SAFETY

ANALYSIS

COST ESTIMATE FOR REACTOR IRRADIATION OF BISIUTH

PRODUCTION PROGRAM COUNCSL

FACTORS RELEVANT TO AN ORALLOY

DEVELOPMENT PROGRAM FOR THE $K$ REACTORS

MBR - OCT 1969

DUN OCT 1969 FORECAST WORKING PAPERS

CASE I

DUN OCT 1969 FORECAST WORKING PAPERS

CASE II

FUELS ACCOUNTING UNIT MONTHLY COST

ANALYSIS REPORT SEP 1969

TECHMICAL MOTE: BASES FOR TUBE PONER

LIHITS FOR THE $K$ REACTORS MATURAL

LOADING
AUTHOR

AR MAEUIRE

AR MACUIRE

Ho CONN

R NILSON

R MILSON

RH SHOEMKER/RE BMARS

RH SHOEMKER

CRD

$10 / 13 / 69$

RH SHOEMAKER/RE BAMRS CRD

$11 / 03 / 69$

RH SHOEMAKER/RE BAMRS

CRD

$11 / 26 / 69$

RU REID

CRD

$10 / 23 / 69$

AE ENGLER

CRD

$02 / 17 / 70$

OC SCHROEDER

CD HARRINGTON

STAFF

CRD

SECR

SRD

STAFF

SRD

CW KUHLMAM

SRD

CH RUHLMAN

SRO

R HILSON

R MILSON

SRD

SECR

SECR

SECR

SECR

SECR

RE BMARS

CRD
$09 / 30 / 69$

10/03/69

$10 / 03 / 69$

$10 / 03 / 69$

$10 / 13 / 69$

08/08/69

10/08/69

$10 / 10 / 69$

$10 / 31 / 69$

$06 / 10 / 71$

$06 / 10 / 71$

06/09/71

$10 / 14 / 69$ 
Page No. 92

$04 / 28 / 93$

LIST OF HANFORD-GENERATED CLASSIFIEO

DOCLMENTS DATED BETWEEN 1/1/61 AND 12/31/72

DOC. NO.

DUN-0635

DUN-06381

DUN-06387

DUN-06390RD

DUN-06391RD

DUN-06398

DUN-06405

DUN-06406REV1

DUW-06410

DUN-06413

DUN-0641801

DUN-0641802

DUN-0641803

DUN-0641804

DUN-0641805

DUN-0641806

DUN-0641807

DUN-0641808

DUN-0641809

DUN-0641810

DUN-0641811

DUN-0641812

DUN-0641813

DUN-0641814

DUN-0641815

DUN-0641816

DUN-0641817

DUN-0641818

DUN-0641819

DUN-0641820

DUN-0641821

DUN-0641822

DUN -0641823

DUH-0641824
TITLE

REACTOR OPERATIONS DAILY REPORT

STANDARDIZING REACTOR OPERATIONS

$K$ HYDRIDING FAILURE AMALYSIS

HDS PLANMING DOCUNENTS WORK PAPERS

HDS PROJECT EQUIPMENT DEVELOPMENT, WORK

PAPERS

CAVITATION PARAMETERS FOR $K$ REACTORS

HITH ESTIMATE OF CRITICAL VALUE FOR FUEL SUPPORTS

BUILDUP-BURNOUT RATIOS FOR C2N \& O3N

FUEL IN K REACTORS

PRCOUCT COST GRAMS GENERATED FIRST

QUARTER FY 1970 SEPTEMBER 30, 1969

DUN FIVE YEAR BLDGET PLAN, FY'S

1972-1976 PROGRAM O2 CAPITAL BUDGET LINE ITEMS

PT PT-NR-118 MARK IV DESIGN PERFORMANCE CHARACTERIZATION TEST

BASIN P INVENTORY BY P -240 CONTENT

BASIN P INVENTORY BY $P-240$ CONTENT

BASIN P INVENTORY BY P -240 CONTENT

BASIN P INVENTORY BY P -240 CONTENT

BASIN $P$ INVENTORY BY $P-240$ CONTENT

BASIN $P$ INVENTORY BY $P-240$ CONTENT

BASIN $P$ INVENTORY BY $P-240$ CONTENT

BASIN $P$ INVENTORY BY $P-240$ CONTENT

BASIN $P$ INVENTORY BY $P-240$ CONTENT

BASIN P INVENTORY BY P -240 CONTENT

BASIN P INVENTORY BY $P-240$ CONTENT

BASIN $P$ INVENTORY BY $P-240$ CONTENT

BASIN P INVENTORY BY $P-240$ CONTENT

BASIN P INVENTORY BY $P-240$ CONTENT

BASIN $P$ INVENTORY BY $P-240$ CONTENT

BASIN PLUTONIUN INVENTORY BY PU-240

CONTENT

BASIN PLUTONILN INVENTORY BY PU-240

CONTENT

BASIN PLUTONIUN INVENTORY BY PU-240

CONTENT

BASIN PLUTONIUM INVENTORY BY PU-240

\section{CONTEAT}

BASIN PLUTONIUM INVENTORY BY PU24O

CONTENT

BASIN PLUTONIUM INL_NTORY BY PU240

CONTENT

BASIN PLUTONIUM INVENTORY BY PU-240 CONTENT

BASIN PLUTONIUN INVENTORY BY PU-240 CONTENT

BASIN PLUTONILA INVENTORY BY PU-240 CONTENT

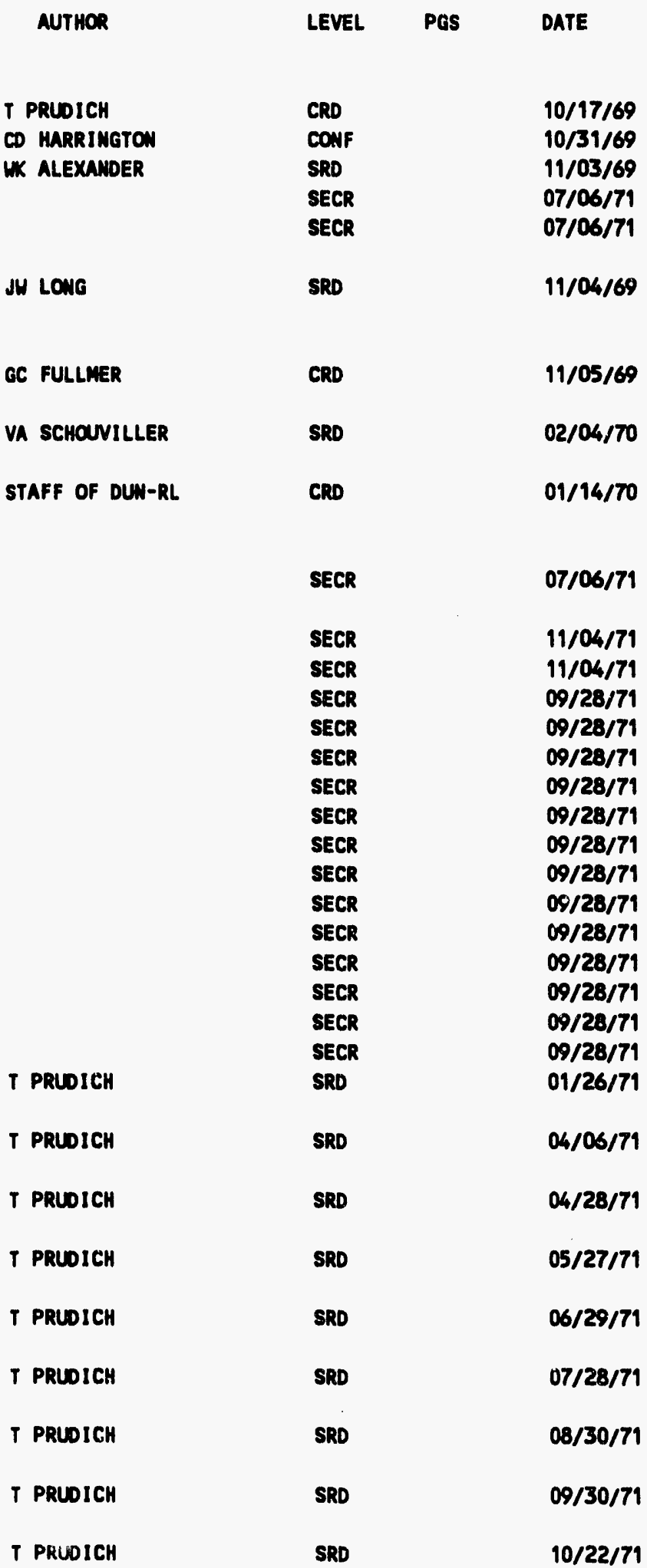


DOC. No.

DUN-0641825

DUN-0641826

DUW-0641827

DUN-0641828

DUN-0641829

DUN-06432

DUW-06434

DUN-06442

DUN-06444

DUN-08447

DUN-06448

DUN-06454

DUN-06471

DUN-06480

DUN-06483

DUN-06485

DUN-06490

DUN-06496RO

DUN-06499

DUN-06501

DUN-06508RD

DUN-06513RD

DUN-06522

DUN-06523

DUN-06524

DUN-06531

DUN-06544

DUN-06544

DUN-06547
TITLE

BASIN PLUTONIUM INVENTORY BY PU-240 CONTENT

BASIN PLUTONIUN INVENTORY, BY PU-240

CONTENT

BASIN PLUTONIUM INVENTORY BY PU-260

CONTENT

BASIN PLUTONIUN INVENTORY BY PU-240

CONTENT

BASIN PLUTONILN INVENTORY BY PU-240

CONTENT

DAILY REPORTS $K$ REACTORS

DEVELOPMENT TEST 197 A AND B RISER

PRESSURE TEST K REACTOR

100 AREAS METAL INVENTORIES

HYDRAULIC AMALYSIS OF EMERGENCY DIESEL

COOLANT SYSTEM CHECK VALVE

REPLACENENT IN $K$ REACTORS

MONTHLY MENT RPTS 313 PROCESS COATROL

$12 / 70$

MONTHLY MENT RPTS 313 PROCESS CONTROL

METAL INVENTORY BY KEYS, 100-KE AREA

FUELS ACCOUNTING UNIT MONTHLY COST

ANALYSIS REPORT OCT 1969

POTENTIAL FOR THERMAL FLUX TAILORING

NITHIN DRIVERELEMENTS

POISON BURNOUT IN REACTOR HCR'S - K

REACTOR

CALORIMETRIC ASSAY OF GOCO SOURCES FROM

C-OVERBOREFACILITIES

MBR - NOV 1969

INCREMENTAL COSTS OF $U-233$

MEMORANDUM OF PROCESS S?ANDARDS 9-13 M

BRITTLE FRACTURE TUFE POWER LIMITS

AMD RISER PRESSURE LIMITS - $K$

REACTORS APPENDIX

PHYSICS ASSISTANCE FOR K-REACTOR

STARTUPS

D.g. OLAMDER HORKIHG PAPERS

WORKING MOTES

PRESENTATION CHARTS SEMI-AMMUAL PROCESS DEVELOPMENT REVIEW NOVEMBER

1969

FLUX TRAVERSIMG K-REACTORS

REVIEU OF E-Q DRIVER CONVERSION

PARANETERS

EFFECT OF $K$ SEQUENTIAL OPERATION ON FY

1970 OPERATING COSTS

P FUEL fabricatió

PLUTONIUM FUEL FABRICATION STUDIES

DOUGLAS UNITED NUCLEAR, INC. SEMIAMHUAL

RESEARCH AND DEVELOPNENT REPORT SUMMURY
NTHOR

T PRUDICH

T PRUDICH

T PRUDICH

T PRLDICH

T PRUDICH

AR MAUIRE

RJ EHLERS

$T$ PRUDICH

AN KUGLER

RV SKINMER

JF MEMEC

$K B \operatorname{sou} A$

LH LANG

RW REID

JP MAMRIC

KE FIELDS

HH HEACOCK

JP HAMARIC

RH MEICHLE

FA KINMEY

JP TOSDALE

JH RICHES
LEVEL PGS

DATE

$11 / 22 / 79$

$01 / 03 / 72$

$01 / 27 / 72$

$02 / 24 / 72$

$03 / 30 / 72$

$11 / 10 / 69$

$11 / 11 / 69$

$11 / 01 / 69$

$10 / 24 / 69$

$04 / 08 / 71$

$04 / 08 / 71$

$05 / 25 / 71$

$06 / 09 / 71$

$11 / 19 / 69$

$11 / 26 / 69$

$12 / 18 / 69$

$11 / 30 / 69$

$11 / 21 / 69$

$11 / 25 / 69$

$11 / 24 / 69$

$07 / 06 / 71$

$12 / 01 / 69$

$12 / 03 / 69$

12/03/69

$12 / 05 / 69$

12/04/69

$09 / 28 / 71$

$01 / 30 / 70$

$12 / 15 / 69$ 
Page No. 94

$04 / 28 / 93$

LIST OF MANFORD-GENERATED CLASSIFIED

DOCLMENTS DATED BETUEEN 1/1/61 AMD 12/31/72

DOC. NO.

TITLE

AUTHOR

LEVEL

PGS

DATE

DUN-06548

DOUGLAS UNITED MUCLEAR, INC. SEMIAMNUAL

JW RICHES

SRD

$12 / 31 / 69$

RESEARCH AMD DEVELOPNENT REPORT

DUN-0655

FUELS ACCOUWTING UNIT MOWTHLY COST

SECR

$06 / 09 / 71$

DUN-06556

AMALYSIS REPORT HOV 1969

COMPOSITION OF ENDING INVENTORY - DEC

SECR

$12 / 31 / 69$

DUN-06557

1969

DUN-06558

MBR - DEC 1969

DUN-06561

DUN-0657ORD PT1

FAILURE TO COMPLY MITH OPERATING LIMITS

AR MCEUIRE

SECR

$12 / 31 / 69$

- CODE COMPLIAMCE RECUIREMENT

CALENDAR-MONTH AND FISCAL-YEAR-TO-DATE JF MARVEY

$12 / 05 / 69$

YIELDS FOR ALSI AMD HDS PROCESSES

DUN PROOUCTION FORECAST 1/1/70 THRU

$12 / 16 / 69$

6/30/74

DUN-06571

DUN-065T7BK1

DUN-06577BK2

DUN-06577BK3

DUN-06577BK4

DUW-065778K5

DUW-06594

DUN-06595

DUN-06596

DUN-06615RD

DUN-06616

DUN-06618

DUN-06622RD

DUN-06625

DUN-06626RD

DUN-06634

DUN-0.6638

DUN-06640

$K$ REACTOR SUPPLY COOLANT TRAMSIENTS

PT ANALYSIS VISUAL A ATTRIBUTE REPORT

PT AMALYSIS PRE-IRRNDIATION REPORT

PT AMALYSIS DIMENSIOMAL CHANEES REPORT

PT AMALYSIS FITTED FLUX REPORT

PT AHALYSIS TUBE OPERATING CONDITIONS

Cro

$\operatorname{sen}$

$06 / 10 / 71$

REPORT

DUA MONTHLY REPORT JAN 1970

DUN MONTHLY REPORT FEB 1970

DUN MONTHLY REPORT MAR 1970

FUELS SECTION MONTHLY RECORD REPCRT JULY

1969 THROUGH JUNE 1970

ALTERMATE N REACTOR OPERATING MOOE

TECHNICAL BASES OF $K$ REACTOR ORALLOY

PROOUCTION TEST

FUEL IRRADIATIONS UNIT MAMACENENT REPORT

FUELS SECTION MONTHLY COST AMALYSIS

REPCRT - 12/69

BUDGET STUDIES FY 1971

K REACTOR VOID COEFFICIENTS

DUN REQUEST NO. 85 DISPOSITION OF

IRRADIATED ORALLOY FUEL ELEMENTS

SEGMENTALLAY DISCHARGING OF REACTOR

FUELS

DUN-06647

DUN-06648

DUN-06650

DUW-06659

DUM-06660

DUN-06661RU

COEI JAN 1970

MBR - JAN 1970

SOURCE OF PROCESS WATER SCANDIUM

INWER EXTRUSION CAMPAICN REJECT SUMMRY

RADIOLOGIC ASPECTS ASSOCIATED WITH THE

GROUND DISPOSAL OF $K$ REACTOR

EFFLUENT

MONTHLY MAMAGEMENT REPORTS PROCESS

CONTROL

SUBSECTION

DUN-06672

DUN-06673RD

DUN-06683

REACTOR \& CRITICALITY SAFETY APPRAISAL

OF KE \& KN REACTORS

WG Cown

12/22/69

$07 / 12 / 71$

$07 / 12 / 71$

$07 / 12 / 71$

$07 / 12 / 71$

$07 / 12 / 71$

AEC-RL

$01 / 18 / 70$

03/18/70

$04 / 17 / 70$

$01 / 07 / 70$

JH FouTs

SECR

SPD

$01 / 09 / 70$

OC SCHROEDER

SECR

$07 / 06 / 71$

JP scimior

$01 / 12 / 70$

SRD

$06 / 10 / 71$

DH BAMCERTER

$01 / 12 / 70$

BN DUNM

T PRLDICH

$01 / 15 / 70$

$11 / 18 / 69$

CE FRANTZ

$01 / 15 / 70$

06/09/71

02/01/70

AF LARRICK

$01 / 20 / 70$

JA ROEDELL

CO COFFER

01/21/70

$01 / 26 / 70$

MANAGEMENT REPORTS K PLANT SECTION

SHUTDOW OF PRCOUCTION REACTOR

AE ENGLER

SRD

$01 / 01 / 70$

CD MARRIMGTON

CRD

$02 / 02 / 70$

AR MACUIRE

OC SCHROEDER

SRD

$01 / 27 / 70$

$01 / 28 / 70$ 
DOC. NO.

DUN-06685RD

DUN-06686 02

DUN-066862

DUN-06692

DUN-06694

DUN-06695RD

DUN-0670301

DUN-0670307

DUN-0670308

DUN-0670310

DUN-0670311

DUN-0670312

DUN-06709

DUN-06713

DUN-06714

DUN-06719

DUN-06727

DUN-06739

DUN-06732

DUN-06735

DUN-06737

DUN-06745

DUN-06754

DUN-06757

DUN-06T73

DUN-06777

DUN-06788

DUN-06790
TITLE

MANAGEMENT REPORTS - FUELS SECTION JANUARY 1970 THROUGH DECEMBER 1970 DUN - MONTHLY REPORT - FEBRUARY 1970 FUELS ENGINEERING

DUN - MONTHLY REPORT - FEBRUARY 1970 FUELS EMGINEERIMG

AVERAGE U-236 CONTENT ON INCONING IRE CORES \& MPR BILLETS FOR CY69 PRODUCTION DATA FOR 26 PIECE KVW SECMENTAL DISCHARGE

FY 1971 OPERATIMG ESTIMATES REACTIVITY BALANCE AND ASSOCIATED REACTOR PHYSICS DATA - JAMUARY 1970 REACTIVITY BALANCE AND ASSOCIATED REACTOR PHYSICS DATA - JULY 1970 REACTIVITY BALANCE AND ASSOCIATED REACTOR PHYSICS DATA - ALGUST 1970 REACTIVITY BALAMCE AMD ASSOCIATED REACTOR PHYSICS DATA - OCTOBER 1970 REACTIVITY BALANCE AND ASSOCIATED REACTOR PHYSICS DATA - MCNEMBER 1970 REACTIVITY BALANCE AND ASSOCIATED REACTOR PHYSICS DATA - DECEMBER 1970 PROOUCT COST GRAMS GENERATED SECOND QUARTER FY 1970 DECEMBER 31, 1969 \& FISCAL YEAR TO DATE

KE REACTOR MON-MATURAL URANIUN LOADIMG STATUS FOR 1970

K EAST REACTOR FACTOR DATA FROM 1970 INTERIM REPORT HALF-PLANT PROCESS WATER TURBIDITY TEST

ANMUAL REPORT OF OPERATING EXPERIENCE PERTINENT TO MUCLEAR SAFETY

COEI - FEB 1970

MBR - FEB 1970

DUN CHARTS USED IM PRESENTATIONS TO FP BARAMOUSKI FEBRUARY 10, 1970

FUELS SECTION MONTHLY COST ANALYSIS REPORT - 01/70

LOADING LIAITATIONS FOR P IRRADIATIONS REACTOR FUEL SUMHARY

PTA DRAFT K REACTOR ORALLOY FUEL

IRRADIATION

REACTOR MATERIAL TRANSFERS BUN - CTO -

FY 1969 - 1971

PTA-208 BISNUTH IRRADIATION IN KE

REACTOR TISR 821

FULL FLON HEAT DECAY TEST: MUMERICAL

RESULTS

IRRADIATED THORIA
NUTHOR

JT STRIMGER

JT STRIMEER

KR MILAER

RH MEICHLE/ER PERL

DH BAMEERTER

RM FULSMAS

DE CLARK

DE CLARK

DE CLARK

DE CLARK

DE CLARK

VA SCHOUVILLER

RM FULSMS

SRD

RM FULSMS

CY MELLS/AP LARRICK

SECR

SECR

SECR

SECR

SECR

SECR

LJ MEWELL

T PRUDICH

CRD

SECR

SRD

SECR
DATE

$01 / 28 / 70$

$02 / 27 / 70$

$02 / 27 / 70$

$01 / 30 / 70$

$02 / 02 / 70$

$01 / 30 / 70$

02/02/70

$08 / 10 / 70$

$09 / 04 / 70$

$11 / 09 / 70$

$12 / 03 / 70$

01/08/71

02/06/70

$02 / 01 / 70$

$02 / 01 / 70$

03/30/70

$02 / 18 / 70$

$06 / 10 / 71$

$03 / 10 / 70$

$02 / 12 / 70$

$06 / 10 / 79$

$09 / 28 / 71$ $02 / 18 / 70$ 07/06/79

$02 / 25 / 70$

$07 / 06 / 71$

$01 / 21 / 71$

07/06/71 
DOC. NO.

DUN -06807 DUN-06812

DUN-06813RD

DUN-06815

DUN-0681501

DUN-06819RD

DUN-06825

DUN-06826

DUN-06832

DUN-06836

DUN-06841

DUW-06848

DUH-06849

DUN-06850

DUN-06862

DUN-06867

DUN-0686701

DUN-06868RD

DUN-06893

DUN-06904

DUN-06905

DUN-06906

DUN-06909

DUN-06910

DUN-06912

DUN-06913

DUN-06914

DUN-06922

DUN-06928RD

DUN-06930
TITLE

GRAPHITE COATING STUDIES

PTA-209 IMPROVED MEPTUNIUN 237 TARGET

ELEMENT CONFIGURATIONS

SHIELD PROTECTION LONDINGS - K REACTOR

PROOUCTION INVENTORY AND MANUFACTURING

STATEMENT FEBRLARY 28, 1970

PROOUCTION INVENTORY AND MANUFACTURING

STATENENT FEBRUARY 1970

SECOND K REACTOR LOAD

COEI - MAR 1970

MBR - MAR 1970

FUELS SECTION MONTHLY COST AMALYSIS

REPORT - 02/70

INTERIM REPORT OF MATER HAMMER TRANSIENT

AMALYSIS FOR K REACTOR IMLET SUPPLY

PIPING SYSTEM

CONVERSION CALCULATIONS FOR THIN-ANMULUS

MEPTUNIUM ELEMENT DESIGN

DUN MONTHLY REPORT APR 1970

DUW MONTHLY REPORT MAY 1970

DUW MONTHLY REPORT JUNE 1970

KTOTAL - TOTAL CONTROL FOR THE K REACTOR

PRODUCTION INVENTORY AND MANUFACTURING

STATEMENT MARCH 31, 1970

PRODUCTION INVENTORY AND MANUFACTURING

STATEMENT MARCH 31, 1970

CLASSIFIED WORKING PAPERS APRIL 11970

DOUGLAS UNITED MUCLEAR, INC. OPERATING

BUDGET FOR FY 1972 AND REVISION OF FY 1971

FUELS SECTION MONTHLY COST AMALYSIS

REPORT - 03/70

PITA-215, AUTHORIZATION FOR UTILIZATION

OF REDESIGNED 50-MIL ARCH RAIL

SELF-SUPPORTS

PTA-216, KE REACTOR LOW FLOU TEST

PONER GENERATION PREDICTIONS USING

HAMMER- HAMHER

BUDGET FOR FY 1972 \& REVISION OF FY 1971 SUMUARY OF 02 SPECIAL MUCLEAR MATERIAL

PROOUCTION AMD UNITCOST ESTIMATES

COEI - APR 1970

MBR - APR 1970

SEGMENTAL CHARGE-DISCHARGE

TERMINATION OF PROJECT DCE-524 HANFORD

HOT DIE SIZE PROOUCTION FACILITY

MONTHLY MANAGEMENT REPORTS - 1970

PROCESS AMALYSISUNIT

NEUTRON FLUX CLASSIFICATION ASPECTS IN A

TAILORED THERMAL MEUTRON ENVIROMMENT
AUTHOR LEVEL PGS DATE

R COOPERSTEIN CONF $04 / 30 / 70$

SECR $\quad 07 / 06 / 71$

RL. MILLER SRD $\quad$ 03/05/70

VA SCHOUVILLER SRD O3/25/70

VA SCHONILLER SRD

WK WRIGHT SRD $03 / 10 / 70$

SECR $\quad 06 / 10 / 71$

SECR $\quad 03 / 31 / 70$

SECR $\quad 06 / 10 / 7$

KJ MATTY SRD

07/01/70

AF KUPINSKI

CONF

$03 / 23 / 70$

$05 / 18 / 70$

$06 / 18 / 70$

$07 / 17 / 70$

JR BAICHTAL

SECR

SECR

$03 / 31 / 70$

VA SCHOUVILLER $\quad$ SRD $\quad 04 / 27 / 70$

VA SCHOUVILLER SRD

$04 / 27 / 70$

T PRUDICH

SECR

$04 / 01 / 70$

DH BANGERTER

CRD

$04 / 13 / 70$

SECR

$06 / 10 / 71$

SECR

$07 / 06 / 71$

RV SKINAER

SECR

$07 / 06 / 71$

SECR

$04 / 20 / 70$

DH BAMGERTER

SRD

$04 / 17 / 70$

WA BLANTON/GF OUSLEY

SECR

$06 / 10 / 71$

SECR

$05 / 11 / 70$

FUELS ENGINEERING/FUELS

SRD

$08 / 24 / 70$

SECTIOW

$04 / 30 / 70$

EE LEITZ

SRD

$04 / 23 / 70$

RV SKINMER

$04 / 27 / 70$ 
Page No. 97

$04 / 28 / 93$

LIST OF MANFORD-GENERATED CLASSIFIED

DOCUMENTS DATED BETWEEN 1/1/61 AND 12/31/72

DOC. NO.

DUN-06954RD

DUN-06959

DUN-06962

DUN-0696201

DUN-06965

DUN-06965D

DUN-06970

OUN-06979

DUN-06972

DUN-06980

DUN-0698001

DUN-06981

DUN-0698101

DUN-06981RD

DUN-06992RD

DUN-06993

DUN-06994

DUN-06999

DUN-07000BO

DUN-OTOOOCO

DUN-07000001

DUN-07000KO

DUN-07000TO SUPK DUN-07000TO1

DUN-070021

DUN-070022

DUN-070023
TITLE

TECHNICAL NOTES ON THE THERMAL HYDRAULIC EFFECTS OF THE $K$ REACTOR PROCESS TUBE DISTORTION

PRODUCT COST GRAMS GENERATED THIRD QUARTER FY 1970 MARCH 31, 1970 \& FISCAL YEAR TO DATE

PROOUCTION INVENTORY AND MANUFACTURING

STATEMENT APRIL 30, 1970

PROOUCTION INVENTORY AND MANUFACTURING

STATEMENT APRIL 30, 1970

N REACTOR TRANSFER STUDY

N TRANSFER STUDY

COEI - MAY 1970

MBR - MAY 1970

FUELS SECTION MONTHLY COST ANALYSIS REPORT - 04/70

PRCOUCTION INVENTORY AND MANUFACTURING

STATEMENT MAY 31, 1970

PROOUCTION INVENTORY AND MANUFACTURING

STATEMENT MAY 31, 1970

PROOUCTION INVENTORY AND MANUFACTURING

STATEMENT JUNE 30, 1970

PROOUCTION INVENTORY AMD MAHUFACTURING

STATEMENT JUNE 30, 1970

PRODUCTION INVENTORY AND MANUFACTURING

STATEMENT JUNE 30, 1970

WORKING PAPERS - M. H. STEMM - MOD $2 C$

EXAMINATION OF FRACTURE POTENTIAL OF THE $K$ REACTOR INLET PIPIMG SYSTEM FINAL REPORT PTA-202

ANALYTICAL DATA: MPR CORES USED IN SRP EXTRACTIONS THIRD SCRUP CASK SHIPMENT PRODUCTION OF MEDICAL-GRADE PU-238 PROGRAM PLAN K REACTOR RISERS PROGRAM FUEL PERFORMANCE CHARACTERIZATION PROGRNM

PLANT life PROgRAM PLAN

K REACTORS ZIRCALOY HYDRIDING PROGRAM

PLAN

K REACTOR RECULATORY PROGRAY (TO)

TO-N REACTOR REGULATORY PROGRAM AUGUST 1969

DUN SINGLE PASS REACTOR \& ENGIMEERING SOURCE DATA F/Y 1971, FOR JULY, AUCUST, SEPTEMBER 1970

DUN SIMGLE PASS REACTOR \& ENGINEERING SOURCE DATA,F/Y 1971, FOR OCTOBER, NOVEMBER, DECEMBER, 1970

SIMGLE PASS REACTOR \& EMGINEERING SOURCE DATA, FY-1971 FOR JAN, FEB \& MAR 1971
NUTHOR

SA 1500

VA SCHOUVILLER

SRD

VA SCHOUVILLER

SRD

SRD

VA SCHOUVILLER

CL ABEL

SECR

SECR

SECR

SECR

SECR

VA SCHOUVILLER

SRD

VA SCHONILLER

SRD

VA SCHOUVILLER

SRD

VA SCHOUVILLER

SRD

VA SCHOUVILLER

SRD

SRD

SECR

R COOPERSTEIN

SRD

SRD

CRD

SECR

CONF

CRD

SRD

CONF

SRD

SRD

SECR
DATE

05/05/70

05/07/70

$05 / 26 / 70$

$05 / 26 / 70$

$06 / 01 / 70$

05/08/70

$06 / 10 / 71$

05/31/70

$06 / 10 / 71$

06/25/70

$06 / 25 / 70$

07/30/70

07/30/70

$07 / 30 / 70$

05/21/70

07/06/71

$05 / 28 / 70$

$06 / 03 / 70$

12/22/69

11/07/6?

$12 / 16 / 69$

10/08/69

$01 / 12 / 70$

$12 / 31 / 69$

$07 / 01 / 70$

07/01/70

$12 / 05 / 72$ 
DOC. NO.

DUN-070024

DUN-07003

DUN-0700404

DUN-070049

DUN-070042

DUN-070043

DUN-07005C

DUN-07005D

DUN-07006

DUN-07007A

DUN-07007B

DUN-07007C

DUN-070070

DUN-07008G

DUN-07008H

DUN-070081

DUN-07008J

DUN-07008K

DUN-07008L

DUN-07009

DUN-07013G
TITLE

DUN SINGLE PASS REACTOR \& ENGIMEERIMG SOURCE DATA FY 1971

DUN SINGLE PASS REACTOR ENGINEERING

REPORT TO COST FISCAL YEAR 1971

DUN SINGLE PASS REACTORS \& ENGINEERING

MONTH END COMPUTER DATA, F/Y 1971,

APRIL, MAY, JUNE 1971

DUN SINGLE PASS REACTORS \& ENGINEERING

MONTH END COMPUTER DATA, F/Y 1971 JULY, AUGUST, SEPTEMBER 1970

DUN SIMGLE PASS REACTORS \& ENGIMEERING

MONTH END COMPUTER DATA, F/Y 1971,

OCTOBER, NOVEMBER, DECEMBER 1970

DUN SIMGLE PASS REACTORS \& ENGINEERING

MONTH END COMPUTER DATA FY 1971 JAN, FEB \& MAR 1971

PRODUCTION FUELSS SOURCE DATA \&

IMVENTORIES FOR SINGLE PASS REACTOR JAN, FEB \& MAR 1971

PRODUCTION FUELS SOURCE DATA \&

AUTHOR

LEVEL

PGS

DATE

INVENTORIES FOR SINGLE PASS REACTOR APR, MAY \& JUN 1971

PROOUCTION FUELS COST REPORT FOR SIHGLE

PASS REACTOR FISCAL YEAR 1971

PRODUCTION FUELS MONTHEND COMPUTER DATA FOR SINGLEPASS REACTOR, F/Y 1971, JULY, AUGUST, SEPTEMBER 1970

PRODUCTION FUELS MONTHEND COMPUTER DATA

FOR SINGLEPASS REACTOR, F/Y 1971,

OCTOBER, NOVEMBER, DECEMBER 1970

PRODUCTION FUELS ME COMPUTER DATA FOR

SINGLE PASS REACTOR JAN, FEB \& MAR 1971 PRODUCTION FUELS MONTHEND COMPUTER DATA FOR SINGLE PASS REACTOR F/Y 1971, APRIL, MAY, JUNE, 1971

MUCLEAR MATERIALS ACCOUNTING CONPUTER OUTPUT RUWS (DMA) FOR MONTH OF JAN 1971 NUCLEAR MATERIALS ACCOUNTING COMPUTER OUTPUT RUNS (DMA) FOR MONTH OF FEB 1971 NUCLEAR MATERIALS ACCOUNTING CONPUTER OUTPUT RUNS (DIM) FOR MARCH 1971 NUCLEAR MATERIALS ACCOUNTING COMPUTER OUTPUT RUNS (DMA) FOR APRIL 1971 NUCLEAR MATERIALS ACCOUNTING COMPUTER OUTPUT RUNS (DMA) FOR MONTH OF MAY 1971 NUCLEAR MATERIALS ACCOUNTING COMPUTER OUTPUT RUNS (DMA) FOR MONTH OF JUNE 1971 MUCLEAR MATERIALS PROOUCTION REPORT FISCAL YEAR 1971

N OPERATIONS - NM REPORTS, INVENTORIES, 1/71 SOURCE DATA FY 1971
SECR

$07 / 01 / 70$

CP DORISS

SRD

$07 / 01 / 70$

CP DORISS

SRD

07/01/70

CP DORISS

SRD

07/01/70

CP DORISS

SRD

07/01/70

SECR

07/01/70

SECR

07/01/70

SECR

$12 / 05 / 72$

CP DORISS

SRD

$07 / 01 / 70$

CP DORISS

SRD

07/01/70

CP DORISS

SRD

07/01/70

SECR

07/01/70

CP DORISS

SRD

07/01/70

SECR

$09 / 28 / 71$

SECR

$09 / 28 / 71$

SECR

$03 / 20 / 72$

SECR

$03 / 20 / 72$

CP DORISS

SRD

07/01/70

CP DORISS

SRD

07/01/70

T PRUDICH

SRD

07/01/70

SECR 
Page No. 99

04/28/93

LIST OF HANFORD-GENERATED CLASSIFIED

DOCLMENTS DATED BETUEEN 1/1/61 AND 12/31/72

DOC. NO.

TITLE

AUTHOR

LEVEL

PGS

DATE

DUN-07013H

N OPERATIONS - MM REPORTS, INVENTORIES,

2/71 SOURCE DATA FY 1971

DUN-07013J

N OPERATIONS - MM REPORTS, INVENTORIES, 3/71 SOURCE DATA FY 1971

DUN-07013K

N OPERATIONS- NUCLEAR MATERIALS REPORTS, INVENTORIES, SOURCE DATA

DUN-07013L

N OPERATIONS- muclear mAterials Reports, INVENTORIES, SOURCE DATA

DUN-07013M

N OPERATIONS MUCLEAR MATERIALS REPORTS, IMVENTORIES AND SOURCE DATA

JUNE 1971 FISCAL YEAR 1971

DUN-07014K

N OPERATIONS- MUCLEAR MATERIALS MONTHLY INVENTORY REPORTS-DMA FY 1971

DUN-07014L

DUN-070144

N OPERATIONS MUCLEAR MATERIALS MONTHLY

INVENTORY-DMA-FY 1971 DATA FY 1971

H OPERATIONS NUCLEAR MATERIALS MONTHLY

INVENTORY REPORTS JUNE 1971 FISCAL YEAR. 1971

DUN-07015A

NUCLEAR MATERIALS BALANCE AND

CONSOLIDATIONS

JULY THEUU SEPT 1970

DUN-07015B

FISCAL YEAR 1971

NUCLEAR MATERIALS BALANCE AMD

CONSOLIDATIONS OCTOBER THRU

DECEMBER 1970 FISCAL YEAR 1971

DUN-07015C

MUCLEAR MATERIALS BALAMCE \&

CONSOLIDATIONS JANUARY THRU MARCH

1971 FISCAL YEAR 1971

DUN-070150

MUCLEAR MATERIALS BALANCE AND

CONSOLIDATIONS APRIL THRU JUNE 1971

FISCAL YEAR 1971

DUN-07018

DUN -07019

DUN-07028

DUN -07033

DUN-07033RD

DUN-07039RD

DUN-07040RD

DUN-07041

DUN-07048

DUN-07049

DUN-07051

DUN-07055

DUH -07056

OUN-07058

DUN-07059

COMPOSITION OF ENDING INVENTORY FISCAL

YEAR 1971

AEC 101 LISTING FISCAL YEAR 1971

SHIELD PROTECTION LOADING KE REACTOR

THORIA DATA ANALYSIS RESULTS

THORIA DATA AMALYSIS

REACTOR OUTAGE TIME \& UTILIZATION

$$
7 / 1 / 7 n \text { - 6/30/71 }
$$

REACTOR OPERATING MONTHLY STATISTICS

SOURCE OF PROCESS MATER SCANDIUM

COEI - JUN 1970

MBR - JUN 1970

IRRADIATION ANALYSIS PTA-213

TISR-WADCO-631

IRRADIATED UO2-PU2

FUEL IRRADIATION

K REACTOR OPERATIONAL PHYSICS PARAMETERS

EFFECTS OF TUBE POAER LIMITS ON $K$

CP DORISS

SECR

$07 / 01 / 70$

SECR

$07 / 01 / 70$

SECR

$12 / 05 / 72$

SECR

$12 / 05 / 72$

SRD

07/01/70

SECR

$12 / 05 / 72$

SECR

12/05/72

CP DORISS

SRD

07/01/70

CP DORISS

SRD

$07 / 01 / 70$

CP DORISS

SRD

07/01/70

CP DORISS

SRD

07/01/70

CP DORISS

SRD

$07 / 01 / 70$

CP DORISS SRD

07/01/70

CP DORISS

RL MILLER

SRD

$07 / 01 / 70$

SRD

$06 / 03 / 70$

$07 / 06 / 71$

07/06/71

M THRESS

SECR

CRD

06/01/70

M THRESS

CRD

07/01/70

AP LARRICK

SRD

SECR

SECR

EC BENNETT

SRD

$06 / 12 / 70$

$06 / 30 / 70$

$06 / 30 / 70$

01/19/71

JF MENEC

SRD

RE BAARS/RH MEICHLE

CRD

$06 / 17 / 70$

06/15/70

REACTOR ADVANCED LOADINGS

DUN HONTHLY REPORT JULY 1970

SECR

SECR

$08 / 17 / 70$

$09 / 25 / 70$ 
Page No. 100

$04 / 28 / 93$

LIST OF HANFORD-GENERATED CLASSIFIED

DOCUNENTS DATED BETWEEN 1/1/61 AND 12/31/72

DOC. NO.

TITLE

AUTHOR

LEVEL

PGS

DATE

DUN-07060

DUN-07061

DUN-07064RD

DUN-07068

DUN-07072

DUN-07075

DUN-07076

DUN-07078

DUN-07079

DUH-07080

DUN-07089RD

DUW-07093

DUN-0709301

DUN-07123

DUN-07127

DUN-07129

DUN-07141

DUN-07153

DUN-07162

DUN-07163

DIJN-0716803

DUN-0716804

DUN-0716805

DUN-0716806

DUN-07194
DUN MONTHLY REPORT SEPT 1970

FUELS SECTION MONTHLY COST AMALYSIS REPORT - 05/70

BRITTLE FRACTURE \& OTHER MISCELLAMEOUS WORKING PAPERS

C AND O SIZE FUEL IRRADIATION IN KE . REACTOR

THERMAL HYDRAULIC LIMITS - O \& C FUELS

IN KE REACTOR

PROCESS CHANGE AUTHORIZATION K-18-70

AUTHORIZATION TO CHARGE FUEL ELEMENTS

NOT PRESENTLY COVERED IN PROCESS

STAMDARDS

REACTOR INVENTORY AND DISCHARGE SUMMARY FY 1971

FUELS SECTION EQUIVALENT PRCOUCTION ANO

COST STATISTICS FY 1971

COEI - JUL 1970

MSR - JUL 1970 (CORRECTED PAGES 2-7)

FEDC MATERIAL FOR 3/70 MEETIMG

PROOUCTION INVENTORY AND MANUFACTURING

STATEMENT JULY AND AUEUST 1970

PRODUCTION INVENTORY AND MANUFACTURING

STATEMENT JULY AND AUEUST 1970

REACTOR INVENTORIES, FY 1971 - 1975

BUCKET LOADING SUMUARIES 100-KE FY 1971

- 1975 7-1-70 THRU 6-30-75

PROCESS CHANGE AUTHORIZATION $K-20-70$

AUTHORIZATION TO CHARGE FUEL ELEAENTS

NOT PRESENTLY COVERED IN PROCESS

STANDARDS

CHARGE-DISCHARGE RATES - K REACTOR

PROVISIONAL MANUFACTURING PROCESS

SPECIFICATIONS AND PROCEDURES FOR THE

FABRICATION OF URANIUA ALUMIMUM

ALLOY FUEL BY THE MOT DIE SIZE PROCESS

CHEMICAL DISCHARGED TO THE COLUMBIA

RIVER FROM DUN FACILITIES FY- 1970

PTA-226, IRRADIATION OF CONTROLLED

TEMPERATURE STRUCTURAL STEEL. CHARPY

IMPACT SPECIMENS

MONTHLY UNIT COST REPORT SEPTEMBER 30 , 1970

MONTHLY UNIT COST REPORT OCTOBER 31, 1970

MONTHLY UNIT COST REPORT NOVEMBER 1970

MONTHLY UNIT COST REPORT 12/70

PRODUCT COST GRAMS GENERATED FOURTH

QUARTER

FY 1970 JUWE 30,1970 \&

FISCAL YEAR TO DATE
AEC-RL

SRD

$10 / 19 / 70$

SECR

$06 / 10 / 71$

PA CARLSON

SRD

$09 / 03 / 70$

AE ENGLER

SRD

$06 / 19 / 70$

RK ROBINSON

CRD

$06 / 22 / 70$

MT LEUIS

SRD

$06 / 23 / 70$

EF FAIRMEATHER

SRD

$07 / 01 / 70$

ML LAWLER

SusI

$07 / 01 / 70$

SECR

$06 / 10 / 71$

$07 / 31 / 70$

$04 / 08 / 71$

VA SCHOUVILLER

SECR

SRD

VA SCHOUVILLER

SRD

$09 / 25 / 70$

F FAIRLEATHER

RP ROWE

SRD

SRD

SRD

$07 / 01 / 70$

MY LENIS

RL MILLER

SRD

$07 / 14 / 70$

EM EPPERSON

CRD

$07 / 30 / 70$

SECR

07/06/71

SECR

07/06/71

VA SCHOUVILLER

SRD

$10 / 15 / 70$

VA SCHOUVILLER

SRD

$11 / 18 / 70$

VA SCHOUVILLER

SRD

$12 / 15 / 70$

VA SCHOUVILLER

SECR

SRD
$07 / 06 / 71$

$08 / 03 / 70$ 
Page No. 101

$04 / 28 / 93$

LIST OF HANFORD-GENERATED CLASSIFIED

DOCUMENTS DATED BETWEEN 1/1/69 AND 12/31/72

DOC. NO.

DUN-07212

DUN-07213

DUN-07214

DUN-07216

DUN-0721710

DUN-0721711

DUN-07223RD

DUN-07225

DUN-07238

DUN-07241

DUN-07242

DUN-07243RD

DUN-07245

DUN-07246

DUN-07253

DUN-07262

DUN-07263

DUN-07266

DUN-07269

DUN-07270

DUN-07271

DUN-07272

DUN-07292

DUN-07296

DUN-07307

DUH-0730701

DUN-07323

DUN-07324

DUN-07325
TITLE

FIMAL REPORT OF WATER HAMAER TRANSIENT ANALYSIS FOR $K$ REACTOR INLET SUPPLY PIPING SYSTEM

MSR - AUG 1970

COEI - AUG 1970

CHANGE IN KE REACTOR DISCHARGE PLAN FOR FY-1971

FUELS SECTION - PROOUCTION COST MAY 1971

FUELS SECTION - PRCOUCTION COST JUNE

1971

EFFECT OF FY 1970 ACTIVITIES ON FY 1971 COSTS

AECOP TASK \#19 SHUTDOWN OF B REACTOR

LIQUID MASTE DISPOSAL REVIEW 100-K PLANT

FY 1969 ANNUAL REPORTS TO AEC AND MISC

OTHER CLOSING ACTIVITIES JUNE 30,

1969

AMNUAL REPORTS TO AEC \& MISC. OTHER

CLOSING ACTIVITIES $6 / 30 / 70$

EXPERIMENTAL ISOTOPIC AMALYSIS OF POINT EXPOSURE DATA IN HANFORD PRODUCTION

REACTOR FUELS

MONTHLY RECORD REPORTS PROCESS COWTROL

UNIT FISCAL YEAR 1971

STATUS OF SPECIAL REACTOR PROCESS TUBE

LONDINGS SEPTEMBER 1, 1970

HALF-PLANT LOW DICHROMTE EVALUATION AT

KW REACTORFIMAL REPORT PROOUCTION

TEST-176

COEl - SEP 1970

MATERIAL STATUS REPORT SEPTEMBER 1970

CONSIDERATIONS FOR DECLASSIFICATION OF

SHUTDOWN REACTORS

DUN MONTHLY REP.ORT OCT 1970

DUN MONTHLY REPORT NOV 1970

DUN HONTHLY REPORT DEC 1970

FUELS SECTION MONTHLY COST ANALYSIS

REPORT - 08,70

SEMIANNUAL REPORT NO. 1 PT-MR-150

MEASUREMENT OF IRRADIATION EFFECTS ON $N$

MOOERATOR

PRCOUCTION YEST-240 - GAMA SPECTROMETER DEVELOPMENT AMD TESTING

PROOUCTION INVENTORY AND MANUFACTURIMG

STATEMENT SEPTEMBER 1970

PROOUCTION INVENTORY AND MANUFACTURING

STATEMENT SEPTEMBER 1970

COEI - OCT 1970

MSR - OCT 1970

FUELS SECTION MONTHLY COST AMALYSIS

REPORT - 09/70
NUTHOR

KJ MATTY

SRD

$08 / 12 / 70$

OC SCHROEDER

RG SPENCER

RG SPENCER

FM KIMMEY

Lx woods

GW MELLS

DH SEMMERA

SECR

$08 / 31 / 70$

SECR

SRD

$06 / 10 / 71$

$08 / 18 / 70$

CRD

06/09/71

$07 / 19 / 71$

$08 / 21 / 70$

$08 / 25 / 70$

$09 / 09 / 70$

$09 / 08 / 70$

$12 / 05 / 72$

H TOFFER/AF KUPINSKI

SRD

09/08/70

JA ROEDEL

SRD

07/01/70

RP ROWE

CRD

$09 / 10 / 70$

AP LARRICK

SRD

$09 / 30 / 70$

$T$ PRUDICH

GC FULLLAER

SECR

SRD

SRO

$06 / 10 / 71$

$09 / 30 / 70$

$09 / 17 / 70$

$11 / 20 / 70$

$12 / 18 / 70$

$01 / 20 / 71$

$06 / 10 / 71$

WK ALEXANDER

$10 / 01 / 70$

RL MILLER

SRD

$09 / 30 / 70$

VA SCHOUVILLER

SRD

$10 / 29 / 70$

VA SCHONVILLER

SRD

$10 / 29 / 70$

SECR

06/10/71

SECR

SECR 
Page No. 102

$04 / 28 / 93$

LIST OF HANFORD-GENERATED CLASSIFIED

DOCUMENTS DATED BETUEEN 1/1/61 AND 12/31/72

TITLE

AUTHOR

LEVEL

PGS

DATE

DUN-0733

IMNER EXTRUSION CAMPAIGH REJECT SUMAARY

JA ROEDEL

SRD

$10 / 19 / 70$

DUN-07333RD

8/12/70 THRU 9/15/70

DOLLARS \& QUANTITIES BY ASSET TYPE AS OF

VA SCHOUVILLER

SRD

$10 / 19 / 70$

DUN-07335A

$6 / 30 / 70$

AC REDISKE

CONF

$01 / 19 / 71$

STUDY C'JRING N REACTOR OPERATION

DUN-07335A1

FINAL REPORT ON PT " 2334 - DUN-7335-A

ELECTRICAL NOISE STUOY DURIMG N REACTOR

RO TOZKARZ

CONF

$01 / 21 / 79$

DUN-07340

DUN-07343

DUN-07348

DUN-07354

DUN-0735401

DUN-07360

\section{OPERATION}

FY-1971 O2 PROGRAM COST PROJECTIOUS

AUTHORIZATION TO CHARGE FUEL ELEMENTS

NOT

PRESENTLY COVERED IN PROCESS

STAMDARDS PCA-K-32-70

PROTECTION AGAINST SABOTAGE

PRODUCT CCIST GRAMS GENERATED IST QUARTER FY 1971

PRCDUCT COST GRAMS GENERATED 2ND QUARTER FY 1971

BORON SPECIFICATION FOR SPR ALUMINUM

COMPONENTS

DUN-07369

DUH-07372RD

DUN-07375

DUN-0737501

DUN-07383

DUN-07398RD

DUN-07401

DUN-07409

DUN-07416

DUN-07433

DUN-0745501

HYDROSTATIC TEST OF HIGH PRESSURE

CROSSTIE LINE IN16E ·KE

RADIOISOTOPES RELEASED IN DUN LIQUID

EFFLUENTS

PRODUCTION INVENTORY AND MANUFACTURING

STATEMENT OCTOBER 1970

PRCOUCTIOH INVENTORY AND MANUFACTURING

STATEMENT OCTOBER 1970

FUELS SECTION MONTHLY COST ANALYSIS

REPORT - 10/70

QUARTERLY CHANGES FOR SS MATERIAL

QUANTITIES AND DOLLARS

MSR - NOV 1970

PROOUCTION FORECAST DOUGLAS UNITED

MUCLEAR, INC. JAMUARY 1, 1979 THROUGH JUNE 30, 1976

EVALUATION OF PITA-215 AUTHORIZATION FOR

FM r.JWMEY

CRD

SRD

$10 / 22 / 70$

MT LEUIS

CD MARRIMGTON

VA SCHOUVILLER

CONF

SRD

$11 / 03 / 70$

$10 / 30 / 70$

SECR

07/06/71

JP SCHMIDT

SRD

$11 / 02 / 70$

LB BRINKANN

SRD

$11 / 20 / 70$

AP LARRICK

SECR

03/26/70

VA SCHOUVILLER

SRD

$12 / 04 / 70$

VA SCHOUVILLER

SRD

$12 / 04 / 70$

SECR

$06 / 10 / 71$

VA SCHOUVILLER

SRD

$11 / 20 / 70$

T PRUDICH

$11 / 30 / 70$

SECR

$01 / 19 / 71$

UTILIZATION OF REDESIGNED 50-MIL ARCH

RAIL SELF-SUPPORIS

MATERIALS DISCHARGED TO THE RIVER

(PRELIMIMARY ESTIMATE)

PRODUCTION INVENTORY AND MAMUEACTURING

STATEMENT NOVEMBER 1970

VA SCHOUVILLER

SRD

07/06/71

COMPOSITION OF ENDING INVENTORY - DEC

1970

MSR - DEC 1970

DUN-07457

DUN-07457EX

DUN-07461

DUN-07462

DUN-07463

MATERIAL BALAMCE REPORT DECEMBER 1970

DUN MONTHLY REPORT JAN 1971

DUN MONTHLY REPORT FEB 1971

DUN MONTHLY REPORT MARCH 1971

SECR

07/06/71

SECR

$12 / 31 / 70$

SECR

$01 / 11 / 71$

T PRUDICH

SECR

SRD

$01 / 07 / 70$

$12 / 07 / 70$

$02 / 18 / 71$

$03 / 17 / 71$

SECR

$04 / 16 / 71$ 
Page No. 103

$04 / 28 / 93$
LIST OF HANFORD-GENERATED CLASSIFIED DOCUMENTS DATED BETWEEN 1/1/61 AND 12/31/72
DOC. NO.

DUN-07465RD

DUN-07469RD1

DUN-07472

DUN-07480RD

DUN-07481

DUN-0748901

DUN-07495RD

DUN-07510

DUN-07518

DUN-07521

DUN-07531RD1

DUN-07535

DUN-07537

DUN-07543

DUN-07544

DUN-07544EX

DUN-07551

DUN-07552

DUN-07552EX

DUN -07560

DUN-07561RD

DUN-07571

DUN-07572

DUN-07572EX

DUN-075T7

DUN-07579

DUN-07589

DUN-07620

DUN-07620EX

DUN-07621

DUN-07632
TITLE

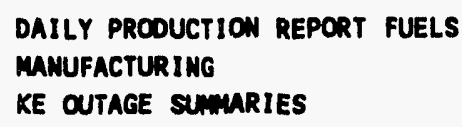
STATEMENT MARCH 1971

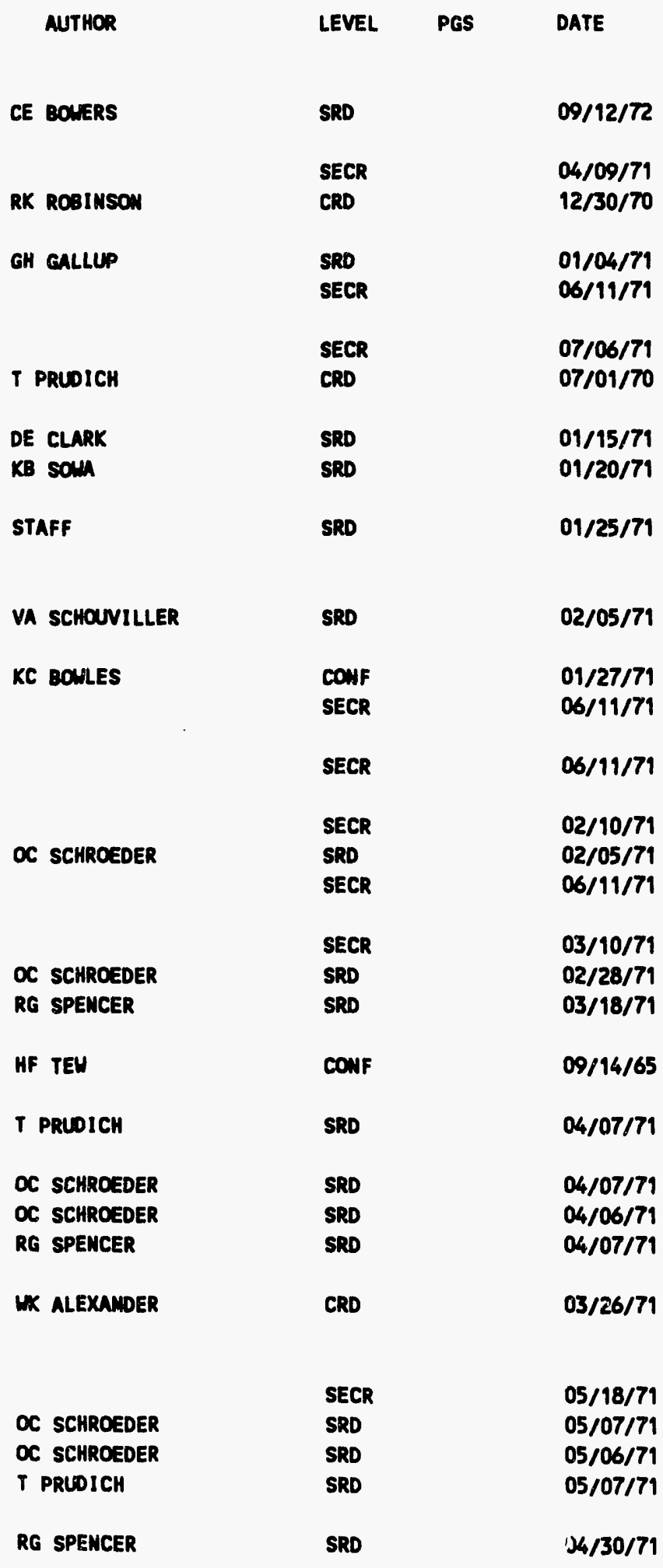


Page No. 104

$04 / 28 / 93$

LIST OF HANFORD-GENERATED CLASSIFIED

DOCUMENTS DATED BETUEEN 1/1/61 AND 12/31/72

DOC. NO.

DUN-076321

DUN-07633RD

DUN-07637

DUN-07645

DUN-07653

DUN-0765301

DUN-07656

DUN-07656EX

DUN-07657

DUN-07682

DUN-07683

DUW-07691

DUN-07696

DUN-07700

DUN-0770001

DUN-07710

DUN-07718

DUN-07721

DUN-07722

DUN-07723

DUN-0TT24

DUN-07725

DUN-07726

DUN-0T727

DUN-07728A

DUN-077288

DUN-07728C
TITLE

PRCOUCTION INVENTORY AMD MANUFACTURING

STATEMENT MARCH 1971

FINAL REPORT - IRRADIATION OF

MPO2-GRAPHITE MAFERS

EFFECTS OF CONTIMUED OPERATION ON $K$ REACTOR GRAPHITE DISTORTION

TRIP REPORT "SHIPPING \& MATERIAL

HANDLING REQUIREMENTS FOR

COPROOUCT TARGET CORES"

DUN PIEN STATEMENT O4/79

PRCOUCTION INVENTORY MANUFACTURING

STAIEMENT APRIL 1971

MATERIAL STATUS REPORT - MAY 1971

MATERIAL STATUS REPORT FOR MAY 1971

COMPOSITION OF ENDING INVENTORY MAY 1971

MATERIAL STATUS REPORT - JUNE 1971

COMPOSITION OF ENDING INVENTORY - JUNE

1971

MUCLEAR MATERIALS COMPOSITION OF ENDING

INVENTORY FISCAL YEAR 1972

FUELS SECTION PROOUCTION COST REPORT

$5 / 71$

PROOUCTION INVENTORY AND MANUFACTURING

STATEMENT MAY 1971

PRCDUCTION \& INVENTORY MANUFACTURING

STATEMENT MAY 1971

TRANSFER VOUCHER O02-HXA-FVA-002 F

N OPERATIONS MUCLEAR MATERIALS DMA 5 \& 6

JULY \& NUCUST 1971 FISCAL YEAR 1972

NUCLEAR MATERIALS PRCOUCTION R..PORT FY

1972

SIMGLE PASS FUELS \& REACTOR SOURCE DATA

JULY, AUCUST, \& SEPTEMBER 1971

FISCAL YEAR 1972

SINGLE PASS FUELS \& REACTOR REPORT TO

COST

FISCAL YEAR 1972

SIMGLE PASS FUELS AND REACTOR DMA 5 \& 6

FOR FISCALYEAR 1972

MUCLEAR MATERIALS STATUS REPORT AND

CONSOLIDATIONSJULY THRU DEC 1971 FISCAL

YEAR 1972

MATERIAL STATUS REPORT - JULY 1971

COMPOSITION OF ENDING INVENTORY - JULY

1971

MUCLEAR MATERIALS ACCOUNTING RUNS, DMA 5

\& 6 FOR JUL 1971

MUCLEAR MATERIALS ACCOUNTING RUNS, DMA 5

\& 6 FOR AUG 1971

MUCLEAR MATERIALS ACCOUNTING RUMS DMA 5

\& 6 FOR SEP 1971
NTHOR

RG SPENCER

TL DEOBALD

A RUSSELL

RA UIMSHIP

SRD

SECR

RG SPENCER

SRD

OC SCHROEDER

OC SCHROEDER

T PRUDICH

OC SCHROEDER

T PRIDICH

CP DORISS

ML LALLER

GH GALLUP

GH GaLLUP

GH GALLUP

Cn DORISS

T PRLOICH

CP DORISS

SRD

SRD

SRD

SRD

SRD

SRD

SRD

SRD

SRD

SECR

SRD

SRD

SRD

CP DORISS

SRD

SRD

SRD

CP DORISS

OC SCHROEDER

$T$ PRUDICH

SRD

SRD

SECR

SECR

SECR
DATE

$04 / 30 / 71$

$04 / 26 / 71$

$04 / 30 / 71$

05/06/71

07/06/71

05/24/71

06/08/71

06/07/71

$06 / 08 / 71$

$07 / 07 / 71$

07/07/71

07/01/71

$06 / 16 / 71$

$06 / 22 / 71$

$06 / 22 / 71$

$06 / 30 / 71$

$07 / 12 / 71$

$07 / 12 / 71$

$07 / 10 / 71$

07/10/71

$07 / 10 / 71$

07/10/71

08/10/71

$08 / 10 / 71$

$02 / 24 / 72$

$02 / 24 / 72$

$03 / 20 / 72$ 
Page No. 105

$04 / 28 / 93$

LIST OF HANFORD-GENERATED CLASSIFIED

DOCUMENTS DATED BETMEEN 1/1/61 AND 12/31/72

DOC. NO.

DUN-0T728D

DUN-07728E

DUN-0T728F

DUN-077286

DUN-07728 H

DUN-07728

DUN-07T28J

Duw-07728K

DUN-0T728L

DUN-07744

DUN-07747

DUN-07747RD

DUN-07753

DUN-07758

DUN-07759

DUN-0776501

DUN-0776502

DUN-0776503

DUN-0776504

DUN-0776505

DUN-0776506

DUN-0776506 RD

DUN-0776507

DUN-0776508

DUN-0776509

DUN-0776510

DUN-0776511
TITLE

MUCLEAR MATERIALS ACCOUNTING RUNS DMA 5

86 FOR OCTOBER 1971

MUCLEAR MATERIALS ACCOUNTING RUNS DMA 5

$\& 6$ FCR NOVEMBER 1971

NUCLEAR MATERIALS ACCOUNTING RUNS DMA 5

2 6 FOR DECEMBER 1971 NUCLEAR MATERIALS ACCOUNTIMG RUNS DMM 5

8 6, FOR JAN 1972

nUCLEAR MATERIALS ACCOUNTING RUNS DMA 5

\& 6, FOR FEB 1972

NM ACCONTING RUNS DMA $5 \& 6$ FOR 3/72

NUCLEAR MATERIALS ACCOUNTING RUNS DMA 5

\& 6 FOR APR 1972

MUCLEAR MATERIALS ACCOUNTING RUWS DMA

586 FOR MAY 1972

MUCLEAR MATERIALS ACCONTING RUNS DHA 5

\& 6 FOR JUNE 1972

FY 1971 CHANGES IN PRODUCT AND

NON-PROOUCTION SS MATERIAL

INVENTORIES

PRODUCTION IEM STATENENT 6/1/71RS

PROOUCTION INVENTORY AND MANUFACTURING

STATEMENT JUNE 1971

TRANSFER VOUCHER 001-HXA-F2C-001

MATERIAL STATUS REPORT - AUCUST 1971

COMPOSITION OF ENDING INVENTORY - AUEUST

1971

PROOUCTION INVENTORY \& MANUFACTURING

STATEMENT JULY 1971

PRODUCTION INVENTORY \& MAMUFACTURING

STATEMENT AUEUST 1971

PROOUCTION INVENTORY \& MANUFACTURING

STATEMENT SEPTEMBER 1971

PRODUCTION INVENTORY \& MANUFACTURING

STATEMENT OCTOBER 1971

PRCOUCTION INVENTORY \& MANUFACTURING

STATEMENT NOVEMBER 1971

PRODUCTIOA IMVENTORY I MMUAACTURIHG

STATEMENT DECEMBER 1971

PROOUCTION INVENTORY AND MANUFACTURIMG

STATEMENT DECEMBER 1971

PROOUCTION INVENTORY AND MANUFACTURIMG

STATEMENT JANUARY 1972

PRCOUCTION IHVENTORY AND MAMUFACTURING

STATEMENT FEBRUARY 1972

PROOUCTION INVENTORY AND MANUFACTURING

STATEMENT MARCH 1972

PROOUCTION INVENTORY AND MANUFACTLRING

STATEMENT APRIL 1972

PROOUCTION INVENTORY AND MANUFACTURING

STATEMENT MAY 1972

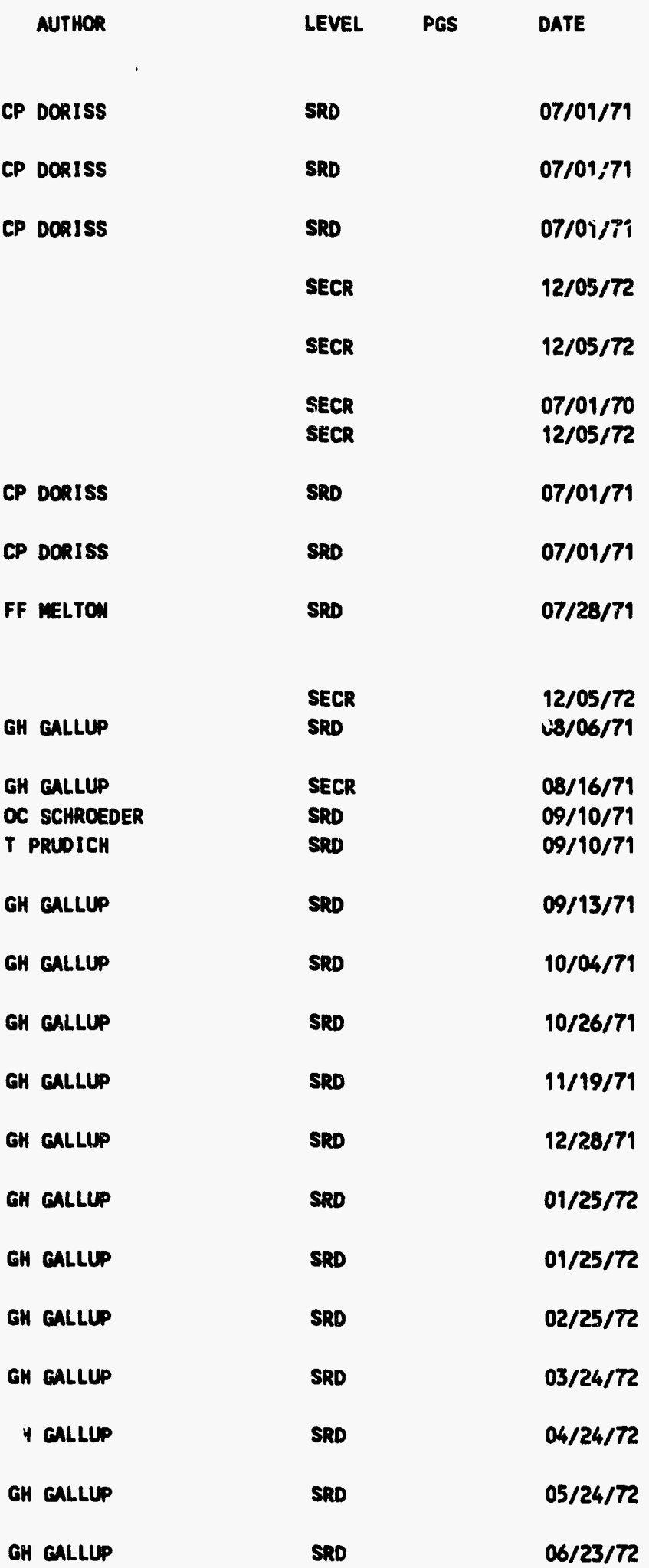


Page No. 106

$04 / 28 / 93$

LIST OF HAMFORD-GENERATED CLASSIFIED

DOCUMENTS DATED BETWEEN 1/1/61 AND 12/31/72

Doc. NO.

TITLE

AUTHOR

LEVEL

PGS

DATE

DUN-0776511 RD

DUN-0776512

DUN-0776512 RD

DUN-07768

DUN-07769

DUN-07T72

DUN-07Tr5

DUN-07T76

DUN-0T780

DUN-07789

DUN-07790

DUN-07801

DUN-07802

DUH-07803

DUN-07809RD

DUN-07810RD

DUN-07811RD

DUN-07825

DUN-07827

DUN-07828

DUN-07829

DUN-07847

DUN-07848

DUN-07849

DUN-07850

DUN-0785001

DUN-07873RD

DUN-07875
PROOUCTION INVENTORY AND MANUFACTURING STATEMENT MAY 1972 JUWE 1972 PROOUCTION INVENTORY \& MANUFACTURING STATEMENT

PRCOUCTION INVENTORY AND MANUFACTURING STATEMENT JUNE 1972

MONTHLY MANAGEMENT REPORTS - PROCESS

EVALUATION UNIT JANUARY 1968 TO JUNE 1971

SIMGLE PASS FUELS \& REACTOR SOURCE DATA FOR SEPTEMBER THRU FEBRUARY 1971

FISCAL YEAR 1972

N OPERATIONS MUCLEAR MMTERIALS DMM 586

SEPTEMBER ROCTOBER 1971, FISCAL YEAR

1972

MATERIAL STATUS REPORT - SEPTEABER 1971

COMPOSITION OF ENDING INVENTORY -

SEPTEMBER 1971

PROOUCTION INVENTORY SUBACCOUNTS AND

DESCRIPTIONS

MATERIAL STATUS REPORT OCTOBER 1979

COMPOSITION OF ENDING INVENTORY -

OCTOBER 1971

N OPERATIONS NUCLEAR MATERIALS DM 5 \&

6,

NOVEMBER \& DECEMBER 1979, F/Y

1972

MATERIAL STATUS REPORT - MOVEMBER 1979

COMPOSITION OF ENDING INVENTORY -

MOVEMBER 1971

PROGRAM MANAGEMENT

PROGRAM MANAGEMENT OUARTERLY REPORTS

PROGRAMS

MUCLEAR MATERIALS STATUS REPORT AMD

CONSOLIDATIONS JANUARY THRU JUNE 1972

FISCAL YEAR 1972

OPERATIONS MUCLEAR MATERIALS DMA 5 \& 6

FOR JANUARYE FEBRUARY 1972, FISCAL YEAR 1972

MATERIAL STATUS REPORT - DECEMBER 1971

COMPOSITION OF ENDING INVENTORY -

DECEMBER 1971

MATERIAL STATUS REPORT - JAMUARY 1972

COMPOSITION OF ENDING INVENTORY -

JANUARY 1972

TRANSFER VOUCHER O02-HXA-HVA-001

TRANSFER VOUVHER 003-HXA-HYA-001

TRANSFER VOUVHER 004-HXA-HYA-002

THERMAL-HYDRAULIC EFFECTS OF THE $K$

REACTOR PROCESSTUBE DISTORTION

TRANSFER VOUCHER O01-HXA-HYA-001
CH GALLUP

SRD

$05 / 24 / 72$

GH GALLUP

SECR

$07 / 25 / 72$

GH GALI.UP

$\sin$

$07 / 25 / 72$

we conn

SRD

09/08/71

CP DORISS

SRD

$09 / 09 / 71$

CP DORISS

SRD

$09 / 10 / 71$

OC SCHROEDER

$T$ PRLDICH

SRD

SRD

CD SHEALEY

SRD

OC SCHROEDER

$T$ PRLDICH

SRD

SRD

CP DORISS

SRD

OC SCHROEDER

$T$ PRUDICH

SRD

SRD

RK ROBINSON

RK ROBIMSON

RK ROBINSON

CP DORISS

CP DORISS

SRD

OC SCHROEDER

SRD

SRO

SRD

OC SCHROEDER

T PRUDICH

GH GALLUP

GH GALLUP

GH GALLUP

RK ROBIHSOH

GH GALLUP
$01 / 01 / 72$

10/11/71

10/11/71

$10 / 26 / 71$

$11 / 10 / 71$

$11 / 10 / 71$

$11 / 11 / 71$

$12 / 10 / 71$

$12 / 10 / 71$

$11 / 22 / 71$

$11 / 22 / 71$

$11 / 22 / 71$

$01 / 01 / 72$

01/10/72

$01 / 10 / 72$

$02 / 10 / 72$

$02 / 10 / 72$

$12 / 31 / 71$

$12 / 31 / 71$

12/31/71

$01 / 13 / 71$

$01 / 31 / 72$ 
Page No. 107

$04 / 28 / 93$

LIST OF HANFORD-GENERATED CLASSIFIED

DOCUMENTS DATED BETUEEN 1/1/61 AMD 12/31/72

DoC. No.

DUN-07876

DUN-07877

DUN-07880

DUN-07881

DUN-07892

DUW-07895

DUW-07897

DUN-07902

DUN-07904

DUN-07904SUP1

DUW-07910

DUN-07911

DUN-07924

DUN-07928

DUN-07929

DUN-07941

DUW-07944

DUN-079/,5

DUN-07964

DUN-07965

DUN-07966

DUN-07991

DUN-07992

DUN-08011A

DUN-08012

DUN-08014A

DUN-08014B

DUN-08014C

DUN-08016

DUN-08017 DUN-C8017EX

DUN-08018
TITLE

AUTHOR

TRANSFER VOUCHER OO2-NYXA-HYA-00!

TRANSFER YOUCHER O03-HXA-FYA-001

MATERIAL STATUS REPORT - FEBRUARY 1972

COMPOSITION OF ENDING INVENTORY -

FEBRUARY 1972

TRANSFER VOUCHER OOL-HXA-HLA-001

SIMGLE PASS FUELS \& REACTOR SOURCE DATA

FOR MARCH THRU JUWE 1972 - F/Y 72

OPERATIONS NUCLEAR MATERIALS DMA $5 \& 6$

FOR MARCH \& APRIL 1972 F/Y 1972

DOUGLAS UNITED MUCLEAR, IMC. MUCLEAR

MATERIALS MAMACEMEMT PLAN

DUN AND THE MATICHAL DEFENSE PROERAM

DUN AND THE MATIONAL DEFENSE PROCRNA

MMTERIAL STATUS REPORT - MARCH 1972

COMPOSITION OF ENDING INVENTORY - MARCH 1972

VALUE OF ENDING INVENTORIES SINGLE PASS REACTOR FUEL

K REACTORS - PLUTONIUN CONVERSION AND

UNIT COSTS

KE REACTOR INCIDENT

DUN AND THE MATIONAL DEFENSE PROCRAM

MATERIAL STATUS REPORT - APRIL 1972

COMPOSITION OF ENDING INVENTORY - APRIL

1972

MATERIAL STATUS REPORT MAY 1972

COAPOSITION OF ENDIMG INVENTORY - MAY

1972

OPERATIONS MUCLEAR MATERIALS DMA $5: 6$

FOR MAY \& JUWE 1972 FISCAL YEAR

1972

MATERIAL STATUS REPORT JUNE 1972

COAPOSITION OF ENDING INVENTORY - JUNE 1972

MUCLEAR MATERIALS CONSOLIDATIONS OF MSR

\& COEI FOR JULY THRU DECEABER 1972

MUCLEAR MATERIALS COMPOSITION OF ENDING INVENTORIES, FISCAL YEAR 1973

MUCLEAR MATERIALS ACCOUTING DATA DAM 5

\& 6 JULY \& MUCUST 1972

MUCLEAR MATERIALS ACCOUHTING DATA DMA 5

\& 6 SEPTEMBER \& OCTOBER 1972

NUCLEAR MATERIALS ACCOUNTING DATA, DMA 5

\& 6 MOVEMBER \& DECEMBER 1972

MUCLEAR MATERIALS DMM 5 \& 6, JULY,

AUEUST, \& SEPT 1972

MAERIAL STATUS REPORT JULY 1972

MATERIAL STATUS REPORT FOR JUL 1972

COMPOSITION OF ENDING IHERETORY - JULY

1972
GH GALLUP

GH GALLUP

OC SCHROEDER

$T$ PRLDICH

GH GALLUP

CP DORISS

CP DORISS

T PRLDICH

CD HARRIMGTON

CD MARRIMGTON

OC SCHROEDER

T PRUDICH

GH GALLUP

GH GALLUP

ET MURPHY

CD MARRINGTON

OC SCHROEDER

T PRUDICH

OC SCHROEDER

T PRUDICH

CP DORISS

OC SCHROEDER

T PRUDICH

CP DORISS

CP DORISS

CP DORISS

CP DORISS

CP DORISS

CP DORISS

OC SCHROEDER

OC SCHROEDER

T PRUDICH
LEVEL

PAS

DATE

$01 / 31 / 72$

$01 / 31 / 72$

$02 / 10 / 72$

$03 / 10 / 72$

02/29/72

$03 / 09 / 72$

03/09/72

$03 / 10 / 72$

$03 / 24 / 72$

$04 / 05 / 72$

$04 / 11 / 72$

$04 / 11 / 72$

$03 / 28 / 72$

$04 / 04 / 72$

$06 / 26 / 68$

$04 / 21 / 72$

05/10/72

$05 / 10 / 72$

$06 / 09 / 72$

06/09/72

$05 / 10 / 72$

$07 / 10 / 72$

07/10/72

$07 / 13 / 72$

$07 / 13 / 72$

$07 / 13 / 72$

$07 / 13 / 72$

$07 / 13 / 72$

$07 / 13 / 72$

$08 / 10 / 72$

08/08/72

$08 / 10 / 72$ 
Page No. 108

04/28/93

LIST OF HANFORD-GENERATED CLASSIFIED

DOCUNENTS DATED BETUEEN 1/1/61 AND 12/31/72

DOC. NO.

DUN-08026

DUN-08036

DUN-08036EX

DUN-08037

DUN-0804201

DUH-0804201 RD

DUN-0804202

DUW-0804202 RD

DUN-0804203

DUN-0804203 RD

DUN-0804204

DUN-0804204 RD

DUN-0804205

DUH-0804205 RD

DUH-08049

DUN-08049EX

DUN-08050

DINN-08063

DUN-08072

DUN-08072EX

DUN-08073

DUN-08082

DUN-08082EX

DUN-08093

DUN-08185RD

DUN-08205

DUN-08286

DUW-08287

DUN-08288

DUH $-M-00028$
TITLE

AUTHOR

FY 1972, YEAR END FIMANCIAL STATMENTS, CHANGES IN PROOUCT \& NONPRCOUCTION SS MATERIALS

MSR - AUG 1972

MATERIAL STATUS REPORT FOR MUG 1972

COMPOSITION OF ENDING INVENTORY - AUG

1972

JULY 1972 PROOUCTION INVENTORY \&

MANUFACTURING STATEMENT

PRODUCTION INVENTORY AMD MANUFACTURING STATEMENT JULY 1972

AUG 1972 PRODUCTION INVENTORY \&

MANUFACTURING STATEMENT

PRODUCTION INVENTORY AMD MANUFACTURING

STATEMENT AUEUST 1972

SEP 1972 PROOUCTION INVENTORY \&

MANUFACTURING STATEMENT

PRODUCTION INVENTORY AND MANUFACTURING

STATEMENT SEPTEMBER 1972

OCT 1972 PROOUCTION INVENTORY 2

MANUFACTURING STATEMENT

PROOUCTION INVENTORY AND MANUFACTURING

STATEMENT OCTOBER 1972

NOV 1972 PROOUCTION INVENTORY \&

MANUFACTURING STATEMENT

PRODUCTION INVENTORY AND MANUFACTURING

STATEMENT NOVEMBER 1972

MSR - SEP 1972

MATERIAL STATUS REPORT FOR SEP 1972

FISCAL YR 1973

COMPOSITION OF ENDIMG INVENTORY - SEP

1972

MUCLEAR MATERIALS DMA $5 \& 6$, OCTOBER, MOVEMBER, \& DECEMBER 1972

MSR - OCT 1972

MATERIAL STATUS REPORT FOR OCT 1972

COMPOSITION OF ENDING IMVENTORY - OCT

\section{2}

MSR - NOV 1972

MATERIAL STATUS REPORT FOR NOV 1972

MSR - DEC 1972

PLUTONILM-238 - PROGRAM ANALYSIS

MONTHLY MANAGEMENT REPORTS - REACTOR

MATERIAL TECHNOLOGY SUBSECTION

ROD MITHORAGAL SEQUENCE MARK I LOAD

PATTERN F'

ROO HITHDRAWAL SEQUENCE PATTERN L

Z -A MELTIHG INTERACTIONS

URANIUN-236 AMD MEPTUNIUA-237 PRCOUCTION TABLES
SECR

$12 / 05 / 72$

OC SCHROEDER

SECR

$09 / 10 / 72$

$09 / 08 / 72$

$09 / 08 / 72$

GH GALLUP

SECR

08/25/72

GH GALLUP

$08 / 25 / 72$

GH GALLUP

$09 / 29 / 72$

GH GALLUP CRD

$09 / 29 / 72$

GH GALLUP SECR

$10 / 24 / 72$

GH GALLUP CRD

$10 / 24 / 72$

GH GALLUP SECR

$11 / 21 / 72$

GH GALLUP CRD

$11 / 21 / 72$

GH GALLUP

$12 / 1 ! / 72$

GH GALLUP CRD

$12 / 19 / 72$

$10 / 09 / 72$

OC SCHROEDER

SECR

$10 / 09 / 72$

CP DORISS

SRD

$10 / 09 / 72$

CD HARRINGTOW

SECR

SRD

SECR

$11 / 07 / 72$

11/07/72

$11 / 09 / 72$

CD HARRINGTON

SECR

SRD

SECR

CONF

CRD

PA CARLSON

DL LARKIN

CONF

12/07/72

$12 / 06 / 72$

01. $19 / 72$

09/23/8́8

$01 / 01 / 69$

CB/07/68

DL LARKIN CONF

WK KRATZER CONF

$10 / 02 / 68$

$10 / 02 / 72$

AF KUPINSKI/ER PERL SRD

07/01/69 
Page No. 109

$04 / 28 / 93$

LIST OF HANFORD-GENERATED CLASSIFIED

DOCUMENTS DATED BETWEEN 1/1/61 AND 12/31/72

Doc. No.

TITLE

AUTHOR

LEVEL

PGS

DATE

DUN-N-00001

DUN-N-00012

DUN-N-00030

DUN-N-00042

DUN-N-00044

DUN-N-00045

DUN-N-O0046

DUN-N-00059

DUN-N-00062

DUN-N-00063

DUN-N-00086

DUN-N-00090

DUN-N-00091

DUN-N-00093

DUN-N-00111

DUN-N-00112

DUN-N-00115

DUN-N-00121

DUN-N-00122

DUN-N-00130

DUN-N-00142

DUN-N-00148

DUN-N-00149

DUN-N-00150

DUN-N-00151

DUN $-N-00178$

DUN-N-00180

DUN-N-00201

DUN-N-00203

DUN-N-00213

DUN-N-00214

DUN-N-00216

DUN-N-00219

DUN-N-00221

DUN-N-00231

DUN-N-00233

DUN-N-00244

DUN-N-00244EX

DUN-N-00247

DUH-N-00248

DUN-N-00251

DUN-N-00257

DUN-N-00259

DUN-N-00265

DUN-N-00282

DUN-N-00288

DUN-N-00289

DUN-N-00291

DUN-N-00292VOL1
PERSONAL MOTES

PERSONAL MOTES

PERSONAL NOTES

PERSOHAL MOTES

PHYSICS NOTES

FUELS NOTES

PERSOMAL NOTES

PERSONAL MOTES

PERSONAL MOTES

PERSONAL MOTES

PERSONAL NOTES

MOSTLY PAST-INS-FUELS

MOSTLY PASTE-IHS-FUELS

PERSONAL NOTES, RRE

PERSONAL NOTES

PERSONAL MOTES

ENGINEERING DATA

PERSONAL NOTES

PERSONAL NOTES

IN CORE FLUX MONITOR DATA - KW

- FUELS STATISTICAL DATA

PERSONAL NOTES

PERSOMAL MOTES

PERSONAL NOTES

PHYSICS NOTES

PERSONAL MOTES

OPERATING STUDIES \& CERT.

PERSONAL MOTES

STLOY MOTES

PERSONAL NOTES

PERSONAL MOTES

FUELS NOTES

MOTES ON FORECASTING SCHEDULE AMD

NUCLEAR MATERIAL

FUELS NOTES

NO TITLE GIVEN

N PRCOUCTIOA DATA MOTES

TECHNICAL NOTES

EXCERPTS FROM SECRET MOTEBOOK

C REACTOR NOTES \& DATA

PHYSICS NOTES

PERSONAL MOTES

SUPERVISOR LOGBOOK, 105-C

SUPERVISOR'S MOTEBOOK

PERSONAL NOTES

PERSONAL NOTES

PERSONAL MOTES

PERSONAL MOTES

PERSONAL NOTES

TOTAL CONTROL PARANETERS VOL 1
DUN-RL

DH BAMGERTER

RE BMARS

FE OWEN

DUN-RL

DUN-RL

EC BENMETT

JP HAMRIC

H TOFFER

ER PERL

DUN-RL

DUN-RL

DUN-RL

DUH-RL

DUN-RL

WF FIFER

DUN-RL

ER PERL

DUN-RL

JE KAVECKIS

DUN-RL

DUN-RL.

RL MILLER

CD EUTHRIE

DUN-RL

EE LEITZ

DUN-RL

RE RUSSELL

HL DEBBAN

TE EDMARDS

JP SCHAIDT

DUN-RI.

T PRUDICH

DUN-RL

DUN-RL

RP ROVE

JP COOKE

JP COOKE

DUN-RL

DUN-RL

DUN-RL

DUN-RL

ML PETERSON

RV SKIMAER

JR LAMGTON

EC BENMETT

DH BAMGERTER

JF MEMEC

JF MENEC

\begin{tabular}{|c|c|}
\hline SECR & $07 / 06 / 71$ \\
\hline SRD & $11 / 01 / 65$ \\
\hline CRD & $11 / 01 / 65$ \\
\hline CRD & $11 / 04 / 65$ \\
\hline SECR & $09 / 01 / 65$ \\
\hline SECR & $11 / 01 / 65$ \\
\hline CRD & $11 / 04 / 65$ \\
\hline CRD & $11 / 13 / 65$ \\
\hline SRD & $11 / 13 / 65$ \\
\hline SRD & $11 / 13 / 65$ \\
\hline SECR & $07 / 06 / 72$ \\
\hline SECR & $11 / 24 / 65$ \\
\hline SECR & $11 / 19 / 65$ \\
\hline SECR & $11 / 13 / 65$ \\
\hline SECR & $04 / 01 / 66$ \\
\hline SRD & $04 / 27 / 66$ \\
\hline SECR & $05 / 20 / 66$ \\
\hline SRD & $08 / 08 / 66$ \\
\hline SECR & $06 / 11 / 71$ \\
\hline CRD & $12 / 13 / 66$ \\
\hline SECR & $02 / 10 / 67$ \\
\hline SECR & $04 / 08 / 71$ \\
\hline CRD & $04 / 04 / 67$ \\
\hline SRD & $04 / 28 / 67$ \\
\hline SECR & $05 / 01 / 67$ \\
\hline SRD & $08 / 11 / 67$ \\
\hline SECR & $10 / 06 / 67$ \\
\hline CRD & $09 / 99 / 67$ \\
\hline CRD & $09 / 19 / 67$ \\
\hline CRD & $10 / 10 / 67$ \\
\hline CRD & $10 / 10 / 67$ \\
\hline SECR & $11 / 02 / 67$ \\
\hline CRD & $11 / 30 / 67$ \\
\hline SECR & $12 / 05 / 67$ \\
\hline SECR & $02 / 26 / 68$ \\
\hline SECR & $03 / 08 / 68$ \\
\hline CONF & $05 / 08 / 68$ \\
\hline conf & $01 / 23 / 69$ \\
\hline SECR & $05 / 31 / 68$ \\
\hline SECR & $06 / 10 / 68$ \\
\hline SECR & $06 / 01 / 68$ \\
\hline SECR & $08 / 01 / 68$ \\
\hline CRD & $08 / 07 / 68$ \\
\hline CRD & $09 / 05 / 68$ \\
\hline CRD & $12 / 03 / 68$ \\
\hline CRD & $01 / 24 / 69$ \\
\hline SRD & $02 / 06 / 69$ \\
\hline CRD & $02 / 26 / 69$ \\
\hline CRD & $02 / 28 / 69$ \\
\hline
\end{tabular}


Page No. 110

$04 / 28 / 93$

LIST OF HANFORD-GENERATED CLASSIFIED

DOCUMENTS DATED BETLEEN 1/1/61 AND 12/31/72

DOC. $N$ No.

DUN-N-00295

DUN-N-00296BK2

DUN-N-00300

DUN-N-00303

DUN-N-00307

DUN-N-00308

DUN-N-00309

DUN-N-00312

DUN-N-00322

DUN-N-00327

DUN-N-00328

DUN-N-00332

DUN-N-00335

DUN-H-00338

DUN-N-00351

DUN-N-00352

DUN-N-00354

DUN-N-00357

DUN-N-00360

DUN-N-00364

DUN-N-D0377

DUN-P-00015

DUN-P-00016

DUN-USU-00004

DUN- MSU-00005

DUN-YSU-00006

DUN-WSU-00007

DUN-WSU-00008

FTS-CLVI-00T51

FTS-CLVI-00755

FTS-CLVI-00756

FTS-CLVI-00757

FTS-XX-02129

FTS $-X X-02130$

FTS-XX-02131

FTS $-X X-02132$

FTS-XX-02133
TITLE

AUTHOR

N FUELS \& REACTOR NOTES

CLASSIFIED NOTES ON ACCOUNTABILITY

PHYSICS (ZTM)

KU PROCESS ENGR LOG

SUPERVISOR LOGBOOK, KH

STRESS AMALYSIS PARAMETERS

STRESS AMALYSIS PARNETERS

SUPERVISOR LOCBOOK, KE

165 KU CONTROL ROOM LOG

105-KM SUPERVISOR LOCBOOK

PERSONAL MOTES

105-KE SUPERVISOR LOGBOOK

105-KW SUPERVISOR LOCBOOK

105-KW SUPERVISOR LOCBOOK

SUPERVISOR'S LOG BOOK

SUPERVISOR'S LOG BOOK

SUPERVISOR'S LOG BOOK

SUPERVISOR'S LOG BOOK

SUPERVISOR'S LOG BOOK 12/6/70 - 1/10/71

SUPERVISOR'S LOG BOOK 1/11/71 - 2/16/71

NOTEBOOK OF C.W. KUHLMAN (MAN-89425)

COLUMBIA RIVER MODEL 8-14 INFRARED IMAGE

VIEW OF COLUMBIA RIVER SHOWING COYOTE RAPIDS

EXTENDED TEMPERATURE STUDIES $C \& K$

DOWCOMER MODELS

DATA COLLECTION NOTEBOOK FOR CONTRACT

DDR-112

$K$-DOMNCOMER STUDIES

THERMAL-HYDRAULIC MOOEL DATA

THERMAL-HYDRAULIC MODEL STUDIES OF THE

COLLMBIA RIVER VOLLAE II

RELATIONSHIP BETUEEN CONVERSION COSTS

INVESTMENT \& PRCOUCTION AT HAPO

STUDY OF THE MUCLEAR MATERIALS OPERATION OF HAPO

CPD - UNIT COST \& PRODUCTION FIQURES

CY-1957 - 69 ACTUAL, CY-1962 COAL,

CY 1963 - 1966 ESTIMATES

MEASUREMENT OF PRODUCTION RETURNS FROM

HANFORD RED PROGRAMS 1056 - 1960

GRAPH - HISTORY OF MD 1945 - 1963

GRAPH - TOTAL PLUTOWIUM PROOUCTION -

RLOO 1945 - 1963

TELETYPE - PU PERCENT OF FORECAST

CONSOLIDATION - COMPARATIVE REACTOR

PERFORMANCE REPORTS JAN 1955 THRU DEC

1956

QUANTITY \& ISOTOPIC CONTENT OF PLUTONIUN

SHIPMENTS HANFORD TO RFP CY 1952 - 1959
DUN-RL

ER PERL

DUN-RL

DUN-RL

DUN-RL

EA LUMD

EA LUND

DUA-RL

RK WAHLEN

DUN-RL

RL MILLER

DUM-RL

DUN-RL

DUN-RL.

RG CLOUEH

RG CLOUEH

RG CLOUEH

RG CLOUEH

RG CLOUEH

RG CLOUGH

DUN-RL

JH BALLONE

JH BALLONE

CC LONXX

ER TINMEY

CC LOMAX

HD copp

Ho COPP

GB VINCENT

GB VIMCENT

SECR
SECR

$04 / 05 / 62$

07/03/63

07/03/63

$07 / 26 / 63$

02/21/65

SECR

$04 / 01 / 69$

04/45/69

06/09/69

$06 / 19 / 69$

$06 / 19 / 69$

$06 / 09 / 69$

$09 / 16 / 69$

$10 / 24 / 69$

$11 / 01 / 69$

$11 / 25 / 69$

$01 / 16 / 70$

$07 / 15 / 70$

$08 / 20 / 70$

$09 / 29 / 70$

$11 / 09 / 70$

12/22/70

$12 / 05 / 72$

$09 / 13 / 67$

12/04/67

$06 / 07 / 68$

$06 / 29 / 67$

$06 / 07 / 68$

$03 / 18 / 68$

$10 / 01 / 68$

$04 / 07 / 62$

01/02/62

$03 / 23 / 62$

SECR

ECR

SECR

$01 / 25 / 68$ 
Page No. 111

$04 / 28 / 93$
LIST OF HANFORD-GENERATED CLASSIFIED

DOCUMENTS DATED BETUEEN 1/1/61 AND 12/31/72
DOC. NO.

FTS $-X X-02134$

FTS-XXX-02135

HAN-100-00016 HAN-100-00069 HAN-100-00075 HAN-100-00076 HAN-100-00109 HAN-100-00127

HAH-100-00156

HAN-100-00232 HAN-100-00233 HAN-100-00278 HAN- $100-00280$ HAN-100-00357

HAN-100-00373 HAN-100-00466 HAN-100-00536 HAN-100-00570

HAN-100-00585 HAN-100-00622

HAN-100-00628

HAN-100-00629 HAN-100-00651 HAN-100-00690

HAN-100-00710 HAN -58378

HAN-60650

HAN-60883

HAN-60888

HAN -61143

HAN-61537

HAN-61679

HAN-6471701
TITLE

MEMO - NONDEFENSE PLUTONIUA PROOUCTION TABLE I - QUANTITY \& ISOTOPIC CONTENT OF PLUTONIUM SHIPMENT TO RF FRON HANFORD CY 1952 - 1959

HAZARDS SUMARY REPORT SUPPLEMENT IV IMFORMATION OF FPCE PLANT (ROUGH DRAFT) N REACTOR PROOUCTION PLUTONILM PRODUCTION HISTORY SHIPPER-RECEIVER DIFFERENCE REPORT MONTHLY STATUS AND PROGRESS REPORT FOR MARCH 1968

PTA 107 IRRADIATION OF HIGH U-236 CONTENT 95 METAL IN KE REACTORS MARK IV FUEL PROGRAM COMPARATIVE PILE PERFORMANCE MARCH 1968 HIGHLY ENRICHED FUEL DEVELOPNENT PROGRAM MP PROCESSING FACTS

MOUTHLY STATUS AND PROCRESS REPORT FOR APRIL 1968

ACCELERATED E-D PRCOUCTION PROCRNM

COMPARATIVE PILE PERFORMANCE APRIL 1968

WASTE TANK HISTORY AND PROJECTED STATUS.

TABLE 1 ESTIMATED MANUFACTURING AND

ADDITIONAL EQUIPMENT COSTS FOR PU STORAGE

SHIPPER-RECEIVER DIFFERENCE REPORT

PROOUCTION OF U-233 FOR LWBR

DEMONSTRATIOA CORE

MOHTHLY STATUS AND PROGRESS REPORT FOR

MAY 1968

SHIPPER-RECEIVER DIFFERENCE REORT

CLASSIFICATION OF RIVER TEMPERATURE DATA

EFFECTIVE PU-238 \& NP-237 MEUTRON

ABSORPTION CROSS SECTION IN N \& K REACTORS

COMPARATIVE PILE PERFORMANCE MAY 1968

$100 \& 300$ AREAS MONTHLY REPORT FOR JAN

1955 THRU AUG 1955

MONTHLY REPORTS - OPERATIONS DIV - MARCH

THRU AUCUST 1954

100 AREA MONTHLY REPORT FOR SEPTEMBER

1955

100 AREA MONTHLY REPORT FOR OCTOBER 1955

100 AREA MONTHLY REPORT FOR NOVEMBER

1955

100 AREA MONTHLY REPORT FOR DECEMBER

1955

MONTHLY REPORTS FOR 100 AREA RPTS \#1

THRU \#12 1956

100 AREA MONTHLY RPT FOR 1/57
AUTHOR

DN SNITH

LEVEL PGS DATE

SEC

$02 / 23 / 68$

$01 / 25 / 68$

SECR

SECR

$01 / 31 / 68$

$01 / 201 / 66$

$03 / 28 / 68$

$03 / 27 / 68$

$04 / 02 / 68$

SECR

SECR

$04 / 11 / 68$

SECR

$12 / 27 / 67$

SECR

SECR

$04 / 16 / 68$

03/01/68

$04 / 17 / 68$

$04 / 23 / 68$

$05 / 13 / 68$

SECR

05/01/68

PA CRAIG

$04 / 01 / 68$

$05 / 24 / 68$

05/28/68

SECR

$05 / 20 / 68$

SECR

$05 / 16 / 68$

SECR

$06 / 13 / 68$

SECR

06/07/68

SECR

$06 / 11 / 68$

$06 / 10 / 68$

KF PAULOVICH

SECR

$06 / 21 / 68$

$11 / 22 / 71$

SECR

$11 / 22 / 71$

SECR

$11 / 22 / 71$

SECR

SECR

$11 / 22 / 71$

$11 / 22 / 71$

SECR

$11 / 22 / 79$

SECR

$11 / 22 / 71$

SECR

$11 / 22 / 71$ 
Page No. 112

$04 / 28 / 93$

LIST OF HANFORD-GENERATED CLASSIFIED

DOCUMENTS DATED BETWEEN 1/1/61 AND 12/31/72

DoC. NO.

TITLE

AUTHOR

LEVEL PGS

DATE

HAN-6471702

HAN -6471703

HAN-6471704

HAN-6471705

HAN-6471706

HAN-6471707

HAN-6471708

HAN -6471709

HAN-6471710

HAN-6471711

HAN -6471712

HAN-67953

HAN-6822101

HAN-6822102

HAN- 6822103

HAN-6822104

HAN-6822105

HAN-6822106

HAN-6822107

HAN-6822108

HAN-6822109

HAN-6822110

HAN-6822111

HAN-6822112

HAN-70316

HAN-7132901

HAN-7132902

HAN-7132903

HAN-7132904

HAN -7132905

HAN -7132906

HAN-7132907

HAN-7132908

HAN-7132909

HAN-7132910

HAN-7132911

HAN-7132912

HAN-72862

HAN-73829

HAN-T7341

HAN -77440

HAN-77681 (RPTS

1-12)

HAN-77687
100 AREA MONTHLY RPT FOR 2/57

100 AREA MONTHLY RPT FOR 3/57

100 AREA MONTHLY RPT FOR 4/57

100 AREA MONTHLY RPT FOR 5/57

100 AREA MONTHLY RPT FOR 6/57

100 AREA MONTHLY RPT FOR 7/57

100 AREA MONTHLY RPT FOR 8/57

100 AREA WOUTHLY RPT FOR 9/57

100 AREA MONTHLY RPT FOR $10 / 57$

100 AREA MONTHLY RPT FOR 11/57

100 AREA MONTHLY RPT FOR 12/57

PROPOSED AEC MAMUAL CHAPTER

0621-ASSESSMENT OF DISASTER DAMMGE

100 AREA MONTHLY OPERATION RPT FOR 1958

100 AREA MONTHLY OPERATION RPT FOR 1958

100 AREA MONTHLY OPERATION RPT FOR 1958

100 AREA MONTHLY OPERATION RPT FOR 1958

100 AREA MONTHLY OPERATION RPT FOR 1958

100 AREA MONTHLY OPERATION RPT FOR 1958

100 AREA MONTHLY OPERATION RPT FOR 1958

100 AREA MONTHLY OPERATION RPT FOR 1958

100 AREA MONTHLY OPERATION RPT FOR 1958

100 AREA MONTHLY OPERATION RPT FOR 1958

100 AREA MONTHLY OPERATION RPT FOR 1958

100 AREA MONTHLY OPERATION RPT FOR 1958 PERSOMAL NOTEBOOK OF PHILLIP A. CRAIG

HANFORD LAB REPORT FOR JAN 1969

HANFORD LAB REPORT FOR FEB 1969

HANFORD LAB REPORT FOR MAR 1969

HANFORD LAB REPORT FOR APR 1969

HANFORD LAB REPORT FOR MAY 1969

HANFORD LAB REPORT FOR JLM 1969

HANFORD LAB REPORT FOR JUL 1969

HANFORD LAB REPORT FOR AUG 1969

HANFORD LAB REPORT FOR SEP 1969

HANFORD LAB REPORT FOR OCT 1969

HANFORD LAB REPORT FOR NOV 1969

HANFORD LAB REPORT FOR DEC 1969

CONVERSION OF KER TEST LOOP FOR MPR

PROTOTYPE TESTIHG FEASIBILITY STLDY

FY 1962 REVISION TO HOO MAMAGER'D DATA BOOK

TABLE OF PRCOUCTION COSTS FOR CASES A2, D2, AND D9

MOWTHLY STATUS AND PROGRESS REPORT -

DECEMBER 1960

REACTOR OPERATIONS BRANCH MONTHLY

REPORTS - JAMUARY 1961 THROUGH DECEMBER, 1961

MONTHLY STATUS \& PROGRESS REPORT FOR JAN 1961
SECR

$11 / 22 / 71$

$11 / 22 / 71$

$11 / 22 / 71$

$11 / 22 / 71$

$11 / 22 / 71$

$11 / 22 / 71$

$11 / 22 / 71$

$11 / 22 / 71$

$11 / 22 / 71$

$11 / 22 / 79$

$11 / 22 / 71$

$01 / 13 / 68$

$11 / 22 / 71$

$11 / 22 / 71$

$11 / 22 / 71$

$11 / 22 / 71$

$11 / 22 / 71$

91/22/71

$11 / 22 / 71$

$11 / 22 / 71$

$11 / 22 / 71$

$11 / 22 / 71$

$11 / 22 / 71$

$11 / 22 / 71$

$10 / 15 / 64$

$11 / 22 / 71$

$11 / 22 / 71$

$11 / 22 / 71$

$11 / 22 / 71$

$11 / 22 / 71$

$11 / 22 / 71$

$11 / 22 / 71$

$11 / 22 / 71$

$11 / 22 / 71$

$11 / 22 / 71$

$11 / 22 / 71$

$11 / 22 / 71$

$03 / 13 / 64$

$12 / 12 / 62$

BURHS \& ROE, INC. SRD

$01 / 13 / 69$

TRAVIS JE

SRD

11

$01 / 13 / 61$

PLUM RL

SRD

144

02/09/61

SECR

$04 / 11 / 63$ 
Page No. 113

$04 / 28 / 93$

LIST OF HANFORD-GENERATED CLASSIFIED

DOCLMENTS DATED BETMEEN 1/1/61 AND 12/31/72

Doc. No.

TITLE

A'JTHOR

LEVEL.

PGS

DATE

HAN-TT72412

HAN-78047

HAN-78689

HAN-80549

HAN-805491

HAN-80582RPT

HAN-80582RPT 10

MAN-80582RPT11

HAH-80582RPT 12

HAN-80582RPT2

HAN-80582RPT3

HAN-80582RPT4

HAK-80582RPT5

HAN-80582RPT6

HAN-80582RPT7

HAN-80582RPT8

HAN-80582RPT9

HAN-80635

HAN-80649

HAN-806491

HAN-80668

HAN -80684

HAN -80715

HAN-80718

HAN-80726

HAN-80727
3000 PROGRAM - WEAPONS PRCDUCTION \& DEVELOPNENT

REPORT ON TURBINE GENERATOR OPTIMIZATION STUDIES EXPORT ELECTRIC PLANT HANFORD NEW PRODUCTION REACTOR

SUPPLEMENTAL REPORT - CONVERSION OF HANFORD NEW PRODUCTION REACTOR TO EXPORT ELECTRIC GENERATION

VALUE OF REACTOR IMPROVEMENTS \& OIXTH LOOP IF FIMANCED BY UPPSS

ESTIMATED PRODUCTION INCREASE DERIVED FROM ADDITION OF GE REACTOR CONVERSION COSTS \& HANFORD MPR SYS CHANGES MONTHLY STATUS AMD PROCRESS REPORT JANUARY THRU DECEMBER 1962 MONTHLY STATUS AND PROCRESS REPORT JANUARY THRU DECEMBER 1962 MONTHLY STATUS AND PROERESS REPORT JAMUARY THRU DECEMBER 1962

MONTHLY STATUS AND PROGRESS REPORT JANUARY THRU DECEMBER 1962 MONTHLY STATUS AND PROGRESS REPORT JAMUARY THRU DECEMBER 1962 MONTHLY STATUS AND PROGRESS REPORT JANUARY THRU DECEMBER 1962 MONTHLY STATUS AND PROGRESS REPORT JANUARY THRU DECEMBER 1962 MONTHLY STATUS AND PROGRESS REPORT JAMUARY THRU DECEMBER 1962 MONTHLY STATUS AND PROCRESS REPORT JAMUARY THRU DECEMBER 1962 MONTHLY STATUS AND PROGRESS REPORT JAMUARY THRU DECEMBER 1962 MONTHLY STATUS AND PROGRESS REPORT JAMUARY THRU DECEMBER 1962 MONTHLY STATUS AND PROGRESS REPORT JAMUARY THRU DECEMBER 1962 MONTHLY STATUS \& PROGRESS REPORT FOR DEC 1961

MENO - $P$ - 238 REQUIREMENT CHART

CHART - $P-238$ REQUIREMENTS VS

PROOUCTION

MONTHLY PROOUCTION REPORT - DEC 1961

MATERIALS BALANCE REPORT DECEMBER 1961

MATERIAL. SPECIFICATIONS

MATERIALS BALANCE REPORT DECEMBER 1961

SUMMARY OF AEC RADIOLOGICAL MONITORIMG METLORK MEETING 1/9/62 AT GERMANTOWN COAPARATIVE REACTOR PERFORMRNCE - DEC 1961
SECR

$01 / 10 / 62$

BURNS \& ROE, INC., NEW SRD 90

03/20/61 YORK IMC.

SRD

SECR

$01 / 19 / 62$

SECR

$01 / 20 / 62$

SECR

$01 / 01 / 62$

SECR

$01 / 01 / 62$

SECR

01/01/62

SECR

$01 / 01 / 62$

SECR

$01 / 01 / 62$

SECR

$01 / 01 / 62$

SECR

$01 / 01 / 62$

SECR

01/01/62

SECR

$01 / 01 / 62$

SECR

01/01/62

SECR

01/01/62

SECR

$01 / 01 / 62$

SECR

$01 / 12 / 62$

SECR

SECR

$01 / 08 / 62$ $01 / 08 / 62$

SECR

SECR

SECR

SECR

SECR

SECR
$01 / 12 / 62$ $01 / 15 / 62$

$01 / 11 / 62$

$01 / 18 / 62$

$01 / 16 / 62$

$01 / 18 / 62$ 
Page No. 114
04/28/93

LIST OF HANFORD-GENERATED CLASSIFIED

DOCUNAENS DATED BETUEEN 1/1/61 AND 12/31/72

DOC. NO.

HAN-80734

HAN-80867

HAN -80896

HAN-8094601

HAN-8094602

HAN-8094602 SUP1

HAN-8094603

HAN-8094604

HAN-8094604 SUP1

HAN-8094605

HAN-8094605 SUP1

HAN -8094606

HAN-8094606 SUP 1

HAN -8094607

HAN-8094608

HAN-8094609

HAN-8094610

HAN-8094611

HAN-8094612

HAN-80963

HAN-80976

HAN-80977

HAN-80986

HAN-81002

HAN-81008

HAN-81065

HAN-81139
TITLE

AUTHOR

LEVE

PGS

DATE

FIVE YEAR ELECTRICAL PONER FORECAST

SECR

$01 / 12 / 62$

PROCESS LOAD HANFORD ATOMIC PRODUCT

OPERATION

HAPO PRODUCTION FORECAST 1/01/62 THRU

6/30/67

PROOUCTION FORECAST 1/01/62 THRU 6/30/67

WE JOHNSON

SRD

$01 / 25 / 62$

FUEL \& METALLURGY BRANCH MONTHLY

REPORTS, O3 PROGRAH WEAPONS

FUEL \& METALLURGY BRANCH MONTHLY

REPORTS, 03 PROGRAM MEAPONS

FUEL \& METALLURGY BRANCH MONTHLY

REPORTS, O3 PROGRAM WEAPONS

FUEL \& METALLURGY BRANCH MONTHLY

REPORTS, 03 PROGRAM WEAPONS

FUEL \& METALLURGY BRANCH MONTHLY

REFORTS, 03 PROGRAM WEAPONS

FUEL \& METALLURGY BRANCH MONTHLY

REPORTS, 03 PROGRAH WEAPOUS

FUEL \& METALLURGY BRAHCH MONTHLY REPORTS, 03 PROGRAM WEAPONS

FUEL \& METALLURGY BRANCH MONTHLY

REPORTS, 03 PROCRAM WEAPONS

FUEL \& METALLURGY BRANCH MONTHLY

REPORTS, O3 PROGRAM MEAPONS

FUEL \& METALLURGY BRANCH MONTHLY

REPORTS, 03 PROGRAM WEAPONS

FUEL \& METALLURGY BRANCH MONTHLY

REPCRTS, 03 PROGRAM MEAPONS

FUEL \& METALLURGY BRANCH MONTHLY

REPORTS, O3 PROGRAM IEAPONS

FUEL \& METALLURGY BRANCH MONTHLY

REPORTS, 03 PROGRAM WEAPOWS

FUEL \& METALLURGY BRANCH MONTHLY

REPORTS, 03 PROGRAM WEAPONS

FUEL \& METALLURGY BRANCH MONTHLY

REPORTS, 03 PROGRAM WEAPONS

FUEL \& METALLURGY BRANCH MONTHLY

REPORTS, 03 PROGRAM IEAPONS

ENRICHED U FOR MATURAL U IN ANTICIPATION

OF BLANKET LONDINGS

INCREMENTAL COSTS FOR BRANDYUIHE

MONTHLY STATUS \& PROGRESS REPORT FOR JAN

1962

MONTHLY PROOUCTION REPORT - JAN 1962

MINUTES OF COMBINED OPERATIONS WORKING

GROUP MEETING OF JAMUARY 18, 1962

THE FISSION PRCOUCT PROGRAM AND ITS

RELATION TO FUEL PROCESSING PROGRAMS

UNCLASSIFIED $P$

COMBINED OPERATIONS
SECR

SECR

SECR

SECR

SECR

SECR

SECR

SECR

SECR

SECR

SECR

SECR

SECR

SECR

SECR

SECR

SECR

SECR

SECR

SECR

SECR

SECR

SECK

SECR

SECR
$02 / 01 / 62$

02/01/62

$03 / 01 / 62$

$03 / 10 / 62$

$04 / 01 / 62$

05/01/62

$05 / 10 / 62$

$06 / 01 / 62$

$06 / 10 / 62$

07/01/62

$07 / 10 / 62$

$08 / 01 / 62$

$09 / 01 / 62$

$10 / 01 / 62$

$11 / 01 / 62$

$12 / 01 / 62$

01/01/63

$02 / 08 / 62$

$02 / 06 / 62$

$02 / 13 / 62$

$02 / 13 / 62$

02/02/62

$02 / 16 / 62$

02/21/62

$02 / 28 / 62$ 
Page No. 115

$04 / 28 / 93$

LIST OF HANFCRD-GENERATED CLASSIFIED

DOCUNENTS DATED BETWEEN 1/1/61 AND 12/31/72

Doc. NO.

HAN-81166

HAN -81170

HAN-81213

HAN-81236

HAN-81252

HAN-81282

HAN-81287

HAN-81366

HAM $1-81387$

HAN-81404

HAN-81540

HAN-81566

HAN-81610

HAN -81799

HAN-81825

HAN-81837

HAN-81839

HAN -81848

HAN-81878

HAN-81939

HAN-81953

HAN-81958

HAN -81960

HAN-82003

HAN -82034

HAN-820691

HAN -820692

HAN-820693

HAN-82093

HAN-82130
TITLE

HAPO 1961 ANMUAL REPORT

P SCRAP RECOVERY

COMPARATIVE REACTOR PERFORMAHCE - JAN

1962

MONTHLY STATUS \& PROARESS REPORT FOR FEB

1962

MANFORD PRCDUCTION PLANNIMG

MONTHLY PRODUCTION REPCRT - FEB 1962

ALTERMATE PROGRAM ASSUMPTIONS FOR FY

64-67

EVALUATION OF UO3 PRCOUCTION PRACTICES

PROO RATES FOR U-236 CONVERSION FACTORS

FOR MATERIAL PROD

COAPARATIVE REACTOR PERFORMANCE - FEB

1962

MONTHLY STATUS \& PROGRESS REPORT FOR MAR

1962

MONTHLY PRCOUCTION REPORT - MAR 1962

PRCOUCTION STUDIES - ALTERAATE QUDGET

COMPARATIVE REACTOR PERFORMANCE - MARCH

1962

MONTHLY STATUS \& PROGRESS REPORT FOR APR 1962

SHIPMENT OF ENRICHED UO3 (YOUR MENO 4/24 AND 4/19/62 MEMO SAPIRIE TO KARL

$3 / 26 / 62$ )

MONTHLY PRODUCTION REPORT - APR 1962

HAPO PROOUCTION FORECAST 4/01/62 THRU

12/31/63

TRANSMITTAL PROOUCTION FORECAST 4/01/62

THRU 12/31/63

COMPARATIVE REACTOR PERFORMANCE - APRIL

1962

PROPOSED SPECIFICATIONS FOR PROOUCTION

REACTOR RETURN UO3 FOR GASEOUS DIFFUSION

PLAMT FEED

MINUTES OF THE 4TH MEETING OF THE

HOO/ALOO PANEL

REDUCTION SCHEDULE FOR UNCLASSIFIED P

REACTOR SAFETY - MPR CONFIMEMENT SYSTEM

(MEMO: 4/3/62 P:EFM)

IPD TWO YEAR ELECTRIC POWER FORECAST FY

$1963 \& 1964$

FLOW SHEETS GROUP I - CASE A

DISCUSSION OF ESTIMATING CASES

TABULATION OF LOADS FOR PU BLENDING

CASES ENTRIS

WEAPON COMPONENTS FABRICATION STUDIES

MONTHLY STATUS \& PROGRESS REPORT FOR MAY 1962

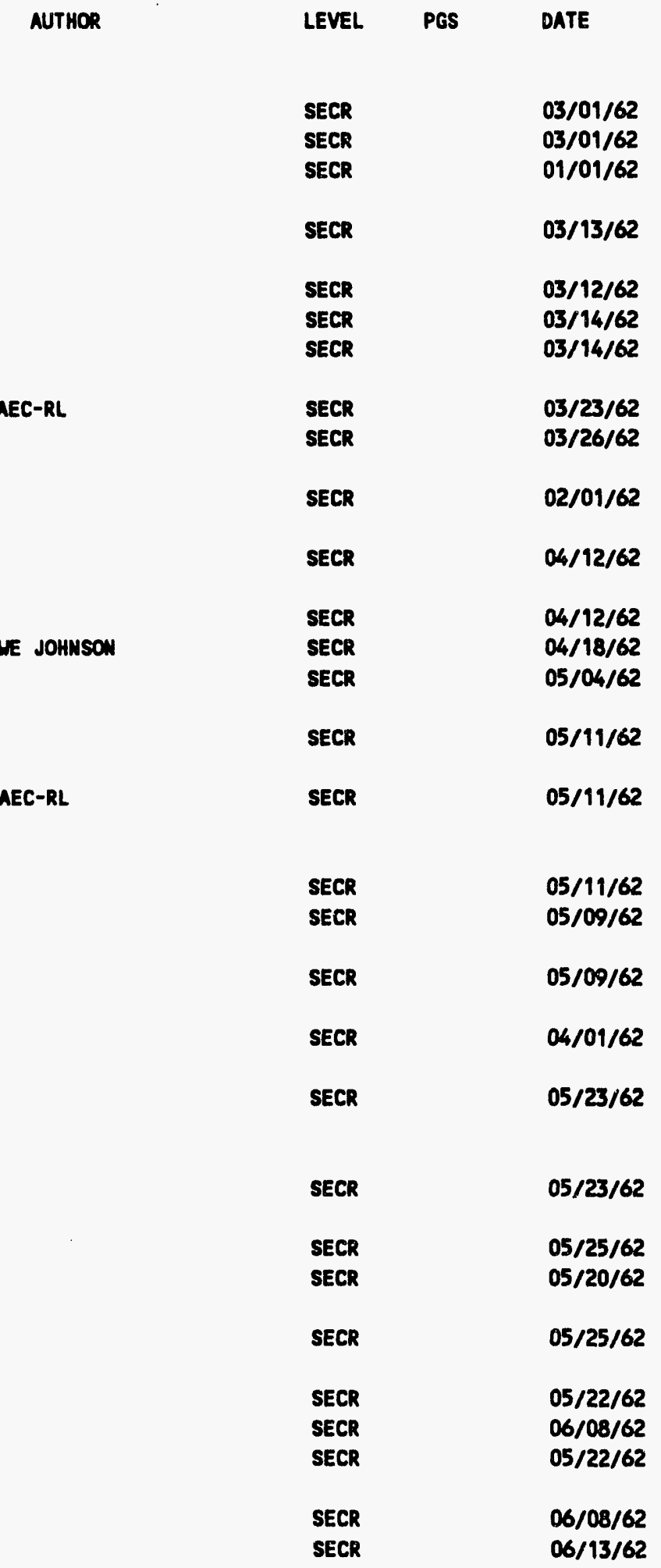


Page No. 116

$04 / 28 / 93$

LIST OF HANFORD-GENERATED CLASSIFIED

DOCUMENTS DATED BETMEEN 1/1/61 AND 12/31/72

DOC. No.

TITI.E

AUTHOR

PGS

DATE

HAN-82147

HAN-82160

HAN -82190

HAN-82224

HAN-82321

HAN-82346

HAN-82373

HAN-82385

HAN-82398

HAN-82406

HAH -82414

HAN-82428

HAN-82466

HAN-82469

HAN-82481

HAN-82513

HAH -82579

HAN-82600

HAN-82636

HAN-82658

HAN-82694

HAN-82789

HAN-82813

HAN-82834

HAN-82848

HAN-82885

HAN-82889

HAN-82891

HAN-82904
MONTHLY PRCOUCTION REPORT - MAY 1962

PLUTONIUN BLENDING

EXPLANATIONS OF PIDS, AIH-OS, AND

ADJUSTMENTS FOR MAY 1962

COLUMBIA RIVER TEMPERATURES

HANFORD PRCOUCTION PLANNIKG STUDY

COAPARATIVE REACTOR PERFORMANCE - MAY

1962

MONTHLY STATUS \& PROGRESS REPORT FOR JUA

1962

MONTHLY PRODUCTION REPORT - JUN 1962

REACTOR SAFETY - OPERATIONAL

CHARGE-DISCHARGE

MATERIAL BALANCE REPORT JUNE 1962

MATERIAL BALANCE REPORT JUNE 1962

PROPOSED SPECIFICATIONS FOR PRCOUCTION

REACTOR RETURMS NO3 FOR GASEOUS

DIFFUSION PLANT FEED

HAPO PROOUCTION FORECAST 7/01/62 THRU

6/30/67

COST STATEMENTS MORMAL URANIUM AMD

THORIUM FOR JUNE 1968

NLO COST STATEMENTS COVERING ACCOUNT

1611 ENRICHED URANIUM FOR JUNE 1962

MINIIUM COST STUDY CHARTS \& TABLES

REACTOR SAFETY-IMPACT OF PROPOSED HIGHER

GRAPHITE TEMPERATURE ON GRAPHITE

DAMAGE/DISLOCATION IN EVENT PROC TUBE

FAILURES

MONTHLY STATUS \& PROGRESS REPORT FOR JUL

1962

MONTHLY PRODUCTION REPORT - JUL 1962

PRODUCTION FORECAST 7/01/62 THRU 6/30/67

COMPARATIVE REACTOR PERFORMANCE - JUNE

1962

IMPACT OF HIGHER GRAPHITE TEMPERATURE ON

REACTOR SAFETY

SPECIFICATIONS FOR PLUTONIUM MITRATE

FROM PRIVATE INDUSTRY

COMPARATIVE REACTOR PERFORMAMCE - JULY

1962

COMPUTATION OF PRICE DEDUCTION FROM

REACTCR PONER FOR OUTAGE OF 7/10/62

MONTHLY STATUS \& PROGRESS REPORT FCR AUG

1962

REACTOR DOWTIME

PRCOUCTION STUDIES

HU-71619: TECHNICAL

SPECIFICATIONS-HANFORD PRODUCTION

REACTORS

\begin{tabular}{|c|c|}
\hline $\begin{array}{l}\text { SECR } \\
\text { SECR }\end{array}$ & $\begin{array}{l}06 / 13 / 62 \\
06 / 15 / 62\end{array}$ \\
\hline & $06 / 20 / 62$ \\
\hline SECR & $06 / 22 / 62$ \\
\hline SECR & $07 / 03 / 62$ \\
\hline SECR & $07 / 06 / 62$ \\
\hline SECR & $07 / 13 / 62$ \\
\hline SECR & $07 / 13 / 62$ \\
\hline SECR & $07 / 11 / 62$ \\
\hline SECR & $07 / 17 / 62$ \\
\hline SECR & $07 / 19 / 62$ \\
\hline SECR & $07 / 20 / 62$ \\
\hline SECR & $07 / 25 / 62$ \\
\hline SECR & $06 / 01 / 62$ \\
\hline SECR & $06 / 01 / 62$ \\
\hline SECR & $07 / 25 / 62$ \\
\hline SECR & $08 / 06 / 62$ \\
\hline SECR & $08 / 13 / 62$ \\
\hline SECR & $08 / 13 / 62$ \\
\hline SECR & $08 / 15 / 62$ \\
\hline SECR & $08 / 20 / 62$ \\
\hline SECR & $08 / 29 / 62$ \\
\hline SECR & $08 / 28 / 62$ \\
\hline SECR & $07 / 01 / 62$ \\
\hline SECR & $09 / 06 / 62$ \\
\hline SECR & $09 / 14 / 62$ \\
\hline SECR & $09 / 12 / 62$ \\
\hline SECR & $09 / 10 / 62$ \\
\hline SECR & $09 / 10 / 6 ?$ \\
\hline
\end{tabular}

JE TRAVIS SECR

$\begin{array}{ccc}\text { SECR } & 07 / 06 / 62 \\ & \text { SECR } & 07 / 13 / 62 \\ \text { SECR } & 07 / 13 / 62 \\ \text { SECR } & 07 / 11 / 62 \\ \text { SECR } & 07 / 17 / 62 \\ \text { SECR } & 07 / 19 / 62 \\ & 07 / 20 / 62 \\ & \text { SECR } & \\ & \text { SECR } & 07 / 25 / 62 \\ & 06 / 01 / 62 \\ \text { SECR } & 06 / 01 / 62 \\ \text { FP BARAMOUSKI } & \text { SECR } & 07 / 25 / 62 \\ & \text { SECR } & 08 / 06 / 62\end{array}$

$08 / 13 / 62$

83/13/62

$08 / 15 / 62$

$3 / 29 / 62$

/28/62

$09 / 10 / 62$ 
Page No. 117

$04 / 28 / 93$

LIST OF HANFORD-GENERATED CLASSIFIED

DOCUMENTS DATED BETUEEN 1/1/61 AND 12/31/72

Doc. NO.

TITLE

AUTHOR

LEVEL PGS

DATE

HAN-82917

MONTHLY PRODUCTION REPORT - AUG 1962

REACTOR SAFETY: OPERATIONAL

SECR

$09 / 15 / 62$

HAN-82918

CHARGE-DISCHARGE

HAN-82999

PP REVIEW SHEET ADDITIONAL. PLUTONIUA

SECR

$09 / 14 / 62$

STORAGE FACILITIES 234-5 BLDG (CGC- 968 )

SECR

$09 / 24 / 62$

HAN-83066

HAPO AMMUAL OPERATING ESTIMATES -

SECR

$10 / 01 / 62$

HAN-83075

EXISTING PLAMTS

COOLING TOMERS ON HANFORD REACTOR

SECR

$10 / 01 / 62$

HAN-83084

EFFLUEMT LIMES

COMPARATIVE REACTOR PERFORMANCE - NUGUST

$08 / 01 / 62$

1962

HAN-83099

PP REVIEH SHEET COC-984 - ELECTRICAL \&

SECR

$10 / 04 / 62$

HYDRAULIC EOUIPNENT - RM LINE

HAN -83101

PP REVIEW SHEET CGC-984 - P INCOTING \&

SECR

10/04/62

HAN-83158

AUXILIARIED FACILITIES

MOWTHLY STATUS \& PROGRESS REPORT FOR SEP

SECR

$10 / 12 / 62$

1962

MAN-83174

MAN-83211

MAN-832111

HAN-83221

MONTHLY PROOUCTION REPORT - SEP 1962

REACTOR COOLING REO \& LAST DITCH COOLANT

$10 / 11 / 62$

SYSTEM CAPABILITIES

TABLE-LAST DITCH COOLANT SYSTEM

COMPARATIVE REACTOR PERFORMANCE - SEPT

1962

HAN-83252

HAN-83254

HAN-83256

HAN-83257

HAN-83277

HAN-83294

HAN-83332

REACTOR SITE MINIMUN COST STUDIES

MINIMUM COST STUDIES BUDCET STUDIES

MINIMUY COST STUDY SUIWURY OF V-E-CASE

A-1

(VE)

MINIMUN COST STUDY-BASE CASE A EXTENDED

HAPO PROOUCTION FORECAST 10/01/62 THRU

$6 / 30 / 64$

PRCOUCTION FORECAST 10/01/62 THRU

$6 / 30 / 64$

COMPILATION OF HH CP SS ACCOUNABILITY

SECR

$10 / 15 / 62$

SECR

$10 / 01 / 62$

SECR

$09 / 01 / 62$

SECR

$10 / 18 / 62$

SECR

$08 / 14 / 62$

SECR

$10 / 22 / 62$

SECR

10/22/62

SECR

$10 / 23 / 62$

SECR

$10 / 24 / 62$

OPERATION SS MATERIAL RECEIVED \& SHIPPED DECAY HEAT TEST AT DR REACTOR

CALCILM PROCUREMENT

MONTHLY STATUS \& PROGRESS REPORT FOR OCT 1962

HAN-83474

HAN-83476

HAN- 83513

HAN-83528

HAN-83592

HAN-83625

HAN-83677

HAN -83690

MONTHLY PRODUCTION REPORT - OCT 1962

ALTERNATE BUDGETS PROO DATA

COAPARATIVE REACTOR PERFORMANCE - OCT

1962

HANFORD WEAPON FACTSHEET KIT

COOLING TONERS ON HANFORD REACTOR

EFFLUENT LIMES

SHIPPING CONTAINER DROP TEST

MONTHLY STATUS \& PROGRESS REPORT FOR NOV

1962

MONTHLY PRCOUCTION REPORT - NOV 1962

PROOUCTION STUDIES - REACTOR SHUTDOWN DATA 
Page No. 118

$04 / 28 / 93$

DoC. NO.

HAN-83740

HAN-83797

HAN-83805RPT 1

HAN-83805RPT 10

HAN-83805RPT11

HAN-83805RPT 12

HAN-83805RPT2

HAN-8380JRPT3

HAN-83805RPT4

HAN-83805RPT5

HAN-83805RPT6

HAN-83805RPT7

HAN-83805RPT8

HAN-83805RPT9

HAN-83807

HAN-83854

HAN-83899

HAN-83901

HAN-83914

HAN-83915

HAN-83925

HAN-83974

HAN-8398601

HAN-8398602

HAN-8398603

HAN-8398604

HAN-8398605
TITLE

HA EXPLANATION OF ACCIDENTAL LOSSES NORMLL OPERATIONAL LOSSES, WRITE-OFFS

MUF

YEAR-END APPRAISAL REPORTS AND INSPECTIOA SCHEDULES

REPORTS AMD AMALYSIS BRANCH MONTHLY REPORT 1963

REPORTS AND ANALYSIS BRANCH MONTHLY REPORT 1963

REPORTS AND ANALYSIS SÃANCH MONTHLY REPORT 1963

REPORTS AND ANALYSIS BRANCH MONTHLY REPORT 1963

REPORTS AND AMALYSIS BRAMCH MONTHLY REPORT 1963

REPORTS ANO ANALYSIS BRANCH MONTHLY REPORT 1963

REPORTS AND ANALYSIS BRANCH MONTHLY REPORT 1963

REPORTS AND AMALYSIS BRANCH MONTHLY REPORT 1963

REPORTS AND ANALYSIS BRANCH MONTHLY REPORT 1963

REPORTS AND ANALYSIS BRANCH MONTHLY REPORT 1963

REPORTS AND ANALYSIS BRANCH MONTHLY REPORT 1963

REPONTS ANO ANALYSIS BRANCH MONTHLY REPORT 1963

COMPARATIVE REACTOR PERFORMANCE - NOV

1962

HANFORD WEAPON FACTSHEET KIT

HQ REQUESTS FOR P MIMIMM COST STLDIES

E-N LITHIUM REQUIREMENTS

MONTHLY PROOUCTION REPORT - DEC 1962

REACTOR SAFETY: REQUESTED FOR INCLUSION

OF MPR DEVELOPMENT POCRAM RESULTS DATA

ON SAFETY REVIEH AGENDA

MONTHLY STATUS \& PROERESS REPORT FOR DEC 1962

MATERIAL BALANCE REORT DECEMBER 1962

03 PROOUCTION \& DEVELOPMENT WEAPONS -

JAN THRU DEC 1963

03 PRCOUCTION \& DEVELOPMENT MEAPONS -

JAN THRU DEC 1963

03 PRODUCTION \& DEVELOPMENT MEAPONS JAN THRU DEC 1963

03 PRODUCTION \& DEVELOPNENT MEAPONS -

JAN THRU DEC 1963

03 PRCOUCTION \& DEVELOPMENT MEAPONS JAN THRU DEC 1963
AUTHOR

LEVEL PGS

DATE

SECR

$12 / 20 / 62$

SECR

$12 / 21 / 62$

SECR

$01 / 01 / 63$

SECR

$01 / 01 / 63$

SECR

01/01/63

SECR

$01 / 01 / 63$

SECR

$01 / 01 / 63$

SECR

$01 / 01 / 63$

SECR

$01 / 01 / 63$

SECR

$01 / 01 / 63$

SECR

$01 / 01 / 63$

SECR

$01 / 01 / 63$

SECR

01/01/63

SECR

$01 / 01 / 63$

SECR

$11 / 01 / 62$

SECR

$12 / 19 / 62$

01/03/63

$12 / 27 / 62$

$01 / 14 / 63$

01/07/63

SECR

SECR

$01 / 14 / 63$

SECR

$01 / 16 / 63$

SECR

02/01/63

SECR

03/01/63

SECR

$04 / 01 / 63$

SECR

05/01/63

SECR

06/01/63 
Page No. 119

$04 / 28 / 93$

LIST OF HAMFORD-GENERATED CLASSIFIED

DOCUMENTS DATED BETUEEN 1/1/61 AND 12/31/72

Doc. No.

HAN-8398606

HAN-8398607

HAN-8398608

HAN-8398609

HAN-8398610

HAN-8398611

HAN-8398612

HAN-83997

HAN-84009

HAN-84048

HAN-8410001

HAN-8410002

HAN -8410003

HAN-8410004

HAN-8410005

HAN-8410006

HAN-8410007

HAN-8410008

MAN-8410009

HAN-8410010

HAN-8410011

HAN -8410012

HAN-84140

HAH-84195

HAN -84202

HAN-84235
TITLE

O3 PRODUCTION \& DEVELOPHENT MEAPONS JAN THRU DEC 1963

03 PROOUCTION \& DEVELOPMENT MEAPOHS -

JAN THRU DEC 1963

03 PRODUCTION \& DEVELOPNENT VEAPONS JAN THRU DEC 1963

O3 PRODUCTION \& DEVELOPNENT MEAPONS JAN THRU DEC 1963

03 PROOUCTION \& DEVELOPMENT MEAPONS -

JAN THRU DEC 1963

03 PRCDUCTION \& DEVELOPNENT MEAPONS JAN THRU DEC 1963

03 PROOUCTION \& DEVELOPNENT MEAPONS JAN THRU DEC 1963

COAPARATIVE REACTOR PERFORMANCE - DEC 1962

EXPLAMATIONS OF AL, MOL, W-O, MUF AND ADJUSTMENTS

ANALYSIS OF MASHINGTON REVISED COST

ESTIMATES FOR FY 1965 AND FY 1966

MONTHLY PRCOUCTION REPORT JAN THRU DEC 1963

MONTHLY PRODUCTION REPORT JAN THRU DEC 1963

MONTHLY PRODUCTION REPORT JAN THRU DEC 1963

MONTHLY PRODUCTION REPORT JAN THRU DEC 1963

MONTHLY PRODUCTION REPORT JAN THRU DEC

1963

MONTHLY PROOUCTION REPORT JAN THRU DEC

1963

MONTHLY PRODUCTION REPORT JAN THRU DEC

1963

MONTHLY PRODUCTION REPORT JAN THRU DEC

1963

MONTHLY PRCOUCTION REPORT JAN THRU DEC 1963

MONTHLY PRCOUCTION REPORT JAN THRU DEC

1963

MONTHLY PRCOUCTION REPORT JAN THRU DEC

1963

MONTHLY PRCOUCTION REPORT JAN THRU DEC

1963

FORECAST OF LARGE SCALE RADIOISOTOPES

REQUIREMENTS \& PROO PLANHIMG

HAPO PRODUCTION FORECAST 1/01/63 THRU

$6 / 30 / 68$

PRODUCTION FORECAST 1/01/63 THRU 6/30/68

MONTHLY STATUS \& PROGRESS REPORT FOR JAN 1963
AUTHOR

LEVEL

PGS

DATE

SECR

07/01/63

SECR

$08 / 01 / 63$

SECR

$09 / 01 / 63$

SECR

$10 / 01 / 63$

SECR

$11 / 01 / 63$

SECR

12/01/63

SECR

$01 / 01 / 64$

SECR

12/01/62

SECR

$01 / 21 / 63$

SECR

$11 / 30 / 62$

SECR

$02 / 14 / 62$

SECR

$03 / 14 / 62$

SECR

$04 / 14 / 62$

SECR

05/14/62

SECR

$06 / 14 / 62$

SECR

$07 / 14 / 62$

SECR

$08 / 14 / 62$

SECR

$09 / 14 / 62$

SECR

$10 / 14 / 62$

SECR

$11 / 14 / 62$

SECR

$12 / 14 / 62$

SECR

$01 / 14 / 63$

SECR

$01 / 10 / 63$

SECR

$01 / 31 / 63$

SECR

$02 / 06 / 63$

SECR 
Page No. 120

$04 / 28 / 93$

LIST OF HANFORD-GENERATED CLASSIFIED

DOCUMENTS DATED BETWEEN 1/1/61 AND 12/31/72

DOC. NO.

HAN-84269

HAN-84304

HAN-84308

HAN-84408

HAN-84443

HAN -84448

MAN -844481

HAN-84453

HAN -84465

HAN -84500

HAN-84523

HAN-84547

HAN-84580

HAN-84625

HAN -84639

HAN -84640

HAN -84658

HAN- 84686

HAN -84700

HAN-84757

HAN-84772

HAN -847721

HAN-84826

HAN-84858

HAN-84921

HAN-84955

HAN-84982

HAN-85001

HAN-85002

HAN-85087

HAN-85097

HAN-85098
TITLE

NPR TRITIUM PRODUCTION

COMPARATIVE REACTOR PERFormanCE - JAN

1963

DETERMINATION OF VEAPON-GRADE PLUTONIUM

BID-ASSAY SAHPLES

PLUTOHIUN BLENDING 9

HAMFORD IRRADIATION RECUEST

PRCOUCTION DIVISION WORK PAPERS FOR

MINIMUN COST STLDIES

PRODUCTION SUMMURY CASES (WORK PAPERS)

FIVE YEAR ELECTRICAL POUER FORECAST

PROCESS LOAD HANFORD ATONIC PRODUCT

OPERATION

REACTOR SITE MINIIUM COST STUDIES

MONTHLY STATUS \& PROGRESS REPORT FOR FEB

1963

PROOUCTION STUDIES - EVALUATION

PERSOMAL NOTE BOOK OF P.A. CRAIG

COMPARATIVE REACTOR PERFORMAMCE - FEB

1963

P PROOUCTION FOR THE VARIOUS MINIMUM COST CASES

RL MINIMUM COST STUDY CASES

MINIMUM COST STUDY CASES AT SV \& RL

AMERICIUM RECOVERY FOR CURIUN PRCOUCTION

BRAMDYHINE STUDY

HANFORD'S CONTRIBUTION TO RIVER HATER

TEMPERATURES

MONTHLY STATUS \& PROGRESS REPORT FOR MAR

1963

U-233 PROGRAM PROPOSAL

REDUCED SCOPE U-233 PROGRAM

COAPARATIVE REACTOR PERFCRMANCE - MARCH

1963

WEAPONS RED SS MATERIAL PROJECTS

HAPO PRODUCTION FORECAST 4/01/63 THRU

$12 / 31 / 64$

TRANSMITTAL PRODUCTION FORECAST 4/01/63

THRU 12/31/64

PUREX-REDOX OPERATION STLDY

MONTHLY STATUS \& PROGRESS REPORT FOR APR

1963

MEAPONS FABRICATION FLON CHART (PHOTO

MUMBER G-122-250)

COMPARATIVE REACTOR PERFORMANCE - APRIL

1963

SUMMARY OF PROOUCTION AND COST EXCLUDING DEPRECIATION

DETAILS OF PRODUCTION AND COSTS

EXCLLDING DEPRECIATION
AUTHOR

LEVEL PGS

DATE

RL PLUM

SECR

$02 / 14 / 63$

SECR

SECR

$02 / 19 / 63$

SECR

CONF

SECR

02/27/63

03/06/63

03/05/63

SECR

SECR

03/07/63

$03 / 04 / 63$

SECR

SECR

SECR

SECR

SECR

SECR

SECR

SECR

SECR

SECR

SECR

SECR

SECR

SECR

SECR

SECR

SECR

SECik

SECR

SECR

SECR

SECR

SECR

SECR
$05 / 02 / 63$

$02 / 26 / 63$

$03 / 13 / 63$

03/15/63

$03 / 19 / 63$

03/20/63

$03 / 21 / 63$

03/01/63

02/27/63

$03 / 29 / 63$

$03 / 28 / 63$

$04 / 02 / 63$

$04 / 11 / 63$

$04 / 11 / 63$

$04 / 11 / 63$

03/01/63

$04 / 16 / 63$

$04 / 29 / 63$

05/02/63

$04 / 26 / 63$

05/13/63

05/09/63

$04 / 01 / 63$

05/02/63 
DoC. Mo.

HAN-85099

HAN-85197

HAN -85205

HAN-85234

HAN-85234?

HAN-85278

HAN-852781

HAN-852782

HAN-85280

HAN -85281

HAN-85282

MAN-85283

HAN-85306

MAN-853061

HAN-85318

HAN-85338

HAN -85339

HAN -85340

HAH -85344

HAN-85376

HAN-85383

HAN-83386

HAN-85392

HAN-85403

HAN-85414

HAN -85415

HAN -85440

HAN- 85483

HAN -85493

HAN-85508

HAN-85522

HAN-8554

HAN-85552
TITLE

PRODUCTION COST ESTIMATES

PRODUCTION STUDIES

MERICIUN RECOVERY STUDY

REPORT OF MEETING OF REACTOR COST TASK

FORCE GERMANTOW MAY 23, 1963

SCHEDULE

REPORT OF REACTOR COST TASK FORCE

DisETING

REPORT OF REACTOR TASK FORCE MEETING MAY

25, 1963

TASK FORCE ASSUMPIIONS AND OBJECTIVES

REACTOR COST STUDY

SCHEDULE MO. TO BE SUPPLIED TO EACH

\section{REACTOR}

SUPPL SCHEDULE EACH SITE SULAIT ONE

REPCRT TO EACH CASE 300 AREA

SUPPL SCHEDULE B EACH SITE SUBMIT ONE

REORT TO EACH CASE 200 AREA

REACTOR COST TASK FORCE

COSTING PLUTONIUM CHARGED TO CURIUM

PROGRAM

MONTHLY STATUS \& PROGRESS REPSA.T FOR MAY 1963

REACTOR COST STUOY

REACTOR COST STUDY

SUPPORTING AND SUPPLEMENTAL DATA FOR

REACTOR COST STLDY

REPORT OF REACIOR COST TASK FORCE

MEETING

UNCLASSIFIEC P PRODUCTION

PROCUREMENT PROURAM FOR PROCESS MEAT

EXCHANGES

COMPARATIVE REACTOR PERFormanCe - maY

1963

PROBLEMS CONTRIBUTING TO REDUCED

MEPTUNIUM SHIPMENT FY 1963

REPORT OF REACTOR COST TASK FORCE

MEETING

REACTOR CASE STUOY ELECTRIC PONER

LEVEL

PGS

DATE

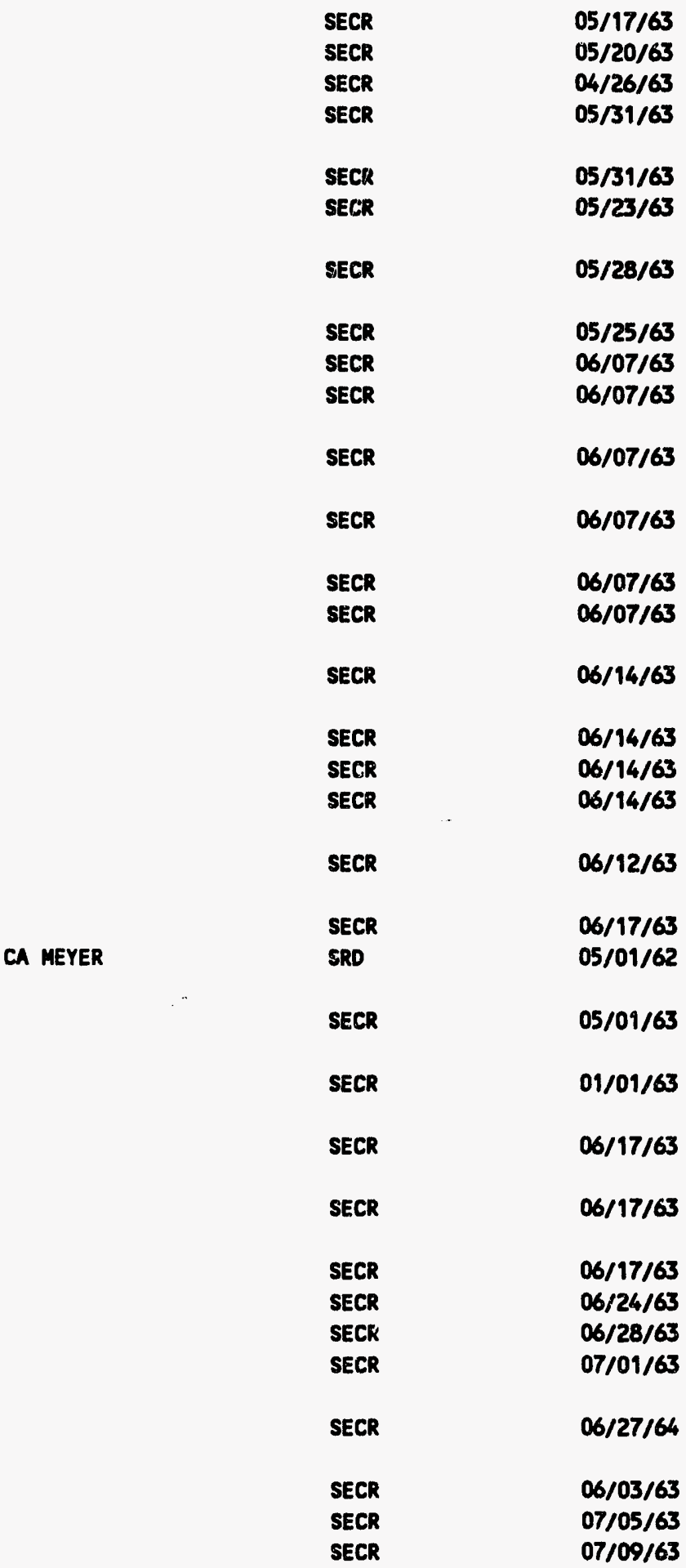


Page Ho. 122

$04 / 28 / 93$

LIST OF HANFORD-GENERATED CLASSIFIED

DOCLMENTS DATED BETWEEN 1/1/61 AND 12/31/72

DOC. NO.

TITLE

AUTHOR

LEVEL

PGS

DATE

HAN-85578

HAN-85581

HAN-85588

HAN-85599

HAN-85600

HAN-85615

HAN-85622

HaN-85624

HAN-85627

HAN -85627

HAN-85629

HAN-85630

HAN-85646

HAN-85647

HAN-85647

HAN-85656

HAN-85706

HAN-85716

HAN-85739

HAN-85756

HAN-85759

HAN-85769

HAN-85772

HAN-85778

HAN-85809

MAN-85811

HAN-85812

HIN-85813

HAN-85814

HAN-85815

HAN-85847

HAN -85850

HAN-85855

HAN-85855 1

HAN-85865
MONTHLY STATUS \& PROGRESS REPORT FOR JUN 1963

REPORT OF REACTOR SITE TASK FORCE

MEETING - HQS

QAP $N-10$ SURVEY

REACTOR COST STUDIES

REACTOR ADVANTAGES

MATERIAL BALANCE REPORT JUNE 1963

MONTHLY PRODUCTION REPORT JUN 1963

PP: DEGREASING \& BRIQUETTING FACILITY

RM LINE - 234-5 BLDG (CAC-109)

PUREX-REDOX OPERATION STLDY

PUREX-REDOX OPERATION STUDY

CONSOLIDATION OF MATERIAL BALANCE REPORT GRO JUNE 1963

EXPLANATION OF AL, NOL, H-OS AND MUF-MBR FOR JUNE 1963 3

REACTOR COST STLDY - GROUP II AND III

TRAMSMITTAL OF REACTOR SITE COST STUDIES

REACTOR SITE COST STUDY

REACTOR COST STLDY QUESTIONS ON PRIORITY

I CASES

REACTOR COST STUDIES

HAPO PRODUCTION FORECAST 7/01/63 THRU

$6 / 30 / 68$

PRCOUCTION FORECAST 7/01/63 THRU 6/30/68

REACTOR COST STUDY PRIORITY GROUP IV

10 YEAR REACTOR STLDY SUPPLEMENT

PRIORITY CASES - MEPTUNIUN 237

PROOUCTION

REACTOR COST STLOY ESTIMATED SUPPORT OF

PERSONMEL BY AEC PROGRAM CLASS

AUDIT OF CASTING OPERATION

COST STUDY REPORT

TEN YEAR REACTOR STUDY SUPPL. PRIORITY

11, III, IV CASES NEPTUNILM 237

PRCOUCTION

SUP. 3 NEH CAPITAL EQUIPMENT ITEMS.

REACTOR SITE STUDY - SUPPLEMENT 4

CASE $X$ SUMMARY AND 100 AREAS ANMUAL

COSTS, EXCLUDING DEPRECIATION

CASE 2 SUPPL. 6

SUPPL. 7 CASE 5

QAIA QUALITY REPORT FOR JULY 1963

MONTHLY STATUS \& PROGRESS REPORT FOR JUL 1963

UMCLASSIFIED P PRODUCTION CYOUR MEMO 22/63)

AMALYSIS \& COMMENTS OF DOC HW-77675

APPENDIX "C" PROOUCTION CAPABILITY
SECR

$07 / 12 / 63$

SECR

$07 / 10 / 63$

SECR

SECR

SECR

SECR

JE TRAVIS

SRD

SECR

SECR

SECR

SECR

SECR

SECR

SECR

SECR

SECR

SECR

SECR

SECR

SECR

SECR

SECR

$07 / 31 / 63$

SECR

SECR

SECR

$07 / 31 / 63$

08/01/63

$08 / 06 / 63$

SECR

$08 / 06 / 63$

SECR

SECR

SECR

SECR

SECR

SECR

SECR

08/06/63

08/06/63

08/06/63

08/06/63

08/09/63

$08 / 13 / 63$

$08 / 12 / 63$

SECR

$08 / 12 / 63$

08/07/63 
Page No. 123

$04 / 28 / 93$
LIST OF HAMFORD-GENERATED CLASSIFIED DOCUMENTS DATED BETWEEN 1/1/61 ANR 12/31/72
DOC. NO.

HAN-85866

HAN-85867

HAN-85868

HAN-85869

HAN-85885

HAN-85926

HAN-85950

HAN-85952

HAN-85969

HAN-85970

HAN-85971

HAN-85996

HAN-86008

HAN-86009

HAN-86010

HAN-86011

HAN-86015

HAN-86021

HAN -86025

HAN-86038

HAN -86060

HAN-86061

HAN-86098

HAK-86106

HAN-86107

HAN-86126

HAN-86130

HAN-861301

HAN-861302

HAN -861303

HAN-86146

HAN-86170

HAN -86204

HAN-86206

HAN-86209

HAN-86226
TITLE

AUTHOR

APPENDIX "E" COAPARISON OF REACTORS

APPENDIX "H" TRITIUN PROOUCTION

$07 / 31 / 63$

CAPABILITY

APPENDIX "J" NEW PRCOUCTION REACTOR

APPENDIX "K" MAINTENANCE OF SHUTDOWN

REACTORS

HEADQUARTER DISCUSSION 8-12-63 ON

REACTOR COMPARISON

MEAPONS MANUFACTURING OPERATION PRODUCT

SPECIAL PROOUCT STUDY - N REACTOR

REACTOR SHUTDON STUDY

DRAFT APPENDIX "C" PRCOUCTION CAPABILITY

GRAPH - FLUX SPECTRUN WITHIN FUEL

REACTOR COST STUDY

P -210 PRODUCTION

MEMORAMDUM PREPARED AT THE REQUEST OF

COWISSIONER J.T. RAMEY

REACTOR COST STUDY

RLO COMMENTS ON DRAFT APPENDIX "B"

COMPARISON OF REACTORS

RLO COMMENTS ON APPENDIX J NEW

PROOUCTION REACTOR

PROOUCTION STUDIES - N REACTOR

REACTOR COST STUDY SR UNIT COSTS

REACTOR COST STUDIES

REACTOR COST STUDIES

COMPARATIVE REACTOR PERFORMANCE - JUME

1963

COMPARATIVE REACTOR PERFORMANCE - JULY

1963

REACTOR COST STUDIES

MONTHLY STATUS \& PROGRESS REPORT FOR AUG

1963

U-233 PRCOUCTION

QAIA QUALITY REPORT FOR AUGUST 1963

SPECIFICATIONS FOR 1807 MEMBRANE

COMPONENTS-DRHG UC-251918 SHEET 8

SHEET 9 DRAHING UC 231918-GEMERAL

SPECIFICATIONS FOR 1807 MEMBRAME

COMPONENTS

INSPECTION SHEET U-205

INSPECTION SHEET U-206

REACTOR COST STUDY - CASE 24

REACTOR COST STUDIES

REACTOR COST STUDY - CASE 23 - FY 1967

COMPARATIVE REACTOR PERFORMANCE - AUG

1963

PU-NITRATE UNIT COST

HANFORD COST STUDIES
SECR

SECR

SECR

SECR

SECR

SECR

LEVEL PGS DATE

SECR

SECR

SECR

SECR

SECR

SECR

SECR

SECR

SECR

SECR

SECR

SECR

SECR

SECR

SECR

SECR

SECR

SECR

SECR

SECR

SECR

SECR

SECR

SECR

SECR

SECR

SECR

SECR

SECR
$07 / 31 / 63$

$08 / 07 / 63$

08/06/63

$08 / 15 / 63$

$07 / 30 / 63$

$08 / 16 / 63$

$08 / 12 / 63$

$09 / 23 / 63$

$08 / 23 / 63$

$08 / 23 / 63$

$08 / 23 / 63$

$08 / 22 / 63$

$08 / 01 / 63$

$08 / 28 / 63$

$08 / 28 / 63$

$08 / 26 / 63$

$08 / 28 / 63$

$08 / 29 / 63$

$08 / 30 / 63$

$06 / 01 / 63$

07/01/63

09/09/63

$09 / 13 / 63$

$09 / 10 / 63$

$09 / 12 / 63$

$09 / 13 / 63$

$09 / 13 / 63$

$09 / 13 / 63$

$09 / 13 / 63$

$09 / 12 / 63$

$09 / 17 / 63$

$09 / 19 / 63$

08/01/63

$09 / 19 / 63$

$09 / 19 / 63$ 
Page No. 124

$04 / 28 / 93$

LIST OF HANFORD-GENERATED CLASSIFIED

DOCUMENTS DATED BETMEEN 1/1/61 AND 12/31/72

DOC. No.

TITLE

AUTHOR

LEVEL

PGS

DATE

ADDITIONAL SEPT 1963 COST AND PROOUCTION

SECR

$09 / 18 / 63$

HAN-86237

HAN-862371

HAN-862372

HAN -86248

HAN-86261

HAN-86315

HAN-86318

HAN-86326

STUDIES

REACTOR COST STUDIES

SECR

$09 / 23 / 63$

EXHIBIT

EXHIBIT II

DESIGN SCOPE DEGREASING \& BRIOUETTING

SECR

$09 / 23 / 63$

$09 / 23 / 63$

FACILITY - RMA FABRICATION LINE 234-5

SECR

$08 / 13 / 63$

BLDG (CAC-109)

UNCLASSIFIED $P$

LI-AL TARGETS

DESIGN SCOPE ADDITIONAL P CASTING

$09 / 27 / 63$

$10 / 02 / 63$

FACILITIES - RMA LINE PP CAC-110 - 234-5

SECR

$09 / 10 / 63$

BLDG

SPECIFICATION \& WORKING DRAUINGS FOR

FABRICATED P METAL

HAN-86333

U-233 PROOUCTION STUDIES

SECR

$10 / 03 / 63$

HAN -86337

HAN-86338

HAN-86359

REACTOR COST STUDIES

REACTOR COST STUDIES

MONTHLY STATUS \& PROGRESS REPORT FOR SEP

1963

HAN-86364

HAN-86365

PP: REVIEW SHEET FOR CAC-109

PP REVIEH SHEET FOR CAC-110

DESIGN SCOPE METAL STABILIZATION 234-5

BLDG (CAC-987)

QAIA QUALITY REPORT FOR SEPT 1963

REACTOR COST STUDIES

N REACTOR DEPARTMENT MPR REACTOR COST

STUDIES

COMPARATIVE REACTOR PERFORMANCE - SEPT

1963

MPR REACTOR COST STUDIES

REACTOR COST STUDY

LI-AL TARGETS

SUMYARY OF UNIT COSTS EXCLLDING

DEPRECIATION

DETAILS OF FY 1963 REACTOR OPERATING

COSTS, UNIT COSTS ANO PRODUCTION

PRODUCTION STUDIES - N REACTOR CASES 22

SECR

SECR

SECR

SECR

$10 / 04 / 63$

$10 / 04 / 63$

$10 / 04 / 63$

$10 / 11 / 63$

AND 22A

REACTOR COST STUDIES

HAN- 86498

PROOUCTION OF SPECIAL NUCLEAR MATERIALS

SECR

SECR

$11 / 09 / 63$

$10 / 09 / 63$

SECR

$09 / 18 / 63$

SECR

$10 / 10 / 63$

SECR

$10 / 14 / 63$

SECR

$10 / 14 / 63$

SECR

09/01/63

SECR

$10 / 16 / 63$

SECR

$10 / 18 / 63$

SECR

10/18/R3

SECR

$10 / 18 / 63$

SECR

$10 / 18 / 63$

SECR

$10 / 18 / 63$

IN N-REACTOR

HAPO PROOUCTION FORECAST 10/01/63 THRU

6/30/65

ADJUSTMENT OF RATE OF PLUTONIUM

PRODUCTION AT HANFORD

U-233 DEVELOPMENT PROGRAM

CO-PRCOUCT DEMONSTRATION - N-REACTOR

TRANSHITTAL PRCOUCTION FORECAST 10/01/63 THRU 6/30/65

SECR

SECR

SECR

SECR

SECR

SECR

SECR
$10 / 22 / 63$

$10 / 21 / 63$

$10 / 25 / 63$

$10 / 25 / 63$

$10 / 25 / 63$

$10 / 20 / 63$

$10 / 25 / 63$ 
DOC. NO.

HAN- 86600

HAN -86603

HAN -86604

HAN-86613

HAN -86648

HAN-86662

HAN-86679

HAN-86695

HAN-86721

HAN-86749

HAN-86767

HAN-86785

HAN-86786

HAN-86787

HAN-86821

HAN-86842

HAN- 86865

HAN-86867

HAN-86868

HAN-86897

HAN-86903

HAN-86959

HAN -869591

HAN -86970

HAN-87001

HAN-870012

HAN -870013

HAN-87007

HAN-87021

HAN -87036

HAN -87048

HAN-87102RPT1

HAN-87102RPT10

HAN-87102RPT11

HAN-87102RPT12
TITLE

REACTOR COST STLDY

MANUFACTURING UNIT COST DATA FOR

HW-HW79375

PUREX-REDOX OPERATIONS STUDY

REACTOR COST STUDIES

HANFORD DIVERSIFICATION PROGRAN

ONE PAGE REVIEH PROPOSAL CAC-115 - RMA

LIME 234-5 BLDG

EXHIBIT "G" BPA-AEC-STUDIES

MOWTHLY STATUS \& PROGRESS REPORT FOR OCT

1963

U-233 DEVELOPMENT PLANS FY-1964 \&

FY- 1965

REACTOR PRODUCTION COST DATA

COMPARATIVE REACTOR PERFORMANCE - OCT

1963

REACTOR COST STUDIES

QAIA QUALITY REPORT FOR OCT 1963

U-233 PROOUCTION STLDIES

FEASIBILITY OF REDOX PLANT SHUT DOW

H REACTOR E-N PROOUCTION COSTS

COST OF HIGH PLUTONIUH

FULL COST OF SEGREGATING A BATCH OF $P$

OF UNUSUAL $P-240 \%$

UNIT COST OF $P$ FROM NATURAL URANIUM

OPERATING LIMITS MANFORD PROOUCTION

REACTORS

COST OF MACHINING THORIUM CORES

AUDIT OF PROCESS RUN CARDS, SEPT AMD OCT 1963 MC 1807 PROGRAM

LIST OF RUN CARDS

MONTHLY STATUS \& PROGRESS REPORT FOR MOV

\section{3}

COST OF HIGH 240 PLUTONIUA

FULL COST OF SEGREGATING A BATCH OF PU

OF UNUSUAL PU-240\%

FULL UNIT COST OF PU FROM MATURAL U

QAIA QUALITY REPORT FOR NOV 1963

COST OF THORIUN CORES

BALANCE REPORTS

COMPARATIVE REACTOR PERFORMANCE - NOV

1963

MONTHLY PRODUCTION REPORT JAMUARY THRU

DECEMBER 1964

MONTHLY PROOUCTION REPORT JANUARY THRU DECEMBER 1964

MONTHLY PROOUCTION REPORT JAMUARY THRU DECEMBER 1964

MONTHLY PROOUCTION REPORT JANUARY THRU DECEMBER 1964

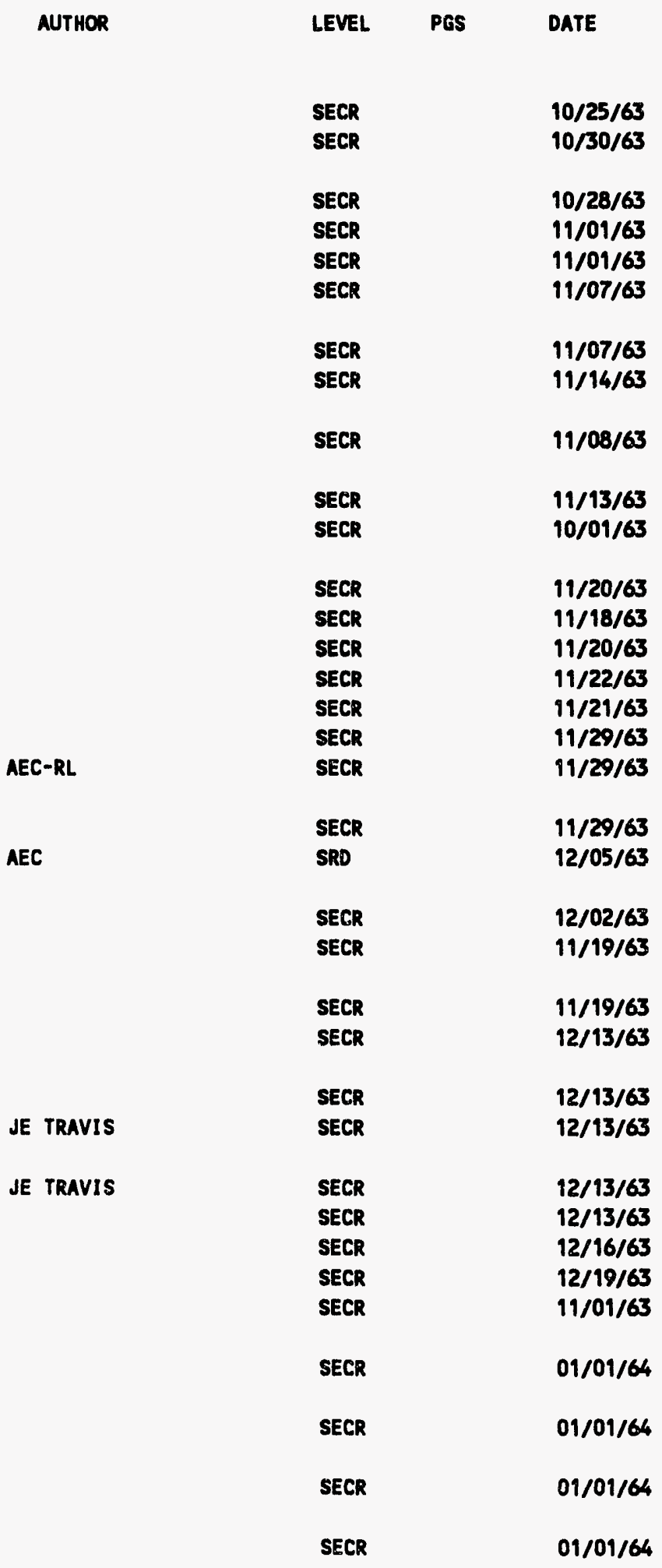


Page No. 126

$04 / 28 / 93$

LIST OF HANFORD-GENERATED CLASSIFIED

DOCUMENTS DATED BETUEEN 1/1/61 AND 12/31/72

DOC. NO.

HAN-87102RPT2

HAN-87102RPT3

HAN-87102RPT4

HAN-87102RPTS

HAN-87102RPT6

HAN-87102RPT7

HAN-87102RPT8

HAN-87102RPT9

HAN-87118

HAN -87183

HAN-87199

HAN-87228

HAN-87229

HAN-8724801

HAN-8724802

HAN -8724803

HAN -8724804

HAN-8724805

HAN -8724806

HAN-8724807

HAN-8724808

HAN-8724809

HAN -8724810

HAN-8724811

HAN-8724812

HAN-87271

HAN-87295
TITLE

MONTHLY PROOUCTION REPORT JAMUARY THRU DECEMBER 1964

MONTHLY PROOUCTIOM REPORT JAMUARY THRU DECEMBER 1964

MONTHLY PRODUCTION REPORT JAMUARY THRU DECEMBER 1964

MONTKLY PRODUCTION REPORT JANUARY THRU DECEPABER 1964

MONTHLY PROOUCTION REPORT JAFUARY THRU DECEMBER 1964

MONTHLY PROOUCTION REPORT JANUARY THRU DECEMBER 1964

MONTHLY PROOUCTION REPORT JANUARY THRU DECEMBER 1964

MONTHLY PRCOUCTION REPORT JAMUARY THRU DECEMBER 1964

REACTOR COST STUDIES

PROOUCTION STUDIES-N REACTOR CASES 22 AND 22A - ADDENDUM

URANIUM INVENTORY WEEKLY REPORT - JAN THRU DEC 1964

MONTHLY STATUS \& PROGRESS REPORT FOR DEC 1963

QAIA QUALITY REPORT FOR DEC 1963

03 PROOUCTION \& DEVELOPNENT IEAPONS -

JAN 1964

03 PRCOUCTION \& DEVELOPMENT WEAPONS -

FEB 1964

03 PROOUCTION \& DEVELOPMENT MEAPONS -

MAR 1964

03 PROOUCTION \& DEVELOPMENT MEAPONS -

APR 1964

03 PRODUCTION \& DEVELOPNENT MEAPONS -

MAY 1964

03 PROOUCTION \& DEVELOPMENT WEAPONS -

JUN 1964

03 PROOUCTION \& DEVELOPNENT MEAPONS -

JUL 1964

03 PROOUCTION \& DEVELOPMENT MEAPONS -

AUG 1964

03 PRODUCTION \& DEVELOPMENT VEAPONS -

SEP 1964

03 PRODUCTION \& DEVELOPMENT MEAPONS -

OCT 1964

03 PRODUCTION \& DEVELOPMENT MEAPONS -

NOV 1964

03 PROOUCTION \& DEVELOPMENT MEAPONS -

DEC 1964

MATERIAL BALANCE REPORT DEC 1963

CONSOLIDATION OF RL MATERIAL BALANCE

REPORT FOR DECEMBER 1963
AUTHOR

SECR

$01 / 01 / 64$

SECR

$01 / 01 / 64$

SECR

$01 / 01 / 64$

SECR

$01 / 01 / 64$

SECR

$01 / 01 / 64$

SECR

$01 / 01 / 64$

SECR

01/01/64

SECR

01/01/64

SECR

SECR

$12 / 31 / 63$

$12 / 18 / 63$

SECR

$01 / 08 / 64$

SECR

$01 / 14 / 64$

SECR

SECR

$01 / 13 / 64$

$02 / 01 / 64$

SECR

03/01/64

SECR

$04 / 01 / 64$

SECR

$05 / 01 / 64$

SECR

$06 / 01 / 64$

SECR

$07 / 01 / 64$

SECR

08/01/64

SECR

$09 / 01 / 64$

SECR

$10 / 01 / 64$

SECR

$11 / 01 / 64$

SECR

$12 / 01 / 64$

SECR

$01 / 01 / 65$

SECR

$01 / 16 / 64$

SECR 
Page No. 127

04/28/93

LIST OF HANFORD-GENERATED CLASSIFIED

DOCUMENTS DATED BETUEEN 1/1/61 AND 12/31/72

Doc. no.

TITLE

AUTHOR

LEVEL

PGS

DATE

HAN-87296

HAN -87380

HAN-87392

HAN-873921

HAN-87432

HAN -87441

HAN-87483

HAN-87501

HAN-87505

HAN-87508

HAN-8752001

HAN-8752002

HAN-8752003

HAN-8752004

HAN-8752005

HAN-8752006

HAN-8752007

HAN -8752008

HAN -8752009

HAN-8752010

HAN-8752011

HAN-8752012

HAN-87561

HAN-87592

HAN-87603

HAN-87637

HAN-87674

HAN-87703

HAN-87723

HAN-87744
EXPLAMATION OF AL, MOL, MUFS AND

ADJUSTMENTS FOR DECEABER 1963

COMPARATIVE REACTOR PERFORMANCE - DEC

1963

SELECTION OF REACTORS FOR SHUTDOWN

REACTOR SHUTDOWN CONSIDERATIONS

U-233 PROOUCTION CAPABILITY

AUDIT OF HATERIAL CERTIFICATION CHEMICAL

AND PHYSICAL PROPERTIES MC 1807 PROGRAM

QAIA OUALITY REPORT FOR JAMUARY 1964

SUMmARY OF CLEAN U-233 PROGRAM

HAPO PROOUCTION FORECAST 1/01/64 THRU

6/30/69

MONTHLIY STATUS \& PROGRESS REPORT FOR JAN

1964

MONTHLY PROOUCTION REPORT JAM THRU DEC

1964

MONTHLY PROOUCTION REPORT JAN THRU DEC

1964

MONTHLY PROOUCTION REPORT JAN THRU DEC

1964

MONTHLY PRCOUCTION REPORT JAN THRU DEC

1964

MONTHLY PRCOUCTION REPORT JAN THRU DEC

1964

MONTHLY PROOUCTION REPORT JAN THRU DEC

1964

MONTHLY PROOUCTION REPORT JAN THRU DEC

1964

MONTHLY PRODUCTION REPORT JAN THRU DEC

1964

MONTHLY PRODUCTION REPORT JAN THRU DEC

1964

MONTHLY PRODUCTION REPORT JAN THRU DEC

1964

MONTHLY PROOUCTION REPORT JAN THRU DEC

1964

MONTHLY PROOUCTION REPORT JAN THRU DEC

1964

NM PROOUCTION REPORT - JAN 1964

LOADING \& OPERATING CONDITIONS FOR PT IP

629-D-NAM $1151 \& 2$ HIGH TEMPERATURE

TRITIU: SHIPMENT FORECAST

TREATMENT OF SHUTDOWW REACTORS

GALLIUM REQUIREMENTS - FY 1965 AND 1966

REVISED OPERATING LIMITS - HANFORD

PROOUCTION REACTORS

AUDIT OF CERTIFICATION FOR DENSITY

MEASUREMENTS MC 1807 PROGRAM

GRAPHITE BURNOUT
SECR

SECR

SECR

SECR

SECR

SECR

SECR

SECR

SECR

SECR

SECR

SECR

SECR

SECR

SECR

SECR

SECR

SECR

SECR

SECR

SECR

SECR

SECR

SECR

SECR

SECR

SECR

JE TRAVIS

CONF

SECR

SECR

GT ORTON
$01 / 21 / 64$

$12 / 01 / 63$

$01 / 30 / 64$

$01 / 30 / 64$

02/03/64

$01 / 29 / 64$

$02 / 10 / 64$

$02 / 10 / 64$

$02 / 11 / 64$

$02 / 14 / 64$

$02 / 14 / 64$

$03 / 14 / 64$

$04 / 14 / 64$

$05 / 91, / 64$

$06 / 14 / 64$

$07 / 94 / 64$

$08 / 14 / 64$

$09 / 14 / 64$

$10 / 14 / 64$

$11 / 14 / 64$

$12 / 14 / 64$

$01 / 14 / 65$

$02 / 11 / 64$

$01 / 27 / 64$

$02 / 20 / 64$

$02 / 24 / 64$

$02 / 25 / 64$

03/03/64

$02 / 26 / 64$ 
Page No. 128

$04 / 28 / 93$

LIST OF HANFORD-GENERATED CLASSIFIED

DOCLMENTS DATED BETWEEN 1/1/61 AND 12/31/72

DOC. NO.

HAN-8T771

HAN-8TT72

HAN-87785

HAN-87793

HAN-87795

HAN-87861

HAN-87869

HAN-8786901

HAN-8786902

HAN -87979

HAN-879791

HAN -87980

HAN-88006

HAN-88020

HAN-88057

HAN-88063

HAN-880631

HAN -88070

HAN-88115

HAN-88176

HAN-88262

HAN-882621

HAN-88272

HAN-88287

HAN-88299

HAN-88312

HAN-88324

HAN-883304

HAN-883305

HAN-88356

HAN -88358

HAN-883581

HAN-883582

HAN-88364 HAN-88365
TITLE

NM PROOUCTION REPORT - FEB 1964

COMPARATIVE REACTOR PERFORMANCE - JAN

1964

MONTHLY STATUS \& PROGRESS REPORT FOR FEB

1964

PUREX PLANT CAPABILITY REQUIREMENTS

ROUTINE PRODUCTION OF U-233

QAP $N-10.0$ SURVEY REPORT

REVISION OF GE COMPANY FORECAST

CHART - FIGURE 1

CHART - FIGURE 2

QAI REPORT FOR FEBRUARY 1964

APPENDIX I

ASSURIHG THAT REACTORS ARE SHUT DOWN

COMPARATIVE REACTOR PERFORMANCE - FEB

1964

AUDIT OF RADIOGRAPHY OPERATIONS 74-C PROCRAM

NM PRODUCTION REPORT - MAR 1964

QAIA REPORT QUALITY REPORT FOR MARCH

1964

APPENDIX I

MONTHLY STATUS \& PROGRESS REPORT FOR MAR

1964

REVS TO SPECS FOR URANIUM METAL BILLETS

FOR MPR FUEL ELEMENTS

EXCESS STARTUP REACTIVITY - H REACTOR

SHUTDOWN OF RL REACTORS

ATTACHMENT SHUTDOWN OF THREE HANFORD

REACTORS

EXCESS STARTUP IN H REACTOR - 4/24/64

HAPO PROOUCTION FORECAST 4/01/64 THRU

12/31/65

INTERIM SUWWARY REPORT POWER RECOVERY

STUOY EXISTING SMALLER MANFORD REACTORS

COMPARATIVE REACTOR PERFORMANCE - MARCH

1964

ROUTINE PRODUCTION OF U-233

DATA SHEET U-205

DATA SHEET U-206

INVITATION FORM PROPOSALS MANFORD

FISSION PRODUCT RECOVERY REPORT AND

OPERATION OF 200 AREA FACILITIES

REACTOR SHUTDOWN

ATTACH I SHUTDONN OF RL REACTORS

ATTACH 2 SHUTDOWN OF THREE MANFORD

REACTORS

NM PROOUCTION REPORT - APR 1964

PROOUCTION FORECAST 4/01/64 THRU

12/31/65

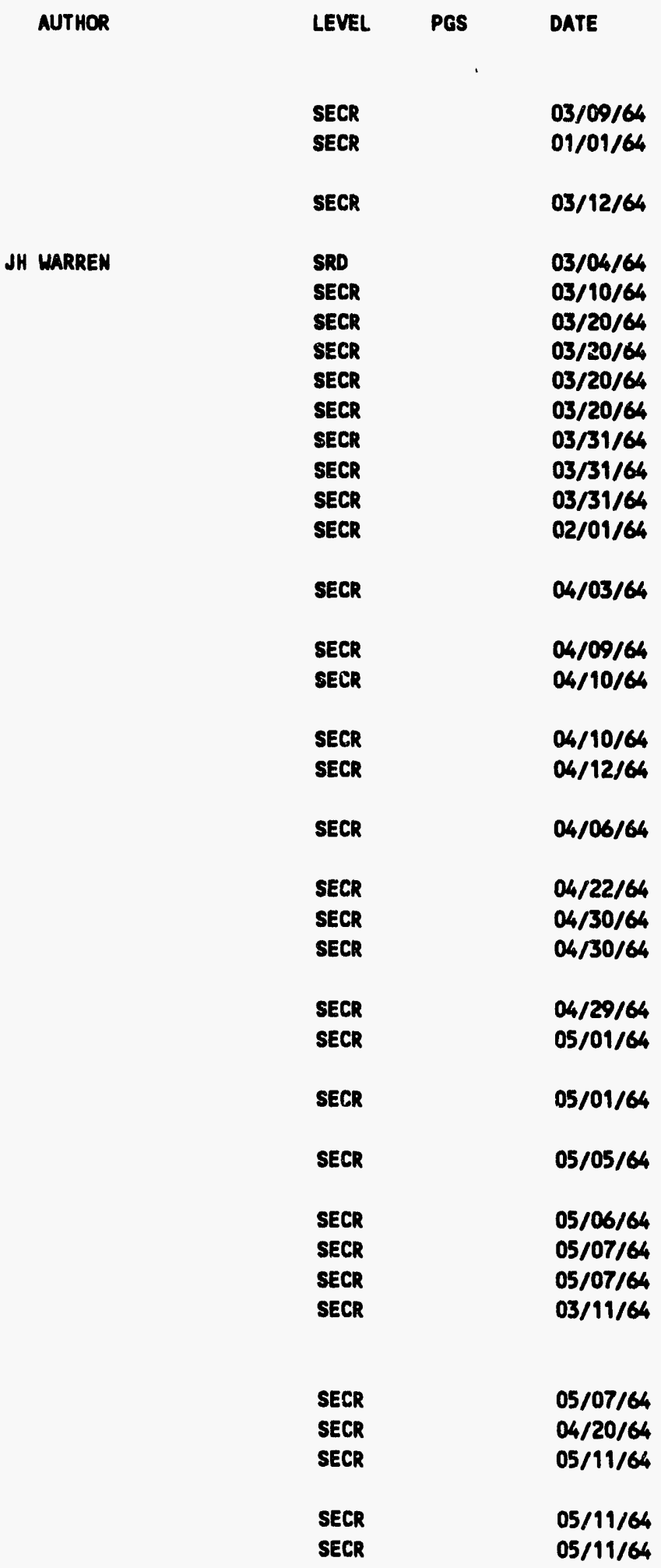


DoC. NO.

HAN-88373

HAN-88385

HAN-8849

HAN-88439

MAN-88440

HAN-88457

HAN-884571

HAN-88574

HAN-88585

HAN-88586

HAN- 88605

HAN-88610

HAN-88612

HAN -88633

WAN -88638

HAN -88640

HAN -88672

HAN-88674

HAN -88683

HAN-88687

HAN -88693

HAN-88710

HAN-88753

HAN -88789

HAN-887891

HAN-88796

HAN-88827

HAN-88915

HAN-88916

HAN-88929

HAN -88930
TITLE

MONTHLY STATUS \& PROGRESS REPORT FOR APR

1964

ANDIY OF INCOYING OPERATIONS 74-C

PROGRAM

ALDIT OF MACHINING OPERATIONS-MC- 1807

- PROGRn

POWER RECOVERY STLDY OF EXISTING SYALLER

HANFORD REACTORS

PP REVIEW CAC-987 REV1 METAL

STABILIZATION FACILITY 234-5 BLDG

QAIA MONTHLY REORT FOR APRIL 1964

APPENDIX I

HAPO PRCOUCTION FORECAST 4/01/64 THRU 12/31/65

SURVEY OF STATION HGE - NO. 21 PART 3

FEASIBILITY OF MRADIOACTIVE MARHEADS"

U-233 PROOUCTION

NM PRODUCTION REPORT - MAY 1964

MONTHLY STATUS \& PROGRESS REPORT FOR MAY 1964

REACTOR SHUTDOWN

COMNENTS OF RLOO STAFF ON INTERIM POWER

STUDIES - SWALLER HANFORD REACTORS

U-233 PRODUCTION STUDIES

SPECIFIC U-233 PROOUCTION ESTIMATES

STATEMENT OF EXPLANATIONS FOR MATERIAL

BALANCE REPORT MAY 1964

COST ESTIMATES U-233 PROOUCTION STUDY GROUP V

CURTAILMENT OF OPERATING \& MAINTENANCE COST WITH RESPECT TO SHUTDOWN OF DR, H AND F REACTORS

TECH BASES U-233 PROOUCTION STLOY GROUP IV

SEPARATIONS PLANT SCHEDULING VS REDOX

SHUTDOWN

ALOIT OF CASTING OPERATIONS-MC- 1807

PROGRAM

QAIA REPORT FOR MAY 1964

APPENDIX I

U-233 PRODUCTION PROGRAM

EXPEDITED REDOX SHUTDOWN

AUDIT OF POWER ROLL FORMING OPERATIONS

74-C PROGRAM

AUDIT OF CONTROL OF PROCESS TEST

IDENTIFICATION ON PROCESS RECORDS,

CHARTS AND IEM REPORTS - MC- 1807 PROGRAM U-233 PRCOUCTION PROGRAM

MONTHLY STATUS \& PROGRESS REPORT FOR JUN 1964
AUTHOR

LEVEL

PGS

DATE

HR FREITAG

AEC-RL

SECR

$05 / 15 / 64$

SECR

05/07/64

SECR

05/07/64

SECR

05/19/64

SECR

$05 / 12 / 64$

SECR

05/22/64

SECR

05/22/64

$06 / 05 / 64$

$06 / 15 / 64$

$06 / 05 / 64$

$06 / 10 / 64$

06/08/64

$04 / 11 / 63$

MH ARNDT

SECR

$06 / 11 / 64$

06/03/64

$06 / 12 / 64$

$06 / 12 / 64$

$06 / 17 / 64$

$06 / 17 / 64$

SECR

$06 / 18 / 64$

SECR

$06 / 15 / 64$

SECR

$06 / 19 / 64$

SECR

$06 / 16 / 64$

SECR

$06 / 26 / 64$

SECR

SECR

SECR

N KARAgIANES

SRD

SECR

$06 / 26 / 64$

$06 / 26 / 64$

$06 / 30 / 64$

07/09/64

SECR

$07 / 10 / 64$

SECR

07/09/64

SECR
07/15/64 
Page No. 130

$04 / 28 / 93$

LIST OF MANFORD-GENERATED CLASSIFIED

DOCUMENTS DATED BETMEEN 1/1/61 AND 12/31/72

Doc. No.

HAN-88941

HAN-88957

HAN-88964

HAN-889641

HAN-88985

HAN-89006

HAN-89044

HAN-89083

HAN-89097

HAN-89117

HAN-89157

HAN-89168

HAN-89203

HAN-89245

HAN-89257

MAN-892571

HAN-89258

HAN-89301

HAN-89378

HAN-89383

HAN- 89400

HAN-89436

HAN-894361

HAN-89449

HAN-894491

HAN-89475

HAN-89484

HAN-89496

HAN-89549

HAN-89586

HAN-89587

HAN-89597
TITLE

MM PROOUCTION REPORT - JUN 1964

MATERIAL BALANCE REPORT JUNE 1964

OAIA OUALITY REPORT FOR JUNE 1964

APPENDIX I

ALLOCATION OF U-233 PRODUCED FROM

DEMONSTRATION TEST

TRANSFER JOURWAL FOR FY 1965

REVS TO SPECS FOR URANIUN BILLETS FOR

N-REACTOR ELEMENTS

COMPARATIVE REACTOR PERFORMANCE - JUME

1964

ISOSTATIC PRESSING - STATUS

PROJECT INVENTORY ACCOUNTS FY 1965

MUDIT OF DIMENSIONAL INSPECTION OPERTION

MC 1807 PROGRAM

MONTHLY STATUS \& PROGRESS REPORT FOR JUL

1964

MM PRODUCTION REPORT - JUL 1964

ALOIT OF DIMENSIONAL IMSPECTION

OPERATIONS - 74-C PROCRAM

OAIA OUALITY REPORT FOR JULY 1964

APPENDIX I

HAPO PRODUCTION FORECAST 7/01/64 THRU

6/30/69

PRCOUCTION FORECAST 7/01/64 THRU 6/30/69

- tRANSMITTAL

MINUTES OF MEETING OF RLO \& US PUBLIC

HEALTH SERVICE ON COLLMBIA RIVER

CONTAMINATION

AUDIT OF PACKAGING OPERATIONS - 74-C

PROGRAM

MONTHLY STATUS \& PROGRESS REPORT FOR NUG

1964

AEC PRODUCTION PROGRAM DRAFT 3

ESTIMATES COST LEVELS DRAFT 1

QAIA QUALITY REPORT FOR AUEUST 1964

APPENDIX I

NM PROOUCTION REPORT - AUG 1964

SURVEY NO.21, PART 4

O3 PROGRAM MEAPONS PROOUCTION \&

DEVELOPHENT - JUL 1964 RPT

CLASSIFIED INFORMATION MEETING ALTERMATE

PRODUCTS FROM THE HANFORD REACTORS

CLASSIFIED INFORMATION MEETING GENERAL

COMNENTS REACTOR AND FUELS

CLASSIFIED INFORMATION MEETING

IRRADIATION PROCESSING DEPT PROO FUELS

SECTION

CLASSIFIED INFORMATION MEETING HEALTH

PHYSICS OPERATIONS STANDPOINT
AUTHOR

LEVEL

PGS

DATE

$07 / 10 / 64$

$07 / 15 / 64$

$07 / 16 / 64$

$07 / 16 / 64$

$07 / 17 / 64$

07/22/64

$07 / 21 / 64$

$07 / 30 / 64$

$07 / 31 / 64$

$08 / 04 / 64$

$08 / 10 / 64$

SECR

SECR

$08 / 16 / 64$

SECR

$08 / 10 / 64$

SECR

$08 / 21 / 64$

SECR

SECR

$08 / 26 / 64$

$08 / 24 / 64$

$08 / 24 / 64$

SECR

$08 / 28 / 64$

SECR

$09 / 04 / 64$

SECR

$09 / 04 / 64$

SECR

$09 / 15 / 64$

SECR

$09 / 09 / 64$

$09 / 10 / 64$

$09 / 11 / 64$

$09 / 11 / 64$

$09 / 11 / 64$

$09 / 18 / 64$

$09 / 18 / 64$

SECR

$09 / 25 / 64$

SECR

$09 / 25 / 64$

SECR

$09 / 22 / 64$

SECR

$09 / 25 / 64$ 
Doc. No.

HAN-89598

HAN-89602

HAN-89659

HAN-89669

HAN-89690

HAN-89699

HAN-89700

HAN-89701

HAN-89702

HAN-89705

HAN-89722

HAN-89734

MAN-89739

HAN-897391

HAN-89766

HAN-89773

HAN-89775

HAN-89780

HAN-89799

HAN-89822

HAN-89833

HAN -89834

HAN -89918

HAN-89954 HAN-89973

HAN-89990

HAN-90123

HAN -90125

HAN-90126

HAN-90142
TITLE

CLASSIFIED INFORMATION MEETING REACTOR OPERATIONS AND MAINTEMANCE DESCRIPTION OF 100 F AREA FACILITIES ALOIT OF INCOTING RECORDS - MC 1807 PROCRAN

MONTHLY STATUS \& PROGRESS REPORT FOR SEP 1964

ANDIT OF PRCOUCTION RECORDS - 74-C PROGRAM - PART I

COMPARATIVE REACTMR PERFORMANCE - APRIL

1964

COMPARATIVE REACTOR PERformanCE - maY

1964

COMPARATIVE REACTOR PERfoRMANCE - JULY

1964

COMPARATIVE REACTOR PERFoRMAMCE - NUG

1964

NA PROOUCTION REPORT - SEP 1964

COST ESTIMATES U-233 PRCOUCTION STLOY

CASES

MONTHLY INVENTORY REPORT - SAN 32500 -

SEP 1964

QAIA OUALITY REPORT FOR SEPT 1964

APPENDIX I

PLUTONIUM, MEPTUNIUN AND AMERJCIUM MASTE

TANK INVENTORIES

SURFACE CONDITION EVALUATION

HAZARDS SUMURY REPORT SUPPLEMENT I

EXTRACT FRON HAN 89419 DATED SEPT 4,

1964

COMPARATIVE REACTOR PERfORMANCE - SEPT

1964

HAPO PRCOUCTION FORECAST 10/01/64 THRU

6/30/66

PROOUCTION FORECAST 10/01/64 THRU

6/30/66

COST ESTIMATES U-233 PROOUCTION

MONTHLY STATUS \& PROCRESS REPORT FOR OCT

1964

WM PROOUCTION REPORT - OCT 1964

TRITIUM SHIPMENT FORECAST

MONTHLY INVENTORY REPORT - SAN 32500 -

OCT 1964

SURVEY "Z22 - FACILITY HZA

MONTHLY STATUS \& PROGRESS REPORT FOR MOV

1964

AUDIT OF PROOUCTION RECORDS - 74-C

PROGRAM - PART II

COMPARATIVE REACTOR PERFORMANCE - OCT 1964

\section{AUTHOR}

LEVEL

PGS

DATE

$09 / 25 / 64$

$09 / 28 / 64$

10/09/64

SECR

$10 / 13 / 64$

SECR

$10 / 14 / 64$

SECR

$04 / 01 / 64$

SECR

$05 / 01 / 64$

SECR

$06 / 01 / 64$

SECR

$08 / 01 / 64$

SECR

10/09/64

10/16/6

SECR
SECR

SECR

$10 / 13 / 64$

SECR

SECR

SECR

10/20/64

$10 / 20 / 64$

$10 / 19 / 64$

SECR

$10 / 23 / 64$

$09 / 15 / 64$

10/23/64

SECR

SECR

$09 / 01 / 64$

SECR

$10 / 28 / 64$

SECR

$10 / 30 / 64$

SECR

10/30/64

SECR

$11 / 13 / 64$

SECR

SECR

SECR

$11 / 19 / 64$

$11 / 16 / 64$

$11 / 16 / 64$

AEC-RL

SRD

$12 / 16 / 64$

SECR

$04 / 11 / 63$

SECR

$12 / 10 / 64$

SECR

$10 / 01 / 64$ 
Page No. 132

$04 / 28 / 93$

LIST OF HANFORD-GEMERATED CLASSIFIED

DOCUMENTS DATED BETUEEN 1/1/61 AND 12/31/72

DOC. NO.

HAN-90160

HAN-90166

HAN-90171

HAN-901711

HAN- 90183

HAN-90195

HAN-90196

HAN-90231

HAN-90237

HAN -90266

HAN- 90291

HAN- 90292

HAN-90326

HAN-903261

HAN-90354

HAN-90367

HAN-90402

HAN- 90419

HAN-90420

HAN-90424

HAN-9044701

HAN- 9044702

HAN -9044703

HAN-9044704

HAN-9044705

HAN- 9044706

HAN -9044707

HAN- 9044708

HAN-9044709

HAN-9044710

HAN-9044711

HAN-9044712

HAN-9044801

HAN-9044802

HAN- 9044803

HAN-9044804

HAN-9044805
TITLE

COMPARATIVE REACTOR PERFORMAMCE - MOV

1964

KW CORE E-Q LOADINOS

QAIA OUALITY REPORT FOR OCTOBER 1964

APPENDIX I

MONTHLY INVENTORY REPORT - SAN 32500 NON 1964

FIMAL SHUTDOWN OF DR REACTOR PLANT

UM PRCOUCTION REPORT - HOV 1964

AUTHORIZATION FOR ADDITIONAL PROOUCTION

OF $\mathrm{U}-233$

USE OF OXIDE FUELS AT HANFORD

BALANCE SHEET CHANGES FY 1964

P - 238 CONVERSION

U-233 PROOUCTION

QAIA OUALITY REPORT FOR MOVEMBER 1964

APPENDIX I

MONTHLY STATUS \& PROCRESS REPORT FOR DEC

1964

HANFORD P FABRICATION OPERATIONS

REDUCTION

MATERIAL BALANCE REPORT DEC 1965

MONTHLY INVENTORY REPORT - SAN 32500 -

DEC 1964

NM PROOUCTION REPORT - DEC 1964

MATERIAL BALANCE REPORT DEC 1964

MOB MONTHLY REPORT FOR JAN 1965

MOB MONTHLY REPORT FOR FEB 1965

MOB MONTHLY REPORT FOR MAR 1965

MOB MONTHLY REPORT FOR APR 1965

MOB MONTHLY REPORT FOR MAY 1965

MOB MONTHLY REPORT FOR JUN 1965

MOB MONTHLY REPORT FOR JUL 1965

MOB MONTHLY REPORT FOR NUG 1965

MOB MONTHLY REPORT FOR SEP 1965

MOB MONTHLY REPORT FOR OCT 1965

MOWTHLY STATUS \& PROGRESS REPORT FOR MOV 1965

MONTHLY STATUS \& PROCRESS REPORT FOR DEC

1965

O3 PRCDUCTION \& DEVELOPNENT MEAPONS -

JAN 1965

03 PRCOUCTION \& DEVELOPMENT VEAPONS -

FEB 1965

03 PROOUCTION \& DEVELOPMENT MEAPONS -

MAR 1965

O3 PROOUCTION \& DEVELOPMENT MEAPONS APR 1965

O3 PRCOUCTION \& DEVELOPMENT MEAPONS maY 1965
AUTHOR

LEVEL PaS

DATE

$12 / 14 / 64$

$12 / 15 / 64$

$12 / 15 / 64$

$12 / 15 / 64$

$12 / 15 / 64$

$12 / 17 / 64$

$12 / 19184$

$12 / 22 / 64$

JE TRAVIS

$12 / 22 / 64$

$09 / 30 / 64$

$12 / 30 / 64$

$12 / 31 / 64$

$12 / 31 / 64$

$12 / 31 / 64$

$01 / 14 / 65$

$01 / 11 / 65$

$01 / 15 / 65$

SECR

01/14/65

SECR

$01 / 14 / 65$

$01 / 19 / 65$

02/01/65

03/01/65

04/01/65

$05 / 01 / 65$

$06 / 01 / 65$

07/01/65

$08 / 01 / 65$

$09 / 01 / 65$

10/01/65

$11 / 01 / 65$

$04 / 11 / 63$

$04 / 11 / 63$

02/01/65

SECR

03/01/65

SECR

$04 / 01 / 65$

SECR

05/01/65

SECR 
DOC. NO.

HAN-9044806

HAN-9044807

HAN-9044808

HAN- 9044809

HAN-9044810

HAN-9044811

HAN- 9044812

HAN-90465

HAN-90482

HAN-90515

HAN-90536

HAN-90539

HAN-905391

HAN- 90542

HAN-90549

HAN- 90561

HAN- 90586

HAN- 90596

HAN- 90629

HAN-90630RPTI

HAN-90630RPT2

HAN-90630RPT3

HAN-90630RPT4

HAN-90630RPT5

HAN-90630RPT6

HAN-90630RPT7

HAN-90630RPT8

HAN-9063101
TITLE

AUTHOR

03 PRODUCTION 2 DEVELOPMENT MEAPONS JUN 1965

03 PROOUCTION \& DEVELOPMENT MEAPONS JUL 1965

O3 PROOUCTION \& DEVELOPNENT VEAPONS NUG 1965

O3 PRODUCTION \& DEVELOPNENT MEAPONS SEP 1965

03 PRCOUCTION \& DEVELOPNENT MEAPONS OCT 1965

O3 PRODUCTION \& DEVELOPMENT MEAPONS NOV 1965

O3 PROOUCTION 2 DEVELLPNENT WEAPONS DEC 1965

PHYSICS TESTS-REACTORS TO BE SHUTOOWM COMPARATIVE REACTOR PERFORMAMCE - DEC 1964

ANIT OF CHEMICAL ASSAYS -MC-1807 PROGRAM

ACRS PRESENTATIONS OF HIGHER $K$ POWER LIGHT

QAIA QUALITY - AUDIT OF CHEMICAL ASSAYS MC-1807 PROGRAM

APPENOIX I

BRANDY SHIPPING DOCUMENTS, APR 1962 JAN 1965

FY 196502 RED MIDYEAR BUDGET REVIEW N-REACTOR OPERATION

TRANSFER JOURWAL FY 1965 FOR HYA

CONTINUED THORIA IRRADIATIONS

PROCESS CHANMEL ENLARGEMENT $B$ \&

REACTORS

PROOUCTION MONTHLY STATUS AND PROCRESS REPORT FOR JANUARY THRU DECEMBER 1965

PROOUCTION MONTHLY STATUS AND PROCRESS REPORT FOR JAMUARY THRU DECEMBER 1965 PROOUCTION MONTHLY STATUS AND PROCRESS REPORT FOR JAMUARY THRU DECIEMBER 1965 PROOUCTION MONTHLY STATUS AND PROCRESS REPORT FOR JAMUARY THRU DECEMBER 1965 PRODUCTION WOWTHLY STATUS AND PROERESS REPORT FOR JAMUARY THRU DECEMBER 1965 PROOUCTION MONTHLY STATUS AND PROGRESS REPORT FOR JAMUARY THRU DECEMBER 1965 PRCOUCTION MONTHLY STATUS AND PROCRESS REPORT FOR JANUARY THRU DECEMBER 1965 PROOUCTION MONTHLY STATUS AND PROGRESS REPORT FOR JAMUARY THRU DECEMBER 1965 WONTHLY PROOUCTION REPORT FOR JAN THRU DEC 1965

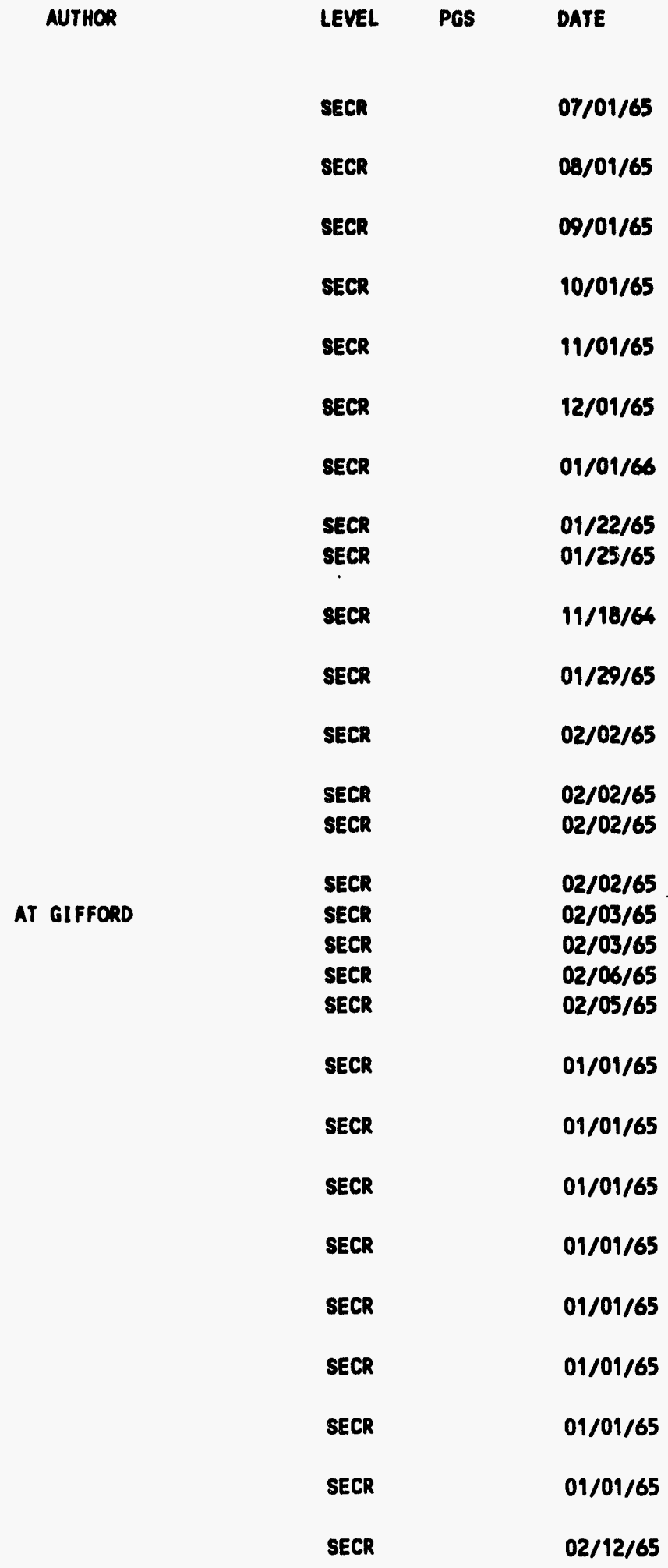


Page No. 134

$04 / 28 / 93$
LIST OF HANFORD-GENERATED CLASSIFIED DOCUMENTS DATED BETMEEN 1/1/61 AND 12/31/72
DOC. NO.

HAN-9063102

HAN-9063103

HAN- 9063104

HAN-9063105

HAN-9063106

HAN-9063107

HAN-9063108

HAN-9063109

HAN-9063110

HAN-9063111

HAN-9063112

HAN-90648

HAN-90649

HAN-90650

HAN-90687

HAN-90688

HAN-90696

HAN-90698

HAN-906981

HAN-90706

HAN-90707

HAN-90776

HAN-90843

HAN-90871

HAN-90872

HAN-90907

HAN-90975

HAN-90977

HAN-909771

HAN-90985
TITLE

AUTHOR

MONTHLY PROOUCTION REPORT FOR JAN THRU DEC 1965

MONTHLY PRCOUCTION REPORT FOR JAN THRU DEC 1965

MONTHLY PRCOUCTION REPORT FOR JAN THRU DEC 1965

MONTHLY PROOUCTION REPORT FOR JAN THRU DEC 1965

MONTHLY PRCOUCTION REPORT FOR JAN THRU DEC 1965

MONTHLY PRCOUCTION REPORT FOR JAN THRU DEC 1965

MONTHLY PRODUCTION REPORT FOR JAN THRU DEC 1965

MONTHLY PRODUCTION REPORT FOR JAN THRU DEC 1965

MONTHLY PRODUCTION REPORT FOR JAN THRU DEC 1965

MONTHLY PROOUCTION REPORT FOR JAN THRU DEC 1965

MONTHLY PRODUCTION REPORT FOR JAN THRU DEC 1965

ALOIT OF EVALUATION CYCLE OPERATION -

88-C PROGRAMS

AUOIT OF PART EVALUATION CYCLE OPERATION

- 1807 PROGRAM

MONTHLY STATUS \& PROGRESS REPORT FOR JAN

1965

HAPO PRODUCTION FORECAST 1/01/65 THRU

6/30/70

NM PRODUCTION REPORT - JAM 1965

COMPARATIVE REACTOR PERForMAMCE - JAN

1965

QAIA QUALITY REPORT FOR JAMUARY 1965

APPENDIX I

PROOUCTION FORECAST 1/01/65 THRU 6/30/70

IRRADIATION PROCESSING DEPARTMENT

REQUEST TO SHIP DEPLETED URANIUM,

RECUEST "CPD-450

CONTIMUED THORIA IRRADIATIONS

AUDIT OF RADIOGRAPHY OPERATION -MC-1807

PROGRAM

ALDIT OF RADIOGRAPHY OPERATION - 88-C

PROGRAMS

MONTHLY STATUS \& PROGRESS REPORT FOR FEB

1965

MM PRODUCTION REPORT - FEB 1965

QAIA QUALITY REPORT FOR FEBRUARY 1965

APPENDIX I

U-233 PROOUCTION MANUFACTURING COSTS
LEVEL Pas DATE

SECR

$03 / 12 / 65$

SECR

$04 / 12 / 65$

SECR

$05 / 12 / 65$

SECR

$06 / 12 / 65$

SECR

$07 / 12 / 65$

SECR

$08 / 12 / 65$

SECR

$09 / 12 / 65$

SECR

$10 / 12 / 65$

SECR

$11 / 12 / 65$

SECR

$12 / 12 / 65$

SECR

$01 / 12 / 66$

SECR

$02 / 16 / 65$

SECR

$02 / 16 / 65$

SECR

$02 / 12 / 65$

SECR

$02 / 11 / 65$

SECR

$02 / 10 / 65$

SECR

01/01/65

SECR

SECR

$02 / 16 / 65$

02/16/65

$02 / 16 / 65$

$02 / 15 / 65$

02/18/65

SECR
SECR

03/03/65

SECR

03/15/65

SECR

$03 / 15 / 65$

SECR

$03 / 12 / 65$

SECR

03/10/65

SECR

$03 / 15 / 65$

03/15/65

$03 / 15 / 65$ 
Page No. 135

04/28/93

LIST OF HANFORD-GENERATED CLASSIFIED

DOCUMENTS DATED BETWEEN 1/1/61 AND 12/31/72

DOC. NO.

HAN-90987

HAN-90991

HAN-91012

HAN-91091

HAN-91095

HAN-91160

HAN-91177

HAN-91178

HAN-91198

HAN -91199

HAN-911991

HAN-91214

HAN- 91216

HAN-91231

HAN-91246

HAN-91282

HAN-91285

HAN-91302

HAN- $\$ 1313$

HAN-91318

HAN-91360

HAN- 91388

HAN-91449

HAN- 91479

HAN-91481

HAN-914811

HAN-91499

HAN-91505

HAN-91513

HAN-915131

HAN-91521

HAN-91547

HAN-91565
TITLE

AUTHOR

COMPARATIVE REACTOR PERfORMANCE - FEB

1965

IPD MONTHLY REPORT FEB 1965

PROGRAM CONSIDERATIONS N-REACTOR

FEASIBILITY REPORT. THE USE OF OXIDE

FUELS AT Hi:..: ORD

COST BUDGET REPORT CONSOLIDATED SUMARY

FOR FEB 1965

U-233 PRODUCT DISTRIBUTION

ALDIT OF DENSITY MEASUREMENT OPERATION MC-1807 PROGRAM

ALDIT OF INGOTING OPERATION - 88-C

PROGRAMS

MONTHLY STATUS \& PROGRESS REPORT FOR MAR

1965

QAIA QUALITY REPORT FOR MARCH 1965

APPENDIX I

NM PRCOUCTION REPORT - MAR 1965

IRRADIATION OF N-REACTOR FUEL LOAD

FORMS USED BY RLO QAIA IN ACCEPTANCE OF PROOUCT

COMPARATIVE REACTOR PERFORMANCE - MARCH

1965

IPD MONTHLY REPORT MARCH :365

FY 1966 ANO 1967 BUDGET ASSUMPTIONS

PP REVIEH SHEET - CAC-109, REV1

PU-238 STATEMENTS IN RL-NRD-150-2

PRESIDENTIAL DIRECTIVE INFORMATION FY

1966

NON-DEFENSE PLUTONIUM PROOUCTION

105-DR DEACTIVATION MONITORING RESULTS

AUDIT OF MOLD PREPARATION \& CASTING

OPERATION - 88-C PROGRAM

QUALITY AND AVAILIBILITY OF FISSION

PRCOUCTS

AUDIT OF QUALITY CONTROL PROGRAM

EFFECTIVENESS-MXC-1807 PROGRAM

EXHIBIT I THRU VI

MONTHLY STATUS \& PROGRESS REPORT FOR APR 1965

MUCLEAR MATERIALS REVIEW MEAPONS

MANUFACTURING OPERATION $1: 2$

QUARTERS - FY-1965

QAIA QUKLITY REPORT FOR APRIL 1965

APPENDIX I

NM PRODUCTION REPORT - APR 1965

HAPO PROOUCTION FORECAST 4/01/65 THRU

12/31/66

AVAILIBILITY OF VERY HIGHEY ENRICHED

URAMIUM HEXAFLUORIDE

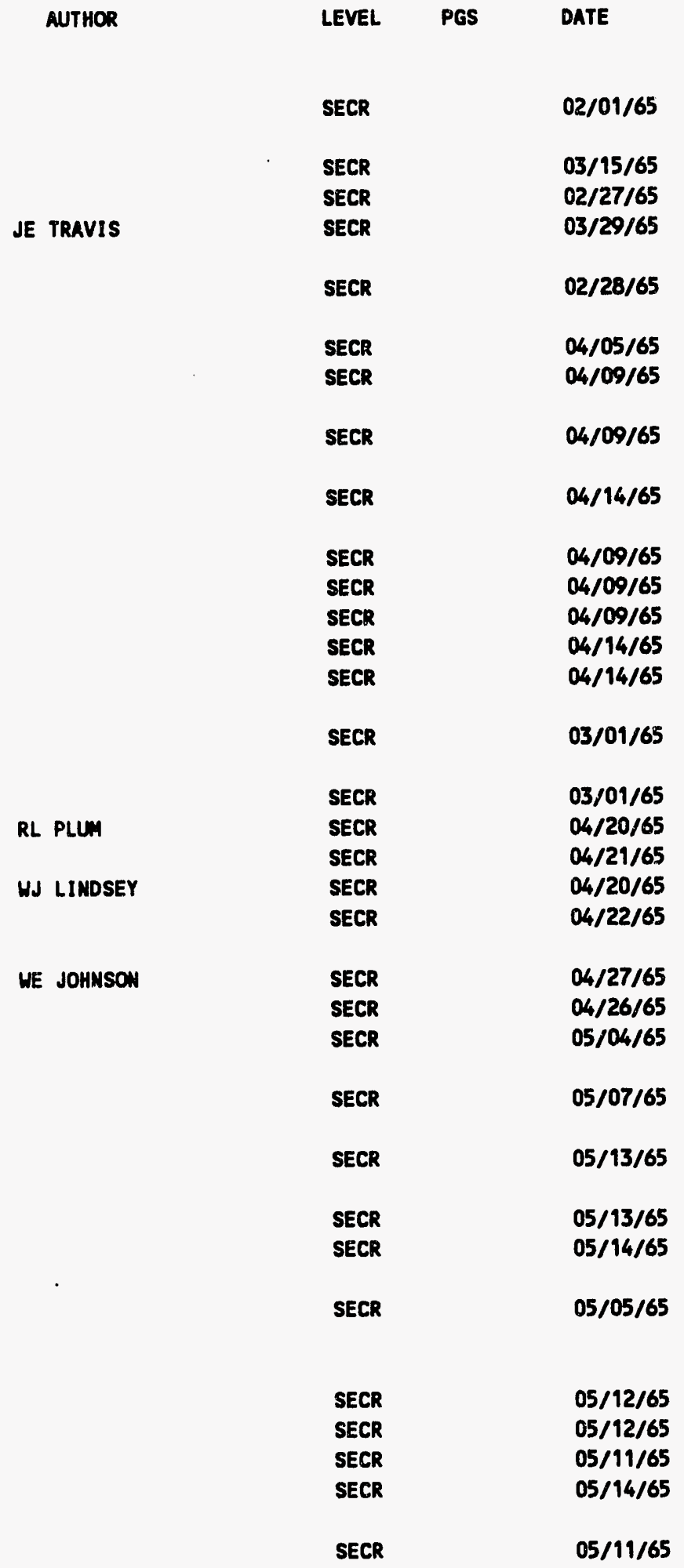


Page No. 136

$04 / 28 / 93$

LIST OF HANFORD-GENERATED CLASSIFIED

DOCLMENTS DATED BETUEEN 1/1/61 AHD 12/31/72

DOC. NO.

HAN-91617

HAN-91618

HAN-91621

HAN-91720

HAN-91758

HAN-91778

HAN-91791

HAN-91824

HAN-918241

HAN-91845

HAN-91903

HAN-91904

HAN-91953

HAN-91995

HAN-91999

HAN-92005

HAN-9200501

HAN-9200502

HAN-92077

HAN-92118

HAN-92119

HAN-92126

HAN-92130

HAN-92141

HAN-92142

HAN-92143

HAN-92167

HAN-92168

HAN-921681

HAN-92176

HAN-92177

HAN-92178

HAN-92i84
TITLE

IPD MONTHLY REPORT APRIL 1965

COMPARATIVE REACTOR PERFORMANCE - APRIL

1965

TRANSMITTAL PRCOUCTION FORECAST 4/01/65

THRU 12/31/66

ENGINEERING STUDY THORIUN PROCESSING

PUREX PART I FLOW SHEET

BMI MONTHLY ACTIVITIES REPORT - APRIL

1965

THORIA REQUIREMENTS FOR 2/1/66 FRINGE

LOADS

MONTHLY STATUS \& PROGRESS REPORT FOR MAY

1965

QAIA QUALITY REPORT FOR MAY 1965

APPENDIX $A$

MM PRODUCTION REPORT - MAY 1965

N-REACTOR DEPARTMENT MONTHLY REPORT -

JUNE 1965

IPD MONTHLY REPORT MAY 1965

REACTOR COST STUDIES

COMPARATIVE REACTOR PERFORMANCE - MAY

1965

DIVISION OF REACTOR LICENSING REPORT TO

ADVISORY COMMITTEE ON REACTOR*

("SAFEGUARLS ON PRCOUCTION REACTORS)

MODIFICATION TO THE 1964 REFINED REACTOR

COST STUDIES TO CONSIDER P -238

PRCOUCTION

APPENDIX "A"

APPENDIX "B"

MONTHLY STATUS \& PROGRESS REPORT FOR JUN

LEVEL

PGS

DATE

1965

COMPOSITION OF ENDING INVENTORY - JUN

1965

MATERIAL BALANCE REPORT JUNE 1965

PRELIMINARY ASSESSMENT OF IPD'S U-233

CAPABILITIES

MAXIMUM CREDIBLE REACTOR ACCIDENT

MM PRODUCTION REPORT - JUN 1965

N-REACTOR DEPT MONTHLY REPORT JUNE 1965

IPD MONTHLY REPORT JUNE 1965

AUDIT OF ANALYTICAL LABORATORY - LASL

PROGRAM

QAIA QUALITY REPORT FOR JUNE 1965

APPENDIX I

MBR/COEI TRANSCRIPTION SHEETS

HAA CONSOLIDATION MATERIAL BALANCE REPORT

HAM COMPOSITION OF ENDING INVENTORY

H-REACTOR STLOIES BY AEC STAFF

\begin{tabular}{|c|c|}
\hline SECR & $05 / 14 / 65$ \\
\hline SECR & $04 / 01 / 65$ \\
\hline SECR & $05 / 21 / 65$ \\
\hline SECR & $05 / 12 / 65$ \\
\hline SECR & $05 / 14 / 65$ \\
\hline SECR & $06 / 08 / 65$ \\
\hline SECR & $06 / 11 / 65$ \\
\hline SECR & $06 / 11 / 65$ \\
\hline SECR & $06 / 11 / 65$ \\
\hline SECR & $06 / 11 / 65$ \\
\hline SRD & $06 / 10 / 65$ \\
\hline SECR & $06 / 14 / 65$ \\
\hline SECR & $06 / 25 / 65$ \\
\hline SECR & $05 / 01 / 65$ \\
\hline SECR & $02 / 05 / 65$ \\
\hline SECR & $07 / 02 / 65$ \\
\hline SECR & $07 / 06 / 65$ \\
\hline SECR & $07 / 06 / 65$ \\
\hline SECR & $07 / 16 / 65$ \\
\hline SECR & $07 / 15 / 65$ \\
\hline SECR & $07 / 15 / 65$ \\
\hline SECR & $07 / 09 / 65$ \\
\hline
\end{tabular}

RL PLUA

$07 / 16 / 65$

$07 / 13 / 65$

$06 / 01 / 65$

$06 / 01 / 65$

$07 / 23 / 65$

$07 / 23 / 65$

$07 / 23 / 65$

$07 / 23 / 65$

$07 / 23 / 65$

SECR

$07 / 23 / 65$

$07 / 26 / 65$ 
Page No. 137

04/28/93
LIST OF HANFORD-GENERATED CLASSIFIED

DOCLIMENTS DATED BETWEEN 1/1/61 AND 12/31/72
DOC. NO.

HAN-92214

HAN-92227

HAN-92252

HAN-92257

HAN-92282

HAN-922821

HAN-92312

HAN-92316

HAN-92346

HAN-92351

HAN-92355

HAN-92377

HAN-92379

HAN-92389

HAN-92395

HAN-92414

HAN-92430

HAN-92469

HAN-92476

HAN-92496

HAN-924961

HAN-92512

HAN-92525

HAN- 92554

HAN -92590

HAN-92591

HAN- 92603

HAN-92621

HAN- 92650

HAN- 92655

HAN-92681

HAN-92693

HAN-92716

HAN-92724
TITLE

COST STATEMENTS PROOUCTION INVENTORIES

AND SS MATERIAL IN RESEARCH

HAPO PRODUCTION FORECAST 7/01/65 THRU

6/30/70

DESIGN STUDY REPORT CO-PROOUCT EXTRA

CTION FACILITY N-REACTORY, 100 AREA

COAPARATIVE REACTOR PERFORMANCE - JUNE

1965

QAIA OUALITY REPORT FOR JULY 1965

APPENDIX I

AUDIT OF MACHINING OPERATIONS - LASL

PROGRAM

MONTHLY STATUS \& PROGRESS REPORT FOR JUL

1965

TRANSMITTAL PRODUCTION FORECAST 7/01/65

THRU 6/30/70

NM PROOUCTION REPORT - JUL 1965

COMPARATIVE REACTOR PERFORMANCE - JULY

1965

IPD MONTHLY REPORT JULY 1965

RELATIVE PRODUCTIVITY OF N -237

MATERIAL BALANCE REPORT - COEI

TRANSCRIPTION SHEET

AEC SF SHIPPING FORM MAN-LZB-1

DATA ON N -237 PRODUCTION

DATA ON N -237 PRODUCTION

AUDIT OF YIELD STRENGTH \& CERTIFICATION

- LASL PROGRAM

MBR/COEI TRANSCRIPTION SHEET

OAIA QUALITY REPORT FOR AUGUST 1965

APPENDIX I

U-233 FOR LSBR CRITICAL EXPERIMENTS

MONTHLY STATUS \& PROGRESS REPORT FOR AUG

1965

NM PRODUCTION REPORT - AUG 1965

DEPLETED URANIUM, JAN - JUN 1965

REPORT OF COEI FOR DEPLETED URANIUM AS

OF $6 / 30 / 65$

IPD MONTHLY REPORT AUG 1965

PRICE SCHEDULE FOR 94\% U SCRAP

DATA ON N -237 PRODUCTION

THORIUN \& U-233 PROGRAM

TT RE: ISOTOPIC COAPOSITION OF KG5O

NUCLEAR MATERIALS REVIEW WEAPONS

MAHUFACTURING OPERATION $3 \& 4$

QUARTERS - FY-1965

A. OIT OF PONER BILL ROLL FORMING

OPERATION - LASL PROGRAM

COMPARATIVE REACTOR PERFORMANCE - AUG

1965
AUTHOR

LEVEL

Pas

date

06/01/65

07/30/65

$06 / 17 / 65$

$06 / 01 / 65$

$08 / 04 / 65$

08/04/65

$08 / 10 / 65$

SECR

$08 / 13 / 65$

08/13/65

SECR

08/11/65

SECR

07/01/65

SECR

SECR

$09 / 13 / 65$

08/16/65

$06 / 30 / 65$

SECR

$07 / 29 / 65$

$08 / 20 / 65$

$08 / 25 / 65$

09/27/65

SECR

SECR

$09 / 03 / 65$

$09 / 03 / 65$

$09 / 07 / 65$

$09 / 08 / 65$

$09 / 13 / 65$

$09 / 95 / 65$

$09 / 25 / 65$

$09 / 25 / 65$

SECR

$09 / 15 / 65$

$09 / 21 / 65$

$09 / 28 / 65$

$09 / 28 / 65$

RL PLUN

SECR

$10 / 01 / 65$

$09 / 27 / 65$

SECR

$10 / 04 / 65$

SECR

$10 / 07 / 65$ 
Page No. 138

$04 / 28 / 93$

LIST OF HANFORD-GENERATED CLASSIFIED

DOCUMENTS DATED BETWEEN 1/1/61 AND 12/31/72

DOC. MO.

TITLE

AUTHOR

LEVEL

PGS

DATE

HAN-92725

HAN-92726

HAN-92744

HAN -92750

HAN-92751

HAN -92766

HAN-92776

HAN-92783

HAN- 92786

HAN-92797

HAN-927971

HAN-92809

HAN-92810

HAN-92830

HAN-92842

HAN-92891

HAN-92898

HAN-92913

HAN-92954

HAN-92975

HAN-92988

HAN-92989

HAN-92993

HAN-92996

HAN-93006

HAN-93008

HAN-93022

HAN-930221

HAN-93095

HAN-93097

HAN-93190

HAN-93219

HAN-93220

HAN-93233

HAN-93234

HAN-93252

HAN-93288

HAN- 93296
N-REACTOR NEPTUNIUN TEST IRRADIATION

U-233 PROOUCTION

IRRADIATION OF NP-237

MONTHLY STATUS \& PROGRESS REPORT FOR SEP 1965

MPR CO-PROOUCTION MEETING AT SR

FORMAL QAP $N-10.0$ SURVEY

N-REACTOR FUEL DISCHARGE SCHEDULE

E-D LOADING STATUS

NM PRODUCTION REPORT - SEP 1965

QAIA QUALITY REPORT FOR SEPT 1965

APPENDIX 1

IPD MONTHLY REPORT SEPT 1965

H-REACTOR DEPARTMENT MONTHLY REPORT

SEPTEMBER 1965

WEAPONS PROGRAM

CO-PRODUCT PROGRAM - SCHEDULE FACTORS

U-233 REQUIREMENTS FOR PROGRAM 04

CO-PRODUCT PROGRAM N-REACTOR RL NRD-298

\& RL NRD-595

TELECON WITH SHOWALTER RE: SPEC. ON U-233 FOR DMA

MOUTHLY STATUS \& PROGRESS REPORT FOR OCT 1965

THORIUM-U 233 SHIPPING

CO-PROOUCT SCHEDULE ALTERMATES N-REACTOR

PROPOSED REV OF $K$ CORE E-Q LONDAING

NM PROOUCTION REPORT - OCT 1965

COMPOSITION OF ENDING INVENTORIES TO DUN

NON DEFENSE PU PROOUCTION N-REACTOR

HAPO PROOUCTION FORECAST 10/01/65 THRU

6/30/67

QAIA QUALITY REPORT FOR OCTOBER 1965

APPENDIX 1

COMPARATIVE REACTOR PERFORMAMCE - SEPT

1965

MEAPONS MANUFACTURING OPERATION MUCLEAR

MATERIAL MEASUREMENT RELIABILITY

COMPARATIVE REACTOR PERFORMANCE - OCT

1965

CO-PRODUCT PROGRAM AT N-REACTOR

PRODUCTION FORECAST 10/01/65 THRU

6/30/67

IPD MONTHLY REPORT OCT 1965

RLO QAIA INSTRUCTION AND PROCEDURES FOR

USE OF QUALITY ASSURANCE INSPECTION

PROPOSED REV OF E-Q LOADING

REFERENCE DATA CO-PRODUCT DISCUSSIONS

$11 / 16 / 65$

QAIA QUALITY REPORT FOR NOVEMBER 1965
HE PARKER

RL DICKEMAN

TH AMBROSE

RL DICKEMAN

$\begin{array}{ll}\text { SECR } & 10 / 07 / 65 \\ \text { SECR } & 10 / 07 / 65 \\ \text { SECR } & 10 / 08 / 65 \\ \text { SECR } & 10 / 13 / 65 \\ & \\ \text { SECR } & 10 / 05 / 65 \\ \text { SECR } & 10 / 07 / 65 \\ \text { SECR } & 10 / 11 / 65 \\ \text { SECR } & 10 / 08 / 65 \\ \text { SECR } & 10 / 13 / 65 \\ \text { SECR } & 10 / 12 / 65 \\ \text { SECR } & 10 / 12 / 65 \\ \text { SECR } & 10 / 01 / 65 \\ \text { SRD } & 09 / 01 / 65\end{array}$

$10 / 29 / 65$

$10 / 25 / 65$

$10 / 28 / 65$

$10 / 29 / 65$

SECR

$11 / 02 / 65$

SECR

$11 / 12 / 65$

SECR

$10 / 28 / 65$

$11 / 10 / 65$

$11 / 05 / 65$

$11 / 11 / 65$

$11 / 08 / 65$

RL DICKEMAN

SECR

SECR

SECR

SECR

SECR

SECR

SECR

SECR

SECR

$10 / 01 / 65$

SECR

$11 / 26 / 65$

SECR

$11 / 26 / 65$

SECR

SECR

$11 / 15 / 65$

$11 / 30 / 65$

SECR

$12 / 01 / 65$

$11 / 12 / 65$

SECR 
DATE

HAN-932\%61

HAN-93300

HAN-93317

HAN -93328

HAN-93334

HAN-93335

HAN-93372

HAN-93379

HAN-93402

HAN -934021

HAN- 93417

HAN- 93458

HAN-93460

HAN-93464

HAN- 93485

HAN-93551

HAN- 93558

HAN-93569

HAN-93628

HAN-93629

HAN-93630

HAN- 93640

HAN-93647

HAN-93648

HAN-93665

HAN-93677

HAN-9372601

HAN-9372602

HAN-9372603

HAN-9372604

HAN-9372605

HAN-9372606

HAN-9372607

HAN -9372608

HAN-9372609

HAN- 9372610

HAN-9372611

HAN-9372612

HAN-93773
APPENDIX 1

MONTHLY STATUS \& PROGRESS REPORT FOR NOV 1965

AMALYSIS OF DISSOLVER SOLUTIONS

U-233 PRODUCTION COST STLDY

CONTINUED FRINGE THORIA LOADINGS

WM PRODUCTION REPORT - NOV 1965

MM PROOUCTION MONTHLY REPORT - NOV 1965

N-REACTOR DEPARTMENT MONTHLY REPORT

MOVEMBER 1965

QAP QUALITY REPORT FOR DECEMBER 1965

APPENDIX 1

DUN MONTHLY REPORT NOVEMBER 1965

U-233 MANUFACTURING COSTS

PROPOSAL FOR MAMAGEMENT IF FACILITY

CAPABILITY PROJECT SAFETY OF HANFORD PRODUCTION REACTORS UTILIZING HIGHTLY ENR FUEL

NONDEFENSE PLUTONIUN RESUME 12/29/65

BRANDY SHIPPING PAPERS 1966

MONTHLY STATUS \& PROGRESS REPORT FOR DEC 1965

NM PRODUCTION REPORT - DEC 1965

ISOCHEM INC COMPOSITION OF TAKEOVER INVENTORY

MATERIAL BALANCE REPORT - DECEMBER 1966 COMPOSITION OF ENDING INVENTORY - DEC 1965

VERIFICATION OF SHUTDOWN PRODUCTION REACTORS

FINAL INVENTORIES CPD- DECEMBER 1965

MATERIAL BALANCE REPORT - DEC 1965

COMPOSITION OF ENDING INVENTORY - DEC

1966

DUN MONTHLY REPORT DECEMBER 1965

NM PROOUCTION REPORT - DEC 1965

METALLURGICAL BRANCH MONTHLY REPORT 1966 METALLURGICAL BRANCH MONTHLY REPORT 1966 METALLURGICAL BRANCH MONTHLY REPORT 1966 METALLURGICAL BRANCH MONTHLY REPORT 1966 METALLURGICAL BRANCH MONTHLY REPORT 1966 METALLURGICAL BRANCH MONTHLY REPORT 1966 METALLURGICAL BRANCH MONTHLY REPORT 1966 METALLURGICAL BRANCH MONTHLY REPORT 9966 METALLURGICAL BRANCH MONTHLY REPORT 1966 METALLURGICAL BRANCH MONTHLY REPORT 1966 METALLURGICAL BRANCH MONTHLY REPORT 1986 METALLURGICAL BRANCH MOMTHLY REPORT 1966 COMPARATIVE REACTOR PERFORMANCE - NOV 1965
SECR

SECR

$12 / 07 / 65$

$12 / 13 / 65$

AEC-RL

SRD

$12 / 07 / 65$

12/10/65

$12 / 13 / 65$

$12 / 10 / 65$

$12 / 09 / 65$

RL DICKEMAN

SECR

SECR

SRD

$12 / 10 / 65$

SECR

SECR

SECR

SECR

SECR

$12 / 20 / 65$

$12 / 20 / 65$

$12 / 15 / 65$

$12 / 29 / 65$

$12 / 29 / 65$

RG RADAR

$\begin{array}{ll}\text { SECR } & 12 / 30 / 65 \\ \text { SECR } & 04 / 12 / 66 \\ \text { SECR } & 01 / 13 / 66 \\ & \\ \text { SECR } & 01 / 10 / 66 \\ \text { SECR } & 01 / 10 / 66 \\ & \\ \text { SECR } & 01 / 13 / 66 \\ \text { SECR } & 01 / 13 / 66 \\ & \\ \text { SECR } & 01 / 07 / 66 \\ & \\ \text { SECR } & 12 / 31 / 66 \\ \text { SECR } & 01 / 11 / 66 \\ \text { SECR } & 01 / 13 / 66 \\ & \\ \text { SECR } & 01 / 14 / 66 \\ \text { SECR } & 01 / 14 / 66 \\ \text { SECR } & 02 / 18 / 66 \\ \text { SECR } & 02 / 18 / 66 \\ \text { SECR } & 02 / 18 / 66 \\ \text { SECR } & 02 / 18 / 66 \\ \text { SECR } & 02 / 18 / 66 \\ \text { SECR } & 02 / 18 / 66 \\ \text { SECR } & 02 / 18 / 66 \\ \text { SECR } & 02 / 18 / 66 \\ \text { SECR } & 02 / 18 / 66 \\ \text { SECR } & 02 / 18 / 66 \\ \text { SECR } & 02 / 18 / 66 \\ \text { SECR } & 02 / 18 / 66 \\ \text { SECR } & 02 / 01 / 66 \\ & \\ & \\ & \\ & \end{array}$


Page No. 140

$04 / 28 / 93$

LIST OF HANFORD-GEMERATED CLASSIFIED

DOCUMENTS DATED BETHEEN 1/1/61 AND 12/31/72

DOC. NO.

TITLE

AUTHOR

LEVEL

PGS

DATE

HAN-93776

HAN-93802

HAN-93803

HAN- 93804

HAN-9381201

HAN-9381202

HAN-9381203

HAN-9381204

HAN-9381205

HAN- 9381206

HAN-9381207

HAN-9381208

HAN-9381209

HAN-9381210

HAN-9381211

HAN-9381212

HAN-93818

HAN-93828

HAN- 93835

KAN-9385501

HAN-9385502

HAN -9385503

HAN- 9385504

HAN-9385505

HAN-9385506

HAN-9385507

HAN-9385508

HAN-9385509

HAN -9385510

HAN-9385511

HAN-93855512

HAN -9386501

HAN-9386502

HAN-9386503

HAN-9386504

HAN -9386505

HAN-9386506

HAN-9386507

HAN- 9386508

HAN-9386509

HAN-9386510

HAN-9386511

HAN-9386512

HAN-93879
PLUTONIUM SCRAP FROM BATTELLE OHIO MONTHLY STATUS \& PROGRESS REPORT FOR JAN 1966

MOOEL 74-C WEAPOUS FLOW --

COMPARATIVE REACTOR PERFORMANCE - DEC

1965

PROOUCTION REPORT FOR YEAR 1966

PROOUCTION REPORT FOR YEAR 9966

PROOUCTION REPORT FOR YEAR 1966

PROOUCTION REPORT FOR YEAR 1966

PRODUCTION REPORT FOR YEAR 1966

PRODUCTION REPORT FOR YEAR 1966

PRCOUCTION REPORT FOR YEAR 1966

PROOUCTION REPORT FOR YEAR 1966

PRODUCTION REPORT FOR YEAR 1966

PRODUCTION REPORT FOR YEAR 1966

PRODUCTION REPORT FOR YEAR 1966

PROOUCTION REPORT FOR YEAR 1966

PLUTONIUM FOR PEACEFUL PROGRAMS

FP BARAMOUSKI

30, 1966

NM PRODUCTION REPORT - JAN 1966

CHEM PROCESSING MONTHLY REPORT - JAN

1966

CHEM PROCESSING MONTHLY REPORT - JAN

THRU DEC 1966

CHEM PROCESSING MONTHLY REPORT - MARCH

1966

200 AREA MONTHLY REPORT - APRIL 1966

200 AREA MONTHLY REPORT - MAY 1966

200 AREA MONTHLY REPORT - JUN 1966

200 AREA MONTHLY REPORT - JUL 1966

200 AREA MONTHLY REPORT - AUG 1966

CPD MONTHLY REPORT - SEP 1966

CPD MONTHLY REPORT - OCT 1966

CPD MONTHLY REPORT - MOV 1966

COB MONTHLY REPORT - DEC 1966

N-REACTOR MONTHLY REPORT - JAN 1966

N-REACTOR MONTHLY REPORT

N-REACTOR MOWTHLY REPORT

N-REACTOR MONTHLY REPORT

N-REACTOR MONTHLY REPORT

N-REACTOR MONTHLY REPORT

N-REACTOR MOWTHLY REPORT

N-REACTOR MONTHLY REPORT

N-REACTOR MONTHLY REPORT

N-REACTOR MONTHLY REPORT

N-REACTOR MONTHLY REPORT

N-REACTOR MONTHLY REPORT

COST ESTIMATE FOR NPR TARGET EXTRACTION

$\begin{array}{ll}\text { SECR } & 02 / 02 / 66 \\ \text { SECR } & 04 / 11 / 63 \\ \text { SECR } & 02 / 04 / 66 \\ \text { SECR } & 12 / 01 / 65 \\ & \\ \text { SECR } & 02 / 12 / 66 \\ \text { SECR } & 03 / 12 / 66 \\ \text { SECR } & 04 / 12 / 66 \\ \text { SECR } & 05 / 12 / 66 \\ \text { SECR } & 06 / 12 / 66 \\ \text { SECR } & 07 / 12 / 66 \\ \text { SECR } & 08 / 12 / 66 \\ \text { SECR } & 09 / 12 / 66 \\ \text { SECR } & 10 / 12 / 66 \\ \text { SECR } & 11 / 12 / 66 \\ \text { SECR } & 12 / 12 / 66 \\ \text { SECR } & 01 / 12 / 67 \\ \text { SECR } & 02 / 03 / 66 \\ \text { SECR } & 02 / 03 / 66 \\ \text { SECR } & 02 / 08 / 66 \\ \text { SECR } & 02 / 10 / 66 \\ \text { SRD } & 03 / 10 / 66\end{array}$

SECR

$04 / 12 / 66$

SECR

SECR

SECR

SECR

SECR

SECR

SECR

SECR

SECR

SECR

SECR

SECR

SECR

SECR

SECR

SECR

SECR

SECR

SECR

SECR

SECR

SECR
$05 / 12 / 66$

$06 / 13 / 66$

$07 / 14 / 66$

$08 / 09 / 66$

$09 / 09 / 66$

$10 / 12 / 66$

$11 / 09 / 66$

$12 / 19 / 66$

$01 / 11 / 67$

$02 / 10 / 66$

$02 / 10 / 66$

$02 / 90 / 66$

02/10/66

$02 / 10 / 66$

$02 / 10 / 66$

$02 / 10 / 66$

$02 / 10 / 66$

$02 / 10 / 66$

$02 / 10 / 66$

$02 / 10 / 66$

$02 / 10 / 66$

$02 / 07 / 66$ 
Page No. 141

04/28/93

DOCUMENTS DATED BETWEEN 1/1/61 AND 12/31/72
DOC. NO.

HAN-938791

HAN-93888

HAN-93894

HAN-93931

HAN-93950

HAN-93973

HAN-94040

HAN-94068

HAN- 94078

HAN-94114

HAN -94127

HAN-94142

HaN- 94154

HAN-94277

HAN-94291

HAN- 94310

HAN-94330

HAN-94333

HAN- 94336

HAN-94350

HAN-94362

HAN-94374

HAN-94406

HAN- 94432

HAN-94453

HAN-94559

HAN $=94591$

HAN -94605

HAN-94607

HAN-94633

HAN-94647

HAN-94671

HAN-94715
TITLE

AMMUAL COST ESTIMATE MPR TARGET

PROCESSING

COMPARATIVE REACTOR PERformanCE - JAN

1966

NM PRODUCTION REPORT - JAN 1966

DUN MONTHLY REPORT JAMUARY 1966

N-REACTOR DEPT MONTHLY REPORT JAN 1965

TRANSFER OF CPD SS INVENTORIES FRON THE

GEMERAL ELECTRIC COMPANY (SS FACILITY

HZA) TO ISOCHEM INC. (SS FACILITY HWA)

MOWTHLY STATUS \& PROGRESS REPORT FOR FEB 1966

NM PRCOUCTION REPORT - FEB 1966

COST ESTIMATE FOR MPR TARGET EXTRACTION

NM PRCOUCTION REPORT - FEB 1966

N-REACTOR DEPT MONTHLY REPORT FEB 1965

COMPARATIVE REACTOR PERFORMANCE - FEB

1966

DUN MONTHLY REPORT FEBRUARY 1966

NPR TARGET EXTRACTION

ESTIMATED COSTS TO SHIP TARGETS TO

DUPONT AT SROO

WASTE DISPOSAL. INSPECTION REPORT OF I SOCHEM INC.

MONTHLY STAYUS \& PROGRESS REPORT FOR MAR 1966

ANALYTICAL RESULTS FOR SHIPMENT

AMA-ASA-5

WASTE DISPOSAL INSPECTION REPORT

MM PROOUCTION REPORT - MAR 1966

HIGH EXPOSURE PLUTONIUM FOR ZPPR

WASTE PACKAGING STUOY COMMERCIAL VS

WASTE PACKAGING

NM PROOUCTION REPORT - MAR 1966

DUN HONTHLY REPORT MARCH 1966

COMPARATIVE REACTOR PERFORMANCE - MARCH

1966

HIGH EXPOSURE PLUTOWIUN FOR ZPPR

MONTHLY STATUS \& PROGRESS REPORT FOR APR 1966

NM PRCOUCTION REPORT - APR 1966

STUDY OF APPLICATIONS FOR HANFORD

PROOUCED COBALT -60

RECYCLE OF NORMAL URANIUN FOR INCREASING

N -237 \& P -238 PROOUCTION

NM PRODUCTION REPORT - APR 1966

DUN MONTHLY REPORT APRIL 1966

N-REACTOR ADDITIONAL BOILER CAPACITY

STEAM ISOLATION GATE VALVES AND PRIMARY

PUMP DRIVE TURBINE REPAIRS

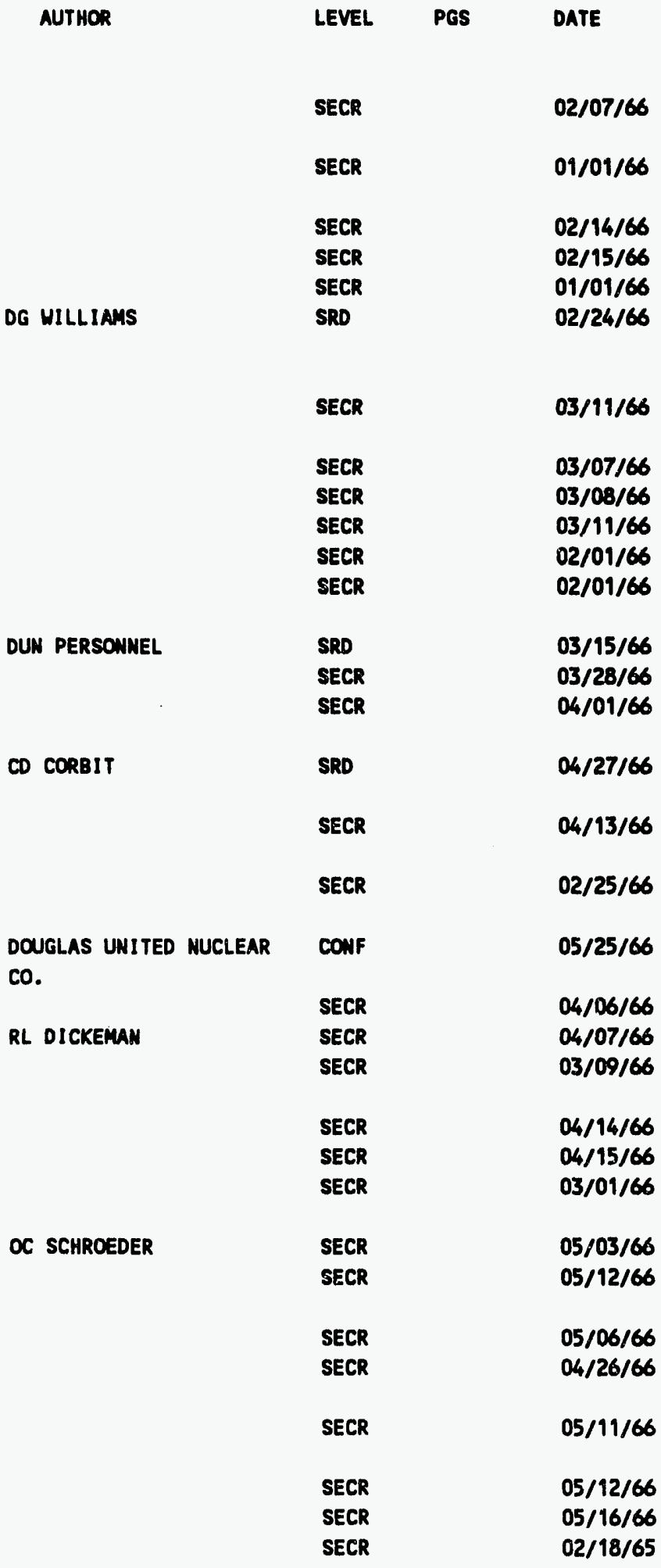


Page No. 142

$04 / 28 / 93$

LIST OF HANFORD-GENERATED CLASSIFIED

DOCUMENTS DATED BETHEEN 1/1/61 AND 12/31/72

DOC. NO.

HAN-947151

HAN- 94747

HAN-94752

HAN-94762

HAN- 94805

HAN-94842

HAN- 94869

HAN- 94887

HAN- 94888

HAN- 94893

HAN- 94911

HAN-94918

HAN-94924

HAN- 94945

HAN-94961

HAN-94976

HAN-95019

HAN-95090

HAN-95105

HAN-95111

HAN-95124

HAN-95135

HAN-95136

HAN-95156

HAN-95157

HAN-95168

HAN-95170

HAN-95171

HAN-95172

HAN-95178

HAN-95184

HAN-95208

HAN-95213
TITLE

N-REACTOR ADDITIONAL BOILER CAPACITY STEAM ISOLATION GATE VALVES AND PRIMARY PUAP DRIVE TURBINE REPAIRS

PUREC SEFOR NOV-DEC 1966

COMPARATIVE REACTOR PERFORMANCE - APRIL

1966

ENGINEERING STUDY THORIUN PROCESSING

PUREX PT 2 NUCLEAR AMD CHEMICAL SAFETY

MON DEFENSE P DURING CO-PRCOUCT

DEMONSTRATION

MONTHLY STATUS \& PROGRESS REPORT FOR MAY 1966

THORIA PROCESSING STATUS REPORT MAY 1966

NM PROOUCTION REPORT - MAY 1966

SUPP CRITICALITY AND CHEM HAZARDS

CONTROL SPEC. TRANSP. AND STORAGE OF

URANIUN 233

U-233 AMALYSIS

HIGH EXPOSURE PLUTONIUM FOR ZPPR

NM PRCOUCTION REPORT - MAY 1966

INCREASED GRAPHITE TEMPERATURE LIMITS B,

C, \& D REACTORS

DUN MONTHLY REPORT MAY 1966

PERSONAL NOTEBOOK OF CARL D. CORBIT

SPECIAL NUCLEAR MATERIALS DRAFT A-03-638

ANALYTICAL DATA FOR N-REACTOR MARK I

FUEL ELEMENTS - KEY NO. 7671

COMPARATIVE REACTOR PERFORMANCE - MAY

1966

MONTHLY STATUS \& PROGRESS REPORT FOR JUN

1966

NM PRODUCTION REPORT - JUN 1966

ANALYSIS OF DISSOLVER SOLUTION

COMPOSITION OF ENDING INVENTORY - JUN

1966

MATERIAL BALANCE REPORT - JUNE 1966

MM PROOUCTION REPORT - JUN 1966

DUN MONTHLY REPORT JUNE 1966

COMPOSITION OF ENDING INVENTORY - JUN

1966

NUCLEAR MATERIALS BALANCE REPORT JUNE

1966

MATERIAL BALANCE REPORT - JUN 1966

DUN COMPOSITION OF ENDING IAVENTORY JUN 1966

BUDGET STUDY

SHIPMENT OF PLUTONIUM METAL TO PACIFIC

NH.

U-233 FOR DNR

ANALYSIS OF LINE 60 AND PU SCRAP

INVENTORY FOR JCAE HEARINGS

\section{AUTHOR}

LEVEL

PGS

DATE

RL DICKEMAN

DG MILLIAMS

CD CORBIT

AEC-RL

SECR

05/23/66

$03 / 24 / 66$

$04 / 01 / 66$

05/27/66

$06 / 01 / 66$

$06 / 13 / 66$

$06 / 08 / 66$

$06 / 13 / 66$

$06 / 13 / 66$

$06 / 13 / 66$

05/26/66

$06 / 15 / 66$

$06 / 16 / 66$

$06 / 15 / 66$

$06 / 21 / 66$

$06 / 21 / 66$

$06 / 02 / 66$

$07 / 18 / 66$

07/18/66

$07 / 15 / 66$

$07 / 13 / 66$

$07 / 14 / 66$

SECR

$07 / 14 / 66$

$07 / 15 / 66$

$07 / 15 / 66$

$07 / 18 / 66$

$07 / 18 / 66$

SECR

$07 / 14 / 66$

$07 / 14 / 66$

WE JOHNSON

SECR

SECR

$10 / 18 / 65$

$07 / 19 / 66$

SECR

07/25/66

$07 / 26 / 66$ 
DOC. NO.

HAN-95218

HAN-95224

HAN-95230

HAN -95239

HAN- 95243

HAN-952431

HAN-952432

HAN-95284

HAN-95296

HAN-95313

HAN- 95322

HAN-95340

HAN-95341

HAN-95347

HAN-95348

HAN- 95350

HAN- 95351

HAN-95393

HAN-95462

HAN-95491

HAN-95502

HAN-95507

HAN-95514

HAN- 95524

HAN-95539

HAN-95555

HAN-95558

HAN-95571

HAN-95575

HAN-95599

HAN-95614

HAN-95623
TITLE

COMPARATIVE REACTOR PERformaMCE - JUNE

1966

COST STATEMENT PROOUCTION INVENTORIES

AND SS MATERIALS IN RESEARCH JUNE 1966

HIGH EXPOSURE PLUTOWIUM FOR ZPPR

APPENOIX J NEW PROOUCTION REACTOR ADDITIONAL BACK-UP TO INDICATED SAVINGS

OF $\$ 1.3$ MILLION IN FUEL COSTS

NOW DEFENCE $P$ DURING CO-PROOUCT

DEMONSTRATION

NON DEFENCE $P$ DURING CO-PRODUCT

DEMONSTRATION

MOWTHLY STATUS \& PROGRESS REPORT FOR JUL

1966

COMPOSITION OF ENDING INVENTORY - JUL

1966

PRCOUCTION SUMMRY

NM PRODUCTION REPORT - JUL 1966

NM PROOUCTION REPORT - JUL 1966

A PROGRAM FOR PROOUCTION OF CERTAIN MON DEFENSE PLUTONIUM MEEDS IN DUN REACTORS

DUN MONTHLY REPORT JULY 1966

RECYCLE OF NORMAL URANIUN AT RL

THORIA PROCESS CAMPAIGN FY 1966

THORIA PROCESS CAMPAIGN FY 1968

NUCLEAR MATERIALS ACCOUNTING FINAL

ENDING INVENTORY REPORT AND TRIAL

BALANCE

SCRAP AND SS MATERIAL WASTE FOR BURIAL

AT RICHLAND

MONTHLY STATUS \& PROGRESS REPORT FOR AUG

1966

COMPARATIVE REACTOR PERFORPANCE - JULY

1966

SHIPMENT OF PLUTONIUM METAL TO PACIFIC

NH. LABORATORY

NM PRODUCTION REPORT - AUG 1966

PLUTONILN ASSAY VARIATION STLOY

STRONTIUN-9O

DUN MONTHLY REPORT AUG 1966

NM PRCOUCTION REPORT - AUG 1966

PRODUCTION REACTOR EQUATIONS

OPERATION KW REACTOR OUTSIDE OPERATING

LIMITS

PRICE SCHEDULE FOR SLIGHTLY ENRICHED

SCRAP

COMPARATIVE REACTOR PERFoRMANCE - AUGUST

1966

RECYCLE OF NORMAL URANIUN FOR INCREASING

N $-237 \&$ P -238 PRODUCTION
AUTHOR

LEVE:

PGS

Date

DG WILLIAMS

SECR

06/01/66

SECR

$06 / 01 / 66$

SECR

$07 / 29 / 66$

$08 / 01 / 66$

$08 / 01 / 66$

SECR

SECR

$10 / 11 / 66$

SECR

SECR

$10 / 11 / 66$

08/01/66

SECR

$08 / 10 / 66$

SECR

SECR

SECR

SECR

SECR

SECR

SECR

SECR

SECR

$08 / 11 / 66$

$08 / 15 / 66$

$08 / 15 / 66$

08/05/66

$08 / 15 / 66$

07/05/66

$08 / 16 / 66$

$08 / 16 / 66$

$08 / 24 / 66$

SECR

$08 / 31 / 66$

SECR

$09 / 15 / 66$

SECR

$09 / 13 / 66$

SECR

$09 / 13 / 66$

RW BOWN

SECR

SECR

SECR

SECR

SECR

SECR

SECR

SECR

SECR

SECR
08/01/66

$09 / 14 / 66$

$09 / 01 / 66$

$09 / 16 / 66$

$09 / 15 / 66$

$09 / 15 / 66$

09/21/66

$09 / 20 / 66$

$09 / 22 / 66$

$09 / 30 / 66$ 
Page No. 144

$04 / 28 / 93$

LIST OF HANFORD-GENERATED CLASSIFIED

DOCUMENTS DATED BETMEEN 1/1/61 AND 12/31/72

DOC. NO.

HAN- 95695

HAN-95710

HAN-95720

HAN-95721

HAN-95754

HAN-95756

HAN-95758

HAN-95T73

HAN- 95806

HAN-95855

HAN-95857

HAN-95863

HAN-95878

HAN-95879

HAN-95880

HAN-95881

HAN-95918

HAN-95979

HAN-95989

HAN- 96016

HAN-96038

HAN-96065

HAN-96071

HAN-96072

HAN-96136

HAN-96143

HAN-96169

HAN- 96176

HAN-96198

HAN- 96204

HAN-96208

HAN-96238

HAN-96247

HAN-96261
TITLE

MONTHLY STATUS \& PROGRESS REPORT FOR SEP 1966

EFFLUENT LIMITS

PACKAGED STRONTIUN AND CESIUN CONTAIMER

CRITERIA AND B-PLANT STRONTIUM AND

CESIUM PROOUCTION AND STORAGE

LIMITATIONS

NM PRCOUCTION REPORT - SEP 1966

DUN MONTHLY REPORT SEPT 1966

ZPPR PROOUCTION SCHEDULE FOLLOUING THE

AVAILIBILITY OF DEPLETED URAMILM

NM PROOUCTION REPORT - SEP 1966

HAPO MONTHLY REPORT SEPT 1966

DISCUSSION OF PP BCE TO 231-2 BLDG

SHEATH PROGRAM AUTHORITY

COMPARATIVE REACTOR PERFORMANCE - SEPT

1966

CHANGE IN FUEL IRRADIATION PROGRAM

B-PLANT SOLUTION STORAGE CAPABILITY.

ISOCHEM PROPOSAL TO PACKACE AND STORE

STRONTIUN AND CESIUM IN FPCE FOR THE

WASTE MAHAGEMENT PROGRAM.

B- -U-PLANT PACKAGING AND STORAGE.

STRONTIUM AND CESIUN FEED AVAILABILITY

FOR GUARANTEES.

MONTHLY STATUS \& PROGRESS REPORT FOR OCT

1966

NM PROOUCTION REPORT - OCT 1966

NM PROOUCTION REPORT - OCT 1966

CLASSIFICATION 100 AREAS CHEMICAL

DISCHARGES

DUN MONTHLY REPORT OCT 1966

COMPARATIVE REACTOR PERFORMANCE - OCT

1966

NOVEMBER 18 MEETING WITH ISOCHEM

DRAFT LETTER OF INTENT

STUOY OF APPLICATIONS FOR HAMFORD

PROOUCED COBALT -60

MONTHLY STATUS \& PROGRESS REPORT FOR MOV 1966

PLUTONIUM ASSAY VARIATION STUDY

MAME PLATE POWER LEVEL CAPABILITY

NM PROOUCTION REPORT - NOV 1966

NM PROOUCTION REPORT - NOV 1966

ASSESSMENT OF PREPAREDNESS TO ENGAGE

CO-PROOUCT LOADINGS IN N-REACTOR

DISPOSAL OF RESIDUAL FISSION PROOUCTS

CONTAIMED IN ZEOLITE CONTAIMERS

HANFORD IRRADIATION REQUEST

DUN MONTHLY REPORT NOV 1966
AUTHOR

LEVEL

PGS

DATE

CD CORBIT

$10 / 13 / 66$

PE REED

SRD

SRD

09/02/66

$10 / 07 / 66$

OC SCHROEDER

SECR

SECR

SECR

10/13/66

$10 / 15 / 66$

$10 / 14 / 66$

SECR

$10 / 14 / 66$

$09 / 01 / 66$

$10 / 20 / 66$

$10 / 18 / 66$

$10 / 28 / 66$

AEC-RL

JN JUDY

SEC?

SRD

SRD

$10 / 27 / 66$

10/31/66

$10 / 31 / 66$

PE REED

SRD

SRD

$10 / 31 / 66$

$12 / 31 / 68$

SECR

$11 / 08 / 66$

SECR

SECR

OC SCHROEDER

SECR

$11 / 11 / 66$

$11 / 15 / 66$

$11 / 18 / 66$

SECR

$11 / 15 / 66$

SECR

10/01/66

SECR

SECR

$11 / 18 / 66$

$11 / 29 / 66$

$11 / 23 / 66$

SECR

$12 / 12 / 66$

HE PARKER

RL PLUM

SECR

SECR

SECR

SECR

SECR

SECR

$12 / 12 / 66$

$12 / 09 / 66$

$12 / 13 / 66$

$12 / 14 / 66$

$12 / 13 / 66$

$12 / 19 / 66$

SECR

SECR
$11 / 08 / 66$

$12 / 15 / 66$ 
Page No. 145

$04 / 28 / 93$

LIST OF MAMFORD-GENERATED CLASSIFIED

DOCLMENTS DATED BETLEEN 1/1/61 AND 12/31/72

DOC. NO.

HAN -96350

HAN- 96368

HAN- 96399

HAN- 96400

HAN- 96403

HAN-96413

HAN-96415

MAN- 96419

HAN- 96420

HAN-96445

HAN- 96454

HAN-96473

HAN- 96483

HAN- 96490

HAN-96506

HAN- 96507

HAN- 96513

HAN-96529

HAN- 96543

HAN- 9655001

HAN-9655002

HAN-9655003

HAN- 9655004

HAN- 9655005

HAN-9655006

HAN-9655007

HAN- 9655008

HAH -9655009

HAN -9655010
TITLE

COMPARATIVE REACTOR PERformance - nOV

1966

MONTHLY STATUS \& PROGRESS REPORT FOR DEC

1966

REVIEN OF CFR TITLE 10 CHARTS $20 \& 100$

FOR POSSIBLE APPLICATION TO HANFORD

REACTOR

COMPOSITION OF ENDING IMVENTORY - DEC

1966

MATERIAL BALANCE REPORT DEC. 1966

COST OF THULILM IRRADIATION

MUCLEAR MATERIALS BALAHCE REPORT

DECEMBER 1966

NM PROOUCTION REPORT - DEC 1966

MATERIAL BALANCE REPORT - DEC 1966

COAPOSITION OF ENDING INVENTORY - DEC

31. 1966

NM PROOUCTION REPORT - DEC 1966

SEMI ANMUAL REPORT - MISSION 1 BASIC RG RADAR

PRCOUCTION

DUN MONTHLY REPORT DEC 1966

FY 1965 LEDGER-NUCLEAR MATERIALS

SUPPLEMENTARY INFO ON MATURAL RECYCLE

MISSION 5 - TRANPLUTONIUN

02 RED SEMIANMUAL REPORT

CO-PROOUCT DEMONSTRATION PROGRAM

SHIELD PROTECTION LOADIMGS HAND-OUTS FOR

BUDGET REVIEW 1967

COMPARATIVE REACTOR PERFORMANCE - DEC

1966

MONTHLY PROOUCTION REPORT JAMUARY THRU

DECEMBER 1967

MONTHLY PROOUCTION REPORT JANUARY THRU

DECEMBER 1967

MONTHLY PROOUCTION REPORT JANUARY THRU

DECEMBER 1967

MONTHLY PROOUCTION REPORT JAMUARY THRU

DECEMBER 1967

MONTHLY PRODUCTION REPORT JANUARY THRU

DECEMBER 1967

MONTHLY PROOUCTION REPORT JANUARY THRU DECEMBER 1967

MONTHLY PROOUCTION REPORT JANUARY THRU

DECEMBER 1967

MONTHLY PROOUCTION REPORT JANUARY THRU

DECEMBER 1967

MONTHLY PROOUCTION REPORT JANUARY THRU

DECEMBER 1967

MONTHLY PRCOUCTION REPORT JANUARY THRU DECEMBER 1967
AUTHOR

RG RADAR

LEVEL POS DATE

SECR

$12 / 28 / 66$

SECR

$01 / 01 / 67$

SECR

$01 / 10 / 67$

SECR

$01 / 13 / 67$

SECR

$01 / 13 / 67$

$01 / 13 / 67$

$12 / 31 / 66$

SECR

$01 / 16 / 67$

$01 / 16 / 67$

01/16/66

01/18/67

$01 / 19 / 67$

$01 / 16 / 67$

$01 / 24 / 67$

01/19/67

$01 / 27 / 67$

01/25/67

$01 / 27 / 67$

$01 / 30 / 67$

RG RADAR

SECR

SECR

$12 / 31 / 66$

SECR

$02 / 15 / 67$

SECR

03/15/67

SECR

$04 / 15 / 67$

SECR

$05 / 15 / 67$

SECR

$06 / 15 / 67$

SECR

$07 / 15 / 67$

SECR

$08 / 15 / 67$

SECR

$09 / 15 / 67$

SECR

$10 / 95 / 67$

SECR

$19 / 15 / 67$ 
Page No. 146

04/28/93

LIST OF MANFORD-GENERATED CLASSIFIED

DOCUNENTS DATED BETWEEN 1/1/61 AND 12/31/72

DOC. NO.

HAN-9655011

HAN-9655012

HAN-96590

HAN-9659501

HAN- 9659502

HAN- 9659503

HAN-9659504

MAN-9659505

HAN-9659506

HAN-9659507

HAN- 9559508

HAN- 9659509

HAN-9659510

HAN-9659511

HAN-9659512

HAN- 9660101

HAN- 9660102

HAN- 9660103

HAN- 9660104

HAN- 9660105

HAN-9660106

HAN- 9660107

HAN- 9660108

HAN- 9660109

HAN- 9660110

HAN-9660111

HAN-9660112

HAN-9662002

HAN-9664001

HAN-9664002

HAN- 9664003
TITLE

MONTHLY PROOUCTION REPORT JANUARY THRU DECEMBER 1967

MONTHLY PROOUCTION REPORT JANUARY THRU DECEMBER 1967

MONTHLY STATUS AND PROGRESS REPORT FOR JAMUARY 1967

METALLURGICAL BRANCH MONTHLY REPORT JAN

- DEC 1967

METALLURGICAL BRANCH MONTHLY REPORT JAN

- DEC 1967

METALLURGICAL BRANCH MONTHLY REPORT JAN

- DEC 1967

METALLURGICAL BRANCH MONTHLY REPORT JAN

- DEC 1967

METALLURGICAL BRANCH MONTHLY REPORT JAN

- DEC 1967

METALLURGICAL BRANCH MONTHLY REPORT JAN

- DEC 1967

METALLURGICAL BRANCH MONTHLY REPORT JAN

- DEC 1967

METALLURGICAL BRANCH HONTHLY REPORT JAN

- DEC 1967

METALLURGICAL BRANCH MONTHLY REPORT JAM

- DEC 1967

METALLURGICAL BRANCH MONTHLY REPORT JAN

- DEC 1967

METALLURGICAL BRANCH MONTHLY REPORT JAN

- DEC 1967

METALLURGICAL BRANCH MONTHLY REPORT JAN

- DEC 1967

CPD MONTHLY REPORT - JAN 1967

CPD MONTHLY REPORT - FEB 1967

CPD MONTHLY REPORT - MAR 1967

CPO MONTHLY REPORT - APR 1967

CPO MONTHLY REPORT - MAY 1967

CPD MONTHLY REPORT - JUN 1967

CPD MONTHLY REPORT - JUL 1967

CPD MONTHLY REPORT - AUG 1967

CPB MONTHLY REPORT - SEP 1967

CPB MONTHLY REPORT - OCT 1967

CPB MONTHLY REPORT - NOV 1967

CPB MONTHLY REPORT - DEC 1967

REACTOR BRANCH MONTHLY REPORT JAN - DEC 1967

N-REACTOR MONTHLY REPORT JAN 1967 THRU

DEC 1967

N-REACTOR MONTHLY REPORT JAN 1967 THRU DEC 1967

N-REACTOR MOWTHLY REPORT JAM 1967 THRU DEC 1967
NUTHOR

LEVEL
SECR

SECR

SECR

SECR

SECR

SECR

SECR

SECR

SECR

SECR

SECR

SECR

SECR

SECR

SECR

SECR

SECR

SECR

SECR

SECR

SECR

SECR

SECR

SECR

SECR

SECR

SECR

RL PLUM

SRD

SECR

SECR

SECR
$12 / 15 / 67$

$01 / 15 / 68$

$02 / 13 / 67$

$02 / 18 / 67$

$02 / 18 / 67$

$02 / 18 / 67$

$02 / 18 / 67$

$02 / 18 / 67$

$02 / 18 / 67$

$02 / 18 / 67$

$02 / 18 / 67$

$02 / 18 / 67$

$02 / 18 / 67$

02/18/67

$02 / 18 / 67$

$02 / 13 / 67$

03/10/67

$04 / 17 / 67$

$05 / 17 / 67$

06/09/67

$07 / 13 / 67$

08/13/67

$09 / 13 / 67$

10/11/67

$11 / 13 / 67$

12/13/67

$01 / 11 / 68$

$03 / 10 / 67$

$01 / 01 / 68$

$01 / 01 / 68$

$01 / 01 / 68$ 
Page No. 147

04/28/93

LIST OF HANFORD-GENERATED CLASSIFIED

DOCUMENTS DATED BETUEEN 1/1/61 AND 12/31/72

TITLE

AUTHOR

LEVEL PGS

DATE

\section{HAN-9664004}

HAN- 9664005

HAN-9664006

HAN- 9664007

HAN- 9664008

HAN- 96643

HAN -96662

HAN- 96692

HAN- $966 \% 4$

HAN- 96700

HAN- 96705

HAN-96706

HAN-96727

HAN-96771

HAN-96780

HAN-96805

HAN- 96910

HAN- 96918

HAN-96927

HAN-96989

HAN-97066

HAN-97163

HAN-97170

HAN-97171

HAN-97259

HAN-97263

HAN -97300

HAN-97392

HAN-97398

HAN-97428

HAN-97486

HAN-97510
N-REACTOR MONTHLY REPORT JAN 1967 THRU DEC 1967

N-REACTOR MONTHLY REPORT JAN 1967 THRU DEC 1967

N-REACTOR MONTHLY REPORT WAN 1967 THRU DEC 1967

N-REACTOR MONTHLY REPORT JAN 1967 THRU DEC 1967

N-REACTOR MONTHLY REPORT JAN 1967 THRU DEC 1967

HANFORD CLASSIFICATION GUIDE REVISIONS

DECRADED DEPLETED 94 URAMIUM

SPECIAL IRRADIATIONS HAMDBOOK

DOUGLAS MONTHLY REPORT JAN 1967

PP DISCHARGE CHUTE CLEARIMG EQUIPMENT $K$ REACTORS

COMPARATIVE REACTOR PERFORMANCE - JAN

1967

PU-238 PROOUCTION DENONSTRATION

HAPO MONTHLY REPORT JAMUARY 1967

PU-REQUIREMENTS AND PRODUCTION ESTIMATES 1963-1972

TABLE - AVERAGE MONTHLY RIVER

TEMPERATURE

MONTHLY STATUS AND PROGRESS REPORT FOR

FEBRUARY 1967

DOUGLAS MOWTHLY REPORT FEB 1967

TARGET SPACE EMHANCEMENT MISSION MEETING

- 3/15/67

PROOUCTION PROGRAM FOR IRRADIATING ALL HANFORD PRODUCED MEPTUNIUM

CO-PROOUCT EXTRACTION FACILITY

MONTHLY STATUS AND PROGRESS REPORT FOR MARCM 1967

THE 68 SPARE CHANMELS AS a genERAL

ISOTOPE PROOUCTION FACILITY

DOUGLAS MONTHLY REPORT MARCH 1967

HAPO MONTHLY REPORT MARCH 1967

NEPTUNIUN SHIPPING PAPERS FOR 1967

COAPARATIVE REACTOR PERFORMANCE - MARCH

1967

MONTHLY STATUS AND PROGRESS REPORT FOR

APRIL 1967

SPECIFICATIONS FOR PU-238

PY AUTHORIZATION -067 150\% SPECIFIC

PONER DEMONSTRATION TEST

NON DEFENSE PLUTONIUN PROOUCTION

COMPARATIVE REACTOR PERFORMANCE - APRIL

1967

HAPD MONTHLY REPORT APRIL 1967

\begin{tabular}{|c|c|c|c|}
\hline \multirow[t]{6}{*}{ AUTHOR } & LEVEL & PGS & DATE \\
\hline & SECR & & $01 / 01 / 68$ \\
\hline & SECR & & $01 / 01 / 68$ \\
\hline & SECR & & $01 / 01 / 68$ \\
\hline & SECR & & $01 / 01 / 68$ \\
\hline & SECR & & $01 / 01 / 68$ \\
\hline \multirow[t]{9}{*}{ RL PLUM } & SECR & & $02 / 10 / 67$ \\
\hline & SECR & & $02 / 15 / 67$ \\
\hline & SECR & & $06 / 15 / 66$ \\
\hline & SECR & & $02 / 15 / 67$ \\
\hline & SECR & & $02 / 20 / 67$ \\
\hline & SECR & & $01 / 01 / 67$ \\
\hline & SECR & & $02 / 11 / 67$ \\
\hline & SECR & & $02 / 10 / 67$ \\
\hline & SECR & & $09 / 03 / 63$ \\
\hline \multirow{3}{*}{$\begin{array}{l}\text { RICHLAND OPERATIOWS } \\
\text { OFFICE }\end{array}$} & SRD & 1 & $03 / 03 / 67$ \\
\hline & SECR & & $03 / 13 / 67$ \\
\hline & SECR & & $03 / 15 / 67$ \\
\hline \multirow[t]{10}{*}{ RG RADAR } & SECR & & $03 / 20 / 67$ \\
\hline & SECR & & $03 / 22 / 67$ \\
\hline & SECR & & $03 / 24 / 67$ \\
\hline & SECR & & $04 / 14 / 67$ \\
\hline & SECR & & $04 / 12 / 67$ \\
\hline & SECR & & $03 / 01 / 67$ \\
\hline & SECR & & $03 / 01 / 67$ \\
\hline & SECR & & $05 / 01 / 67$ \\
\hline & SECR & & $03 / 01 / 67$ \\
\hline & SECR & & $05 / 12 / 67$ \\
\hline \multirow[t]{2}{*}{ CHRISTY JT } & SRD & 2 & $05 / 10 / 67$ \\
\hline & SECR & & $05 / 10 / 67$ \\
\hline \multirow[t]{3}{*}{ RL. PLUM } & SECR & & $05 / 12 / 67$ \\
\hline & SECR & & $04 / 01 / 67$ \\
\hline & SECR & & $04 / 01 / 67$ \\
\hline
\end{tabular}


Page No. 148

$04 / 28 / 93$

LIST OF HANFORI)-GENERATED CLASSIFIED

DOCLMENTS DATED BEIWEEN 1/1/61 AND 12/31/72

Doc. No.

HAN-97568

HAN-97620

HAN-97642

HAN-97791

HAN-97845

HAN-97851

HAN-97978

HAN- 98008

HAN-98031

HAN-98039

HAN-98040

HAN- 98047

наN-98050

HAN-98063

HAN-98077

HAN-98163

HAN-98194 HAN-98195

HAN-98196

HAN-98198

HAN-98208

HAN-98233

HAN-98245

HAN-98247

HAN-98265

HAN-98282

HAN- 98290

HAN-98343

HAN-98409

HAN- 98464

HAN-98483

HAN-98529
TITLE

AUTHOR

JOUGLAS MONTHLY REPORT APRIL 1967

COMPARATIVE REACTOR PERFORMANCE - FEB

1967

K REACTOR ADAINISTRATIVE LIMITS

DELIVERIES FYS 1964 - 1968

MONTHLY STATUS AND PROGRESS REPORT FOR

MAY 1967

CRITICAL MASS ANALYSIS OF THE PROCESSING

OF ZIRCALOY-CLAD FUEL WITH U235

ENRICHMENTS TO 1.15 PER CENT

COMPARATIVE REACTOR PERFORMANCE - MAY

1967

RL NEPTUNIUN PROOUCTION

ISOTOPIC CONTENT OF WEAPONS GRADE

PLUTONIUN

DOUGLAS MONTHLY REPORT MAY 1967

HAPD MONTHLY REPORT MAY 1967

NEPTUNIUN FORECAST RECONCILIATION

MEPTUNIUN CONVERSION RATIOS

THULIUN COAPARABILITY, RL-GETR

THE CASCADE CONSTANTS FOR THE "UO3

STAMDARD VALUES"

MONTHLY STATUS AND PROGRESS REPORT FOR JUNE 1967

MATERIAL BALANCE REPORT

COMPOSITION OF ENDING INVENTORY - JUN

1967

MATERIAL BALANCE REPORT JUME 1967

NUCLEAR MATERIAL BALANCE REPORT JUNE

1967

COEI

UO3 STANDARD VALUES

COMPARATIVE REACTOR PERFORMANCE - JUNE 1967

DOUGLAS MONTHLY REPORT JUNE 1967

PTA-069 NEPTUNIUN 237 PRODUCTION FROM

MATURAL URANIUM HITH A HIGH U-236

CONTENT

DISCARD REQUEST WHL-1080 \&HL-1082

COST STATEMENT PROOUCTION INVENTORIES

AND SS MATERIAL IN RESEARCH JUNE 1967

MONTHLY STATUS AND PROGRESS REPORT FOR JULY 1967

COMPARATIVE REACTOR PERFORMANCE - JULY

1967

DOUGLAS MONTHLY REPORT JULY 1967

ANHUAL APPRAISAL BUDGET PREPARATION AND EXECUTION-DUN

MONTHLY STATUS AND PROGRESS REPORT FOR AUGUST 1967

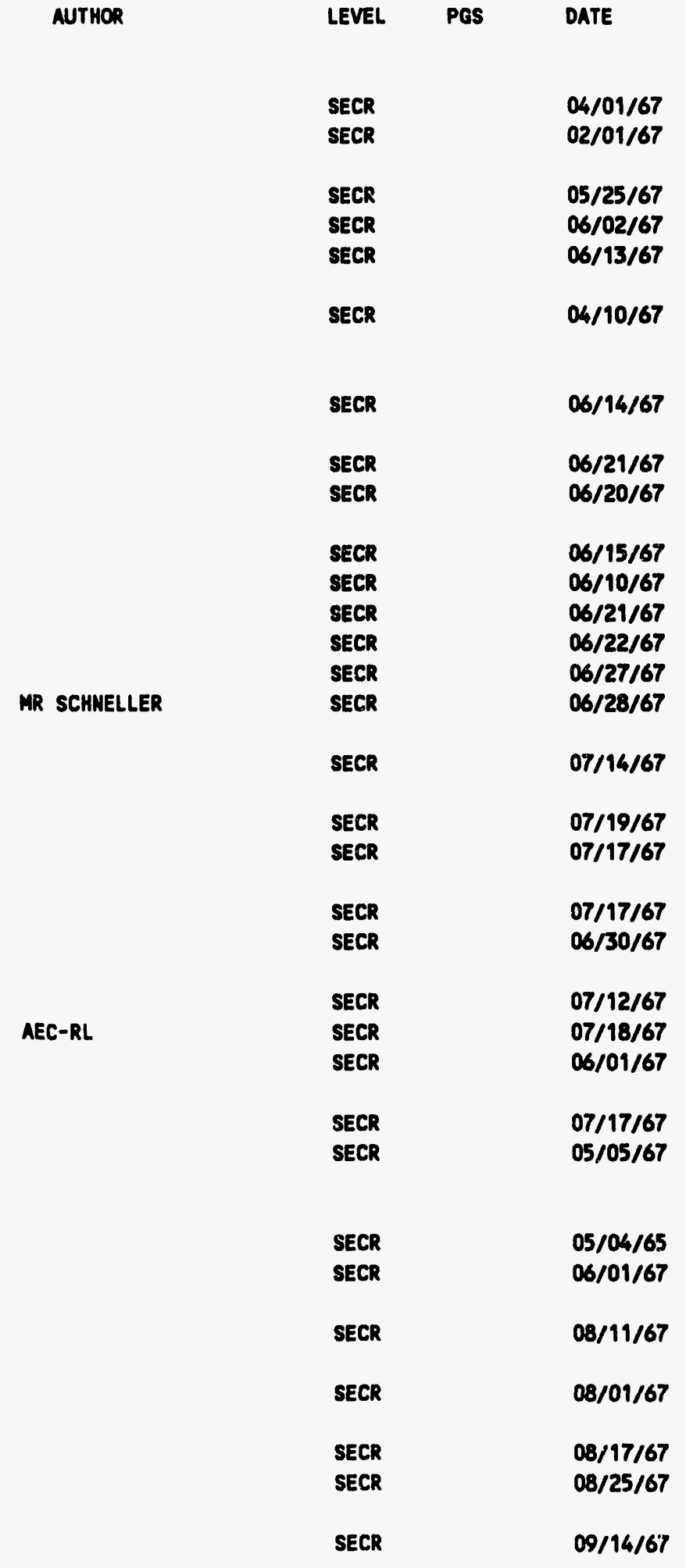


Page No. 149

$04 / 28 / 93$

LIST OF HAMFORD-GEMERATED CLASSIFIED

DOCUMENTS DATED BETWEEN 1/1/61 AND 12/31/72

DOC. NO.

HAN-98530

HAN-98550

HAN-98665

HAN-98677

HAN-98749

HAN-98762

HAN-98819

HAN-98829

HAN-98843

HAN -98860

HAN-98864

HAN-98881

HAN-98918

HAN-98948

HAN-98977

HAN-98980

HAN- 99004

HAN- 99018

HAN- 99049

HAN-99062

HAN-99074

HAN- 99084

HAN- 99085

HAN-99106

HAN- 99123

HAN- 99196

HAN-99250

HAN- 99289

HAN- 99290

HAN- 99297

HAN- 99329

HAN-99396
TITLE

AUTHOR

DG WILLIAMS

INC. (SS FACILITY HWA) TO ARHCO (SS

FACILITY HVA)

MATERIAL BALANCE REPORT SEMI-FISCAL YEAR

ENDING DECEMBER 31, 1967

COAPARATIVE REACTOR PERFORMUNCE - aUG

1967

DOUELAS MONTHLY REPORT AUG 1967

MONTHLY STATUS AMD PROGRESS REPORT FOR SEPTERABER 1967

DESIGN STUDY TRITIUM EXTRACTION FACILITY

100-N INCREASED CAPABILITIES

URANIUM OXIDE RETURNS TO FERMALD

COMPARATIVE REACTOR PERFORMANCE - SEPT

1967

DOUGLAS MONTHLY REPORT SEPT 1967

PWL MONTHLY ACTIVITIES REPORT FOR

SEPTEMBER 1967

COST STATEMENT PRODUCTION INVENTORIES

AND SS MATERIAL IN RESEARCH SEPT 1967

USE OF VERY SLIGHTLY ENRICHED URANIUM

FUEL

MONTHLY STATUS AND PROGRESS REPORT FOR

OCTOBER 1967

DATA OH SAMDERS THULIUM IRRADIATION

URAMIUM OXIDE RETURMS TO FERMALD

U-236 FONECASTING

U-236 FORECASTING

STORAGE OF PLUTONIUM

CHEMICAL AMALYSIS OF DRIVERS FROM 2.1

COPROOUCT BLOCK (PT-66)

COMPARATIVE REACTOR PERFORMANCE - OCT

1967

USE OF SR V-R TAILS

VARING ENRICHMENT OF $V-R$ TAILS

BOILING TANK STATUS AND PROGRAM CHARTS.

N REACTOR CO-PRODUCT DEMONSTRATION

DOUGLAS MONTHLY REPORT OCT 1967

MONTHLY STATUS AND PROGRESS REPORT FOR NOVEMBER 1967

COMPARATIVE REACTOR PERFORMANCE - MOV

1957

DOUGLAS MONTHLY REPORT MOV 1967

PUREX PLANT THORIUM PROCESS OPERATION

REPORT

PROCESSING OF N-REACTOR MATERIAL

UTILIZATION OF SR V R TAILS IN THE $K$ REACTORS

MONTHLY STATUS AMD PROERESS REPORT FOR DECEMBER 1968
SRD

$09 / 01 / 67$

LEVEL PGS DATE

SECR

09/03/67

SECR

$09 / 22 / 67$

SECR

$09 / 18 / 67$

$10 / 12 / 67$

SECR

10/05/67

SECR

10/12/67

SECR

$10 / 19 / 67$

SECR

10/18/67

SECR

10/25/67

SECR

$10 / 01 / 67$

SECR

$10 / 26 / 67$

SECR

$11 / 14 / 67$

SECR

$11 / 03 / 67$

$11 / 09 / 67$

$11 / 10 / 67$

$11 / 17 / 67$

$11 / 09 / 67$

08/02/67

SECR

$1 / 20 / 67$

SECR

$11 / 20 / 67$

$11 / 21 / 67$

$11 / 30 / 67$

$11 / 22 / 67$

$11 / 20 / 67$

$12 / 13 / 67$

SECR

$12 / 15 / 67$

$12 / 15 / 67$

$12 / 01 / 67$

$12 / 21 / 67$

SECR

$12 / 15 / 67$

SECR

$01 / 12 / 68$ 
Page No.

$04 / 28 / 93$

LIST OF HANFORD-GEMERATED CLASSIFIED

DOCUMENTS DATED BETUEEN 1/1/61 AND 12/31/72

DOC. NO

HAN-99423

HAN- 99434

HAN- 99439

HAN- 99442

HAN- 99444

HAN- 99455

HAN-99471

HAN- 99484

HAN-9951401

HAN-9951402

HAN- 9951403

HAN-9951404

HAN-9951405

HAN-9951406

HAN-9951407

HAN-9951408

HAN- 9951409

HAN- 9951410

HAN-9951411

HAN-9951412

HAN- 99595

HAN-9959701

HAN-9959702

HAN -9959703

HAN-9959704

HAN-9959705

HAN-9959706

HAN- 9959707

HAN-9959708

HAN- 9959709

HAN- 9959710

HAN-9959711

HAN-9959712

HAN-9960301

HAN- 9960302

HAN- 9960303

HAN-9960304

HAN- 9960305

HAN-9960306

HAN- 9960307

HAN- 9960308

HAN-9960309

HAN- 9960310

HAN- 9960311

HAN- 9960312

HAN- 99604

HAN-99624
TITLE

COMPOSITION OF ENDING INVENTORY - DEC 31, 1967

MATERIAL BALANCE REPORT - DEC 1967

MBR - DEC 1967

COEI - DEC 1967

COMPARATIVE REACTOR PERFORMANCE - DEC

1967

MEPTUNIUN-237 SPECIFICATIONS

DOUGLAS MONTHLY REPORT DEC 1967

PRCOUCTION TEST AUTHORIZATION 103

N-REACTOR MONTHLY REPORT JAN 1968

N-REACTOR MONTHLY REPORT

N-REACTOR MONTHLY REPORT

N-REACTOR MONTHLY REPORT

N-REACTOR MONTHLY REPORT

N-REACTOR MONTHLY REPORT

N-REACTOR MONTHLY REPORT

N-REACTOR MONTHLY REPORT

N-REACTOR MOWTHLY REPORT

N-REACTOR MOWTHLY REPORT

N-REACTOR MONTHLY REPORT

N-REACTOR MOWTHLY REPORT

ENHANCEMENT OF N -237 PRODUCTION

COP MONTHLY REPORT - JAN 1968

CPD MONTHLY REPORT - FEB 1968

200 AREA MONTHLY REPORT - MAR 1968

COB MONTHLY REPORT - APR 1968

COB MONTHLY REPORT - MAY 1968

COB MONTHLY REPORT - JUN 1968

COB MOWTHLY REPORT - JUL 1968

COB MONTHLY REPORT - AUG 1968

COB MONTHLY REPORT - SEP 1968

COB MONTHLY REPORT - OCT 1968

COB MONTHLY REPORT - NOV 1968

COB MONTHLY REPORT - DEC 1968

MONTHLY PRODUCTION REPORT FOR JAN 1968

MONTHLY PRCOUCTION REPORT FOR FEB 1968

MONTHLY PRODUCTION REPORT FOR MAR 1968 MONTHLY PROOUCTION REPORT FOR APR 1968 MONTHLY PRODUCTION REPORT FOR MAY 1968 MONTHLY PRODUCTION REPORT FOR JUN 1968 MONTHLY PROOUCTION REPORT FOR JUL 1968 MONTHLY PRODUCTION REPORT FOR AUG 1968 MONTHLY PRCOUCTION REPORT FOR SEP 1968 MONTHLY PRODUCTION REPORT FOR OCT 1968 MONTHLY PROOUCTION REPORT FOR NOV 1968 MONTHLY PROOUCTION REPORT FOR DEC 1968 MONTHLY STATUS AND PROGRESS REPORT FOR JAMUARY 1968

TRANSFER JOURNAL FOR FY 1968

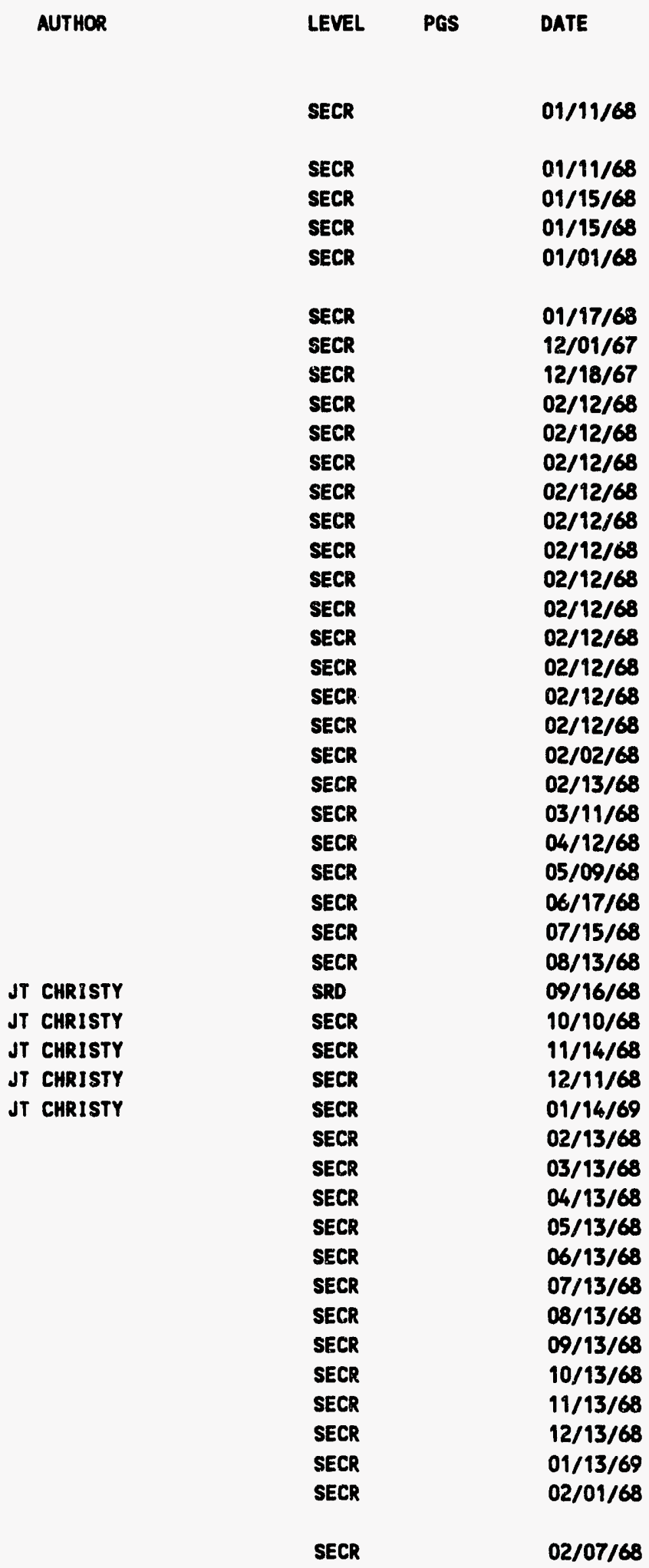


Doc. NO.

HAN-99705

HAN- 99737

HAN- 99765

HAN- 99766

HAN- 99779

HAN-99861

HAN-99870

HAN- 99883

HAN- 99890

HAN- 99965

HAN-99972

HAN-99981

HU-68047

HH-68066

HW-68083

HH-68089

HW-68101

HW-68121

HW- 68124

HU-68126

HH- 68134

HW- 68138

$H W-68176$

HW- 68184

$H W-68197$

HW- -68214

HH-68225
TITLE

COMPARATIVE PILE PERFORMANCE JAMUARY 1968

KE REACTOR FLUX MONITOR TRIP SETTINGS

NONDEFENSE PLUTONILM PROOUCTION

USE OF O-TYPE FUEL INVENTORY

CLASSIFICATION GUIDANCE

NEPTUNIUN DIVERSION FOR $R$ \& D UNDER P

-238 MISSION

MONTHLY STATUS AND PROCRESS REPORT FOR

FEBRUARY 1968

PROGRAM \& FINAMCIAL PLAN PROJECTS \&

BUDGET ASSUMPTIONS FOR FY-1970

RL PLUTONIUN DEMONSTRATIONS

PRICING OF ENRICHED "U" PRCOUCTION SCRAP

COMPARATIVE PILE PERFORMANCE FEBRUARY

1968

DUN LONG RANGE PLAN (ONERVIEW)

PLUTONIUM METALLURGY OPERATION

CONTRIBUTION TO BLDGET FISCAL YEAR 1963

AND REVIEW OF FISCAL YEAR 1962 - 3000

ACCOUNT RESEARCH \& DEVELOPEMEMT

CONVERSION RATIO CONSIDERATION IN THE

E-N FRINGE LOADING

DATA FOR FUEL COST COMPUTATIONS - MPR

PHASES I, II, \& III

EXAMIMATION OF BRAZED CLOSURES,

PT-IP-309-A, SUPP B

100-K AREA DOWNCOMER TEST DATA - PROJECT CGI -883

C REACTOR OVERBORE GRAPHITE TEMPERATURES PROJECT MHITNEY - PLUTONIUM

METALLURGICAL DEVELOPMENT - MONTHLY

REPORT - DECEMBER 1960

CPD PLANT IMPROVEMEKT PROGRAMS

PRODUCTION TEST IP-387-D, IRRADIATION

SERVICE REQUEST HAPO-255, IRRADIATION OF COBALT IN THE KW BOTTOM FRONT-TO-REAR MAGAZINE FACILITY(0074)

USE OF MUCLEAR REACTORS TO PRODUCE HYDRAZINE

FUEL PERFORMANCE COMPARISON OLD REACTORS

- MARCH 1960 THRU DECEMBER 1960

E-N AND BLANKET CONVERSIONS FROM ANALYSIS OF TUBES IRRADIATED AT $H$ SIMPLIFIED PLUTONIUN CONVERSION EXPRESSION

HEAT TRANSFER EXPERIMENTS SIMULATING A FAILURE OF THE INLET PIPING TO A D REACTOR PROCESS TUBE SOME MPE ṔTOOLCTION VARTIABLES
AUTHOR

LEVEL

PGS

DATE

\begin{tabular}{|c|c|c|c|}
\hline & SECR & & $02 / 01 / 68$ \\
\hline & SECR & & $02 / 20 / 68$ \\
\hline & SECR & & $02 / 02 / 68$ \\
\hline & SECR & & $02 / 22 / 68$ \\
\hline \multirow[t]{8}{*}{ DEROUIM JP } & SRD & 2 & $02 / 27 / 68$ \\
\hline & SECR & & $03 / 04 / 68$ \\
\hline & SECR & & $03 / 13 / 68$ \\
\hline & SECR & & $02 / 27 / 68$ \\
\hline & SECR & & $03 / 07 / 68$ \\
\hline & SECR & & $03 / 01 / 68$ \\
\hline & SECR & & $03 / 01 / 68$ \\
\hline & SECR & & $03 / 05 / C$ \\
\hline NELSON TC & SRD & 4 & $01 / 05 / 69$ \\
\hline
\end{tabular}

BOWERS CE

SRD

10

$01 / 09 / 61$

WJ DOWIS

SRD

9

$01 / 09 / 61$

SMITH EA, TVERBERG JC

CRD

17

$01 / 27 / 61$

HUTTON PH

SRD

5

$01 / 10 / 61$

LARSON JR

SRD

WICK OJ

SRD

11

$01 / 13 / 61$

$01 / 18 / 61$

Demers AE

SECR

$06 / 30 / 70$

SRD

5

$01 / 12 / 61$

\begin{tabular}{|c|c|c|c|}
\hline ALBAUGH, FH & SRD & 14 & $01 / 13 / 61$ \\
\hline BLOONSTRAND RR & SRD & 5 & $01 / 16 / 61$ \\
\hline LANG LH, NECHODOH, WS & SRD & 19 & $02 / 10 / 61$ \\
\hline JENSEN RD & SRD & 9 & $01 / 18 / 61$ \\
\hline $\begin{array}{l}\text { WATERS ED, FITZSIMWOWS, } \\
\text { DE }\end{array}$ & SRD & 16 & $01 / 20 / 61$ \\
\hline Sinfsom oE & 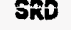 & 3 & $01 / 20 ; 61$ \\
\hline
\end{tabular}


Page No. 152

$04 / 28 / 93$

LIST OF MANFORD-GENERATED CLASSIFIED

DOCUMENTS DATED BETUEEN 1/1/61 AND 12/31/72

DoC. NO.

TITLE

AUTHOR

LEVEL

PGS

DATE

HW- 68260

A STANDARD ALSI BRAZE WETTING TEST FOR

BURGESS, CA

CRD

10

$02 / 01 / 61$

ALUAINUA COAPONENTS USED IN THE LEAD-DIP PROCESS

HU-68262 RD

PROPOSAL FOR CHARGING THE SIXTH RUPTURE FUEL ELEMENT EXPERIMENT: GEH-10: 46, 47

CALL RL, KAULITZ DC CRD

$01 / 25 / 61$

HN-68263

INTERIM REPORT: SOME TEMPERATURE AND SWELLING DATA ON ZIRCOLOY 2 CLAD COEXTRUDED FUEL ELEMENTS

HW-68273

HEAT TRANSFER EXPERIMENTS SIMULATING FRONT HEADER PRESSURE REDUCTIONS TO A K-REACTOR PROCESS TUBE

$H W-68279$ RD HW-68285 HU-68315 ECONONIC PLANT DIRECTION INVENTION REPORT A FISSIOH-PROOUCT SECURITY PROBLEM PRELIMIMARY EVALUATION

HW-68337 APP ARTIFICIAL COOLING OF THE COLUMBIA RIVER BY DAM RECULATION-1960-BENEFITS RESULTING FROM USE OF COLD MATER FROM THE DEPTHS OF GRAND COULEE RESERVOIR- 1960

$H W-68345$ G CHEMICAL PROCESSING DEPARTMENY RESEARCH AND ENGINEERING OPERATION MONTHLY REPORT JANUARY 1961

HW-68357 REVISED REQUIREMENTS FOR CONTINUOUS BIRCH REQUIREMENTS AT REDOX

HU- 68374

THIRD SET OF LONDIHG AND OPERATING CONDITIONS FOR NIN-1 ANO NIE-1 ELEMENTS IN THE KER LOOPS UNDER PT-IP-377-A

HH-68403 IRRADIATION TEST DATA FOR DEVELOPMENT TEST IP-212-D, IRRADIATION SERVICE REQUEST HAPO-212, THE IRRADIATION OF ZIRCONIUN TENSILE SPECIMENS AT INTERMEDIATE TEMPERATURES IN THE KE MAGAZINE FACILITY

HH- 68404 IRRADIATION TEST DATA FOR PT-IP-2\%-D, SWELLIMG TEST OF MATURAL AND THREE PER CENT ENRICHED URANIUM CAPSULES NOS. 4,5, AND 6

HW-68409 SONE FIRE AND FISSION PROOUCT RELEASE POSSIBILITIES FOR DISCHARGED FUEL SLUGS SPECIAL REACTOR MOOIFICATION STUDY

HW- 68418 HWH-68438

$H W-68448 A$ BATCHES FOR THE REVIEW OF PLUTONIUA FORMATION RATES AS A FUNCTION OF MND DESIGN OF PRODUCTION TEST IP-310-A-FP, DETERMINATION OF THE DIMENSIONAL

CALL RL, GEERING GT SRD 4

$01 / 25 / 61$

WATERS ED, FITZSIMMONS DE SRD · 19

$02 / 20 / 61$

LANG LH

SRD

$01 / 30 / 61$

MELSON RD

MCKEE RH

SRD

SRD

$01 / 26 / 61$

$02 / 20 / 61$

SRD

11

$02 / 15 / 61$

BARMES RG

SRD

12

$02 / 02 / 61$

KRATZER WK

SRD

6

$02 / 02 / 61$

MARSHALL RK

SRD

$02 / 06 / 61$

MARSHALL RK

SRD

2

$02 / 06 / 61$

STABILITY OF URANIUM FUEL CORES

CLASSIFIED BY THE FLEL CORE TESTER.

(UT-2)

$\begin{array}{llll}\text { ZIMA GE } & \text { SRD } & 17 & 02 / 07 / 61 \\ \text { TUPPER WJ } & & & \\ \text { ZIMMER WH } & \text { SRD } & 7 & 02 / 20 / 61 \\ & \text { SRD } & 10 & 02 / 09 / 61 \\ \text { HOOGSON WH, CLINTON MA } & & & \\ & \text { SRD } & 13 & 02 / 15 / 61\end{array}$


Page No. 153

$04 / 28 / 93$

LIST OF HANFORD-GENERATED CLASSIFIED

DOCUMENTS DATED BETUEEN 1/1/61 AMD 12/31/72

DOC. NO.

TITLE

AUTHOR

LEVEL

PGS

DATE

$H H-68448 C$

HW-68448 D

HW-68452

HW-68458

HW-68458 REV

HW-68459

HW-68459 REV

HW-68462

HW- 68469

HW-68474

HH-68476

$H W-68486$

HH- 68487

HW- 68499

HU- 68518

HW-68536

HW -68544

HW -68550

HW-68597

HW- 68598

HW-68601
PRODUCTION TEST IP-310-A-FP, DETERMIMATION OF THE DIMENSIONAL STABILITY OF URANIUN FUEL CORES CLASSIFIED BY THE FUEL CORE TESTER (UT-2)

DAILY DATA SHEETS - UT-2 TEST, PT-IP-310-A-FP, D REACTOR MUCLEAR SAFETY SPECIFICATION - REDUOOD CAR

BLAMKET AND E-N ECONOMICS - A LIMEAR PROGRAM STUDY

BLANKET AND E-N ECONONICS - A LIMEAR PROGRAM STLDY

BLANKET AND E-N ECONOMICS - BACKGROUND

DATA AND LINEAR PROGRAM FORIULATIOH

BLANKET AND E-N ECONONICS - BACKGROUND

DATA AND LIMEAR PROGRAM FORIMLLATION

FINAL REPORT ON PRODUCTION TEST

IP-289-I, SUPPLEMENT 1, H REACTOR EXPORT FLOW TEST

PRODUCTION TEST IP-394-I - USE OF PT-216 FUEL COLLANS FOR ROITIME AXIAL FLUX DETERMIMATION

REVISED VARIABLE GOAL EXPOSURE PLANS FOR C-II-N AND C-II-E MATERIAL.

INTERIM GOAL EXPOSURE PLANS FOR 0-1II-NB AND O-III-EB MATERIAL FOR B, D, OR, AND F REACTORS

QUARTERLY PROGRESS REPORT PLUTONIUM

METALLURGY OPERATION JANUARY, FEBRUARY, MARCH, 1961

ATOMIC WEAPON DATA SUPPLEMENT QUARTERLY PROGRESS REPORT - PLUTONIUM METALLURGY OPERATION - JANUARY - MARCH, 1961

100-C WATER PLANT

KW WET CRITICAL LOADING THREE GROUP FLUX FINAL RESULTS OF PRCOUCTION TEST IP-348-I - K AREA LOW-FLON CALIBRATION TEST

IRRADIATED URANIUM FIRE HAZARD

RADIOCHEMISTRY FOR THE RUPTURE OF A ZIRCALOY-2 CLAD URANIUM FUEL ELEMENT IN KER-1

CURRENT CODE WORDS - THEIR DEFINITIONS AND USAGE

PROOUCTICN TEST IP-397-A-FP - COEXTRLDED

ZIRCALOY CLAD ENRICHED I \& E FUEL ELEMENTS

HATER SOLUBLE PROTECTIVE COATING FOR

FUEL ELEAENTS - FINAL REPORT -

PRCOUCTIOA TEST IP-308-F
CLINTON MA

SRD

13

$04 / 27 / 61$

CLINTON MA

SRD

08/17/61

GAST PF

NEEF MI

SRD

2

$02 / 10 / 61$

MEEF MI

SRD

24

$02 / 20 / 61$

SRD $\quad 13$

$09 / 01 / 61$

MEEF MI

SRD

25

$03 / 01 / 61$

MEEF MI

SRD

8

$09 / 01 / 61$

BREMER BR

SRD

5

$07 / 10 / 61$

GRAVES SM

SRD

12

$02 / 13 / 61$

BLOOMSTRAMD RR

CRD

3

$02 / 13 / 61$

BLCOMSTRAMD RR

SRD

4

$02 / 13 / 61$

HICK OJ

SRD

83

$04 / 15 / 61$

MICK OJ

SRD

40

$04 / 94 / 61$

AGAR JF

SIMPSON DE

FULLER ME

SRD

SRD

SRD

$02 / 20 / 61$

$02 / 15 / 61$

02/16/61

REID RH

DEMAITT TF

SRD

SRD

$02 / 15 / 61$

$02 / 28 / 61$

JAYMES RE

SRD

3

$02 / 22 / 61$

KUSLER LE, CLINTON MA

SRD

$03 / 27 / 61$

NESSELSON EJ, COOKE JP SRD 
Page No. 154

$04 / 28 / 93$

LIST OF HANFORD-GENERATED CLASSIFIED

DOCUMENTS DATED BETWEEN 1/1/61 AND 12/31/72

DOC. NO.

TITLE

AUTHOR

LEVEL PGS

DATE

HW- 68605

$H H-68624$ RD $H H-68667$

HW-68674

$H W-68697$

$H W-68707$

$H W-68711$

$H W-68720$

$H 4-68737$

$H W-68746$

$H W-68761$

HW- 68793

HW-68800 REV $H W-68815$

HW-68825

HW-68838 A HH- 68855

$H W-68903$

$H W-68908$ RD HH- 68970

$H H-68970$ B

$H W-69011$
PROJECT WHITNEY - PLUTONIUM

METALLURGICAL DEVELOPNENT MONTHLY REPORT

- JAMUARY 1961

REACTOR MCOIFICATION PROGRAM DATA

IRRADIATION TEST DATA FOR PRODUCTION

TEST IP-149-D

IRRADIATION SERVICE REQUEST HAPO-215, THE IRRADIATION OF URANIUM DIOXIDE

IPD PREDICTED VS CPD MEASUREMENT DIFFERENCES TWELVE TW-TON TEST BATCHES MARCH 29, 1961

COMPARATIVE RETURN FOR OVERBORE AMD E-N LOADINGS

B,D, F, DR REACTORS

POST IRRADIATION EXAMINATION OF

ELLIPTICAL FUEL ELEMENT (RM-416)

COMPARATIVE RETURM FOR OVERBORE AND E-N LOADINGS

CONBINED OPERATION STLDY -- HAPO

EXPOSURE COST RELATIONSHIPS FOR N AND

EXISTING REACTORS

RE-EVALUATION OF METAL PERFORMANCE

LEVELS OF C-II-N AND C-II-E MATERIAL

MEETIHG NOTES ON IMPROVEMENT FROM BUMPER SLUGS

REVIEW MATERIAL FOR REACTOR PHYSICS

PRIMER

INCENTIVES FOR NICKEL PLATING

ALUMINUM-JACKETED FUEL ELEMENTS

1961 PLANT IMPROVEMENT PROGRAM

PROJECT UHITNEY - PLUTONIUM

METALLURGICAL DEVELOPMENT - MONTHLY

REPORT - FEBRUARY, 1961

BUDGET FOR FY 1963 AND REVISION OF

BUDGET FOR FY 1962 PROPOSAL FOR

REASEARCH AND DEVELOPMENT GENERAL

MEAPONS DEVELOPMENT -03-16-04

HAPO PROCESS ECONONICS I

PRODUCTION TEST IP-401-A, IRRADIATION OF ZIRCALOY-2 JACKETED U02 TUBULAR ELEMENTS IN THE KER LOOPS

SAFE DESIGN AND OPERATION OF HANFORD

PROOUCTION REACTORS

OFFSITE EXTRUSION PROGRAM SUMMARY REPORT PROOUCTION TEST IP-402-A, IRRADIATION OF ZR-2 CLADDING STLDIES CAPSULES

PT-IP-402-A ALUMINUM CAPSULES IN DR

DAILY DATA SHEETS

PROJECT CGC-897 - TITLE I DESIGN FISSION PROOUCT STORAGE IN B-PLANT
WICK OJ

FIFER MF

MARSHAL RK

SRD

SRD

67

$03 / 17 / 61$

$02 / 27 / 61$

HOBA DH

SRD

8

03/29/61

MECHCDOM US

SRD

5

$03 / 22 / 6$

GRUBER WJ

CRD

SRD

3

GENINGER AB

SRD

21

$04 / 05 / 61$

BLOOMSTRAND RR

SRD

15

$03 / 03 / 61$

SPENCER HG

SRD

5

$03 / 17 / 61$

LOCKLOOD EH

SRD

124

03/01/61

JACKY GF

SRD

41

$03 / 20 / 61$

HICK OJ

SRD

SRD

26

$04 / 14 / 61$

$03 / 15 / 61$

COOPER VR, SMITH AE

SRD

19

$03 / 10 / 61$

HOUGH CG, SPENCER HG

KRATZER WK

SRD

SRD

84

$05 / 01 / 61$

$03 / 16 / 61$

BROWN JH

SRD

5

03/17/61

hICKOLAUS JH, GUAY AE

CRD

KRATZER WK

SRD

MEBER JW

SRD

CAUDILL HL, ZAHN LL

45

$04 / 01 / 61$

03/24/61

$10 / 04 / 61$

$04 / 03 / 61$ 
Page No. 155

04/28/93

LIST OF HANFORD-GENERATED CLASSIFIED

DOCUNENTS DATED BETHEEN 1/1/61 AND 12/31/72

DOC. NO

HW-69019

HW-69064

HW- 69068

HW-69085

HW- 69087

HW- 69088

HW-69154

HH-69155

HW-69158

HH-69173 RD

HH-69194RD

HH-69202

HW-69216

HW-69223 RD

$H H-69236$ RD

$H W-69285$

HW-69314

HW- 69320

HW-69321

HW-69359

HW-69365

HW-69378

$H W-69390$

HH -69404

HW-69419
TITLE

GORTON LATHE AUTOMATIC SPEED CONTROL POST-IRRADIATION EXAMINATION OF BUMPER ELEMENTS WITH HIGH IN-REACTOR WEIGHT LOSSES (RM-418)

IN-TANK SOLIDIFICATION OF INTERMEDIATE ACTIVITY WASTES

QUARTERLY PROGRESS REPORT FUELS DEVELOPMENT OPERATIOW JULY, AUGUST, SEPTEMBER, 1960

METALLOGRAPHY OF PIT 81 COMPONENTS LOADING AND OPERATING CONDITIONS FOR PT-IP-401-A AND PT-IP-363-A IN KER -1 SUPPLEMENTARY REPORT OF PRELIMINARY TESTING PROOUCTION TEST IP-234-AE SUPPLEMENT 2, PROJECT CG-775, PROTOTYPE TESTING OF THE 190-K PROCESS PLMPING UNITS

CHAPTER 18 OF PRODUCTION CAPACITY STUDY NO. 2 HANFORD

DETERMIMATION OF PU240 BY MEUTRON COUNTING

PROPOSAL FOR CHARGING GEH-10-43 NINI

INTERIM MEASUREMENT TEST IN THE ETR NOTES ON PUREX PALM RECOVERY PROCESS WATER FLOW TESTS - C REACTOR PROJECT MHITMEY - PLUTONIUM METALLURGICAL DEVELOPMENT - MONTHLY REPORT - MARCH, 1961

2D-500 BALL RCD AND PLUG ROD CASTING NOVEMBER, 1960 THROUGH FEBRUARY, 1961 PRELIMINARY STUDY - MINT RECOVERY FACILITIES

POST-IRRADIATION EXAMINATION OF CHEMICALLY NICKEL-PLATED FUEL ELEMENTS FROM PT-IP-263-A (RM-414)

PROCESSING DATA FOR NIE AND NIN KER LOOP CHARGES (PT 3T7)

STUDY PROGRAM - REACTOR MOOIFICATIONS FOR INTERIM FLOW INCREASES

40-TUBE OVERBORE FACILITY LOCATION - C REACTOR

C REACTOR OVERBORE STUDY

REVISION TO OPTIMIZATION CONSTANTS

SUPPLEMENT G, PROOUCTION TEST IP-314-A, MEASUREMENT OF FUEL ELEMENT TEMPERATURE CHANGES AS THE RESULT OF FILM DEPOSITION PCCF FLON ANALYSIS - DR REACTOR SUPPLEMENTAL DATA FOR THE TOPICAL REPORT ON MGCR-HDR-1, 2, AND 3 TESTS

PHYSICS DATA FOR PROPOSED HAPO H-1 LOOP IRRAOIATION

\begin{tabular}{|c|c|c|c|}
\hline AUTHOR & LEVEL & PGS & DATE \\
\hline INKERTON JM & SRD & 16 & $03 / 29 / 61$ \\
\hline SRUBER HJ & CRD & 10 & $04 / 03 / 61$ \\
\hline AMPBELL BF & SRD & 25 & $04 / 03 / 61$ \\
\hline $\begin{array}{l}\text { ADUELL JJ, TOBIN JC, } \\
\text { AST GA, EVANS EA, MINOR } \\
\text { IE }\end{array}$ & CRD & 148 & $10 / 15 / 60$ \\
\hline IELSON TC & CRD & 18 & $04 / 03 / 61$ \\
\hline KRATZER WK & SRD & 3 & $04 / 05 / 61$ \\
\hline СНАСК МН & SRD & 8 & $04 / 06 / 61$ \\
\hline
\end{tabular}

GARREN JH

SRD

19

$04 / 22 / 60$

WING NS

SRD

4

$04 / 04 / 61$

GEERING GT, HECK EN

CRD

6

$04 / 07 / 61$

HAMILTON WD

WICK OJ

SECR

SRD

SRD

$06 / 30 / 70$

$04 / 19 / 61$

$04 / 21 / 61$

BOND RH

SRD

20

$04 / 07 / 61$

GRAF WA

SRD

2

$04 / 12 / 61$

GRUBER Wd

SRD

14

$04 / 17 / 61$

KUSLER LE, HAYS DD

CRD

17

$04 / 19 / 61$

CORLEY JP, HUTTON PH,

SRD

23

$06 / 01 / 61$

PORTER M

NILSON R, NECHODON HS

SRD

4

$04 / 19 / 61$

BUNCH WL

MEYELL LJ, SPENCER HG

SRD

SRD

41

$05 / 15 / 61$

KRATZER WK

SRD

$04 / 25 / 61$

$04 / 25 / 61$

CALKIN JF

CRD

SRD

$04 / 26 / 61$

BENNETT EC

SRD

3

$04 / 27 / 61$

FRANKLIN FC 
Page No. 156

$04 / 28 / 93$

LIST OF HANFORD-GENERATED CLASSIFIED

DOCUMENTS DATED BETUEEN 1/1/61 AND 12/31/72

DOC. NO.

HW-69422

HW-69437

$H W-69499$

HW-69517

HW-69522

HW-69525 RD

$H W-69530$

$H W-69550$ RD

H4-69552

HW-69561

$H W-69566$

HW-69570 RD

HW-69573 RD

HW-69585

HW-69592

HW-69600Z-R3 PT10

HW-69600Z-R3 PT11

HH-69600Z-R3 PT12

HW-69600Z-R3 PT7

HW-696002-R3 PT8

HH-696002-R3 PT9

HW- 69615

HW-69628
TITLE

MEASURED CADMIUM BURNUP IN C REACTOR HCR'S

IRRADIATION TESTING OF TUBULAR FUEL ELEMENTS: FINAL REPORT: PT-IP 292A INTERIM FLOW INCREASES AT B, D, DR, $F$, AND H REACTORS RELATED TO THE SHORT-RANGE WATER PLANT MODIFICATION PROGRAM

PUREX PLANT BIRCH ISOLATION RUN - APR

1961

RECOMIENDED HIGH-TANK TEMPERATURES FOR

MAINTEMANCE OF HIGH-TASK BACKUP SUPPORT, REVISION 1

MATERIAL BUCKLING MEASUREMENTS OF GRAPHITE URANIUN SYSTEMS AT HANFORD

FLON INCREASE - C REACTOR

OLD PILE OPERATION MITH VARING AMOUNTS

OF E-METAL

ACCOUNTABILITY EQUATIONS FOR PLUTONIUN

AND TRITIUN YIELDS FOR PT-IP-305-C AT

105-H

HAZARD ANALYSIS FOR CESIUM SHIPMENTS

PHYSICS OF E-N LOAD COMPARED TO MATURAL URANIUM LOAD AT H REACTOR

HANFORD REWORK CAPABILITY

TRIP REPORT - MEETING ON ISOTOPE SEPARATION AGRONWE MATIONAL LABORATORY MAY 8, 1961

SAFETY REVIEH OF NUCLEAR FUEL MANUFACTURING OPERATIOUS FUELS PREPARATION DEPARTMENT

REPORT ON THE PROJECTION WELDED BRAZED CLOSURE FOR 2IRCALOY-2 CLAD FUEL

ELEMENTS

FINISHED PRCOUCTS - PRODUCT RECOVERY

SOURCE DATA APRIL 1962.

FINISHED PRCOUCTS - PRCDUCT RECOVERY SOURCE DATA MAY 1962

FINISHED PRODUCTS - PRODUCT RECOVERY SOURCE DATA JUNE 1962

FINISHED PROOUCTS - PRODUCT RECOVERY SOURCE DATA JANUARY 1962.

FINISHED PROOUCTS - PRODUCT RECOVERY SOURCE DATA FEBRUARY 1962.

FINISHED PROOUCTS - PRCDUCT RECOVERY SOURCE DATA MARCH 1962.

CRITICAL MASS ESTIMATE FOR STACKED MODEL 81 CASTINGS

FRACTIONAL HEAT GENERATION RATES IN HANFORD REACTORS AFTER SHUTDOWN
AUTHOR

CHITHOOO RA

GEERING GT

BENSON JL, GRAVES SH

SRD

SECR

$06 / 30 / 70$

GREAGER OH

SRD

05/08/61

1000 DE

CRD

05/08/61

TUPPER HJ

LANG LH

SRD

SRD

NECHODON US

SRD

2

$05 / 10 / 61$

HATSON EG, JUNKINS RL, CRD

$05 / 11 / 61$

FUOUAY JJ

MONWIE DI

HOPKINS HH, JR.

VOILAND EE, TOMLINSON RE

SRD

15

$05 / 11 / 61$

SRD

31

$05 / 26 / 61$

$05 / 12 / 61$

MORLTON, DC

CRD

29

$05 / 19 / 61$

05/15/61

GB XUKLINSKI

SECR

$04 / 01 / 62$

GB KUKLINSKI

SECR

05/01/62

GB KUKLINSKI

SECR

06/01/62

GB KUKLINSKI

SECR

$01 / 01 / 62$

GB XUKLIMSKI

SECR

02/01/62

GB KUKLINSKI

SECR

03/01/62

ISAACSON RE, STEVENSON RL SRD

5

$05 / 16 / 61$

NILSON R, MEICHLE RH CRD

20

05/17/61 
Page No. 157

$04 / 28 / 93$

LIST OF HANFORD-GENERATED CLASSIFIED

DOCUMENTS DATED BETUEEN 1/1/61 AND 12/31/72

DOC. NO.

HU-69694 RD

HW-69699

HW-69717

HW-69730

HW-69751

HH-69754

HH-69755

HH- 69766

HW- 69811

HM-69857

HW- 69860

HW -69868

HW-69897

HH-69899

HHY -69904

HW-69912

HW-69918

HW-69934

HW-69936

HU- 69940

HW-69952

HU-69964 A

$H W-69964 C$
TITLE

GEH-4-63, 64

PROPOSAL FOR IRRADIATION OF PRCOUCTION BRAZED ZIRCALOY-2 TIAD FUEL ELEMENTS EVALUATION OF BULK OUTLET TEMPERATURE LIMITS

C-REACTOR GRAPHITE BURNOUT INTERIM REPORT 1P-25A(PT-105-532-E)

CRITICAL MASS CONTROL SPECIFICATION FOR STORING PLUTONIUM METAL IN ROON 172. 234-5 BUILDING

RPR DELAYED NEUTRON FRACTIONS AND DECAY CONSTANTS

GRAPHITE TEMPERATURE PARAMETERS - C

REACTOR OVERBORE

CGI-844 DIESEL PLAP FLON TRANSIENTS

CLASSIFICATION: PLUTONILM

PROJECT HHITMEY - PLUTONIUM

METALLURGICAL DEVELOPMENT - MONTHLY

REPORT - APRIL, 1961

KER-3 OPERATING REPORT TEST K-3-13

PT-IP-363-A

ECONOMICS OF LEAD DIP AND SSDB PROCESSES

TRIP REPORT ROCKY FLATS PLANT AND LOS

ALAMOS SCIENTIFIC LABORATORY MAY 15-18, 1961

PROOUCTION TEST IP-416 HORIZONTAL ROD

CALIBRATIONS - H REACTOR

DR-1 GAS LOOP THERMAL AND FLUID FLON

ANALYSES CLASSIFIED SUPPLEMENT

NOTES ON REACTOR OPERATION WITHIN A

PROOUCT SPECIFICATION

PROOUCT QUALITY AND AN INTERIM GOAL

EXPOSURE PLAN

PERFECT FUEL-ELEMENT INCENTIVES

PRCOUCTION YIELD DATA AS A FUNCTION OF GRAPHITE TEMPERATURE

PROJECT UHITNEY - PLUTONIUM

METALLURGICAL DEVELOPMENT - MONTHLY

REPORT - MAY, 1961

ANALOG PROGRAM IN REACTOR SPEED OF

CONTROL STUDIES

RADIOGRAPHIC PROCEDURES FOR PARTS 8105,

$8106,8107,8108$ AND 8109

DESIGN OF PRODUCTION TEST-IP-409-A-FP

PILOT TEST OF SELF-SUPPORTED FUEL

ELEMENTS IN K SIZE SMOOTHBORE ZIRCONIUM PROCESS TUBES

PRODUCTION TEST-IP-409-A-FP PILOT TEST

OF SELF-SUPPORTED FUEL ELEMENTS IN $K$

SIZE SMOOTH-BORE ZIRCONIUM PROCESS TUBES

\begin{tabular}{|c|c|c|c|}
\hline AUTHOR & LEVEL. & PGS & DATE \\
\hline TVERBERG JC & CRD & 15 & $05 / 18 / 61$ \\
\hline POOR CF & SRD & 21 & $05 / 23 / 61$ \\
\hline RYAN BA & SRD & 20 & $04 / 05 / 61$ \\
\hline MEEKER LM & SRD & 6 & $06 / 01 / 61$ \\
\hline ALLEN CH & CRD & 7 & $05 / 26 / 61$ \\
\hline AEAR JD & SRD & 12 & $06 / 12 / 61$ \\
\hline ACAR JD & SRD & 21 & $08 / 10 / 61$ \\
\hline BOREMIER BJ & SRD & 2 & $06 / 01 / 61$ \\
\hline WICK OJ & SRD & 8 & $04 / 30 / 61$ \\
\hline RICHARDSON CA & SRD & 12 & $06 / 12 / 61$ \\
\hline $\begin{array}{l}\text { DARSOW DY, KREIG SA } \\
\text { BARBER AE }\end{array}$ & $\begin{array}{l}\text { SRD } \\
\text { SRD }\end{array}$ & $\begin{array}{l}23 \\
9\end{array}$ & $\begin{array}{l}05 / 05 / 61 \\
06 / 12 / 61\end{array}$ \\
\hline
\end{tabular}

VAUGKM AD

SRD

9

$06 / 09 / 69$

BMARS RE

SRD

45

$06 / 16 / 61$

LANG LH, MEEF HI

SRD

7

06/09/61

PRLDICH T

SRD

2

$06 / 12 / 61$

SHIMER RD

MONTAGUE DG

SRD

SRD

3

$06 / 12 / 61$

HICK OJ

SRD

8

$06 / 01 / 61$

CAMERON WD, TILLER RE SRD 32

$06 / 14 / 61$

WILBUR GE

SRD

15

$06 / 14 / 61$

CLINTON MA, HODGSON WH SRD

9

$07 / 26 / 69$

$06 / 19 / 61$ 
DoC. NO.

HW- 69975

HW-69979

HW-69985

HW-69999

HW-70096

HW-70107

HW-70128

HH-70136 HW-70137

HW-70150 HW-70160

HH-70184

$H W-70198$

HW-70215

HW-70219

HW-70223 A

HW-70295

HU-70312

HH-70313

HW-70317

HW-70356
TITLE

SUPPLEMENT A: PT IP-105-C GRAPHITE PONER COEFFICIENT DETERMINATION SPLINE-INDUCED 1960 PROOUCTION GAINS REDUCTION OF THE MMOUNT OF DICHROMTE ADDED TO REACTOR COOLIMG MATER INTERIM REPORT, PRCOUCTION TEST-IP-321-A PLANNED MORMALIZATION OF PLUTONIUN YIELD PREDICTIONS TO THE TWELVE THO-TON TEST BATCH MEASUREMENTS BUMPER FUEL INCENTIVES UNDER A PROOUCT SPECIFICATION

IN-REACTOR OPERATING CONDITIONS FOR THREE CHARGES OF KSE-3 ELEMENTS IN TH KER LOOPS

PROCESS TUBE EXPANSION - 105-C CONVERSION TO SELF-SUPPORTED FUEL ELEMENTS

SLIGHTLY ENRICHED U STUDY CASES ANALYSIS OF RADIATION INDUCED STRESSES IN C REACTOR GRAPHITE ECONOMICS OF FUEL CORE LENGTH INCREASES PROOUCTION TEST IP-412-AI B AND C REACTORS EXPORT SYSTEM TEST

105-C OVERBORE 40 TUBE TEST PROCESS TUBE ASSEMBLY FLON AND PRESSURE DROP CALIBRATION TEST

ANALOG SIMULATION OF VSR WITHDRAMAL

RATES

SUMMARY OF THE OPERATIONAL STATUS OF REACTOR CONTROL INSTRUMENTATION - REPORT NO. 2

MISCELLANEOUS PU240 CONCENTRATION DATA FOR THE C PILE OVERBORE STUOY

DESIGN OF PROOUCTION TEST IP-423-A-FP EVALUATION OF URAMILN FUEL CORES HAVING VARIOUS HEAT TREATMENTS

PROCESS IMPROVEMENT TRANSITION AUTHORIZATION IP-17-AI, REDUCTION OF THE AMOUNT OF DICHROMATE ADDED TO PROCESS HATER

QUARTERLY PROGRESS REPORT PLUTONIUM METALLURGY OPERATION APRIL, MAY, JUNE, 1961

ATOMIC WEAPON DATA SUPPLEMENT QUARTERLY PROGRESS REPORT PLUTONIUN METALLURGY OPERATION APRIL - JUNE, 1961

PRODUCTION TEST IP-376-D, SUPPLEMENT B IRRADIATION OF MGCR-HDR-3 TEST ELEMENT PROJECT WHITNEY - PLUTONILA METALLURGICAL DEVELOPMENT - MONTHLY REPORT - JUNE 1961

\begin{tabular}{|c|c|c|c|}
\hline AUTHOR & LEVEL & PAS & DATE \\
\hline CARTER RD & SRD & 7 & $06 / 16 / 61$ \\
\hline CARTER RD & SRD & 6 & $06 / 19 / 61$ \\
\hline MESSELSON EJ & SRD & 20 & $06 / 19 / 61$ \\
\hline HOBA DW, VAUGHN AD & SRD & 12 & $06 / 07 / 61$ \\
\hline HOUGH CG, SEHMEL GA & SRD & 18 & $06 / 23 / 61$ \\
\hline KRATZER WK & SRD & 13 & $06 / 26 / 61$ \\
\hline MOLLERUS FJ, JR. & SRD & 14 & $08 / 09 / 61$ \\
\hline LANG LH & SRD & 11 & $06 / 28 / 61$ \\
\hline YOSHIKALA НH & SRD & 8 & $08 / 14 / 61$ \\
\hline NECHODON WS & SRD & 3 & $06 / 29 / 61$ \\
\hline BENSON JL, JONES SS & SRD & 19 & $08 / 02 / 61$ \\
\hline ETHERIDGE EL & SRD & 8 & $07 / 06 / 61$ \\
\hline NILSON R & SRD & 5 & $07 / 06 / 61$ \\
\hline STEMART SL & CRD & 11 & $07 / 10 / 61$ \\
\hline NECHODON WS, BLYCKERT WA & SRD & 11 & $07 / 07 / 61$ \\
\hline HOOGSON WH, CLINTON MA & SRD & 15 & $01 / 05 / 62$ \\
\hline MESSELSON EJ, SHIMER RD & SRD & 6 & $07 / 14 / 61$ \\
\hline HICK OJ & CRD & $\%$ & $07 / 14 / 61$ \\
\hline HICK OJ & SRD & 29 & $07 / 14 / 61$ \\
\hline BAMRS RE & SRD & 11 & $07 / 11 / 61$ \\
\hline WICK OJ & SRD & 6 & $07 / 25 / 61$ \\
\hline
\end{tabular}


Page Ho. 159

$04 / 28 / 93$

LIST OF HANFORD-GENERATED CLASSIFIED

DOCUMENTS DATED BETUEEN 1/1/61 AMD 12/31/72

DOC. NO.

HW-70357 REV

HW-70357 REV 2

HW-70361

$H W-70394$ RD

HW-70408

HW-70413

HW- 70432

HW-70520

HW-70521

HH-70556

HH-70557

$H W-70577$

HW-70578

HW-70602

HW-70630

HW-70642

HW-70648

HW-70659

HW-70695RD

HW-70697

HW-70702

HW-70703

HW-70707 11
TITLE

AUTHOR

LEVEL

PQS

DATE

COMBINED OPERATION STLDY BLANKET AND E-N LONDINGS AT HAPO

COMBINED OPERATION STUDY BLANKET AND E-N LOADINES AT HAPO

THE PROCESSING OF PU SCRAP AT MANFCRD

GEH-4-68, 69, 70: PROPOSAL FOR VARIABLE BRAZE THICKMESS IRRADIATION

SUMUARY OF CHEMICAL PROCESSING DEPARTMENT SS MATERIALS CONTROL DURING FY 1961 AND FUTURE CONTROL PROGRAM

E-N AND PLANT EXPOSURES

SOME EFFECTS OF THE PU-240 SPECIFICATION

ON THE C REACTOR OVERBORE PROCRAM

RADIOCHEMISTRY FOR THE RUPTURE OF A

ZIRCALOY-2 CLAD URANIUM FUEL ELEMENT IN KER-2

RADIOCHEMISTRY FOR THE RUPTURE OF A

2IRCALOY-2 CLAD, MATURAL URAMILA

THERMOCOUPLE FUEL ELEMENT IN KER-1

QUARTERLY PROGRESS REPORT FUELS

DEVELOPMENT OPERATION JAMUARY, FEBRUARY, MARCH 1961

QUARTERLY PROGRESS REPORT FUELS DEVELOPMENT OPERATIOM APRIL, MAY, JUME 1961

PLUTONIUN FABRICATION DEVELOPMENT

MONTHLY REPORT - JULY 1961

LETTER TO WJ RAMSEY RE: HANFORD

CONTRIBUTION FOR FOURTH PLUTONIUM

MEETING

MATER LEAK PREVENTION PROGRAM

SPEED-OF-CONTROL LIMITS - 105-DR

ANALYSIS OF SRL PURIFIED MEPTUNIUM NITRATE STANDARD

TECHNICAL BASES AND CRITERIA EXPANSION OF OVERBORE TEST FACILITIES C-REACTOR

FINAL REPORT: IRRADIATIOH PERFORMANCE OF ENRICHED ZIRCALOY-2 CLAD SEVEA-ROO CLUSTER FUEL ELEMENTS, PROOUCTION TEST IP-214-A, (RM-295)

NOTES ON P SCRAP RECOVERY DISCUSSIONS HELD AT GERMANTOWN JULY 17 18, 1961 ULTRASONIC AND RESISTANCE MELDING OF ZR SUPPORTS TO ZR FUEL ELEMENT CLADDING PRODUCTIOA TEST IP-431-A EXPANSION OF OVERBORE TEST FACILITIES C REACTOR PROJECT WHITNEY - PLUTONIUM METALLURGICAL DEVELOPMENT - MONTHLY REPORT - JULY, 1961

UNIT COST COMPARISON BY REACTOR MAY, 1962

\begin{tabular}{|c|c|c|c|}
\hline LANG LH & SRD & 26 & $08 / 28 / 61$ \\
\hline LANG LH & SRD & 25 & $02 / 09 / 62$ \\
\hline GRIIM KG & $\mathbf{S R O}$ & 36 & $07 / 13 / 61$ \\
\hline TVERBERG JC, RUSLER LE & CRD & 16 & $06 / 27 / 61$ \\
\hline DOERR RH, JOHNSON WH & SRD & 57 & $08 / 18 / 61$ \\
\hline $\begin{array}{l}\text { HOUGM CG } \\
\text { GILBERT WD }\end{array}$ & $\begin{array}{l}\text { SRD } \\
\text { SRD }\end{array}$ & $\begin{array}{l}13 \\
21\end{array}$ & $\begin{array}{l}07 / 17 / 61 \\
07 / 18 / 61\end{array}$ \\
\hline DEMAITT TF & SRD & 5 & $05 / 29 / 61$ \\
\hline DEMIITT TF & SRD & 6 & $06 / 05 / 61$ \\
\hline $\begin{array}{l}\text { CADWELL JJ, TOBIN JC, } \\
\text { LAST OA, EVANS EA, MINOR } \\
\text { JE }\end{array}$ & SRD & 129 & $04 / 14 / 61$ \\
\hline $\begin{array}{l}\text { CADIELL JJ, TOBIN JC, } \\
\text { LAST GA, EVANS EA, MIHOR } \\
\text { JE }\end{array}$ & CRD & 133 & $07 / 15 / 61$ \\
\hline HICK O.J & SRD & 4 & $08 / 01 / 61$ \\
\hline HICK OJ & SRD & 2 & $07 / 28 / 61$ \\
\hline YOUNG JR & CRD & 10 & $08 / 01 / 61$ \\
\hline NECHODON WS & SRD & 4 & 08/01/61 \\
\hline SCHMEIDER RA & SRD & 2 & $08 / 03 / 61$ \\
\hline VAN WORMER FW & SRD & 11 & $05 / 22 / 61$ \\
\hline $\begin{array}{l}\text { RL CALL, TT CLAUDSON, R } \\
\text { TEATS }\end{array}$ & CONF & & $05 / 21 / 62$ \\
\hline & SECR & & $06 / 30 / 70$ \\
\hline STEINKAMP HI & CRD & 4 & $08 / 08 / 61$ \\
\hline VAN HORMER FW & SRD & 12 & $08 / 11 / 61$ \\
\hline HICK OJ & SRD & 10 & $08 / 23 / 61$ \\
\hline REED CE & SRD & 1 & $06 / 14 / 62$ \\
\hline
\end{tabular}


DOC. NO.

HW-70708

HW-70710

HN-70722

HW-70723

HW-70724

HW-70728

HW-70737

HU-70740

HW-70743

HW-70744

HW-70745

HU-70766

HW-70769

HH-70772 C

HW-70778

HW-70780

HW-70807

$H W-70809-R D$

HW-70828

HU-70851 REV
TITLE

CURRENT STATUS OF H REACTOR TOTAL CONTROL

FLOW EFFECTS FOLLOWING A SUDDEN LEAK ON A C-OVERBORE FRONT MOSSLE ASSEMBLEY QUARTERLY PROCRESS REPORT PLUTONIUN METALLURGY OPERATION JULY, AUCUST, SEPTEMBER, 1991

ATONIC WEAPON DATA SUPPLEMENT OUARTERLY PROGRESS REPORT PLUTONIUN METALLURGY OPERATION JULY - SEPTEMBER, 1961

TRIP REPORT - NUCLEAR SAFETY DISCUSSIONS AT SRL, ORNL, Y-12, ORCDP, LASL, AND RFP MEASUREMENT PROCEDURES FOR PURIFIED BIRCH

DECISION CRITERIA AND FACTORS RELATED TO REACTOR FRONT FACE CRITICAL COAPONENT FAILURE RECOMMEHDED E-N CHARGE MOOIFICATION (SECOND CYCLE PT-IP-350-C E-N DEMONSTRATION LOAD)

ESTABLISHMENT OF COOE WORD MMRG" POST IRRADIATION EXAMINATION OF SELF SUPPORT ELEMENTS WITH SEVERE IN-REACTOR CLADDING CORROSION (RH425)

HOT PRESS FORGING OF PLUTONILM DIAMETRICAL CLEARANCE CONSIDERATIONS FOR C OVERBORE PROJECT HEAT TRANSFER EXPERIMENTS SIMULATING A FAILURE OF THE INLET PIPING TO A BDF REACTOR PROCESS TUBE

PROOUCTION TEST IP-432-A-FP, EVALUATION OF THE CORROSION CHARACTERISTICS OF ELLIPTICAL BUMPER RAILS RADIOCHEMISTRY FOR THE RUPTURE OF $A$ 2IRCALOY-2 CLAD THERMOCOUPLED FUEL ELEMENT IN KER LOOP-1 ON MAY 12, 1961 AN OPERATIONAL REACTIVITY ACCOUNTING SYSTEM BASED ON ONE-GROUP DIFFUSION THEROY

RADIOCHEMISTRY FOR THE RUPTURE OF ZIRACALOY-2 CLAD HEAVY-HALLED TUBULAR FUEL ELEMENT IN KER LOOP-3 PROPOSED PRODUCTION TEST FOR REDUCING MINIMUM DOWTIME

THE PREPARATION AND IRRADIATION BEHAVIOR OF CHEMICALLY-NICKEL PLATED ALUMINUM-JACKETED FUEL ELEMENTS (A PAPER FOR PRESENTATION AT THE 14TH AMMUAL JOINT US-CANADIAN SHEATH CONFERENCE) WATER SHUT-OFF TIMES FOR C OVERBORE CHARGES

$$
\text { AUTHOR }
$$

VAUGHM AD

WATERS ED

HICK OJ

WICK OJ

ISSACSON RE

BUCKIMGHAM JS

GRAVES SN

CARTER RD

JAYMES RE

GRUBER WJ

KING RR

BUNCH UL

WATERS ED, KREITER MR

CLINTON MA

SRD

SRD

8

GF BAILEY

SRD

PERRICO LD

SRD

8

JAKLEVICK JF

SRD

JACKY GF

CRD

DATE

$10 / 15 / 61$

08/08/61

$10 / 16 / 61$

$10 / 13 / 61$

$08 / 14 / 61$

08/14/61

$10 / 01 / 61$

08/15/61

$08 / 16 / 61$ 08/28/61

$08 / 15 / 61$ 08/30/61

$08 / 16 / 61$

$10 / 02 / 61$

08/18/61

$04 / 01 / 62$

08/21/61 
Page No. 161

04/28/93

LIST OF MANFORD-GENERATED CLASSIFIED

DOCUMENTS DATED BETWEEN $1 /: 161$ AND 12/31/72

DOC. NO.

HU-70856

HL-70858

HW-70875 RD HW-70895

HW-70926

HH-70926 A

HW-70926 B

HH-70941

HW-71003

HW-71034

HW-71036

NU-71052

HU-71061

HW-71062

HW-71074

HW-71075

HW-71096

HW-71100

HW-71119

HW-71124

HW-71131 RD

HW-71134

HW-71138

HH-71141

HW-71154

HW-71165
TITLE

REACTOR OPTIMIZATION WITH PULP AND LINEAR PROGRAMIING

POST IRRADIATION EXAMINATION OF TRAMSVERSELY CRACKED RUPTURE FROM 2986 DR (RM427)

REPORT TO THE WORKING COMITTEE INPURITIES IN MOOEL 81-C COMPONENTS INTERIM REPORT ON SHIPPING CONTAINER SECONO INTERIM REPORT ON SHIPPING CONTAIMER ENVIRONMENTAL STLDIES THIRD INTERIM REPORT ON SHIPPING CONTAINER ENVIRONMENTAL STLDIES POST IRRADIATION EXAMIMATION OF LOW EXPOSURE OVERBORE ELEMENTS (RM 410) POWER CALCULATION ACCURACY WITH DUAL DOMNCOMER OPERATION

FABRICATION COSTS

REPORT OF THE WORKING COMITTEE OF THE FUEL ELEMENT DEVELOPMENT COMITTEE REVISED COOE WORDS

PERFORMANCE CHARACTERISTICS MOOIFIED LOW LIFT PLMPS 100-K AREA

OPERATIONAL FUELING INCENTIVES CURSORY ESTIMATE

EXPERIMENTAL BOILING BURNOUT CONDITIONS FOR HANFORD PROOUCTION REACTORS RESULTS OF LABORATORY HEAT TRANSFER EXPERIMENTS FOR C-REACTOR OVERBORE FUEL CHANMELS

ENGINEERING BASES FOR PONER LEVELS AND EXPOSURES OCTOBER 1961 THRU DECEMBER

\section{2}

DETERMINATION OF THE PU24O CONTENT OF

PLUTONIUN BUTTONS BY NEUTRON COUNTING

PONER EXCURSION IN A HANFORD REACTOR DUE TO A POSITIVE REACTIVITY RAMP

PROCESSING DATA FOR COEXTRUED FUEL

ELEMENTS IN ETR, KER AND MTR LOOPS

A FIVE-YEAR ADVANCED RESEARCH AND

DEVELLPMENT PROGRAM IN PLUTONIUN

METALLURGY

B AND D DONCOMER PROTOTYPE DATA

EQUIPMENT DESCRIPTION OF ADDITIONAL

PLUTONIUM FOUNDRY FACILITIES - 234-5

PROOUCTION TEST IP-442-A HALF-PLANT

REDUCTION IN PROCESS MATER PH, 105-D

MPR PILE GAS PURIFICATION

MUMERICAL RESULTS OF PROOUCTION TEST

IP-326-1,LON FLON CALIBRATION TESTS AT

DR AND C REACTORS AND OF PRODUCTION TEST

IP-395-I, REMOVAL OF HIGH TANK DISCHARGE

LINE STRAIMGER GATES AT B,D, AND F

REACTORS

\begin{tabular}{|c|c|c|c|}
\hline AUTHOR & LEVEL & PGS & DATE \\
\hline NEEF WI & SRD & 35 & $09 / 12 / 61$ \\
\hline GRUBER WJ & CRD & 7 & $08 / 28 / 61$ \\
\hline STRIMGER JT, MIMOR JE & SRD & 28 & $08 / 29 / 61$ \\
\hline KNIGHTS LM & SRD & 5 & $08 / 30 / 61$ \\
\hline MELSON TC & SRD & 9 & $08 / 31 / 61$ \\
\hline MELSON TC & SRD & 5 & $09 / 18 / 61$ \\
\hline NELSON TC & SRD & 4 & $09 / 27 / 61$ \\
\hline GRUBER MJ & CRD & 8 & 09/05/61 \\
\hline RENBERGER OL & SRD & 6 & $09 / 11 / 61$ \\
\hline LANG LW & $\begin{array}{l}\text { SRD } \\
\text { SRD }\end{array}$ & $\begin{array}{l}5 \\
130\end{array}$ & $\begin{array}{l}09 / 13 / 61 \\
09 / 12 / 61\end{array}$ \\
\hline JAYMES RE & SRD & 2 & $09 / 14 / 61$ \\
\hline KEENE LM & SRD & 6 & $09 / 21 / 61$ \\
\hline 1000 EC & CRD & 6 & $09 / 15 / 61$ \\
\hline BATCH JM, MATERS ED & SRD & 9 & $09 / 20 / 61$ \\
\hline MATERS ED, KREITER MR & SRD & 19 & $11 / 10 / 61$ \\
\hline $\begin{array}{l}\text { PROCESSING ENGINEERING } \\
\text { UNIT }\end{array}$ & SRD & 15 & $09 / 18 / 61$ \\
\hline HING NS & CRD & 6 & $09 / 19 / 61$ \\
\hline JOWES ED & SRD & 45 & $09 / 20 / 61$ \\
\hline ROBINSOW RK & CRD & 13 & $11 / 08 / 61$ \\
\hline HICK OJ & SRD & 17 & $09 / 22 / 61$ \\
\hline CORLEY JP & SRD & 3 & $09 / 22 / 61$ \\
\hline WOORICH DD & SRD & 17 & $09 / 22 / 61$ \\
\hline VAN WORMER FW & SRD & 8 & $05 / 08 / 62$ \\
\hline STEPMEWSKI DD & CRD & 15 & $10 / 19 / 61$ \\
\hline BENSOW JL & SRD & 11 & 09/29/61 \\
\hline
\end{tabular}


Page No. 162

$04 / 28 / 93$

LIST OF HAHFORD-GENERATED CLASSIFIED

DOCUNENTS DATED BETMEEN 1/1/61 AND 12/31/72

Doc. No.

HU-71174

HU-71179

HW-71201 RD

HW-71201 RD REV 1

HH-71202

HW-71206

HW-71207

HW-71233

HW-71238

HW-71253

HW-71267

HW-71271

$H W-71274$ RD

HW-71280

HW-71284

HW-71305

HH-71321

HW-71331

HW-71338

HW-71344 RD

HH- 71345

HW-71357

HW-71377

$H W-71378$
TITLE

NPR REACTOR SHIELD CALCULATIONS

HANFORD FISSION PRODUCTS - CONTEMPLATED FACILITY PLAN

OWER-OPERATOR PROCESS AND FUNCTIOMAL REQUIREMENTS INTERIM MOOIFICATIONS FOR IMPROVED COOLANT BACKUP 100-B,C,D,DR,F, AND H REACTORS. PROJECT CGI-905

OWNER-OPERATOR PROCESS AND FUNCTIONAL REQUIREMENTS INTERIM MOOIFICATIONS FOR IMPROVED COOLANT BACKUP 100-B, C, D, DR, F, AND H REACTORS, PROJECT CGI-905

ERRORS IN DETERMINING OPTIMMMS TEST OF MONPOISONOUS SPLIMES REACTIVITY AND GRAPHITE TEMPERATURE EFFECTS OF A HELILM-NITROGEN ATMOSPHERE FINAL REPORT OF PT IP-385-AC

REAR FACE PIPING STLDY AT ELEVATED BULK OUTLET TEMPERATURES - KE REACTOR ACTIVATION OF NPR CONTROL ROO TITANIUM HANDLING FUEL IN REACTOR BASIN (INCENTIVE FOR IMPROVEMENT) SUPPLEMENT B, PROOUCTION TEST IP-377-A, IRRADIATION OF N-REACTOR INWER FUEL. TUBES IN THE KER LOOPS

REPLACEMENT OF ORIGINAL TUBES TO PREVENT VAN STONE FLANGE FAILURES

MILITARY THERMAL CYCLING OF 81 COMPONENTS

REACTOR EFFLUENT ACTIVITY REDUCTION PROCESS MATER TREATMENT TESTS: PART 2 CORROSION EFFECTS

PROOUCTION TEST IP-447-C ROO DROP TRANSIENT COMPARISON D AMD DR REACTORS NEPTUNIUM-237 CONTENT OF E-METAL CESIUM-137 AS AM IMDEX OF THE NUCLEAR MATERIALS CONTENT OF COATING WASTE 81-C STABILITY IMPROVEMENTS PRCOUCTION TEST IP-449-A EFFLUENT ACTIVITY STUDIES IN THE 1706-KE IN-REACTOR FACILITY

TOE WORK SHEETS

CRITICAL MASS ESTIMATES FOR ARRAYS OF CASTINGS

DEVELOPMENT OF SCANMING TYPE FUEL

FAILURE DETEC TION SYSTEM

POSSIBILITIES FOR RELAXING PROCESS TUBE FLOU TRIP REQUIREMENTS FOR HANFORD REACTORS

PROJECT HHITNEY - PLUTONILM

METALLURGICAL DEVELOPMENT - MONTHLY

REPORT - SEPTEMBER, 1961

\begin{tabular}{|c|c|c|c|}
\hline AUTHOR & LEVEL & PQS & DATE \\
\hline PETERSON EG & $\begin{array}{l}\text { SRD } \\
\text { SECR }\end{array}$ & 13 & $\begin{array}{l}09 / 27 / 61 \\
06 / 30 / 70\end{array}$ \\
\hline SCHACK MH, TUPPER WJ & SRD & 20 & 10/02/61 \\
\hline SCHACK MH, TUPPER WJ & SRD & 21 & $10 / 23 / 61$ \\
\hline $\begin{array}{l}\text { SPENCER, HG } \\
\text { FERCUSON JH } \\
\text { BAILEY GF }\end{array}$ & $\begin{array}{l}\text { SRD } \\
\text { CRD } \\
\text { SRD }\end{array}$ & $\begin{array}{l}10 \\
6 \\
12\end{array}$ & $\begin{array}{l}10 / 02 / 61 \\
09 / 29 / 61 \\
09 / 29 / 61\end{array}$ \\
\hline FRIELING DH, HUTTON PH & SRD & 12 & $11 / 09 / 61$ \\
\hline BUNCH ML & CRD & 5 & $10 / 04 / 61$ \\
\hline GILBERT RD & $\mathbf{S}$ & 6 & $10 / 12 / 61$ \\
\hline KRATZER WK & SRD & 7 & $10 / 06 / 61$ \\
\hline CLEMANS WH, YOUMG JR & C & 11 & $10 / 06 / 61$ \\
\hline SMITH AE & SRD & 5 & $10 / 13 / 61$ \\
\hline MESSELSON EJ & SRD & 11 & 10/09/61 \\
\hline VAUGHN AD & SRD & 9 & $10 / 16 / 61$ \\
\hline SCHAEIDER RA & SRD & 3 & $10 / 10 / 61$ \\
\hline SCHNEIDER RA & CRD & 12 & $10 / 11 / 61$ \\
\hline KURTZ EF & SRD & 20 & 10/12/61 \\
\hline GEIER RG & SRD & 14 & $10 / 14 / 61$ \\
\hline CHATTEN JCL & SRD & 18 & $09 / 14 / 61$ \\
\hline $\begin{array}{l}\text { CHITWOOO RB, ISACSON RE, } \\
\text { STEVENSON RL }\end{array}$ & SRD & 8 & $10 / 16 / 61$ \\
\hline JL STRIMGER, EM SHEEN & CRD & & $06 / 01 / 62$ \\
\hline $\begin{array}{l}\text { WATERS ED, HESSON CN, } \\
\text { BATCH JM }\end{array}$ & SRD & 26 & $10 / 17 / 61$ \\
\hline HICK OJ & SRD & 10 & $10 / 30 / 61$ \\
\hline
\end{tabular}


Page No. 163

$04 / 28 / 93$

LIST OF HANFORD-GENERATED CLASSIFIED

DOCUMENTS DATED BETHEEN 1/1/61 AMD 12/31/72

\begin{tabular}{|c|c|c|c|c|c|}
\hline DOC. NO. & TITLE & AUTHOR & LEVEL & PGS & DATE \\
\hline$H W-71408$ VOL2 RD & $\begin{array}{l}\text { NPR HAZARDS REVIEW - (PHASE I - } \\
\text { PRCOUCTION ONLY - APPENDIXES) }\end{array}$ & MILLER NR, TRUNBLE RE & SRD & 376 & $08 / 15 / 62$ \\
\hline$H W-71408-R D$ & $\begin{array}{l}\text { HPR HAZARDS REVIEH - VOLUME I (PHASE I - } \\
\text { PRODUCTION ONLY) }\end{array}$ & MILLER NR, TRUNBLE RE & SRD & 232 & $10 / 19 / 61$ \\
\hline HW-71431 & ERROR ANALYSIS OF THE TAI EQUATION & CREMER BR & SRD & 7 & $10 / 23 / 61$ \\
\hline HW-71457 & REVISED COOE WORDS & JAYNES RE & SRD & 3 & $10 / 27 / 61$ \\
\hline HW-71546 & $\begin{array}{l}\text { OPERATING PROCEDURE AND PROCESS } \\
\text { SPECIFICATIONS DIMENSIONAL STABILITY } \\
\text { EVALUATION }\end{array}$ & KURTZ EF, SMITH AE & CRD & 5 & $10 / 30 / 61$ \\
\hline$H H-71556$ & $\begin{array}{l}\text { SAVANMAH RIVER MUCLEAR INSTRLMENTATION } \\
\text { REPORT OF TRIP TO SAVANMAH RIVER PLANT } \\
\text { AUGUST } 10 \text { AND } 11,1961\end{array}$ & HILDRETH NT, STIEDE WL & SRD & 15 & $10 / 31 / 61$ \\
\hline HW-71581 & ECONOMICS OF REUSABLE CRUCIBLES & CURRAN RG & SRD & 8 & $11 / 01 / 61$ \\
\hline HU-71586 & $\begin{array}{l}\text { PRCOUCTION TEST IP- } 455-A \text { COMPARISON OF N } \\
\text { REACTOR INNER FUEL. TUBES MITH DIFFERENT } \\
\text { PRE-IRRADIATION WARP CONDITINS }\end{array}$ & KRATZER WK & SRD & 6 & $11 / 02 / 61$ \\
\hline HW-71591 & $\begin{array}{l}\text { ADVANCED PLUTONIUN METALLURGY RESEARCH } \\
\text { AND DEVELOPMENT PROGRAM }\end{array}$ & HICK Od & SRD & 18 & $11 / 03 / 61$ \\
\hline $\mathrm{HH}-71592$ & BLENDING ECONONICS & LANG LH & SRD & 22 & $11 / 28 / 61$ \\
\hline HH-71619 & $\begin{array}{l}\text { TECHNICAL SPECIFICATIONS HANFORD } \\
\text { PRCOUCTION REACTORS }\end{array}$ & GILBERT W & SRD & 19 & $06 / 25 / 62$ \\
\hline HW-71619 RD & MUCLEAR HEALTH AND SAFETY & GILBERT W & SRD & 23 & $11 / 06 / 61$ \\
\hline HW-71619 RD REV1 & $\begin{array}{l}\text { NUCLEAR HEALTH AND SAFETY TECHAICAL } \\
\text { SPECIFICATIONS }\end{array}$ & GILBERT WD & SRD & 14 & $12 / 07 / 61$ \\
\hline$H H-71619$ RD REV3 & $\begin{array}{l}\text { TEC' ICAL SPECIFICATIONS - HANFORD } \\
\text { PROOUCTION REACTORS }\end{array}$ & GILBERT WD & SRD & 24 & $02 / 01 / 62$ \\
\hline HW-71619 RD REV4 & $\begin{array}{l}\text { TECHNICAL SPECIFICATIONS HANFORD } \\
\text { PROOUCTION REACTORS }\end{array}$ & GIL BERT WD & SRD & 23 & $02 / 16 / 62$ \\
\hline$H W-71665$ & $\begin{array}{l}\text { DIMENSIONAL STABILITY OF ALPHA PLUTONIUM } \\
\text { COMPONENTS }\end{array}$ & TOALINSON RE & SRD & 6 & $11 / 10 / 61$ \\
\hline HH-71709 & $\begin{array}{l}\text { RELATIVE XENOW INSTABILITY IN THE } \\
\text { HANFORD K AND N REACTORS }\end{array}$ & MCDANIELS DK & SRD & 18 & $91 / 16 / 61$ \\
\hline HW-71751 & $\begin{array}{l}\text { D-REACTOR GRAPHITE BURNOUT INTERIM } \\
\text { REPORT-IP-25A(PT-105-532-E) }\end{array}$ & RYAN BA & SRD & 20 & $11 / 10 / 61$ \\
\hline HW-71770 & $\begin{array}{l}\text { PROJEL'T HHITNEY - PLUTONIUM } \\
\text { METALLURGICAL DEVELOPMENT - MONTHLY } \\
\text { REPORT - OCTOBER } 1961\end{array}$ & HICK OJ & SRD & 8 & $11 / 28 / 61$ \\
\hline$H W-71809$ & $\begin{array}{l}\text { PRODUCTION TEST IP-467-C REDUCING } \\
\text { MINIMUR DOWTIME }\end{array}$ & JAKLEVICK JF & SRD & 18 & $12 / 06 / 61$ \\
\hline HW-71877 & $\begin{array}{l}\text { PRODUCTION TEST IP-466-A TEST OF THE } 190 \\
\text { TURBINE PUMPS AT KE(KW) REACTOR }\end{array}$ & JOMES SS & CRD & 11 & $02 / 01 / 62$ \\
\hline HW-71880 & $\begin{array}{l}\text { PROCESS DESIGN BASIS FOR THE PLUTONIUM } \\
\text { THERMAL TREATMENT FACILITIES - } 234-5 \\
\text { BUIILING }\end{array}$ & WODRICH DD & SRD & 10 & $01 / 11 / 62$ \\
\hline$H W-71917$ & PANELLIT GAUGE REQUIREMENTS - $K$ REACTORS & POOR CF & SRD & 3 & $12 / 04 / 61$ \\
\hline HW-71917 REV1 & PANELLIT GAUGE REQUIREMENTS - $K$ REACTORS & POOR CF & SRD & 3 & $02 / 16 / 62$ \\
\hline$H W-71913$ & $\begin{array}{l}\text { HAZARDS EVALUATION STUDY - CHEMICAL } \\
\text { PROCESSING DEPARTIMENTT }\end{array}$ & EG PIERICK & SRD & & 05/01/62 \\
\hline HW-71918 REV & $\begin{array}{l}\text { EVALUATION OF HAZARDS CHEMICA! } \\
\text { PROCESSIHG DEPARTMENT }\end{array}$ & PIERICK EG, TOMLINSON RE & SRD & 314 & $11 / 02 / 64$ \\
\hline
\end{tabular}


DOC. NO.

HW-71924

HW-71950

HW-71957

HN-71964

HH-71968

KH-71974

HW- 71978

HH-71990

HU-71997

HH-72073

HW-72074

HH-72075

HW-72077 RD

HW-72095

HW-72105

HW- 72106

HH-72110

HH-72115

HW-72162

HH-72167

HW-72187

HH-72222 RD

$H H-72224 R D$

HH-72239
TITLE

PROJECT PROPOSAL: ADDITIONAL PLUTONIUM STORAGE FACILITIES - 234-5 BLDG (CGC-968)

LOADING AND OPERATING CONDITIONS FOR FIVE 23-INCH MIE-1 ELEMENTS IN KER-1 AND KER-2 UNDER PT-IP-455-A REDUCING MINIMU DOWNTIME A MUCLEAR SAFETY ANALYSIS MEUTRON SHIELDING FOR PLUTONIUM METAL STORAGE HOODS

PROJECT WHITNEY - PLUTONIUA

METALLURGICAL DEVELOPMENT - MONTHLY REPORT - NOVEMBER, 1961

CLASSIFICATION: URANIUNA RECYCLE PLUTONIUM STABILITY STUDIES STATUS REPORT 1

PROCESS SUWMARY OF PUREX PLANT OPERATION JAMUARY 1962 THRU DECEMBER 1962

SOLIDS IN RECUPLEX WASTE

SPEED-OF-CONTROL LIMITS FOR XENON

OVERRIDE PROOUCTION TEST

EFFECT OF SPECIAL URANIUM FUEL ON HANFORD REACTORS

NP-237 PROOUCTION IN THE HANFORD IPD REACTORS

REAR FACE LEAK REPAIR STUDY - 105-C

A STORAGE CONCEPT FOR PLUTONIUA COMPONENTS

CROSSHEADER TEMPERATURE STUDY

PROJECT WHITAEY - PLUTONIUM

METALLURGICAL DEVELOPMENT - MONTHLY

REPORT - DECEMBER 27, 1961

DIVERSITY VARIABLES INVESTIGATION AND

CHROMATE COATING FORMATIONS

PLUTONILM FABRICATION DEVELOPMENT

MONTHLY REPORT - DECEMBER 1961

ATOMIC MEAPON DATA SUPPLEMENT OUARTERLY

PROGRESS REPORT

SUMUARY OF US-UK EXCHANGE MEETINGS AT

AWRE, ALDERMASTON, ENGLAND

OPERATIONAL FUELING TESTS AT 105-KE

REACTOR, ON DECEMBER 27, 1961

REPORT OF THE GENERAL ELECTRIC COMPANY TO THE WORKIHG COMMITTEE

$Z$ PLANT MEEKLY REPORT RECUPLEX, INCINERATOR AND TASK ONE AND TWO JAMUARY THROUGH DECEMBER 1962

EFFECTS OF BOHD QUALITY AND DIMENSIONAL STABILITY ON SIDE RUPTURE RESISTANCE OF NON-BUMPER FUEL ELEMENTS
AUTHOR

LW FINCH

SRD

$04 / 27 / 62$

KRATZER WK

SRD

4

$12 / 05 / 61$

JAKLEVICK JF, OUSLEY GF SRD 16

05/09/62

KNIGHTS LM

CRD 5

12/07/61

HICK OJ

SRD

$12 / 08 / 61$

BOREMIER Bd

WICK OJ

CRD

SRD

JUDSON BF, RATHVON HC

SRD

KNIGHTS LM

TILLER RE

NILSON R

NILSON R

RUDOCK ER

MCKEE DH

STCDDARD JA

HICK OJ

CREAIN RH

HICK OJ

HICK OJ

SHITH AE

MCCARTHY PB, HALKINS KG

SRD

MINOR JE, STRIMGER JT

SRD

SECR

CRD

19

01/05/62

$12 / 08 / 61$ $12 / 01 / 61$

01/11/62

12/11/61 $01 / 04 / 62$

12/21/61

$05 / 25 / 64$

$12 / 20 / 61$

$12 / 26 / 61$

$12 / 29 / 61$

$12 / 27 / 61$

$01 / 10 / 62$

$01 / 02 / 62$

01/15/62

$01 / 02 / 62$

$12 / 29 / 61$

01/01/62

$01 / 02 / 62$

BLOOMSTRAND RR

47 
Page No. 165

04/28/93

LIST OF HANFORD-GENERATED CLASSIFIED

DOCUNENTS DATED BETUEEN 1/1/61 AND 12/31/72

DOC. NO.

$H H-72240$

HW-72242

HW-72249 RD

HW-72269

HW- 72283

HW-72291

HU-72296

HW- 72310

HU- 72343

HUV- 72345

HW- 72347

HH-72361

HW-72366

HW-72371

$H W-72374$

HW-72374 SUP 1

HW-72391

HH-72402

$H 4-72411$

$H W-72420$

HH-72496

HH-72497

HW-72506

HU-72507 RD
TITLE

EFFECT OF EXPOSURE ON CRITICALITY IH WATER LATTICES - NPR AND I\&E FUEL

ELEMENTS

PLUTONIUN SCRAP RECOVERY

RESUME OF RUPTURED SLUG EXAMIKATION

EXPERIENCE HITHIN RADIO-METALLURGY -

1948 - 1955

PRCOUCTION TEST IP-4T7-A, IRRADIATION OF N REACTOR FUEL ELEMENTS IN KER LOOPS 3

\section{AND 4}

CHEMICAL PROCESSING NUCLEAR MATERIALS

CONTROL - DEC 1961

DEVELOPMENT AND EVALUATION OF SPIRE

PULSE FOR ALSI LEAD-DIP CANHIHG

FABRICATION PROCESS SPECIFICATIONS

MUMERICAL RESULTS OF PT IP-412-AI, B AND

C REACTORS EXPORT SYSTEM TEST

PROCESS CONTROL LOGBOOK

MPR PHYSICS STARTUP TESTING PROGRAM

QUARTERLY PROGRESS REPORT FUELS

DEVELOPMENT OPERATION JANUARY, FEBRUARY, MARCH, 1962

ANALYSIS OF WASTE STREAMS - 234-5

BUILDING

INTERIM REPORT REAR FACE PIPING STLDY AT ELEVATED BULK OUTLET TEMPERATURE - KE REACTOR

K REACTOR INLET ASSEMBLY CAVITATION STUDY HEADER VENTURI VERSUS HOZZLE VENTURI

PRELIMINARY HAZARDS REVIEW OVERBORING HANFORD REACTORS

PRELIMIMARY HAZARDS REVIEW OVERBORING HANFORD REACTORS (CONDENSED VERSIOW) PRCOUCT LOG

FINAL EVALUATION REPORT -GE-312 COMPUTER TEST PROGRAM - 234-5 BUILDING

FIMAL REPORT - PT IP-416 MEASUREMENT OF THE HORIZONTAL ROD STRENGTH AT H REACTOR PROGRESS REPORT - FINISHED PROOUCTS FABRICATION TECHNOLOGY OPERATION, DEC 1961 - JAN 1962

PHYSICS ASPECTS OF OPERATION IN EVENT OF A LARGE REACTIVITY LOSS

CHARACTERISTICS OF SPLIMES FOR FINE DISTRIBUTION CONTROL

PRODUCTION TEST IP-486-D, IRRADIATION OF CORRUGATED FUEL ELEMENTS

PROPOSAL FOR CHARGING THE EIGHT FUEL. RUPTURE EXPERIMENT GEH 10-57

\begin{tabular}{|c|c|c|c|}
\hline AUTHOR & LEVEL & PGS & DATE \\
\hline TILLER RE, JOHMSON DL & SRD & 10 & $01 / 30 / 62$ \\
\hline & SECR & & $06 / 30 / 70$ \\
\hline O'KEEFE DP & SRD & 61 & $04 / 26 / 56$ \\
\hline KRATZER, WK & SRD & 10 & $01 / 09 / 62$ \\
\hline RH DOERR & SECR & & $01 / 12 / 62$ \\
\hline HANSON GR & CRD & 8 & $01 / 17 / 62$ \\
\hline AE SNITH & SRD & & $01 / 12 / 62$ \\
\hline BENSON JL & SRD & 29 & $02 / 20 / 62$ \\
\hline FA SWYDER & SECR & & $01 / 17 / 62$ \\
\hline BOMERS CE & SRD & 87 & $04 / 25 / 62$ \\
\hline $\begin{array}{l}\text { CADWELL JJ, TOBIN JC, } \\
\text { LAST GA, EVANS EA, MINOR } \\
\text { JE }\end{array}$ & SRD & 136 & $04 / 16 / 62$ \\
\hline LA KHIGHTS & CONF & & $01 / 18 / 62$ \\
\hline FRIELING DH, HUTTON PH & SRD & 39 & $01 / 17 / 62$ \\
\hline KELLEY LR & SRD & 21 & $01 / 22 / 62$ \\
\hline NILSON R, CARLSON PA & SRD & 47 & $07 / 25 / 62$ \\
\hline MILSON R, CARLSON PA & SRD & 14 & $08 / 28 / 62$ \\
\hline RM HALSEY & SECR & & $01 / 22 / 62$ \\
\hline KOLB JH & SRD & 48 & $12 / 29 / 61$ \\
\hline VAUGHN AD & SRD & 21 & $01 / 24 / 62$ \\
\hline AEC-RL & SRD & & $01 / 25 / 62$ \\
\hline HEIPLE RB & SRD & 11 & $01 / 29 / 62$ \\
\hline CARTER RD & SRD & 14 & $01 / 29 / 62$ \\
\hline MARSHALL RK, KRATZER MK & SRD & 12 & $01 / 26 / 62$ \\
\hline KAULITZ DC, CALL RL & CRD & 36 & $01 / 29 / 6$ \\
\hline
\end{tabular}


Page No. 166 $04 / 28 / 93$
LIST OF HANFORD-GENERATED CLASSIFIED

DOCUMENTS DATED BETWEEN 1/1/61 AND 12/31/72
DOC. NO.

HW-72541 A

$H H-72541 C$

HW-72543

HW-72549

HW-72574

HW-72575

HW- 72577

HW-72591

HH -72605

HW-72ธ28

HW- 72632

$H W-72664$

HH-72675

HU-72676

$H W-72680$

HW-72692

$H W-72699$

HW-72701

HH- 72710

HW -72717

HW- 72728

$H W-72733$ RD $H W-72738$

$H W-72742$

HW-72745
TITLE

DESIGN OF PRODUCTION TEST IP-468-A-FP, PROGRAM FOR EVALUATION OF ALPHA EXTRLOED FUEL CORES

PROOUCTION TEST IP-468-A-FP, PROGRAM FOR EVALUATION OF ALPHA EXTRUDED FUEL CORES RMO MONTHLY REPORT - JANUARY 1962 CESIUM-137 INDEX FOR MUCLEAR MATERIALS CONTENT OF COATING WASTE

PROJECT MHITNEY - PLUTONIUN

METALLURGICAL DEVELOPMENT - MONTHLY REPORT - JANUARY, 1962

REAR CROSSHEADER EXPANSION JOINTS STARTUP CALCULATIONS (NOTEBOOK) INSTRUMENTATION STUDIES - STATUS REPORT HIGH PRESSURE AUTOCLAVE DEVELOPMENT FACILITY

INTERIM REPORT - PY IP-447-C ROD DROP TRANSIENT COMPARISON D AND DR REACTORS RE-DEFINITION OF DR VERTICAL SAFETY SYSTEM STRENGTH

COMPARISON OF MEIGHT PER CENT PU-240 DETERMINED BY NEUTRON COUNTING AND BY MASS SPECTROMETRY

DESIGN, DEVELOPMENT AND PRODUCTION

EVALUATION OF I\&E CONTOUR-BASE COMPONENTS

PRELIMIMARY FUEL ELEMENT DESIGN STUDIES

FOR 2000 PROGRAM TASK FORCE

DESIGH SCOPE OF THE 2 PLANT METAL

CONTROL FACILITY PROJECT CGC-944

KER LOOP FUEL TESTING PROGRAM AND SCHEDULE - CY-1962

EFFECT OF PROCESS TUBE MATERIAL CHANGES ON REACTOR ECONOMICS

REACTOR PROCESS FLOU TECHNICAL DATA

BLENDIHG STUDY

RESULTS OF TEN TUBE OPERATIONAL FUELING ON FEBRUARY 9, 1962

TRIP REPORT: AWRE, ALDERMASTON, BERKSHIRE, ENGLAND - NOVEMBER 6-10, 1961 SUMMIARY STATUS REPORT INTERNAL CORROSION OF RIBBED ALUMINUM PROCESS TUBES

$K$ REACTOR TUBE REPLACEMENT

RIVER CONTAMINATION REDUCTION STUDIES PROGRAM OUTLINE AND SCHEDULE

PROJECT CGI-883 MOOEL TEST OF INLET TO K DONCOMER HH-WSU-7

AMALYSIS OF THREE BATCHES OF PB (PINCH BOTTLE)- 1 MATERIAL - DECEMBER 1961

\begin{tabular}{|c|c|c|c|}
\hline AUTHOR & LEVEL & PGS & DATE \\
\hline CLINTOW MA, HODGSON WH & SRD & 14 & $01 / 28 / 62$ \\
\hline CLINTON MA & SRD & 11 & $01 / 31 / 62$ \\
\hline $\begin{array}{l}\text { AJ STEVENS } \\
\text { SCHNEIDER RA, ZIMMER WH }\end{array}$ & $\begin{array}{l}\text { SRD } \\
\text { CRD }\end{array}$ & 4 & $\begin{array}{l}01 / 31 / 62 \\
02 / 01 / 62\end{array}$ \\
\hline HICK OJ & SRD & 9 & $02 / 02 / 62$ \\
\hline $\begin{array}{l}\text { HUTTON PH } \\
\text { DG ALBERTSON }\end{array}$ & $\begin{array}{l}\text { SRD } \\
\text { SECR }\end{array}$ & 15 & $\begin{array}{l}02 / 08 / 62 \\
02 / 02 / 62\end{array}$ \\
\hline $\begin{array}{l}\text { STIEDE WL, MONHIE DI } \\
\text { AJ KARNIE }\end{array}$ & $\begin{array}{l}\text { CRD } \\
\text { CONF }\end{array}$ & 49 & $\begin{array}{l}03 / 29 / 62 \\
02 / 02 / 62\end{array}$ \\
\hline VAUGHA AD & SRD & 5 & $02 / 06 / 62$ \\
\hline ZIMUER UH & SRD & 9 & 02/07/62 \\
\hline PADGETT EV & CRD & 14 & $02 / 13 / 62$ \\
\hline HEEB CM, BLYCKERT WA & SRD & 15 & $02 / 13 / 62$ \\
\hline HABERMAN HD & SRD & 29 & $03 / 12 / 62$ \\
\hline EVAMS TH, KRATZER HK & CRD & 14 & $02 / 14 / 62$ \\
\hline BLYCKERT WA & SRD & 11 & $02 / 15 / 62$ \\
\hline $\begin{array}{l}\text { JOWES SS, MORRISSEY JE, } \\
\text { MAMUEL JL }\end{array}$ & CRD & 56 & $02 / 15 / 62$ \\
\hline LAKG LH & SRD & 15 & $02 / 21 / 62$ \\
\hline $\begin{array}{l}\text { HAMHOND RS, MCCARTHY PB, } \\
\text { HAWKINS KG }\end{array}$ & SRD & 9 & $02 / 15 / 62$ \\
\hline STEWART RH & SRD & 10 & $02 / 16 / 62$ \\
\hline $\begin{array}{l}\text { CARLSON PA, CURTISS DH, } \\
\text { MILLER MR, VANWORMER FH }\end{array}$ & SRD & 96 & $02 / 19 / 62$ \\
\hline THOMAS RE & SRD & 8 & $02 / 20 / 62$ \\
\hline SCHACK MH & SRD & 8 & $02 / 20 / 62$ \\
\hline LM KEENE & SRD & & $02 / 20 / 62$ \\
\hline RINGLE RP, STEIHHAUSER JC & SRD & 4 & $02 / 20 / 62$ \\
\hline
\end{tabular}


Page No. 167

$04 / 28 / 93$

LIST OF HANFORD-GENERATED CLASSIFIED

DOCUMENTS DATED BETHEEN 1/1/61 AHD 12/31/72

DOC. NO.

$H W-72748$ RD

HH-72771

HU-72773 RD

HW-72809

HW- 72810

HW- 72814

HW-72825

HW-72842

HW- 72848

HW-72855

HW-72856

HW- 72860

HH-72896

HW-72901

HW-72919

HW- 72946

HW-72977 RD

HW-72979

$H W-72980$

HW-72988RD

HH-73005

HW-73005 EX1
TITLE

AUTHOR

LEVEL

PGS

DATE

TABLE OF FUEL ELEMENT PONERS, TEMPERATURES AND EXPOSURES, TEST $K-1-14$, PT-IP-455-A

DISASTER PLANHING STUDY

2101-M BUILDING USE - FEASIBILITY STUDY

PROJECT HHITMEY - PLUTONIUM

METALLURGICAL DEVELOPMENT - MONTHLY

REPORT - FEBRUARY, 1962

PREREQUISITE REQUIREMENTS FOR HIGHER

GRAPHITE TEMPERATURE LIMITS AMD/OR

NITROGEN ATMOSPHERE AT ALL REACTORS

CPD FPO PROGRESS REPORT - FPFTO -

FEBRUARY 1962

LOADING AND OPERATING CONDITIONS FOR A

CHARGE OF FIVE 23-INCH MAE-1 ELEMENTS IN

KER-3 OR KER-4 UNDER PT-IP-477-A

PRESSURE DROP AND CRITICAL FLON OF

REPAIR CAP ASSEMBLY FOR B, D, AND F REAR HEADERS

TECHNOLOGY, ECONONICS AND INCENTIVES FOR

A SOLID STATE DIFFUSION BONDING PROCESS

- hOT DIE SIZING

REACTIVITY AND EFFICIENCY TRENDS VS

OPERATING TRENDS FOR B, D, DR, AND F

REACTORS - 1956 THROUGH 1960

REACTIVITY AND EFFICIENCY TRENDS VS OPERATING TRENOS FOR C, H, KE, AMD KW

REACTORS - 1956 THROUGH 1960

LEAK PLUGGING WITH FIBROUS MATERIALS

IRRADIATION OF A TUBULAR, UO2 FUEL

ELEMENT IN THE KER-1 LOOP

PRELIMINARY REPORT ON THE TENSILE

PROPERTIES OF A PLUTONIUM ALLOY

SEGMENTAL DISCHARGE ECONONICS FOR

NATURAL URANILM FUEL AT A $K$ REACTOR

TEN-YEAR IMPROVEMENT PROGRAM - P

CONVERSION AND FABRICATION

THE EFFECT OF THERMAL AND MECHANICAL

TREATMENTS ON THE DENSITY OF UNALLOYED

PLUTONIUN

BUDGET FOR FY 64 \& REVISED BUDGET FOR FY

63 - SEPARATIONS RED PROGRAM (02-53-04)

BUDGET FOR FY 64 \& REVISED BUDGET FOR FY

63 - PLUTONIUM PROCESSING

105-C REACTOR OVERBORE PROGRAM

PROCEEDINGS OF THE PLUTONIUM IHFORMATION MEETING HELD AT HANFORD ATOMIC PRODUCTS OPERATION ON FEBRUARY 6 AND 7, 1962

DENSITY AND STRAIN CHANGES FOLLONING CASTING
KRATZER WK

SRD

2

$02 / 21 / 62$

WE JOHNSOW CRD

CRD

GP, MCKEE DH, KURTZ EE

HICK OJ

SRD

19

$02 / 22 / 62$

03/09/62

$03 / 06 / 62$

GRAVES SM

SRD

7

02/26/62

AEC-RL

SRD

$02 / 26 / 62$

KRATZER WK

SRD

$02 / 28 / 62$

MOEN RH

CRD

6

$02 / 28 / 62$

BURGESS CA

SRD

25

03/01/62

PERL ER

SRD

57

03/01/62

PERL ER

SRD

55

03/01/62

SANDWITH CJ

SRD

HORN GR, MILLHOLLEN MK

SRD

11

02/07/62

03/05/62

GARDNER HR

SRD

7

03/08/62

HEID RL

SRD

25

$03 / 29 / 62$

SECR

$06 / 30 / 70$

MELSON TC, KING RR

SRD

26

$19 / 01 / 62$

SECR

$06 / 30 / 70$

SECR

$06 / 30 / 70$

HW HEACOCK

SRD

$03 / 12 / 62$

HICK OJ

SRD

159

$03 / 12 / 62$

MELSON TC

SRD

30

$03 / 12 / 62$ 
Page Ho. 168

$04 / 28 / 93$

LIST OF HANFORD-GENERATED CLASSIFIED

DOCUMENTS DATED BETHEEN 1/1/61 AND 12/31/72

DOC. NO.

HH-73005 EX2

HW-73008

HW-73010

HW-73017

HH-73062

HU-73064

HW-73070

HW-73093 A

HW-73093 C

HU-73111

HW- 73114

HW-73118

$H W-73131$

HW-73132

$H H-73133$

HW- 73148

HU-73149

HW- 73154
TITLE

PLUTONIUM-STRESS MEASUREMENTS AND STRESS RELAXATION

BUDGET FOR FY 1964 AND REVISION OF BUDGET FOR FY 1963 PROPOSAL FOR RESEARCH AND DEVELONENT GENERAL WEAPONS DEVELOPMENT - 03-16-04

PRE-IRRADIATION URAMIUA FUEL CORE CHARACTERISTICS AND IRRADIATION DATA FOR SAVANHAH RIVER AND HANFORD PRCDUCTION FUEL. ELEMENTS - JULY 1959 THROUGH DECEMBER 1960

ECONONICS OF HIGHER GRAPHITE TEMPERATURES

EXAMINATION OF OVERBORE ELEMENTS WITH SEVERE CORROSION

THE EFFECT OF FRINGE POISON ON HEAT GENERATION IN THE SHIELD COMPLEX BUDGET FOR FY 9964 AND REVISION OF BUDGET FOR FY 1963. PROPOSAL FOR RESEARCH AND DEVELOPMENT GENERAL MEAPONS DEVELOPMENT - 03-16-04 - DETAILS DESIGN OF PRODUCTION TEST IP-490-A-FP EVALUATION OF DIFFUSION BONDED FUEL ELEMENTS

PRCOUCTION TEST IP-490-A-FP, EVALUATION OF DIFFUSION BONDED FUEL ELEMENTS SUPPLEMENTAL SPECIFICATIONS OF LABORATORY HOT PRESS PROCESS - FOR CV SIZE SELF-SUPPORTED IRE FUEL ELEMENTS LOADING AND OPERATING CONDITIONS FOR A CHARGE OF ELEVEN OR FOURTEEN 23-INCH MAE-1 ELEMENTS IN KER-3 AND KER-4 UNDER PT-IP-477-A

STATISTICAL STATEMENTS \& ASSOCIATED DATA FOR 234-5 BLDG, FINAL PRCDUCT SHIPPED DURING THE 4TH QUARTER OF CY 1961 RADIOGRAPHIC PROCEDURES FOR PARTS 8105, $8106,8107,8108$ AND 8109

RADIOGRAPHIC PROCEDURES FOR PARTS U 205 AND U 206

TRITIUM PROOUCTION IN HIGH-TEMPERATURE ( 300 C) REACTORS BY IRRADIATION OF LITHIUM LITERATURE REVIEW

COEXTRUDED K-REACTOR ENRICHED I8E FUEL ELEMENT PROGRAM

STATUS OF ALLOYED DINGOT PROGRAM JANUARY, 1963

RELOCATION OF RADIAL ENRICHMENT AT THE K REACTORS TO DECREASE ENRICHMENT INVENTORY

\begin{tabular}{llll}
\multicolumn{1}{c}{ AUTHOR } & LEVEL & PGS & DATE \\
BAILEY HJ & & & \\
FRAHK HS, SMITH AE & SRD & 32 & $03 / 15 / 62$ \\
& & & \\
& & & \\
STRINGER JT & SRD & 172 & $04 / 24 / 62$ \\
& & & \\
JENSEN RD & & & $03 / 15 / 62$ \\
GRUBER WJ & SRD & 51 & $03 / 19 / 62$ \\
PETERSON EG, BUNCH WL & SRD & 8 & $03 / 19 / 62$ \\
FRANK US, SMITH AE & SRD & 22 & $03 / 20 / 62$
\end{tabular}

HODGSON WH, CLINTON MA SRD $12 \quad 03 / 26 / 62$

$\begin{array}{llll}\text { CLINTON M } & \text { SRD } & 8 & 05 / 02 / 62\end{array}$

BURGESS CA, SCHWEIKHARDT CRD $41 \quad$ 06/01/64 CM

KRATZER HK

SRD

$03 / 22 / 62$

AE SHITH

SRD

$03 / 23 / 62$

HILBUR GE

SRD

14

$03 / 26 / 62$

HILBUR GE

SRD

7

$03 / 26 / 62$

SMITH EA

SRD

$03 / 30 / 62$

NORWOOD KW

SRD

14

$03 / 26 / 62$

WEAKLEY EA

SRD

55

$01 / 11 / 63$

KOSMATA HR

SRD

9

$03 / 28 / 62$ 
Page No. 169

$04 / 28 / 93$

LIST OF HANFORD-GENERATED CLASSIFIED

DOCUMENTS DATED BETUEEN 1/1/61 AND 12/31/72

Doc. NO.

TITLE

AUTHOR

LEVEL

DATE

$H W-73162$

$H W-73170$

HW-73171

HW-73185

HH-73195

$H W-73200$ RD

HW-73209

HW-73229

HW- 73238

HW- 73261

HW-73262

$H W-73265 R D$

HW- 73275

HW- 73310

HW- 73319

HW- 73331

HW-73339

$H W-73380$

HU-73383 RD

HW-73397

$H W-73439 R D$

$H W-73451 R D$
FLON CAPACITY TESTS AT 183-B AND POSSIBLE MODIFICATIONS FOR INCREASED FLOM

MOOIFICATION OF K REACTOR ZONE 12 ORIFICES

FPFTO - PROGRESS REPORT MARCH 1962

REFUELING AN OPERATING REACTOR PROGRESS REPORT FOR THE PROJECTION WELDED BRAZED CLOSURE SCOPE AND JUSTIFICATION FOR AN NPR ANALOG SIMULATION FACILITY

A METHOO TO DETERMINE OPTIMUM VENTURI SIZE

FLOW TESTING REAR FACE HARDWARE COMBINATIONS

INGOT-DINGOT COMPARISON

PROJECT HHITNEY - PLUTONILA

METALLURGICAL DEVELOPMENT - MONTHLY

REPORT - MARCH, 1962

GOAL EXPOSURE PLANS FOR BUMPER AND

NON-BUMPER MATERIAL

SURVEY OF INCIDENTS INVOLVING MUCLEAR

SAFETY AT THE HANFORD PRODUCTION REACTORS

IRRADIATION EFFECTS ON MUCLEAR FUEL ELEMENTS IN THE HANFORD REACTORS

SUPPLEMENTARY INSTRUCTIONS AND SPECIFICATIONS FOR PREPARATION OF OVERBORE FUEL - PT-IP-381-A-FP ATOMIC HEAPON DATA SUPPLEMENT QUARTERLY PROGRESS REPORT PLUTONIUN METALLURGY OPERATION JANUARY - MARCH, 1962 DESIGN CHANGE 538 ADDITIONAL PUMPING CAPACITY - 181-D

PONER LEVEL RECONMENDATIONS INTERIM TO THE CURRENT TUBE REPLACEMENT PROGRAM AT $B, D, D R, F, A M D$ H REACTORS REPORT TO THE WORKING COMIITTEE FROM THE GENERAL ELECTRIC COMPAMY

OPERATING CONDITIONS FOR FIVE NIE-1

ELEMENTS IRRADIATED IN KER-2,

PT-IP-377-A, TEST K-2-14

EXAMINATION OF FUEL CHARGING LIMITATIONS ASSOCIATED HITH GRAPHITE DISTORTION - B, D, F REACTOR

CPD DAILY PROO REPORTS - 1/2/62 THRU $3 / 31 / 62$

PT-105-600-A-FIRST MEASUREMENT OF GRAPHITE TEMPERATURE REACTIVITY COEFFICIENT AT KE (COMPILATION OF DATA)
BRINKMAN LB

MOEN RH

FPO PERSONMEL

MCCARTHY PB

VANCOTT LR

TRLABLE RE

RADTKE WH

HAUN FE, JR.

BLOOMSTRAND RR

HICK OF

SHIMER RD

RD MARNER

HAGIE LT, JAECH dL

SRD

STRAND CA

CRD

HICK OJ, MELON TC,

SRD

STEWART RW, THOMS ID

HEDGES JH

SRD

SPARKS GR

STRINGER JT, MIMOR JE

KRATZER WK

RUSSELL $A$

SRD

RE ROBERTS

SRD

GUMPRECHT RO
SRD
54

05/01/62

5

$03 / 23 / 62$

65

$04 / 16 / 62$

22

$04 / 17 / 62$

$04 / 20 / 62$

$04 / 02 / 62$

$03 / 28 / 62$

$03 / 29 / 62$

$04 / 20 / 62$

04/05/62

$03 / 28 / 62$

$04 / 02 / 62$

$06 / 01 / 62$

$05 / 21 / 62$

$04 / 04 / 62$

$04 / 04 / 62$

$04 / 04 / 62$

$04 / 11 / 62$

$04 / 12 / 62$

$04 / 16 / 62$

SRD

$05 / 13 / 55$ 
Page No. 170

$04 / 28 / 93$

LIST OF HANFORD-GENERATED CLASSIFIED

DOCUMENTS DATED BETHEEN 1/1/61 AND 12/31/72

DOC. NO.

TITLE

AUTHOR

LEVEL

PGS

DATE

HW -73467

HW-73483 SUP1

HW- 73489

HW- 73508

Hบ- -73530

HU- 73545

HW- 73553

HW- 73564

HW-73579

HW- 73580

HW- 73590

HW-73595

HW-73615

HW-73615 SUP2

HN-73615 SUP3

HW-73638

HW-73642 RD

HW- 73650

HW-73650 REV 1

HW-73656

HW-73679

$H W-73700$

HW-73704 RD

HH-73707 APP1
A CONTOUR COMPLETION ALGORITHM SCRAM RECOVERY DATA PRECEDING "C" OVERBORE FUEL FAILURES

BUDGET FOR FY 64 \& REVISED BUDEET FY 63 - SUPPLEMENTARY SEPARATIONS RED PROGRAM (02-53-04\& 02-53-05)

STUDY OF MPR OPTIMUN EXPOSURE AHD UNIT PRODUCT COST

PLUTONIUN FABRICATION DEVELOPMENT

MONTHLY REPORT - APRIL 1962

OPERATIONAL FUELING AT CONSTANT

PRODUCTION

PROPOSAL FOR THE IRRADIATION OF CLADDING

STUDIES CAPSULES - SERIES II

PROJECT WHITNEY - PLUTONIUM

METALLURGICAL DEVELOPMENT - MONTHLY

REPORT - APRIL 1962

SPECIAL PLUTONIUN RECYCLE AT PUREX

C REACTOR OVERBORE FUEL FAILURES

PROGRESS REPORT - FPFTO - APRIL 1962

HYDRAULIC CHARACTERISTICS OF THE UNIFORM

WALL BDF PROCESS TUBE WITH O-111-N FUEL

ELEMENTS

TECHNICAL EVALUATION OF E-N

DEMONSTRATION LOADING (INTERIM REPORT, PROOUCTION TEST (P-350-C)

SUPPLEMENTARY E-N LOAD CONVERSION RATIO

DATA

FINAL REPORT ON E-N CONVERSION RATIO

DATA PRODUCTION TEST IP-350-C

ASSEMBLY AMD PERFORMANCE OF FUEL

ELEMENTS FOR H-REACTOR E-N DEMONSTRATION LOAD

EQUIPMENT FOR PLUTONILM FABRICATION

REACTOR ECONONIC INCENTIVES FOR NICKEL

BONDED FUEL ELEMENTS

REVISED REACTOR ECONONIC INCENTIVES FOR

NICKEL-BONDED FUEL ELEMENTS

STATISTICAL. STATEMENTS \& ASSOCIATED DATA FOR 234-5 BLDG, FINAL PRCOUCT SHIPPED

DURING THE 1ST QUARTER OF CY 1962

CRITICAL MASS CONTROL SPECIFICATION HOOO 7-A

TRANSIENT CALCULATION TECHNIQUES

PLUTONIUM HANDBOOK SECTION IV CHEMICAL PROCESSING OF PLUTONIUM CHAPTERS 1,2 AND 3

ARTIFICIAL COOLING OF THE COLUMBIA RIVER BY DAM REGULATION 1961
DEAN RY

WOOD SA

SRD

SRD

10

9

$04 / 24 / 62$

05/15/62

SECR

$06 / 30 / 70$

LANG LH, MCAURRY JW, DOWIS UJ

HICK OS

JENSEN RD

MEBER JH

SRD

28

$06 / 25 / 62$

SRD

4

05/01/62

SRD

7

05/02/62

05/03/62

WICK OJ

SRD

$$
8
$$

SRD

5

05/03/62

JUDSON BF, RATHVON HC AMBROSE TH, GRAVES SM AE SMITH

$05 / 16 / 62$

ROTAN KF, WATERS ED

SRD

$04 / 30 / 62$

05/08/62

$05 / 01 / 62$

CARTER RD, NECHODON US, SRD

SHIMER RD, FULLMER GC

MECHOOOM US

SRD

CARTER RD, BLYCKERT WA

SRD

BLANTON WA, HODGSON WH

SRD

ALBAUGH FH

HEEB CM

SRD

SRD

HEEB CM

SRD

AE SHITH

SRD

$11 / 21 / 63$

05/11/62

MEEKER LM

CRD

14

05/16/62

BRUGSE RO, STAMP SR, WORTHINGTON JT

CLEVELAND JM

SRD

133

$12 / 20 / 56$

CRD

138

05/18/62

KRAMER HA

$06 / 15 / 62$ 
Page No. 171

$04 / 28 / 93$

LIST OF HANFORD-GENERATED CLASSIFIED

DOCLMENTS DATED BETUEEN 1/1/61 AND 12/31/72

DOC. NO.

HW- 73713

HW- 73724

HW- 73725

HW- 73743

HH- 73754

HW- 73763

HW- 73764

$H W-73780$

HU-73784 RD

HW- 73853

HW- 73897

HW-73906 RD

HW-73906 RD2

HW- 73917

HW- 73930

$H W-73930$ RD

HW-73936

HH- 73956

HW- 73958

$H W-7396,3$ RD

HW- 73981

HH- 73991

HH-74029
TITLE

DESCRIPTION OF THE MET RETURN

OPTIMIZATION COMPUTER PROGRAM - IBM

7090, FORTRAN MONITOP

IRRADIATION PROCESSING DEPARTMENT

OPERATING REPORT PERIOD ENDED MAY 31, 1962

ADJUSTMENT OF TOTAL CONTROL PARAMETERS

FOR C REACTOR

TRIP REPORT TO ROCKY FLATS

CPD - PLANT IMPROVEMENT PROGRAMS

HORIZONTAL CONTROL ROO CORROSION - KW

REACTOR

PLUTONILM FABRICATION DEVELOPMENT

MONTHLY REPORT - MAY 1962

PROGRESS REPORT - FPFTO - MAY 1962

PROOUCTION TEST IP-508-D, IRRADIATION OF

A POROUS STAINLESS STEEL CLAD FUEL

ELEMENT ASSEMBLY

CALCULATED FUEL ELEMENT MODEL PARAMETERS

KVNS AND KVES

NITROGEN IN REACTOR ATMOSPHERE

DESIGN CRITERIA MODIFICATIONS FOR USE OF

ZIRCONIUN TUBES 100-K REACTOR

DESIGN CRITERIA MODIFICATIONS FOR USE OF

ZIRCONIUM TUBES $100 \mathrm{~K}$ REACTORS

PROJECT WHITNEY - PLUTOHIUM

METALLURGICAL DEVELOPMENT - MONTHLY

REPORT - MAY, 1962

PROOUCTION TEST IP-467-C SUPPLEMENT B

REDUCING MINIMUM DONTIME - $K$ REACTORS

PRODUCTION TEST IP-467-C SUPPLEMENT B

REDUCING MINIMUM

PROOUCTION TEST IP-510-A, IRRADIATION OF

CLADDING STUDIES CAPSULES, SERIES II

EXAMPLES OF THE CHANGES IN TEMPERATURE, PRESSURE AND FLOW FOLLONING A REACTOR POWER SURGE

RUPTURED SLUG DATA JANUARY 1, 1962, THROUGH APRIL 30, 1962

PROCESS FLON SHEET--PONER-ROLL FORMING (FLOTURN PROCESS) FOR ALLOYED AND

UNALLOYED PLUTONIUM

POISON COMPENSATION WITH THE XENON

OVERRIDE

PROCESS IMPROVEMENT TRANSITION

AUTHORIZATION IP-20-I INCREASED GRAPHITE

TEMPERATURE LIMITS AND USE OF NITROGEN

IN THE K REACTORS

PLUTONILM FABRICATION DEVELOPMENT

MONTHLY REPORT - JUNE 1962

$$
\text { AUTHOR }
$$

SPARKS GR

SRD

66

$05 / 21 / 62$

CRD

65

$06 / 07 / 62$

VAUGHN AD

SRD

8

$05 / 22 / 62$

BELLARTS HJ

RENBERGER DL.

HICK OJ

AE SAITH

MARSHALL RK, COX JH

SRD

SECR

SRD

$$
6
$$

05/22/62

$06 / 30 / 70$

05/25/62

06/01/62

SRD

5

05/29/62

SRD

SRD

14

05/28/62

HEEB CM

SRD

4

05/29/62

AB GRENINGER

SCHACK MH

SCHACK MH

CRD

CRD

16

06/04/62

$06 / 11 / 62$

CRD

20

$08 / 02 / 62$

HICK OJ

SRD

$$
6
$$

06/05/62

CHITUOOO RA

SRD

13

06/06/62

CHITWOOD RA

SRD

12

07/05/62

KRATZER WK

SRD

10

$06 / 11 / 62$

KREITER MR, BATCH JM SRD

7

06/07/62

MEUELL LJ

SRD

10

06/08/62

SMITH AE

SRD

6

$06 / 11 / 62$

OUSLEY GF

SRD

7

06/12/62

BAARS RE

SRD

23

$06 / 13 / 62$

HICK OJ

SRD

6

07/02/62 
Page No. 172

$04 / 28 / 93$

LIST OF HANFORD-GENERATED CLASSIFIED

DOCUMENTS DATED BETUEEN 1/1/61 AND 12/31/72

DOC. NO.

$H W-74031$ RD

HW-74048 REV1

HW-74068

HW-74078

HW-74095 VOL2

HW-74096

HW-74104

HW-74104 RD

HW-74109

HW-74114

HW-74121

HW-74122

HW-74125 RD

HW-74133

HW-74150

HW-74151 G

HW-74155

HH-74156

HW-74163

HW-74164

HW-74165

$H W-74188 R D$

HW-74193
TITLE

ANALYSIS OF DIMENSIONAL DIFFERENCES OF PLUTONIUM METAL PARTS - FINISHED PROOUCTS OPERATION UCLRL CLASSIFICATION CUIDANCE PROPOSAL HANFORD ASSISTANCE TO UCLRL FY-63 NEW MATERIALS DEVELOPMENT TRANSMITTAL OF PROPOSAL (HH-74068) FOR HANFORD ASSISTANCE TO UCLRL HAZARDS SUMHARY REPORT VOL2 PROCESS CONTROL \& TECHNICAL DATA HANFORD $K$ PROOUCTION REACTORS

THE APPLICATION OF AN OPERATIONAL REACTIVITY ACCOUHTING SYSTEM BASED ON ONE-GROUP DIFFUSION THEORY

TECHNICAL CRITERIA AND BASES FOR A ZIRCONIUM-TUBED $K$ REACTOR TECHNICAL CRITERIA AMD BASES FOR A ZIRCONIUM-TUBED $K$ REACTOR SURVEY OF GRAPHITE DISTORTION AND BURNOUT IN HANFORD REACTORS PROJECT HHITMEY - PLUTONIUA METALLURGICAL DEVELOPMENT - MONTHLY REPORT - JUNE, 1962

BULK OUTLET TEMPERATURE AND RELATED REQUIREMENTS

A REVIEH OF HANFORD PONER LEVEL LIMITS CONVERSION OF C REACTOR TO NITROGEN-HELIUM ATMOSPHERE REACTOR AND METALLURGY RED PROGRAMS IRRADIATION PROCESSING DEPARTMENT FY-1963 - FY-1964

2244 DATA LOG BOOK Wh MONTHLY REPORT CHEMICAL PROCESSIMG DEPARTMENT, RESEARCH AND ENGINEERING OPERATION - JUME $19<2$ CONTRACTION OF GRAPHITE: A COMPARISON OF LABORATORY AND PRCOUCTION REACTOR DATA PART I - B, D, F, H, OR, AND C REACTORS

RHO - MONTHLY REPORT FOR JUNE 1962 ATOMIC WEAPON DATA SUPPLEMENT OUARTERLY PROGRESS REPORT PLUTONILM METALLURGY OPERATION APRIL - JUNE, 1962

PROGRESS REPORT - FPFTO - JUNE 1962

A SUMMARY OF THE IRRADIATION PERFORMANCE OF ROO CLUSTER FUEL ELEMENTS

TABULATION OF LOADS, OPERATING FUND COST \& CAPITAL ADDITIONS FOR P BLENDING CASES

PRELIMIMARY HAZARDS REVIEW FUELS RECYCLE PILOT PLANT
AUTHOR

MCHAHON WH

CRD

22

$06 / 14 / 62$

BORCHIER BJ

ALBAUGH FW

SRD

SRD

PARKER MM

SRD

HAPO

CRD

STEWART SL

SRO

19

$07 / 02 / 62$

CURTISS DH

SRD

22

SRD

20

16

SRD

12

07/02/62

POOR CF, SPENCER HG

SRD

7

$06 / 28 / 62$

VANLORMER FU

SRD

CRD

24

$06 / 28 / 62$

DUNN RE

SRD

49

$09 / 14 / 62$

RY LYOW

FRANK Us

SECR

CRD

13

07/02/62

07/06/62

GIBERSON RC, MORGAN WC

SRD

25

$08 / 06 / 62$

AN STEVENS

SECR

WICK OJ, NELSON TC,

SRD

STEWART RH, THOWAS ID

AE SMITH

SRD

CALL RL, CLAUDSON TT

CRD

28

$07 / 03 / 62$

$07 / 01 / 62$

SECR

$06 / 30 / 70$

SCHNEIDER KJ
SRD

138

07/05/62 
Page No. 173

$04 / 28 / 93$

LIST OF HANFORD-GENERATED CLASSIFIED

DOCUMENTS DATED BETUEEN 1/1/61 AND 12/31/72

DOC. NO.

TITLE

AUTHOR

LEVEL

PGS

DATE

HW- 74207

HW-74209

HW-74211

HW-74239

HW-74255

HW-74258

HW-74284

HW-74297

HW-74305

HW-74335

HW-74341

HW-74377

$H W-74378$

HH-74381

HW-74384

HW-74395

HW- 74403

HW-74406

HW-74407
PROPOSAL FOR CHARGING TEST FUEL ELEMENT WITH CORRUGATED OUTER JACKET GEH-10-62 PLUTONIUM BLENDING AT Z PLANT JUSTIFICATION FOR ZIRCONIUM TUBE INSTALLATION 100-K REACTOR FRIMGE CHANMELS

PLUTONIUM FABRICATION DEVELOPMENT MONTHLY REPORT - JULY 1962

HANFORD LABORATORIES SS MATERIAL REPORT MAY 31, 1962

CRITICAL MASS ESTIMATE FOR A REFLECTED ARRAY OF CASTINGS

CP NUCLEAR MATERIALS CONTROL - JUNE 1962 CHAPTER 18 OF PRCDUCTION CAPACITY STUDY NO 4

PROOUCTION TEST IP-520-A IN-REACTOR TESTING HITH COOLANT PREPARED IN THE MATER TREATMENT PILOT PLANT

RADIOMETALLURGY EXAMINATION OF THREE BUMPERED FUEL FAILURES FROM D REACTOR/RM 449/

FINAL REPORT: IRRADIATION PERFORMANCE OF ENRICHED SEVEN-ROD CLUSTER FUEL ELEMENTS WITH 20- AND 30-MIL-THICK CLADDING, SUPPLEMENT B TO PRODUCTION TEST IP-226-A, (RM-562)

QUARTERLY PROGRESS REPORT FUELS DEVELOPMENT OPERATION APRIL, MAY, JUNE, 1962

QUARTERLY PROGRESS REPORT FUELS DEVELOPMENT OPERATION JULY, AUCUST, SEPTEMBER, 1962

TECHNICAL AND ECONOMIC STUDY ENLARGED REAR FACE HARDWARE - OLD REACTORS FINAL REPORT: IN-REACTOR RUPTURE TESTING OF ZIRCALOY-2-CLAD, COEXTRLDED FUEL ELEMENTS IN THE ENGINEERIMG TEST REACTOR

100-C REACTOR ZIRCONIUM TUBE INSTALLLATION BOTTOH SIX RONS FINAL REPORT: IRRADIATION PERFORMANCE OF TWO EIGHTEEN INCH LONG TUBE-IN-TUBE FUEL ELEMENTS, PT-IP-292-A (RM-572) FINAL. REPORT: LOW EXPOSURE IRRADIATION OF AN ENRICHED SEVEN-ROD CLUSTER IN KER OOP 1, PT-IP-246-A

FINAL REPORT: IRRADIATION PERFORMANCE OF A COEXTRLDED, ZIRCALOY-2-CLAD THREE-ROD CLUSTER FUEL ELEMENTS, PT-IP-186-A

\begin{tabular}{|c|c|c|c|}
\hline WHEELER RG, HECK EN & CRD & 26 & $07 / 06 / 62$ \\
\hline SWAIN EO & SRD & 20 & $07 / 16 / 62$ \\
\hline YOWNG JR & SRD & 6 & $07 / 10 / 62$ \\
\hline HICK OJ & SRD & 5 & $08 / 01 / 62$ \\
\hline CLOUSE he & SRD & 14 & $07 / 12 / 62$ \\
\hline CLAYTON ED, STEVENSON RL & SRD & 7 & $07 / 26 / 62$ \\
\hline $\begin{array}{l}\text { CJ SHORTESS JR } \\
\text { WARREN JH }\end{array}$ & $\begin{array}{l}\text { SECR } \\
\text { SRD }\end{array}$ & 33 & $\begin{array}{l}07 / 18 / 62 \\
07 / 16 / 62\end{array}$ \\
\hline GEIER RG & SRD & 9 & $07 / 16 / 62$ \\
\hline TEATS R & CRD & 21 & $07 / 27 / 62$ \\
\hline CALL RL & CRD & 34 & $07 / 01 / 62$ \\
\hline $\begin{array}{l}\text { CADUELL JJ, TOBIN IC, } \\
\text { LAST GA, EVANS EA, MINOR } \\
\text { JE }\end{array}$ & SRD & 129 & $07 / 20 / 62$ \\
\hline $\begin{array}{l}\text { CADUELL JJ, TOBIN JC, } \\
\text { LAST GA, EVAHS EA, MINOR } \\
\text { JE }\end{array}$ & SRD & 196 & $10 / 15 / 62$ \\
\hline FRIELIMG DH & SRD & 26 & $08 / 03 / 62$ \\
\hline CALL RL, KAULITZ DC & CRD & 62 & $07 / 01 / 62$ \\
\hline YOUNG JR & SRD & 6 & $07 / 25 / 62$ \\
\hline CALL RL & SRD & 13 & $07 / 25 / 62$ \\
\hline CALL RL & SRD & 3 & $07 / 25 / 62$ \\
\hline CALL RL & SRD & 4 & $07 / 25 / 62$ \\
\hline
\end{tabular}


Page No. 174

$04 / 28 / 93$

LIST OF HANFORD-GENERATED CLASSIFIED

DOCUMENTS DATED BETWEEN 1/1/61 AND 12/31/72

DOC. NO.

HW-74408

HW-744472-R3 PTI

HU-744472-R3 PT10

HW-744472-R3 PT11

HW-744472-R3 PT12

HW-744472-R3 PT2

HW-74447Z-R3 PT3

HU-74447Z-R3 PT4

HU-744472-R3 PT5

HN-74447Z-R3 PT6

HW-744472-R3 PT7

HW-744472-R3 PT8

HU-744472-R3 PT9

HW-74467

HW-74479

HW-74479 ADD

HU-74483

HW-74484RD

HW-74498

HW-74509

HW-74510

HU-74518

HW-74519

HU-74535

HN-74545
TITLE

REVISED GOAL EXPOSURE PLAN

FINISHED PROOUCTS PRODUCT RECOVERY

SOURCE DATA JULY 1962

FINISHED PROOUCTS - PRODUCT RECOVERY

SOURCE DATA APRIL 1963.

FIHISHED PRODUCTS - PRCDUCT RECOVERY SOURCE DATA MAY 1963.

FINISHED PRCDUCTS - PRCOUCT RECOVERY SOURCE DATA JUNE 1963.

FINISHED PROOUCTS PRODUCT RECOVERY

SOURCE DATA AUCUST 1962

FINISHED PRCOUCTS PRCOUCT RECOVERY

SOURCE DATA SEPTEMBER 1962

FINISHED PRCOUCTS PRCOUCT RECOVERY

SOURCE DATA OCTOBER 1962

FINISHED PRCOUCTS PROOUCT RECOVERY

SOURCE DATA NOVEMBER 1962

FINISHED PROOUCTS - PRCOUCT RECOVERY

SOURCE DATA DECEMBER, 1962.

FINISHED PROOUCTS - PRCOUCT RECOVERY

SOURCE DATA JAMUARY 1963.

FINISHED PRCOUCTS - PRODUCT RECOVERY

SOURCE DATA FEBRUARY 1963.

FINISHED PROOUCTS - PRODUCT RECOVERY

SOURCE DATA MARCH 1963.

PERFORMANCE RATES

ALTERMATIVE CONSTANT PROOUCTION CASES

ALTERMATIVE CONSTANT PRCOUCTION CASES

(ONE REACTOR OVERBORE CASES)

FINAL REPORT: IRRADIATION PERFORMANCE

OF AN NIN-1 FUEL ELEMENT IN THE

ENGIMEERING TEST REACTOR, GEH-10-43, (RM-596)

PILE PHYSICS MONTHLY REPORT

PROGRESS REPORT - FPFTO - JULY 1962

ECONONICS OF $K$ REACTOR RETUBING

STATISTICAL STATEMENTS \& ASSOCIATED DATA

FOR 234-5 BLDG, FINAL PRCOUCT SHIPPED

DURING THE 2ND QUARTER OF CY 1962

RMO - MONTHLY REPORT FOR JULY 1962

QUARTERLY SUMUARY REPORT \& ASSOCIATED

DATA FOR 234-5 BLDG, FIMAL PRODUCT

SHIPPED DURING THE IST \& 2ND QUARTER OF

CY 1962

PROJECT WHITNEY - PLUTONIUH

METALLURGICAL DEVELOPMENT - MONTHLY

REPORT - JULY, 1962

SUMARY OF CPD NUCLEAR MATERIALS CONTROL DURING FY-1962 AND FUTURE CONTROL PROGRAM.

$$
\text { AUTHOR }
$$

SHIMER RD

GB RUKLIMSKI

GB KUKLIMSKI

GB KUKLINSKI

GB RUKLINSKI

GB KUKLINSKI

GB RUKLINSKI

GB RUKLINSKI

GB KUKLIMSKI

GB KUKLINSKI

GB KUKLINSKI

GB KUKLINSKI

GB KUKLIMSKI

LJ MEWELL

LANG LW, MCCURRY JH

LANG LH, MCCURRY JH

CALL RL

DE MEMBROUGH

AE SNITH

BAILEY GF

AE SMITH

AJ STEVENS

AE SNITH

HICK OJ

RU DOERR
LEVEL PQS

DATE

08/07/62

07/01/62

$04 / 01 / 63$

$05 / 01 / 63$

06/01/63

$08 / 01 / 62$

09/01/62

$10 / 01 / 62$

$11 / 01 / 62$

12/01/62

$01 / 01 / 63$

$02 / 01 / 63$

03/01/63

$07 / 30 / 62$

$07 / 31 / 62$

08/09/62

$07 / 31 / 62$

$07 / 31 / 62$

$08 / 03 / 62$

$08 / 17 / 62$

08/08/62

$07 / 31 / 62$

08/03/62

SRD
SRD

SRD 7

08/07/62

SECR

$08 / 10 / 62$ 
Page No. 175

$04 / 28 / 93$

LIST OF HANFORD-GENERATED CLASSIFIED

DOCUMENTS DATED BETHEEN 1/1/61 AMD 12/31/72

DOC. NO.

TITLE

AUTHOR

LEVEL PGS

DATE

HW-74547

$H H-74570$

HW-74577

$H W-74577$ ADD

HW-74583

HU-74604

HW-74607

HU-74626 RD

HW-74628

HW-74645

$H W-74660$

$\mathrm{HH}-74665$

HW-74669

$H W-74681$

HW-74682

HW-74684

HW-74694

$H W-74695-R D$

HW-74698

HW-74699

HH-74706 RD

HW-74714

HW-74717 REV
MUNERICAL RESULTS OF PROOUCTION TEST IP-466-A TEST OF IHE 190 TURBINE PUMPS AT KE (KH) REACTOR (PROJECT CGI-844) PROOUCTION TEST IP-524-AL TESTING IN 1706-KE SINGLE-PASS TUEES OF OUACHROM gLuCOSATE AS A REPLACEMENT FOR DICHROMATE

KER LOOP $\uparrow$ TUBE TEMPERATURES

KER LOOP I TUBE TEMPERATURES

10 YEAR PLAN FOR MODERNIZATION AND REPLACEMENT OF MACHINE TOOLS AND RELATED EOUIPMENT

EVALUATION OF LITHIUA AS A CONTROL ROO POISON

PROJECT PROPOSAL, REVISION I ADDITIONAL PLUTONIUN STORAGE FACILITIIES - 234-5 BLDG. PROJECT CGC-968

PRELIMINARY REVIEW OF CPD THORIUM PROCESSING POTENTIALS

DESCRIPTION OF ADDITIONAL PLUTONIUM FABRICATION FACILITIES - 234-5 BUILDING THE EFFECTS OF IRRADIATION ON CORROSION IN HIGH-TEMPERATURE HATER - INTERIM REPORT NO. 2

PRECISION OF PLUTONIUM STOCKPILE ESTIMATES

UTILIZING INCREASED MATER PLANT CAPACITY BY DECREASING FUEL ELEMENT SIZE AT THE OLD REACTORS

RESULTS OF LABORATORY HEAT TRANSFER EXPERIMENTS FOR $K$ REACTOR FUEL CHAMMELS EXAMINATION OF RUPTURE FRON 1372-H (RM 454)

EXAMINATION OF ENRICHED RUPTURED ELEMENT FRON 2955C (RM 452)

PRCOUCTION IN THE NPR

PPP-METAL STABILIZATION FACILITY - 234-5

BLDG (PROJECT CGC-987)

PRELIMIMARY SURVEY POTENTIAL EQUIPMENT

IMPROVEMENTS 333 BUILDIMG

FABRICATION CAPABILITY PROGRAM

A FEASIBILITY STUDY THE APPLICATION OF COOLING TONERS FOR REDUCTION OF HANFORD PLANT HEAT LOAD TO THE COLUMBIA RIVER INCENTIVE FOR BY-PROOUCT ROOS

FALL SAMPLING PLAN

CRITICAL MASS CONTROL SPECIFICATION 7.35 WEIGHT PERCENT PU-AL ALLOY FUEL ELEMENT STORAGE GAS CYLINDER STORAGE AREA, 234-5 BUILDING

\begin{tabular}{|c|c|c|c|}
\hline RENBERGER DL, SMIT WR & SRD & 32 & $08 / 03 / 62$ \\
\hline $\begin{array}{l}\text { DICKINSON DR, VANLORMER } \\
\text { FM }\end{array}$ & SRD & 10 & $08 / 07 / 62$ \\
\hline AGAR JD & SRD & 20 & $10 / 01 / 62$ \\
\hline ACAR JD & SRD & 5 & $12 / 07 / 62$ \\
\hline OLSON RE & SRD & 11 & $08 / 08 / 62$ \\
\hline CARTER RD & SRD & 2 & $08 / 10 / 62$ \\
\hline GE LISH & SRD & & $09 / 17 / 62$ \\
\hline REY G & SRD & 19 & $10 / 08 / 62$ \\
\hline BELLARTS HJ & SRD & 57 & $09 / 07 / 62$ \\
\hline LARRICK AP & SRD & 27 & $08 / 13 / 62$ \\
\hline GRANOUIST DP & SRD & 10 & $08 / 13 / 62$ \\
\hline HEEB CM & SRD & 8 & $08 / 17 / 62$ \\
\hline
\end{tabular}

KREITER MR, HAMYND JE SRD $18 \quad 10 / 09 / 62$

$\begin{array}{llll}\text { GRUBER WJ CRD } & 5 & 09 / 12 / 62\end{array}$

$\begin{array}{llll}\text { GRUBER HJ SRD } & 13 & 08 / 21 / 62\end{array}$

EVANS TH, SHIELDS RJ SRD $\quad 7 \quad 08 / 21 / 62$

LH FINCH SRD $10 / 15 / 62$

$\begin{array}{llll}\text { DRUMHELLER } K & \text { SRD } & 16 & 08 / 22 / 62\end{array}$

$\begin{array}{llll}\text { SMITH AE } & \text { SRD } & 21 & 08 / 30 / 62\end{array}$

PECK GE SRD $34 \quad 08 / 24 / 62$

LANG LW

SLOAT RJ 
DOC. NO.

HW-74718

HW-74719

HW-74738

HW-74741

HW-74755

HW-74789 RD HW-74811

$H W-74821$ RD

HW-74822

HW-74891

HW-74932

HW-74945

HW-74953

HW-74964

$H W-74965$

HW-74970

$H W-74988$

$H W-74997$

HH- 75044

$H W-75053$

HW-75070

$H W-75131$

$H H-75131$ RD
TITLE

QUARTERLY PROGRESS REPORT

ATOMIC WEAPOW DATA SUPPLEMENT OUARTERLY PROGRESS REPORT PLUTONIUN METALLURGY OPERATION JULY, AUGUST, SEPTEMBER, 1962 PROJECT MHITMEY - PLUTONILN METALLURGICAL DEVELOPMENT - MONTHLY REPORT - AUCUST, 1962 PROGRESS REPORT - FPFTO - AUEUST 1962 IRRADIATION EFFECTS ON THE CORROSION \& HYDRIDING OF ZIRCALOY IN A CONTAMIMATED HELIUM ATMOSPHERE

PROCESS FLON SHEET, ROLLING AND DRAHING RHO - MONTHLY REPORT FOR AUCUST 1962 HANFORD CONTRIBUTION TO FEDC WORKING COMIITTEE REPORT

REPCRT OF THE WORKING COMMITTEE OF THE FUEL ELEMENT DEVELOPNENT COMITTEE SEPTEMBER 18-19-20, 1962

ALTERMATE PROCESS STUOY TRIP REPORT JULY 17-20, 1962

INTERIM REPORT 105-K ZIRCONIUM PROCESS TUBE DISTORTION TEST

PROJECT PROPOSAL: ADDITIONAL PLUTONIUA

CASTING FACILITIES - RMA - 234-5 BLDG (CGC-988)

6.6 PH PROCESS WATER AT 100-B,DR, F, AND

PROJECT PROPOSAL ELECTRICAL AND

HYDRAULIC EQUIPMENT MEZZANINE RMA LINE 234-5 BLDG - PROJECT CGC-984

PROJECT PROPOSAL PLUTONIUM INGOTING AMD AUXILIARIES FACILITY- RMA LIME - 234-5 BLDG - PROJECT CGC-983

INTERIM REPORT OF PRODUCTION TEST IP-394-I USE OF PT-216 FUEL COLUNAS FOR ROUTINE AXIAL FLUX OETERMINATION

STATUS REPORT - PROJECTION FUEL ELEMENT TESTING PROGRAM

REDOX TEST PROGRAM, FY 1963

PLUTONIUM FABRICATION DEVELOPMENT

MONTHLY REPORT - SEPTEMBER 1962

RESEARCH AND DEVELOPMENT INCENTIVES PLUTOWIUM PROCESSING

PROGRESS REPORT - WEAPONS PROCESS ENG SEPTEMBER 1962

STRESS CONSIDERATIONS ON A CYLINDRICAL BLOCK WITH A HEMISPERICAL CAVITY UNDER INTERNAL PRESSURE

STRESS CONSIDERATIONS ON A CYLINDRICAL BLOCK WITH A HEMISPHERICAL CAVITY UNOER INTERNAL PRESSURE
MUTHOR LEVEL PGS DATE
Ma

HICK Od, NELSOW TC, STEWART RH, THOMAS ID WICK OJ, NELSON TC,

$10 / 15 / 62$

STEWART RH, THOMAS ID

SRD $\quad 80$

$10 / 15 / 62$

HICK OJ

SRD

8

$08 / 28 / 62$

AE SMITH

SRD

09/05/62

DW SHANMON, RS MOPE

CRD

$02 / 02 / 63$

SHITH AE

AJ STEVENS

SRD

SRD

SRD

6

$08 / 30 / 62$

NORLOOO KH

62

SRD

150

$09 / 18 / 62$

BURGESS CA, STENOUIST DR SRD

$09 / 04 / 62$

RUSSELL A

CRD

12

$09 / 12 / 62$

NM RAILE

SRD

$10 / 15 / 62$

YOUNG JR

SRD

13

$09 / 13 / 62$

MN RAILE

SRD

$09 / 20 / 62$

MN RAILE

SRD

$09 / 20 / 62$

NIELSON SM

SRD

37

$09 / 17 / 62$

PEACOCK DH, nibrose TH

SRD

21

$07 / 31 / 62$

RG BARNES

CRD

$09 / 21 / 62$

WICK OJ

SRD

5

$10 / 01 / 62$

HH HOPKINS

$10 / 01 / 62$

AE SAITH

$10 / 22 / 62$

RIDER RL

23

$07 / 24 / 62$

RIDER RL

SRD

$07 / 24 / 62$ 
Page No. 177

$04 / 28 / 93$

LIST OF HANFORD-GENERATED CLASSIFIED

DOCUMENTS DATED BETWEEN 1/1/61 AND 12/31/72

DOC. HO.

HW- 75159

$H H-75164$

$H H-75206$

HW-75207

HW- 75222

HH- 75246

HW- 75247

HW- 75259 RD

HW- 75280

$H W-75288$

$H W-75291$

KW- 75327

HW-75339

HW- 75364

$H W-75375$

HW-75378

HH- 75383

HW- 75387

$\mathrm{HW}-75407$

HH-75410 A

$H W-75412$

$H W-75413$

HU- 73414
TITLE

PROJECT UHITNEY - PLUTONILA

METALLURGICAL DEVELOPMENT - MOWTHLY

REPORT - SEPTEMBER, 1962

CGI-844 - 100-K COOLANT BACKUP SYSTEM

DESIGN BASIS

INTERIM REPORT PROOUCTION TEST IP-467-C, SUPPLEMENT A HORIZONTAL ROO CALIBRATION

AT B REACTOR DURING XENOW OVERRIDE

STARTUP

REACTOR DESIGN AMALYSIS MONTHLY REPORT NUGUST \& SEPTEMBER, 1962

CRITICAL MASS CONTROL - CONTIMLOUS

NEPTUNIUN

CPD MEAPONS MANUFACTURING COSTS PER UNIT CALEMDAR YEAR TO DATE

CPD REDOX MANUFACTURING COST PER UNIT CALENDAR YEAR TO DATE

REVIEW OF C OVERBORE INCENTIVE

PRCOUCTION TEST IP-536-A, IRRADIATION OF N-REACTOR INWER FUEL TUBES IN KER LOOPS

1 AND 2

HANFORD CONTRIBUTION FOR THE 15TH HIGH

TEMPERATURE FUELS COMIITTEE MEETING,

NOVEMBER 1962

PERFORMANCE TEST ADDITIONAL PUMPING

CAPACITY, 181-D

REORIFICING PROPOSAL O REACTOR

PITA-IP-22 E-N DEMONSTRATION LOAD

HAPO COST, PROOUCTION AND UNIT COST DATA

FINAL REPORT - TEST NO. 2 PRCOUCTION

TEST IP-461-E

RESULTS OF PROOUCTION TEST IP-528-I F

REACTOR HIGH TANK DRALDON TEST

SUPPLEMENT B, PRCOUCTION TEST IP-536-A

IRRADIATIOH OF N-REACTOR INMER FUEL

TUBES IN KER LOOPS 1 AND 2

PRODUCTION TEST IP-541-A REACTOR

EFFLUENT STUDIES

FINAL REPORT PT IP-447-C ROD DROP

TRANSIENT COMPARISON, D AND DR REACTORS

REDEFINITION OF DR VERTICAL SAFETY

SYSTEM STRENGTH

FINAL REPORT: PROOUCTION TEST IP-543-A

EFFLUENT SAMPLING - OVERBORE TUBE

PLUTONILA FABRICATION DEVELOPNENT

MONTHLY REPORT - OS,TOBER, 1962

PROJECT WHITHEY - PLUTOHILA

METALLURGICAL DEVELOPMENT - MONTHLY

REPORT - OCTOB'.R, 1962

KER-3 OPERAT ING REPORT TEST K-3-16 PT IP- $477-A$
AUTHOR

HICK OJ

WATSON DF

VAUGHA AD

SRD

15

$11 / 13 / 62$

$10 / 09 / 62$

DL COADOTTA

CRD

$10 / 09 / 62$

BARNES RG

SRD

10

05/09/62

DL MORRELL

SECR

$10 / 16 / 62$

DL MORRELL

SECR

$10 / 16 / 62$

NEEF MI, MELELL LJ

WX KRATZER

SRD

9

10/12/62

$10 / 16 / 62$

LAST GA

CRD

11

$11 / 02 / 62$

HEDGES JH

SRD

9

$10 / 30 / 62$

HOLLIFIELD PJ

CARTER RD

LESSOR LC

LESSOR LC

WK KRATZER

GEIER RG

VAUGHN AD

GEIER RG

HICK OJ

H:Ex oj

QUIAN hT
SRD

12

$10 / 29 / 62$

SRD

11

$10 / 10 / 62$

SRD

8

$01 / 09 / 64$

SRD

6

$11 / 01 / 62$

$10 / 19 / 62$

$10 / 22 / 62$

$06 / 30 / 70$

$10 / 24 / 62$

$10 / 24 / 62$

$10 / 24 / 62$

SRD

5

$10 / 30 / 62$

SRD

8

$11 / 20 / 62$ 
Page No. 178

$04 / 28 / 93$

LIST OF HANFORD-GENERATED CLASSIFIED

DOCUNENTS DATED BETWEEN 1/1/61 AND 12/31/72

DOC. NO.

HW -75415

HW- $75419 \mathrm{C}$

HW- 75421

HU-75444

$H H-75449$ RD

HH- 75450

HW- 75452

HW-75456

$H W-75460$

HW- 75465

HW- $75465 \mathrm{C}$

$H W-75465 E$

$H W-75465 G$

$H W-75466$ RD

HM- 75473

$H W-75487$

$H H-75490$

$H W-75493$

HW -75534

$H U-75542$

HW- 75557
TITLE

KER-4 OPERATING REPORT TEST $K-4-13$ PT

IP-4T7-A

EO - FUEL PREPARATION DEPT MONTHLY REPORT OCTOBER 1962

CRITICAL MASS CALCULATIONS FOR LOH DENSITY METAL ARRAYS

PROGRESS REPORT - MEAPONS PROCESS ENG OCTOBER 1962

MEASURED VS CALCULATED RIVER T

PRELIMINARY ANALYSIS MOOIFICATION OF PRE-K REACTORS

TRITIUN IN HANFORD SAGEBRUSH SAMPLES

PRCOUCTION TEST IP-544-A, IRRADIATION OF

1.6\% ENRICHED THICK HALLED SINGLE TUBE

ELEMENTS IN KER-1 AND 2

PROJECT WHITNEY MEW MATERIALS

DEVELOPMENT BI-MONTHLY REPORT,

SEPTEMBER, OCTOBER, 1962

PROOUCTION TEST IP-546-A IRRADIATION OF HOT DIE SIZE DIFFUSION BONDED FUEL

ELEMENTS

FABRICATION OF HOT DIE SIZE DIFFUSION BONDED FUEL ELEMENTS FOR PRODUCTION TEST IP-546-A

SUPPLEMENT A TO PRODUCTION TEST IP-546-A IRRADIATION OF DIFFUSION-BONDED FUEL ELEMENTS

FABRICATION OF HOT DIE SIZED DIFFUSION BONDED FUEL ELEMENTS FOR SUPPLEMENT "A" TO PRODUCTION TEST IP-5:46-A

REACTOR INSTRUMENTATION AND SAFETY CIRCUIT STATUS REVIEH AND PROGRAM DOCUMENT

RE: HU-75370, "BY-PROOUCT PRCDUCTION IN HCR'S", HEACOCK, H.H., 10/24/62

QUARTERLY SUMAARY REPORT \& ASSOCIATED DATA FOR :34-5 BLDG, FINAL PRODUCT SHIPPED DURI I THE 3RD QUARTER OF CY 1962

HYDRAULIC CHARACTERISTICS OF C-IIIN \& C- $I I I-E$ FUEL ELEMENTS IN A "C" UNIFORM WALL PROCESS TUBE

REVIEH OF REACTOR ERAPHITE DISTORTION PROBLEMS

REACTOR DESIGA ANALYSIS MONTHLY REPORT OCTOBER 1962

190-DR STEAM TURBINE BACKUP ADECUACY REPORT

DECONTAMINATION OF the PLUTONILM RECYCLE TEST REACTOR

\begin{tabular}{|c|c|c|c|}
\hline AUTHOR & LEVEL & PGS & DATE \\
\hline DAVIS JP & SRD & 17 & $10 / 29 / 62$ \\
\hline LM LOEB & SRD & & $11 / 07 / 62$ \\
\hline STEVENSON RL & SRD & 6 & $10 / 29 / 62$ \\
\hline AE SMITH & SRD & & $11 / 21 / 62$ \\
\hline CORLEY JP & SRD & 6 & $10 / 25 / 62$ \\
\hline BAILEY GF & SRD & 4 & $10 / 30 / 62$ \\
\hline BROUNS RJ & SRD & 7 & $10 / 31 / 62$ \\
\hline KRATZER WK, HISE MJ & SRD & 7 & $12 / 12 / 62$ \\
\hline HICK OJ & SRD & 6 & $10 / 31 / 62$ \\
\hline HLADEK KL, BURGESS CA & SRD & 11 & $06 / 27 / 63^{\prime}$ \\
\hline STRAND CA & SRD & 14 & $10 / 10 / 63$ \\
\hline HLADEK KL & SRD & 7 & $02 / 04 / 64$ \\
\hline Strand Ca & CRD & 8 & $10 / 05 / 64$ \\
\hline DEICHMAN JL & CRD & 82 & $02 / 15 / 63$ \\
\hline LANG LH & SRD & 2 & $11 / 01 / 62$ \\
\hline AE SMITH & SRD & & $11 / 01 / 62$ \\
\hline
\end{tabular}

MR KREITER, KF ROTAN, ED CRD

$12 / 31 / 62$ WATERS

COUGHREN KD, KEMPF FJ, SRD

46

$11 / 19 / 62$

MUNRO CA

DL CONDOTTA

CRD

$11 / 07 / 62$

RADTKE WH, KEPLINGER ER SRD

i

$11 / 08 / 62$

AYRES JA, DEEMITT TF, CRD

95

05/01/63 HAYDEN KD, PERRIGO LD, HEED 
Page No. 179

$04 / 28 / 93$

LIST OF HANFORD-GENERATED CLASSIFIED

DOCUMENTS DATED BETWEEN 1/1/61 AND 12/31/72

DOC. NO.

$H W-75559$

HW- 75588

HW- 75589

$H W-75590$

HH- $\mathbf{3 5 9 9 1}$

HW- 75603

HW-75607

$H W-75607$ A

HW-75611

HW-75619

HH- 75628

Hแ⿰纟- 75639

HW- 75648

$H W-75651$

$H W-75667$

HW-75672

HW- 75697

HW- 75700

HW- 75713

HH- 75727

HU- 75743

HW-75752

배-75753

$H 4-75755$
TITLE

PHYSICS CONSIDERATIONS OF $K$ REACTOR CHANMEL STRAIGHTENING

ESTIMATED OUTAGE REQUIREMENTS -

ZIRCONIUM RETUBING PROGRAM - TYPICAL OLD REACTORS

K-REACTOR RETUBING PROGRAM GRAPHITE TEMPERATURE STUDY

PCA \#2-142 EXCESSIVE VSR'S OUT OF SERVICE, B REACTOR

DESIGN CRITERIA HIGH PRESSURE AUTOCALVE DEVELOPMENT FACILITY PROJECT CAF-954 FRINGE TUBE THICKMESS AND CORROSION RATES - $X$ REACTORS

PRCDUCTION TEST IP-550-I ROUTINE MONITORING FOR MODERATOR OXIDATION RATES PRODUCTION TEST IP-550-1, SUPPLEMENT A ROUTINE MONITORIHG FOR MODERATOR OXIDATION RATES

C REACTOR OVERBORE FUEL EXAMIMATION

EFFECT OF PROCESS TUBE DIMENSIONS ON TUBE LIFE

PROOUCTION PLANNING STUOY (TYPICAL REACTOR)

PLUTONIUM PROOUCT DEVELOPMENT OPERATION

MONTHLY REPORT - NOVEMBER, 1962

PROCESS DESIGN BASES METAL BLANK

PREPARATION RMA LIME - 234-5 BUILDING

PROJECT HHITHEY - PLUTONIUM

METALLUURGICAL DEVELOPMENT - MONTHLY

REPORT - NOVEMBER, 1962

PROGRESS REPORT - WEAPONS PROCESS ENG NOVEMBER 1962

INSTRUMENTATION STLDIES STATUS REPORT II

(HIGH LEVEL AMD RATE-OF-RISE)

RMO - MONTHLY REPORT FOR NOVEMBER 1962

1962 ANHUAL REPORT

PROPOSAL FOR IRRADIATING A TEST FUEL

ELEMENT WITH FLUTED OUTER SURFACE: GEH

10-67 AND GEH 10-68

MUMERICAL RESULTS OF PT-IP-338A AND

SUPPLEMEAT A DR REACTOR HEAT DECAY TEST

AT HIGH OUTLET WATER TEMPERATURES

SWELLING BEHAVIOR OF N-REACTOR FUEL

ELEMENTS (AN INTERIM REPORT)

PONER GENERATION OF SAR FUEL ASSEMBLIES

IN KER LOOP

HYDRAULIC CHARACTERISTICS OF ORNL-184

FUEL PIECES

105-DR DAILY PHYSICS DATA - MOTEBOOK
AUTHOR

BAILEY GF

BALLONE JH

AGAR JD

HAROIM AK

KARMIE AJ

BENSON RD

BMARS RE

BAARS RE

HLADEK KL, TEATS R, WEAKLEY EA

HOUGH CG, FULLER NE, JENSEN RD

BALLONE JH

WICK OJ

BORGESON ME, SWAIN EO, MODRICH DD

HICK OJ

AE SMITH

STIEDE HL, MONMIE DI

AJ STEVENS

MARSHALL RK, O'KEEFE DP

SRD

SRD

CRD

SRD

SRD

SRD

8

SRD

SRD

SRD

SRD

SRD

SRD

9

SRD

SRD

SRD

SRD

CRD

JOMES SS, MANUEL JL

SRO

40

$12 / 03 / 62$

GOFFARD JU

SRD

FRY WE

CRD

KREITER MR

CRD

SECR

DATE

$11 / 12 / 62$

$11 / 14 / 62$

$12 / 26 / 62$

$11 / 14 / 62$

03/06/63

$11 / 16 / 62$

$04 / 01 / 63$

$04 / 29 / 64$

$04 / 18 / 63$

$12 / 03 / 62$

$11 / 26 / 62$

12/01/62

$11 / 30 / 62$

$11 / 27 / 62$

$12 / 14 / 62$

$11 / 28 / 62$

$11 / 30 / 62$

03/15/63

$12 / 03 / 62$

SH SKIDMORE
$12 / 04 / 62$

$06 / 01 / 56$

$11 / 13 / 62$

$12 / 06 / 62$ 
DATE

HH-75762

HH- 75774

HH- 75779

HH-75802

HH-75888

HW-75889

$H W-75899$ RD

HH-75902

HW- 75904

HW- 75915

HH-75921

HW- 75937

HW- 75938

HH-75942

$H W-75955$

$H W-75958$ RD

HUH-75962

HW- 75963

$H W-75972$

HW-75973
ATTACK THREAT CONTROL FACILITIES CHEMICAL PROCESSING DEPARTMENT PRTR LAYAGAY CONSIDERATIONS MINIMIZING CONSEQUENCES OF AN ATTACK THREAT

STABILIZATION OF HANFORD PLANT FOR PURPOSE OF MINIMIZING CONSEQUENCES OF AN ENEMY ATTACK THREAT

TRIP REPORT MEVADA TEST SITE NOVEMBER 29 THROUGH DECEMBER 5, 1962

NEUTRON AND GAMM DOSE RATES AS A FUNCTION OF PLUTONIUN-240 AND 241 COAPOSITIOY

SUMMIARY EVALUATION OF POTENTIAL LIABILITY FROM ACTIVITIES AT HANFORD INFORMATION FOR ONE-MONTH STUDY MULTI-PRODUCT OPERATION OF NPR REACTIVITY ACCOUNTING BY "CALHOT", A ONE-GROUP ONE-DIMENSION BUCKLING COOE PROOUCTION TEST IP-555-A SOOILN SILICATE AS A CORROSION INHIBITOR

TEST RESULTS AND ECONOMIC ANALYSIS FOR H REACTOR E-N CORE LOADINGS

MODIFICATION OF SHIPPING FACILITIES AND RAILROAD CARS FOR COMBINED 103 AND METAL TRANSPORTATION

IPD REACTOR TRAINING TEXT CLASSIFIED SUPPLEMENT

DEVELOPMENT TEST IP-556-D, IRRADIATION SERVICE REQUEST HAPO-278, OUTGASSING RATE OF TRITIUM AT HIGH TEMPERATURES PROCESS IMPROVEMENT TRANSITION AUTHORIZATION IP-23-I, EVALUATION OF OIL-QUENCHED, MOLLO-BLANK, HEAT-TREATED URANIUM CORES

SELECTED RADIOISOTOPES' CONCENTRATIONS IN INTEGRATED REACTOR EFFLUENT WATER SAMPLES

SPECIAL REVIEW OF SELECTED REACTOR CONSTRUCTION, OPERATION, AND PROGRAM DATA

H PROCESS TUBES FOR EX-REACTOR EXAMIMATION

PROGRAM AND ASSOCIATED EQUIPMENT REQUIREMEATS FOR UCLRL MATERIAL DEVELOPMENT PROGRAM

DEVELOPMENT TEST NO. IP-558- $Y$ GAS SAMPLING FACILITY CORRELATED WITH FRONT TO REAR THERAAOCOUFLE STRIHGER = WH REACTO:

\begin{tabular}{|c|c|c|c|}
\hline $\begin{array}{l}\text { BRADEN DE, LARIVIERE JR, } \\
\text { ZAHN LL }\end{array}$ & SRD & 13 & $12 / 10 / 62$ \\
\hline KOBERG DR, HITTENBROCK NG & SRD & 3 & $12 / 05 / 62$ \\
\hline PRIOOE CA & SRD & 7 & $12 / 07 / 62$ \\
\hline LL GERMAN & SECR & & $12 / 14 / 62$ \\
\hline FAUST LG & SRD & 4 & 12/92/62 \\
\hline FAUST LG, ENDRES GWR & SRD & 12 & $11 / 15 / 63$ \\
\hline WATSON EC & SRD & 38 & 12/11/62 \\
\hline DOWIS WJ & SRD & 7 & $12 / 14 / 62$ \\
\hline CHITWOOD RA, TOYOOKA RT & SRD & 27 & $12 / 14 / 62$ \\
\hline GEIER RG, CLINTON MA & CRD & 9 & $12 / 17 / 62$ \\
\hline LANG LH & SRD & 8 & $12 / 18 / 62$ \\
\hline & SECR & & $06 / 30 / 70$ \\
\hline STAFF & SRD & & $01 / 01 / 63$ \\
\hline DEMERS AE & SRD & 6 & $12 / 21 / 62$ \\
\hline NEEF HI & SRD & 8 & $01 / 04 / 63$ \\
\hline JACKSON PO & SRD & 265 & $12 / 01 / 62$ \\
\hline $\begin{array}{l}\text { BALLOWE JH, BRASFIELD RL, } \\
\text { FIFER NF }\end{array}$ & SRD & 5 & $12 / 26 / 62$ \\
\hline HOUGH CG & SRD & 3 & $12 / 21 / 62$ \\
\hline STEWART RU & SRD & 3 & $12 / 26 / 62$ \\
\hline COOKE JP, BAARS RE & SRD & 7 & $12 / 20 / 62$ \\
\hline
\end{tabular}


DOC. NO.

HW- 75983

HW- 75986

HW- 75987

$H W-75988$

HW-75997

HW-76001

HH-76009

HH-76026

HW-76040

HW-76060

HW-76074

HW-76078

$H W-76078 C$

HH-76078 F

HU-76101

$H H-76110$ RD

HW-76132

HW-76135

$H W-76136$

HW-76162RD
TITLE

ECONOMICS OF E-N LOADINGS AND PROGRAM DIRECTION

PLUTONIUM PROOUCT DEVELOPMENT OPERATION MONTHLY REPORT - DECEMBER, 1962

PROJECT MHITMEY - PLUTONIUM

METALLURGICAL DEVELOPNENT - MONTHLY

REPORT - DECEMBER, 1962

PROJECT HHITMEY - PLUTONILM

METALLURGICAL DEVELOPMENT - MONTHLY

REPORT - JANUARY 1963

FLOW TESTS OF C REACTOR OUTLET ELBOW

REPLACEMENT FITTINGS

BAUXITE-SULFURIC ACID COACULANT FEED SYSTEMS 100-K - PRELIMINARY EMGINEERING REPORT

ESTIMATED COSTS FOR PROOUCTION OF HXPU FRON SIX/6/SRP PU-AL SPIKES

IMPROVEMENTS TO THE GAMMA MONITOR SYSTEMS, 105-KE \& 105-KW REACTORS (PROJECT CGI-998)

PROGRESS REPORT - WEAPONS PROCESS ENG DECEMBER 1962

CONTRACTION OF GRAPHITE: A COMPARISON OF LABORATORY AND PRCOUCTION REACTOR

DATA - PART II - KE AND KW REACTORS

DISTRIBUTION OF TIME BETMEEN UNSCHEDULED OUTAGES

PROOUCTION TEST IP-560-A HALF-PLANT LOW

DICHROMATE - LON PH HATER TREATMENT AT C REACT'S

INTERIM REPORT 1, PRODUCTION TEST

IP-560-A, HALF-PLANT LOW DICHROMATE -

LON PH HATER TREATMENT AT C REACTOR

INTERIM REPORT 111, PRODUCTION TEST

IP-560-A HALF-PLANT LOW DICHROMATE - LOW PH WATER TREATMENT AT C REACTOR

ADVANCEMENTS IN REACTOR CONTROL. EFFICIENCY POISON SPLINE SUPPLEMENTARY SYSTEM

EQUIPMENT REQUIREMENTS FOR WEAPOW FABRICATION AND ASSEMBLY

REPORT TO THE WORKING COMNITTEE FROM THE GENERAL ELECTRIC COMPANY, HAPO

EXCERPTS FROM PROJECT HHITMEY MONTHLY REPORT - NOVEMBER, 1962

EXCERPTS FROM PROJECT WHITMEY MONTHLY REPORT - DECEMBER, 1962

2 PLANT MEEKLY REPORT - TASK I-II AND INCIMERATOR-FROM JAMUARY 1, 1963 TO DECEMBER 31, 1963

\begin{tabular}{|c|c|c|c|}
\hline AUTHOR & LEVEL & PGS & DATE \\
\hline JOHNSON WE & CRD & 4 & $12 / 01 / 62$ \\
\hline HICK OJ & SRD & 5 & $01 / 02 / 63$ \\
\hline HICK OJ & SRD & 9 & $12 / 31 / 62$ \\
\hline HICK OJ & SRD & 12 & $01 / 31 / 63$ \\
\hline WATERS ED & CRD & 3 & $07 / 20 / 62$ \\
\hline ETHERIDGE EL & SRD & 20 & $01 / 14 / 63$ \\
\hline BRADLEY JG & SRD & 3 & $12 / 14 / 62$ \\
\hline M JANOS & CRD & & 03/28/63 \\
\hline AE SMITH & SRD & & $01 / 17 / 63$ \\
\hline GIBERSOW RC, MORGAN WC & SRD & 19 & 01/02/63 \\
\hline JAECH JL, BURKE RC & SRD & 17 & $01 / 02 / 63$ \\
\hline GEIER RG & SRD & 8 & $02 / 18 / 63$ \\
\hline GEIER RG & SRD & 6 & $04 / 10 / 63$ \\
\hline GEIER RG & SRD & 13 & $11 / 07 / 63$ \\
\hline FULLMER GC, CARTER RD & SRD & 7 & $01 / 03 / 63$ \\
\hline SHAIN EO, HCORICH DD & SRD & 15 & $12 / 31 / 62$ \\
\hline STR!HGER JT, MIMOR JE & SRD & 51 & $01 / 07 / 63$ \\
\hline HICK OJ & SRD & 4 & $01 / 08 / 63$ \\
\hline HICK OJ & SRD & 6 & $01 / 08 / 63$ \\
\hline UF UNZICKER & SECR & & $01 / 02 / 63$ \\
\hline
\end{tabular}


Page No. 182

$04 / 28 / 93$

LIST OF HANFORD-GENERATED CLASSIFIED

DOCUMENTS DATED BETHEEN 1/1/61 AND 12/31/72

DOC. NO.

HW-76170

$H W-76206 R D$

HW-76214

HW-76227

HW-76227 ADD

HU-76227 RD

HW-76237

HW-76245

HW-76259

HW-76271

$H W-76272$ RD

$H H-76273$ RD

HW-76274 RD

HW-76297

HW-76305

HW-76316

HW-76327 RD

HW-76327 RD REV2

HH-i $\tilde{3} 327 R D$ REV3

HW-76332

HW-76333

HW-76356

HW-76361

liW-76361 APP
TITLE

AUTHOR

PROCESS TUBE CORROSION AND BULK OUTLET TEMPERATURE

DRAFT OF PHYSICS SECTIONS TO 100-N TECHNICAL MANUAL

POST-IRRADIATION DATA ON FUEL ELEMENTS

FROM KER LOOP 4

FEBRUARY 1963 PROOUCTION STUDIES

FEBRUARY 1963 PRODUCTION STLDIES ADDENDUN

FEBRUARY 1963 PROOUCTION STUDIES

ADVANCED CONCEPT - NPR PRODUCTION

1963 REVISION OF CESIUM-137 INDEX FOR

NUCLEAR MATERIALS CONTENT OF COATING

WASTE

190-H DRALDOWN TEST

PUREX SECTIONALIZED CONCENTRATOR

CALCULATIOHS

TRANSFORMATION OF RETAIMED PHASES IN PLUTONIUM

RETAINED BETA AND MICROCRACK FORMATION

IN PLUTONIUM

METASTABILITY OF ALPHA PLUTONIUM

THE PRODUCTION OF TRITIUN AT

INTERMEDIATE TEMPERATURES BY MEANS OF

THE LI6(N.Q)H3 REACTION (A LITERATURE REVIEW)

LOADING, OPERATING CONDITIONS, AND WATER SHUTOFF TIMES FOR A CHARGE OF TWELVE ENRICHED SINGLE TUBE ELEMENTS, PT-IP-544

A

REPORT OF INVENTION

OPERATING LIMITS HANFORD PRCOUCTION

REACTORS OPERATING AMD PERFORMANCE

RESTRICTIONS

OPERATING LIMITS HANFORD PROOUCTION REACTORS

OPERATIMG LIMITS HANFORD PRCOUCTION REACTORS

SUMMARY OF K REACTOR GRAPHITE TREATMENT

FOR THE ZIRCONIUM TUBE PROGRAM

PLUTONILM PRODUCT DEVELOPMENT OPERATION

MONTHLY REPORT - JAMUARY 1963

FIMAL REPORT: HARP FAILURE OF $A$

THIRTY-SIX INCH LONG URAHIUN - 2 WT. $\%$

ZIRCONIUA ALLOY TUBULAR FUEL ELEMENT,

PT-IP-250-A, (RM-568)

CP-NUCLEAR MATERIALS TRANSFER

MEASUREMENT STATISTICS

CP-NUCLEAR MATERIALS TRANSFER

MEASUREMENT STATISTICES

\begin{tabular}{|c|c|c|c|}
\hline MORRISSEY JE & SRD & 23 & $04 / 01 / 63$ \\
\hline NILSON R & SRD & 142 & $01 / 25 / 63$ \\
\hline BENNETT EC & SRD & 9 & $01 / 10 / 63$ \\
\hline LANG LH, MCCURRY JH & SRD & 33 & $02 / 11 / 63$ \\
\hline LANG LH, MCCURRY JW & SRD & 6 & $02 / 13 / 64$ \\
\hline $\begin{array}{l}\text { LANG LH, MCCURRY JH } \\
\text { RL DICKEMAN }\end{array}$ & $\begin{array}{l}\text { SRD } \\
\text { SECR }\end{array}$ & 31 & $\begin{array}{l}01 / 18 / 63 \\
01 / 25 / 63\end{array}$ \\
\hline ZIMMER HH & SRD & 3 & $01 / 24 / 63$ \\
\hline $\begin{array}{l}\text { CREMER BR, BOKISH KP } \\
\text { LR MICHELS }\end{array}$ & $\begin{array}{l}\text { SRD } \\
\text { SECR }\end{array}$ & 3 & $\begin{array}{l}01 / 17 / 63 \\
08 / 01 / 62\end{array}$ \\
\hline NELSON RD & SRD & 10 & $01 / 21 / 63$ \\
\hline NELSON RD & SRD & 5 & $01 / 21 / 63$ \\
\hline $\begin{array}{l}\text { NELSON RD } \\
\text { GELEZUMAS VL }\end{array}$ & $\begin{array}{l}\text { SRD } \\
\text { SRD }\end{array}$ & $\begin{array}{l}8 \\
26\end{array}$ & $\begin{array}{l}01 / 21 / 63 \\
01 / 21 / 63\end{array}$ \\
\hline
\end{tabular}

KRATZER WK, MISE MJ SRD 8 01/21/63

LANG LH

OUSLEY GF

SRD

3

$01 / 23 / 63$

SRD

$01 / 23 / 63$

OUSLEY GF

SRD

22

05/20/63

OASLEY GF

SRD

24

$08 / 02 / 63$

COUGHREN KD

CRD

37

$02 / 08 / 63$

WICK OJ

SRD

6

02/01/63

KUHLKEN LE

CRD

23

$01 / 25 / 63$

STEVES LH

SRD

59

$12 / 01 / 63$

STEVES LH

SRD

26

$12 / 01 / 63$ 
DOC.

HW-76362RD

HW-76368

HU-76372

$H M-76385$

HW-76404

HW-76417

HW-76420

HW-76421

$H W-76429$

HH-76435

HW-76470

HW-76481

$H W-76481-C$

HH-76501

HW-76509

HW-76528

HW-76528 B

HW-76528 C

$H W-76528$ D

HH-76528 E
TITLE

IPD - PRODUCTION, RECEIPTS \& INVENTORIES LOADING, OPERATING CONDITIONS, AND WATER SHUTOFF TIMES FOR A CHARGE OF TUELVE 17-INCH N INMER FUEL TUBES OR TMELVE 18-INCH UI-2 W/O 2R ELEMENTS. PT-IP-536-A AND PT-IP-536-A, SUPPLEMENT B

PROGRESS REPORT - VIEAPONS PROCESS ENG JAMUARY 1963

BACKUP WATER SUPPLIES TO K REACTOR 1963

SUMMARY REPORT DISSOLVED AIR IN PROCESS WATER STUOY

ECONONIC ANALYSIS OF 10-INCH AND 12-INCH

FUEL ELEAENT PRCOUCTION FOR THREE

SEPARATE CONDITIONS

PRELIMINARY PROJECT PROPOSAL: PONER-ROLL FORMIMG FACILITY - RMC LINE (CGC-996)

TEST RESULTS FOR UNRESTRAINED REACTOR GUNBARREL-NOZZLE ASSEMBLIES INCREASED LENGTH FUEL ELEMENTS

A DISCUSSION ON THE DETERMINATION OF THE ORIGIN OF A SAMPLE OF PLUTONIUM BASED ON AN ISOTOPIC ANALYSIS

POISON SPLINE SUPPLEMENTARY SYSTEM HISTORY OF DEVELOPMENT AND APPLICATION PROOUCTION TET IP-572-A EFFECT OF ECCENTRICITY ON THE IRRADIATION BEHAVIOR OF KVNS FUEL ELEMENTS

SUPPLEMENT A TO PT-IP-572-A EFFECT OF ECCENTRICITY ON THE IRRADIATION BEHAVIOR OF KVNS FUEL ELEMENTS

PRELIMIMARY REPORT ON CX-2 N-IMNER FUEL TUBE NUMBER 1055-8

FINAL REPORT PT IP-513-C RECALIBRATION

OF HORIZONTAL CONTROL ROOS AT H REACTOR INTERIM REPORT ONE TO PROOUCTION TEST-IP-549-A, HALF-PLANT LON ALUM FEED WATER TREATMENT AT F REACTOR

INTERIM REPORT II, PRODUCTION TEST IP-549-A, HALF-PLANT LOW ALUM FEED MATER TREATMENT AT F REACTOR

INTERIM REPORT III, PRODUCTION TEST

IP-549-A HALF-PLANT LOW ALUM FEED WATER TREATMENT AT F REACTOR

INTERIM REPORT IV, PRODUCTION TEST

IP-549-A HALF-PLANT LOW ALUM FEED WATER

TREATMENT AT F REACTOR

INTERIM REPORT $V$, PROOUCTION TEST

IP-549-A MALF-PLANT LON ALUM FEED WATER

TREATMENT AT F REACTOR

$\begin{array}{llll}\text { AUTHOR } & \text { LEVEL } & \text { PGS } & \text { DATE } \\ & & & \\ \text { WW MOBLEY } & \text { SRD } & & 01 / 25 / 63 \\ \text { KRATZER WK, HISE MJ } & \text { SRD } & 5 & 01 / 28 / 63\end{array}$

\begin{tabular}{|c|c|c|c|}
\hline AE SNITH & SRD & & $02 / 19 / 63$ \\
\hline HICKANA RD & SRD & 8 & $01 / 29 / 63$ \\
\hline RADTKE WH & SRD & 10 & $01 / 21 / 63$ \\
\hline GRUBB FW & SRD & 11 & $01 / 28 / 63$ \\
\hline NN RAILE & SRD & & $03 / 04 / 63$ \\
\hline COFF JM, JR. & SRD & 9 & $02 / 14 / 63$ \\
\hline WHEATON RH & SRD & 9 & $02 / 04 / 63$ \\
\hline GRANOUIST DP & CRD & 8 & $02 / 01 / 63$ \\
\hline
\end{tabular}

FULLMER GC, CARTER RD SRD $17 \quad 02 / 05 / 63$

CARLSON PA, HLADEK KL SRD $13 \quad 02 / 06 / 63$

CARLSON PA， HLADEK KL SRD $10 \quad 04 / 12 / 63$

KUHLKEN LE, WHEELER RG SRD $36 \quad 02 / 07 / 63$

$\begin{array}{llll}\text { VAUGHN AD } & \text { SRD } & 16 & 02 / 08 / 63\end{array}$

CLINTON MA, GEIER RG SRD $502 / 11 / 63$

$\begin{array}{llll}\text { GEIER RG } & \text { SRD } & 7 & 04 / 26 / 63\end{array}$

$\begin{array}{llll}\text { GEIER RG } & \text { SRD } & 8 & 06 / 11 / 63\end{array}$

$\begin{array}{llll}\text { GEIER RG } & \text { SRD } & 9 & 08 / 01 / 63\end{array}$

$\begin{array}{llll}\text { GEIER RG } & \text { SRD } & 11 & 10 / 18 / 5:\end{array}$ 
Pege No. 184

$04 / 28 / 93$

LIST OF HANFORD-GENERATED CLASSIFIED

DOCUMENTS DATED BETWEEN 1/1/61 AND 12/31/72

DOC. NO.

TITLE

AUTHOR

LEVEL PGS

DATE

HW-76528 F

HW-76528 G

HU-76536

HW-76563

HW-76571

HW-76571 DEL

HH-76571 REV

HW-76572

HW- 76586

HW- 76618

HH-76619

HW-76634

HW-76650

HW-76688

$H W-76690$

HW-76784

HW-76785

HW-76794

HH-76842

HU-76847

HW-76858

HW-76884

$H W-76887$ ADD
INTERIM REPORT VI, PRCOUCTION TEST IP-549-A HALF-PLANT LON ALUM FEED WATER TREATMENT AT F REACTOR

INTERIM REPORT VII, PRODUCTION TEST IP-549-A HALF-PLANT LON ALUM FEED WATER TREATMENT AT F REACTOR 190-C CLEARWELL DRALDOW TEST RESULTS. STATUS OF HARVEY ALUMIMUM COMPONENT EVALUATION - PROOUCTION TEST IP-12-A CRITICALITY HAZARDS CONTROL SPECIFICATIONS REDOX PLANT

CRITICALITY HAZARDS CONTROL SPECIFICATIONS REDOX PLANT CRITICALITY HAZARDS CONTROL SPECIFICATIONS REDOX PLANT MONTHLY REPORTS KW PROCESSING OPERATION FROM JAN 1963 - 2/1/63 PERSONAL NOTES \& WORKING PAPERS PROJECT WHITMEY PLUTONIUN METALLURGICAL DEVELOPMENT MONTHLY REPORT - FEBRUARY, 1963

PROJECT HHITHEY PLUTONIUN METALLURGICAL DEVELOPMENT MONTHLY REPORT - MARCH, 1963 EXCERPTS FRON PROJECT WHITHEY MONTHLY REPORT - JAMUARY, 1963

IRRADIATION TESTING (TL DEOBALD) VERTICAL SAFETY ROO CHANNEL DISTORTION DATA C REACTOR

FISSION PROOUCT PRODUCTION PLANT ENGINEERING STUOY BASIS - CHEMICAL FLOUSHEET

GRAVITY FED WATER SUPPLY TO REACTOR STORAGE BASINS

STATUS REPORT CARBON-14 IN THE CONDENSATE FROM THE SILICA GEL DRYERS OF THE PROOUCTION REACTORS

FLON TESTS OF PROOUCTION MOOELS OF C-REACTOR OUTLET REPLACEMENT HARDWARE

SINGLE TUBE MELTDOWN INCIDENT PROGRESS REPORT - MEAPONS PROCESS ENGINEERING - FEBRUARY 1963 PROOUCTION OF U-233 AND PROGRAM PROPOSAL

INTERIM REPORT ON HOT DIE SIZING VARIABLES TEST

ATOMIC WEAPON DATA SUPPLEMENT QUARTERLY PROGRESS REPORT METALLURGY DEVELOPMENT OPERATION OCTOBER, NOVEMBER, DECEMBER, 1962

ARTIFICIAL COOLING OF THE COLUMBIA RIVER BY DAM RECULATION 1962

\begin{tabular}{|c|c|c|c|}
\hline AUTHOR & LEVEL & PGS & DATE \\
\hline GEIER RG & SRD & 8 & $01 / 07 / 64$ \\
\hline GEIER RG & SRD & 8 & $03 / 18 / 64$ \\
\hline $\begin{array}{l}\text { WOOD SA, GEORGE DK } \\
\text { BLANTON WA }\end{array}$ & $\begin{array}{l}\text { SRD } \\
\text { SRD }\end{array}$ & $\begin{array}{l}2 \\
14\end{array}$ & $\begin{array}{l}02 / 07 / 63 \\
02 / 13 / 63\end{array}$ \\
\hline ISACSON RE & SRD & 16 & $02 / 13 / 63$ \\
\hline ISAACSON RE & CRD & 15 & $07 / 01 / 63$ \\
\hline RE ISAACSON & SRD & & $12 / 16 / 63$ \\
\hline RF CORLETT & SECR & & $02 / 14 / 63$ \\
\hline JM BYLAND & SECR & & $02 / 14 / 63$ \\
\hline WICK OJ & SRD & 9 & $02 / 28 / 63$ \\
\hline HICK OS & SRD & 9 & $03 / 29 / 63$ \\
\hline HICK OJ & SRD & 5 & $02 / 20 / 63$ \\
\hline DEOBALD TL & CRD & 9 & $02 / 18 / 63$ \\
\hline $\begin{array}{l}\text { MOORE RL, RICHARDSON GL, } \\
\text { SUIFT UH, VANTUYL HH }\end{array}$ & SRD & 29 & $03 / 13 / 63$ \\
\hline HEDGES JW & SRD & 13 & $u_{4} / 01 / 63$ \\
\hline BARTON GB & CRD & 8 & $03 / 14 / 63$ \\
\hline KREITER MR & CRD & 4 & $12 / 04 / 62$ \\
\hline TRUABLE RE & CRD & 7 & $02 / 01 / 60$ \\
\hline & SRD & 13 & $03 / 05 / 63$ \\
\hline $\begin{array}{l}\text { LANG WL, MEICHLE RH, } \\
\text { NILSON R }\end{array}$ & SRD & 49 & $03 / 21 / 63$ \\
\hline STRAND CA & SRD & 44 & $05 / 20 / 63$ \\
\hline $\begin{array}{l}\text { LAST GA, MINOR JE, MELSON } \\
\text { TC, STEWART RH }\end{array}$ & SRD & 64 & $03 / 06 / 63$ \\
\hline KRAMER HA & SRD & 6 & $03 / 07 / 63$ \\
\hline
\end{tabular}


Page No. 185

$04 / 28 / 93$

LIST OF HANFORD-GENERATED CLASSIFIED

DOCUMENTS DATED BETWEEN 1/9/61 AND 12/31/72

DOC. NO.

HW-76902

HW-76909

HW-76910

HW-76912

HH-76913 RD

HW-76920

HW-76922 RD HW-76926

HH-76930 VOL 2 RD2

$H W-76942$ RD

HW-76972

HW-76977

HH-76980

HH-76994

HW-76996 A

HH-76996 B

$H W-77040 R D$ HW- 77043

HU-77058

HW-77064

HW- 77070

HW- 77091

HW-77101
TITLE

105-KE-SPECIALIST LOG \#2

TRIP REPORT - ROCKY FLATS PLANT JANUARY 7 ANO 8, 1963

THE EFFECT OF HIGH EXPOSURE ON

PROOUCTION EFFICIENCY

PUREX PROCESS PERFORMANCE SUMURY JANUARY 1963 THRU DECEMBER 1963

PU HIGHER ISOTOPE PROPOSAL

BUDGET FOR FY 1965 AND REVISION OF BUDGET FOR FY 1964 - PROPOSAL FOR RESEARCH AMD DEVELOPMENT - GEMERAL MEAPONS DEVELOPMENT - 03-25-04-01 EXPANDED USES OF IPD FACILITIES PROJECT PROPOSAL: MCOIFICATIONS FOR USE OF BAUXITE, KE \& KW WATER PLANTS (CGI-105)

N-REACTOR HAZARDS SUMMARY REVIEH (PHASE

IB, PROOUCTION ONLY) VOLUNE 2 REACTOR AND REACTOR AUXILIARY SYSTEMS

CPD'S POTENTIAL FOR PROCESSIHG THE HIGHER TRANSURANICS

JUSTIFICATION FOR ELLIPTICAL BUMPERS

PROPOSAL FOR IRRADIATION OF TARGE'

ASSEMBLIES

IPD - COST \& PRODUCTION ANALYSIS -

FEBRUARY 1963

ENGINEERING REVIEH OF STAINLESS STEEL

CLAD THERMOCOUPLE ELEMENTS

INTERIM REPORT I - PKOOUCTION TEST

IP-581-A HALF-PLANT HIGH FLOCCULATION PH TEST AY B REACTOR

INTERIM REPORT II - PRODUCTION TEST

IP-581-R HALF-PLANT HIGH FLOCCULATION PH TEST AT B REACTOR

THE PLUTONIUN NITRIDE PROBLEM REPORT TO THE WORKING COMMITTE FROM THE GENERAL ELECTRIC COMPANY, MAPO MEASUREMENTS OF REDOX PROOUCED NEPTUNIUM HANFORD CONTRIBUTION FOR THE 16TH HIGH TEMPERATURE FUELS COMIITTEE MEETING MAY, 1963

PROOUCTION TEST IP-580-AL. IRRADIATION OF "C" AND "J" FUEL ELEMENTS AND THERMOCOUPLE SLUGS FOR CORROSION TESTS IN THE 1706-KE SINGLE-PASS FACILITY THE N-REACTOR FUEL TESTING PROGRAM IN THE KER LOOPS

BUDGET FOR FY 1965 AND REVISION OF BUDGET FOR FY 1964 - 02 PROGRAM RESEARCH AND DEVELOPMENT

\begin{tabular}{llll}
\multicolumn{1}{c}{ AUTHOR } & LEVEL & PGS & DATE \\
& & & \\
$\begin{array}{l}\text { RG CLOUGH } \\
\text { CROCKER HM, FISHER FD, } \\
\text { KINGSLEY RS } \\
\text { NEEF HI }\end{array}$ & $\begin{array}{l}\text { SECR } \\
\text { CRD }\end{array}$ & 6 & $03 / 08 / 63$ \\
BF JUDSON & SRD & 3 & $02 / 15 / 63$ \\
SECR & & $01 / 15 / 63$
\end{tabular}

LANG LH

FRANK US, SMITK AE

SRD 23

$03 / 12 / 63$

SRD $\quad 37$

$03 / 21 / 63$

KUSLER LE

SRD

29

$03 / 15 / 63$

JV LOFTUS

SRD

$06 / 07 / 63$

MECHODON US

SRD

58

$04 / 04 / 63$

TONLINSCN RE

SRD

9

$03 / 14 / 63$

NEEF HI

SRD

1

$03 / 15 / 63$

GELEZUNAS VL

SRD

13

$03 / 18 / 63$

AS TERRY

SRD

$03 / 21 / 63$

FITZPATRICK VF

SRD

14

$03 / 25 / 63$

GEIER RG

SRD

6

$10 / 31 / 63$

GEIER RG

SRO

7

$02 / 11 / 64$

BOND RH

SRD

MINOR JE, RICHES JW,

SRD

22

$03 / 22 / 63$

STRINGER JT

JS BUCKINGHAM

LAST GA

SRD

CRD

14

03/25/63

$04 / 17 / 63$

DICKIMSON DR, GEIER RG SRD

18

$01 / 17 / 64$

EVANS TH

SRD

15

$04 / 01 / 63$

SECR 
DOC. HO.

HW-77105

HW- 77116

$H W-77121$

HW- 77123

HW- 77125

HU- 7716

HW- 77177

HW- 77178

HW-77182

HW- 77183

HW-77184

HW- 77185

HW-77186

HH- 77202

HH-77202 RD

HW-77212

HW-77230

HW-77239

HW -77253

HW- 77257

HWH-77258

HW- 77268

HW-77276

HW- 77278

HW-77290

HH- 77298
TITLE

K REACTOR "SHUTDOW COSTS"

PROGRESS REPORT - WEAPONS PROCESS ENG MARCH 1963

INTERIM REPORT EXPERIENCE WITH PT IP-467-C REDUCING MINIMUM DOWTIME DR BALL 3X DROP OPERATIONAL PHYSICS REPORT

PHASE III - B PLANT OPERATIONS

PLUTONIUN PRODUCT DEVELOPMENT OPERATION

- MONTHLY REPORT - MARCH, 1963

PITA IP-22, SUP I E-N DONONSTRATION LOAD PRELIMIMARY ENGIMEERIMG PROGRAM FOR RENOVATION OF THE HORIZONTAL CONTROL ROD SYSTEM - K REACTORS

ATONIC MEAPON DATA SUPPLERENT QUARTERLY PROGRESS REPORT METALLURGY DEVELOPMENT OPERATION JANUARY, FEBRUARY, MARCH, 1963 PROVISIONAL PROCESS SPECIFICATIONS FOR FABRICATION OF KIT \& KIIT TARGET ELEMENTS FOR $K$ REACTOR E-N LOAD (PT-IP-561-C)

PROJECT WHITHEY PLUTONIUN METALLURGICAL DEVELOPMENT MONTHLY REPORT - APRIL, 1963 PROJECT WHITMEY PLUTONIUM METALLURGICAL DEVELOPMENT MONTHLY REPORT - MAY, 1963 EXCERPTS FROM PROJECT HHITHEY MONTHLY REPORT - FEBRUARY, 1963

PROPOSAL TO PRCDUCE TRAMSURANIUM ISOTOPES IN HANFORD PROOUCTION REACTORS PROPOSAL TO PRODUCE TRANSURANILM ISOTOPES IN HANFORD REACTORS GOAL EXPOSURE FOR RUPTURE-PRONE FUEL ELEMENT LOTS

PROOUCTION TEST IP-582-D, IRRADIATION OF DEPLETED URANIUM IN THE KW SNOUT

FACILITY, HAPO-275

INSPECTION STUDY ENVELOPE VS. AVERAGE

PROPOSED A-LINE PROCESS

TECHNICAL CRITERIA AND BASES REACTOR GAS SYSTEM - 100-K

NUCLEAR GRAPHITE - REFERENCES AND ROUGH

NOTES

ESTIMATED PLUTONIUN SCRAP RECOVERY COSTS

TECHNICAL STUDIES SUPPORTING IPD

DISASTER PLAHNING

IPD - COST \& PRODUCTION ANALYSIS - MARCH 1963

FINAL REPORT PT IP-535-C TEST OF SMALLER VSR'S IN DR REACTOR

RADIOGRAPHIC PROCEDURES FOR 74C, 79C, AMD $88 C$ PLUTONIUM COMPONENTS

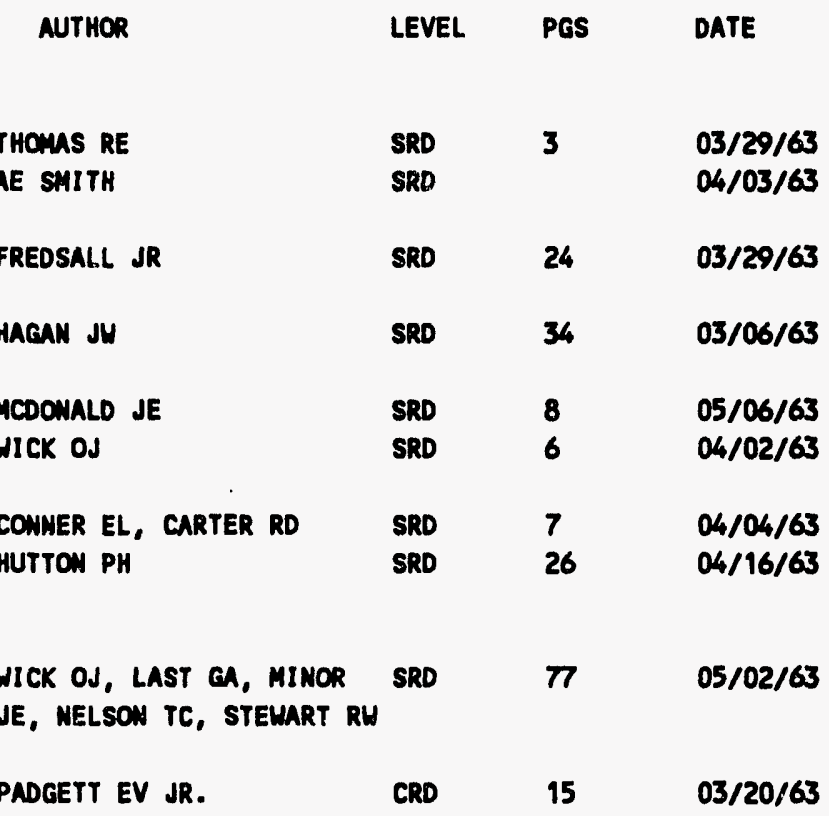

WICK O

SRD

8

$04 / 30 / 63$

WICK OJ

SRD

11

$05 / 31 / 63$

HICK OJ

SRD

5

04/05/63

KUSLER LE, GUAPRECHT RO SRD

$04 / 12 / 63$

KUSLER LE

SRD

15

04/05/63

MEWELL LJ, SHIMER RD

SRD

7

$04 / 08 / 63$

$\operatorname{coX} \mathrm{JH}$

SRD

11

$04 / 12 / 63$

MCGREGOR LL

RE VANDERCOOK

RUSSELL A, BMARS RE

SRD

SRD

CRD

6

8

Nightimgale Re

SRD

464

KG GRIMW

CARLSOW PA, JASKE RT

CRD

SRD

25

AS TERRY

SRD

VAUGHN AD

SRD

WILBUR GE

SRO
$12 / 07 / 62$

$03 / 27 / 63$

$04 / 16 / 63$

$04 / 10 / 63$

$04 / 12 / 63$

08/22/63

$08 / 05 / C 3$

$04 / 17 / 63$

$04 / 17 / 63$ 
Page No. 187

$04 / 28 / 93$

LIST OF HANFORD-GENERATED CLASSIFIED

DOCLMENTS DATED BETMEEN 1/1/61 AMD 12/31/72

DOC. NO.

TITLE

AUTHOR

LEVEL

DATE

HW-TT298 REV

HW-77325

HW- 77338

HU- 77339

HW- 77367

HW-T7378

HW-77401

HH-77402

HU- 77403

HW-77413

HW-77472

HW- 77474

HN- 77475

HW-77494

$H H-77523$ RD

HU- 77528

$H W-77537$

HW- 77554

HW-77556

HW- 77574

HU- T7578

HW- 77579

HU- 77579
RADIOGRAPHIC PROCEDURES FOR 74C, 79C, AND 8BC PLUTONIUN COMPONENTS

FUEL USAGE AUTHORIZATION IP-01-I, IRRADIATION OF DINGOT URANIUM FUEL ELEMENTS

EVALUATION OF THE B-CASK IN ACCORDANCE HITH PROPOSED RECULATIONS OF THE AEC AND ICC FOR OFF-SITE SHIPMENTS OF IRRADIATED BISWUTH

ENGIMEERING STUDY MEAPON FABRICATION FACILITIES EXPANSION 234-5 BUILDING PROPOSED IRRADIATION OF SUBSTAMDARD FUEL ELEMENTS - PHASE II

PRESSURE AND FLON DATA PT IP-573-1, 100-K FLOH TESTS

SANL 727-3992705

EXCERPTS FROM PROJECT MHITNEY MONTHLY REPORT - APRIL, 1963

FINAL REPORT - PITA-18 USE OF NOWPOISONOUS SPLINES FOR LONGITUDINAL FLUX TRAVERSING

PROGRESS REPORT - WEAPONS PROCESS ENG APRIL 1963

PRODUCTION TEST IP-586-AIE EXTENDED COAL EXPOSURE FOR SELECTED KUNS FUEL COLUMNS PLUTONIUM PROOUCT DEVELOPMENT OPERATION MONTHLY REPORT - APRIL, 1963

TRIP REPORT - CRITICALITY CONTROL DISCUSSIONS AT ROCKY FLATS, MARCH 25, 26, 27, 1963

NOTEBOOK - SPECIALISTS LOGBOOK 105-B PROCESSING

B HIGH TANK ADEQUACY

CRLD DEPOSITION IN KER LOOPS AT PH 10

LIOH

QUARTERLY SUMARY REPORT \& ASSOCIATED

DATA FOR 234-5 BLDG, FINAL PRCOUCT

SHIPPED DURING THE IST QUARTER OF CY 1963

PLANT STABILIZATION PLANS

N-REACTOR FUTURE COWSIDERATIONS

FILTERED WATER SYSTEM STUDY 105-H.

BUILDING

USE OF DEPLETED URANIUM FOR HIGHER

ISOTOPE PU

PRCDUCTION TEST IP-584-D, IRRADIATION OF TARGET ASSEMBLIES IN THE KER LOOPS

PRODUCTION TEST IP-584-D, SUPPLEMENT B IRRADIATION OF TARGET ASSEMBLIES IN THE KER LOOPS

\begin{tabular}{|c|c|c|c|}
\hline HILBUR GE & SRD & 8 & $07 / 18 / 63$ \\
\hline NEEF HI & SRD & 8 & $04 / 22 / 63$ \\
\hline PECK GE & SRD & 31 & $07 / 12 / 63$ \\
\hline WOORICH DD & SRD & 39 & $04 / 30 / 63$ \\
\hline BLANTON WA, MEAKLEY EA & CRD & 19 & $04 / 22 / 63$ \\
\hline LESSOR LC & SRD & 18 & $04 / 24 / 63$ \\
\hline STEMART RW & SRD & 2 & $04 / 29 / 63$ \\
\hline HICK OJ & SRD & 3 & $05 / 13 / 63$ \\
\hline ALBERTSON DG, BONERS CE & CRD & 34 & 05/01/63 \\
\hline AE SNITH & SRD & & 05/03/63 \\
\hline $\begin{array}{l}\text { HLADEK KL, MCCARTHY PB, } \\
\text { SPENCER HG }\end{array}$ & SRD & 9 & 05/01/63 \\
\hline HICK OJ & SRD & 9 & 05/01/63 \\
\hline $\begin{array}{l}\text { BROWN CL, KIEL GR, } \\
\text { STEVENSON RL }\end{array}$ & SRD & 11 & $04 / 29 / 63$ \\
\hline SN GRAVES & SECR & & $04 / 30 / 63$ \\
\hline ZIMMERMAN PJ & SRD & 3 & $03 / 22 / 63$ \\
\hline DICKIMSON DR, DEMIITT TF & CRD & 11 & 05/03/63 \\
\hline AE SNITH & SRD & & $05 / 06 / 63$ \\
\hline LL GERMAN & CRD & & $05 / 06 / 63$ \\
\hline CREMER BR, MEISLOGEL LD & SRD & 15 & $04 / 08 / 63$ \\
\hline LANG LW & SRD & 2 & 05/08/63 \\
\hline NEIDNER R & SRD & 11 & $05 / 08 / 63$ \\
\hline DEOBALD TL & SRD & 9 & $04 / 28 / 64$ \\
\hline
\end{tabular}


Page No. 188

$04 / 28 / 93$

LIST OF HANFORD-GENERATED CLASSIFIED

DOCUNENTS DATED BETUEEN 1/1/61 AND 12/31/72

DOC. NO.

HW-77611

$H W-77642$

HM-77654

HW-77674

HW-77675

HN-77675 ADD

HU- 77675 RD

HU -77680

HW- 77686

HU-77687

HU- 77688

HW- 77692

HW-TT700Z-R3 PT1

HW-T7700Z-R3 PT10

HU-777002-R3 PT11

HW-777002-R3 PT12

HW-TT7OOZ-R3 PT2

HW-TT700Z-R3 PT3

HH-7T700Z-R3 PT4

HW-TT700Z-R3 PT5

HH-777002-R3 PT6

HW-TT700Z-R3 PT7

HW-TT700Z-R3 PT8

HH-TTTOOZ-R3 PT9

HW- 77706

HW- 77713

HW- 77718
TITLE

CORROSION OF ZIRCALOY-2 BY PH 10 LIOH IN HEATED CREVICES

PRELIMIMARY REPORT A STUOY OF THE PROPERTIES AND BEHAVIOR OF PLUTONIUM ALLOY

RADIOGRAPHIC EXAMINATION OF PROOUCTION

FUELS

EFFECT OF 6.6 PH PROCESS MATER ON

PROCESS TUBE AMD FUEL ELEMENT CORROSION

DEPLETED URANILN IRRADIATIONS

UNCLASSIFIED PLUTONIUN

DEPLETED URANIUN IRRADIATIONS

AGENDA FOR MEETING OF JUNE 6-7

MUAERICAL RESULTS OF A D REACTOR FUEL

STUDY

PROJECT PROPOSAL EMERGENCY STORAGE BASIN

COOLANT (PROJECT CGI-108)

IRRADIATION AND PERFORMANCE OF DEPLETED URANIUN

REVIEW OF BUMPER ECONOAICS

FINISHED PROOUCTS PRODUCT RECOVERY SOURCE DATA JULY 1963

FINISHED PRODUCTS PRCOUCT RECOVERY

SOURCE DATA APRIL 1964

FINISHED PROOUCTS PROOUCT RECOVERY

SOURCE DATA MAY 1964

FINISHED PRODUCTS PROOUCT RECOVERY

SOURCE DATA JUNE 1964

FINISHED PROOUCTS PRODUCT RECOVERY

SOURCE DATA AUCUST 1963

FINISHED PRODUCTS PROOUCT RECOVERY

SOURCE DATA SEPTEMEER 1963

FINISHED PRCOUCTS PROOUCT RECOVERY SOURCE DATA OCTOBER 1963

FINISHED PRCDUCTS PRODUCT RECOVERY

SOURCE DATA NOVEMBER 1963

FINISHED PROOUCTS PROOUCT RECOVERY

SOURCE DATA DECEMBER 1963

FINISHED PRCOUCTS PRODUCT RECOVERY

SOURCE DATA JANUARY 1964

FINISHED PRODLICTS PROOUCT RECOVERY

SOURCE DATA FEBRUARY 1964

FINISHED PROOUCTS PROOUCT RECOVERY

SOURCE DATA MARCH 1964

REDOX NEPTUNIUM ML..SUREMENTS

SUPPORT FOR THE STRATEGIC TRADE CONTROL

PROGRAM A TECHMICAL EVALUATION OF

SELECTED COMHOOITIES

OPERATING PHYSICS FACTORS WITH ZIRCONIUM TUBES AT THE $K$ REACTORS
AUTHOR

DICKIMSON DR

GARDNER HR

BLASEMITZ AG

YOUNG JR

LANG LW

LANG LH, MCCURRY JW

LANG LH

BROUMS RJ

HOLLIFIELD PJ

LEVE

Pas

DATE

SECR

SRD

8

SRD

14

SRD

SRD

SRD

SRD

SRD

14

8

14

2

HEEB CM, KNIGHT FW, SPENCER HG

JENSEN RD, MEEF MI

SRD

5

SRD

SECR

SECR

SRD

SECR

SECR

SRD

SECR

SECR

SECR

SRD

SRP

SECR

MALOOY CW

BENMETT CA

SRD

CRD

3

14

TILLER RE, VAUGHN AD
SRO
$05 / 23 / 63$

$05 / 23 / 63$

$11 / 12 / 63$

$05 / 16 / 63$

$05 / 17 / 63$

$05 / 20 / 63$

$05 / 21 / 63$

$07 / 11 / 63$

05/21/63

$05 / 21 / 63$

$05 / 21 / 63$

$07 / 17 / 63$

$05 / 21 / 63$

$05 / 22 / 63$

07/01/63

$04 / 01 / 64$

05/01/64

06/01/64

$08 / 01 / 63$

09/01/63

$10 / 01 / 63$

$11 / 01 / 63$

$12 / 01 / 63$

$01 / 01 / 64$

$02 / 01 / 64$

03/01/64

$05 / 24 / 63$ 
Page No. 189

$04 / 28 / 93$

LIST OF HANFORD-GENERATED CLASSIFIED

DOCUMENTS DATED BETUEEN 1/1/61 AND 12/31/72

DOC. NO.

HW- TT730

HL- T7731

HH-TT733

HW- 77735

HW- 77742

HW-T7755

HW- 77765

HW- 77769

HU- 77773

HW-T7803

HU- 77803 RD

HW-77803 RO1

HW- 77811

HW-77811RD

HW- 77815

HW- 77820

$H W-77838$ RD

$H W-77841$

$H W-77848$ RD

HW-77859

HW- 77860

HW- 77861

HW-T7862

$H W-77866$

HW- 77872

HW- 77875
TITLE

AUTHOR

CHRISTENSEN GP

KER-2 OPER

KER-2 TUBE HISTORY

PLUTONIUM PRODUCT DEVELOPNENT OPERATION MONTHLY REPORT - MAY, 1963

PRELIAIMARY REPORT - PROPERTY

DETERMIMATIONS ON MATERIAL PONER ROLL FORMED IN A $50 \%$ MODIFIED UNIFORM SHEAR DESIGN

PROCESS CHANGE AUTHORIZATION $\mathbf{\$ 3 - 4 3}$

GRAPHITE TEMPERATURE LIMITS KE REACTOR

PROGRESS REPORT - WEAPONS PROCESS ENG -

MAY 1963

AMERICIUM RECOVERY STUDY

EVALUATION OF THE "MACHIMED PARTS" CASK

FOR OFF-SITE SHIPMENTS OF IRRADIATED

LITHIUN

FORECAST REACTOR MATER LEAKS

DESIGN BASES BAUXITE-SULFURIC ACID FEED

FACILITIES 100-K AREA

DESIGN BASES, BAUXITE-SULFURIC ACID FEED FACILITIES,

DESIGN BASES BAUXITE-SULFURIC ACID FEED FACILITIES 100-K AREA

MAY 28 GERMANTOWN MEETING AND TRIP REPORT

MAY 28 GERMANTOWN MEETING \& TRIP REPORT RESULTS OF PRCOUCTION TEST IP-528-1 SUPPLEMENT A B AND D REACTORS HIGH TANK DRALDOWN TEST

UNH TRANSFER STUOY

EMPIRICAL PLUTONIUN COISTANTS 7090

PROGRAM

PLUTONILN FABRICATION N/O ASSEMBLY STLOY REORIENTATION OF GENERAL WEAPONS RESEARCH AMD DEVELOPMENT PROGRAM

PROJECT HHITMEY PLUTONILM METALLURGICAL DEVELOPMENT MONTHLY REPORT - JUNE, 1963 PROJECT WHITNEY PLUTONIUN METALLURGICAL DEVELOPMENT MONTHLY REPORT - JULY, 1963 EXCERPTS FROM PROJECT UHITNEY MONTHLY REPORT - MAY, 1963

EXCERPTS FRON PROJECT WHITNEY MONTHLY

REPORT - JUME, 1963

NUCLEAR ASPECTS OF MEAVY ISOTOPE

PROOUCTION IN N-REACTOR

PLESSURE MEASUREMENTS ON 19-1/2

CROSSHEADER - - DR REACTOR

DESCRIPTION OF REACTOR OPERATING LIMITS (ROL) AND REACTOR MASTER FILE (RMF)

REPORTS

\begin{tabular}{|c|c|c|c|}
\hline AUTHOR & LEVEL & POS & DATE \\
\hline CHRISTENSEN GP & SRD & 13 & $08 / 13 / 63$ \\
\hline BANISTER WC & SRD & 6 & $08 / 16 / 63$ \\
\hline HICK OJ & SRD & 9 & $05 / 29 / 63$ \\
\hline GARDNER HR & SRD & 13 & $05 / 29 / 63$ \\
\hline AK HARDIN & SECR & & $05 / 28 / 63$ \\
\hline AE SMITH & SRD & & $06 / 05 / 63$ \\
\hline & SECR & & $06 / 30 / 70$ \\
\hline GLENN E PECK & SRD & 15 & $07 / 12 / 63$ \\
\hline Young JR & SRD & 9 & $06 / 03 / 63$ \\
\hline ETHERIDGE EL & SRD & 21 & $06 / 10 / 63$ \\
\hline ETHERIDGE EL & SRD & 17 & $06 / 10 / 63$ \\
\hline ETHERIDGE EL & SRD & 17 & $06 / 10 / 63$ \\
\hline LANG LH & SRD & 5 & $06 / 05 / 63$ \\
\hline LH LANG & SRD & & $06 / 05 / 63$ \\
\hline $\begin{array}{l}\text { HOLLIFIELD PJ, ZINWERMAN } \\
\text { PJ }\end{array}$ & SRD & 10 & $06 / 05 / 63$ \\
\hline FRENCH RB & SRD & 30 & $06 / 17 / 63$ \\
\hline JENSEN RD & SRD & 22 & $10 / 16 / 61$ \\
\hline GARTIA HJ & SRD & 7 & $06 / 17 / 63$ \\
\hline HICK OJ & SRD & 4 & $06 / 10 / 63$ \\
\hline HICK OJ & SRD & 8 & $06 / 28 / 63$ \\
\hline HICK OJ & SRD & 9 & $07 / 31 / 63$ \\
\hline MICK OJ & SRD & 6 & $06 / 18 / 63$ \\
\hline HICK OJ & SRD & 4 & $07 / 19 / 63$ \\
\hline NICHOLS PF & SRD & 7 & $06 / 11 / 63$ \\
\hline RAND JL & SRD & 3 & $06 / 12 / 63$ \\
\hline WOOD SA & SRD & 8 & $05 / 20 / 63$ \\
\hline
\end{tabular}


Page No. 190

$04 / 28 / 93$

L.IST OF HANFORD-GENERATED CLASSIFIED

DOCUMENTS DATED BETWEEN 1/1/61 AND 12/31/72

DOC. NO.

TITLE

AUTHOR

LEVEL

PGS

DAre

HW-77911

PROPOSAL HANFORd ASSISTANCE TO UCLRL -

ALBAUGH FW

SRD

$06 / 14 / 63$

$H W-77917$ RD

FY-64 MEW MATERIALS DEVELOPMENT

COMMENT ISSUE N-REACTOR STARTUP PHYSICS

TEST PROGRAM TEST PROCEDURES APPROACH TO

HAGAN JW

CRD

61

$06 / 26 / 63$

CRITICAL AMD MIMIMMM CRITICAL SIZE

HU- 77920

THE FUEL ELEMENT STORY A VALUE

WA BLANTON

CRD

$06 / 17 / 63$

HW- 77929

ACHIEVEMENT

URANIUA BURNOUT VALUES

HH- 77942

RECOWNENDED MU FACTORS FOR TRITIUM

YIELD PREDICTIONS

HN- 77946

HW- 77947

STUDY OF HANFORD PROOUCTION CAPABILITY

THROUGH FISCAL YEAR 1973

HW-T7985

HU- 77988

INSPECTION METHCD FOR TSETSE COMPONENTS

PROPOSED GRAPHITE CORING PATTERNS FOR

$B, D, F, D R$, AND H REACTORS

KER-4 OPERATING REPORT TEST $K-4-14$ PT

IP-477-A PT IP 482-A

HW- 78000

HW- 78000

HW- 78017

HU-78079

ALTERMATE USES FOR HAKFORD FACILITIES

ALTERHATE USES FOR HANFORD FACILITIES

PROPERTY EVALUATION OF PLUTONIUA SHEET

USED IN CAPACITOR DISCHARCE STUDIES

PLUTONILM PRCOUCT DEVELOPMENT OPERATION

MOWTHLY REPORT - JUNE, 1963

N-REACTOR CAPABILITY APPRAISAL

HW-78085

HW-78091

HW-78095

$H W-78100$

HW-78128

PROGRESS REPORT - MEAPOUS PROCESS EHG -

JUNE 1963

DEVELOPMENT TEST IP-556-D, SUPPLEMENT $A$, IRRADIATION SERVICE REQUEST MAPO-278

OUTGASSING RATE OF TRITIUA AT HIGH

TEMPERATURE

HANFORD REACTOR AND SEPARATIONS FACILITY ADVANTAGES

FLOUSHEET FOR NMERICIUN RECOVERY AND PURIFICATION

$H W-78143$ RD

HW-78148

CALCULATIONS ON CLOSE-COUPLED PROCESSING

FOR PU-238 RECOVERY

DIBASIC ALUMINUM nITRATE FOR THE REDOX

PROCESS

HW-78157

QUARTERLY PROCRESS REPCRT METALLURGY

DEVELOPNENT OPEKATION APRIL, MAY, JUNE, 1963

HW-78158

ATOAIC WEAPON DATA SUPPLEMENT QUARTERLY PROGRESS REPORT METALLURGY DEVELOPMENT OPERATION APRIL, MAY, JUNE, 1963

HH-Tล170

HW-78177 TUBE MALL THICKNESS ISOTOPE PROOUCTION TUBES

QUARTERLY SUMARY REPORT \& ASSOCIATED DATA FOR 234-5 BLDG, FINAL PRCOUCT SHIPPED DURING THE 2ND QUARTER OF CY 1963

\begin{tabular}{|c|c|c|c|}
\hline SAITH Wo & SRD & 20 & $06 / 17 / 63$ \\
\hline HANDSHUH JH, VAUGHN AD & SRD & 3 & $06 / 18 / 63$ \\
\hline GRENINGER AB & SRD & 10 & $06 / 19 / 63$ \\
\hline MCERECOR LL & SRD & 17 & $06 / 21 / 63$ \\
\hline AGAR JD & SRD & 21 & $06 / 20 / 63$ \\
\hline \multirow{3}{*}{ CaERG KC } & SRD & 19 & $07 / 03 / 63$ \\
\hline & SECR & & $05 / 10 / 63$ \\
\hline & $\operatorname{sed}$ & 106 & $05 / 10 / 63$ \\
\hline TAYLOR JM, GARDNER MR & 520 & 8 & $06 / 25 / 63$ \\
\hline \multirow[t]{2}{*}{ HICK OJ } & SRD & 9 & 07/01/63 \\
\hline & SRD & 78 & $07 / 02 / 63$ \\
\hline AE SMITH & SRD & & $07 / 03 / 63$ \\
\hline \multirow[t]{2}{*}{ DEMERS AE } & SRD & 6 & $07 / 05 / 63$ \\
\hline & SRD & 39 & $08 / 27 / 63$ \\
\hline SZULINSKI MJ, CURTIS MH & SRD & 15 & $09 / 03 / 63$ \\
\hline COPPINGER EA & SRD & 14 & $07 / 10 / 63$ \\
\hline RA YODER & SECR & & $07 / 09 / 63$ \\
\hline $\begin{array}{l}\text { HICK OJ, LAST GA, MIMOR } \\
\text { JE, MELSON TC, STEWART RH }\end{array}$ & CRD & 64 & $09 / 18 / 63$ \\
\hline $\begin{array}{l}\text { HICK OJ, LAST GA, MINOR } \\
\text { JE, MELSON TC, STEWART RH }\end{array}$ & SRD & 31 & $09 / 18 / 63$ \\
\hline YOUNG JR & SRD & 7 & $07 / 10 / 63$ \\
\hline AE SMITH & SRD & & $07 / 10 / 63$ \\
\hline
\end{tabular}


Page No. 191

O4/28/93

LIST OF HANFORD-GENERATED CLASSIFIED

DOCUMENTS DATED BETWEEN 1/1/61 AND 12/31/72

TITLE

AUTHOR

LEVEL

PaS

DATE

HIH-78198RD

CORRECTION FOR MEASUREMENT BIAS SLAG AND

RU DOERR

SECR

$07 / 15 / 63$

CRUCIBLE FROM SCRAP PONDER TASK III

REDUCTION

HH-78266

PROJECT PROPOSAL: ADOITIONAL PLUTONIUM

NN RAILE

SRD

$07 / 17 / 63$

HH-78273RD

HU-78319 RD

HW-78333

HW- 78337

HW-78340

HU-78351

HW-78355

CASTING FACILITIES RMC LIME - 234-5 BLDG (CAC-110)

MONTHLY REPORTS REACTOR PHYSICS

PROCESS FLOU SHEET, ISOSTATIC PRESSING

MONTHLY TECHNICAL REPORT APRIL, 1963

PLUTONIUM PRODUCT DEVELOPNENT OPERATION

MONTHLY REPORT - JULY, 1963

MONTHLY TECHNICAL REPORT MAY, 1963

HAZARDS ANALYSIS DT IP-556-D,SUP. A.

HAPO-278 OUTGASSING RATE OF TRITIUH AT

HIGH TEMPERATURES

IPD REACTOR TRAINING TEXT CLASSIFIED

SUPPLEMENT CHAPTER II DESIGN AND

CONSTRUCTION OF THE HANFORD PRODUCTION

REACTORS

HU-78356

MEUTRON LIFETIMES IN THE HANFORD

LATTICES

HH-78363

TEN YEAR REACTOR STLOY SUPPLEMENT

PRIORITY I CASES MEPTUHIUM-237

PRCOUCTION

HU-78366

RECOMMENDED EMERGENCY PROCEDURE FOR EXTENDED OUTAGE AT ALL IPD PRODUCTION REACTORS

HH-78368

HW-78374

HW-78374 1

HW-78375 RD

HW-783T7REV

HW-78423

HH-78423 REV2

HW-78443

HW-78452

EFFECTIVE DELAYED MEUTRON FRACTIONS AMD DECAY CONSTANTS IN THE HANFORD PROOUCTION REACTORS

DESIGN SCOPE ARC MELTIMG FACILITY

DESIGN SCOPE ARC MELTING FACILITY

LOW FLON TUBES

PLUTONIUM ISOTOPIC DETERMINATION -

SUPPLEMENT

HAPO ECONOMICS FOR OPERATING ONE KE

REACTOR PLUS N REACTOR

HAPO ECONOMICS FOR OPERATING ONE KE

REACTOR PLUS N REACTOR

PROGRESS REPORT - MEAPONS PROCESS ENG -

JULY 1963

PROOUCTION TEST IP-603-I EVALUATION OF

ACCIDENTAL FUEL FLUSHING POTENTIAL D-DR REACTORS

HW-78488

REPORT OF THE WORKING COMMITTEE OF THE

FUEL ELEMENT DEVELOPNENT COMAITTEE

HW-78488 RD

REPORT TO THE WORKING COMITTEE OF THE

FUEL ELEMENT DEVELOPMENT COMIITTEE FROM THE GENERAL ELECTRIC COMPANY - HANFORD

HW- 78489

PROJECT PROPOSAL, ARC MELTING FACILITY RAM LINE - 234-5 BUILDING (PRDJECT

CAC-115)

\begin{tabular}{|c|c|c|c|}
\hline H TOFFER & SRD & & $06 / 01 / 63$ \\
\hline SMITh AE & SRD & 4 & $07 / 18 / 63$ \\
\hline NICHOLS PF & SRD & 5 & $05 / 01 / 63$ \\
\hline WICK OJ & SRD & 43 & $07 / 31 / 63$ \\
\hline NICHOLS PF & SRD & 6 & $06 / 03 / 63$ \\
\hline DEMERS AE & SRD & 3 & $07 / 05 / 63$ \\
\hline WNDE IGE & SRD & 90 & $07 / 02 / 63$ \\
\hline TILLER RE & SRD & 3 & $07 / 23 / 63$ \\
\hline \multirow[t]{2}{*}{ GRIMA KG } & SRD & 3 & $07 / 24 / 63$ \\
\hline & SRD & 11 & $07 / 29 / 63$ \\
\hline TILLER RE & SRD & 5 & $07 / 24 / 63$ \\
\hline $\begin{array}{l}\text { KENDALLL WR, MIITALA MF } \\
\text { KENDAI.L WR, UIITALA MF } \\
\text { HUFFMAIN IL }\end{array}$ & $\begin{array}{l}\text { SRD } \\
\text { SRD } \\
\text { SRD } \\
\text { SECR }\end{array}$ & $\begin{array}{l}33 \\
33 \\
5\end{array}$ & $\begin{array}{l}07 / 24 / 63 \\
07 / 24 / 63 \\
07 / 24 / 63 \\
06 / 30 / 70\end{array}$ \\
\hline $\begin{array}{l}\text { STENQUIST DR, MCCURRY JH, } \\
\text { LANG LW }\end{array}$ & SRD & 5 & $07 / 26 / 63$ \\
\hline $\begin{array}{l}\text { STENCUIST DR, MCCURRY JW, } \\
\text { LANG LW }\end{array}$ & SRD & 5 & 08/09/63 \\
\hline AE SinITH & SRD & & 08/05/63 \\
\hline \multirow[t]{2}{*}{ HOLLIFIELD PJ, GEORGE DK } & SRD & $\mathbf{5}$ & $07 / 30 / 63$ \\
\hline & SRD & 157 & $10 / 01 / 63$ \\
\hline $\begin{array}{l}\text { MINOR JE, RICHES JH, } \\
\text { STRINGER JT }\end{array}$ & SRD & 18 & $09 / 09 / 63$ \\
\hline RAILE MN & SRD & 17 & $09 / 20 / 63$ \\
\hline
\end{tabular}


HW-78505

HW-78511

HW- 78514

HW- 78525

HW-78547

HH-78551

HH-78552

HW-78553

HW-78566

HW-78569

HH-78571

HW-78573 RD

HU-78582

HW- 78583

HW-78585

HW-78589

HW-78595

HW-78596

HW-78604

HW-78632

HW-78634

HH-78634 1

HW-78638

HW-78639
MUCLEAR METERIALS REVIEW 200 AREA SUMMARY - FY 1963

TRIP REPORT AND MINUTES OF COMBINED OPERATIONS MEETIMG HELD OCTOBER 16, 1962 DETRMINING INITIAL COMPOSITION OF REGENERATIMG IN-CORE MEUTRON FLUX DETECTORS

HAPO ISOTOPE PROGRAM - PO-210 PRODUCTION

COAPARISON OF PHYSICS PARAMETERS FOR $N$ AND K REACTORS

POSSIBLE CONSEQUENCES OF OPERATION HITH KIVN FUEL ELEMENTS IN $K$ ZIRCALOY PROCESS TUBES

PRCOUCTION TEST IP-601-D EVALUATION OF CRLD-FORMING CHARACTERISTICS OF KER LOOP COOLANT CONTAINING HH4 OH FOR PH CONTROL UNUSUAL IMCIDENT REPORT MO. 25 CROSSHEADER DAMAGE POTENTIAL PROOUCTION OF U-233 AT HANFORD FY 1964 MEASUREMENT FACTORS AND LIMITS MEAPONS MANUFACTURING OPERATION 234-5 BUILOING

POTENTIAL PRODUCTION OF U-233

PROCESS FLOW SHEET - STABILIZING OF ALLOYED PLUTONIUN MEAPON COMPONENTS SPECIALISTS LOG 105-D REACTOR (PERICD: 8/7/63 THRU -.--)

FUEL ELEMENT DEVELOPMENT COMMITTEE AMNUAL REPORT FROM THE GENERAL ELECTRIC COMPANY HANFORD

PROJECT PROPOSAL INCREASED STORAGE BASIN OVERFLOW 105NE/KW PROJECT CGT-112

MOLD TEMPERATURE CALIBRATION FOR MOOEL

74-C GRAPHITE MOLDS IN HA-19-B1

E-METAL USAGE AND ITS OPTIMIZATION

TRITIUM PRCOUCTION HORIZONTAL CONTROL RODS

HAPO ISOTOPE PROGRAM - P -210 PROOUCTION INSPECTION OF OBJECTS OF REVOLUTION FROM POINTS OTHER THAN THE DESIGN CENTER DESIGN SCOPE DEGREASING AND BRIQUETTING FACILITY RMA FABRICATION LINE, 234-5 BUILDING PROJECT CAC-109

DESIGN SCOPE DEGREASING AND BRIQUETTING FACILITY RMA FAVRICATION LINE, 234-5 BUILDING PROJECT CAC-109

EXCERPTS FROM PROJECT MHITHEY MOWTHLY REPORT - JULY, 1963

EXCERPTS FROA PROJECT WHITHEY MONTHLY REPORT - AUEUST, 1963

\begin{tabular}{|c|c|c|c|}
\hline $\begin{array}{l}\text { DOERR RH, STEVES LH, } \\
\text { WIRFS LF }\end{array}$ & SRD & 22 & $07 / 31 / 63$ \\
\hline LANG LH, MCCURRY JH & SRD & 9 & $08 / 01 / 63$ \\
\hline BUNCH WL & SRD & 27 & $09 / 11 / 63$ \\
\hline $\begin{array}{l}\text { LANG LH, KUSLER LE, } \\
\text { RATHVON HC }\end{array}$ & SRD & 12 & $08 / 07 / 63$ \\
\hline NICHOLS PF, NILSON R & SRD & 4 & $08 / 02 / 63$ \\
\hline CARLSON PA & SRD & 5 & $08 / 06 / 63$ \\
\hline NEIDNER R & SRD & 12 & $08 / 06 / 63$ \\
\hline MAGUIRE AR & SRD & 6 & $08 / 06 / 63$ \\
\hline WARREN JH & SRD & 4 & $08 / 06 / 63$ \\
\hline DOERR RU & SRD & 14 & $07 / 01 / 63$ \\
\hline WARREN JH & SRD & 3 & $08 / 08 / 63$ \\
\hline SAITH AE & SRD & 4 & $08 / 07 / 63$ \\
\hline JT BAKER & SECR & & $08 / 07 / 63$ \\
\hline \multirow[t]{2}{*}{ LM LEOB } & SECR & & $08 / 09 / 63$ \\
\hline & SECR & & $10 / 03 / 63$ \\
\hline BARBER AE & CRD & 7 & $08 / 08 / 63$ \\
\hline \multirow{3}{*}{$\begin{array}{l}\text { CARTER RD, MEEF UI } \\
\text { HEACOCK HW }\end{array}$} & SRD & 23 & $08 / 15 / 63$ \\
\hline & CRD & 2 & $08 / 09 / 63$ \\
\hline & SECR & & $06 / 30 / 70$ \\
\hline MCERECOR LL & SRD & 8 & $08 / 13 / 63$ \\
\hline BORGESON ME & SRD & 24 & $08 / 13 / 63$ \\
\hline BORGESON ME & SRD & 24 & $08 / 13 / 63$ \\
\hline WICK OJ & SRD & 4 & $08 / 15 / 63$ \\
\hline HICK Od & SRD & 3 & $09 / 13 / 63$ \\
\hline
\end{tabular}


DOC. NO.

HH- 78640

HW-78641

HW- 78642

HW-78648

HW-78650

HW- 78664

HW-78664 I

HW-78671

HW-78673

HU-78678

HW- 78689

HH- 78693

$H W-78695$ RD

HW-78695 RD REV

HH-78704 RD

HW-78708

HW-78708 1

HW-78712

HW-78713

HH-78733 RD2

HW-78740

HแM-78741

HW-78746 RD

HW-78753RD

HW-78759

$H_{1}-78764$
TITLE

AUTHOR

HICK OJ

PROJECT HHITMEY PLUTONIUN METALLURGICAL DEVELOPMENT MONTHLY REPORT - AUGUST, 1963

PROJECT WHITNEY PLUTONIUN METALLURGICAL DEVELOPMENT MONTLHY REPORT - SEPTEMBER, 1963

A CRITICAL REVIEH OF THE MPR FUEL

ELEMENT AUTOCLAVE CYCLE

HUNT N PECK - A PROGRAM TO PERFORM ZONE FLATTENING CALCULATIONS ON THE IBM-7090

$K$ AND N REACTOR CAPABILITIES

DESIGN SCOPE METAL STABILIZATION

FACILITY 234-5 BUILDING PROJECT CAC-987

DESIGN SCOPE METAL STABILIZATION

FACILITY 234-5 BUILDING PROJECT CAC-987

SPECIAL PRCDUCT STLOY N REACTOR

PITA, IP-22, SUP II DEMONSTRATION LOAD

POTENTIAL PRODUCTION OF U-233 AT HANFORD

SIZCAN - A FORTRAN CODE TO AID IN SIZING CYLINDRICAL OR AMNULAR FISSION PROOUCT CONTAINERS

PLUTONILM PROOUCT DEVELOPNENT OPERATION

MONTHLY REPORT - AUGUST, 1963

ESTIMATIOA OF RADIOACTIVE LEVELS IN ANIMAL THYROIDS

ESTIMATION OF RADIOIODINE LEVELS IN ANIMAL THYROIDS

ACRS PRESENTATIONS ON N-REACTOR AUEUST

14-15, 1963

DESIGN SCOPE ADDITIONAL PLUTONIUN

CASTING FACILITIES RMA LINE, PROJECT

CAC-110 234-5 BUILDING

DESING SCOPE ADDITIONAL PLUTONIUN

CASTING FACILITIES RMA LINE, PROJECT

CAC-110 234-5 BUILDING

A PROGRAM FOR THE MUMERICAL CONTROL OF A PULSE INCREMENT SYSTEM

SURVEY OF POSSIBLE SUPPORT ATTACHMENT

PROCESSES FOR N-REACTOR FUELS

ANALYSIS AND EVALUATION OF THE N-REACTOR

ZONE TEMPERATURE MONITORING SYSTEM

COST OF REMOVING PROCESS TUBES FROM

SERVICE

NUCLEAR SAFETY EXPERIENCE - HANFORD

PRCOUCTION REACTORS

INTERIM PUMPING MOOIFICATION REVIEW SPECIAL COST STUDY

PRODUCTION TEST-IP-610-A EVALUATION OF

INDUCTION HEAT TREATED FUEL CORES

PROGRESS REPORT - MEAPONS PROCESS

ENGINEERING - AUCUST 1963

\begin{tabular}{|c|c|c|c|}
\hline HICK Od & SRD & 9 & $08 / 30 / 63$ \\
\hline HICK OJ & SRD & 11 & 09/30/63 \\
\hline ROBINSON RK & CRD & 26 & $12 / 23 / 63$ \\
\hline GRUMAE LL & SRD & 74 & $08 / 14 / 63$ \\
\hline $\begin{array}{l}\text { LANG LH, DONIS WJ } \\
\text { WOORICH DD }\end{array}$ & $\begin{array}{l}\text { SRD } \\
\text { SRD }\end{array}$ & $\begin{array}{l}11 \\
34\end{array}$ & $\begin{array}{l}08 / 16 / 63 \\
09 / 18 / 63\end{array}$ \\
\hline WOORICH DD & SRD & 34 & $09 / 18 / 63$ \\
\hline RL DICKEMAN & SECR & & $08 / 16 / 63$ \\
\hline CONMER EL & SRD & 8 & $08 / 16 / 63$ \\
\hline HARREN JH & SRD & 4 & $08 / 19 / 63$ \\
\hline DEMIER W & CRD & 33 & $08 / 23 / 63$ \\
\hline HICK OJ & SRD & 7 & $08 / 30 / 63$ \\
\hline BROUWS RJ & SRD & 34 & $08 / 14 / 63$ \\
\hline BROUNS RJ & SRD & 30 & $06 / 04 / 64$ \\
\hline TRUABLLE RE & SRD & 1 & $08 / 21 / 63$ \\
\hline SHAIN EO, HOORICH DD & SRD & 28 & $09 / 10 / 63$ \\
\hline SHAIN EO, HODRICH DD & SRD & 28 & $09 / 10 / 63$ \\
\hline GRAY UC & SRD & 148 & $08 / 21 / 63$ \\
\hline TB CORREY & CRD & & $09 / 03 / 63$ \\
\hline PHILIPP LD & CRD & 43 & $11 / 22 / 63$ \\
\hline MEEF WI & SRD & 6 & $08 / 23 / 63$ \\
\hline GREAGER OH & SRD & 7 & $08 / 27 / 63$ \\
\hline HEDGES JH & $\begin{array}{l}\text { SRD } \\
\text { SECR }\end{array}$ & 5 & $\begin{array}{l}08 / 23 / 63 \\
06 / 30 / 70\end{array}$ \\
\hline HLADEK KL & SRD & 9 & $11 / 19 / 63$ \\
\hline EA SMITH & SRD & & $09 / 05 / 63$ \\
\hline
\end{tabular}


Page No. 194

$04 / 28 / 93$

LIST OF HANFORD-GEMERATED CLASSIFIED

DOCUMENTS DATED BETWEEN 1/1/61 AND 12/31/72

DOC. NO.

HW- 78770

HW-78T77

$H H-78778$ RD

HW-78782

HU-78789

HW-78795

HW-78803RD

HH-78836

HW-78845

HW- 78859

HW- 78864

HW-78868

HH-78869

$H W-78873$

HW-78874

HW-78883

HW-78889

HW-78901

HW-78918

HW-78930

HW-78942

Hแ-78959

HW-78959SUP1

$H W-78960$

HW-78960 B
TITLE

DESIGN CRITERIA BAUXITE-SULFURIC ACID

FEED FACILITIES 100-K AREA

FEASIBILITY OF THE USE OF INDUCTION

HEATING EQUIPMENT FOR PILOT PLANT

DEVELOPMENT WORK

MEMO PREPARED AT THE REQUEST OF

COMMISSIONER JAMES T. RAMEY PRODUCTION

STUOY - N REACTOR

HIGHER ISOTOPE CASE STUDY

PROOUCTION TEST IP-614-A IRRADIATION OF THORIUM TARGET ELEMENTS

REACTIVATION OF SHUTDCMN REACTOR PLANTS OBSERVATIONS ON NUCLEAR FUEL BUSIMESS

TIMS DATA PROCESSING FORHAT DEFINITION O3 RESEARCH AND DEVELOPMENT PROGRAM FOR FY'S 1964 AND 1965

SANL. 128-000

A PROGRAM FOR MULTI-PROOUCT OPERATION OF THE N-REACTOR

DOWNCOMER FOR 105KW AND KE PROJECT

CA-512-R

END CLOSURE OF HOT DIE SIZE DIFFUSION BONDED FUEL ELEMENTS

STATUS REPCRT OF IRRADIATED NPR FUEL

ELEMENT RUPTURE STUDIES IN THE IRP DEVIATION REQUESTS FOR HANFORD WEAPON COMPONENTS

HYDRAULIC TESTS OF SPLIME INSERT

MODIFICATIONS - K REACTOR

EMERGENCY STORAGE BASIN COOLANT - DESIGN

CRITERIA FOR ARCHITECT-ENGINEER USAGE

PROVISIONAL SPECIFICATIONS FOR

PROTOTYPICAL LITHIUN-ALUMINUM TARGET

ELEMENT O5T

PROOUCTION STUDIES N REACTOR CASES 1, 20

\& 24

TOPICS FOR OCTOBER PHIEG MEETING

FEASIBILITY STUDY BY-PROOUCT HORIZONTAL CONTROL ROOS - ALL REACTORS

AMALYSIS OF IRRADIATED THORIUM OXIDE

(SERIES I-DEVELOPMENT TEST IP-588-D)

ANALYSIS OF IRRADIATED THORIUM OXIDE -

SERIES 11 - DEVELOPMENT TEST IP-588-D

PRODUCTION TEST-IP-616-A IRRADIATION OF ENRICHED HOT-DIE-SIZE DIFFUSION-BONDED FUEL ELEMENTS

FABRICATION OF ENRICHED HOT DIE SIZED DIFFUSION BONDED FUEL ELEMENTS FOR PROOUCTION TEST IP-616-A
AUTHOR

ETHERIDGE EL

PONERS HG

RL DICKEMAN

SRD

08/22/63

KUSI.ER LE, LANG LH, SRD

MCCURRY JH

HLADEK KL, KUSLER LE SRD $98 / 29 / 63$

SNITH EA

RL DICKEMAN

WALLACE DA

FRANK US

SRD $\quad 10$

$08 / 27 / 63$

$08 / 30 / 63$

$09 / 04 / 63$

$09 / 06 / 63$

STEMART RH

SRD

SRD

2

5

SRD 3

CRD 18

09/05/63

$09 / 09 / 63$

ROBINSON TF, MARINOS D SRD $28 \quad 11 / 12 / 52$

STRAHD CA

CRD

62

$01 / 14 / 64$

HAYDEN KD

CRD

10

$09 / 09 / 63$

US FRAMK

CONF

$08 / 29 / 63$

WATERS ED

CRD

BRIMKMAN LB

CRD

4

$09 / 10 / 63$

HICK JJ, JR.

SRD

12

$09 / 12 / 63$

09/02/63

SECR

$10 / 10 / 63$

SMITH AE

SRD

SRD

MATSUMOTO WY, WEILER MR, SRD SCHAEIDER RA

SECR

3

24

$09 / 13 / 63$

$02 / 10 / 64$

6

$09 / 17 / 63$

$06 / 30 / 70$

HLADEK KL

SRD

11

$09 / 17 / 63$

STRAND CA

CRD

8

$10 / 02 / 64$ 
Page No. 195

04/28/93

LIST OF HANFORD-GENERATED CLASSIFIED

DOCUMENTS DATED BETWEEN 1/1/61 AND 12/31/72

DOC. NO.

HU-78975

HW-78985

HW-78986

HH-79007

HW- 79034

HW- 79040

HW-79055 RD

HW-79056

HW-79058

HW- 79078

HW-79107

HW- 79120

$H H-79156 R D$

HW-79161

HW-79166

$H W-79168$

HH-79169

$H W-79170$

HW-79171

HW-79172

HW-79173

HW-79177
TITLE

REACTOR FLATTENING OPTIMIZATION ATOMIC WEAPON DATA SUPPLEMENT QUARTERLY PROGRESS REPORT METALLURGY DEVELOPMENT OPERATIOH JULY, AUEUST, SEPTEMBER, 1963 INTERIM REPORT I, PROOUCTION TEST IP-442-A HALF-PLANT REDUCTIN IN PROCESS WATER PH, 105-D

PLUTONIUM PROOUCT DEVELOPNENT OPERATION MONTHLY REPORT - SEPTEMBER, 1963

THE APPLICATION OF DIMENSIONAL STABILITY EVALUATION DATA TO DETERMIME ACCEPTABILITY OF PLUTONIUM PARTS

N FUEL FAILURE BEHOVIOR AND THE ROLE OF THE PRTR RUPTURE LOOP IN THE N FUEL FAILURE TESTING PROGRAM HIGH PU-240-CONTENT PLUTONIUN CHEMICAL PROCESSING COST ESTIMATES

FINAL REPORT ON THE EVALUATION OF HARVEY ALLAINMM COMPONENTS UNDER PITA-IP-12-I MACHIMABILITY OF PLUTONIUM

PROGRESS REPORT - WEAPONS PROCESS ENG SEP 1963

N-REACTOR DEPARTMENT MONTHLY REPORT SEPTEMBER 1963

INTERNAL GRAPHITE MODERATOR FORCES

STUDY, C AND K REACTORS

HOOD 9 HOURLY LOG - 1963

REACTOR DESIGN ANALYSIS MONTHLY REPORT SEPTEMBER4, 1963

EXCEEDING ALLAYS-SAFE RATIO IN F-2 AMD

F-1 TANKS - (NUWBER 2, CY-63) REDOX PROCESSING - REDOX OPERATION

EXCERPTS FROM PROJECT WHITNEY MONTHLY

REPORT - SEPTEMBER, 1963

EXCERPTS FROM PROJECT HHITNEY MONTHLY

REPORT - OCTOBER, 1963

EXCERPTS FRON PROJECT UHITHEY MONTHLY

REPORT - NOVEMBER, 1963

PROJECT WHITNEY PLUTONIUA METALLURGICAL

DEVELOPMENT MONTHLY REPORT - OCTOBER, 1963

PROJECT MHITNEY PLUTONIUM METALLURGICAL DEVELOPMENT MONTHLY REPORT - NOVEMBER, 1963

PROJECT HHITMEY PLUTONIUM METALLURGICAL DEVELOPMENT MONTHLY REPORT - DECEMBER, 1963

CPD REQUIREMENTS IN SUPPORT OF MULTI-PROOUCT ONERATION - N-REACTOR
AUTHOR

LEVEL

PGS

DATE

NEEF HI

15

$09 / 18 / 63$

HICK OJ, LAST GA, MINOR SRD

69

$10 / 18 / 63$

JE, MELSON TC, STEWART RH

GEIER RG

SRD

14

$09 / 20 / 63$

HICK OJ

SRD

9

$09 / 30 / 63$

OBERG GC

SRD

5

$09 / 24 / 63$

EVANS TW

CRD

30

$09 / 30 / 63$

MCDONALD JE, OLSON RE, SRD RATHVOW HC

HUBERT RV

SRD

10

$09 / 26 / 63$

RECTOR JH, ARMSTRONG DA, SRD PETERSON RWE

AE SHITH

SRD

$10 / 02 / 6$

$10 / 07 / 63$

COOLEY DE

SRD

43

$10 / 28 / 63$

RJ SLOAT

SRD

30

$091 / 01 / 63$

HR KOSHATA

MALADOY CY

SRD

3

$10 / 07 / 63$

HICK OJ

SRD

5

$10 / 14 / 63$

NICK OJ

SRD

4

$11 / 11 / 63$

WICK OJ

SRD

HICK OJ

SRD

5

$12 / 11 / 63$

$10 / 31 / 63$

WICK OJ

SRD

11

$11 / 27 / 63$

HICK OJ

SRD

9

$12 / 31 / 63$

REINKER PH

$10 / 08 / 63$ 
Page No. 196

$04 / 28 / 93$

LIST OF HANFORD-GENERATED CLASSIFIED

DOCLMENTS DATED BETWEEN 1/1/61 AND 12/31/72

DOC. NO.

HH-79183

HW-79209

HH-79211 RD

HW- 79213

HW-79228 REV

HH-79261

HW-79265

$H H-79266$ RD

HW-79305

HW-79306

HH- 79328

HU- 79331 RD

HW- 79338

$H W-79341$

HW-79357

HW- 79363

HW-79370 RD

HW- 79376

HW- 79384

HH-79385

HW-79424

HH-79428

HW-79448

HW -79450

HW- 79465

HW- 79468
TITLE

MONTHLY TECHNICAL REPORT SEPTEMBER, 1963

REACTOR PHYSICS

OLD PILE HCR OPERATIHG TEMPERATURES

CHARGING PROCEDURES

E-N LOADS AT THE $K$ REACTORS

NUCLEAR SAFETY CRITERIA FOR SPECIAL TEST LOADS N-REACTOR

COST IMPROVEMENT PROPOSAL INCREASED

COOLANT FLON THROUGH BLANKET POISON TUBES (H REACTOR)

FABRICATION PROCESS TEST EVALUATION DISTRIBUTION OF NITROGEN AND OTHER IMPURITIES IN CAST SHAPES

AN ENGINEERING REVIEH OF FABRICATION PROCESSES

PROOUCTICN OF ZIRCONIUN-NIOBIUN-95 AND SCAMDIUM-46 AT HANFORD

PROOUCTION OF SPECIAL MUCLEAR MATERIALS IN N-REACTOR

MANUFACTURING DIVISIONS SEMI-ANNUAL SUMMAARY REPORT (PERIOD ENDIMG JUNE 30 , 1963)

$\mathrm{U}-233$

PLUTONIUM PROOUCT DEVELOPNENT OPERATION

MONTHLY REPORT - OCTOBER, 1963.

RADIOMETALLLURGY EXAMINATION OF THREE OVERBORE SPLIT FAILURES FROM 3062C (RM C-422)

A-LINE PRODUCT CHARACTERISTICS

MOOEL 74-C SHIPPING PROBLEM STATUS COMPARISON OF PU + TR CASES

ANALYSIS OF TRITILM-ONLY CASES AND WEAPONS-GRADE MULTI-PROOUCT ALTERMATIVES N REACTOR

OUARTERLY SUMMARY REPORT \& ASSOCIATED

DATA FOR 234-5 BLDG, FIMAL PRCDUCT

SHIPPED DURING THE 3RD QUARTER OF CY 1963

STATISTICAL STATEMENTS \& ASSOCIATED DATA FOR 234-5 BLDG, FINAL PRODUCT SHIPPED DURING THE 3RD QUARTER OF CY 1963

FABRICATION PROCESS TEST - FPT-63-28 REDUCTION OF IN-PROCESS INVENTORY FPT-63-27 - DIMENSIONAL STABILITY, MOOEL 1807 SHAPE CASTINGS

N-REACTOR DEPARTMENT MONTHLY REPORT

OCOTBER 1963

U-233 PROOUCTION STUDIES

INCREASED COOLANT FLON FOR H REACTOR

FUEL ELEMENT PERFORMANCE
AUTHOR

NICHOLS PF

AGAR JD

GERDES KW

MEEF HI

MECHODOM, WS, MILLER NR

HUFFMAN IL

SRD

HALKER CI

SRD

VANDERCOOK RE

SRD

WARREN JH

SRD

CONDOTTA DL, PIERICK EG SRD

TW HAUFF

SRD

SNITH AE

WICK OJ

SRD

SRD

TEATS R

AE SHITH

SRD

BARBER AE

PIERICK EG, STENOUIST DR

RICE JE

AE SNITH

FISHER JG, LEHIS LI, PINKERTON JM

WALKER CM

GRENIMGER AB

HUFFMAN IL

HAGIE LT
18

SRD

SRD

SRD

$10 / 28 / 63$

CRD

CRD

SRO

EVEL PGS

DATE

$10 / 01 / 63$

$10 / 10 / 63$

05/10/63

$10 / 10 / 63$

04/05/65

$06 / 26 / 63$

$10 / 04 / 63$

$10 / 15 / 63$

$10 / 18 / 63$

$10 / 21 / 63$

$10 / 22 / 63$

10/22/63

$10 / 31 / 63$

$10 / 22 / 63$

$10 / 24 / 63$

$10 / 24 / 63$

$10 / 25 / 63$

$10 / 29 / 63$

$10 / 18 / 63$

SRD

SRD

CRO
$10 / 30 / 63$

$10 / 18 / 63$

$11 / 07 / 63$

$11 / 01 / 63$

$10 / 23 / 63$

$01 / 02 / 58$ 
Page No. 197

$04 / 28 / 93$

LIST OF HANFORD-GENERATED CLASSIFIED

DOCUMENTS DATED BETWEEN 1/1/61 AND 12/31/72

DOC. NO.

HH- 79481

HW-79506 RD

HW- 79517

HW- 79526

HU-79528

HW-79529

HW-79542

HW-79577

HU-79593

HH-79602

HW-79602

HW-79611

$H W-79623$ RD

HiW-79624

$H H-79630$

HW-79635

HW-79641

HW- 79642

HW-79645

HW-79650

HW-79653

HW-79665

HL-79675

$H W-79677$

HH- 79678

HW-79679
TITLE

DESIGN CRITERIA PONER ROLL FORMING FACILITY RMC FABRICATION LINE 234-5 BUILDING

PRODUCTION VALUES for PLANT PLANNINg PROPOSALS

DIPPER MATERIALS MEETING

MONTHLY TECHNICAL REPORT OCTOBER, 1963

REACTOR PHYSICS

RADIATION EXPOSURE FROM INCONEL

HORIZONTAL CONTROL ROOS

DESIGN CRITERIA PLUTONIUM THERMAL

TREATMENT FACILITY A FABRICATION LIME 234-5 BUILDING

A REVIEH OF THE PHILOSOPHY AND FUTURE USE CF THE BALL-3X SYSTEM IN THE IPD REACTORS

FABRICATION PROCESS TEST EVALUATIOA -

SHIPPING, HI-74-SL

PRELIMINARY SURVEY REACTOR FORMATION OF

RHENIUM-TUNGSTEN ALLOY

A PRELIMIMARY STUDY OF PRODUCTION OF TUNGSTEN-RHENIUN ALLOYS IN N-REACTOR PRCOUCTION OF TUNGSTEN-RHENIUM ALLOYS IN N-REACTOR

SEPARATIONS COST DISTRIBUTION

NOTES FROM PUIEG MEETING - OCTOBER 15

AND 16, 1963

SUGGESTED STARTUP PLAN WITH HIGH CO2

FUEL ELEMENT DESIGN FOR CO-PRCOUCT PILOT

LOAD

LONGER ENRICHED FUEL ELEMENTS FOR H

REACTOR

COINCIDENT TRIP PRESSURE MONITORS

SUMMARY REPORT FLIXIBLE VSR'S AND VSR

CHANMEL SLEEVE DEVELOPMENT PROGRAMS

PROCESS DESIGN SCOPE CRITERIA PUREX

MULTIPURPOSE DISSOLVERS

PRODUCTION OF ZIRCONIUM-NIOBILN-95 AMD

SCANDIUM-46 AT HANFORD ADDENDUM TO

$H H-79404$

E-N SUPER FUEL ELEMENTS

PRELIMINARY STUDY OF MULTIPLE FUEL

PROCESSING AT THE PUREX PLANT

THE REACTIVITY EVALUATION OF PITA IPZ2

DEMONSTRATION LOAD, SUPP. III

DESIGN CEITERIA LINEAR POWER

RATE-OF-RISE INSTRUMENTATION

POTENTIAL PROBLEMS IN U-233 PROOUCTION

PLUTONILM PROOUCT DEVELOPMENT OPERATION

MONTHLY REPORT - NOVEMBER, 1963
AUTHOR

HOLLINGSHEAD RC

LANG LH

STEWART RW

NICHOLS PF

KOOP WN

HOORICH DD

NILSON R

BARBER AE

LANG LH, MEICHLE RH

RICHES JH, PIERICK EG

RICHES NH

MCDONALD JE, SMITH WG

SMITH AE

GROSS PD

SHIELDS RJ

HUFFMAN IL

MEMELL LJ, SPENCER HG

KEMPF FJ

EHRLICH RD, LARIVIERE JR SRD

JOHNSON WE

HICK JJ NIELSON SH

ESSIG TH

HERRMAN BH

KOFOED RJ

HICK OJ
SRD

4

$11 / 15 / 63$

LEVEL PGS

DATE

0/31/63

$11 / 04 / 63$

$11 / 05 / 63$

$11 / 01 / 63$

$11 / 08 / 63$

$11 / 20 / 63$

$11 / 06 / 63$

$11 / 08 / 63$

$11 / 11 / 63$

$11 / 19 / 63$

$11 / 11 / 63$

$11 / 13 / 63$

$11 / 12 / 63$

$11 / 12 / 63$

$11 / 11 / 63$

$09 / 18 / 63$

$11 / 14 / 63$

$11 / 15 / 63$

$10 / 15 / 63$

$11 / 13 / 63$

$11 / 26 / 63$

$11 / 18 / 63$

$01 / 16 / 64$

$11 / 19 / 63$

$11 / 30 / 63$ 
Page No. 198

$04 / 28 / 93$

LIST OF HANFORD-GENERATED CLASSIFIED

DOCUNENTS DATED BETHEEN 1/1/61 AND 12/31/72

DOC. NO.

HH-79706

HW- 79710

HW- 79715

HW-79718

HW- 79734

HH-79745

HW- 79752

HW- 79756

HW- 79758

HW-79759

HH-79775

HW-79797

HW-79801

HL-79808

$H W-79813$

$H H-79826$

HW-79858

HW-79915

HW-79916

HW-79917

HH-79931

HW-79932

HWH-79944

HH- 79953

HW- 79957

HW-79974

HW- 79978

$H W-79980$

HW- 80005
TITLE

AUTHOR

BLANTON MA

DEVELOPMENT STATUS AND INCENTIVES FOR

THE HOT DIE SIZIMG PROCESS

E-N PROOUCTION COSTS INTERIM REPORT

COST BUDGET REPORT OCTOBER 1963

ANALYSIS OF E-N TARGET CONVERSION DATA

LONG-TERM NEUTRON ACTIVATION PROOUCTS OF

NICKEL -58

A-LINE PRCOUCT CHARACTERISTICS OCTOBER

1963

COMPARATIVE REACTOR FLUX SPECTRA

REDUCTIONS IN BUMPER FUEL USAGE

FABRICATION PROCESS TEST EVALUATION

REMOVAL INTERMETALLIC COMPOUNDS DURING

INCOTING

U233 FABRICATION

PRODUCTION TEST IP-584-D, SUPPLEMENT A, IRRADIATION OF TARGET ASSEMBLIES IN THE KER LOOPS

PITA IP-22, SUP III DEMONSTRATION LOAD

PROGRESS REPORT - WEAPONS PROCESS ENG NOVEMBER 1963

PRODUCTION TEST IP-638-D, OPERATION OF KER-1, -2, -3, AND -4 SIMULATING N REACTOR CONDITIONS

DIPPER MATERIALS PROGRAM AT HAHFORD

LABORATORIES

PROGRESS REPORT ON MASA CERMET STUDIES

IDENTIFICATION SNC-12, NOVEMBER 1963

EMPIRICAL PLUTONIUN CONSTANTS 7090

PROGRAM

INVENTION RPT: REACTOR DESIGMS FOR

PRODUCING \& USING CLEAN U-233

INVENTION RPT: PROOUCTION OF CLEAN U-233

INVENTION RPT: FUEL CYCLE THAT PERMITS

REPROCESSING CLEAN U-233 BUT MINIMIZES

RADIATION FROM CONTAMINATING U-232

QUALITY CONTROL PROGRAM FOR THE

PLUTONIUN REDUCTION PROCESS

FABRICATION PROCESS TEST FPT-63-31-ROON

TEMPERATURE ISOSTATIC PRESS

RADIOLOGICAL ASPECTS OF DISSOLVING

IRRADIATED :I I-A?

ESTIMATED HELIUN SAVINGS CO2 PRODUCTION

TEST

COST OF HIGH-24O PLUTONIUM

CAPABILITY TESTS OF 181-H RIVER PUMP

HOUSE

URANIUN COST AND PRICE SCHEDULES

MONTHLY TECHNICAL REPORT NOVEMBER, 1963

REPORT OF INVENTION TERTIARY NIINE

SOLVENT EXTRACTION-RECOVERY OF $P$ \&

FROM PUREX PROCESS IWN SOLUTION

$\begin{array}{llll}\text { LANG LH } & \text { SRD } & 10 & 11 / 20 / 63 \\ \begin{array}{l}\text { RA CONNELL } \\ \text { CARTER RD }\end{array} & \begin{array}{l}\text { CONF } \\ \text { MORGAN WC }\end{array} & & 11 / 21 / 63 \\ & \text { SRD } & 3 & 11 / 21 / 63 \\ \text { SRD } & 9 & 12 / 11 / 63 \\ \text { AE SMITH } & \text { SRD } & & 11 / 26 / 63 \\ \text { GUAPRECHT RO } & \text { SRD } & 10 & 11 / 27 / 63 \\ \text { SPENCER HG } & \text { SRD } & 13 & 11 / 27 / 63 \\ \text { MALKER CM } & \text { SRD } & 8 & 11 / 27 / 63 \\ & & & \\ \text { GILL SH } & & & 12 / 06 / 63 \\ \text { KNIGHT JT } & \text { SRD } & 3 & 12 / 05 / 63\end{array}$

CONMER EL, JR.

SRD

7

$12 / 03 / 63$

AE SNITH

SRD

$12 / 04 / 63$

MEIDNER $R$

CRD

6

$12 / 05 / 63$

WOODFIELD FW

JENSEN RD
HICK OJ

SRD

CRD

SRD

SECR

SECR

SECR

LH LANG

KNIGHTS LM

SRD

15

$11 / 12 / 63$

MALKER CM

CRD

OWEN FE

SRD

SRD

SRD

WE JOHNSOW

FRYMIER JH

SRD

DOWIS HJ, GARCIA IMA

NICHOLS PF

SRD

CRD

CONF

$12 / 04 / 63$

$12 / 05 / 63$

$12 / 09 / 63$

$12 / 10 / 63$

$12 / 10 / 63$

$12 / 10 / 63$

WW SCHULZ
$12 / 10 / 63$

$12 / 11 / 63$

$12 / 11 / 63$

$12 / 16 / 63$

$12 / 12 / 63$

$12 / 13 / 63$

12/02/63

$12 / 13 / 63$ 
DOC. NO.

HW-80014

HH-80015

HW-80016

HW-80023

HW-80039

HW- 80040

HW-80070

HW-80078

HU-80089

HW-80109

HW-80112

HW-80113

HW-80125 PT1

HH-80125 PI1 SUP A

HW-80125 PT2

$H W-80159$

$H W-80160$

HW-80163

HH-80164

HW-80168

HW-80172

HW-80177

HW-80202

HH-80205 C
TITLE

PROOUCTION TEST IP-644-D FUEL SURFACE CRUD MONITOR IRRADIATION

PHYSICS STUDY OF PO 210 PROOUCTION

CAPABILITIES IN N REACTOR

ASSURANCE OF SURFACE "V" FLATNESS

MC-1807 COMPONENTS

FABRICATION PROCESS TEST EVALLATION PLUG

POUR INGOTIHG OF TURNIMGS BRIQUETTES

COMPARISON AND ANALYSIS OF N REACTOR PRODUCTION STLDIES PLUTONIUN-ONLY CASES

COMPARISON \& AMALYSIS OF N REACTOR PROOUCTION STUDIES MULTI- \&

ALTERHATE-PROOUCT CASES

PLUTONIUN PRODUCT DEVELOPMENT OPERATION

MONTHLY REPORT - DECEMBER, 1963

PROOUCTION REACTOR PONER CONVERSION STUDIES

INCREASED LENGTH FUEL ELEAENTS

PRELIMINARY COMNENTS ON IAEA INSPECTION QUARTERLY PROGRESS REPORT METALLURGY DEVELOPMENT OPERATION OCTOBER, MOVEMBER, DECEMBER, 1963

ATOMIC WEAPON DATA SUPPLEMENT QUARTERLY PROGRESS REPORT

PITA IP-27-1, PART I INCREASED COOLANT FLOW - H REACTOR

SUPPLEMENT A PITA IP-27-l, PART I INCREASED COOLANT FLOH - H REACTOR

PITA IP-27C - PART II INCREASED COOLANT FLOW - H REACTOR OPERATIONAL PHYSICS REQUIREMENTS

A-LINE PROOUCT CHARACTERISTICS MOVEMBER 1963

STATUS OF CANHING CYCLE OPTIMIZATION STUDY

PROPOSAL TO FABRICATE A MUCLEAR DEVICE REAR FACE HARDUARE REPLACEMENT 105-B, D, DR, F, AND H

CAMPAIGN RELORK OF Z-PLANT MATERIAL AT PUREX

REPORT TO THE WORKING COMITTEE OF THE FUEL ELEMENT DEVELOPMENT COMITTEE FROM THE GENERAL ELECTRIC COMPANY - HANFORD DEVELOPMENT TEST IP-646-D, IRRADIATION SERVICE REOUEST HAPO 278, OUTGASSING

RATE OF TRITIUM AT HIGH TEMPERATURES AND HYDRIDING CORROSION OF ZIRCALOY-2 REDOX MEEKLY PROCESS REPORTS JANUARY THROUGH DECEMBER, 1964

SUPPLEMENT B TO PRODUCTION TEST

IP-648-AC EVALUATION OF THORIU OXIDE AS A FRINGE LOADING

\begin{tabular}{|c|c|c|c|}
\hline AUTHOR & LEVEL & PGS & DATE \\
\hline EIDNER R & CRD & 11 & $12 / 16 / 63$ \\
\hline IETRICH RA & SRD & 11 & $11 / 19 / 63$ \\
\hline INKERTON JM & SRD & 3 & $12 / 16 / 63$ \\
\hline ALKER CH & SRO & 10 & $12 / 17 / 63$ \\
\hline EAL ML. & SRD & 27 & $12 / 19 / 63$ \\
\hline EAL ML & SRD & 28 & $01 / 31 / 64$ \\
\hline ICK OJ & SRD & 8 & $12 / 31 / 63$ \\
\hline C SCHROEDER & CRD & & $12 / 20 / 63$ \\
\hline $\begin{array}{l}\text { WYDER DA } \\
\text { ENMETT CA, GRAMQUIST JP } \\
\text { AST GA, MINOR JE, NELSON } \\
\text { C, STEWART RW }\end{array}$ & $\begin{array}{l}\text { SRD } \\
\text { CRD } \\
\text { SRD }\end{array}$ & $\begin{array}{l}12 \\
5 \\
99\end{array}$ & $\begin{array}{l}12 / 27 / 63 \\
12 / 20 / 63 \\
01 / 20 / 64\end{array}$ \\
\hline $\begin{array}{l}\text { AST GA, MINOR JE, MELSON } \\
\text { C, STEWART RH }\end{array}$ & SRD & 59 & $03 / 02 / 64$ \\
\hline LIATON MA, SPICKA RE & SRD & 13 & $12 / 20 / 63$ \\
\hline PICKA RE & SRD & 5 & $02 / 05 / 64$ \\
\hline
\end{tabular}

AE SNITH

SRD

$12 / 23 / 63$

HUTTON TW

CRD

39

$12 / 23 / 63$

HICK OJ

SRD

$12 / 26 / 63$

BARKER CR, YOUNG JR SRD 12

$12 / 31 / 63$

JUDSON BF, KENDALL JB SRD 7

$12 / 26 / 63$

LEUIS M, MINOR JE, SRD STRIMGER JT

DEMERS AE

SRD

6

$12 / 30 / 64$

ISAMCSON RE

SRD

217

$01 / 02 / 64$

HLADEK KL

SRD

$04 / 21 / 64$ 
Page No. 200

04/28/93

LIST OF HANFORD-GENERATED CLASSIFIED

DOCUMENTS DATED BETUEEN 1/1/61 AND 12/31/72

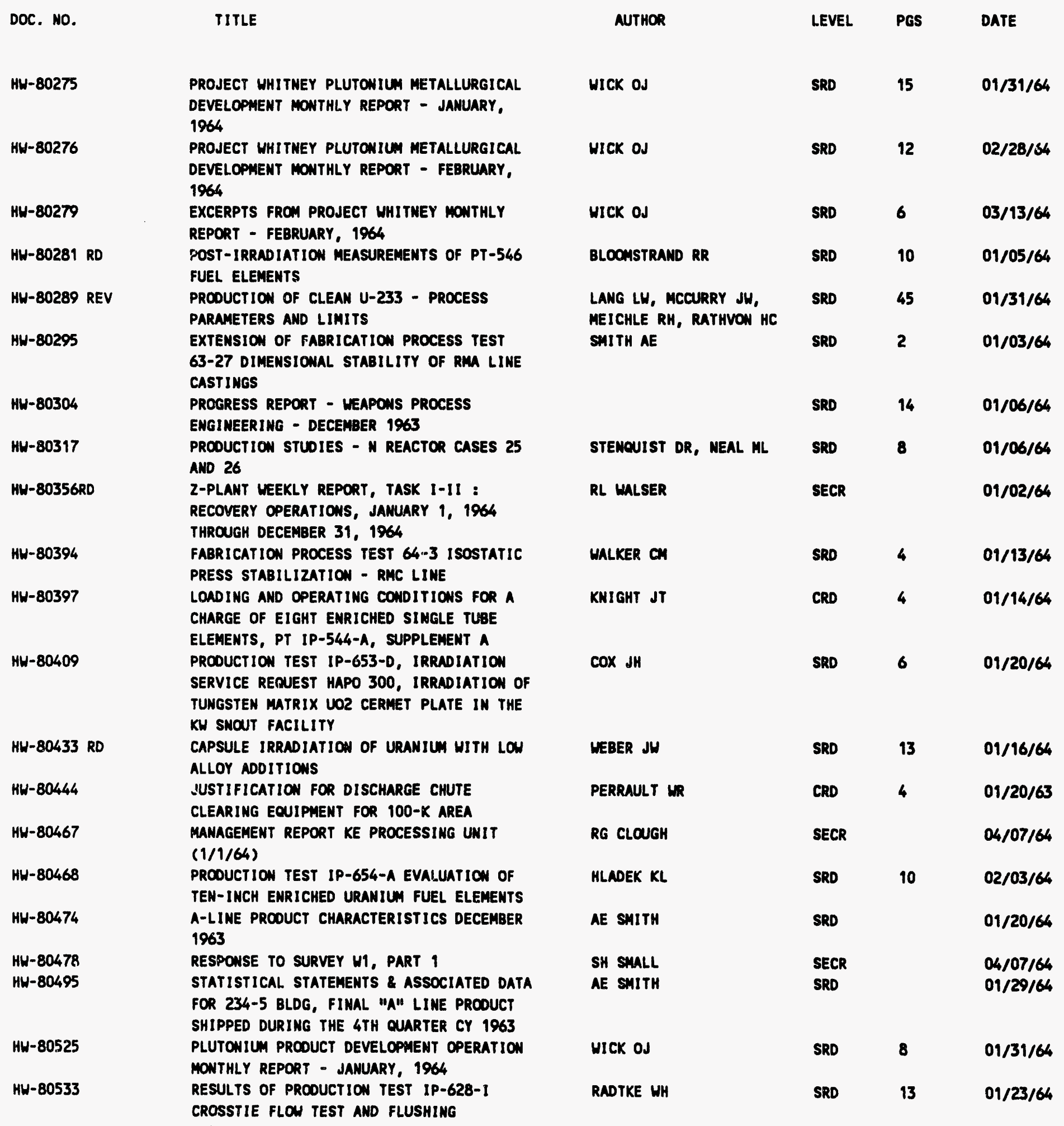

CALIBRATION 
Page No. 201

$04 / 28 / 93$

LIST OF HAMFORD-GENERATED CLASSIFIED

DOCUMENTS DATED BETUEEN 1/1/61 AND 12/31/72

DOC. NO.

HW-80575

HW-80626

HU-80629

HW-80659

$H W-80668$ RD

HW-80676

HW-80677

HU-80685RD

HM-80704

HW- 80710

HW-80729

HW-80753

HW- 80766

$\mathrm{HH}-80774$

HW- 80800

HW-80802

$\mathrm{HW}-80804$

$H H-80817$

HW- 80826

HW-80827

HW-80828

HW-80828 SUP A

HW-80830

HW-80836

HW-80847

HW-80853

HM-80854
TITLE

TEMPERATURE CALCULATIONS FOR A MEHLY DESIGNED FLEXIBLE HCR FOR THE $K$ REACTORS EFFECTS OF CORROSION UPON THE ADEQUACY OF THE 105-KE AMD 105-KU EMERGENCY COOLANT BACKUP SYSTEM

URANIUM BURNOUT VALUES

CAPITAL COST ESTIMATES FOR ZIRCALOY CLAD PONER FUELS

JAMUARY 1964 DATA SUMMARY - MEAPONS PROCESS ENGIMEERING

REPORT OF GETHC MEETING DECEMBER 1963 REVIEH OF IPD BALL-3X SAFETY PHILOSOPHY FIMAL REPORT B,D,F REACTOR SIDE SHIELD HOLE BORING TECHNOLOGY

R \& E MONTHLY MANAGEMENT REPORTS - JAN

THRU DEC 1963

COMPARISON OF ACTUAL UNIT COST

PERFORMANCE HITH REACTOR STUDIES FY 1964 BASE CASE

MEAPONS PROCESS ENGINEERING TECHNOLOGY

REPORT - JAMUARY 1964

REPORT OF INVENTION INCREASING AMOUNTS

OF PU-241 ISOTOPE

INVENTION RPT: NUCLEAR REACTORS AS A

GAMWA RADIATION FACILITY

U-233 PRCOUCTION STATUS

MUMERICAL RESULTS OF PRODUCTION TEST

IP-651-AI, TRIPOUT TEST - H REACTOR

PROPOSED TEST FOR USING ALSI MADE FROM

8001 SCRAP

INTERIM MORMAL LIMITS DR REACTOR

AMALYSIS OF THE THIRD H-REACTOR CORE LOND

AXIAL FLUX DATA FOR FUEL MEASUREMENT

HANFORD PROOUCTION OF THULIUA-17O

BUTTON LINE PROCESS - IRON

DECONTAMINATION

PROOUCTION TEST IP-659-AC EVALUATION OF A THORIUN OXIDE LOADING

PT IP-659-AC, SUPPLEMENT A TRAMSITION TO MORMAL DISCHARGE PLAN USING STRIPED TARGET COLUAMS

ECOMOAICS OF HIGHER 100-K REACTOR

PROCESS HATER PH

RESULTS OF AIR ENTRY CONTROL TEST \#I DR REACTOR

PROGRESS REPORT ON MASA CERMET STUDIES

IDENTIFICATION SNC-12, JAMUARY 1964

PWIEG PAPERS

SOME DESIGN DETAILS FOR THE TARGET COAPONENT OF THE CO-PRCOUCER TEST ELEMENT

\begin{tabular}{|c|c|c|c|}
\hline AUTHOR & LEVEL & PGS & DATE \\
\hline AGAR JD & SRD & 22 & $01 / 27 / 64$ \\
\hline WATSON DF & SRD & 12 & $01 / 30 / 64$ \\
\hline SHITH We & SRD & 50 & $01 / 30 / 64$ \\
\hline DALIMG RE & CRD & 10 & $01 / 31 / 64$ \\
\hline SNITH AE & SRD & 11 & $02 / 03 / 64$ \\
\hline MILSON R, MMBROSE TW & SRD & 22 & $02 / 03 / 64$ \\
\hline CLEMANS NH & CRD & 25 & $02 / 03 / 64$ \\
\hline OH GREAGER & SECR & & $02 / 04 / 64$ \\
\hline \multirow[t]{2}{*}{ GRENIMGER AB } & SRD & 4 & $02 / 04 / 64$ \\
\hline & SRD & 8 & $02 / 04 / 64$ \\
\hline LANG LH & SRD & 4 & $01 / 28 / 64$ \\
\hline LW LANG & SECR & & $01 / 28 / 64$ \\
\hline ScOTt AJ & SRD & 7 & $02 / 06 / 64$ \\
\hline MORRISSEY JE & SRD & 25 & $03 / 12 / 64$ \\
\hline MEAKLEY, EA & CRD & 11 & $02 / 12 / 64$ \\
\hline GEORGE DK & CRD & 6 & $02 / 10 / 64$ \\
\hline RINGLE RP & SRD & 4 & $02 / 10 / 64$ \\
\hline POPOVICH RP & SRD & 8 & $02 / 11 / 64$ \\
\hline LANG LH, MEICHLE RH & SRD & 5 & $03 / 27 / 64$ \\
\hline WALSER RL & SRD & 24 & $02 / 17 / 64$ \\
\hline CARTER RD, HLADEK KL & SRO & 15 & $02 / 28 / 64$ \\
\hline MASCHE GC & SRD & 9 & $04 / 08 / 64$ \\
\hline YOUNG JR & SRD & 9 & $02 / 14 / 64$ \\
\hline GEORGE DK & CRD & 8 & $02 / 10 / 64$ \\
\hline CADWELL JJ & CRD & 8 & $02 / 13 / 64$ \\
\hline SMITH AE & SRD & 4 & $02 / 13 / 64$ \\
\hline EVANS TH & SRO & 3 & $02 / 12 / 64$ \\
\hline
\end{tabular}


Page No. 202

$04 / 28 / 93$

LIST OF HANFORD-GENERATED CLASSIFIED

DOCUAENTS DATED BETUEEN 1/1/61 AND 12/31/72

DOC. NO.

HW-80862

HW-80864

HW-80888

HW-80894

HU- -80899

HW-80901

HW-80908

HW-80927 8

HH-809279

HW-80935

HW-80937

HW-80942

HW-80955

$H W-80996$

HW-81007

HW-81015

HH-81026

HH-81027

HW-81028

HW-81041
TITLE

INTERMAL GRAPHITE MOOERATOR FORCES STUDY SUPPLEMENTAL INFORMATION

FINAL REPORT PT IP-603-1, EVALUATION OF ACCIDENTAL. FUEL FLUSHING POTENTIAL D-DR REACTORS

EFFECTS OF HANFORD OPERATIONS ON COLUMBIA RIVER TEMPERATURES - INTERIM REPORT NO. 2

NEW K REACTOR ALUMINUM HCR - TEMPERATURE STUDY

A-LIME CHEMICAL AMALYTICAL DATA ON PARTS NOT SHIPPED DURING THE FOURTH QUARTER OF CY 1963

BUDGET FOR FY 1966 AND REVISION OF

BUDGET FOR FY 1965 O2 PROGRAM --

RESEARCH AND DEVELOPMENT

N-REACTOR DEPARTMENT RESEARCH AND

DEVELOPMENT BUDGET FOR FY 1966 AND

REVISION OF BUDGET FOR FY 1965

MONTHLY REPORT OF RADIOISOTOPES RESEARCH AND DEVELOPMENT - HANFORD LABORATORIES AUGUST 1964

MONTHLY REPORT OF RADIOISOTOPES RESEARCH AND DEVELOPMENT - MANFORD LABORATORIES SEPTEMBER 1964

PLUTONIUM PRODUCT DEVELOPMENT OPERATION

MONTHLY REPORT - FEBRUARY, 1964

FISSION PROOUCT FORMATION IN FUEL ELEMENTS

RESULTS OF THERMAL-HYDRAUL.JC EXPERIMENTS WITH KVNS SELF-SUPPORTED FUEL IN A ZIRCALOY TUBE -- K REACTOR

DEVELOPMENT OF PROCESS PARAMETERS FOR ISOSTATIC PRESS STABILIZATION DEVELOPMENTAL REQUIREMENTS FOR CANHING A LOW DENSITY THORIA DEMONSTRATION LOND A-LINE PROOUCT CHARACTERISTICS JAMUARY 1964

PRODUCTION TEST IP-665-A, EVALUATION OF HOT-DIE-SIZED END BONDING PROCESSES LETTER TO DH CURTISS GETR GRAPHITE IRRADIATION CAPSULES: $H-4,5,6$ EQUIVALENT N-REACTOR EXPOSURE

TECHNICAL NOTE ON RESULTS OF PROOUCTION TEST IP-628-AL, CROSSTIE FLON TEST AND FLUSHING CALIBRATIOW, AND THE EARLIER PRODUCTION TESTS IP-5T3-AI AND IP-499-AI TECHNICAL NOTE ON THE DETERMIMATION OF K-REACTOR LAST-DITCH COOLING ADEQUACY

K REACTOR MIXER RECOMWENDATION ALUMINUM SPLINE TUBES

\begin{tabular}{|c|c|c|c|}
\hline AUTHOR & LEVEL & PGS & DATE \\
\hline COOLEY DE & CRD & 9 & $02 / 14 / 64$ \\
\hline GEORGE DK, HOLLIFIELD PJ & SRD & 7 & $03 / 18 / 64$ \\
\hline CrRLEY JP & SRO & 93 & $12 / 03 / 64$ \\
\hline AGAR JD & SRD & 5 & $02 / 17 / 64$ \\
\hline \multirow[t]{3}{*}{ AE SMITH } & SRD & & $02 / 18 / 64$ \\
\hline & SRD & 219 & $04 / 14 / 64$ \\
\hline & SRD & 69 & $03 / 25 / 64$ \\
\hline HALLIING MT & CRD & 4 & $10 / 15 / 64$ \\
\hline MALLIING MT & CRD & 4 & $10 / 30 / 64$ \\
\hline HICK OJ & SRD & 8 & $02 / 28 / 64$ \\
\hline SHIELDS RJ & SRD & 3 & $02 / 19 / 64$ \\
\hline WATERS ED, SHOCKLEY ME & SRD & 18 & $02 / 03 / 64$ \\
\hline WALKER CH & SRD & 4 & $02 / 20 / 64$ \\
\hline $\begin{array}{l}\text { HUFF, GA, KMIGHT FW, } \\
\text { PADGETT EV, POWERS HG }\end{array}$ & SRD & 10 & $01 / 25 / 64$ \\
\hline AE SMITH & SRD & & $02 / 25 / 64$ \\
\hline HLADEK KL & SRD & 10 & $03 / 30 / 64$ \\
\hline HELM JW & SRD & & $03 / 05 / 64$ \\
\hline JONES SS & SRD & 37 & $06 / 15 / 64$ \\
\hline JONES SS & SRD & 26 & $02 / 28 / 64$ \\
\hline CG HOUGH & SRD & 18 & $02 / 27 / 64$ \\
\hline
\end{tabular}


Page No. 203

$04 / 28 / 93$

LIST OF HANFORD-GENERATED CLASSIFIED

DOCUMENTS DATED BETUEEN 1/1/61 AND 12/31/72

Doc. No.

WW-81050

HW-81074

HW-81104

HW-81136

HW-81139

HW-81140

HW-81141

HU-81142

$H W-81170$ RD

HW-81194

HW-81198

HW-81200

HW-81202

HW- 81203

HW-81213

$H H-81213$ RD

$H W-81230$

$H W-81240$

HH- 81242

HW- 81243

HW- 81272

HW-81283RD

HH-81329

$H W-81354 R D$

HW- 81378

$H H-81378$ RD

HH-81379
TITLE

FISSION PRODUCT SECURITY PROBLEM ECONOMIC AMALYSIS OF CHARGE-DISCHARGING THORIUM AT SAMLLER REACTORS

PROJECT NO. RL-325N4-D1-1 REQUEST NO. HL-1029

WEAPONS PROCESS ENGINEERIMG TECHNOLOGY REPORT FEBRUARY 1964

EXCERPTS FRON PROJECT WHITHEY MONTHLY

REPORT - MARCH, 1964

EXCERPTS FROM PROJECT MHITMEY MONTHLY

REPORT - APRIL, 1964

PROJECT UHITNEY PLUTONILM METALLURGICAL

DEVELOPMENT MONTHLY REPORT - APRIL, 1964

PROJECT MHITMEY PLUTOWIUM METALLURGICAL

DEVELOPMENT MONTHLY REPORT - MAY, 1964

PRELIMIMARY ENGINEERING STUOY

INTERMEDIATE RANGE INSTRUMENTATION

SS MATERIAL REQUEST

DESIGN AND FABRICATION OF TARGET

ELEMENTS FOR PT-645-D

BUDGET STUOY MULTIPRCDUCT EQUIPMENT FOR

100 N \& CPD PLANTS

FABRICATION PROCESS TEST EVALUATION -

PPT-63-27 DIMENSIONAL STABILITY OF

UMALLOYED PLUTONIUN

OPERATING HISTORY

INTERIM REPORT POWER RECOVERY STUDY

EXISTING HANFORD REACTORS

INTERIM REPORT PONER RECOVERY STUOY

EXISTING HANFORD REACTORS

OBSERVATIONS ON THE USE OF ARC MELTED

FEED

CORROSION OF PLUTONIUM METAL UHEN STORED IHCONTAINERS HAVING PLASTIC COMPONENTS

BUTTON SPECIFICATIONS

INCREASED C REACTOR FLOW

PLUTONIUM PRCOUCT DEVELOPMENT OPERATION

MONTHLY REPORT - MARCH, 1964

BACK-UP DATA FOR 17 CASE STLOY

THE PRODUCTION OF PU-238 AND CH-242 FROM REACTOR-GRADE PU

105-KE MONTHLY REPORTS

REPORT OF VISIT OF DR. L.C. MATSCH MARCH 11 AND 12, INCLUDING DISCUSSION AT MEETING AT THE C REACTOR CONFERENCE ROON REPORT OF VISIT OF DR. LC MATSCH MARCH 11 AMD 12, INCLUDING DISCUSSION AT MEETING AT THE C REACTOR CONFERENCE ROOM PRODUCTION TEST IP-669-AC DEPLETED URANIUN IRRADIATION SWALLER REACTORS

\author{
AUTHOR \\ WARREN JH \\ HEDGES JH
}

BYLAND JH

HICK os

WICK Od

MICK OJ

WICK OJ

HERRMAN BU

BENHETT CA

TVERBERG JC

JF NESBITT

WALKER CH

LJ MEUELL

KIMG RR, JR.

BOND RH

VANDERCOOK RE HOOPER RH

UICK OJ

VR CHAPNAN

LANG LH

RE CLOUGH

COOKE JP

COOKE JP

GROSS PD, HLADEK KL
LEVEL PGS

DATE

$02 / 27 / 64$

03/02/64

$03 / 03 / 64$

03/04/64

$04 / 16 / 64$

$06 / 01 / 64$

$04 / 30 / 64$

$05 / 28 / 64$

$03 / 04 / 64$

$03 / 04 / 64$ $03 / 09 / 64$

03/09/64

03/05/64 $\begin{array}{lll}\text { CRD } & & 03 / 05 / 64 \\ \text { SRD } & 125 & 05 / 15 / 64\end{array}$

SRD $212 \quad 03 / 13 / 64$

SRD $\quad 12 \quad 03 / 09 / 64$

SRD 32

$03 / 06 / 64$

SRD 5

SRD 14

03/09/64

$02 / 26 / 64$

$03 / 31 / 64$

$03 / 10 / 64$ $03 / 20 / 64$

$04 / 07 / 64$ 03/13/64

SRD

7

$03 / 13 / 64$

SRD

12

$03 / 13 / 64$

$06 / 01 / 64$ 
Page No. 204

$04 / 28 / 93$

LIST OF HANFORD-GENERATED CLASSIFIED

DOCUMENTS DATED BETHEEI $1 / 1 / 61$ AND 12/31/72

DOC. No.

HW-81379

HU-81379 G

HW-81379 H

HW-81411

HW-81420 RD

HW-81445

HH- 81446

HW-81455

HH-81456

HW-81456 REV

$H H-81458$ RD

HW- 81470

HW-81485

HW-81489

HW-81502

HW-81515

$H W-81530$

HH -81537

HW- 81538

HW-81547 RD

HH-81548 RD
TITLE

PRCOUCTION T ST IP-669 DEPLETED URANIUM IRRADIATION NOTEBOOK

SUPPLEMENT B TO PRODUCTION TEST IP-669-AC DEPLETED URANIUM IRRADIATION SWALLER REACTORS

SUPPLEMENT C TO PROOUCTION TEST IP-669-AC DEPLETED URANIUN IRRADIATION SMALLER REACTORS

PRODUCT CHARACTERISTICS

2 PLANT FABRICATION PROOUCTION DATA PROCESSING SYSTEM

BUDGET FOR FY 1966 AND REVISED BUDGET

FOR FY 1965 SEPARATIONS RESEARCH AMD

DEVELOPMENT PROGRAN (02 5010151 1)

BUDGET FOR FY 1966 AND REVISED BUDGET

FOR FY 1965 PLUTONIUN PROCESSING

RESEARCH AMD DEVELOPMENT PROGRAM CO2 50

1020 1)

BLDGET FOR FY 1966 AND REVISION OF

BUDGET FOR FY 1965 - PROPOSAL FOR

RESEARCH AND DEVELOPMENT - GENERAL

MEAPONS DEVELOPMENT

21A-6 BUDGET FOR FY 1966 AND REVISION OF BLDGET FOR FY 1965 - PROPOSAL FOR

PROCESS DEVELOPMENT

BUDGET FOR FY 1966 AND REVISION OF BUDGET FOR FY 1965 PROPOSAL FOR 03

PROCESS DEVELOPMENT (21A-6)

OVERBORE FUEL FAILURE SUMAMAY

MATERIAL EVALLATION CRITERIA

ATOMIC HEAPCN DATA SUPPLEMENT QUARTERLY

PROGRESS REPORT METALLURGY DEVELOPMENT

OPERATION JANUARY, FEBRUARY, MARCH 1964

FABRICATION PROCESS TEST EVALUATION TEST NO. 63-30

A-LINE PROOUCT CHARACTERISTICS FEBRUARY 1964

KER-2 OPERATING REPORT TEST K-2-17 PT IP $-484-D$

ALOO - FACTSHEETS FY-1966 ECUIPMENT

BLDGEY SUBMISSION - MEAPONS

MUCLEAR MATERIALS REVIEW, DEPLETED URANIUN SYSTEM, JULY THROUGH DECEMBER 1963

NUCLEAR MATERIALS REVIEW ENRICHED URANIUM SYSTEM JULY THROUGH DECEMBER 1963

N-REACTOR FUEL DEVELOPMENT PP 51 THROUGH 57 - $\mathrm{HH}-80908$

CO-PROOUCT DEVELOPNENT FOR N-REACTOR PP 58 THROUGH 64 - HH-80908

\begin{tabular}{|c|c|c|c|}
\hline NUTHOR & LEVEL & PGS & DATE \\
\hline KL HLADEL & SECR & & $04 / 08 / 64$ \\
\hline GROSS PD, HLADEK KL & SRD & 6 & $06 / 14 / 65$ \\
\hline GROSS PD & SRD. & 4 & $10 / 22 / 65$ \\
\hline $\begin{array}{l}\text { SMITH AE } \\
\text { JACOBS MC }\end{array}$ & $\begin{array}{l}\text { SRD } \\
\text { SRD }\end{array}$ & $\begin{array}{l}6 \\
18\end{array}$ & $\begin{array}{l}03 / 19 / 64 \\
04 / 01 / 63\end{array}$ \\
\hline FRANK US & SRD & 59 & $04 / 01 / 64$ \\
\hline FRANK US & CRD & 27 & $04 / 01 / 64$ \\
\hline FRANK US, SHITH AE & SRD & 13 & $03 / 31 / 64$ \\
\hline FRANK US, SMITH AE & SRD & 30 & $03 / 31 / 64$ \\
\hline WATKINS RM & SRD & 30 & $04 / 23 / 64$ \\
\hline HLADEK KL & SRD & 3 & $03 / 23 / 64$ \\
\hline REARDON WA, HARRIS RA & SRD & 8 & $03 / 23 / 64$ \\
\hline $\begin{array}{l}\text { WICK OJ, LAST GA, MIHOR } \\
\text { JE, NELSON TC, STEWART RW }\end{array}$ & SRD & 112 & $05 / 20 / 64$ \\
\hline ARMSTRONG DA & SRD & 10 & $03 / 24 / 64$ \\
\hline SHITH AE & SRD & 11 & $03 / 25 / 64$ \\
\hline CHRISTENSEN GP & SRD & 12 & $03 / 26 / 64$ \\
\hline GRIMn KG & SRD & 7 & $03 / 27 / 64$ \\
\hline LF WIRFS & SECR & & $03 / 25 / 64$ \\
\hline \multirow[t]{3}{*}{ CJ SHORTESS } & SECR & & $03 / 25 / 64$ \\
\hline & SRD & 7 & $03 / 30 / 64$ \\
\hline & SRD & 7 & $03 / 30 / 64$ \\
\hline
\end{tabular}


Page No. 205

04/28/93

LIST OF HANFORD-GENERATED CLASSIFIED

DOCLMENTS DATED BETUEEN 1/1/61 AND 12/31/72

DOC. NO.

TITLE

MUTHOR

LEVEL

PGS

DATE

HW-81565

HW-81569

HW-81579 RD

HH-81612 1

HU-81612 2

HW-81695

HW-81657

HU-81658

$H W-81660$

HW-81662

HW-81663

HW-81663 REV

HH-81679

HW-81708

$H W-81712$ RD

HU-81721

HW-81730

HW-81735

HW-81736

HW- 81740

HW-81741 RD
PROCESSING OPERATING PROCEDURES

C REACTOR OVERBORE TEST FACILITY REVIEH HANFORD CONTRIBUTION FOR THE EIGHTEENTH HIGH TEMPERATURE FUELS COMNITTEE MEETING, MAY 19-21, 1964

PROJECT PROPOSAL: COOLANT BACKUP MODIFICATION BY REACTOR DEACTIVATION THREE SHALL REACTORS (CGI-142)

PROJECT PROPOSAL: COOLANT BACKUP MODIFICATION BY REACTOR DEACTIVATION THREE SMALL REACTORS (CGI-142) REPORT TO THE WORKING COMMITTEE OF THE FUEL ELEMENT DEVELOPMENT COMMITTEE FROM THE GENERAL ELECTRIC COMPAMY - HANFORD OPTIMUN COOLANT PH

SUPPLEMENTARY DATA TO 03 PROGRAM PROOUCTION BUDGET SUBMISSION BUDGET FOR FY 1966 AND REVISION OF BUDGET FOR FY 1965

AN EVALUATION OF THE EXPERIMENTAL DETERMIMATION OF THE EFFECTS OF BURNOUT AND CORROSION ON SPLINE WORTH

AMALYSIS OF ALTERNATIVES: PLUTONIUM BUTTON-LINE IMPROVEMENTS

21A-5 SUMARY AMALYSI OF PROPOSED PROCESS DEVELOPMENT COSTS FOR FY 1966 AND REVISION OF FY 1965

BUDGET FOR FY 1966 AND REVISION OF BUDGET FOR FY 196503 PROCESS DEVELOPNENT COSTS FOR FY 1966 ND REVISION OF FY 1965 (21A-5)

MEAPONS PROCESS ENGINEERING TECHNOLOGY REPORT - MARCH 1964

FABRICATION PROCESS TEST CASTING MODEL

74-C PREFORMS IN DOUBLE CAVITY MOLDS

POWER ROLL FORM DEFORHATION COMPARED TO CONVENTIONAL ROLLING

FABRICATIOW PROCESS TEST 64-7 TEST OF CO-REDUCED ALLOY IN THE FABRICATION OF MODEL 74-C COMPONENTS

CRITICAL MASS CONTROL SPECIFICATION FOR

FABRICATION PROCESS TEST 64-10 CASTING MODEL 74-C PREFORMS IN DOUBLE CAVITY MOLDS, HA-20-MB, HA-28-C, HC-2, HC-3 AND HC-19-A1

C- 1 LOOP OPERATING LOG

C-1 LOOP ENGINEERING LOGBOOK

NFS PROCESSING OC N REACTOR FUEL

HANFORD ISOTOPES PROOUCTION PLANT
GE-RL

CARLSON PA, NOLSON $R$

LAST GA

TH LYONS

TH LYONS

LEWIS M, MIMOR JE, STRINGER JT

GEIER RG

DOBBS BM

BLYCKERT MA

JOHASON BM, SWITH VW, SWAIN EO, SZULIMSKI Md, ZAHN LL

FRANK US, SHITH AE SRD

WATKINS RM

SMITH AE

HAYS DD

FIELD ST

BARBER AE

$04 / 08 / 64$

EC BENNETT

EC BENMETT

STEHOUIST DR

SMITH CW
SRD

SRD

SRD

4

$04 / 23 / 64$

SRD

$$
7
$$

SRD

SRD

SRD

SRD

5

$04 / 01 / 64$

$04 / 24 / 64$

05/05/64

$05 / 04 / 64$

05/04/64

$04 / 07 / 64$

$04 / 02 / 64$

$04 / 06 / 64$

$07 / 13 / 64$

05/01/64

$03 / 31 / 64$

$04 / 03 / 64$

$04 / 07 / 64$

$04 / 08 / 64$

$04 / 06 / 64$

SECR

SECR

CRD

SRD
$04 / 09 / 64$ $04 / 09 / 64$ $04 / 06 / 64$ $04 / 06 / 64$ 
Page No. 206

$04 / 28 / 93$

LIST OF HANFORD-GENERATED CLASSIFIED

DOCLMENTS DATED BETMEEN 1/1/61 AND 12/31/72

DOC. NO.

HW-81742 RD

HW- -81743

Hแ- 81747

HW-81747 RD REV1

HW-81779 PT2 1

HW-81801

HW-81832

HW-81838

HW- 81840

HW-81861

HU- 81870

HW-81875

HH-81876

HW-81939

HW- 81947

HW-81958

$H W-81962$

HW-81988

HH-82000

HH-82004

HW-82006

HH- 82046

HW-82047

$H W-82047$ ADD1

HH-82049
TITLE

PUREX 4.0 STUDY

TRIP REPORT - MEETING OF THE NONDESTRUCTIVE TESTING REPRESENTATIVES OF THE METALS FABRICATING AGENCIES LASL.

DESIGN CRITERIA FOR COOLANT BACKUP THREE REMAINING SMALLER REACTORS

DESIGN CRITERIA FOR COOLANT BACKUP THREE REMAINING SWALLER REACTORS

DESIGN CRITERIA FISSION MASTE CONTAINER STORAGE FACILITIES

PROPOSAL TO FABRICATE A nUCLEAR DEVICE PREL'MINARY STUOY CONVERSION OF 105-H REACTOR FOR PROOUCTION OF RADIOISOTOPES KW - AMALYIST LOG (NOTEBOOK)

PRODUCTION TEST IP-677-A HOT-DIE-SIZED TUBE CLADDING

THULIUN OXIOE IRRADIATION

U-233 STLOY - DIVISION OF REACTOR DEVELOPMENT REQUEST

N-REACTOR CO-PRODUCT TARGET ELEMENT

RUPTURE DETECTION STUDY

DT-IP-434-D: IRRADIATION EFFECT ON CERAMIC MATERIALS

GOAL EXPOSURE RECOMWENDATIONS

GRAPHITE TEMPERATURE AN IMPORTANT

ECONOMIC PARAMETER

FABRICATION PROCESS TEST FPT-64-14 INVERTED CAVITY MOLDS FOR RMC LINE SHAPE CASTING

REVIEH - PROCESS DEVELOPAENT 1.25\%

ENRICHED CO-PROOUCT DRIVER FUEL

A-LINE PROOUCT CHARACTERISTICS MARCH

1964

CONTINUOUS ELECTRONINNING OF PLUTONIUN

METAL PROGRESS REPORT

PLUTONIUM PRODUCT DEVELOPMENT OPERATION

MONTHLY REPORT - APRIL, 1964

74-C PITTING PROBLEMS

ALSI SLOPOVERS STUDIES, METHOD OF

ELIMINATION, \& RESULTS

HAPO PLANT ACQUISITION AND CONSTRUCTION

BUDGET FOR FY 1966 AND REVISION OF

BUDGET FOR FY 1965

HAPO PLANT \& CAPITAL EQUIPMENT BUDGET

FOR FY 1966 \& REVISION OF BLOGET FOR FY 1965 EQUIPMENT NOT RELATED TO CONSTRUCTION PROJECTS

K REACTOR ENRICHMENT RING RELOCATION
AUTHOR

SHITH CH

HERSHEY RB, OBERG GC, MILBUR GE

BRINKMAN LB

BRINKWHN LB

BARNES JH

MICK OJ

FIFER MF

RV MYERS

HLADEK KL, BAARS RE

KUSLER LE

LANG LH, MCCURRY JW

ALLRED DO

COOLEY DE

FULLER NE

BMARS RE, SHIMER RD, SPENCER HG

HALKER CM

SHAW CH, CURRAN RG, HENDRICKSEN MA, HUDSON WG, KEENAK JP

SMITH AE

MH CURTIS

HICK OJ

FRAMK US

SCHAFFER JD

CONMELL RA

MCDONALD JE

BOWERS CE, SPENCER HO
LEVEL PGS

DATE

SRD

120

$04 / 06 / 64$ $04 / 09 / 64$

$08 / 12 / 64$

$06 / 09 / 64$

05/01/64

$04 / 14 / 64$

$05 / 15 / 64$

$04 / 16 / 64$

$05 / 06 / 64$

$04 / 17 / 64$

$04 / 22 / 64$

$04 / 27 / 64$

$04 / 21 / 64$

$04 / 22 / 64$ $04 / 22 / 64$

$04 / 22 / 64$

05/01/64

$04 / 24 / 64$

$04 / 30 / 64$

$04 / 30 / 64$

$04 / 27 / 64$ $12 / 07 / 63$

CRD 10

SRD $109 \quad 04 / 28 / 64$

SRD

19

$05 / 12 / 64$ 
Page No. 207

$04 / 28 / 93$

LIST OF HANFORD-GENERATED CLASSIFIED

DOCUMENTS DATED BETUEEN 1/1/61 AND 12/31/72

DOC. NO.

HW- 82060

HW-82067

HW- 82080

HW-82083

HW-82103 RD

HW-82132RD

HH-82143 RD

HW-82143 RD REV1

HU-82149

HU-82162

HW-82166

HU-82171

$H W-82172$

HU-82179

HW-82205 RD

HW-82212

$H H-82260$

HH-82273

HH-82278

HW- 82290

HW-82321

HW-82335

HW-82336

HW-82338

HW- 82349
TITLE

100-KEW COOLANT BACKUP ADEQUACY

SPEED-OF-CONTROL LIMITS FOR H REACTOR

TEMPORARY SPEED-OF-COHTROL LIMITS FOR C REACTOR

NOTEBOOK - 105-B SPECIALISTS LOG

LABORATORY STUDIES ON THE USE OF

URANIUM(IV) AS A PLUTONIUN REDUCTANT IN

A PUREX PROCESS

MONTHLY MANAGEMENT REPORTS -KE REACTOR-

1/1/64 THRU 12/31/64

INVESTIGATION REPORT - H REACTOR

MISCHARGING INCIDENT

INVESTIGATION REPORT H REACTOR

MISCHARGING INCIDENT

WEAPONS PROCESS ENGINEERING TECHMOLOGY

REPORT - APRIL 1964

HAZARDS REVIEU - N-REACTOR $1.25 \%$

CO-PRODUCER FUEL ELEMENT TEST

HIGH EXPOSURE PLUTOHIUM

C-1 LOOP TEST FACILITY LOGBOOK (PERIDO:

8/26/63 TO $4 / 20 / 64$ )

C-1 LOOP LOGBOOK (PERICD: 11/1/63 TO $4 / 17 /(4)$

BASE CASE CONDITIONS FOR ASSISTANCE TO HANFORD PROGRAM, K-EFFLUENT SYSTEM

ANALYSIS - ATH-1P-5-64

COMBINED OPERATIONS DATA

N-REACTOR PRIMARY SYSTEM WITH FCUR PUMPS

AT 10\% PONER LEVEL - ANALOG COMPUTER

STUDY OF CONTROLLER SETTINGS

CHANGES IN N-REACTOR - UNIT COST MITH

EXPOSURE INCREASES FOR PLUTONIUM-ONLY

PRCOUCTION

PLUTONIUN ECONONICS FOR THE PROOUCTION REACTORS

K REACTOR LAST-DITCH BACKUP ADEQUACY

100-K REACTOR ZIRCONIUN SPLIME TUBES

LOADING AND OPERATING CONDITIONS FOR A

CHARGE OF FIVE NIE-1 FUEL TUBES AND TWO

TARGET ASSEMBLIES IN KER-1 OR KER-2,

PROOUCTION TEST IP-584-D, SUPPLEMENT B

MONTHLY TECHNICAL REPORT - FEBRUARY 1964

KER-4 OPERATING REPORT TEST $K-4-15$, PT

IP-4T7-A, SUPPLEMENT. B

A-LJNE PROOUCT CHARACTERISTICS APRIL

1964

RECOMMENDED HIGH-TANK TEMPERATURES FOR

MAINTENANCE OF HIGH-TANK BACKUP SUPPORT, REVISION 3
AUTHOR

HEACOCK HU
HANRIC JP
HAMRIC JP
RC HAYMES
MENDEL JE

SA 1000

VINTHER AP

VINTHER AP

MILLER NR, NECHODON US

nOOOS UK

EC BENNETT

EC BENNETT

GRAVES SY

LANG LH

SRD

KD COUGHREN, PJ DIONNE, CRD GG THIEME

MaxSON RK

SRD

9

CON

T

SHIMER RD

GREAGER OH

YOUNG JR

DEOBALD TL

NICHOLS PF

OBERG KC

SRD

29

SRD

SRD

SRD

6

8

6

AE SMITH

GREAGER OH
CRD

SRD

SRD

SRD
$05 / 15 / 64$

05/25/64

$04 / 29 / 64$

$04 / 30 / 64$

$04 / 30 / 64$

$05 / 01 / 64$

07/30/65

05/05/64

05/01/64

05/01/64

$05 / 04 / 64$

$07 / 13 / 64$

05/06/64

05/06/64

$05 / 06 / 64$

05/07/64

05/01/64

05/07/64

05/28/64

$06 / 25 / 64$

$05 / 08 / 64$

$02 / 28 / 64$ $02 / 08 / 64$

$05 / 19 / 64$

05/20/64 
Page No. 208

04/28/93

LIST OF HANFORD-GEMERATED CLASSIFIED

DOCUMENTS DATED BETWEEN 1/1/61 AND 12/31/72

DOC. NO.

$H W-82373$

HH- 82383 RD

HW-82386

HU-82406 PT1

HW-82424

HH-82425

HW- 82458

HW-82458 RD

HW-82462

HW-82466

HW-82472

HW- 82481

HW-82486

$H W-82512$

HW- 82513

HW-82521

HW-82530

HW-82534

HW-82549 SUP1

HW-82559

HH- 82570

HW-82597

HW-82623

HM-82647

HU-82657

HW-82665
TITLE

B, D, F, DR, H REACTOR NEW ALUMINUN HCR CONCEPT - TEMPERATURE STLOY

DRD U-233 STUDY

PLUTONILM PRODUCT DEVELOPMENT OPERATION MONTHLY REPORT - MAY, 1964

TECHNICAL NOTE ON LAST-DITCH COOLING OF THE SMALL HANFORD REACTORS - PART I HIGH TAMKS

ADDITIONAL PONER RECOVERY STUDIES

EXISTING HANFORD REACTORS

FINAL REPORT - POWER RECOVERY STUDY

PROGRAM, EXISTING SMALLER HANFORD

REACTORS

HANFORD WEAPON FABRICATION CAPABILITIES HAMFORD MEAPON FABRICATION FACILITIES INGOT DENSITY SPECIFICATION

CRITICAL MASS HAZARDS CONTROL

SPECIFICATIONS REDOX C-2 POT-TYPE

DISSOLVER

PROOUCTION TEST IP-684-A, HOT-DIE-SIZED

PARAMETERS EVALUATION

IRRADIATION TESTING LOG \#1

IMPACT PROPERTIES OF PLUTONIUM 1\%

GALLIUM ALLOY

PROJECT HHITNEY PLUTONIUM METALLURGICAL DEVELOPMENT MONTHLY REPORT - JUNE, 1964

PROJECT UHITNEY PLUTONIUN METALLURGICAL DEVELOPMENT MONTHLY REPORT - JULY, 1964 POTENTIAL GAINS IN E-RING EFFICIENCY WEAPONS PROCESS ENGINEERING TECHNOLOGY REPORT - MAY 1964

ELLIPTICAL BUMPER FLON INCREASES

N-DATA 400 AREA GRID AMALYSES

105-H REACTOR CHARGE MAKE-UP AND HANDLING

PLUTONIUA PRCOUCT DEVELOPMENT OPERATION

MONTHLY REPORT - JUNE, 1964

NUCLEAR MATERIALS REVIEW,DEPLETED URAMIUM SYSTEM, JULY 1963 THROUGH APRIL 1964

FABRICATION PROCESS TEST 64-22 CHILL CASTING OF 74-C PREFORMS

SUMUARY - 74-C SURFACE CONDITION PROBLEM AHSWERS TO QUESTIOHS OF THE ADVISORY COMMITTEE ON REACTOR SAFECUARDS RELATIVE TO THE MEED FOR RETENTION OF GOVERMMENT LAND

TECHNICAL BASES U-233 PRCDUCTION STUDY GROUP V
AGAR JD

SRD

15

13

LAMBERT RW, MARTELL RT, SRD RATHVON HC

HICK OJ

SRD

6

SRD

34

$07 / 01 / 64$

JONES SS

SRD

81

$07 / 08 / 64$

SRD

658

1

GARTIN WJ

SRD

26

GARTIN WJ

KRAUTTER EF

RE ISMACSON

SRD

SRD

SRD

32

6

(2)

05/27/64

05/26/64

05/26/64

HLADEK KL

SRD

9

$07 / 16 / 64$

AE DEMERS

GILL SM

SECR

SRD

HICK OJ

SRD

HICK OJ

SRD

CARTER RD

SRD

SRD

HOLLIFIELD PJ

NICHOLSON UL

SRD

SRD

STINSON AN

SRD

HICK OJ

SRD

$06 / 30 / 64$

LF WIRFS

SECR

$05 / 27 / 64$

SNITH AE

SRD

4

$06 / 04 / 64$

BARBER AE

JUNKINS RL

SRD

CRD

10

49

$06 / 08 / 64$ $06 / 08 / 64$

woods UK

SRD

$06 / 09 / 64$ 
Page No. 209

$04 / 28 / 93$

LIST OF HANFORD-GENERATED CLASSIFIED

DOCUMENTS DATED BETUEEN 1/1/61 AND 12/31/72

DOC. NO.

HW-82697A

$H W-82697 B$

$H W-82725$

$H W-82726$

HW-82744

HW-82744 RD

HH-82744 SUP1

HW-82744 SUP2

HW-82764 RD

HW-82789

HW-82790

HW-82869

HW-82896

HW-82897

HU-82914

HW-82915

HW-82949

HW-82972

HH-82977 RD HW-82977 RD2 HW-82977 RD3 REV HU-82977 RD4 HW-82983A RD

HW-82983B RD

$H H-82983 C$ RD
TITLE

PRODUCTION TEST IP-691, HAPO 300 SUPPLEMENT A IRRADIATION OF H-U02 OR H-PUO2 PLATE CAPSULES IN THE KE OR KH REACTOR SNOUT FACILITIES

PRODUCTION TEST IP-691, HAPO-300, SUPPLEMENT B IRRADIATION OF W-UOZ OR W-PUO2 PLATE CAPSULES IN THE KE OR KW REACTOR SMOUT FACILITIES

ALTERMATE FEED PREPARATION METHODS FOR RMA LINE PLUTONILN ALLOY

REPORT ON REACTOR COST STLOY MEETINGS

JUNE 3 \& 4, 1964 AT GERMANTON, WD PRCOUCTION TEST IP-694-A - DEPLETED URANIUM IRRADIATION - $K$ REACTORS PRCOUCTION TEST IP-694-A - DEPLETED URANIUM IRRADIATION - $K$ REACTORS

SUPPLEMENT I TO PRODUCTION TEST IP-694-A

- DEPLETED URANIUM IRRADIATION - $K$

REACTORS

SUPPLEMENT II TO PRODUCTION TEST

IP-694-A - DEPLETED IIRANIUM IRRADIATION

- $K$ REACTORS

RADIOISOTOPIC ANALYSIS OF ALUMINUM

CONTROL ROOS

PROOUCTION TEST IP-695-AC, THORIUM OXIDE IRRADIATION - K REACTOR

PROOUCTION TEST IP-696 EVALUATION OF ARCH-RAIL SUPPORTS

K REACTOR MODERATOR DISTORTIOW STLOY FISSION PROOUCT RATIOS OF THE PUREX

FILTER

PROPOSAL FOR A COMPUTERIZED MODEL OF THE AEC COMPLEX

THROUGH-REACTOR FLOU CALCULATEO FROM HIGH TANK-HIGH VELOCITY FLUSH DATA URANIUM BURNOUT VALUES

REPORT OF VISIT TO THE SAVANMAH RIVER

PLANT JUNE 1, 2, 1964

TECHNICAL BASES - U-233 PROOUCTION STUDY GROUP IV-A

IPD PROOUCTION PROJECTIONS

IPD PROOUCTION PROJECTIONS

IPD PROOUCTION PROJECTIONS

IPD PRODUCTION PROJECTIONS

PU RECLAMATION OPERATION URANILM THORIUM AND PLUTONIUM SOURCE DATA FY 1965

PU RECLAMATION OPERATION URANIUM THORIUM AND PLUTONIUM SOURCE DATA FY 1965

PU RECLAMATION OPERATION URANIUM THORIUM AND PLUTONIUM SOURCE DATA FY 1965
AUTHOR

EC BENMETT

EC BENMETT

CRD

$08 / 25 / 65$

FIELD ST

JE MCDONALD

GROSS PD, HLADEK KL SRD

GROSS PD, HLADEK KL SRD

GROSS PD, HLADEK KL SRD

GROSS PD

SRD

4

$10 / 22 / 65$

GROSS PD, HLADEK KL

SRD

$\operatorname{con}$

T

SRD

17

SRD $\quad 12$

HLADEK KL

WK ALEXANDER, A RUSSELL

CRD

MEILER MR

CRD

LANG LW, SPENCER HG, SRD

STENQUIST DR

MORRISSEY JE

MCDONALD JE

EVANS TH, KEMPER RS NAYLOR TD

WOODS IK

LAMG LH

LANG LW

LANG LH

LANG LW

WL BURGE

WL BURGE

SRD

SRD

SRD

SRD

SRD

SRD

SRD

SRD

SECR

SECR

SECR

05/25/65

$06 / 11 / 64$

$06 / 11 / 64$

$08 / 17 / 64$

$07 / 20 / 64$

$06 / 14 / 65$

$06 / 01 / 64$

07/07/64

08/07/64

$06 / 22 / 64$ $06 / 12 / 64$

07/06/64

$08 / 20 / 64$

$06 / 22 / 64$ $06 / 23 / 64$

$06 / 24 / 64$

$06 / 25 / 64$ 07/08/64 $08 / 03 / 64$ 08/04/64 07/01/64

08/01/64

WL BURGE

$09 / 01 / 64$ 
Page No. 210

04/28/93

LIST OF HANFORD-GENERATED CLASSIFIED

DOCUMENTS DATED BETUEEN 1/1/61 AND 12/31/72

DOC. NO

TITLE

AUTHOR

LEVEL

PGS

DATE

HW-82983D RD

HH-82983E RD

HW-82983F RD

HW-82983G RD

HU-82983H RD

HU-82983J RD

HW-82983K RD

HW-82983L RD

HW-82983M RD

HW-83017

HW-83022

HW-83028

HH-83029 RD

HW-83091

HW-83095

HW-83103

HW-83150 RD

HW- 83150 REV 1

HW- 83152

HW-83168 RO

HW-83189 RD

HW-83193
PU RECLAMATION OPERATION URANIUM THORIUM AND PLUTONIUM SOURCE DATA FY 1965

PU RECLAMATION OPERATION URANIUN THORIUM AND PLUTONIUM SOURCE DATA FY 1965

PU RECLAMATION OPERATION URANIUN THORIUM AND PLUTONIUM SOURCE DATA FY 1965

PU RECLAMATION OPE' 'TION URANIUN THORIUM AMD PLUTONILM SOURCE DATA FY 1965 JAN 1965

PU RECLAMATION OPERATION URANILN THORILM AND PLUTONIUN SOURCE DATA FY 1965 FEB 1965

PU RECLAMATION OPERATION URANIUM THORIUM AND PLUTONIUM SOURCE DATA FY 1965 MAR 1965

PU RECLAMATION OPERATION URANIUN THORIUM AND PLUTONIUM SOURCE DATA FY 1965 APR 1965

PU RECLAMATION OPERATION URANIUM THORILM AND PLUTONIUN SOURCE DATA FY 1965 PU RECLAMATION OPERATION URANIUH THORIUM AND PLUTONIU: SOURCE DATA FY 1965 JUN 1965

SPECIFICATION FOR ENRICHED URANIUN BILLETS FOR A COPRCOUCT DEMONSTRATION IN N-KEACTOR

KER-2 OPERATING REPORT, TEST K-2-21

FABRICATION PROCESS TEST EVALUATION -

FPT-64-14 INVERTED CAVITY MOLDS FOR RMC LINE SHAPE CASTING

PRODUCTION TEST IP-695-A - THORIUA OXIDE IRRADIATION - K REACTORS

BIBLIOGRAPHY - COEXTRUSION PROCESS

GAMMA IN-CORE STAINLESS STEEL CHAMBERS KW REACTOR

WEAPONS PROCESS ENGINEERING TECHNOLOGY REPORT - JUNE 1964

COST OF A MK - YEAR

COST OF A MKK - YEAR

BALL DROP IMPLICATIONS AT $K$ REACTORS OPERATIONAL PHYSICS AMALYSIS

IRRADIATION ANALYYSIS, PRCOUCTION TEST IP-697, HAPO 306, IRRADIATION OF 102 FUEL ELEMENTS FOR CONTAINMENT STUDIES, KE ANO KH SHOUT FACILITIES

SUMAARY REPORT - PONER RECOVERY STUDY PROGRAM EXISTING SMALLER HANFORD REACTORS

PHYSICS CALCULATIONS FOR MIXED PUOZ-UOZ NPR LOADIMGS
HL BURGE

SRD

$10 / 01 / 64$

WL BURGE

SECR

$12 / 10 / 64$

WL BURGE

SECR

$12 / 01 / 64$

HL BURGE

SECR

$01 / 01 / 65$

HL BURGE

SECR

02/01/65

HL BURGE

SECR

03/01/65

WL BURGE

SECR

$04 / 01 / 65$

WL BURGE

SECR

$05 / 31 / 65$

WL BURGE

SECR

06/01/65

MICKOLAUS JH

SRD

4

$06 / 25 / 64$

CHRISTENSEN GP

HALKER CM

SRD

SRD

13

7

$06 / 25 / 64$

$06 / 25 / 64$

HLADEK KL, GROSS PD

16

$06 / 26 / 64$

B THOMA

07/01/64

BW HERRMAN, PJ HORVATH SRD

07/01/64

OUSLEY GF

OUSLEY GF

VAUGHN AD

SRD

11

$07 / 01 / 64$

PREVO PR

SRD

SRO

SRD

SRD

$\begin{array}{ll}6 & 07 / 02 / 64 \\ 6 & 10 / 07 / 64\end{array}$

19

$06 / 30 / 64$

$07 / 06 / 64$

SRD

30

$07 / 31 / 64$

BENHETT CL
24

$06 / 30 / 64$ 
Page No. 211

$04 / 28 / 93$

LIST OF HANFORD-GENERATED CLASSIFIED

DOCLMENTS DATED BETWEEN 1/1/61 AND 12/31/72

DOC. NO.

TITLE

HW-83197

HW-83198

HW-83203

HW- 83211

HW-83222

HW-832251

HW-83236

HW- 83242

HW-83246

HW- 83250

HW- 83277

HW- 83318

HW-83330

HW-83343 RD

HW-83364

HH- 83365

HW-83367

HW-83387

HW-83411

HW- 83412

HW- 83418

HW-83426

HW- 83454

HW-83454 D 1964 HENRY 1964-73 1964 REACTORS

$$
\text { 74-C }
$$

QUARTERLY PROGRESS REPORT METALLURGY DEVELOPMENT OPERATION APRIL, MAY, JUHE,

ATOMIC MEAPON DATA SUPPLEMENT QUARTERLY PROGRESS REPORT METALLURGY DEVELOPNENT

OPERATION APRIL, MAY, JUHE, 1964

NOTEBOOK - B REACTOR OPERATING DATA

U-233 FABRICATION DISCUSSIONS WITH C

THE PULP-3 PROGRAM AND REACTOR

OPTIMIZATION OF USING LIMEAR PROGRAMING A-LINE PROOUCT CHARACTERISTICS JUNE 1964 MEPTUNIUM-237 PRCOUCTION FORECASTS - IPD

MANUFACTURING SECTION-SEMI-AMNUAL

SLMMARY REPORT, PERIOD ENDING JUME 30,

BUTTON LINE PROCESS, OVER-ALL IMPURITY DECONTAMIMATION

EXPEDITED REDOX SHUTDONW

MOWTHLY TECHNICAL REPORT JUNE, 1964

REACTOR OPERATIONS DAILY REPORT FORM BM-5000-052 (7-64)

SSFE CHARGING MACHIMES FOR THE $K$

STATUS REPORT - INVESTIGATION OF INNER

FUEL ELEMENTS FAILURE, PROCESS TUBE NO.

3361, JULY 7, 1964 (STATUS AT 5:00 PM, JULY 20, 1964)

DOANCOMER FLOW RESTRICTIONS - C REACTOR

REDOX SOLVENT EXTRACTION CONTROL SYSTEM

CALIBRATION MANUAL

N-REACTOR SHUTDOWN FOR FUEL RUPTURE

INDICATIONS - TUBE 3361

ALDIT OF PONER ROLL FORMING OPERATION -

PRELIMINARY HAZARDS EVALUATION FOR

ENRICHED URANIUAH-THORIA (E-Q) LOADING HANFORD IPD REACTORS

SEMI-ANHUAL SUMMARY REPORT - REACTOR

POTENTIAL GAIMS \& OPERATING EFFICIENCIES

THROUGH JUNE 1964

EFFECTS OF SEVERAL EQ FUEL LOADINGS ON ALUMIMUM PROCESS TUBE LIFE

PLUTONIUM PROOUCT DEVELOPMENT OPERATION MONTHLY REPORT - JULY 1964

PROOUCTION TEST IP-708, OIL-OUENCHED

HOT-DIE-SIZED ELEMENT EVALUATION

FABRICATION OF HOT DIE SIZED DIFFUSION BONDED FUEL ELEMENTS FROM OIL AMD MATER QUENCHED CORES FOR PRODUCTION TEST

IP-708-A
AUTHOR

LEVEL

PGS

DATE

CRD

56

$07 / 20 / 64$

SRD

65

$07 / 20 / 64$

CE HUGHEY

GILL SH

NEWELL. LJ, SHIMER RD

SECR

SRD

$$
3
$$

$07 / 09 / 64$ $07 / 08 / 64$

A. SMITH

NILSON R

SRD

115

$07 / 10 / 64$

TH HAUFF

SRD

SRD

4

$07 / 17 / 64$

$07 / 10 / 64$

SECR

$10 / 31 / 64$

RL HALSER

CONF

07/29/64

AB GRENINGER

NICHOLS PF

DENEAL DL.

SRD

CRD

CRD

MCCARTHY PB

SRD

GUAY AE

CRD

45

$07 / 28 / 64$

07/17/64

REID RH

RA WATROUS

SRD

SRD

RENBERGER DL

CRD

14

KNIGHTS LM

SRD

3

$07 / 22 / 64$

NILSON R, CARLSON PA, SRD $15 \quad 07 / 23 / 64$

OASLEY GF

PO GROSS

SRD

$07 / 23 / 64$

HOWGH CG

SRD

16

$07 / 24 / 64$

WICK OJ

SRD

7

$07 / 31 / 64$

HLADEK KL

SRD

9

$08 / 24 / 64$

Strand CA
CRD

20

$09 / 95 / 64$ 
Page No. 212

$04 / 28 / 93$

LIST OF HANFORD-GENERATED CLASSIFIED

DOCUMENTS DATED BETUEEN 1/1/61 AND 12/31/72

\section{DOC. NO. \\ $H W-83455$ A \\ HW-83455 B \\ HW-83455 C \\ $H W-83455 \quad D$ \\ HU-83455 E \\ HW-83455 F \\ NW-83473 \\ HU-83487 \\ HW-83504 \\ HW-83504 REV \\ HW- 83519 \\ HW- 83531}

$H W-83568$ RD

HW- 83569

HH- 83580

HW- 83585

HH- 83593

HW-83596

HW-83612

HW-83624

HN-83638

HH-83687

HW-83693
TITLE

PUREX OPERATION MATERIAL BALANCE \& NET PROOUCTION REPORT FISCAL YEAR 1965 REDOX OPERATION MATERIAL BALANCE \& MET PRODUCTION REPORT FISCAL YEAR 1965 URANIUM OXIDE OPERATION MATERIAL BALANCE 8. NET PROOUCTION REPORT FISCAL YEAR 1965 PLUTONIUM REDUCTION OPERATION MATERIAL BALAMCE \& NET PRCOUCTION REPORT FISCAL YEAR 1965

PLUTONIUN RECLAMATION OPERATION MATERIAL BALANCE \& NET PRODUCTION REPORT FISCAL YEAR 1965

PLUTONIUN FABRICATION OPERATION MATERIAL BALANCE \& NET PRODUCTION REPCRT FISCAL YEAR 1965

WEAPONS PROCESS ENGINEERING TECHNOLOGY

REPORT - JULY 1964

MOOIFIED TUBULAR SUPPORT PATTERNS --

RIBBED AND RIBLESS PROCESS TUBES

CORROSION STUDIES ON PLUTONIUN IAN

INTERIM REPORT/

CORROSION STUDIES ON PLUTONIUN /AN

INTERIM REPORT/

KER-2 OPERATING REPORT TEST K-2-22

LOADING AND OPERATING CONDITIONS FOR A CHARGE OF THREE NIE-1 FUEL TUBES AMD

FOUR CERAMIC FUELED CRLD PROBES IN KER-1 AND KER-2, PROOUCTION TEST IP-601, SUPPLEMENTS A AND B

IRRADIATION AMALYSIS, PRODUCTION TEST

IP-712, NMA-117, HIGH TEMPERATURE

IRRADIATION OF SNAP-8 FUEL CAMDIDATES

REACTOR PHASE-OUT DISCHARGE PLANHIMG

CUTTING SPEED AND FEED RATE CALCULATIONS FOR WEAPON PROCESSES

EFFECT OF LARD OIL ON PU HYDRIDING

ARCH-T SELF-SUPPORTS AT $K$ PLANT

PHYSICS ASPECTS OF REMOVAL OF OVERBORE

TEST FACILITY

PROJECT HHITMEY A SUMARY OF HANFORD

LABORATORIES ACHIEVEMENT HITHIN THIS

PROJECT UNDER GENERAL ELECTRIC 1954-1964 105-DR SPECIALIST LOGBOOK - NOTEBOOK

FABRICATION PROCESS TEST EVALUATION

FPT-64-10 - CASTING MODEL 74-C PREFORMS

IH DOUBLE CAVITY MOLDS

FUEL ELEMENT DEVELOPMENT COMAITTEE ANMUAL REPORT FROM THE GENERAL ELECTRIC COMPAMY - HANFORD

PLUTONILN PRODUCT DEVELOPMENT OPERATION MONTHLY REPORT - AUCUST, 1964

\begin{tabular}{|c|c|c|c|}
\hline AUTHOR & LEVEL & PGS & DATE \\
\hline BURGE WL & SRD & 85 & $08 / 12 / 64$ \\
\hline BURGE WL & SRD & 93 & $08 / 12 / 64$ \\
\hline BURGE UL & SRD & 54 & $08 / 12 / 64$ \\
\hline BURGE WL & SRD & 17 & $08 / 12 / 64$ \\
\hline BURGE ML & SRD & 86 & $08 / 12 / 64$ \\
\hline \multirow[t]{2}{*}{ BURGE ML } & SRD & 37 & $08 / 12 / 64$ \\
\hline & SRD & 8 & $07 / 30 / 64$ \\
\hline AMGLE CH & CRD & 14 & $08 / 25 / 64$ \\
\hline KATAYAMA YB & SRD & 10 & $08 / 03 / 64$ \\
\hline KATAYAMA YB & SRD & 9 & $08 / 03 / 64$ \\
\hline CHRISTEMSEN GP & SRD & 12 & $08 / 03 / 64$ \\
\hline DEOBALD RL & CRD & 5 & $07 / 16 / 64$ \\
\hline
\end{tabular}

BENMETT EC

SRD

16

$08 / 20 / 64$

DUNN RE

PINKERTON JH

SRD

- SRD

6

22

$08 / 06 / 64$

BARBER AE

WOOD SA

BAILEY GF

SRD

SRD

SRD

3

08/11/64

STEWART RH

SRD

51

$12 / 01 / 64$

GL MADSEN

SECR

SRD

$08 / 11 / 64$

BARBER AE

4

$08 / 14 / 64$

LEUIS M, MINOR JE, STRINGER JT

SRD

40

$08 / 14 / 64$

HICK OJ

SRD

8

$08 / 31 / 64$ 


\begin{abstract}
DOC. NO.
\end{abstract}
TITLE

MTHOR

PROJECT MHITMEY PLUTONIUM METALLURGICAL

HWH-83694

HW-83695

$H W-83698$

HW-83697

HH- 85713

HW-83725

HW-83731

HU-83735

H4-83736

HW-83770

HW-83806

HW-83812

HW-83834

$H W-83861$ RD

HW-83869

HW-83870

HW-83877 RD HW-83901A RD

HH-83901B RD
DEVELOPMENT MONTHLY REPORT - AUGUST, 1964

PROJECT WHITNEY PLUTONIUM METALLURGICAL DEVELOPMENT MONTHLY REPORT - SEPTEMBER, 1964

PROJECT WHITHEY PLUTONILN METALLURGICAL DEVELOPMENT MONTHLY REPORT - OCTOBER 1964

PROJECT HHITMEY PLUTONIU⿴ METALLURGICAL DEVELOPMENT MONTHLY REPORT - NOVEMBER 1964

$K$ REACTOR MIXER STUDY ALUMINUM SPLINE TUBES

FISCAL 1965 REVISION OF CESIUM-137 INDEX FOR MUCLEAR MATERIALS COWTENT OF COATING WASTE

A-LINE PROOUCT CHARACTERISTICS JULY 1964 PROPOSAL: DIRECT IMGOTTING OF UNPICKLED BUTTONS FOR ECONOMIC AND PROOUCT CUALITY GAIMS

PREPARATION OF DELTA-STABILIZED

PLUTONIUN BY DIRECT ALLOY BLEND TECHNIQUES

INTERIM REPORT HIGH-SILICON ALUMINUN

CLADDING FOR HOT DIE SIZED FUEL ELEMENTS NUCLEAR MATERIALS REVIEH, DEPLETED URANIUA SYSTEM, FISCAL YEAR 1964 MUAERICAL RESULTS OF THE DUAL AREA TRIPOUT OF B AND C REACTORS' PROCESS PUNPS

ZIRCALOY-2 PROCESS TUBE ACTIVITY - C REACYOR

IRRADIATION AMALYSIS - PT-IP-714 IRRADIATION OF SOLID ALUAINUN IN KER-1, KER-2, KER-3, AND KER-4 HITH PROCESS WATER COOLING

IRRADIATED FUEL AGE DETERMIMATION STUDY HYDRAULIC SUPPLY AND DENAND CHARACTERISTICS OF THORILM TARGET ELEMENTS IN B, C, D, AND $K$ PROCESS TUBES OUTLINE - N-REACTOR CAPABILITY REPORT IRRADIATION AMALYSIS PRCOUCTION TEST IP691 HAPO30OSUPPLEMENT A IRRADIATION OF W-U02 OR W-PU02 PLATE CAPSULE IN THE KE OR KU SNOUT FACILITIES

IRRADIATION AMALYSIS PRCOUCTION TEST IP691 HAPO 300 SUPPLEMENT B IRADIATION OF W-U02 OR W-PU02 PLATE CAPSULES IN THE KE OR KW REACTOR SNOUT FACILITIES

\begin{tabular}{|c|c|c|c|}
\hline HICK OJ & SRD & 10 & $08 / 31 / 64$ \\
\hline HICK OJ & SRD & 12 & $08 / 30 / 64$ \\
\hline WICK OJ & SRD & 10 & $10 / 30 / 64$ \\
\hline HICK OJ & SRD & 11 & $11 / 30 / 64$ \\
\hline HOUEH CG & SRD & 18 & $08 / 18 / 64$ \\
\hline ZIMER WH & CRD & 3 & $08 / 19 / 64$ \\
\hline $\begin{array}{l}\text { AE SMITH } \\
\text { BONO RH }\end{array}$ & $\begin{array}{l}\text { SRD } \\
\text { SAD A }\end{array}$ & 8 & $\begin{array}{l}08 / 19 / 64 \\
09 / 21 / 64\end{array}$ \\
\hline BOND RH & SRD & 22 & $09 / 21 / 64$ \\
\hline STRAND CA & CRD & 9 & $09 / 17 / 64$ \\
\hline LF WIRFS & SECR & & $09 / 01 / 64$ \\
\hline HOOD SA & SRD & 31 & $10 / 21 / 64$ \\
\hline ESSIG TH & CRD & 8 & $08 / 26 / 64$ \\
\hline GERDES KH & SRD & 4 & $08 / 27 / 64$ \\
\hline COOLEY DE & CRD & 18 & $08 / 31 / 64$ \\
\hline ANGLE CH & CRD & 21 & $09 / 21 / 64$ \\
\hline $\begin{array}{l}\text { DICKENAN RL } \\
\text { EC BENNETT }\end{array}$ & $\begin{array}{l}\text { SRD } \\
\text { CRD }\end{array}$ & 9 & $\begin{array}{l}09 / 01 / 64 \\
05 / 25 / 65\end{array}$ \\
\hline
\end{tabular}

EC BENNETT

CRD

$08 / 25 / 65$ 
Page No. 214

$04 / 28 / 93$

LIST OF HANFORD-LENERATED CLASSIFIED

DOCLNENTS DATED BETWEEN 1/1/61 AMD 12/31/72

Doc. No.

TITLE

AUTHOR

LEVEL

Pas

DATE

HW-83906A RD

$H W-83906 B$ RD

HW- 83908

HW-83921

HW-83922

HW-83936

HW- $\mathbf{3 3 9 3 7}$

HU-83939

HW- 83940

HW-83975

HU-83987 RD

HH- 83996

HU-83997

HW- 84000

HW-84001

HW-84021

HW-84021 SUPG

HU-84073

$H W-84080$ RD

HW-84086

HW- 84107

$H H-84116 C$

$H W-84118$ RD

HU- 84139
CPO 200 VEST AREA TANK FARM INVENTORY \& WASTE REPORTS 6/22/53 THRU 12/22/53

CPD 200 WEST AREA TANK FARM INVENTORY \& WASTE REPORTS JAN 1956 THRU DEC 1956 MEAPONS PROCESS ENGINEERING TECHNOLOGY REPORT - AUEUST, 1964

SANDIA CORPORATION RESEARCH PROPOSAL

241-SX TAMK FARM MASTE STORACE IMPROVED MEPTUNIUN ACCMULATION FLOUSHEET

NUCLEAR MATERIALS REVIEH, EMRICHED URAMIUM SYSTEM - F'SCAL YEAR 1964

EFFECT OF HORIZONTAL CONTROL ROOS IN TOTAL CONTROL COMPENSATION THREE KILOGRAM POLONIUN PRODUCTION STUDY

PLUTONIUM CONCENTRATION AMD STORAGE ENGINEERING STUDY FOR PUREX L-CELL PACKACE REPLACEMENT

MONTHLY REPORT SUWWARY NUEUST 1964 REPORT TO THE WORKING COMITTEE FROA THE GENERAL ELECTRIC COMPANY - HANFORD REPORT OF THE WORKING COMMITTEE OF THE FUEL ELEMENT DEVELOPNENT COMMITTEE HANFORD CAPABILITIES IN MEAPONS FABRICATION AND IN MEAPONS RESEARCH \& DEVELOPMENT

ACTIVITY OF IRRADIATED RECULAR METAL IN BUCKETS

PITA-31 FRINGE BLANKET IRRADIATION OF THORIUM OXIDE

PITA-31 FRINGE-BLANKET IRRADIATION OF THORIUM OXIDE SUPPLEMENT VI MUCLEAR MATERIALS REVIEW MEAPONS MANUFACTURING OPERATION FISCAL YEAR 1964 IRRADIATION AMALYSIS, PRODUCTION TEST IP-672, HAPO 288, IRRADIATION OF IMPACTED UO2-PUO2 FUEL ROD BUNDLES IN C REACTOR

105-F INTERMEdIATE RANGE TEST HOLE PARAMETERS

REVIEW OF PROPOSED FLOUSHEET AMO ROUTINGS FOR THE IMPROVED MEPTUNIUN ACCUMULATED FLOUSHEET - REDOX BLISTERS IN BDF-TYPE CONTROL ROOS FIMAL SUMARY REPORT ENGIMEERING HANFORD CONTRIBUTION FOR THE NINETEENTH HIGH TEMPERATURE FUELS COMITTEE MEETING, NOVEMBER 16, 17, 18, 1964 THE USE OF CHEMICAL ADDITIVES TO IMPROVE FIRST CYCLE DECONTAMIMATION AT PUREX

\begin{tabular}{|c|c|c|c|}
\hline MK BAYLESS & SECR & & $09 / 09 / 64$ \\
\hline MK BAYLESS & SECR & & $09 / 09 / 64$ \\
\hline GARTIN WG & SRD & 6 & $08 / 28 / 64$ \\
\hline PAUL RS & SRD & 4 & $09 / 03 / 64$ \\
\hline HAMSON GL & CRD & 3 & $09 / 03 / 64$ \\
\hline ISAMCSON RE & CRD & 5 & $09 / 03 / 64$ \\
\hline LF UIRFS & SECR & & $09 / 01 / 64$ \\
\hline VAUGHN AD & SRD & 8 & $09 / 04 / 64$ \\
\hline $\begin{array}{l}\text { KUSLER LE, RATHVON HC, } \\
\text { BIRD JM }\end{array}$ & SRD & 16 & $09 / 04 / 64$ \\
\hline SNITH CH & SRD & 36 & $09 / 08 / 64$ \\
\hline \multirow{3}{*}{$\begin{array}{l}\text { ALBAUEH FW } \\
\text { LEMIS M, MIMOR JE, } \\
\text { STRINGER JT }\end{array}$} & $\begin{array}{l}\text { CRD } \\
\text { SRD }\end{array}$ & $\begin{array}{l}10 \\
29\end{array}$ & $\begin{array}{l}09 / 08 / 64 \\
09 / 21 / 64\end{array}$ \\
\hline & SRD & 148 & $10 / 01 / 64$ \\
\hline & SRD & 24 & $07 / 09 / 64$ \\
\hline
\end{tabular}

SMITH RH

SRD

6

$09 / 14 / 64$

PD GROSS

SRD

$09 / 11 / 64$

GROSS PD, HLADEK KL

SRD

7

07/01/65

DOERR RU

SRD

$\operatorname{cox} \mathrm{JH}$

SRD

4

$09 / 14 / 64$

ERICKSON GL

CRD

9

$11 / 16 / 64$

SECR

$09 / 15 / 64$

RIEDEMAN GW

SRD

21

$08 / 30 / 65$

LAST GA

CRD

20

$10 / 21 / 64$

MCKENZIE TR

CRD

14

$09 / 25 / 64$ 
Page No. 215

$04 / 28 / 93$

LIST OF HANFORD-GENERATED CLASSIFIED

DOCUMENTS DATED BETWEEN 1/1/61 AND 12/31/72

Doc. No.

HU-84161

$H W-84170$

HW-84172

HW-84178

HW-84198

HIM -84199

HU-84200

HM-84234

HW- 84235

HW- 84243

HU-84249

$1 H-84271$

HW- 84284

HW-84317

HW- 84318

$H W-84319 R D$

HW- 84339

HW- 84351

HU-84357

HW-84358REV

HW-84365

$H W-84370$

$H W-84381$

$H W-84389$

HW- 84396

HW- 84403
TITLE

PROPOSED PROCESS TEST TO EVALUATE DIRECT INCOTING OF UNPICKLED BUTTOUS

NOTEBOOK - 105-KE SPECIALISTS LOG LETTER TO GH TENNEY FROM LM KNIGHTS PROCESS SPECIFICATIONS FOR TAREET ELEMENTS PRCOUC TION TEST MR-13 CO-PRCOUCER DEMONSTRATION TEST (1.96) INTERIM REPORT: SWELLING BEHAVIOR OF KSE-5 FUEL ELEMENTS, KER TEST K-2-21 INTERIM REPORT: SWELLING BEMAVIOR OF KSE-5 FUEL. ELEMENTS, KER TEST K-1-22 INTERIM REPORT: SWELLIMG BEHAVIOR OF CO-PRCOUCT ORIVER ELEMENTS: KER TEST $\mathrm{K}-3-19$

PLUTONIUM PROOUCT DEVELOPNENT OPERATION MONTHLY REPORT - SEPTEWBER 1964 MIXER PLACEMENT AT H REACTOR DEFECT TEST OF TARGET COMPONENT OF CO-PROOUCER TEST ELEMENT

EVALUATION REPORT - FPT-64-7, TEST OF CO-REDUCED ALLOY IN THE FABRICATION OF MOOEL 74-C COMPONENTS

U-233 REACTOR COSTS FOR 60\% TO $90 \%$ DENSITY THORIA

H REACTOR LOADS FY 1964

SEPARATIONS CAPABILITY

ANTICIPATED SERVICE LIFE OF REDOX

DISSOLVERS

SECRET CALCULATIONS

HOT TEST HOLE TEMPERATURES KE \& KU

REACTORS

URANIUN BURHOUT VALUES FOR LONG RANGE

STUDIES

INFORMATION REPORT ON DOWNCOMER

INSTRUNENTATION AND DATA

PRELIMINARY ENGIMEERIMG REPORT

UNDERPONER RELAYING 100-K AREA

ATONIC MEAPON DATA SUPPLEMENT OUARTERLY

PROGRESS REPORT, METALLLURGY DEVELOPNENT

OPERATION, JULY, AUEUST, SEPTEMBER, 1964

HIGHLIGHT REPORT OF THE WORKIMG

COMITTEE TO THE FUEL ELEMENT

DEVELOPMENT COMMITTEE

TECHMOLOGICAL HAZARDS EVALUATION CORE

E-Q LOAD

EXAMINATION OF FOUR IRRADIATED DVMS FUEL

ELEMENTS - RM C-440

REFINED REACTOR COST STUDIES - CASE I

COMPARISON OF HAPO-SR SEPARATIONS COST

CUIULATIVE EXPOSURE - HCR \#2 - 105-B
AUTHOR

LEVE

PGS

DATE

BOND RH

RG CLOUEH

KNIGHTS LH

DH MALKER

CRD

7

$09 / 21 / 64$

SECR

SRD

CRD

$09 / 21 / 64$

$09 / 21 / 64$

$10 / 01 / 64$

GOFFARD JH

SRD

6

$09 / 23 / 64$

COFFARD JU

SRD

COFFARD JH

SRD

5

$09 / 23 / 64$

MICK OJ

SRD

9

$09 / 30 / 64$

HOUGH CG

WEBER JW

SRD

SRD

12

$09 / 24 / 64$

$09 / 01 / 64$

FIELD ST

SRD

4

$09 / 23 / 64$

KUSLER LE

SRD

8

$09 / 28 / 64$

MCCURRY JW, SPENCER HG

HARMON MK

SRD

SRD

RE ISMCSON

SRD

JD AGAR

CRD

SECR

5

$09 / 29 / 64$

$10 / 08 / 64$

$10 / 01 / 64$

$10 / 01 / 64$ $10 / 02 / 64$

WILSOW R, TOFFER H

SRD

114

$10 / 05 / 64$

ZIMMERMAN PJ

SRD

5

$10 / 05 / 64$

WR THORSON

CRD

$06 / 04 / 65$

SRD

71

$10 / 20 / 64$

SRD

17

$10 / 07 / 64$

GREAGER OH

SRD

17

$10 / 07 / 64$

GRUBER WJ

SRD

9

$09 / 01 / 64$

SMITH WG

SRD

4

$10 / 07 / 64$

BOMERS CE
SRD 
Page No. 216

$04 / 28 / 93$
LIST OF HANFORD-GENERATED CLASSIFIED

DOCUMENTS DATED BETWEEN 1/1/61 AND 12/31/72
Doc. Hо.

HW-84407

HU- 84410

HW- 84415

HW-84427

HU-84456

HW-84457

HU-84459

HW-84497

HH- 84526

HYY-84583

$H W-84590$

$H 4-84606$

HW-BMI -10

HW-GEL- 1

HW-SB-10

HW-SC-2

HW-USCM-1

HW-USCM-2

HH-USCM-3

HW-USCM-5

HW-USCM-6

HW-WSU-1

HW-WSU-1 REV1

HW-HSU-2

HU- WSU-3
TITLE

INTERIM REPORT 1, PROOUCTION TEST IP-634 HALF-PLANT LON PH TEST MATER TREATMENT AT D REACTOR

POLISHING OF TYPE 74-C PARTS

MPR LIQUID RADIOACTIVE WASTE

ONE-GROUP XENON REACTIVITY CALCULATIONS

THE PREPARATION OF STRONTIUN SILICIDE (HUIR-17T3)

PLUTONIUN PRODUCT DEVELOPNENT OPERATION

MONTHLY REPORT - OCTOBER, 1964

HAPO RESEARCH AMD DEVVELOPNENT OCTOBER

1964

ALVA SPECIMEN IDENTIFICATION

PLUTONIUM PRCOUCT DEVELOPMENT OPERATION

MONTHLY REPORT - NOVEMBER 1964

PROJECT WHITMEY PLUTONILA METALLURGICAL

DEVELOPMENT MONTHLY REPORT - DECEMBER,

1944

PLUTONIUM PRCOUCT DEVELOPMENT OPERATION

MONTHLY REPORT - DECEMBER 1964

TRIP REPORT: SHEATH CONFERENCE HELD AT

SAVANNAH RIVER LABORATORY - MOVEMBER 24, 1964

GAS PRESSURE BONDING AND EVALUATION OF

ALUMINUM-CLAD NICKEL-PLATED URANIUM FUEL ASSEMBLIES

FINAL REPORT GRAPHITE DISTORTION "C" REACTOR

PROJECTION MELDING OF END CAPS TO

CO-EXTRLDED ZIRCALOY-2 TUBES

HOT PRESSURE BONDING OF I8E CORES

CHEMICAL MILLING OF COUNTERBORE

PRECESSES IN THE URANIUN MALL OF

ZIRCALOY-2 CLAD URANILN TUBES - PROGRESS

REPORT MO. 1

ORAFT PROGRESS REPORT ON GE PROJECT

INTERIM REPORT GENERAL ELECTRIC PROJECT

CONSULTANT AGREEMENT CA-264

FINAL REPORT - RESEARCH PROJECT CHEMICAL MILLING OF COUNTERBORE RECESSS IN THE URANIUM WALL OF ZIRCALOY-2 CLAD URANIUM TUBES

EXPERIMENTAL RESULTS URAMILM CHEMICAL

MILLIMG

AMALYTICAL CONSIDERATIONS FOR

K-DONHCOMER AND BELLOUS

AMALYTICAL CONSIDERATIONS FOR

$K$-DONNCOMER AND BELLOWS FOR GE COMPANY

BOILING TESTS ON DR-DOWNCONER

EXPANSION JOINT TESTS

$$
\text { AUTHOR }
$$

LEVEL

PGS

DATE

GEIER RG

SRD

13

$10 / 08 / 64$

SMITH AE

DICKEMAN RL

FREDSALL JR, BOUERS CE

SRD

CRD

RP POPOVICH

SRD

CRD

HICK OJ

SRD

PARKER MM

HAYS DD

HICK OJ

HICK OJ

HICK OJ

SRD

WEBER JW

JE DAVIS

CRD

$1000 \mathrm{NH}$

CRD

SCIAKY RESEARCH DIV.

CRD

MEYER E

ATKINS DC

CRD

CRD

ATKINS DC

VINTON CJ

CRD

CRD

ATKINS DC

CRD

30

CRD

6

31

41

$06 / 28 / 61$
SRD

SRD

Lomax CC

Lomax CC

Lown CC

Lomax CC

SRD

CRD
6

$06 / 16 / 61$

$06 / 20 / 61$ 
Page No. 217

04/28/93

LIST OF HANFORD-GENERATED CLASSIFIED

DOCUMENTS DATED BETUEEN 1/1/61 AND 12/31/72

DoC. NO

HW-WSU-4

HW-WSU-6 RD

HW- USH-?

HW-WSU-8

ISO-00009A

IS0-000098

Is0-00009C

150-000090

1SO-00009E

iso-00009F

Iso-00011

150-00012

I50-00013A

150-000938

150.00013C

$150-000130$

1SO-00013E

Is0-00013F

ISO-00035

ISO-00039RD ISO-0005/RD

ISO-DCU057RD

150-00063

$150-00069$
TITLE

MOOEL TEST'S OF K-DOWNCOMER ORIFICE

PLATES FOR GENERAL ELECTRIC COMPANY MODEL STLDIES OF CROSS-OVER PIPING TO BRD DOAHCOMERS

MODEL TEST OF THE IMLET TO K-DOWNCOMER

INTERPRETATION OF K-DOWICOMER INLET LOW FLON TEST DATA

PLUTONIUM REDUCTION SOURCE DATA FY 1966

- JamunRY 1966

PLUTONIUM REDUCTION SOLPCE DATA FY 1966

- FEBRUARY 1966

PLUTONIUM REDUCTIOA SOURCE DATA FY 1966

- MARCH 1966

PLUTONILN REDUCTION SOURCE DATA FY 1966

- APRIL 1966

PLUTONIUM REDUCTION SOURCE DATA FY 1966

- mar 196.

PLUTONIUN REIUCTION SOJRCE DATA FY 1966

- JUAE 1966

FABR:CAT:ON SOURCE DATA, FY 1966 -

JANUARY THROUGH JUNE, 1966

FABRICATION MONTH END REPORTS, FY 1966 -

JANUARY THROUGH JUNE, 1966

PLUTONIUM RECLAMATIOY SOURCE DATA, FY

1966 - JANUARY 1966

PLUTONIUA RECLANATION SOURCE DATA, FY

1966 - FEBRUARY 1963

PLUTONIUN RECLAMATIOH SOURCE DATA, FY

1966 - MARCH 1966

PLUTON:UN RECLAMATION SOURCE DATA, FY

1966 - APRIL, 1966

PLUTONIUN RECLAMATION SOURCE DATA, FY

1966 - MAY, 1966

PLUTONIUN RECLAMAIION SOURCE DATA, FY

1966 - JUNE, 1966

QUARTERLY PROOUCTION FORECAST - JAMUARY

1. 1966 THROUGH JUNE 30, 1971 250, DUN,

GE CO, RL-AEC

NUCLEAR MATERIAL CONTROL DATA

2 PLANT REPORT - TASK I-II : RECOVERY

OPERATIONS - JAMUARY 1, 1966 THRU

DECEMBER 31, 1956

2 PLANT REPORT TASK I-II \& RECOVERY

OPERATIONS JANUARY ?, 1966 THROUGH

DECEMBER 31, 1966

PROCESS TEST EVALUATION, FPY-65-2 -

TENSILE MACHINE INSTRLLATION CHECKOUT NUCLEAR MATERIALS ACCOUNTING - SHIPPING AND RECEIVING REPORT - JANUARY THROUGH JUNE, 1966

\begin{tabular}{|c|c|c|c|}
\hline AUTHOR & LEVEL & PGS & DATE \\
\hline Loinax CC & SRD & 69 & $07 / 06 / 61$ \\
\hline Lorax CC & SRD & 44 & $10 / 23 / 61$ \\
\hline LonnX CC & SRD & 47 & $01 / 31 / 62$ \\
\hline TINMEY ER & CRD & 27 & $05 / 29 / 62$ \\
\hline JG MURPHY & SECR & & $01 / 01 / 66$ \\
\hline JG MURPHY & SECR & & $02 / 01 / 66$ \\
\hline JG MURPHY & SECR & & $03 / 01 / 66$ \\
\hline JG MURPHY & SRD & & $04 / 01 / 66$ \\
\hline JG MURPHY & SECR & & $05 / 01 / 66$ \\
\hline JG MURPHY & SECR & & $06 / 01 / 66$ \\
\hline JG MURPHY & SECR & & $01 / 01 / 66$ \\
\hline JG MURPHY & SECR & & $01 / 01 / 66$ \\
\hline JG AURPHY & SECR & & $01 / 01 / 66$ \\
\hline JG MURPHY & SECR & & $02 / 01 / 66$ \\
\hline JG MURPHY & SECR & & $03 / 01 / 66$ \\
\hline JG IURPHY & SECR & & $04 / 01 / 66$ \\
\hline JG MURPHY & SECR & & $05 / 01 / 66$ \\
\hline \multirow[t]{2}{*}{ JG MURPHY } & SECR & & $06 / 01 / 66$ \\
\hline & SECR & & $01 / 24 / 66$ \\
\hline \multirow[t]{2}{*}{ JW FILLMORE } & $\begin{array}{l}\text { SECR } \\
\text { SECR }\end{array}$ & & $\begin{array}{l}01 / 12 / 66 \\
01 / 01 / 66\end{array}$ \\
\hline & SECR & & $01 / 01 / 66$ \\
\hline IN TAYLOR, JR. & SECR & & $01 / 20 / 66$ \\
\hline JG MURPHY & SECR & & $01 / 25 / 66$ \\
\hline
\end{tabular}


Page No. 218

$04 / 28 / 93$

LIST OF HANFORD-GENERATED CLASSIFIED

DOCLMENTS DATED BETHEEN 1/1/61 AND 12/31/72

DOC. NO

150-00073

150-00077

150-00091

ISO-00113

150-00114

$150-00114$

$150-00119$

ISO-001211

150-001212

$150-001213$

150-001214

150-001215

ISO-001216

ISO-00123

150-00153

150-00164

ISO-00164

I $50-00194$

150-00213

ISO-00216

150-00216

150-00221

ISO-0026IRD

150-00263RD
TITLE

QUALITY CONTROL LOG BOOK

CPD RECEIVING AND SHIPPING REPORTS -

JANUARY THRU JUNE, 1966

REDOX S-R DIFFERENCE

NUCLEAR MATERIALS REVIEW - CPD DEPLETED

URANIUM SYSTEM - FIRST AND SECOND

QUARTERS FISCAL YEAR 1966

RESEARCH AND DEVELOPMENT SS MATERIAL

PROJECTS - JANUARY 1966

RESEARCH AND DEVELOPMENT SS MATERIAL

PROJECTS - JANUARY 1966

NUCLEAR MATERIALS REVIEW - CPD ENRICHED

URANIUM SYSTEM - FIRST AND SECOND

QUARTERS FISCAL YEAR 1966

ISCOHEM INC. PRODUCTION INVENTORY AND

MANUFACTURING STATEMENT - JAMUARY 31.

1966

PROOUCTION INVENTORY AND MANUFACTURING

STATEMENT - FEBRUARY 28, 1966

ISOCHEM INC. PROOUCTION INVENTORY AND

MANUFACTURING STATEMENT-MARCH 31, 1966

PRODUCTION INVENTORY AND MANUFACTURING

STATEHEAT - APRIL 30, 1966

PRODUCTION INVENTORY AND MANUFACTURING STATEMENT MAY 31, 1966

PRODUCTION INVENTORY AND MANUFACTURING

STATEMENT - JUNE 30, 1966 - FY-1966

ISOCHEM INC. - 200 AREAS OPERATION -

CHEMICAL PROCESSING DIVISION PUREX

MANUFACTURING COST PER UNIT.

MUCLEAR MATERIALS REVIEH - PLUIDNIUN

FINISHING SECTION - FIRST : SECOND

QUARTERS FISCAL YEAR 1966

RESEARCH AND DEVELOPMENT SS MATERIAL

PROJECTS - FEBRUARY, 1966

RESEARCH AND DEVELOPMENT SS MATERIAL

PROJECTS - FEBRUARY, 1966

CRITICAL MASS SAFETY CALCULATIONS

QUARTERLY PROOUCTION FORECAST-APRIL 1, 1966 THROUGH DECEMBER 31, 1967

RESEARCH AMD DEVELOPNENT SS MATERIAL PROJECTS-MARCH 1966

RESEARCH AND DEVELOPMENT SS MATERIAL

PROJECTS-MARCH 1966

200 AREAS OPERATION-PRODUCTION AND UNIT COST CHARTS

CPD PRCOUCTION GRAPHS-JANUARY 1, 1966

THROUGH JUNE 30, 1966

CPD CHARGING INFORMATION-JANUARY 11966 THRU

\begin{tabular}{|c|c|c|c|}
\hline AUTHOR & LEVEL & PGS & DATE \\
\hline RA YCOER & SRD & & $01 / 01 / 66$ \\
\hline JG MURPHY & SECR & & $01 / 28 / 66$ \\
\hline LH TAYLOR & SECR & & $01 / 01 / 66$ \\
\hline LF WIRFS & SECR & & $02 / 02 / 66$ \\
\hline JG MURPHY & CONF & & $02 / 14 / 66$ \\
\hline JG MURPHY & SRD & & $02 / 14 / 66$ \\
\hline LH TAYLOR & SECR & & $02 / 14 / 66$ \\
\hline DA PETERSON & SRD & & $02 / 22 / 66$ \\
\hline DA PETERSON & SECR & & $03 / 21 / 66$ \\
\hline DA PEterson & SECR & & $04 / 22 / 66$ \\
\hline DA PETERSON & SECR & & $05 / 16 / 66$ \\
\hline DA PETERSON & SECR & & $06 / 20 / 66$ \\
\hline DA PETERSON & SECR & & $07 / 22 / 66$ \\
\hline HF PATTERSON & SECR & & $02 / 19 / 68$ \\
\hline
\end{tabular}

RW DOERR

SECR

$02 / 28 / 66$

JG MURPHY

CONF

$03 / 11 / 66$

JG MURPHY

SRD

$03 / 11 / 66$

RD CARTER

SECR

$03 / 30 / 66$

SECR

$04 / 28 / 66$

JG MURPHY

CONF

$04 / 11 / 66$

JG MURPHY

. SRD

$04 / 11 / 66$

WA LANE

SECR

$06 / 01 / 66$

D mCDONALD

SECR

$01 / 01 / 66$

D MCDONALD

SRD 
Page No. 219

$04 / 28 / 93$

LIST OF HANFORD-GENERATED CLASSIFIED

DOCUMENTS DATED BETHEEN 1/1/61 AND 12/31/72

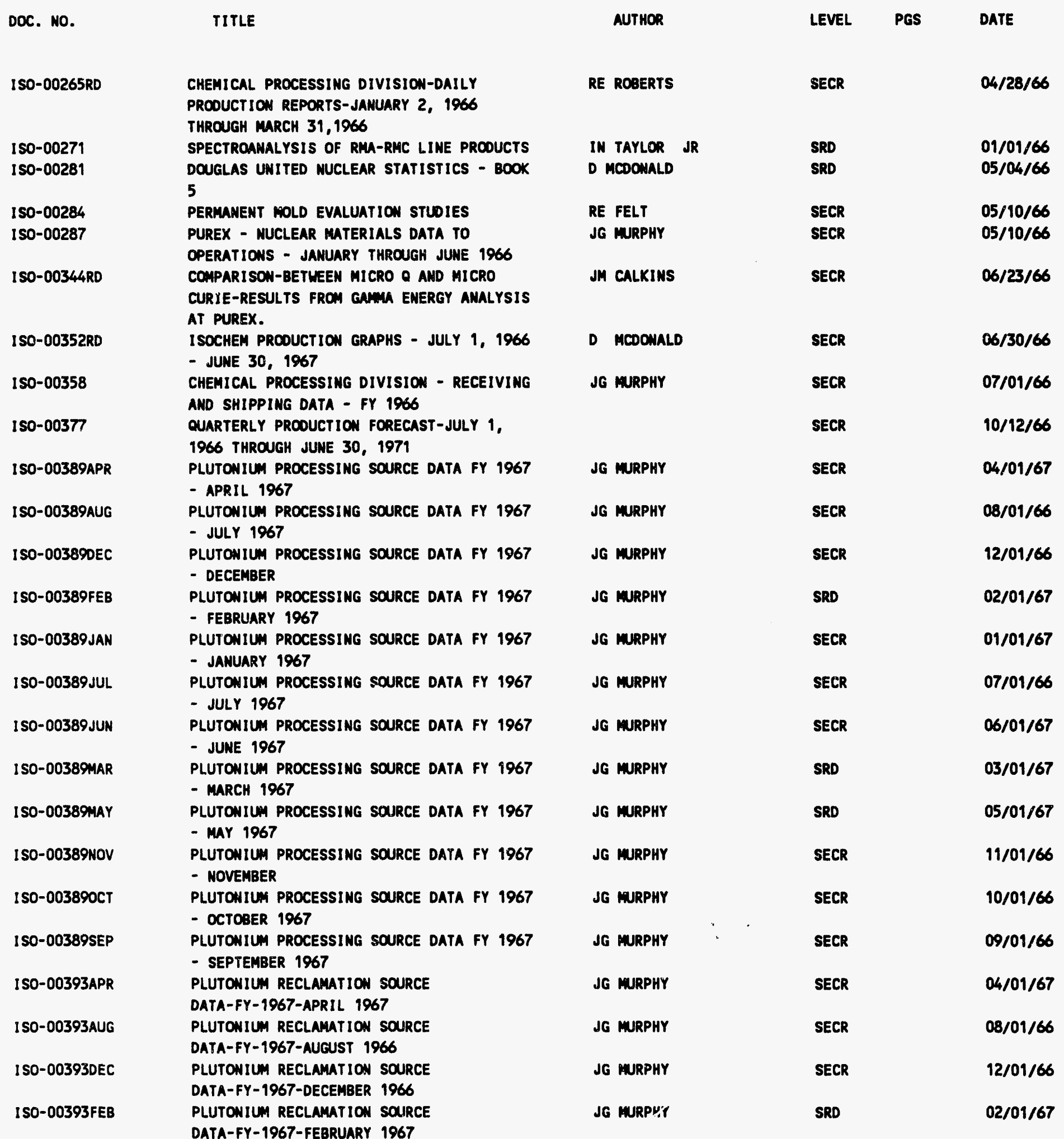


Page No. 220

$04 / 28 / 93$

LIST OF HANFORD-GENERATED CLASSIFIED

DOCUMENTS DATED BETHEEN 1/1/61 AND 12/31/72

DOC. NO.

150-00393JAN

150-00393JUL

ISO-00393JUN

ISO-00393MAR

ISO-00393maY

150-00393NOV

ISO-003930CT

150-00393SEP

$150-00432$

150-00436

150-00442

150-004521

ISO-0045210

150-0045211

Iso-0045212

ISO-004522

150-004523

I50-004524

IsO-004525

ISO-004526

150-004527

Iso-004528

Is0-004529

ISO-00470RD
TITLE

AUTHOR

PLUTONIUN RECLAMATION SOURCE

DATA-FY-1967-JANUARY 1967

PLUTONIUM RECLAMATION SOURCE

DATA-FY-1967-JULY 1966

PLUTONIUM PROCESSING SOURCE DATA-FY

1967-JUNE, 1967

PLUTONIUM RECLAMATION SOURCE

DATA-FY-1967-MARCH 1967

PLUTONIUM RECLAMATION SOURCE

DATA-FY-1967-MAY 1967

PLUTONIUN RECLAMTION SOURCE

DATA-FY-1967-NOVEMBER 1966

PLUTONIUM RECLAMATION SOURCE

DATA-FY-1967-OCTOBER 1966

PLUTONIUN RECLAMATION SOURCE

DATA-FY-1967-SEPTEMBER 1966

MUCLEAR MATERIALS REVIEW - CPD DEPELTED

URANIUM SYSTEM - FISCAL YEAR 1966

NUCLEAR MATERIALS REVIEU - CPO ENRICHED

URANIUM SYSTEM - THIRD AND FOURTH

QUARTERS - FISCAL YEAR 1966

FY 1967 REVISION OF CESIUN-137 - INDEX

FOR NUCLEAR MATERIALS - CONTENT OF

COATING WASTE

PROOUCTION INVENTORY AND MANUFACTURING

STATEMENT - JULY 31, 1966

PROOUCTION INVENTORY AND MANUFACTURING STATEMENT APRIL 30, 1967.

PROOUCTION INVENTORY AND MAMUFACTURING

STATEMENT - MAY 31, 1967

PROOUCTION INVENTORY AND MANUFACTURING

STATEMENT JUNE 30, 1967

PROOUCTION INVENTORY AND MANUFACTURING

STATEMEKT - AUGUST 31, 1966

PRODUCTION INVENTORY AND MANUFACTURING

STATEMENT - SEPTEMBER 30, 1966

PROOUCTION INVENTORY AND MANUFACTURING STATEMENT - OCTOBER 31, 1966

PROOUCTION INVENTORY AND MANUFACTURING

STATEMENT - NOVEMBER 30, 1966

PROOUCTION INVENTORY AND MANUFACTURING

STATEMENT - DECEMBER 31, 1966

PROOUCTION INVENTORY AND MANUFACTURING

STATEMENT JANUARY 31, 1967.

PROOUCTION INVENTOEY AND MANUFACTURING

STATEMENT FEBRUARY 28, 1967.

PROOUCTION INVENTORY AND MANUFACTURING STATEMENT MARCH 31, 1967

CPD CHARGING GRAPHS JULY 1,1966 - JUNE 30,1967

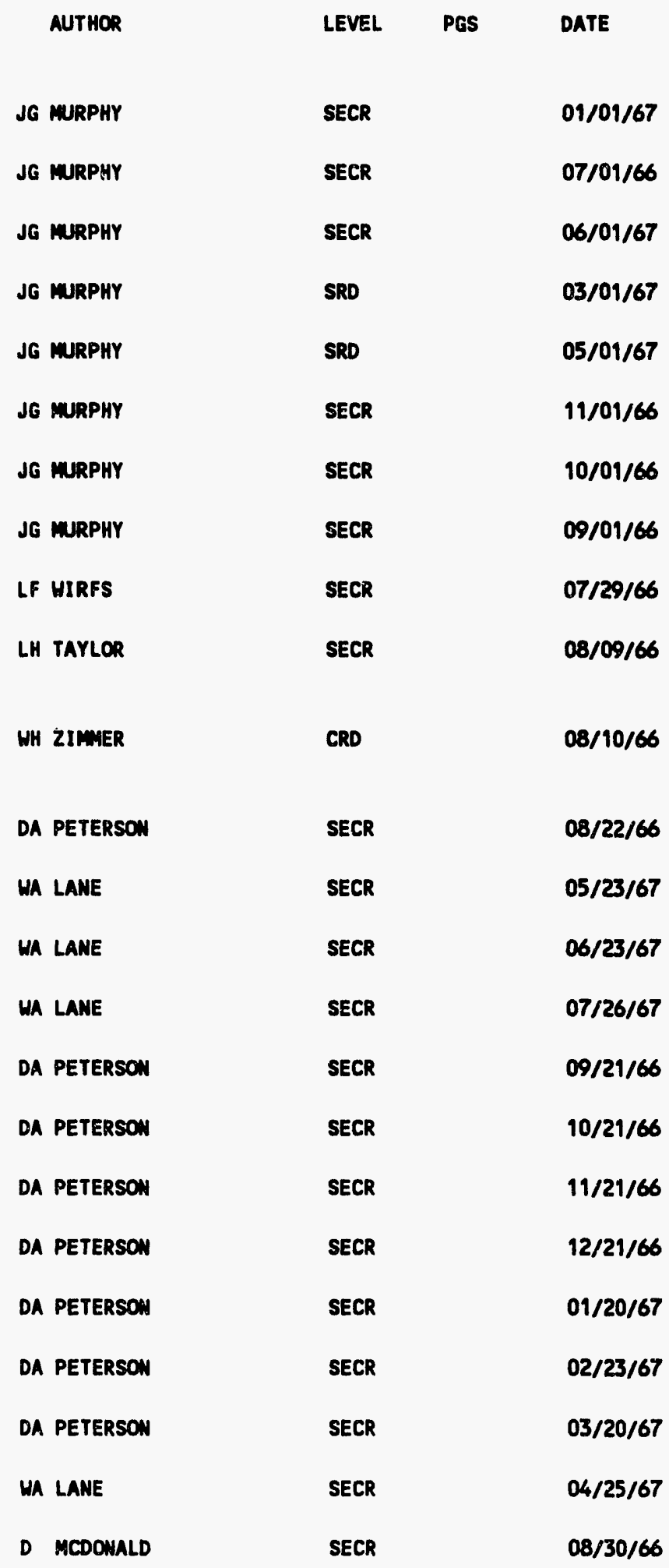


DOC. NO.

ISO-00507

Iso-00508

ISO-00509VOLI

150-00509VOL2

150-00509VOL3

ISO-00568RD

ISO-00569RD

150-00598

$150-00648$

ISO-00659RD

150-00659RD

150-00697

$150-00699$

$150-00736$

ISO-00743

150-00751

150-00758

150-00761

I SO-00783

ISO-00794
TITLE

AUTHOR

CC JONES

SRD

WORKING PAPERS - UNCERTAINTY

CALCULATIONS - PLUTONIUM RECLAMATION

INVENTORY - JAMUARY - DECEMBER 1966

WORKING PAPERS - MH MEASUREMENTS - DATA

EVALUATION SYSTEM - PLUTONIUN FINISHING OPERATION - JANUARY - JUNE 1966

WORKING PAPERS - MM MEASUREMENTS - DATA

EVALUATION SYSTEM - PLUTONIUM FINISHING OPERATION - JULY-DECEMBER 1966

WORKING PAPERS - MM MEASUREMENTS DATA

EVALUATION -PLUTONIUN FINISHING

OPERATION JAMUARY - JUNE 1967

CPD DAILY PROOUCTION REPORTS-APRIL 3,

1966 THROUGH JUNE 30, 1966

CPD DAILY PRODUCTION REPORTS-JULY 4,

1966 THROUGH SEPTEMBER 30, 1966

PLUTONIUM FINISHING SECTION -

MANUFACTURING COST IT - CY TO DATE

QUARTERLY PROOUCTION FORECAST - JANUARY

1, 1967 THROUGH JUNE 30, 1972.

Z PLANT REPORT - TASK I-II : RECOVERY

OPERATIONS - JAMUARY 19967 THROUGH

DECEMBER 311967

2 PLANT REPORT - TASK I-II : RECOVERY

OPERATIONS - JAMUARY 11967 THROUGH

DECEMBER 31967

CPD DAILY PRCDUCTION REPORTS - OCTOBER

2, 1966 DECEMBER 31, 1966

CPD DAILY PRCOUCTION REPORTS JANUARY,

1967 THROUGH MARCH, 1967

MEPTUNIUM MATERIAL BALANCE - REDOX - CY

1966

PLUTONIUM METAL BUTTON QUALITY.

NUCLEAR MATERIALS REVIEH, CPD DEPLETED

URANIUM SYSTEM - FIRST AND SECOND

QUARTERS FISCAL YEAR 1967.

NUCLEAR MATERIALS REVIEW - CPD ENRICHED

URANIUN SYSTEM - FIRST AND SECOND

QUARTERS FISCAL YEAR 1967.

POLONIUN PROOUCTION COSTS AND CAPABILITY MUCLEAR MATERIALS REVIEW - PLUTONIUM

FINISHING SECTION FIRST AND SECOND

QUARTERS FISCAL YEAR 1967.

CPO DAILY PROOUCTION REPORTS APRIL, 1967

THROUGH JUNE, 1967.
SRD

$09 / 27 / 66$

CC JONES

SRD

$09 / 27 / 66$

CC JONES

SRD

$09 / 27 / 66$

CC JONES

SRD

$04 / 06 / 67$

CC JONES

SECR

$11 / 04 / 66$

VR CHAPMAN

SECR

$11 / 04 / 66$

EG GRAVES

SECR

$11 / 29 / 66$

SECR

$01 / 24 / 67$

LM KNIGHTS

SECR

$01 / 01 / 67$

LM KNIGHTS

SECR

01/01/67

VR CHAPMAN

SECR

$01 / 04 / 67$

HW IURRAY

SECR

$04 / 27 / 67$

JW JORDAN

SRD

$02 / 16 / 67$

LM KNIGHTS

SECR

SECR

02/27/67

$02 / 28 / 67$

LH TAYLOR

SECR

03/06/67

HILL OF, SPINK JR RU DOERR

SRD

SECR

11

03/08/67

03/15/67

HW MURRAY

SECR

$04 / 27 / 67$ 
DOC. NO.

150-00811

IS0-00843

$150-00844$

$150-00850$

150-00850

ISO-00855RD

150-00918A

150-00919A

Iso-00922AUG

150-00922JUL

ISO-00924AUG

$150-00924 \mathrm{JUL}$

150-00926A

ISO-00927A

I50-00934

ISO-00944AUG

ISO-00944JUL

Iso-00945

ISO-00947

ISO-00948

150-00949

$150-00950$

1SO-00953RD
TITLE

AUTHOR

QUARTERLY PRODUCTION FORECAST, APRIL 1, 1967 THROUGH JUNE 30, 1969.

UNCERTAINTY CALCULATIONS PLUTONIUA REDUCTION INVENTORY JANUARY-DECEMBER 1967

UNCERTAINTY CALCULATIONS PLUTONIUN RECLAMATION INVENTORY JANUARY - DECEMBER 1967

STUDY PROPOSAL, FUEL PROCESSING - GAS

PURIFICATION.

STUDY PROPOSAL - FUEL PROCESSING - GAS

PURIFICATION

ESSENTIAL MATERIALS REQUIREMENTS AND

COST FOR WASTE MAMAGEMENT AND FISSION PROOUCT. THROUGH FY-69

TECHNICAL SERVICES-SOURCE DATA-FY 1968 JULY THROUGH SEPTEMBER, 1967

TECHNICAL SERVICES MONTH-END REPORTS -FY

1968 JULY THROUGH SEPTEMBER, 1967

PLUTONIUM PROCESSING SOURCE DATA - FY 1968 - AUGUST 1967

PLUTONIUN PROCESSING SOURCE DATA - FY

1968-JULY 1967

PLUTONIUM RECLAMATION SOURCE DATA - FY

1968 - AUGUST 1967

PLUTONIUM RECLAMATION SOURCE DATA-FY

1968 JULY, 1967

RESEARCH AND ENGINEERING SOURCE DATA-FY

1968 JULY THROUGH SEPTEMBER, 1967

RESEARCH AND ENGINEERING MONTH-END REPORTS-FY 1968 JULY THROUGH SEPTEMBER, 1967.

CPD DAILY PRODUCTION REPORTS JULY, 1967 THROUGH SEPTEMBER, 1967.

WORKING PAPERS NUCLEAR MATERIALS REPORT CONSOLIDATIONS-FY 1968-AUGUST, 1967.

WORKING PAPERS NUCLEAR MATERIALS REPORT CONSOLIDATIONS FY 1968.

PUREX SS MATERIALS GEMERAL LEDGER FY

1968

PLUTONIUM PROCESSING SS MATERIALS

GENERAL LEDGER FY 1968

PLUTONIUM RECLAMATION SS MATERIALS

GENERAL LEDGER FY 1968.

RESEARCH AND ENGINEERING SS MATERIALS

GENERAL LEDGER FY 1968

TECHNICAL SERVICES SS MATERIALS GENERAL LEDGER FY 1968

CPD CHARGING GRAPHS JULY 1, 1967-JUAE 30, 1968

\begin{tabular}{|c|c|c|c|}
\hline AUTHOR & LEVEL & PGS & DATE \\
\hline & SECR & & $04 / 26 / 67$ \\
\hline CC JONES & SRD & & $01 / 30 / 67$ \\
\hline CC JONES & SRD & & $01 / 30 / 67$ \\
\hline LR MICHELS & SECR & & $05 / 05 / 67$ \\
\hline MICHELS LR & SRD & 6 & 05/05/67 \\
\hline DE LARSON & SECR & & $05 / 10 / 67$ \\
\hline JG MURPHY & SECR & & $07 / 01 / 67$ \\
\hline JG MURPHY & SECR & & $07 / 01 / 67$ \\
\hline JG MURPHY & SECR & & $08 / 01 / 67$ \\
\hline JG AJRPHY & SECR & & $07 / 01 / 67$ \\
\hline JG MURPHY & SECR & & $08 / 01 / 67$ \\
\hline JG MURPHY & SECR & & $07 / 01 / 67$ \\
\hline JG MURPHY & SECR & & $07 / 01 / 67$ \\
\hline JG MURPHY & SECR & & $07 / 01 / 67$ \\
\hline HW IMURRAY & SECR & & $06 / 23 / 67$ \\
\hline JG MURPHY & SECR & & $08 / 01 / 67$ \\
\hline JG FURPHY & SECR & & $07 / 01 / 67$ \\
\hline JG MURPHY & SECR & & $07 / 01 / 67$ \\
\hline JG MURPHY & SECR & & $07 / 01 / 67$ \\
\hline JG MURPHY & SECR & & $07 / 01 / 67$ \\
\hline JG MURPHY & SECR & & $07 / 01 / 67$ \\
\hline JG MURPHY & SECR & & $07 / 01 / 67$ \\
\hline D MCDONALD & SECR & & $07 / 01 / 67$ \\
\hline
\end{tabular}


DOC. NO.

ISO-00954RD

$150-00963$

$150-01014$

$150-01022$

$150-01025$

150-01026

$150-01027$

150-0104

$150-01040$

150-01042

ISO-01067

ISO-01070RD

150-01071

$150-01074$

150-01082

150-01092

ISO-01093

150-01094RD

ISO-01103RD

ISO-01105RD

150-01106RD

ISO-01107RD

ISO-N-00005 ISO-N-00017 $150-N-00019$ ISO-N-00023
TITLE

ANTHOR

D MCDOMALD

ISOCHEM PRODUCTION
1967-JUNE 30, 1968

QUARTERLY PROOUCTION FORECAST, JULY 1, $1967 T$ TROUGH JUNE 30, 1972.

CPD, PUREX - ESSENTIAL MATERIAL

UTILIZATION

PUREX - NUCLEAR MATERIALS DATA TO

OPERATIONS FY 1968.

PUREX PROCESS WASTE SUMUARY

NUCLEAR MATERIALS REVIEH - PLUTONIUM

FINISHING SECTION THIRD AND FOURTH

QUARTERS FISCAL YEAR 1967.

MUCLEAR MATERIALS REVIEW, CPD ENRICHED

AND DEPLETED URANIUM SYSTEM, THIRD AND

FOURTH QUARTERS FISCAL YEAR 1967.

EVALUATION OF SHEAR STRENGTH MEASUREMENT OF ALLOYED PLUTONIUN PARTS

PUREX PLANT DECEMBER 1966 AND APRIL-MAY

1967 SEFOR CAMPAIGN RESULTS.

PROOUCTION INVENTORY AND MANUFACTURING

STATEMENT - JULY 31, 1967.

HISTORICAL DATA, CPD COST, PRCOUCTION,

AND UNIT COST DATA

MATERIALS HANDLING HITH RESPECT TO

FILTRATE CONCENTRATE-DUALITY

TRANSFER OF MUCLEAR MATERIALS

INVENTORIES-ISOCHEM (SS FACILITY HLA) TO ARHCO (SS FACILITY HVA).

$\checkmark$ AND PU FLOU CHART.

PRODUCTION INVENTORY AND MANUFACTURING

STATEMENT AUGUST 31, 1967

URANIUM OXIDE SUBSECTION CAR SHIPMENT

SUAMARY DATA LOT NO. E-122 THRU E-223.

REDOX DAILY PERFORMANCE REPORTS.

REDOX PLANT ESSENTIAL MATERIALS

PERFORMANCE CY-1965

CPD MONTHLY REPORT SUMMARIES. JANUARY

1966 - AUGUST 1967.

PROOUCT INVENTORY AND MANUFACTURING

STATEMENT WORKING PAPERS FY-1966 JANUARY THRU JUNE.

PROOUCT INVENTORY AND MANUFACTURING

STATEMENT WORKING PAPERS FY 1966 JULY

THRU DECEMBER.

PRODUCT INVENTORY AND MANUFACTURING

STATEMENT WORKING PAPERS FY-1967.

PROOUCTION DATA

LABORATORY NOTES

QUALITY CONTROL NOTES

QUALITY CONTROL NOTES

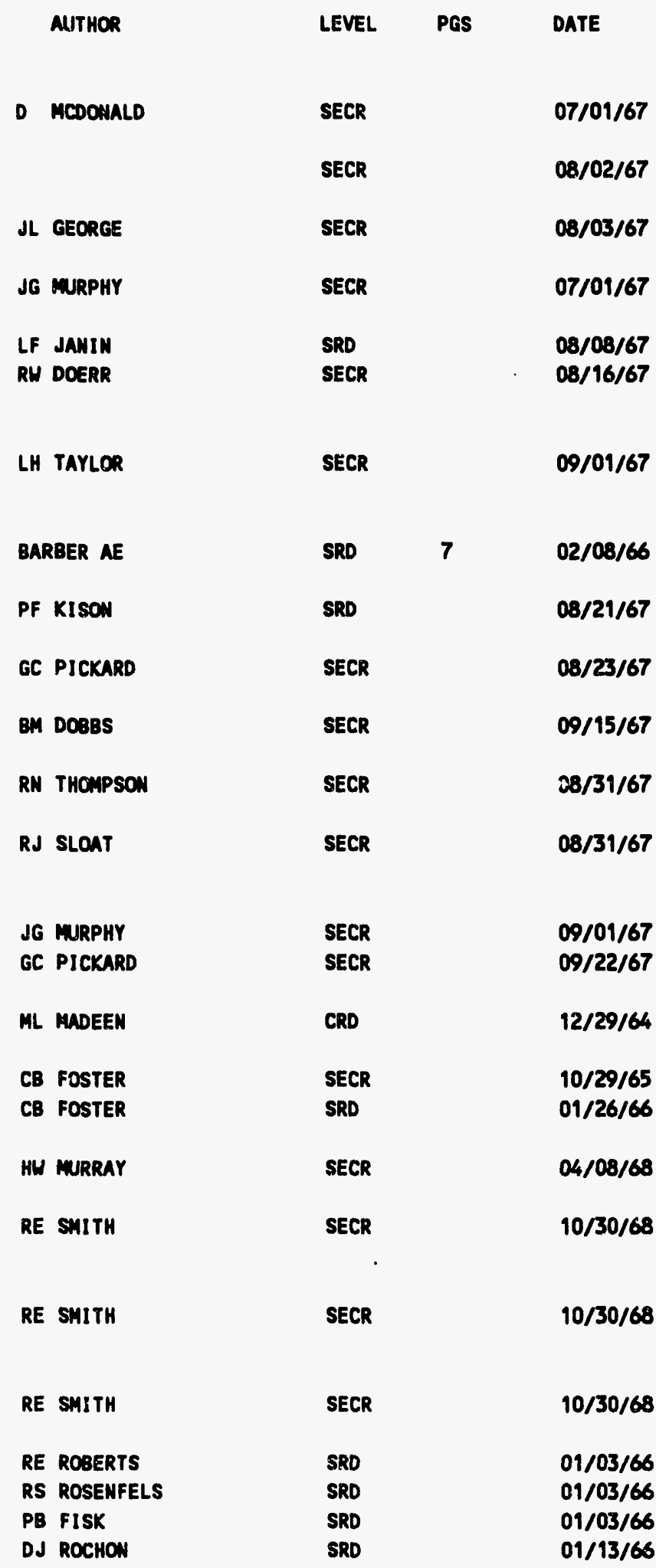


Page No. 224

$04 / 28 / 93$

LIST OF HANFORD-GENERATED CLASSIFIED

DOCLMENTS DATED BETWEEN 1/1/61 AND 12/31/72

DOC. NO.

ISO-N-00027

ISO-N-00037

150-N-00038

ISO-N-00040

I $50-N-00041$

ISO-N-00042

ISO-N-00044

IsO-N-00046

$150-N-00047$

ISO-N-00048

ISO-N-00052

I $50-N-00057$

$150-\mathrm{N}-00064$

$150-N-00066$

I $50-N-00069$

$150-N-000 \% 5$

ISO-N-00086

I $50-N-00087$

ISO-N-00090

ISO-R-00003

ISO-R-00009

ISO-R-00010

ISO-R-00014

I SO-R-00015

I SO-R-00020

I SO-R-00028

1SO-R-00037

150-R-00039

ISO-R-00041

ISO-R-00048

ISO-R-0005

ISO-R-00054

ISO-R-00058

ISO-R-00059

ISO-R-00082RD

PPD -004051

PPD-0040510

PPD-0040511

PPD -0040512

PPD-004052
TITLE

AUTHOR

UH ZIMWER

LABORATORY MOTES

MASS SPECTROMETRY LOG

PERSONAL NOTES

COMPUTOR RUNS

CLASSIFICATION MOTES

PERSONAL MOTES

FISSION PRODUCT PROCESSING STUDIES

PERSONAL NOTES

HEAD-END MEASUREMENTS

MUF WORK SHEETS

PERSONAL NOTES

PERSONAL NOTES

TECHNICAL MOTES

TECHNICAL MOTES

TECHNICAL NOTES

NOTEBOOK - PUREX PLANT PLUTONIUM

INVENTORY

PERSONAL NOTES

PERSONAL MOTES

TECHNICAL NOTES

PLUTONIUM SCRAP FROM LIVERMORE

SHIPMENT "HWA-ASA-10-4

SHIPMENT NO. HUA-ASA-5

EFFECTS ON PROOUCTION OF PLANT OUTAGES

EFFECTS ON PROOUCTION OF PLANT OUTAGES

FY 1967 - 02 RED FUNDS

FY-1967 - 02 FUNDS.

FORMATS FOR REPORTS OF COMPOSITION OF ENDING INVENTORIES

RECOVERY OF PU-238 SCRAP

FY 1967 finANCIAL PLAN NO. 1 FOR OPERATIONS

FY 1967 FIMANCIAL PLAN NO. 2 FOR OPERATIONS

FY 1967 finANCIAL PLAN NO. 3 FOR OPERATIONS

RESEARCH AND DEVELOPMENT FUNDIHG FOR

FY-68.

FY-1967 FINANCIAL PLAN NO. 4 FOR

OPERATIONS.

FY 1969 PROGRAM 02 BLDGET ASSUMPTIONS.

ALBUQUERQUE WORKING PAPERS.

RL MONTHLY PROUCTION REPORT JANUARY 1971

RL MONTHLY PROUCTION REPORT OCTOBER 1971

RL MONTHLY PROUCTION REPORT NOVEMBER 1971

RL MONTHLY PROUCTION REPORT DECEMBER 1971

RL MONTHLY PROUCTION REPORT FEBRUARY 1971
DM CREIGHTON

CC JONES

RD CARTER

UP MCCUE

GL RITTER

JS BUCKINGHAM

UC SCHMIDT

LH TAYLOR

LH TAYLOR

VR CHAPMAN

VH SMITH

HA MOULTHROP

PW SNITH

HA MOULTHROP

JP DUCKNORTH

VO DONIHEE

RL WALSER

WL GOOFREY

HE PARKER

HE PARKER

HE PARKER

DG MILLIAMS

DG HILLIAMS

HR FREITAG

JT CHRISTY

HE PARKER

he PARKER

OJ ELGERT

DG HILLIAMS

CRD

OJ ELGERT

SECR

D mCDONALD

JG MURPHY

JG MURPHY

JG MURPHY

SECR

SECR

SECR

SECR

SECR

JG MURPHY

SECR
$12 / 01 / 71$

$01 / 18 / 66$

$01 / 05 / 66$

$01 / 18 / 66$

$01 / 18 / 66$

$01 / 18 / 66$

$01 / 18 / 66$

01/18/66

$01 / 18 / 66$

$01 / 13 / 66$

$01 / 13 / 66$

$01 / 22 / 66$

$01 / 31 / 66$

$02 / 21 / 66$

$02 / 24 / 66$

$03 / 03 / 66$

04/01/66

07/07/66

07/07/66

07/27/66

$02 / 03 / 66$

$02 / 16 / 66$

03/02/66

$04 / 01 / 66$

$04 / 06 / 66$

$04 / 13 / 66$

05/17/66

$07 / 28 / 66$

08/01/66

08/05/66

$11 / 23 / 66$

$01 / 13 / 67$

$03 / 28 / 67$

$04 / 12 / 67$

$04 / 01 / 67$

02/11/71

10/01/71

$11 / 01 / 71$

03/11/71 
Page No. 225

$04 / 28 / 93$

LIST OF MANFORD-GENERATED CLASSIFIED

DOCUMENTS DATED BETWEEN 1/1/61 AND 12/31/72

Doc. No.

PPD-004053

PPD-004054

PPD-004055

PPD-004056

PPD-004057

PPD-004058

PPD-004059

PPD-0041101

PPD-0041102

PPD -0041103

PPD-0041104

$P P D-0041105$

PPD-0041106

PPD-0041107

PPD -0041108

$P P D-0041109$

PPD-0041110

PPD-0041111

PPD -0041112

$P P D-00413$

PPD-00421

PPD-00429

PPD -00436

$P P D-00445$

$P P D-00448$

PPD-00451

$P P D-00453$

PPO-00457

$P P D-00458$

PPD 00471

$P P D-00483$

$P P D-00485$

PPD-00489

PPD-004931

PPD-0049310

PPD-0049311

PPD-004932
TITLE

RL MONTHLY PROUCTION REPORT MARCH 1971

RL MONTHLY PROUCTION REPORT APRIL 1979

RL MONTHLY PROUCTION REPORT MAY 1971

RL. MONTHLY PROUCTION REPORT JUNE 1971

RL MONTHLY PROUCTION REPORT JULY 1971

RL. MONTHLY PROUCTION REPORT AUEUST 1971

RL MONTHLY PROUCTION REPORT SEPTEMBER

1971

COS MOWTHLY REPORT - JAN 1971

COB MONTHLY REPORT - FEB 1971

COB MONTHLY REPORT - MAR 1971

COB MONTHLY REPORT - APR 1971

MONTHLY REPORT - MAY 1971

MONTHLY REPORT - JUN 1971

MONTHLY REPORT - JUL 1971

COB MONTHLY REPORT - AUG 1971

MONTHLY REPORT - SEP 1971

COB MONTHLY REPORT - OCT 1971

MONTHLY REPORT - NOV 1971

MONTHLY REPORT - DEC 1971

RL MONTHLY STATUS AND PROGRESS REPORT, JANUARY 1971

RL MONTHLY STATUS AND PROGRESS REPORT, FEBRUARY 1971

RL MONTHLY STATUS AND PROGRESS REPORT, MARCH 1971

PLUTONIUM BLENDING PLANS

RL MONTHLY STATUS AND PROGRESS REPORT, MAY 1971

PATENT IDEA

PLUTONIUM METAL SPECIFICATIONS

RL MONTHLY STATUS AND PROGRESS REPORT, JUNE 1971

PROCUREMENT BY LASL FOR RL

RL MONTHLY STATUS AND PROGRESS REPORT, JULY 1971

RL. MONTHLY STATUS AND PROGRESS REPORT, SEPT 1971

PU MANAGEMENT FY 1972-1974

RL MONTHLY STATUS AMD PROGRESS REPORT, NOV 1971

RL MONTHLY STATUS AND PROGRESS REPORT, DEC 1971

RL MONTHLY STATUS AND PROGRESS REPORT, JAN 1972

RL MONTHLY STATUS AND PROGRESS REPORT OCTOBER 1972

RL MONTHLY STATUS AND PROGRESS REPORT NOVEMBER 1972

RL MONTHLY STATUS AND PROGRESS REPORT, FEB 1972

AUTHOR
JG MURPHY
JG MURPHY
JG MURPHY
JG MURPHY
JG MURPHY
JG MURPHY
JG MURPHY
JT CHRISTY
JY CHRISTY
JT CHRISTY
JT CHRISTY
JT CHRISTY
JT CHRISTY
JT CHRISTY
JT CHRISTY
JT CHRISTY
JT CHRISTY
JT CHRISTY
JT CHRISTY

DG MILLIAMS

CJ ELGERT

\begin{tabular}{|c|c|}
\hline SECR & $04 / 13 / 71$ \\
\hline SECR & $05 / 15 / 71$ \\
\hline SECR & $05 / 01 / 71$ \\
\hline SECR & $06 / 01 / 71$ \\
\hline SECR & $07 / 01 / 71$ \\
\hline SECR & $08 / 01 / 71$ \\
\hline SECR & $10 / 01 / 71$ \\
\hline SRD & $02 / 17 / 71$ \\
\hline SECR & $03 / 24 / 71$ \\
\hline SECR & $04 / 14 / 71$ \\
\hline SECR & $05 / 15 / 71$ \\
\hline SECR & $06 / 11 / 71$ \\
\hline SECR & $07 / 11 / 71$ \\
\hline SECR & $08 / 23 / 71$ \\
\hline SECR & $09 / 15 / 71$ \\
\hline SECR & $10 / 19 / 71$ \\
\hline SECR & $11 / 15 / 71$ \\
\hline SECR & $12 / 20 / 71$ \\
\hline SECR & $01 / 19 / 72$ \\
\hline SECR & $02 / 11 / 71$ \\
\hline SECR & $03 / 11 / 71$ \\
\hline SECR & $04 / 13 / 71$ \\
\hline SECR & $04 / 28 / 71$ \\
\hline SECR & $06 / 11 / 71$ \\
\hline SECR & $07 / 06 / 71$ \\
\hline SECR & $07 / 14 / 71$ \\
\hline SECR & $07 / 14 / 71$ \\
\hline SECR & $08 / 10 / 71$ \\
\hline SECR & $08 / 16 / 71$ \\
\hline SECR & $10 / 14 / 71$ \\
\hline SRD & $05 / 20 / 71$ \\
\hline SECR & $12 / 14 / 71$ \\
\hline SECR & $12 / 01 / 71$ \\
\hline SECR & $01 / 01 / 72$ \\
\hline SECR & $11 / 13 / 72$ \\
\hline SECR & $12 / 14 / 72$ \\
\hline SECR & $02 / 01 / 72$ \\
\hline
\end{tabular}


Page No. 226

$04 / 28 / 93$

LIST OF MANFORD-GENERATED CLASSIFIED

DOCANENTS DATED BETMEEN 1/1/61 AND 12/31/72

DOC. NO.

PPO-004933

PPD-004934

PPD-004935

PPD-004936

PPD-004937

PPD-004938

PPD-004939

PPD-0049401

$P P D-0049402$

$P P D-0049403$

PPO-0049404

PPD -0049405

PPD -0049406

PPD-0049407

$P P D-0049408$

PPD-0049409

PPD -0049410

$P P D-0049411$

$P P D-00498$

PRD -00003

PRD -00005

PRD -00013

PRD -00017

PRD-00021

PRD -00023

PRD -00032

PRD-00035

PRD-0004101

PRD -0004102

PRD -0004103 PRD-0004104 PRD -0004105 PRD -0004106 PRD -0004107 PRD-0004 108 PRD -0004109
TITLE

RL MONTHLY STATUS ANO PROGRESS REPORT, MARCH 1972

RL MONTHLY STATUS AMD PROGRESS REPORT, APRIL 1972

RL MONTHLY STATUS ANO PROGRESS REPORT, MaY 1972

RL MONTHLY STATUS AND PROGRESS

REPORT, . JUNE 1972

RL MONTHLY STATUS AND PROGRESS REPORT JULY 1972

RL MONTHLY STATUS AND PROGRESS REPORT AUEUST 1972

RL MONTHLY STATUS AND PROGRESS REPORT

AUTHOR

SEPTEMBER 1972

MONTHLY REPORT - JAN 1972

MONTHLY REPORT - FEB 1972

MONTHLY REPORT - MAR 1972

MONTHLY REPORT - APR 1972

MONTHLY REPORT - MAY 1972

MOWTHLY REPORT - JUN 9972

MONTHLY REPORT - JUL 1972

MONTHLY REPORT - NUG 1972

MOWTHLY REPORT - SEP 1972

MOWTHLY REPORT - OCT 1972

MONTHLY REPORT - NOV 1972

SHIPMENTS OF FUTURE VIRGIN PLUTONIUA

COMPARATIVE PILE PERFORMUNCE AUCUST 1968

COMPARATIVE PILE PERFORMANCE SEPTEMBER

1968

U-233 PRCOUCTION PROGRAM

SPIKE FUEL FOR UPPSS

URANIUM OXIDE SHIPMENTS

COMPARATIVE PILE PERFORMANCE OCTOBER

1968

COMPARATIVE PILE PERFoRMANCE NOVEMBER

1968

COMPARATIVE PILE PERformanCE DECEMBER

1968

COMPARATIVE PILE PERFORMANCE JANUARY

1970

COMPARATIVE PILE PERFORMANCE FEBRUARY

1969

COMPARATIVE PILE PERFORMAMCE MARCH 1969 COMPARATIVE PILE PERFORMAMCE APRIL 1969 COMPARATIVE PILE PERFORMANCE MAY 1969 COAPARATIVE PILE PERFORMAMCE JUNE 1969 COMPARATIVE PILE PERFORMANCE JULY 1969 COMPARATIVE PILE PERFORMANCE AUGUST 1969 COMPARATIVE PILE PERFORMANCE SEPTEMBER 1969

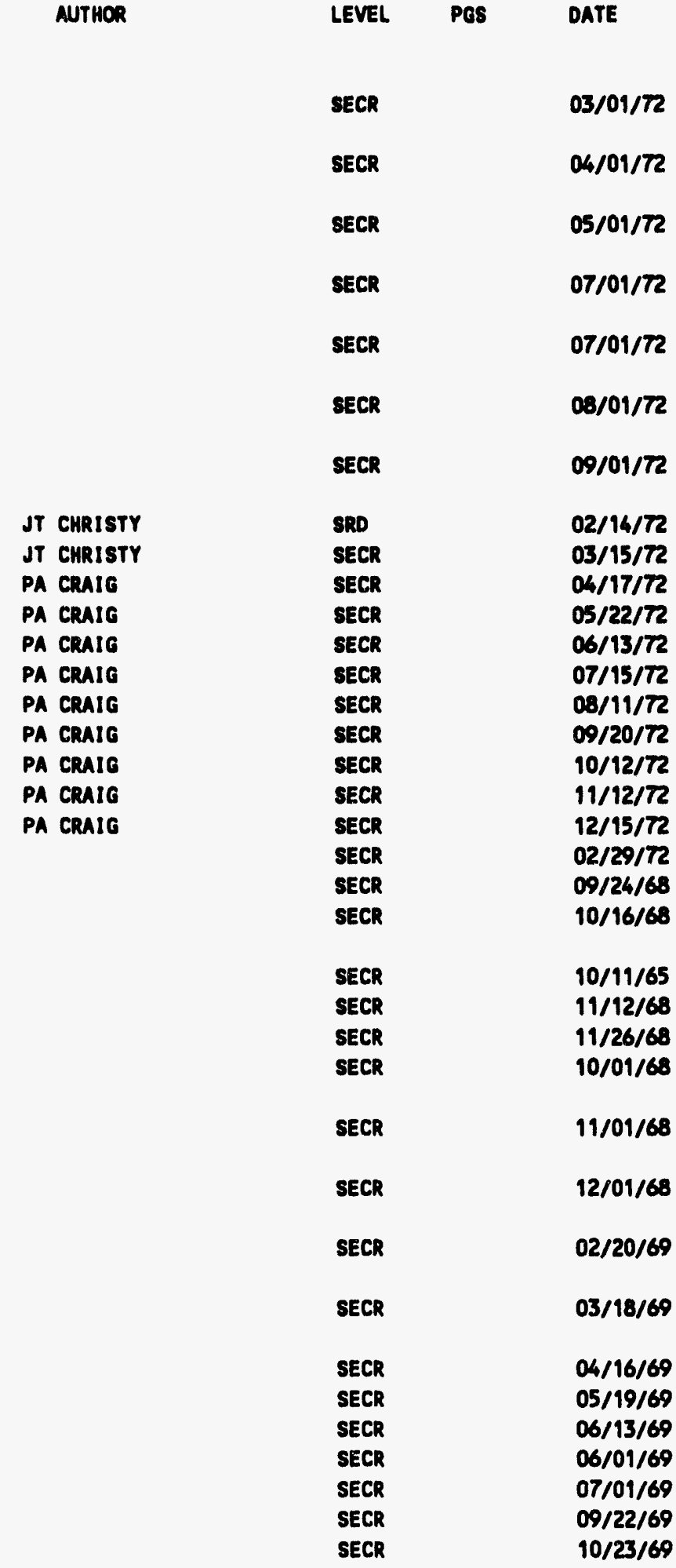


Page No. 227

$04 / 28 / 93$

LIST OF HANFORD-GENERATED CLASSIFIED

DOCUNENTS DATED BETWEEN 1/1/61 AND 12/31/72

DOC. NO.

\section{PRD-0004110}

PRD-0004111

PRD-0004113

PRD-0004114

PRD-0004915

PRD-0004116

PRD-0004117

PRD-0004118

PRD-0004119

PRD -0004120

PRD-0004121

PRD-00045

PRD -00050

PRD -00054

PRD -00056

PRD-00094

PRD -00113

Pun-00516

PLM-00530ADD

PLAM-00602

PLM-00603

PLAM-00616

PLIN-00617

PLII-00618

RL-0000208

$R L-00004-8$

$R L-000040$

$R L-0000410$

$R L-000050$

RL-0000601

RL- 0000602

$R L-C A O-00089$
TITLE

COMPARATIVE PILE PERformance OCtober 1969

COMPARATIVE PILE PERFORMANCE MOVEMBER 1969

COMPARATIVE PILE PERFORMANCE JANUARY 1970

COAPARATIVE PILE PERformanCE february 9970

COMPARATIVE PILE PERFCRMANCE MARCH 1970 COMPARATIVE PILE PERFORMANCE APRIL 1970 COAPARATIVE PILE PERFORMANCE MAY 1970 COAPARATIVE PILE PERFORMANCE JUNE 1970 COMPARATIVE P?LE PERforMaNCE JULY 1970 COMPARATIVE PILE PERForManCE AUEUST 1970 COMPARATIVE PILE PERFORMANCE SEPTEMBER 1970

U-233 PRCOUCTION PROGRAM

U-233 PRCOUCTION PROCRAM

THE DNR THORIA IRADIATION PROGRAM

U-233 PROOUCTION PROGRAM

SCHEDULE OF URANIUM OXIDE SHIPMENT TO

FERMALD

PRICING OF SPECIAL IRRADIATIONS

BAtTELLE ANO ARHCO DOCUMENTS ON PLOUSHARE

HANFORD RADIOACTIVE WASTE MAMAGEMENT

PLANS - AUCUST 1972

COMPARATIVE PILE PERFORMANCE JULY 1968

COMPARATIVE PILE PERFORMANCE JUNE 1968

SHIPPING \& RECEIVING REPORT - SS

MATERIAL

SHIPPING \& RECEIVING REPORT - SS

MATERIAL

SHIPPING \& RECEIVING REPORT - SS

MATERIAL

RICHLAND FIVE YEAR O2 RED PROGRAM CO-60 PROGRAM

RICHLAND FIVE-YEAR O2 RED PROGRAM

RICHLAMD FIVE-YEAR O2 RED PROGRAM SUMUARY

RICHLAND FIVE-YEAR O2 RED PROGRAM COLUMBIA RIVER STUDIES

RL 02 RED PROGRAM ANNUAL REPORT SUMMARY

RL 02 RED PROGRAM SEMIANMUAL REPORT SUMARY

RL 02 RED PROGRAM ANNUAL REPORT SUMUARY NUCLEAR MATERIALS REVIEH, CPO DEPLETED URANIUM SYSTEM, FIRST-SECOND QUARTERS FISCAL

\begin{tabular}{|c|c|c|}
\hline \multirow[t]{16}{*}{ AUTHOR } & LEVEL POS & DATE \\
\hline & SECR & $11 / 18 / 69$ \\
\hline & SECR & $11 / 01 / 69$ \\
\hline & SECR & $01 / 01 / 70$ \\
\hline & SECR & $03 / 17 / 70$ \\
\hline & SECR & $04 / 13 / 70$ \\
\hline & $\begin{array}{l}\text { SECR } \\
\text { SECR }\end{array}$ & $\begin{array}{l}04 / 01 / 70 \\
05 / 01 / 70\end{array}$ \\
\hline & SECR & $07 / 15 / 70$ \\
\hline & SECR & $08 / 01 / 70$ \\
\hline & SECR & $09 / 01 / 70$ \\
\hline & SECR & $09 / 01 / 70$ \\
\hline & SECR & $03 / 07 / 69$ \\
\hline & SECR & $04 / 04 / 69$ \\
\hline & SECR & $04 / 21 / 69$ \\
\hline & SECR & $05 / 16 / 69$ \\
\hline & SECR & $01 / 02 / 70$ \\
\hline $\begin{array}{l}\text { RICHLAND OPERATIONS } \\
\text { OFFICE, AEC }\end{array}$ & SRD & $04 / 23 / 70$ \\
\hline JE COODWIN & SRO & $06 / 29 / 72$ \\
\hline \multirow[t]{6}{*}{ NT KARAGIANES } & SECR & $08 / 01 / 72$ \\
\hline & SECR & $07 / 01 / 68$ \\
\hline & SECR & $06 / 01 / 68$ \\
\hline & SECR & $06 / 18 / 69$ \\
\hline & SECR & $12 / 13 / 68$ \\
\hline & SECR & $01 / 08 / 68$ \\
\hline CE BOWERS & SRD & $06 / 16 / 66$ \\
\hline ARHCO, PNL, UNC & SRD & $06 / 30 / 68$ \\
\hline OK EESTSON & SRD & $06 / 30 / 68$ \\
\hline \multirow[t]{4}{*}{ RG GEIER, JF HONSTEAD } & CRD & $06 / 30 / 68$ \\
\hline & SECR & $06 / 30 / 69$ \\
\hline & SECR & $12 / 31 / 69$ \\
\hline & SECR & $06 / 30 / 70$ \\
\hline LF MIRFS & SRD & $02 / 09 / 65$ \\
\hline
\end{tabular}


DOC. NO.

$R L-C A O-00091$

RL-CAO-00129

$R L-C A O-00152$

$R L-C 10-00165$

$R L-C A O-00165$

$R L-C A O-00191 A$

$R L-C A O-001918$

RL-CAO-00191C

$R L-C A O-001910$

RL-CAO-00191E

RL-CAO-00191F

RL-CAO-00219

$R L-C A O-00240$

RL-CAO-00243

$R L-C A O-00244$

$R L-C A O-00247$

$R L-C A O-00253$

$R L-C A O-00272$

$R L-C A O-00283$

$R L-C 10-00295$

$R L-C A O-00303$
TITLE

MUCLEAR MATERIALS REVIEH, CPO ENRICHED URANIUN SYSTEM, FIRST-SECOND QUARTERS FISCAL 1965

HISTORICAL PU MUF TREND AR.'LYSIS PURES AMD REDOX JULY 1960 - DEC 1964

MUCLEAR MATERIALS REVIEW - MEAPONS MAMUFACTURING - FIRST AND SECONO QUARTERS FISCAL 1965

HISTORICAL REVIEW PLUTONIUA RATIO RECEIPT MEASURENENT JULY 1960 THROUGH DECEMBER 1964.

HISTORICAL REVIEW PU RATION RECEIPT MEASUREMENT JULY 1960 - DEC 1964 PU RECLAMATION SOURCE DATA, FY 1966 JULY, 1965

PU RECLAMATION SOURCE DATA, FY 1966, AUEUST, 1965.

PU RECLAMATION SOURCE DATA, FY 1966 SEPTEMBER, 1965

PU RECLAMATION SOURCE DATA, FY 1966 OCTOBER, 1965.

PU RECLAMATION SOURCE DATA, FY 1966 NOVEMBER, 1965

PU RECL.AMATION SOURCE DATA, FY 1966, $12 / / 65$

HISTORICAL PLUTONIUM MUF TREND AMALYSIS, WEAPONS MANUFACTURING - JULY 1960 DECEMBER 1964

CPD MATERIAL BALANCE AND NET PRODUCTION REPORTS - MONTH OF JULY 1965 - FISCAL 1966

NUCLEAR MATERIALS REVIEH, CPO DEPLETED URANIUA SYSTEM, FISCAL YEAR 1965.

NUCLEAR MATERIALS REVIEU, MEAPOWS MARUFACTURING - THIRD AND FOURTH QUARTERS FY 1965.

NUCLEAR MATERIALS REVIEW, ENRICHED URANIUM SYSTEM - FISCAL YEAR 1965 CPD MATERIAL BALANCE AND MET PROOUCTION REPORT - MONTH OF AUEUST 1965 - FISCAL YEAR 1966

CPD - MATERIAL BALANCE ANO MET PROOUCTION REPORT MONTH OF SEPTEMBER 1965 - FISCAL YEAR 1966

CPD - MATERIAL BALANCE AND MET PROOUCTION REPORTS - MONTH OF OCTOBER 1965 - FISCAL YEAR 1966

CPD MATERIAL BALANCE AND NET PROOUCTION REPORTS - NOVEMBER, 1965 - FY 1966

CPD MATERIAL BALANCE AND NET PRCOUCTION REPORTS -DECEMBER 1965 - FY-1966

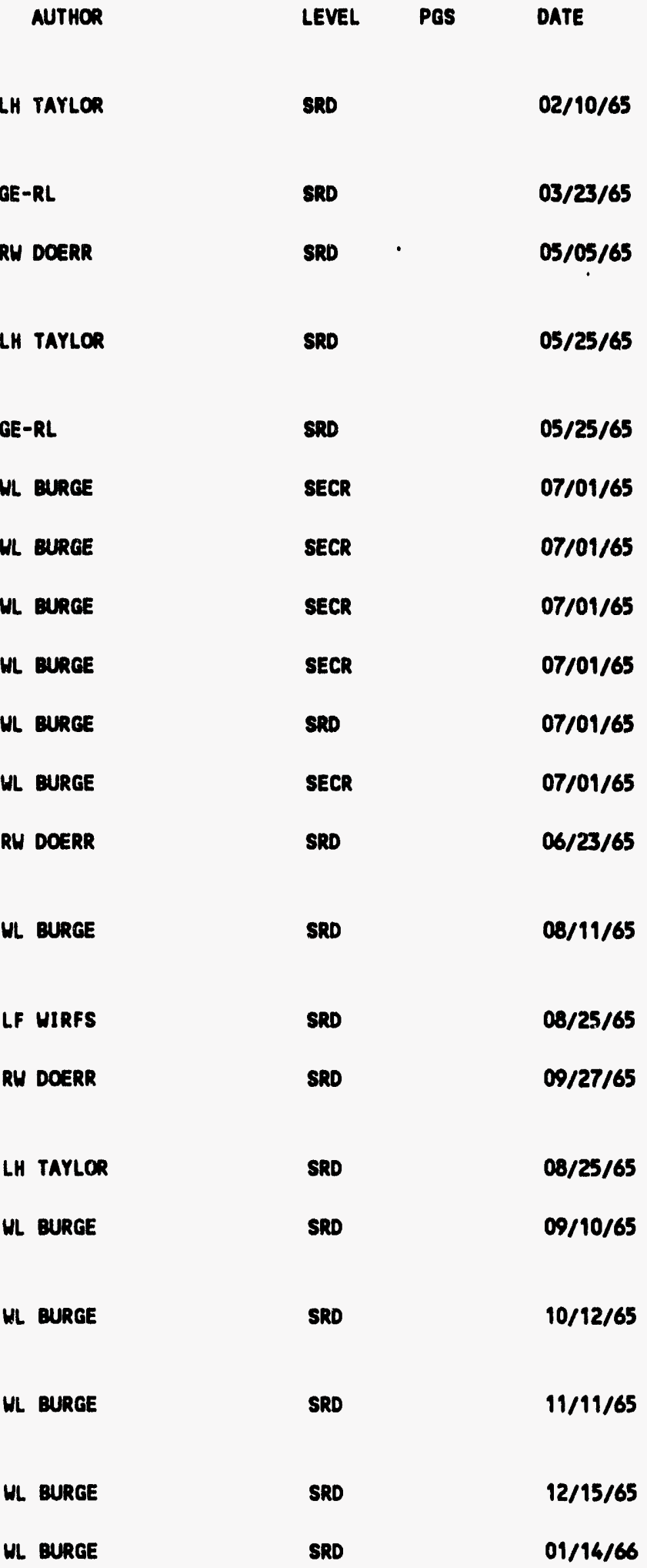


Page No. 229

$04 / 28 / 93$

LIST OF HANFORD-GENERATED CLASSIFIED

DOCUMENTS DATED BETWEEN 1/1/61 AND 12/31/72

DOC. NO

TITLE

$R L-C A O-00303$

RL-CAO-00310

$R L-C A O-00311$

RL-CAO-00312

$R L-C A O-N-00003$

RL-CPD-00001 REELI

RL-CPO-00001 REEL2

RL-CPD-00001 REEL3

RL-CPD-00001 REEL4

RL-CPO-00001 REEL5

RL-CPO-00002 REEL1

RL-CPD-00002 REEL2

RL-CPD-00002 REEL3

RL-CPD-00002 REEL4

RL-CPD-00002 REEL5

RL-CPD-00002 REEL6

RL-CPD-00003 REEL 1

RL-CPD-00004 REEL1

RL-CPD-00004 REEL2

RL-CPD-00004 REEL3

RL-CPD-00004 REEL4

RL-GEN-00022REV1

RL-GEN-00022SUP1

REV1

RL-GEN-00022SUP 10

REVI

RL-GEN-00022SUP 11 REV1 PROGRAM

PROGRAM
CPD MATERIAL BALANCE AND NET PRODUCTION REPORTS -DECEMBER 1965 - FY-1966

ISOCHEM INC. - COMPOSITION OF TAKEOVER INVENTORY

FIMAL ENDIMG INVENTORIES - CPD DECENBER, 1965

MUCLEAR MATERIALS PRCOUCTION REPORT DECEMBER 1965

PERSONAL NOTES

SEPTEMBER REACTOR \& SEPARATIONS

CLASSIFIED MEeETIMG tAPES (U)

SEPTEMBER REACTOR \& SEPARATIONS

CLASSIFIED MEEETING TAPES (U)

SEPTEMBER REACTOR \& SEPARATIONS

CLASSIFIED MEEETING TAPES (U)

SEPTEMBER REACTOR \& SEPARATIONS

CLASSIFIED MEEETING TAPES (U)

SEPTEMBER REACTOR \& SEPARATIONS

CLASSIFIED MEEETING TAPES (U)

SEPTEMBER REACTOR \& SEPARATIONS

CLASSIFIED MEEETIMG TAPES (U)

SEPTEMBER REACTOR \& SEPARATIONS

CLASSIFIED MEEETIMG TAPES (U)

SEPTEMBER REACTOR \& SEPARATIONS

CLASSIFIED MEEETING TAPES (U)

SEPTEMBER REACTOR \& SEPARATIONS

CLASSIFIED MEEETING TAPES (U)

SEPTEMBER REACTOR \& SEPARATIOWS

CLASSIFIED MEEETING TAPES (U)

SEPTEMBER REACTOR \& SEPARATIONS

CLASSIFIED MEEETING TAPES (U)

SEPTEMBER REACTOR \& SEPARATIONS

CLASSIFIED MEEETING TAPES (U)

SEPTEMBER REACTOR \& SEPARATIONS

CLASSIFIED MEEETIMG TAPES (U)

SEPTEMBER REACTOR \& SEPARATIONS

CLASSIFIED MEEETING TAPES (U)

SEPTEMBER REACTOR \& SEPARATIONS

CLASSIFIED MEEETING TAPES (U)

SEPTEMBER REACTOR \& SEPARATIONS

CLASSIFIED MEEETING TAPES (U)

RICHLAND INTEGRATED PROCESS DEVELOPMENT

RICHLAND INTEGRATED PROCESS DEVELOPMENT

PROGRAM DEFENSE PLUTONIUM MISSION

RICHLAND INTEGRATED PROCESS DEVELOPMENT

PROGRAM - HIGH PONER DENSITY FUEL

RICHLAND INTEGRATED PROCESS DEVELOPMENT

PROGRAM - SMALL REACTOR MOOERNIZATION

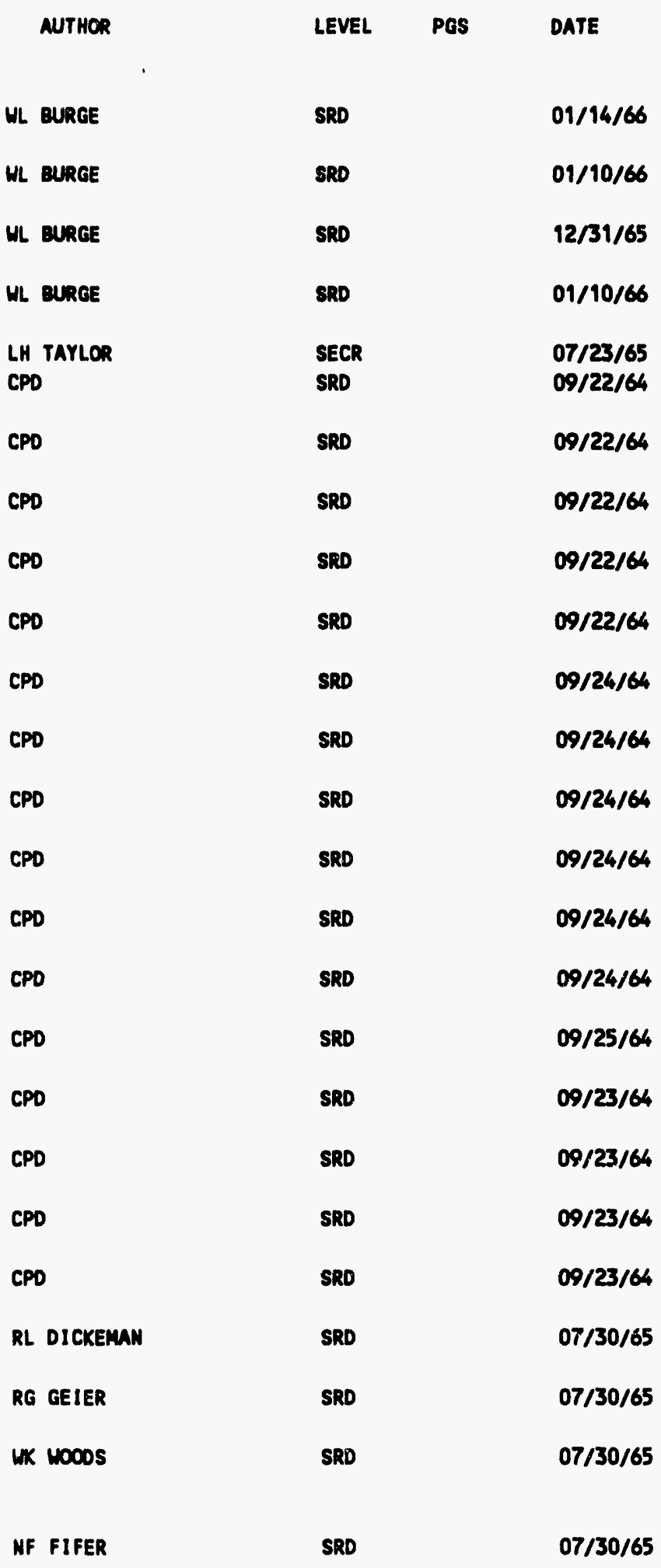

UNCLASSIFIED 
Page No. 230

$04 / 28 / 93$

LIST OF HANFORD-GENERATED CLASSIFIED

DOCUMENTS DATED BETWEEN 1/1/61 AMD 12/31/72

TITLE

AUTHOR

LEVEL.

PGS

DATE

RL-GEN-00022SUP12

REV1

RL-GEN-00022SUP13

REV1

RL-GEN-00022SUP 14

REV1

RL-GEN-00022SUP2

REV1

RL-GEN-00022SUP3

REV1

RL-GEN-00022SUP4

REVI

RL-GEN-00022SUP5

REV1

RL-GEN-00022SUP6

REV1

RL-GEN-00022SUP7

REV1

RL-GEN-000225UP8

REV1

RL-GEN-00022SUP9

REV1

RL-GEN-00026

RL-GEN-00028

RL-GEN-0022 REV1

RL-GEN-0022 SUP1

REV1

RL-GEN-0022 SUP10

REV1

RL-GEN-0022 SUP11

REVI

RL-GEN-0022 SUP12

REV1

RL-GEN-0022 SUP 14

REV1

RL-GEN-0022 SUP2

REV1

RL-GEN-0022 SUP3

REV1

RL-GEN-0022 SUP4

REV1

RL-GEN-0022 SUP5

REV1

RL-GEN-0022 SUP6

REVI

RL-GEN-0022 SUP7

REV1
RICHLAND INTEERATED PROCESS DEVELOPNENT PROGRAM - FUNDAMENTAL AND GENERAL STUDIES

RICHLAND INTEGRATED PROCESS DEVELOPMENT

PROGRAM - WULLEAR SAFETY PROCRAM

RICHLAND INTECRATED PROCESS DEVELOPMENT

PROGRAM - COLLOABIA RIVER STUDIES

RICHLAND INTEGRATED PROCESS DEVELOPMENT

PROGRAM COPRODUCT MISSIOM

RICHLAND INTEGRATED PROCESS DEVELOPMENT

PROGRAM INTEGRATED SITE OPERATION

RICHLAND INTEGRATED PROCESS DEVELOPMENT

PROGRAM - MON-DEFENSE PLUTONIUM MISSION

RICHLAND INTEGRATED PROCESS DEVELOPNENT

PROGRAM PLUTONIUM BURNING PROGRAM

RICHLAND INTEGRATED DEVELOPMENT PROGRAM

\section{P 238 PROGRAM}

RICHLAND INTEGRATED PROCESS DEVELOPMENT PROGRAM U-233 PROCRAM

RICHLAND INTEGRATED PROCESS DEVELOPNENT

PROGRAM - CO-60 PROGRAM

RICHLAND INTEGRATED PROCESS DEVELOPMENT

PROGRAM - PO-210 PROGRAM

PLUTONIUN COSTS FOR NON-STANDARD

ISOTOPIC ASSAYS

BUDGET STLOY

RICHLAND INTEGRATED PROCESS DEVELOPNENT PROGRAM

RICHLAND INTEGRATED PROCESS DEVELOPHENT PROGRAM DEFENSE PLUTONILN MISSION

RICHLAMD IMTEGRATED PROCESS DEVELOPMENT

PROGRAM HIGH POUER DENSITY FUEL PROGRAM

RICHLAND INTEGRATED PROCESS DEVELOPMENT

PROGRAM SMALL REACTOR MODERNIZATION

RICHLAND INTEGRATED PROCESS DEVELOPMENT

PROGRAM FUNDAMENTAL AND GENERAL STUDIES

RICHLAND INTEGRATED PROCESS DEVELOPMENT

PROGRAM COLUMBIA RIVER STUDIES

RICHLAND INTECRATED PROCESS DEVELOPMENT PROGRAM COPRODUCT MISSION

RICHLAMD INTEGRATED PROCESS DEVELOPMENT PROGRAM INTEGRATED SITE OPERATION

RICHLAND INTEGRATED PROCESS DEVELOPNENT PROGRAM HOW-DEFENSE PLUTONILN MISSION RICHLAND INTEGRATED PROCESS DEVELOPMENT PROGRAM PLUTONIUM BURNING PROGRAM

RICHLAND INTEGRATED PROCESS DEVELOPMENT PROGRAM PU-238 PROGRAM

RICHLAND INTEGRATED PROCESS DEVELOPMENT PROGRAM U-233 PROGRAM
RS PAUL.

CUSI

$07 / 30 / 65$

MC LEVERETT

SRD

RG GEIER

SRD

GC FULLMER, EG PIERICK

SRD

OF MILL

SRD

RO GUMPRECHT

SRD

GF OUSLEYI

SECR

R NILSON

SRD

WK wooos

SRD

LE KUSLER

SRD

WA BLANTON

SRD

WE JOHMSON

SRD

WE JOHMSON

SRD

SRD

SRD

GEIER RJ

woods ux

SRD

FIFER NF, 1000S WK

SRD

CRD

22

GEIER RL', FOSTER RF

CRD

29

FULLMER GC, PIERICK EG

SRD

HILL OF

SRD

38

43

GUMPRECHT RO, RICHES JH SRD 18

18

OUSLEY GF, NILSON R

SRD

19

NILSON R

SRD

63

SRD

18

07/30/65

$07 / 30 / 65$

$07 / 30 / 65$

07/30/65

$07 / 30 / 65$

07/30/65

07/30/65

$07 / 30 / 65$

07/30/65

07/30/65

09/01/65

10/15/65

$07 / 30 / 65$

$07 / 30 / 65$

07/30/65

$07 / 30 / 65$

$07 / 30 / 65$

$07 / 30 / 65$

$07 / 30 / 65$

$07 / 30 / 65$

$07 / 30 / 65$

$07 / 30 / 65$

$07 / 30 / 65$

wo00s UK

07/30/65 
Page No. 231

$04 / 28 / 93$

LIST OF HANFORD-GENERATED CLASSIFIED

DOCUMENTS DATED BETHEEN 1/1/61 AND 1i2/31/72

DOC. NO.

TITLE

RL-GEN-0022 SUP8

REV1

RL-GEN-0022 SUP9

REV1

RL-GEN-0033

RL-GEN-0088803

RL-GEN-0088804

RL-GEN-0088805

RL-GEN-0088806

RL-GEN-0088807

RL-GEN-0088809

RL-GEN-0088810

RL-GEN-0088812

RL-GEN-00900

RL-GEN-00912

RL-GEN-00916

RL-GEN-00956

RL-GEN-009601

RL-GEN-009602

RL-GEN-00969

RL-GEN-00974

RL-GEN-00976

RL-GEN-01023

RL-GEN-01035

RL-GEN-01037

RL-GEN-01038 5

RL-GEN-01038 6

RL-GEN- 210387

RL-GEN-01038 8

RL-GEN-01065 RD 1966 1966

1966
RICHLAND INTEGRATED PROCESS DEVELOPNENT PROGRAM CO-60 PROGRAM

RICHLAND INTEGRATED PROCESS DEVELOPMENT PROGRAM PO-210 PROGRAM

RICHLAND INTEGRATED PROCESS DEVELOPMENT PROGRAM DEFENSE PLUTONIUM MISSION HANFORD ATOAIC PRODUCTS DEPARTMENT MCNTHLY REPORT MARCH 1966

HRW ORD ATOMIC PRCOUCTS DEPARTMENT MO::THLY REPORT APRIL 1966

HANFORD ATOMIC PRCOUCTS DEPARTMENT MONTHLY REPORT MAY 1966 HANFORD ATOAIC PRODUCTS DEPARTMENT MONTHLY REPORT JUNE 1966 HANFORD ATOMIC PROOUCTS DEPARTMENT MONTHLY REPORT JULY 1966 HANFORD ATOMIC PRODUCTS DEPARTMENT MONTHLY REPORT

HANFORD ATOAIC PRODUCTS DEPARTMENT MOWTHLY REPORT OCTOBER 1966 HANFORD ATOMIC PRODUCTS DEPARTMENT MOWTHLY REPORT DECEMBER 1966 REVIEW OF N REACTOR COPRODUCT DEMONSTRATION PROGRAM GEMERAL FINAMCIAL STATEMENTS - MARCH

COST BUDGET REPORY - MARCH 1966

REPORT TO THE FEDC WORKING COMMITTEE FOR

THE PERIOD JANUARY TO APRIL 1966

RADIOLOGICAL STATUS OF THE N-REACTOR FOR MARCH 1966

RADIOLOGICAL STATUS OF THE N-REACTOR FOR APRIL 1966

SUPPLEMENT FUNDING COPRODUCT TARGET EXTRACTION

COMPOSITION OF ENDING INVENTORIES APRIL

COST BUDGET REPORT - APRIL 1966

COMPOSITION OF ENDING INVENTORIES MAY

GENERAL ELECTRJC COMPANY HANFORD ATOMIC PRODUCTS DEPARTMENT BUDGET BOOK FY-1967-68

COST BUDGET REPORT MAY 1966

COPRCDUCT TWO LOAD DEMONSTRATION SCHEDULE

COPRODUCT DEMONSTRATION SCHEDULE COPROOUCT DEMONSTRATION SCHEDULE COPROOUCT DEMONSTRATION SCHEDULE PRCOUCTION TEST PT-NR-TS COPRCOLLT THO LOAD
AUTHOR

LEVEL

PAS

DATE

\begin{tabular}{llll} 
KUSLER LE, RICHES JH & SRD & 11 & $07 / 30 / 65$ \\
BLANTON WA & SRD & 24 & $07 / 30 / 65$ \\
$\begin{array}{l}\text { LEVERETT MC, NMBROSE IiW, } \\
\text { TOMLINSON RE } \\
\text { RL DICKEMAN }\end{array}$ & 34 & $01 / 17 / 66$ \\
RL DICKEMAN & SRD & & $03 / 01 / 66$ \\
RL DICKEMAN & SRD & $04 / 01 / 66$ \\
RL DICXEMAN & SRD & & $05 / 01 / 66$ \\
RL DICKEMAN & SRD & $06 / 01 / 66$ \\
\hline
\end{tabular}

RL DICKEMAN SRD

$09 / 01 / 66$

RI. DICKEMAN SRD

$10 / 01 / 66$

RL DICKEMAN SRD

$12 / 01 / 66$

RL DICKEMAN

SECR

$04 / 01 / 66$

EF HULTGRENM

CRD

CRD

JL GNmion

M LEUIS

MEN HENICKSOM

CRD

HENDRICKSON

CONF

MC LEVERETT

SRD

JW HANDSHUH

SRD

JL GANAON

JW MANDSHUH

CRD

SRD

RE MIDDNUGH

SRD

IL GAMHON

CRD

EE LEITZ/DL PURSLEY

SRD

EE LEITZ/DL PURSLEY

SRD

EE LEIT2

EE LEITZ/DL PURSLEY

EE LEITZ/EG PIERICK
$04 / 13,66$

$04 / 13 / 66$

05/06/66

$04 / 08 / 66$

05/13/66

05/09/66

05/10/66

$05 / 11 / 66$ $06 / 15 / 66$

$06 / 15 / 66$

$06 / 09 / 66$ 12/22/66

$02 / 10 / 67$ $03 / 24 / 67$ $06 / 27 / 67$ $07 / 20 / 66$ 
Page No. 232

$04 / 28 / 93$

LIST OF HANFORD-GEMERATED CLASSIFIED

DOCUMENTS DATED BETHEEN 1/1/61 AND 12/31/72

DOC. NO.

RL-GEN-01087

RL-GEN-01102

RL-GEN-01116

RL-GEN-0113

RL.-GEN-0113:

RL-GEN-01152

RL-GEN-01155

RL-GEN-01168

RL-GEN-01172

RL-GEN-01180

RL-GEN-01180 REV1

RL-GEN-01202

RL-GEN-01214

RL-GEN-01218

RL-GEN-01219

RL-GEN-01235 RD

RL-GEN-01247

RL-GEN-01251

RL-GEN-01271 RD

RL-GEN-01281

RL-GEN-01298

RL-GEN-0130001

RL-GEN-0130003

RL-GEN-0130004

RL-GEN-0130005

RL-GEN-01407

RL-GEN-01467
TITLE

COST 3UDGET REPORT JUNE 1966

COMPOSITION OF ENDING INVENTORIES JULY 1966

PROGRAM FOR ATTAINING ELECTROREDUCTION

AND CASTING CAPABILITIES FOR THE

N-REACTOR FUEL STREAM AT HANFORD

COST ANALYSES - N REACTOR

TRIP REPORT SHIPPING AND MATERIAL

HANDLING REQUIREMENTS FOR

COPROOUCT TARGET CORES

COMPOSITION OF ENDING INVENTORIES AUQUST

1966

a PROPOSEd PLAN FOR INTEgRATED PLANT

AUTOMATION

COPRODUCT EXTRACTION FACILITY

N FINANCE INPUT FOR REVISED COPRODUCT DEMONSTRATION PROCRAM

(RL-GEN-900)

PROGRAM CONSIDERATIONS N-REACTOR OCTOBER 3. 1966

PROGRAM CONSIDERATIONS N-REACTOR

CALCULATION OF MUCLEAR HEAT GENERATION IN A CONTROL ROD RESEARCH AND DEVELOPMENT ALLICATION STREAMLIHE THE N-FUEL CYCLE

CHANGE IN FUEL IRRADIATION PROGRAM PRELIMINARY STATISTICAL AMALYSIS OF MARK I FUEL PERFORMANCE REPORT ON COST SAVINGS FROM PROCESS DEVELOPNENT

SUPPORTING INFORMATION "PROGRAM CONSIDERATIONS - N-REACTOR C-REACTOR OVERBORE FACILITY - TUBE TEMPERATURE STLOY HANFORD ATOMIC PROOUCTS DEPARTMENT SEMIANMUAL REPORT OF RESEARCH AND DEVELOPMENT

PROGRAM O2 PLANNING - FY 1969 THROUGH FY 1973

HANFORD ATOMIC PROOUCTS DEPARTMENT MONTHLY REPORT JAMUARY 1967 HANFORD ATOAIC PRODUCTS DEPARTMENT MONTHLY REPORT MARCH 1967 HANFORD ATOMIC PROOUCTS DEPARTMENT MONTHLY REPORT APRIL 1967

HANFORD ATOMIC PROOUCTS DEPARTMENT MONTHLY REPORT MAY 1967

N REACTOR PROFITABILITY ANALYSIS RADIATION INCIDENT
AUTHOR

JL GAMHON

JH HANDSHUH

AC CALLEN

RL DICKEMAN

FE HATCH

JW HAMDSHUH

SRD

DH LEIBY/CF POOR

CRD

RL DICKEMAN

SRD

J MILNE

RL DICKEMAN

SRD

RL DICKEMAN

Ca mansius

SRD

SRD

RL DICKEMAN

CRD

HE JACKEL/TD MAYLOR/JH RICHES

RL DICKEMAN

RR BLOOMSTRAND

J MILNE

WJ DOWIS, RD SHIMER

JD AGAR

R\&E STAFF

RL DICKEMAN

SRD

RL DICKEMAN

SRD

SRD

SRD

SRD

SRD

CONF
DATE

$07 / 15 / 66$ $08 / 15 / 66$

$08 / 09 / 66$

$08 / 30 / 66$ $08 / 31 / 66$

$09 / 15 / 66$

$01 / 17 / 67$

$09 / 21 / 66$

$09 / 27 / 66$

$10 / 03 / 66$

$11 / 15 / 66$

$12 / 09 / 66$

$10 / 26 / 66$

$10 / 28 / 66$

$10 / 27 / 66$

$11 / 03 / 66$

$11 / 14 / 66$

$11 / 16 / 66$

$11 / 30 / 64$

$12 / 15 / 66$

$12 / 19 / 66$

$02 / 10 / 67$

03/01/67

04/01/67

$05 / 01 / 67$

$02 / 01 / 67$

$02 / 23 / 67$ 
Page No. 233

$04 / 28 / 93$

LIST OF HANFORD-GENERATED CLASSIFIED

DOCUMENTS DATED BETWEEN 1/1/61 AND 12/31/72

DOC. NO.

RL-GEN-01482

RL-GEN-01510

RL-GEN-01534

RL-GEN-01551

RL-GEN-01567

RL-GEN-01571

$R L-G E N-01573$

RL-GEN-01578

RL-GEN-01583

RL-GEN-01658

RL-GEN-01676

RL-GEN-01748

RL-GEN-01750

RL-GEN-01758

RL-GEN-01772

RL-GEN-01778

RL-GEN-0883

RL-GEN-0888 10

RL-GEN-0888 12

RL-GEN-0888 3

RL-GEN-0888 4

RL-GEN-0888 5

RL-GEN-0888 6

RL-GEN-0888 7

RL-GEN-0888 9

RL-GEN-0956

RL-GEN-1155
TITLE

PROOUCTION PROGRAM FOR IRRADIATING ALL HANFORD-PRCOUCED NEPTUNIUA

OPERATING COST COMIITMENTS MADE IN RL-MRD-00298 AND RL-GEN-900 AVERAGE POWER DENSITIES FOR VARIOUS REACTCRS

COPROOUCT EXTRACTION FACILITY

THE 68 SPARE CHAMNELS AS A GENERAL

ISOTOPE PRCOUCTION FACILITY

EXTRACTION OF TRITIUN FRON (L A102)

TARGETS

FLUORINE CONTENTS OF LITHIUM ALLMIMATE PONDER AND SINTERED PELLETS

UNIT PRODUCT COST

FY-1968 - 02 RESEARCH AND DEVELOPMENT

FUNDING

CAPITAL AND OPERATING ESTIMATES FOR FIVE PROOUCTION CASES LIAIO 2 TARGET FACILITY HUIR-1868

CLASSIFICATION OF DOCUMENT RL-GEN-01748

$$
\text { "SUMAARY - PUR-241- COPRODUCT }
$$

WARP EVALUATION"

DUAL PURPOSE PLANT ECONONICS

MAJOR OBJECTIVES AND COMITMENTS FOR THE COPRCDUCT PROGRAM

N PLANT TRADEOFF OF PU PROOUCTION FOR STEAM PRESSURE IMPROVEMENT TO INCREASE ELECTRIC POUER CAPABILITY

AEC CASE S-35, 174 - HWIR-1874

5-HZA-HYA-3-66-1F

HANFORD ATOMIC PRODUCTS DEPARTMENT

MONTHLY REPORT OCTOBER 1966

HANFORD ATOMIC PROOUCTS DEPARTMENT

MONTHLY REPORT DECEMBER 1966

HANFORD ATOMIC PRODUCTS DEPARTMENT

MONTHLY REPORT MARCH 1966

HANFORD ATOMIC PRCOUCTS DEPARTMENT

MONTHLY REPORT APRIL 1966

HANFORD ATOAIC PROOUCTS DEPARTMENT MONTHLY REPORT MAY 1966

HANFORD ATONIC PRODUCTS DEPARTMENT

MOWTHLY REPORT JUNE 1966

HANFORD ATONIC PRODUCTS DEPARTMENT

MONTHLY REPORT JULY 1966

HANFORD ATOMIC PRODUCTS DEPARTMENT

MONTHLY REPORT SEPTEMBER 1966

REPORT TO THE FEDC WORKING COWIITEE FOR

THE PERICD JAMUARY TO APRIL 1966

A PROPOSED PLAN FOR INTEGRATED PLANT AUTOMATION
AUTHOR

LEVEL

PGS

DATE

$03 / 22 / 67$

$12 / 15 / 66$

03/21/67

RP SHARP

SRD

$03 / 24 / 67$

RL DICKEMAN

SRD

SRD

$04 / 12 / 67$

$04 / 10 / 67$

RR STUDER

$04 / 10 / 67$

WJ DOWIS

$04 / 11 / 67$

RL DICKEMAN

SRD

CRD

SRD

SRD

R COOPERSTEIN

JP KEENAN

CRO

WJ DOUIS/RD SHIMER

RD SHIMER

SRD

CRD

$06 / 16 / 67$

$06 / 23 / 67$

HJ DONIS

SRD

$04 / 10 / 67$

R COOPERSTEIN

BRONN DP

DICKEMAN RL

SRD

$06 / 30 / 67$

$03 / 31 / 66$

DICKEMAN RL

DICKEMAN RL

SRD

DICKEMAN RL

SRD

68

SRD

67

$06 / 10 / 66$

DICKEMAN RL

SRD

61

$07 / 10 / 66$

DICKEMAN RL

SRD

67

$08 / 10 / 66$

DICKEMAN RL

SRD

65

$10 / 10 / 66$

LEWIS M

SRD

34

$05 / 06 / 66$

POOR CF

CRD

43

$01 / 17 / 67$ 
Page No. 234

$04 / 28 / 93$

LIST OF HANFORD-GENERATED CLASSIFIED

DOCLMENTS DATED BETUEEN 1/1/61 AND 12/31/72

DOC. HO.

RL-GEN-1180 REV1

RL-GEN-1214

RL-GEN-125

RL-GEN-1281

RL-GEN-1300

RL-GEN-1300 3

$R L-G E N-13004$

RL-GEN-1300 5

RL-GEN-1398

RL-GEN-1482

RL-GEN-1574

RL-GEN-1578

RL-GEN- 1674

RL-GEN-1699 RD

RL-GEN- 1768

RL-GEN-919

RL-GEN-978

RL-GEN-H-00011

RL-GEN-N-00021

RL-GEN-N-00022

RL-MRD-00025

RL-NRD-00027RD

RL-NRD-00031

RL-NRD-00066SUP1

RL-NRD-00066SUP1

RL-NRD-00066SUP2

RL-NRD-00075RD

RL-NRD-001 1
TITLE

PROGRAM CONSIDERATIONS N-REACTOR RESEARCH AND DEVELOPMENT ASSOCIATION SUPPORTING INFORMATION - PROGRAM CONSIDERATION - N-REACTOR HANFORD ATONIC PROOUCTS DEPARTMENT SEMIANNUAL REPORT OF RESEARCH AND DEVELOPMENT

HANFORD ATONIC PRODUCTS DEPARTMENT MONTHLY REPORT JANUARY, 1967 HANFORD ATONIC PROOUCTS DEPARTMENT MOWTHLY REPORT MARCH 1967 HANFORD ATONIC PROOUCTS DEPARTMENT MOWTHLY REPORT APRIL 1967 HANFORD ATOMIC PROOUCTS DEPARTMENT MONTHLY REPORT MAY 1967

BORESCOPE EXANINATION OF PROCESS TUBE 2959

PRCDUCTION PROGRAM FOR IRRADIATING ALL HANFORD-PROOUCED MEPTUNIUM HANFORD ATOMIC PRCDUCTS DEPARTMENT PLANT AND CAPITAL EQUIPMENT BUDGET SUBMISSION FOR FY 1969 AND REVISION OF FY 1968 UNIT PRODUCT COST

TRITIUM RELEASE FROM EXPOSED METAL-ALLOY TARGETS

CONTRIBUTIONS TO RL-3-0 SUMUARY NUCLEAR SAFETY PROGRAM - MISSION 8 BASIC HAZARDS IHFORMATION

OUTER ANNULUS IMBALANCE STUDY - MARK I FUEL

CLAD THICKMESS VARIATION N-REACTOR FUEL ELEMENTS

CO-PRODUCT DEMONSTRATION NOTES

PERSONAL NOTES

PERSONAL MOTES

BY-PRCOUCT HCR TEMPERATURE STLOY -

HANFORD REACTORS

DEPRECIATION SCHEDULES

PENALTY FOR BLENDING $.947: 1.25$ NPR

FUEL DURING CHEMICAL PROCESSING

DESIGN STUDY REORT COPRODUCT EXTRACTION FACILITY N-REACTOR, 100-N AREA

DESIGN STUDY REORT COPRODUCT EXTRACTION

FACILITY N-REACTOR, 100-N AREA

BUDGET STUDY REPORT TRITIUM EXTRACTION

FACILITY N-REACTOR 100-N AREA

COMPARISON OF SRP 1963 AND 1964 REACTOR COST STUDIES

N-REACTOR DEPARTMENT MONTHLY REPORT OCTOBER, 1964

$\begin{array}{llll}\text { AUTHOR } & \text { LEVEL } & \text { PGS } & \text { DATE } \\ & & & \\ \text { OICKEMAN RL } & \text { SRD } & 41 & 11 / 15 / 66 \\ \text { DICKEMAN RL } & \text { CRD } & 3 & 10 / 26 / 66 \\ \text { OWIS HJ, SHIMER RD } & \text { SRD } & 32 & 11 / 16 / 66 \\ & & & \\ & \text { SRD } & 61 & 12 / 15 / 66\end{array}$

DICKEMAN RL

SRD

70

$02 / 10 / 67$

DICKEMAN RL

SRD

$04 / 10 / 67$

DICKEMAN RL

SRD

74

$05 / 10 / 67$

DICKEMAN RL

SRD

51

$06 / 10 / 67$

COOPERSTEIN R, MEUBY FM CRD

5

$01 / 26 / 67$

CONSTABLE DH, CURTISS DH SRD

17

$03 / 22 / 67$

JONES AM, IMGRAM NE

SRD

34

$04 / 19 / 67$

DOWIS WJ

COOPERSTEIN $R$

SRD

CRD

$04 / 11 / 67$

$05 / 16 / 67$

MILLER MR

SRD

$06 / 19 / 67$

HENDRICKSON

SRD

LONG JH

CRD

SMITH EA

CRD

SECR

EE LEITZ

SECR

CONF

SRD

JD AGAR

SRD

SRD

RD SHIMER

CE LOVE, MG PATRICK SRD

$06 / 17 / 65$

CE LOVE, MG PATRICK SRD

$06 / 17 / 65$

CE LOVE - R COOPERSTEIN - SRD

$03 / 24 / 67$

DD STEPMEUSKI

RD SHIMER

SRD

$11 / 18 / 64$

DICKEMAN RL

SRD

46

$11 / 10 / 64$ 
Page No. 235

$04 / 28 / 93$

LIST OF HANFORD-GENERATED CLASSIFIED

DOCUMENTS DATED BETUEEN 1/1/61 AND 12/31/72

DOC. NO.

IITLE

AUTHOR

LEVEL

PGS

DATE

RL-NRD-001 1C

RL-NRD-001 2C

RL-NRD-0013C

RL-NRD-00116

RL-NRD-00139

$R L-N R D-00150$

RL-NRD-00150 2C

$R L-N R D-001505 C$

RL-NRD-00150 6C

$R L-M R D-001507 C$

RL-NRD-0015004

RL-NRD-0015005

RL-NRD-0015009

RL-NRD-0015019

RL-NRD-00153

RL-NRD-00171

RL-NRD-00173PT2 REV

RL-NRD-00174

RL-NRD-00178RD

RL-NRD-00179RD

RL-NRD-0018602

RL-NRD-00197

RL-NRD-00208

RL-NRD-00249

$R L-M R D-00250 R D$
MONTHLY REPORT RESEARCH AND ENGINEERING N-REACTOR DEPARTMENT

MONTHLY RECORD REPORT RESEARCH AND

ENGINEERING N-REACTOR DEPARTMENT

MONTHLY RECORD REPORT RESEARCH AND ENGINEERING N-REACTOR DEPARTMENT TRIP REPORT

TOP SHIELD TEMPERATURES - C AND K REACTORS

MONTHLY RECORD REPORT RESEARCH AND ENGINEERING $N$-REACTOR DEPARTMENT MONTHLY RECORD REPORT RESEARCH AND ENGINEERING N-REACTOR DEPARTMENT MONTHLY RECORD REPORT RESEARCH AHD ENGINEERING OPERATION N-REACTOR DEPARTMENT

MONTHLY REOCRD REPORT RESEARCH AND ENGINEERIMG OPERATION N-REACTOR DEPARTMENT

MONTHLY REOCRD REPORT RESEARCH AMD ENGINEERING OPERATION N-REACTOR DEPARTMENT

N-REACTOR DEPARTMENT MONTHLY REPORT APRIL 1965

N-REACTOR DEPARTMENT MONTHLY REPORT JUNE 1965

N-REACTOR DEPARTMENT MONTHLY REPORT SEPTEMBER 1965

N-REACTOR DEPARTMENT MONTHLY REPORT NOVEMBER 1965

NONDEFENSE PLUTONIUM REQUIREMENTS FUEL ELEMENT WARP

N-REACTOR DEPARTMENT HANFORD ATONIC PROOUCTS OPERATIOH

PRCOUCTION OF POLONIUN-290 IN N-REACTOR CONVERSION OF A SAVAHNAH RIVER REACTOR TO A RESONANCE REACTOR FOR MAXIMM NP-237 PRODUCTION

N-REACTOR CONVERSION RATIO ESTIMATES VS. OBSERVED VALUES

PRCOUCTION INVENTORY \& MANUFACTURING STATMENT N REACTOR DEPARTMENT FEBRUARY 1966

N-REACTOR DEPARTMENT GOALS CY-1965 PROOUCT COST-GRAMS GENERATED BASIS INFORMATION ON CONBINED OPERATION STUDIES

N-REACTOR GRAPHITE MODERATOR: PREDICTED BEHAVIOR AND DEVELOPMENT PROGRAM ANALYSIS OF 1964 PRODUCTION STUDY TRITIUN CASES

\begin{tabular}{|c|c|c|c|}
\hline LEVERETT MC & SRD & 16 & $10 / 31 / 64$ \\
\hline L.EVERETT MC & SRD & 14 & $11 / 30 / 64$ \\
\hline LEVERETT MC & SRD & 15 & $12 / 31 / 64$ \\
\hline DR STENQUIST & SRD & & $12 / 11 / 64$ \\
\hline JD AGAR & SRD & & $12 / 28 / 64$ \\
\hline MC LEVERETT & CRD & & $03 / 31 / 65$ \\
\hline MC LEVERETT & SRD & & $02 / 28 / 65$ \\
\hline MC LEVERETT & SRD & & $05 / 39 / 65$ \\
\hline MC LEVERETT & SRD & & $06 / 30 / 65$ \\
\hline MC LEVERETT & SRD & & $07 / 31 / 65$ \\
\hline RL DICKEMAN & CRD & & $05 / 10 / 65$ \\
\hline RL DICKEMAN & SRD & & $06 / 10 / 65$ \\
\hline RL DICKEMAN & SRD & & 09/01/65 \\
\hline RL DICKEAAM & SRD & & $12 / 10 / 65$ \\
\hline DR STENQUIST & SRD & & $01 / 06 / 65$ \\
\hline EA SMITH & CRD & & $01 / 12 / 65$ \\
\hline MC LEVERETT & SRD & & $09 / 30 / 65$ \\
\hline $\begin{array}{l}\text { JM BIRD } \\
\text { RD SHIMER }\end{array}$ & $\begin{array}{l}\text { SRD } \\
\text { SRD }\end{array}$ & & $\begin{array}{l}01 / 20 / 65 \\
01 / 14 / 65\end{array}$ \\
\hline RA BENMETT & SRD & & $12 / 01 / 64$ \\
\hline DP BROWN & SRD & & $03 / 22 / 66$ \\
\hline RL DICKEMAN & SRD & & $01 / 25 / 65$ \\
\hline WJ DOWIS & SRD & & $01 / 28 / 65$ \\
\hline DH CURTISS & CRD & & $03 / 12 / 65$ \\
\hline RR BLOOMSTRAND & SRD & & $02 / 23 / 65$ \\
\hline
\end{tabular}


Page No. 236

$04 / 28 / 93$

LIST OF HANFORD-GENERATED CLASSIFIED

DOCUMENTS DATED BETUEEN 1/1/61 AND 12/31/72

DOC. NO.

RL-HRD-00258RD

RL-NRD-00266

RL-NRD-00273

RL-NRD-00276RD

RL-MRD-00282RD

$R L$-MRD-00310RD

RL-NRD-00315RD

RL-NRD-00332 10

RL-NRD-00332 11

RL-NRD-00332 12

RL-NRD-00332 3

RL-NRD-00332 4

RL-NRD-00332 5

RL-NRD-00332 6

$R L-N R D-003327$

RL-NRD-00332 8

RL-NRD-003329

RL-NRD-00344

$R L-N R D-00349$

$R L-N R D-00354$

RL-NRD-00362RD

$R L-N R D-00375 R D$

RL-NRD-00405RD

RL-NRD-00418

$R L-N R D-00432 R D$

RL-NRD-00447

RL-NRD -00480
TITLE

N REACTOR FUEL COST REDUCTION INCENTIVE $N$-REACTOR DEPARTMENT RESEARCH AND DEVELOPMENT BUDGET FOR FY- 1967 AND REVISION OF BUDGET FOR FY-1966 PROGRAM CONSIDERATIONS N-REACTOR NPR PLUTONIUN UNIT COST ESTIMATES 1958 TO DATE OXIDE FUEL OPERATION AT SAVAMMAH RIVER MEETING NOTES ON COMPUTER SIMULATION OF THE AEC COAPLEX

UNIT COST CCMPARISONS '63' AND '64' PRODUCTION STUDIES

N-REACTOR DEPARTMENT PRCOUCT COST GRAMS GENERATED OCTOBER 1965

N-REACTOR DEPARTMENT PRODUCT COST GRAMS GEMERATED NOVEMBER 1965

N-REACTOR DEPARTMENT PRODUCT COST GRAMS GENERATED DECEMBER 1965 N-REACTOR DEPARTMENT PROOUCT COST GRAMS GEMERATED MARCH 1965 N-REACTOR DEPARTMENT PRODUCT COST GRAMS GENERATED APRIL 1965

N-REACTOR DEPARTMENT PROOUCT COST GRAMS GENERATED MAY 1965

N-REACTOR DEPARTMENT PRODUCT COST GRAMS GENERATED JUME 1965

N-REACTOR DEPARTMENT PRODUCT COST GRAMS GENERATED JULY 1965 N-REACTOR DEPARTMENT PRODUCT COST GRAMS GENERATED AUCUST 1965 N-REACTOR DEPARTMENT PRODUCT COST GRAMS GEMERATED SEPTEMBER 1965

N-REACTOR DEPARTMENT SEMIANNUAL REPORT OF RESEARCH AND DEVELOPMENT

USE OF NITROGEN IN N-REACTOR ATMOSPHERE PROGRAM CONSIDERATIONS - N-REACTOR OUTLINE - HANFORD INTEGRATED PROCESS DEVELOPNENT PROGRAM AEC COMPLEX COMPUTER COOE FOR COST STUDIES APRIL 26 AND 27 MEETING NOTES PRESENTATION OF INTEGRATED FIVE-YEAR PROGRAM - 02 PROCESS DEVELOPMENT MAY 31 AND JUNE 21965

OFF-SITE GRAPHITE IRRADIATION PROGRAM IN SUPPORT OF N-REACTOR

REACTOR COST STUDIES MEETING OF APRIL 27, 1965

CONVERSION RATION TABLES

PORTIONS OF SECTION II OF THE CLASSIFIED MINUTES OF THE SIXTEENTH MEETING OF THE GE-THC HELD IN SAN FRANCISCO, CALIFORNIA OCTOBER 15-16, 1964
AUTHOR

RD SHIMER

STAFF N-REACTOR

DEPARTMENT

RL DICKEMAN

WJ DOW!S

RD SHIMER

RD SHIMER - RR

BLOOHSTRAND

RR 8LOCMSTRAND

NE INGRAM

NE INGRAM

DP BROW

ME INGRAM

ME INGRAM

NE IMGRAM

ME IMGRNM

NE IMGRAM

NE INGRAM

NE INGRAM

RESEARCH \& ENGIMEERING

STAFF

EE LEIT2

RL DICKEMAN

RL DICKEMAN

RD SHIMER

RL DICKEMAN

RL DICKEMAN

CRD

RR BLOOMSTRAND - WJ DOWIS SRD

SRD

RA CHITWOOO

SRD
06/22/65

03/03/65

$04 / 19 / 65$

$02 / 27 / 65$

$03 / 16 / 65$

$03 / 18 / 65$

$04 / 19 / 65$

$04 / 16 / 65$

$12 / 03 / 65$

$12 / 20 / 65$

$01 / 17 / 66$

$04 / 20 / 65$

05/19/65

$06 / 18 / 65$

07/20/65

08/27/65

$09 / 21 / 65$

$10 / 21 / 65$

05/19/65

05/03/65

05/06/65

05/12/65

$05 / 14 / 65$

06/04/65

$06 / 18 / 65$

$07 / 30 / 65$

$07 / 31 / 65$ 
Page No. 237

$04 / 28 / 93$

LIST OF HANFORD-GENERATED CLASSIFIED

DOCUMENTS DATED BETWEEN 1/1/61 AND 12/31/72

DOC. NO

TITLE

AUTHOR

LEVEL

PGS

DATE

RL-NRD-00631
RL-NRD-00644
RL-NRD-00650
RL-NRD-0066002
RL-NRD-0066106
RL-NRD-00664
RL-NRD-00685
RL-NRD-00706
RL-NRD-00710

RL-NRD-00719

$R L-N R D-00721$

$R L-N R D-00724$

RL-NRD-00733

$R L-N R D-00738$

RL-NRD-00742 6

RL - NRD - 00764

RL-MRD-00801

RL-NRD-00802

RL-NRD-0081601

RL-NRD-0081602

RL-NRD-0081603

RL-NRD-0081604

RL-NRD-0081605
REFERENCE DATA - COPRCOUCT DISCUSSIONS NOVEMBER 16, 1965

RESEARCH AND DEVELOPMENT ALLOCATIONS AND PLANT ASSISTANCE SUPPORT FY-1966

TRANSFER VOUCHER 1-HZA-HXA-11-66-1 N-REACTOR DEPARTMENT MONTHLY REPORT FEBRUARY 1966

N REACTOR DEPARTMENT MEEKLY REPORT FEBRUARY 8, 1966

US ATOMIC ENERGY COMIISSION TRANSFER

VOUCHER NO. 7-HZA-HXA-11-66-2

N-REACTOR DEPARTMENT SEMIANMUAL REPORT OF RESEARCH AND DEVELOPMENT

TRANSFER VOUCHER - 11-HZA-HXA-12-66-1-F REPORT TO THE FEDC WORKING COMMITTEE FOR THE PERIOD SEPTEMBER 1965 TO JANUARY 1966

TECHMICAL REQUIREMENTS AND CRITERIA FOR THE COPRODUCT TARGET ELEMENT

MINUTES OF MEETINGS TO DISCUSS EXTRACTION TEST STUDIES OF CERAMIC COPRCOUCT TARGETS

N-REACTOR NP-237 - PU-238 PROGRAM

N-REACTOR DEPARTMENT FY-1965 PERFORMANCE COMPARED WITH 1964 REFIMED REACTOR COST STUDIES

K REACTOR UNCOOLED HCR - EQUILIBRIUNA TEMPERATURE STUDY

REACTOR DESIGN ANALYSIS MONTHLY RECORD

REPORT - JUNE, 1966

TRIP REPORT COPRODUCT EXTRACTION

DISCUSSIONS HITH SAVANMAH RIVER

LABORATORY JAMUARY 14, 1966

MATERIAL BALANCE REPORT JAMUARY 1966

COMPOSITION OF ENDING INVENTORIES

JANUARY 1966

PRODUCTION INVENTORY AND MAYUFACTURING

STATEMENT N REACTOR DEPARTMENT JANUARY

1966

PRCOUCTION INVENTORY AND MANUFACTURING

STATEMENT A REACTOR DEPARTMENT FEBRUARY 1966

PROOUCTION INVENTORY AND MANUFACTURING STATEMENT HANFORD ATOMIC PRODUCTS DEPARTMENTS MARCH 1966

PROOUCTION INVENTORY AND MANUFACTURING STATEMENT - HANFORD ATOMIC PROOUCTS

DEPARTMENT APRIL 1966

PRODUCTION INVENTORY AMD MANUFACTURING STATEMENT HANFORD ATOMIC PROOUCTS

DEPARTMENT MAY 1966

$\begin{array}{lll}\text { RL DICKEMMN } & \text { SRD } & 11 / 12 / 65 \\ \text { MC LEVERETT } & \text { CRD } & 11 / 22 / 65 \\ \text { JD RYAN } & \text { SRD } & 11 / 23 / 65 \\ \text { RL DICKEMMN } & \text { SRD } & 02 / 01 / 66 \\ \text { RL DICKEMMN } & \text { SRD } & 02 / 08 / 66 \\ \text { JD RYAN JR } & & 12 / 12 / 65 \\ & \text { SRD } & 12 / 15 / 65 \\ \text { RESEARCH AND ENGINEERING } & \text { SRD } & 12 / 30 / 65 \\ \text { STAFF } & & 01 / 05 / 66 \\ \text { JD RYAN } & \text { SRD } & \\ \text { M LEMIS } & \text { SRD } & \end{array}$

TH EVANS

SRD

$01 / 07 / 66$

R COOPERSTEIN

SRD

$01 / 03 / 66$

RL DICKEMAN

CRD

$01 / 10 / 66$

$J$ MILNE

CRD

$11 / 03 / 65$

JD AGAR

SRD

$01 / 24 / 66$

RK ROBINSON

CRD

$06 / 21 / 66$

R COOPERSTEIN - M LEWIS SRD

01/19/66

JH HAMDSHUH

SRD

JW HAMDSHUH

SRD

$02 / 15 / 66$

02/15/66

DP BROWW

SRD

$02 / 21 / 66$

DP BRON

SRD

$03 / 22 / 66$

RE MIDDAUGH

SRD

$04 / 26 / 66$

RE MIDDAUGH

SRD

$05 / 23 / 66$

DP BRONN

SRD

$06 / 23 / 66$ 
Page No. 238

$04 / 28 / 93$

LIST OF HANFORD-GENERATED CLASSIFIED

DOCUMENTS DATED BETWEEN 1/1/61 AND 12/31/72

DOC. NO.

RL-NRD-008221

RL- - NRD - 00837

RL-MRD-00840

RL-NRD-00848

RL-NRD-00852

$R L-N R D-00866$

RL-NRD-066 SUP2

RL-NRD-139

RL-MRD-150 10C

RL-NRD-150 11

$R L-N R D-15011 \mathrm{C}$

$R L-N R D-1502 C$

$R L-N R D-1503 C$

RL-NRD-150 4C

$R L-M R D-1505 C$

$R L-N R D-1506 C$

RL-NRD-150 7C

RL-NRD-150 9C

$R L-N R D-163$

RL-NRD-173 PT2 REV

$R L-M R D-174$

RL-NRD-179 RD

$R L-N R D-266$

$R L-M R D-266$ EX5

$R L-N R D-266$ EX6
TITLE

RADIOLOGICAL STATUS OF THE N-REACTOR FOR JAN \& FEB 1966

11-HZA-HXA-2-66-1F

FINAL REPORT UARP PROBLEM STUDIES PREPARATION OF FUEL ELEMENTS COST PRODUCTION AND UNIT COST

UNCOOLED SPLINE PROPOSAL FOR B AMD C REACTORS

CLASSIFICATION GUIDANCE LITHIUN ALUMINUM COMPOUNDS AND ASSOCIATED FABRICATION BUDGET STUDY REPORT TRITIUM EXTRACTION FACILITY N-REACTOR, 100-N AREA TOP SHIELD TEMPERATURES - C AND $K$ REACTORS

MONTHLY RECORD REPORT RESEARCH AND ENGINEERING N-REACTOR DEPARTMENT N-REACTOR DEPARTMENT MONTHLY REPORT, NOVENBER 1965

MONTHLY RECORD REPORT RESEARCH AND ENGINEERING OPERATION N-REACTOR DEPARTMENT MONTHLY RECORD REPORT RESEARCH AND ENGINEERING N-REACTOR DEPARTMENT MONTHLY RECORD REPORT RESEARCH AND ENGINEERING N-REACTOR DEPARTMENT MONTHLY RECORD REPORT RESEARCH AND ENGINEERING N-REACTOR DEPARTMENT MONTHLY RECORD REPORT RESEARCH AND ENGINEERING N-REACTOR DEPARTMENT MONTHLY RECORD REPORT RESEARCH AMD ENGINEERIMG N-REACTOR DEPARTMENT MONTHLY RECORD REPCRT RESEARCH AND ENGIHEERING N-REACTOR DEPARTMENT MONTHLY RECORD REPORT RESEARCH AHD ENGINEERIMG N-REACTOR DEPARTMENT N-FUELS ENGINEERING PROGRAMS - CY-1965 ALTERNATE FUEL CYCLES FOR PONER-ONLY OPERATION OF N-REACTOR

PRODUCTION OF POLONIUN-210 IN N-REACTOR H-REACTOR CONVERSION RATIO ESTIMATES VS OBSERVED VALUES

N-REACTOR DEPARTMENT RESEARCH AND DEVELOPMENT BUDGET FOR FY-1967 AND REVISION OF BUDGET FOR FY- 1966

M-1 N-REACTOR FUEL DEVELOPMENT EXCERPTS FRON N-REACTOR DEPARTMENT RED BLDGET FOR FY-1967 AND REVISION OF BLOGET FOR FY-1966

C-1 COPROOUCT DEVELOPMENT FOR N-REACTOR EXCERPTS FROM N-REACTOR DEPARTMENT RED BUDGET FOR FY-1967 AND REVISION OF BUDGET FOR FY-1966

\begin{tabular}{|c|c|c|c|}
\hline AUTHOR & LEVEL & PGS & DATE \\
\hline HENDRICKSON & CONF & & $03 / 24 / 66$ \\
\hline DP BROWN & SRD & & $02 / 28 / 66$ \\
\hline EA SMITH & CRD & & $03 / 02 / 66$ \\
\hline DP BRON & SECR & & $03 / 14 / 66$ \\
\hline JA WHITE & SRD & & $03 / 14 / 66$ \\
\hline LC ClosSEY & CRD & & $03 / 21 / 66$ \\
\hline $\begin{array}{l}\text { LOVE CE, COOPERSTEIN R, } \\
\text { STEPNEWSKI DD }\end{array}$ & SRD & 22 & $03 / 24 / 67$ \\
\hline AGAR JD & SRD & 22 & $12 / 28 / 64$ \\
\hline LEVERETT MC & CRD & 20 & $10 / 31 / 65$ \\
\hline DICKEMAN RL & SRD & 58 & $12 / 10 / 65$ \\
\hline LEVERETT MC & SRD & 27 & $11 / 30 / 65$ \\
\hline LEVERETT MC & SRD & 19 & $02 / 28 / 65$ \\
\hline LEVERETT MC & CRD & 19 & $03 / 31 / 65$ \\
\hline LEVERETT MC & CRD & 26 & $04 / 30 / 65$ \\
\hline LEVERETT MC & SRD & 21 & $05 / 31 / 65$ \\
\hline LEVERETT MC & SRD & 21 & $06 / 30 / 65$ \\
\hline LEVERETT MC & SRD & 18 & $07 / 31 / 65$ \\
\hline LEVERETT MC & SRD & 19 & $09 / 30 / 65$ \\
\hline NICKOLAUS JH & CRD & 9 & $01 / 08 / 65$ \\
\hline LEVERETT MC, DICKEMAN RL & SRD & 52 & $09 / 30 / 65$ \\
\hline \multirow[t]{4}{*}{$\begin{array}{l}\text { BIRD JH, RICHES JH } \\
\text { BENNETT RA }\end{array}$} & $\begin{array}{l}\text { SRD } \\
\text { SRD }\end{array}$ & $\begin{array}{l}17 \\
1\end{array}$ & $\begin{array}{l}01 / 20 / 65 \\
12 / 01 / 64\end{array}$ \\
\hline & CRD & 76 & $04 / 19 / 65$ \\
\hline & SRD & 7 & $04 / 19 / 65$ \\
\hline & SRD & 9 & $04 / 19 / 65$ \\
\hline
\end{tabular}


Page No. 239

$04 / 28 / 93$

LIST OF HANFORD-GENERATED CLASSIFIED

DOCUMENTS DATED BETHEEN 1/1/61 AND 12/31/72

DOC. NO.

$R L-N R D-344$

RL-NRD-362 RD

RL-NRD-405 RD

RL - NKD -447

$R L-N R D-481$

RL-MRD -512

$R L-N R D-550$

$R L-H R D-575$

RL-MRD-602

$R L-N R D-644$

$R L-M R D-652$

RL-NRD-657 SUP B

RL-NRD-660 1 A

$R L-N R D-6602$

$R L-N R D-663$

$R L=N R D-685$

$R L-N R D-699$

$R L-N R D-707$

$R L-N R D-710$

RL-NRD - 719

$R L-M R D-721$

$R L-M R D-724$

RL-NRD-738

RL-NRD-742 6

RL-NRD-764
TITLE

N-REACTOR DEPARTMENT SEMIANMUAL REPORT OF RESEARCH AND DEVELOPMENT

OUTLINE - HANFORD INTEGRATED PROCESS DEVELOPMENT PROGRAM

PRESENTATION OF INTEGRATED FIVE-YEAR

PROGRAM - 02 PROCESS DEVELOPMENT MAY 31 AND JUNE 21965

CONVERSION RATIO TABLES

TRIP REPORT, AIH REACTOR SITE, MATIONAL

REACTOR TEST STATION, IDAHO FALLS, IDAHO

NUCLEAR SAFETY SPCIFICATIONS FOR

N-REACTOR FUELS FOR 2.4 W/O ENRICHED

URANIUM OXIDE FUEL

URANIUN SWELLING IN N-REACTOR /PT-NR-4, SUP. A, REPORT NO. 1

FABRICATION HISTORY OF FUEL 1829-02

REPORT OF VISIT TO SAVANWAH RIVER PLANT, SEPTEMBER 16-17, 1965

RESEARCH AND DEVELOPMENT ALLOCATIONS AND PLANT ASSISTANCE SUPPORT FY-1966

4-HZA-KYA-11-66-1

SUPPLEMENT B, PRODUCTION TEST PT-NR-57

COPROOUCER TARGET ELEMENT EVALUATION

MONTHLY RECORD REPORT RESEARCH AND

ENGIMEERING OPERATION N-REACTOR

DEPARTMENT

N-REACTOR DEPARTMENT MONTHLY REPORT, FEBRUARY 1966

U.S. ATOMIC ENERGY COMMISSION TRANSFER

VOUCHER NO. 5-HZA-HYA-11-66-2

N-REACTOR DEPARTMENT SEMIANMUAL EPORT OF RESEARCH AND DEVELOPMENT

4-HZA-HYA-12-66-2

3-HZA-HYA-12-66-1

REPORT TO THE FEDC WORKING COMITTEE FOR THE PERIOD SEPTEMBER 1965 TO JAHUARY

1966

TECHNICAL REQUIREMENTS AND CRITERIA FOR THE COPRODUCT TARGET ELEMENT

MINUTES OF MEETINGS TO DISCUSS EXTRACTION TEST STUDIES OF CERAMIC COPRODUCT TARGETS

N-REACTOR, NP-237 - PU-238 PROGRAM

K REACTOR UNCOOLED HCR - EQUILIBRIUM

TEMPERATURE STUDY

REACTOR DESIGN ANALYSIS MONTHLY RECORD

REPORT - JUNE, 1966

TRIP REPORT, COPROOUCT EXTRACTION

DISCUSSIONS WITH SAVANWAH RIVER

LABORATORY, JANUARY 14, 1966
AUTHOR

LEVEL PGS

DATE

DICKEMAN RL

DICKENAN RL

CHITLOOD RA

KRATZER WK, MAZUR DW, LARRICK AP, SCOTT WA

BLACK BR

CRD

55

SRD

33

SRD

116

$06 / 04 / 65$

SRD

CRD

25

07/02/65

CRD

GUAY AE

CRD

15

$09 / 16 / 65$

KENMEDY BA

PATRICK MG, LEHIS M

CRD

SRD

LEVERETT MC

CRD

SRD

RYAN JD

EVAMS TH

SRD

LEVERETT MC

SRD

30

SRD

62

$03 / 10 / 66$

RYAN JD JR

SRD

1

$12 / 02 / 65$

SRD

42

$12 / 15 / 65$

RYAN JD

SRD

$12 / 31 / 65$
RYAN JD

SRD

SRD

LEWIS $M$

EVAHS TH

SRD

15

$01 / 07 / 66$

COOPERSTEIN $R$

SRD

3

$01 / 03 / 66$

DICKEMAN RL

CRD

SRD

9

15

$01 / 10 / 66$

AGAR JD

CRD

13

$06 / 21 / 66$

COOPERSTEIN R, LEHIS M SRD

$12 / 31 / 65$

$01 / 05 / 66$

ROBIMSON RK

3

$01 / 19 / 66$ 
Page No. 240

$04 / 28 / 93$

LIST OF HANFORD-GENERATED CLASSIFIED

DOCUMENTS DATED BETWEEN 1/1/61 AND 12/31/72

DOC. NO.

RL - NRD - T78

RL-MRD-838

RL-NRD-N-00001

$R L-N R D-N-00004$

RL-NRD-N-00010

RL $-M R D-N-00013$

RL-NRD $-N-00017$

$R L-N R D-N-00018$

RL-NRD $-N-00020$

RL-REA-00015

RL-REA-00026

RL-REA-00058

RL-REA-00082

RL-REA-00083

RL-REA-00092

RL-REA-00096

RL-REA-00165

RL-REA-00210

RL-REA-00253

RL-REA-00256

RL-REA-00321

RL-REA-00364RD

RL-REA-00365RD

RL - REA-00472

RL-REA-00473

RL-REA-00474

RL-REA-00475

RL-REA-00476

RL-REA-00477

RL-REA-00560RD

RL-REA-00600
TITLE

6-HZA-HYA-1-66-1F

12-HZA-HYA-2-66-1F

PERSONAL NOTES

PERSONAL NOTES

PERSONAL MOTES

PERSONAL NOTES

PERSONAL MOTES

PERSONAL MOTES

PERSONAL NOTES

MULTI-PRODUCT ALLOCATION OF U-235

BURNOUT AMD REACTOR COSTS

REACTOR SHUTDOWN INSPECTION SYSTEM

TECHNICAL. DEVELOPMENT AND EVALUATION

NUETRON RIMETICS CONSTANTS IN THE

HANFORD IPD REACTORS FOR A STANDARD

WEAPONS GRADE FUEL LOAD AND AN

ENRICHED-THORIA LOAD

RUPTURE REMOVAL FROM ZIRCALOY TUBES

DEALING MITH UNCOOLED OR BURNING

IRRADIATED URANIUM

FLOW BLOCKAGE TUBE 3069-KW

WATER TEMPERATURE RISE IN A MELL CAR

LOADED WITH FRESHLY DISCHARGED FUEL

RESULTS OF DESIGN TEST 1062 EVALUATION

OF THE GE-APED NEUTRON FLUX MONITOR

SEMIANMUAL REPORT REASEARCH AND

DEVELOPMENT IRRADIATION PROCESSING

DEPARTMENT

230/13.8 KV SYSTEM VOLTAGE STUDY POST

DEACTIVATION OF F, H AMD DR

105 KE OPERATIMG DATA

U-233 PRCDUCTION SCHEDULE PROPOSAL II

MONTHLY REPORTS - REACTOR PHYSICS 1965

MANAGEMENT REPORTS - REACTOR PHYSICS 1965

IRRADIATION PROCESSING DEPARTMENT

MONTHLY REPORT JAAUARY 1965

IRRADIATION PROCESSING DEPARTMENT MONTHLY REPORT FEBRUARY 1965

IRRADIATION PROCESSIMG DEPARTMENT MONTHLY REPORT MARCH 1965

IRRADIATION PROCESSING DEPARTMENT MONTHLY REPORT APRIL 1965

IRRADIATION PROCESSING DEPARTMENT MONTHLY REPORT MAY 1965

IRRADIATION PROCESSING DEPARTMENT

MONTHLY REPORT JUNE 1965

PRCDUCTION SUB-SECTIOW CLASSIFIED WORKING PAPERS

MOTEBOOK - CLASSIFIED NOTES ON NUCLEAR SAFETY

\begin{tabular}{llll}
\multicolumn{1}{c}{ AUTHOR } & LEVEL & PGS & DATE \\
& & & \\
RYAN RD & & & \\
BROW DP & SRD & 1 & $01 / 31 / 66$ \\
UK KRATZER & SRD & 1 & $02 / 28 / 66$ \\
RR BLOCNSTRAND & SECR & & $10 / 27 / 64$ \\
DH CONSTABLE & SECR & & $11 / 11 / 64$ \\
CA MUNSIUS & SECR & & $12 / 21 / 64$ \\
DP BROWN & SECR & & $03 / 18 / 65$ \\
DP BROWW & SECR & & $04 / 08 / 65$ \\
RU HANKE & SECR & & $04 / 08 / 65$ \\
HG SPENCER & SECR & & $06 / 11 / 65$ \\
& SRD & & $10 / 13 / 64$ \\
GE-RL & & & \\
& CRD & & $11 / 25 / 64$ \\
JP HANRIC & & & \\
& SRD & & $10 / 27 / 64$
\end{tabular}

WK ALEXANDER

CRD

$11 / 05 / 64$

RE BAARS

SRD

$08 / 20 / 65$

EJ FILIP

SRD

$11 / 06 / 64$

DE BUSH

SRD

$11 / 16 / 64$

PJ MORVATH

CRD

$11 / 20 / 64$

OH GREAGER

CRD

12/01/64

R THOMPSON/FJ MOLLERUS

CRD

$03 / 29 / 65$

SR.

CD BOSTON

PD GROSS - KL HLADEK

R NILSON

R NILSON

HANFORD ATOHIC PROOUCTS

OPERATION

HANFORD ATOMIC PRODUCTS SRD

OPERATION

HANFORD ATCHIC PRODUCTS SRD

OPERATION

HANFORD ATOAIC PRODUCTS

OPERATION

HANFORD ATOMIC PRODUCTS SRD

OPEATION

HANFORD ATOMIC

SRD

PROOUCTIONS OPERATION

RE DUNW

SRD

AD VAUGHN

SECR
$01 / 28 / 65$

$12 / 08 / 64$

$12 / 22 / 64$

$01 / 04 / 65$

01/04/65

$11 / 06 / 65$

$11 / 06 / 65$

$11 / 06 / 65$

$05 / 14 / 65$

$06 / 14 / 65$

$07 / 14 / 65$

02/04/65 
Page No. 241

$04 / 28 / 93$

LIST OF MAMFORD-GEMERATED CLASSIFIED

DOCUNENTS DATED BETWEEN 1/1/61 AND 12/31/72

\section{DoC. NO. \\ RL-REA-00625 \\ RL-REA-00665 \\ RL-REA-00668 \\ RL-REA-00735RD \\ RL-REA-00737RD \\ RL-REA-00739RD \\ RL-REA-00741RD \\ RL-REA-00743RD \\ RL-REA-00745RD \\ RL-REA-00747RD \\ RL-REA-00749RD \\ RL-REA-00838 \\ RL-REA-00851 \\ RL-REA-00899 \\ RL - REA-00919 \\ RL-REA-00931 \\ RL-REA-00937 \\ RL-REA-00941 \\ RL-REA-00975 \\ RL-REA-01006 \\ RL-REA-01014 \\ RL-REA-01031 \\ RL-REA-01032}

RL-REA-01032 A

RL-REA-01032 B

RL-REA-01033 A RD

RL-REA-01033 B RD
TITLE

OPTIMMM PROCESS WATER PH B,C, AND D REACTORS

REVISION OF THORIA LOSS ON IGNITION SPECIFICATION

BUDGET STUDY REACTOR EFFLUENT ACTIVITY REDUCTION

DAILY PONER REPORT 100-KE

MAIN CONTROL DAILY LOG - EOUIPMENT PANEL 100-KE

MAIN CONTROL DAILY LOG - ELECTRICAL

PANEL 100-KE

LOW LIFT I SERVICE MATER PUMP DAILY LOG

- 100-KE

RIVER PUMP DAILY LOQ - 100-KE

FILTER PERFORMANCE DAILY LOG - 100-KE

WATER TREATMENT DAILY LOG - 100-KE

FILTER PERFORMANCE - 100-KE

105-DR AND N REACTOR GROSS CAMM

DISTRIBUTION AND DECAY DATA

REACTOR SAFETY CIRCUIT AUDIT MITH

RESPECT TO SAFETY

GRAPHITE TEMPERATURE DATA KE REACTOR

$$
11 / 3 / 62 \text { TO }
$$

SHUTDOWN HEAT OUTPUT

STM GRID STUDY SYALL HANFORD IPD REACTORS

105 KE CONSOLE LOG PERIOD 4/1/65 THRU

6/30/65

IRRADIATED METAL MONITOR DEVELOPMENT

105-KW REACTOR IN CORE FLUX MONITOR

CHARTS PERIOD - NOVEMBER 17, 1961 TO MAY

3L, 1965

CLASSIFICATION - REFIMENENT OF PRACTICES

ALTERING HCR WITHBRAWAL. RATES

STATUS REPORT IRON-MASONITE SHIELD

ATTENUATION EFFECTIVENESS

PROOUCTION TEST IP-T72, HAPO 327

IRRADIATION OF MO-UC2 \& NB-LOZ PLATES IN

A HELIUN ATMOSPHERE

PROOUCTION TEST IP-TT2, HAPO 327,

SUPPLEMENT A IRRADIATION OF MO-LOZ AMD

MB-UO2 PLATES IN A HELIUN ATMOSPHERE

PROOUCTION TEST IP-T72, HAPO 327,

SUPPLEMENT B IRRADIATION OF MO-LO-2 ANO NB-LO2 PLATES IN A RELIUN ATMOSPHERE

IRRADIATION ANALYSIS FOR PROOUCTION TEST IP-T72 HAPO 327, SUPPLEMENT A -

IRRADIATION OF MO-LOZ \&B-LOZ PLATES IN

A HELIUN ATMOSPHERE

IRRADIATION ANLYSIS PROOUCTION TEST

IP-772, HAPO 327, SUPPLEMENT B,

IRRADIATION OF MO-LOZ AND NB-UOZPLATES

IN A HELIUM ATMOSPHERE
AUTHOR

LEVEL

PGS

DATE

JR YOUNG

SRD

02/15/65

WK ALEXAMDER

CRD

02/15/65

EL ETHERIDEG

SRD

$02 / 26 / 65$

JC MCLAUGHLIN

SRD

03/04/65

JC MCLAUGHLIN

SRD

$03 / 04 / 65$

JC MCLAUGhLIM

SRD

$03 / 04 / 65$

JC MCLAUGHLIN

SRD

03/04/65

JC MCLAUGHLIN

03/04/65

JC MCLAUEHLIM

JC MCLAUERLIN

JC MCLAUGHLIM

GL ERICKSON

W DALOS

SA 1000

DE BUSH

JP HAMRIC

RG CLOUGH

PJ HORVATH

PJ HORVATH

TH MNUFF

GC FULLMER - R NILSOM

PO EROSS

SRD

SECR

$04 / 07 / 65$

$04 / 15 / 65$

EC BENMETT

CRD

$04 / 12 / 65$

EC BENMETT

CRD

$04 / 12 / 65$

EC BENNETT

CRD

$06 / 22 / 65$

EC BENNETT

CRD

$04 / 12 / 65$

EC BENNETT

CRD

$06 / 22 / 65$ 
Page No. 242

$04 / 28 / 93$

LIST OF MANFORD-GENERATED CLASSIFIED

DOCUMENTS DATED BETMEEN 1/1/61 AMD 12/31/72

DOC. NO.

RL-REA-01033 RD

RL-REA-01068

RL-REA-02005

RL-REA-02038

RL-REA-02105

RL-REA-02134RD

RL-REA-02190

RL-REA-02256

RL-REA-02280

RL-REA-02309

RL-REA-02326

RL-REA-02419

RL-REA-02432

RL-REA-02460

RL-REA-02518

RL-REA-02519

RL-REA-02530

RL-REA-02539

$R L-R E A-02562$

RL-REA-02589

RL-REA-02599

RL-REA-02603

RL-REA-02624

RL-REA-02675

RL-REA-1016

RL-REA- 1025

RL. -REA-1031
TITLE

IRRADIATION ANALYSIS PRODUCTION TEST OP-T72, HAPO-327 IRRADIATION OF MO-LO2 \& NB 102 PLATES IN A HELIUN ATMOSPHERE 105-N - CHARGE - DISCHARGE FOR 1965 A MOOEL TO OPTIMIZE PU-238 PROOUCTION BY RECYCLE

PROCRAN FOR INVESTICATION OF ZIRCALOY-2 HYORIDING

WELL CAR CASK SHIELDING

MATERIAL PERTINENT TO $X$ REACTOQ TALKS

1965 TRAMSIENT STABILITY STUDY EFFECT OF BPA DISTURBANCES ON HAPO OPERATIONS

105-KE CONSOLE LOG - PERICD 7/1/65 THRU 9/30/65

THORIUA RECYCLE - CASE STUDIES 300-A CONSIDERATIONS

UNIT COST PER GRAM GENERATED FY 1961 THRU FY 1966

EFFECTIVE STRENGTH OF THE SAFETY SYSTEMS OF HANDORD REACTORS

A OUICK METHCO TO CHECK FUEL LONDIMG

LIMITS

MAXIMU CREDIBLE REACTOR ACCIDENT

FUEL ELEMENT DEVELOPMENT COMNITTEE

ANMUAL REPORT FROM HANFORD

PROOUCTION TEST IP-803 IRRADIATION OF

ENRICHED URANIUN OXIDE FUEL ELEMENTS

UNUSUAL REACTOR OPERATION REPORTS

SECURITY - TELEPHONE COMUNICATIONS

1500 HP LOW LIFT PUMP MOTORS K PLANT

BUSES A AND D DISARM UNDERVOLTAGE TRIPS

REPORT OF THE WORKING COMMITTEE OF THE

FUEL ELEMENT DEVELOPNENT COMNITTEE

INPUT PARAMETERS AND BLENDING SCHEMES

FOR INCREMENTAL NEPTUNILM

PROOUCTION FOR THE 1965 COST

STUDIES

105-KE CONSOLE LOG - PERIOD 10/1/65 THRU 12/31/65

FULL RANGE PROTECTION FOR B, C, \& D

REACTORS

FINAL REPORT - PT IP-754 PHYSICS TESTING

OF H REACOTR

EXTENDED PLUTONIUM IRRADIATION

STATUS OF SPECIAL REACTOR PROCESS TUBE

LONDINGS APRIL 1, 1965

REPORT TO THE WORKING COMITTEE FROM

HANFORD

STATUS REPORT IRON-MASONITE SHIELD

ATTENUATION EFFECTIVENESS
AUTHOR

EC BENMETT

CONF

$06 / 12 / 65$

LEVE

PGS

DATE

RH BOW

SRD

$04 / 15 / 65$

CW SHOWALTER SRD

$04 / 28 / 65$

SN GRAVES - IK ALEXANDER SRD

$05 / 05 / 65$

FE ONEN

CRD

05/18/65

07/01/65

$08 / 20 / 65$

FJ MOLLERUS/MR THORSON CRD

RG CLOUGH

SRD

07/01/65

WA BLANTON

SRD

$07 / 09 / 65$

RE THOWS

SRD

$07 / 14 / 65$

ND VAuenM

CRD

07/01/65

AD VAushi

CRD

TW Ambrose

SRD

08/05/65

M LEEHIS - JE MINOR - JT SRD

$08 / 06 / 65$

$08 / 18 / 65$

$10 / 18 / 65$

STRINGER

PA CARLSON - KL HLADEK SRD

$09 / 07 / 65$

$09 / 09 / 65$

$10 / 29 / 65$

TW HAUFF

SRD

SRD

HANFORO ATONIC PKCOUCTS SRD

OPERATION

$09101 / 65$

H TOFFER

SRD

$10 / 12 / 65$

RG CLOUGH

SRD

$10 / 01 / 65$

JP HAMRIC

CRD

$10 / 04 / 65$

AD VAUGHN/GC MASCHE

CRD

$10 / 07 / 65$

PD GROSS

SRD

10/22/65

BOWN RM

SRD

$04 / 09 / 65$

LEWIS M, MIMOR JE, STRINGER JT

GROSS PO

SRD

SRD

$04 / 12 / 65$

$04 / 15 / 65$ 
Page No. 243

$04 / 28 / 93$

LIST OF HANFORD-GENERATED CLASSIFIED

DOCUMENTS DATED BETHEEN 1/1/61 AND 12/31/72

DOC. NO.

RL-REA-1086

RL-REA-14

RL-REA-14 SUP A

RL-REA-14 SUP B

RL-REA-15

RL-REA-159

RL-REA- 185

RL-REA-188

RL-REA-2005

RL-REA-2008

RL-REA-2029

RL-REA-2032

RL-REA-2038

RL-REA-2075

RL-REA-20T7

RL-REA-2088

RL-REA-2092

RL-REA-210

RL-REA-2108

RL-REA-2150

RL-REA-2156

RL-REA-2182

RL-REA-2199 REV1

RL-REA-220

RL-REA-223
TITLE

LETTER, RE SKAVDAHL FROM SH GRAVES

PROOUCTION TEST IP-T25

PROOUCTION TEST IP-725, SUPPLEMENT A

IMCREASED GRAPHITE TEMPERATURE LIMIT $F$ REACTOR

PROOUCTION TEST IP-725, SUPPLEMENT B INCREASED GRAPHITE TEMPERATURE LIMIT F REACTOR

MULTI-PRCOUCT ALLOCATION OF U-235

BURMOUT AND REACTOR COSTS

COMERCIAL ISOTOPE PRODUCTION IN A SMALL GRAPHITE MODERATED REACTOR

ADDITIONAL PRCOUCTION OF URANIUM-233

PRODUCTION TEST IP-734, HAPO 309, HIGH

CORE TEMPERATURE CAPSULES IN THE KW

SNOUT FACILITY, SERIES II

A MODEL TO OPTIMIZE PU-238 PROOUCTION BY RECYCLE

FINAL REPORT - PROOUCTION TEST IP-740

PHOSPHATE CAPTURE SUBSECUENT TO

FILTERING

PU-238 PRCOUCTION AMALYSIS

CRITICALITY PREDICTIONS IN THE HANFORD

REACTORS

PROGRAM FOR INVESTIGATION OF ZIRCALOY-2 HYDRIDING

STATUS OF SPECIAL REACTOR PROCESS TUBE

LONDINGS MAY 1, 1965

DEVELOPMENT TEST IP-T78 KE DOWNCOMER INCREASED FLOU TEST

MEUTRON FLUX MEASUREMENTS IN THE HANFORD

TEST IRRADIATION FACILITIES

NOTE ON DOCUNENT RL-REA-556 REGARDING

REACTOR OVERBORE

SEMIAMNUAL REPORT RESEARCH AMD

DEVELOPMENT IRRADIATION PROCESSING

DEPARTMENT

FINAL REPORT - PRCOUCTION TEST IP-745

HALF-PLANT VALIDITY TEST

INTEGRATED SITE STLDY-TECHNOLOGICAL CONSIDERATIONS

SEMIANNUAL RESEARCH AND DEVELOPMENT REPORT IRRADIATION PROCESSING DEPARTMENT

STATUS OF SPECIAL REACTOR PROCESS TUBE LOADINGS JUNE 1, 1965

GRAPHICAL DETERMINATION OF $K$ EMERGENCY

FLOUS UNDER VARIED SUPPLY CONDITIONS REACTIVITY MEASUREMENTS OF VARIOUS

TARGET ELEMENTS

OPERATING REPORT - CORROSION TESTING OF ALUMINUM-CLAD PU-AL FUEL ELEMENTS IN THE C-1 LOOP

\begin{tabular}{|c|c|c|c|}
\hline AUTHOR & LEVEL & PGS & DATE \\
\hline GRAVES SM & SRD & 2 & $04 / 23 / 65$ \\
\hline RUSSELL a & SRD & 16 & $12 / 01 / 64$ \\
\hline RUSSELL A & SRD & 13 & $03 / 19 / 65$ \\
\hline RUSSELL A & SRD & 5 & $06 / 18 / 65$ \\
\hline SPENCER HG & SRD & 13 & $10 / 13 / 64$ \\
\hline $\begin{array}{l}\text { KUSLER LE, CUMPRECHT RO, } \\
\text { FIFER NF, RATHVOH HC }\end{array}$ & SRD & 19 & $11 / 12 / 64$ \\
\hline SCHROEDER OC & SRD & 2 & $11 / 20 / 64$ \\
\hline $\operatorname{cox} \mathrm{JH}$ & SRD & 8 & $11 / 20 / 64$ \\
\hline SHOWALTER CW, NILSON R & SRD & 22 & $04 / 28 / 65$ \\
\hline FRYMIER JH, PITMAN RH & SRD & 17 & $05 / 05 / 65$ \\
\hline & SRD & 42 & $05 / 10 / 65$ \\
\hline SKIDMORE SH, BOWERS CE & SRD & 11 & $03 / 31 / 65$ \\
\hline GRAVES SM, ALEXANDER WK & SRD & 26 & $05 / 05 / 65$ \\
\hline BOWN RH & SRD & 14 & $05 / 11 / 65$ \\
\hline JOWES SS $\quad ' \cdot$ & SRD & 14 & $05 / 20 / 65$ \\
\hline PREVO PR, MONTCOMERY MH & SRD & 7 & $05 / 12 / 65$ \\
\hline woods ux & SRD & 5 & $05 / 14 / 65$ \\
\hline GREAGER OH & CRD & 47 & $12 / 01 / 64$ \\
\hline FRYMIER JH & SRD & 6 & $05 / 20 / 65$ \\
\hline $\begin{array}{l}\text { LEWIS M, STRINGER JT, } \\
\text { SZULIMSKI MJ }\end{array}$ & SRD & 19 & $05 / 28 / 65$ \\
\hline AMBROSE TH & SRD & 37 & $06 / 01 / 65$ \\
\hline BOWN RW & SRD & 12 & $06 / 08 / 65$ \\
\hline JONES SS & SRD & 26 & $10 / 18 / 65$ \\
\hline BLYCKERT WA & SRD & 13 & $12 / 01 / 64$ \\
\hline BENAETT EC & SRD & 11 & $12 / 04 / 64$ \\
\hline
\end{tabular}


Page No. 244

$04 / 28 / 93$

LIST OF MANFORD-GENERATED CLASSIFIED

DOCUMENTS DAYED BETUEEN 1/1/61 AND 12/31/72

DOC. NO.

RL-REA-2244

RL-REA-2248 D

RL-REA-2248 J

RL-REA-2249 D

RL-REA-2249 J

RL-REA-2250 D

RL-REA-2250 J

RL-REA-2251 D

RL-REA-2251 J

RL-REA-229

RL-REA-2315

RL-REA-2335

RL-REA-2385

RL-REA-2432

RL-REA-2460

RL-REA-2483 RD

RL-REA-250

RL-REA-2515

RL-REA-2518

RL-REA-2526

RL-REA-2535
TITLE

FRONT-FACE HARDWARE MOOIFICATION 105-C REACTOR

MONTHLY RECORD REPORT - JULY 1965

RESEARCH AND ENGIMEERING OPERATION

IRRADIATION PROCESSING DEPARTMENT

MONTHLY RESEARCH AND DEVELOPMENT REPORT, JULY 1965, IRRADIATION PROCESSING DEPARTMENT

MONTHLY RECORD REPORT - AUCUST 1965, RESEARCH AND ENGIMEERING OPERATION IRRADIATION PROCESSING DEPARTMENT MONTHLY RESEARCH AND DEVELOPMENT REPORT - AUGUST 1965, IRRADIATION PROCESSING DEPARTMENT

MONTHLY RECORD REPORT - SEPTEMBER 1965, RESEARCH AND ENGINEERING OPERATION IRRADIATION PROCESSING DEPARTMENT MONTHLY RESEARCH AND DEVELOPMENT REPORT SEPTEMBER 1965, IRRADIATION PROCESSING DEPARTMENT

MONTLY RECORD REPORT - OCTOBER 1965. RESEARCH AND ENGINEERING SECTION IRRADIATION PROCESSING DEPARTMENT MONTHLY RESEARCH AND DEVELOPNENT REPORT OCTOBER 1965, IRRADIATION PROCESSIHG DEPARTMENT

BALL DROP IMPLICATIONS AT C REACTOR OPERATIONAL PHYSICS ANALYSIS

STATUS OF SPECIAL REACTOR PROCESS TUBE LOADINGS JULY 1, 1965

VERTICAL BOHING MEASUREMENTS, F REACTOR PROGRESS REPORT - PT-1P-759 CHANMEL

CAULKING AT C REACTOR VSR CHANMEL 17-C, JUNE 18-19, 1965

MAXIMUM CREDIBLE REACTOR ACCIDENT

FUEL ELEMENT DEVELOPMENT COMAITTEE, ANNUAL REPORT FROM HANFORD HORIZONTAL CONTROL ROD RENOVATION PROGRAM ALL PROOUCTION REACTORS REACTIVITY BALANCE AND ASSOCIATED REACTOR PHYSICS DATA - NOVEMGER 1964 PROOUCTION TEST IP-BO2 IN-REACTOR TESTING WITH COOLANT PREPARED IN THE WATER TREATMEMT PILOT PLANT PRODUCTION TEST IP-803 IRRADIATION OF ENRICHED URANIUM OXIDE FUEL ELEMENTS REPORT TO THE WORKING COMAITTEE FRON HAMFORD

STATUS OF SPECIAL REACTOR PRCCESS TUBE LONDINGS, SEPTEMBER 1, 1965
NUTHCR

1000 RH

AMBrose TH

ambrose tH

AMBrose TH

AMBROSE TH

AMBrose TW

AMBROSE TH

AMBROSE TH

AMBrose TH

VAUGHM AD

BOW RH

DEAERS AE

COOKE JP

AMBROSE TU

LEWIS M, MIMOR JE, STRIMGER JT HUTTON PH

CLARK DE

GEIER RG, HEID RL.

CARLSON PA, HLADEK KL

LEWIS M, MINOR JE, STRINGER JT BOWN RH
SRD

SRD

CRD

SRD

CRD

SRD

SRD

10

12

LEVEL

PGS

DATE

$09 / 01 / 65$

$08 / 04 / 65$

$08 / 06 / 65$

09/03/65

$09 / 03 / 65$

10/05/65

$10 / 06 / 65$

11/06/65

$11 / 04 / 65$

$12 / 01 / 64$

$07 / 19 / 65$

$07 / 02 / 65$ $08 / 02 / 65$

08/06/65 08/18/65

08/23/65

$12 / 04 / 64$

$09 / 03 / 65$

$10 / 18 / 65$

09/21/65

SRD

$09 / 94 / 65$ 
DOC. NO.

RL-REA-2546

$R L-R E A-2552$

RL-REA-2562

RL-REA-2585

RL-REA-2624

RL-REA-2625

RL-REA-2668

RL-REA-2675

RL-REA-2679

RL-REA-2707

RL-REA-275

RL-REA-279

RL-REA-297

RL-REA-316

RL-REA-328

RL-REA-329

RL-REA-332

RL-REA-413

RL-REA-448

RL-REA-467

RL-REA-473 D

RL-REA-474 D

RL-REA-475 D

RL-REA-476 D
TITLE

INTERIM REPORT I PRCDUCTION TEST IP-728, HALF-PLANT SOOILAM SILICATE TEST IRRADIATION PROCESSING DEPARTMENT AND PROCESS TECHNOLOGY PROGRAMS - FY-1966 REPORT OF THE WORKING COMMITTEE OF THE FUEL ELEMENT DEVELOPMENT COMITTEE PROOUCTION TEST PT-IP-810 INSTALLATION OF FLAT ALUMINUM HORIZONTAL CONTROL ROD, D REACTOR

FINAL REPORT - PT IP-754 PHYSICS TESTING OF H REACTOR

STATUS OF SPECIAL REACTOR PROCESS TUBE LOADINGS, OTTOBER 1, 1965

A HANFORD CLASSIFICATION GUIDE REVISION SECTION 600

EXTENDED PLUTONIUN IRRADIATION

KE DOWNCOMER DEVELOPMENT TEST - IP-T78, INTERIM REPORT

1-HZA-HYA-10-66-1

CARBON DIOXIDE FOR PH ADJUSTMENT 900-K REACTORS

DESIGN TEST REQUEST NO. 1263 K REACTOR GRAPHITE KEY AHD VSR CHANNEL SLEEVE TEST THERMAL SHIELD COOLING TUBE SURVEILLANCE $F, C, D$, AND K REACTORS

SAFETY AMALYSIS OF THE C-1 LOOP

KER-1 OPERATING REPORT - TEST K-1-23 PT IP-601 AND PT IP-601, SUPPLEMENTS A AND $B$

KER-3 OPERATING TEST K-3-18, PT IP-645 REPORT TO THE WORKING COMIITEE OF THE FUEL ELEMENT DEVELOPMENT COMMITTEE FROM THE GENERAL ELECTRIC COMPAMY - HAMFORD REACTIVITY BALANCE AND ASSOCIATED REACTOR PHYSICS DATA

INTERIM REPORT - PT-IP-G74 INVESTIGATION OF TERMINAL CORROSION RATES IN ALUMINUM PROCESS TUBES

STATUS OF SPECIAL REACTOR PROCESS TUBE LOADINGS

MOWTHLY RECORD REPORT - FEBRUARY 1965 RESEARCH AMD ENGINEERING OPERATION IRRADIATION PROCESSIMG DEPARTMENT

MONTHLY RECORD REPORT - RW:'CH 1965 RESEARCH AND ENGINEERING OPERATION IRRADIATION PROCESSING DEPARTMENT

MONTHLY RECORD REPORT - APRIL 1965 RESEARCH AND ENGINEERING OPERATION IRRADIATION PROCESSING DEPARTMEKT MONTHLY RECORD REPORT - MAY 1965 RESEARCH AND EMGIMEERING OPERATION IRRADIATION PROCESSING DEPARTMENT

\begin{tabular}{|c|c|c|c|}
\hline AUTHOR & LEVEL & PGS & DATE \\
\hline EIER RG & SRD & 11 & $09 / 16 / 65$ \\
\hline LSON R, STRINGER JT & CRD & 15 & $09 / 01 / 65$ \\
\hline & SRD & 130 & $09 / 01 / 65$ \\
\hline LEXAMDER WK, HUTTON PH & SRD & 19 & $10 / 91 / 65$ \\
\hline IAUGHN AD, MASCHE GC & CRD & 106 & $10 / 07 / 65$ \\
\hline OWN RH & SRD & 19 & $10 / 08 / 65$ \\
\hline IAUFF TW & CRD & 6 & $10 / 20 / 65$ \\
\hline ROSS PD & SRD & 27 & $10 / 22 / 65$ \\
\hline IONES SS & SRD & 49 & $12 / 01 / 65$ \\
\hline CHOUVILLER VA & SRD & 1 & $10 / 30 / 65$ \\
\hline IOUNG JR & CRD & 9 & $12 / 15 / 64$ \\
\hline IEMPF FJ & CRD & 32 & $12 / 10 / 64$ \\
\hline DONHAM CE & CRD & 19 & $11 / 19 / 64$ \\
\hline $\begin{array}{l}\text { SARLSON PA, TL DEOBALD } \\
\text { ALLLAS MA }\end{array}$ & $\begin{array}{l}\text { SRD } \\
\text { SRD }\end{array}$ & $\begin{array}{l}54 \\
4\end{array}$ & $\begin{array}{l}12 / 31 / 64 \\
12 / 23 / 64\end{array}$ \\
\hline
\end{tabular}

QUINN HT

SRD

$12 / 23 / 64$ MINOR JE, LEHIS $M$, STRINGER JT

CLARK DE

SRD

5

$01 / 06 / 65$

HOUGH CG

CRD

13

$12 / 15 / 64$

BOWN RH

SRD

22

01/01/65

GREAGER OH

SRD

20

$03 / 03 / 65$

GREAGER OH

SRD

25

$04 / 05 / 65$

GREAGER OH

SRD

20

05/05/65

GREAGER OH 
Page No. 246

$04 / 28 / 93$

LIST OF HANFORD-GENERATED CLASSIFIED

DOCUMENTS DATED BETUEEN 1/1/61 AND 12/31/72

DCC. NO.

TITLE

AUTHOR

LEVEL

PGS

DATE

RL-REA-477 D

RL-REA-576

RL-REA-585

RL-REA-59

RL-REA-59 REV

RL-REA-590

RL-REA-602

RL-REA-606

RL-REA-606 SUP

RL-REA-607

RL-REA-614

$R L-R E A-623$

$R L-R E A-625$

RL-REA-628

RL-REA-659 1

RL-REA-659 2

RL-REA-659 3

RL-REA-659 4

$R L-R E A-6611$

RL-REA- 6613

RL-REA-661 4

RL-REA-662 1

RL-REA-663

RL-REA-681 1
MONTHLY RECORD REPORT - JUNE 1965 RESEARCH AND ENGINEERING OPERATION IRRADIATION PROCESSING DEPARTMENT IRRADIATION PROCESSING FUEL AND PROOUCT CONVERSION TABLES /REVISION OF MU-50300-8 REV. $1 /$.

INTERIM REPORT II - PRODUCTION TEST IP-634 HALF-PLANT LOW PH TEST WATER TREATMENT AT D REACTOR - PROCESS TUBE EXAMINATION RESULTS

STATUS OF DEVELOPMENT WORK ON THE HOT DIE SIZE FUEL ELEMENT GRONTH PROBLEM STATUS OF DEVELOPMENT WORK ON THE HOT DIE SIZE FUEL ELEMENT GRONTH PROBLEM ANALYSIS OF PT-684-A HOT DIE SIZE PROCESS PARAMETERS EVALUATION

FINAL REPORT PROOUCTION TEST IP-688 THE IRRADIATION OF COATED FUEL ELEMENTS PROOUCTION TEST IP-T54 PHYSICS TESTING AT H REACTOR DEACTIVATION PRCOUCTION TEST IP-754, SUPPLEMENT 1 PHYSICS TESTS OF SMALL COMPOSITE FUEL AND TARGET LOADINGS PROPOSAL FCR ELIMIMATION OF 100 PERCENT INSPECTION FOR URANIUN GRAIN SIZE REACTIVITY BALANCE AND ASSOCIATED REACTOR PHYSICS DATA JANUARY, 1965 KER-1 OPERATIHG REPORT TESTS $K-1-18$, $K-1-19, K-1-20, K-1-21, \& K-1-22$ PT IP-584, 601, AND 544, SUPPLEMENTS A \& $B$ OPTIMUM PROCESS WATER PH B, C, AND D REACTORS

STAYUS OF SPECIAL REACTOR PROCESS TUBE LOADINGS FEBRUARY 1, 1965

VERTICAL BOWING MEASUREMENTS, C REACTOR HORIZONTAL DISTORTION MEASUREMENTS, C REACTOR

VERTICAL BOWING MEASUREMENTS, C REACTOR VERTICAL BONING MEASUREMENTS, C REACTOR HORIZONTAL DISTORTION MEASUREMENTS, B REACTOR

VERTICAL BOWING MEASUREMENTS, B REACTOR TUBE 4574

VERTICAL BONING MEASUREMENTS, B REACTOR TUBE 1575

VERTICAL BONING MEASUREMENTS, D REACTOR TECHNICAL FEASIBILITY OF USING URANIUA OXIDE FUELS AT HANFORD

STATUS OF PLANT ASSISTANCE IRRADIATIONS AS OF FEBRUARY 13, 1965

\begin{tabular}{|c|c|c|c|}
\hline GREAGER OH & SRD & 21 & $07 / 06 / 65$ \\
\hline PERL ER, VAUGHN AD & SRD & $\begin{array}{l}\text { CON } \\
T\end{array}$ & $06 / 01 / 65$ \\
\hline HOUGH CG, PAGLIERI JN & SRD & 14 & $01 / 01 / 65$ \\
\hline STRAND CA & CRD & 18 & $11 / 05 / 64$ \\
\hline STRAND CA & CRD & 22 & $02 / 18 / 65$ \\
\hline BOUIE $N$ & SRD & 4 & $02 / 04 / 65$ \\
\hline GEIER RG & SRD & 9 & $02 / 04 / 65$ \\
\hline MASCHE GC, VAUGHN AD & SRD & 64 & $02 / 01 / 65$ \\
\hline $\begin{array}{l}\text { MASCHE GC, VAUGHW AD, } \\
\text { OUSLEY GF }\end{array}$ & CRD & 19 & $04 / 01 / 65$ \\
\hline STEVENSON MF & SRD & 11 & $03 / 30 / 65$ \\
\hline CLARK DE & SRD & 5 & $02 / 05 / 65$ \\
\hline DALLAS HA & SRD & 20 & $02 / 09 / 65$ \\
\hline YOUNG JR & SRD & 14 & $02 / 15 / 65$ \\
\hline BOWN RH & SRD & 20 & $02 / 12 / 65$ \\
\hline FERCUSON JH & CRD & 2 & $02 / 25 / 65$ \\
\hline DEMERS AE & CRD & 2 & $03 / 24 / 65$ \\
\hline DEMERS AE & CRD & 2 & $07 / 23 / 65$ \\
\hline DEMERS AE & CRD & 2 & $07 / 06 / 65$ \\
\hline DEMERS AE & CRD & 2 & $03 / 24 / 65$ \\
\hline DEMERS, AE & CRD & 2 & $08 / 25 / 65$ \\
\hline DEMERS, AE & CRD & 2 & $09 / 15 / 65$ \\
\hline \multirow[t]{2}{*}{ DEMERS, AE } & CRD & 2 & $07 / 23 / 65$ \\
\hline & SRD & 35 & $02 / 15 / 65$ \\
\hline FERGUSOW JH & SRD & 17 & $03 / 01 / 65$ \\
\hline
\end{tabular}


Page No. 247

$04 / 28 / 93$

LIST OF HANFORD-GENERATED CLASSIFIED

DOCUMENTS DATED BETHEEN 1/1/61 AND 12/31/72

Doc. No.

RL-REA-681 2

RL-REA-681 3

RL-REA-681 4

RL-REA-681 5

RL-REA-6816

RL-REA-681 7

RL-REA-681 8

RL-REA-688

RL-REA-689

RL-REA-697

RL-REA-709 RD RL-REA-710 RD

RL-REA-711 RD

RL-REA-715 RD

RL-REA-T76

RL-REA-784 RD

RL-REA-785 RD VOL2

RL-REA-788

RL-REA-837

RL-REA-879

RL-REA-893

RL-REA-931

RL-REA-H-C0005

RL-REA-N-00010

RL-REA-N-00020

RL-REA-N-00041

RL-REA-N-00048BK1

RL-REA-N-00049

RL-REA-WSU-00001

REVI
TITLE

STATUS OF PLANT ASSISTAMCE IRRADIATIONS AS OF MARCH 12, 1965

STATUS OF PLANT ASSISTANCE IRRADIATIONS AS OF APRIL 9, 1965

STATUS OF PLANT ASSISTANCE IRRADIATIONS AS OF MAY 14, 1965

STATUS OF PLANT ASSISTANCE IRRADIATIONS AS OF JUME 11, 1965

STATUS OF PLANT ASSISTANCE IRRADIATIONS

AS OF JULY 16, 1965

STATUS OF PLANT ASSISTANCE IRRADIATIONS

AS OF AUGUST 13, 1965

STATUS OF PLANT ASSISTANCE IRRADIATIONS

AS OF SEPTEMBER 10, 1965

MINUTES -- GRAPHITE DISTORTION, VSR, HCR SERVICEABILITY PROGRAM MEETIMG FEBRUARY

4. 1965

RIVER TEMPERATURE FLUCTUATION PROBLEM

PT-IP-759 CHANMEL CAULKING TESTS - C

REACTOR

URANIUN TEMPERATURES FOR KER TEST $K-3-19$ EXPOSURE DATA, Y TEST HOLE - C REACTOR SWELLIHG CAPSULE EXPOSURE DATA

HAPO 303 DATA SHEETS, B REACTOR

REACTIVITY BALANCE AND ASSOCIATED

REACTOR PHYSICS DATA FEBRUARY, 1965

URANIUU SWELLING CAPSULE DATA

CREEP CAPSULE DATA

TH-228 CONTAMINATION IN IRRADIATED THORIA

STATUS OF SPECIAL REACTOR PROCESS TUBE LOADINGS MARCH 1, 1965

KE DOWNCOMER FLOW TEST AMD PRELIMIMARY

FLOW ANALYSIS

PROOUCTION TEST IP-766 IRRADIATION OF

ALUMINUM-PLUTONIUM FUEL ELEMENTS

ZTM GRID STLDY - SMALL HANFORD IPD REACTORS

PERSONAL NOTES

PERSONAL NOTES

PERSONAL NOTES - AUDIT (NOTEBOOK)

PERSONAL NOTES

PERSONAL NOTES

PERSOMAL NOTES

C DOWWOAER MODEL STUDIES
AUTHOR

FERCUSON JH

FERCUSON JH

FERCUSON JH

FERGUSON JH

FERCUSOW JH

FEREUSON JH

FERCUSON JH

PETERSON RS

GUSTAFSON LD

COOKE JP

DEOBALD TL

FERCUSON JH

HAMSE DW

FERGUSON JH

CLARK DE

HAASE DW

HAMSE DH

Hocos in

BOW RH

JONES SS

BAARS RE, OUSLEY GF

HAMRIC JP

RL MILLER

PJ HOLLINFIELD

LH RICE

AP VINTHER

H BOHIE

CC LOMAX
LEVEL PGS

DATE

$03 / 25 / 65$

$04 / 23 / 65$

SRD $\quad 16$

$06 / 15 / 65$

07/07/65

08/03/65

$08 / 30 / 65$

$09 / 28 / 65$

02/17/65

$02 / 26 / 65$

$03 / 19 / 65$

02/25/65

02/25/65

$02 / 18 / 65$

cor

CON

03/12/65

03/05/65

$03 / 12 / 65$

08/23/65

03/08/65

$03 / 10 / 65$

$03 / 22 / 65$

03/26/65

03/05/65

$11 / 19 / 64$

$12 / 07 / 64$

$01 / 20 / 65$

$04 / 14 / 65$

$09 / 08 / 65$

09/08/65

06/01/66 
Page No. 248

$04 / 28 / 93$

LIST OF HANFORD-GENERATED CLASSIFIED

DOCUMENTS DATED BETWEEN 1/1/61 AND 12/31/72

DoC. NO.

RL-REA-WSU-2

RL-SEP-00004

RL-SEP-00012RD

RL-SEP-00021

RL-SEP-00039

RL-SEP-00057 RD

RL-SEP-00093RD

RL-SEP-00111

RL-SEP-00130 RD

RL-SEP-00142

RL-SEP-00150

RL-SEP-00177 REV

RL-SEP-00195

RL-SEP-00237

RL-SEP-00243

RL-SEP-00249RD

RL-SEP-0027

RL-SEP-00277

RL-SEP-00283

RL-SEP-00297

RL-SEP-00298

RL-SEP-00299

RL-SEP-00304

RL-SEP-00312

RL-SEP-00314

RL-SEP-00325
TITLE

HYDRAULIC MODEL STLDIES OF K-DOWCOMER PRODUCT LOSSES TO REDOX $H-10$ COATING WASTE

PUREX \& REDOX WASTE LOSSES JAMUARY 1957 L-CELL PACKAGE REDESIGN WEAPONS PROCESS ENGINEERING TECHMOLOGY REPORT - SEPTEMBER \& OCTOBER, 1964 SECRET WORKSHEETS PUREX FLEXIBILITY PROJECT

DATA FROM METAL PURIFICATION STUDY WEAPONS PROCESS ENGINEERING TECHNOLOGY REPORT - NOVEMBER 1964 REDOX HEAD-END TREATMENT

"A" LIME PROCESS REVISIONS

FPT-63-15 EVALUATION: USE OF TANTALUM CRUCIBLES FOR RMC LINE INGOT PREPARATION

TENSILE PROPERTIES DETERMIMATION FOR RHA LINE PRCOUCT

EVALUATION OF AIR-ACTUATED ELECTROJET (FPT-64-29)

TANTALLM SHAPE CASTING MELT CRUCIBLES INTERIM SUMAARY OF FABRICATION PROCESS TEST FPT-64-26

REDOX CHEMICAL FLOUSHEET HH-7 AND HW-8

2 PLANT REPORT TASK I AND II AND

RECOVERY OPERATIONS JAMUARY 1 - DECEMBER 31, 1965

POURING RATE TESTS IN SIIULATED RMA LINE CASTING CONDITIONS

PLUTONIUN TO URANIUN DETERMINATIONS WEAPONS PROCESS ENGINEERING TECHNOLOGY REPORT - DECEMBER 1964 - JAMUARY 1965 REDOX WEEKLY PROCESS REPORTS JANUARY THROUGH DECEMBER 1965

FABRICATION PROCESS TEST EVALUATION

FPT-64-34, POLISHING OF 74-C PARTS

WITH NYLON SCORING PAD

ADDENDUN I TO FPT-64-22 - CHILL. CASTING OF 74-C PREFORMS

HARDNESS MEASUREMENT FOR DETERMINIMG PRODUCT CHARACTERISTICS

SEMI-ANNUAL REPORT 03 PROCESS

DEVELOPMENT JULY 1, 1964 THROUGH DECEMBER 1, 1964

BLDGET DESIGN CRITERIA - U-233

FABRICATION - "C" FABRICATION LINE -

234-5 BUILOING

SEMI-ANNUAL PROGRESS REPORT O3 RED JULY

1, 1964 THROUGH DECEMBER 1, 1964

\begin{tabular}{llll}
\multicolumn{1}{c}{ AUTHOR } & LEVEL & PGS & DATE \\
& & & \\
LOMX C (EDITOR) & SRD & 32 & $11 / 30 / 65$ \\
RE ISMCSON & SRD & & $10 / 12 / 64$ \\
MCDOHALD & & & \\
CH SMITH & SRD & & $10 / 14 / 64$ \\
HAPO & SRD & & $10 / 16 / 64$ \\
& SRD & $10 / 29 / 64$ \\
JH MORDAHL & & \\
& SRD & & $11 / 03 / 64$ \\
RH BOND & & \\
HAPO & CRD & & $11 / 24 / 64$ \\
& SRD & $12 / 01 / 64$ \\
ARHCO & & \\
AE SMITH & SRD & $01 / 01 / 64$ \\
CM HALKER & SRD & $12 / 10 / 64$ \\
\end{tabular}

IN TAYLOR, JR/JF BERGGREN SRD

$04 / 29 / 65$

LL MCGREGOR

SRD

$01 / 04 / 65$

CM HALKER

SRD

$01 / 07 / 65$

RE ISAACSON

CRD

$01 / 19 / 65$

RL HALSER

SPD

$01 / 01 / 65$

BARBER AE

SRD

10

$10 / 14 / 64$

GL HANSON

CRD

$01 / 28 / 65$

HAPO

02/02/65

RA YOOER

SRD

01/04/65

AE BARBER

SRD

$02 / 04 / 65$

ST FIELD

SRD

$02 / 04 / 65$

CJ BRONKEE

SRD

02/03/65

RE TOMLINSOH

SRD

$02 / 15 / 64$

RC HOLLINGSHEAD

SRD

$02 / 15 / 65$

RE TOMLIMSON

SRD

$02 / 18 / 65$ 
Page No. 249

04/28/93

LIST OF HANFORD-GENERATED CLASSIFIED

DOCLMENTS DATED BETWEEN 1/1/61 AND 12/31/72

DOC. NO

TITLE

AUTHOR

LEVEL

PGS

DATE

RL-SEP-0033

INTERIM REPORT ON FPT-64-22 AND 64-25

FIELD ST

18

$10 / 12 / 64$

CHILL CASTTING ANO ALTERNATE FEED

SOURCES FOR 74-C COMPONENTS

RL-SEP-00330RD

OPERATING PLAN, JaNUARY 1965 - JUNE

WJ GARTIN

SRD

03/01/65

RL-SEP-00348

1967, MEAPONS MANUFACTURING OPERATION.

MEAPONS PROCESS ENGINEERING TECHNOLOGY

HAPO

03/03/65

RL-SEP-00356

RL-SEP-00361

MC JACOBS

03/05/65

SLUDGE DISSOLUTION PROCEDURE EVALUATION.

FABRICATION PROCESS TEST

FPT-65-2 TENSILE MACHINE

IMSTALLATION CHECKOUT

RL-SEP-00366

21A-6 BUDGET FOR FY 1967 \& REVISION OF

BUDGET FOR FY 1966 - PROPOSAL FOR

PROCESS DEVELOPMENT

RL-SEP-00367

BUDGET FOR FY 1967 \& REVISION OF BUDGET

FOR FY 1966 - PROPOSAL FOR RESEARCH \&

JF BERGGREN

SRD

$03 / 12 / 65$

DEVELOPMENT - GEMERAL MEAPONS

DEVELOPMENT

RL-SEP-00379

A-LINE PROCESS DEFINITION

PUREX PLANT STUDY FLOUSHEET, 4.0

RE TOMLINSON/AE SMITH

SRD

$03 / 31 / 65$

CAPACITY FACTOR

RL-SEP-0039 HEAPONS PROCESS ENGINEERING TECHNOLOGY

REPORT - SEPTEMBER \& OCTCBER 1964

RL-SEP-00404

RL-SEP-00417

WEAPONS PROCESS ENGINEERING TECHNOLOGY

REPORT - MARCH 1965

21A-5 SUMWARY ANALYSIS OF PROPOSED

PROCESS DEVELOPMENT COSTS FOR FY

1967 \& REVISION OF BLDGETFOR FY 1966

RL-SEP-00425

SUPPLEMENTARY DATA TO 03 PROGRAM

PROOUCTION BUDGET SUBMISSION BUDGET FOR

FY67 AND REVISION OF BLDGET FOR FY66

RL-SEP-00450

SEMI-ANNUAL PROGRESS REPORT O3 PROCESS

DEVELOPMENT DECEMBER 1, 1964 TO MAY 1,

1965

RL-SEP-00451

SEMINANNUAL PROGRESS REPORT O3 R\&D DECEMBER 1, 1964 TO MAY 1, 1965

RL-SEP-00473

WEAPONS PROCESS ENGINEERING TECHNOLOGY

REPORT - APRIL 1965

RL-SEP-004741

DESIGN CRITERIA - MEW BUTTON LINE

EQUIPMENT - 234-5 BUILDING

RL-SEP-00481

FABRICATION PROCESS TEST 64-25

EVALUATION FPT-64-25

RL-SEP-00510

FABRICATION PROCESS TEST 64-28

EVALUATIONO MECHANICAL CLEANING OF PLUTONIUM BUTTONS.

RL-SEP-00513

WEAPONS PROCESS ENGINEERING TECHNOLOGY

REPORT MAY 1965

RL-SEP-00582

MEASUREMENT FACTORS FOR MATERIAL BALANCE

ACCOUNTING - WEAPONS FABRICATION

RE TOMLINSOM/AE SHITH

SRD

$03 / 29 / 65$

AE SWITH

SM NIELSON

SRD

05/05/65

$04 / 15 / 65$

SRD

SRD

$10 / 29 / 64$

HAPO

$04 / 01 / 65$

RE TOMLINSON/AE SNITH

SRD

$03 / 31 / 65$

BM DOBBS

SRD

$04 / 14 / 65$

RE TOMLINSON

SRD

$04 / 19 / 65$

RE TOALIMSON

SRD

$04 / 19 / 65$

HAPO

$04 / 28 / 65$

DC MELSON

$05 / 12 / 65$

ST FIELD

$04 / 30 / 65$

CM WALKER

06/01/65

OPERATION. 
Page No. 250

$04 / 28 / 93$

LIST OF HANFORD-GENERATED CLASSIFIED

DOCUMENTS DATED BETUEEN 1/1/61 AND 12/31/72

DOC. NO.

RL-SEP-00584

RL-SEP-00586

RL-SEP-00587

RL-SEP-00588

RL-SEP-0059

RL-SEP-00596

RL-SEP-00602

RL-SEP-00608

RL-SEP-00633

RL-SEP-00658

RL-SEP-00669PT 1

RL-SEP-00669PT3

RL-SEP-00669PT 4

RL-SEP-00676

RL-SEP-00680

RL-SEP-00682

RL-SEP-00685

RL-SEP-00703

RL-SEP-00705

RL-SEP-00709

RL-SEP-00746

RL-SEP-00778

RL-SEP-00820

RL-SEP-00831
TITLE

- DR. AE KAY

WEAPONS FABRICATION - UNIT COST AND

VARIANCE REPORT, MAY 1965 FISCAL YEAR TO DATE

O3 PROCESS BUDGET FOR FY 1966-67 UNDER SHUTDOWN CONDITIONS

WEAPONS PROCESS ENGINEERING TECHNOLOGY REPORT - JUNE 1965

02 SPECIAL MUCLEAR MATERIAL PROGRAM -

AEC PROOUCTION DIVISION FISCAL YEAR 1967 BUDGET REVIEW

WEAPONS PROCESS ENGINEERING TECHNOLOGY

REPORT - JULY 1965

DEVELOPMENT OF A PONER-ROLL-FORMING

PROCESS? PART 1, FOUNDRY DEVELOPMENT AND EXPERIENCE

DEVELOPMENT OF A PONER-ROLL-FORMING

PROCESS - PART III - DETERMINATION OF FORMING PARAMETERS

DEVELOPMENT OF A PONER-ROLL-FORMING

PROCESS PART IV, MACHINING FORMED

WEAPOHS COAPONENTS

TRITIUM EXTRACTION FACILITY STUDY - 1965

SEMI-ANNUAL PROCESS REPORT O3 RED MAY 1, 1965 TO DECEMBER 1, 1965

FISCAL 1966 REVISION OF CESILM-137 INDEX FOR NUCLEAR MATERIALS CONTENT OF COATING WASTE

COST COMPARISON FOR ALTERNATE FEED

PREPARATION METHOOS

PLUTONIUN SHIPPER-RECEIVER DIFFERENCE

INVESTIGATION

WEAPONS PROCESS ENGINEERING TECHNOLOGY

REPORT - AUCUST 1965

O3 PROGRAM SUPPORT PROPOSAL

WEAPONS PROCESS ENGIMEERING TECHNOLOGY

REPORT - SEPTEMBER 1965

EVALUATION REPORT: FPT 64-22, ADDENDUM 1

03 DEVELOPMENT RUNS RED-256-301

THROUGH 6-310

THORIUM AND N-REACTOR

WEAPONS PROCESS ENGINEERING TECHNOLOGY

REPORT - OCTOBER 1965
AUTHOR

LM KNIGHTS

HH BRAYMAN

UH BRAYMaN

HH BRAYMAN

SMITH AE

DL MORRELL

LEVEL PGS DATE

SRD

SRD

$06 / 18 / 65$

$06 / 16 / 65$

$06 / 16 / 65$

$06 / 16 / 65$

SRD

3

$11 / 03 / 64$

$06 / 22 / 65$

BM DOBBS

$06 / 25 / 65$

HAPO

$06 / 28 / 65$

JH WARREN

07/08/65

HAPO

$07 / 30 / 65$

AE BARBER

$12 / 30 / 65$

WL LAYTON

08/03/65

JM PINKERTON

$12 / 10 / 65$

GP KESEL

RE TOMLINSON

08/20/65

12/01/65

WH ZIMMER

08/09/65

ST FIELD

SRD

$08 / 13 / 65$

EL KELLEY

SRD

$03 / 31 / 66$

HAPO

$08 / 31 / 65$

AE SMITH

HAPO

$09 / 03 / 65$

$09 / 30 / 65$

ST FIELD

11

D mCDOMALD

HAPO 
Page No. 251

$04 / 28 / 93$

LIST OF HANFORD-GENERATED CLASSIFIED

DOCUMENTS DATED BETWEEN 1/1/61 AND 12/31/72

DOC. NO.

RL-SEP-00844

RL-SEP-00855

RL-SEP-00864

RL-SEP-00877

RL-SEP-00879

RL-SEP-00880

RL-SEP-00881

RL-SEP-00885

RL-SEP-00891

RL-SEP-00893RD

RL-SEP-00899

RL-SEP-00905

RL-SEP-00912

RL-SEP-00922

RL-SEP-00928

RL-SEP-00932RD

RL-SEP-00934RD

RL-SEP-00935RD

RL-SEP-00936RD

RL-SEP-00939RD

RL-SEP-00940RD

RL-SEP-0111

RL-SEP-0136

RL-SEP-0142

RL-SEP-0150

RL-SEP-0177 REV

RL-SEP-0184 RD
TITLE

PERMANENT SHAPE MOLD DEVELOPMENT PROGRAM HISTORICAL EVENTS OF THE CHEMICAL PROCESSING DEPARTMENT

CRITICAL MASS AMALYSIS OF E-METAL PROCESSING - PUREX PLANT WEAPONS PROCESS ENGINEERING TECHNOLOGY REPORT - NOVEMBER 1965

EVALUATION: FPT-65-3 INGOTING OF UNALLOYED PLUTONIUM BUTTOWS SEMI-ANHUAL PROGRESS REPORT O3 RRD MAY 1, 1965 TO DECEMBER 1, 1965 SEMIANMUAL PROGRESS REPORT O3 PROCESS DEVELOPMENT MAY 1, 1965, TO DECEMBER 1, 1965

IPB PROGRAM AND INSTRUCTION DECK BUTTON LINE DATA PROCESSING.

AMERICIUM IN HANFORD-PRODUCED PLUTONILM

PRELIMINARY COST ANALYSES FOR FY-1965 STEAM AND ESSENTIAL MATERIALS AT THE PUREX PLANT

TRIP REPORT VISAM 247 \& EVIR 37. PERSONAL NOTES-POLONIUM MEAPONS PROCESS ENGINEERING TECHNOLOGY REPORT - DECEMBER 1965

CPD - ISOCHEM INC. UNIT COST COMPARISONS

- FY 1966 MIDYEAR BLDGET REVIEH

PUREX SEFOR RUNS - NOVEMBER-DECEMBER 1965

CHEMICAL PROCESSING DIVISION MONTHLY REPORT SUMMARIES. JUL 1963-DEC 1965 FPT-64-24, POLE AREA STRENGTHENING WORKING PAPERS-1964

PONER ROLL FORMING PROCESS DEVELOPMENT, HARDNESS DATA WORKING PAPERS 1962 - 64

YIELD STRENGTH DATA, MISCELLANEOUS PLOTS AND GRAPHS, RFP DATA 1963 - 65

HAPO TRITIUM PROOUCTION FACILITIES STUDY - 1958 - STUDY REPORTS NUMBERS 1 : 2. PRODUCTION GRAPHS CY 1957 - CY 1965 WEAPONS PROCESS ENGINEERING TECHNOLOGY REPORT - NOVEMBER 1964

PLUTONIUM AND 70-58 CONTENT CONTROL AND CERTIFICATION - RMA LIME

"A" LINE PROCESS REVISIONS

FPT-63-15 EVALUATION: USE OF TANTALUM CRUCIBLES FOR RMC LINE INGOT PREPARATION TENSILE PROPERTIES DETERMINATION FOR RMA LINE PRODUCT

PLUTONIUM CHEMISTRY LABORATORY PROPOSAL FOR EXPERIMENTAL WORK IN SUPPORT OF WEAPONS FABRICATION

\begin{tabular}{|c|c|c|c|}
\hline AUTHOR & LEVEL & PGS & DATE \\
\hline EM MALKER & SRD & & $11 / 08 / 65$ \\
\hline JA LANE & SRD & & $11 / 12 / 65$ \\
\hline JB KENDALL & CRD & & $11 / 19 / 65$ \\
\hline AAPO & SRD & & $12 / 01 / 65$ \\
\hline OH WALKER & SRD & & $01 / 30 / 65$ \\
\hline RE TOMLINSON & SRD & & $12 / 01 / 65$ \\
\hline RE TONLINSON & SRD & & $12 / 01 / 65$ \\
\hline W KINNEY & SRD & & $12 / 07 / 65$ \\
\hline AE BARBER & SRD & & $12 / 09 / 65$ \\
\hline DE PETERSON & SRD & & $12 / 09 / 65$ \\
\hline AE SMITH & CRD & & $12 / 16 / 65$ \\
\hline TR MCKENZIE & SRD & & $12 / 20 / 65$ \\
\hline HAPO & SRD & & $12 / 30 / 65$ \\
\hline BM DOBBS & SRD & & $01 / 13 / 66$ \\
\hline JP DUCKMORTH & SRD & & $03 / 24 / 66$ \\
\hline HY MURRAY & SRD & & $04 / 08 / 68$ \\
\hline IN TAYLOR, JR. & CRD & & $07 / 09 / 68$ \\
\hline IN TAYLOR, JR. & SRD & & $07 / 09 / 68$ \\
\hline IN TAYLOR, JR. & SRD & & $07 / 09 / 68$ \\
\hline GP KESEL & SRD & & $11 / 12 / 68$ \\
\hline HW MURRAY & SRD & & $12 / 10 / 70$ \\
\hline SHITH AE & SRD & 8 & $12 / 01 / 64$ \\
\hline OBERG GC & SRD & 7 & $12 / 07 / 64$ \\
\hline SMITH AE & SRD & 12 & $12 / 10 / 64$ \\
\hline WALKER CM & SRD & 11 & $12 / 14 / 64$ \\
\hline TAYLOR IN, BERGGREN JF & SRD & 6 & $04 / 29 / 65$ \\
\hline HOPKINS HH, FELT RE & SRD & 22 & $02 / 11 / 65$ \\
\hline
\end{tabular}


Page No. 252

$04 / 28 / 93$

LIST OF HANFORD-GENERATED CLASSIFIED

DOCUMENTS DATED BETHEEN 1/1/61 AMD 12/31/72

DOC. NO.

RL-SEP-0185

RL-SEP-0187

RL-SEP-0195

RL-SEP-0198

RL-SEP-0237

RL-SEP-0239

RL-SEP-0243

RL-SEP-0247

RL-SEP-0261

RL-SEP-0264

RL-SEP-0283

RL-SEP-0298

RL-SEP-0299

RL-SEP- 0304

RL-SEP-0311

RL-SEP-0312

RL-SEP- 0325

RL-SEP-0348

RL-SEP-0361

RL-SEP-0366

RL-SEP-0367

RL-SEP-0372

RL-SEP-0378
TITLE

A-LINE PRODUCT CHARACTERISTICS NOVEMBER

1964

MEAPONS FABRICATION - UNIT COST AND

VARIANCE REPORT, NOVEMBER 1964

EVALUATION OF AIR-ACTUATED ELECTRON JET

AVERAGING PROCEDURE AND EVALUATION

METHOD FOR TSETSE COMPONENTS

TANTALUM SHAPE CASTING MELT CRUCIBLES

INTERIM SUMURY OF FABRICATION PROCESS

TEST FPT-64-26

BY-PROOUCT RECOVERY DURING WASTE

PROCESSIMG EMGIMEERING STLOY

REDOX CHEMICAL FLOUSHEET HW NO. 7 AND HW NO. 8

A-LINE PRODUCT CHARACTERISTICS DECEMBER

1964

DETERMINATION OF THE 88-C MEUTRON COUNTING FACTOR

INTERMEDIATE PRODUCT CHARACTERISTICS

WEAPONS PROCESS ENGINEERING TECHNOLOGY

REPORT - DECEMBER 1964 - JANUARY 1965

FABRICATION PROCESS TEST EVALUATION

FPT-64-34, POLISHIHG OF 74-C PARTS WITH

NYLON SCOURING PAD

ADDENDUM 1 TO FPT-64-22 - CHILL CASTING OF 74-C PREFORMS

HARDNESS MEASUREMENT FOR DETERMINING

PRODUCT CHARACTERISTICS

A-LINE PRODUCT CHARACTERISTICS JANUARY

1965

SEMI-ANNUAL REPORT 03 PROCESS

DEVELOPMENT JULY 1, 1964 TO DECEMBER 1, 1964

SEMI-ANNUAL PROGRESS REPORT O3 RED JULY

1, 1964 TO DECEMBER 1, 1964

WEAPONS PROCESS ENGINEERING TECHNOLOGY

REPORT - FEBRUARY, 1965

FABRICATION PROCESS TEST FPT-65-2

TENSILE MACHINE INSTALLATION CHECKOUT

21A-6 BUDGET FOR FY 1967 AND REVISION OF BUDGET FOR FY 1966-PROPOSAL FOR PROCESS DEVELOPMENT

BUDGET FOR FY 1967 AND REVISION OF BUDGET FOR 1966 - PROPOSAL FOR RESEARCH AND DEVELOPMENT - GEMERAL MEAPONS DEVELOPMENT

A-LINE PRCOUCT CHARACTERISTICS FEBRUARY 1965

MEAPONS FABRICATION - UNIT COST AMD VARIANCE REPORT, FEBRUARY 1965 FISCAL YEAR TO OATE
AUTHOR

SMITH AE

MORRELL DL

MCGREGOR LL

PINKERTON JH

WALKER CM

BARKES JH, SMITH PH

CRD

ISMACSON RE

CRD

SMITH AE

SRD

HOMI CS, OBERG GC

SMITH AE

BARBER AE

FIELD ST

SRD

SRD

BRONKEN CJ

SMITH AE

TOMLINSON RE

TOMLIMSON RE

SRD

SRD

CRD

BERGGREN JF

TOMLIMSON RE, SMITH AE SRD

TOMLINSON RE, SHITH AE

SRD

14

$03 / 29 / 65$

SMITH AE

SRD

SRD

PGS

DATE

$12 / 21 / 64$

$12 / 23 / 64$

$01 / 04 / 65$

01/05/65

$01 / 07 / 65$

$01 / 15 / 65$

$01 / 19 / 65$

$01 / 14 / 65$

$01 / 18 / 65$

$01 / 22 / 65$

02/02/65

$02 / 04 / 65$

02/04/65

02/03/65

$02 / 17 / 65$

$02 / 15 / 65$

$02 / 18 / 65$

03/03/65

$03 / 19 / 65$

$03 / 31 / 65$

MORRELL DL 
Page No. 253

$04 / 28 / 93$

LIST OF HANFORD-GENERATED CLASSIFIED

DOCUMENTS DATED BETWEEN 1/1/61 AND 12/31/72

DOC. NO.

TITLE

AUTHOR

LEVEL

PGS

DATE

RL-SEP-0379

RL-SEP-0404

RL-SEP-0407

RL-SEP-0409

RL-SEP- 0417

RL-SEP- 0450

RL-SEP- 0451

RL-SEP-0467

RL-SEP- 0473

RL-SEP- 0481

RL-SEP-0493

RL-SEP-0500

RL-SEP-0510

RL-SEP-0513

RL-SEP- 0578

RL-SEP-0586

RL-SEP-0587

RL-SEP-0588

RL-SEP-0608

RL-SEP-0610 RD

RL-SEP-0616 RD

RL-SEP-0648

RL-SEP-0650 RD

RL-SEP-065

RL-SEP-0658
A-LINE PROCESS DEFINITION

MEAPONS PROCESS ENGINEERING TECHNOLOGY

REPORT - MARCH 1965

A-LIME PRODUCT CHARACTERISTICS MARCH

1965

BUDGET FOR FY 1967 AND REVISED BUDGET

FOR FY 1966 SEPARATIONS RESEARCH ANO

DEVELOPMENT PROGRAM /O2 501015 1/

21A-5 SUAWARY ANALYSIS OF PROPOSED

PROCESS DEVELOPMENT COSTS FOR FY 1967

AND REVISION OF BUDGET FOR FY 1966

SEMIANMUAL PROGRESS REPORT O3 PROCESS

DEVELOPMENT DECEMBER 1, 1964 TO MAY 1, 1965

SEMIANMUAL PROGRESS REPORT O3 RRD

DECEMBER 1, 1964 TO MAY 1, 1965

NEUTRON COUNTING FACTOR FOR 88-C

COMPONENTS

MEAPONS PROCESS ENGINEERING TECHMOLOGY

REPORT APRIL 1965

FABRICATION PROCESS TEST $64-25$

EVALUATION

A-LIME PROOUCT CHARACTERISTICS APRIL 1965

MEAPONS INSPECTION 88-C PROCEDURE MANUAL

FABRICATION PROCESS TEST 64-28

EVALUATION: MECHANICAL CLEANING OF

PLUTONIUM BUTTONS

MEAPONS PROCESS ENGINEERING TECHNOLOGY

REPORT - MAY 1965

A-LINE PROOUCT CHARACTERISTICS MAY 1965

SPECIAL YIELD DATA AMALYSES FOR CORE

ANALYSIS MATERIAL KEY NO. 6887-KW-E

SPECIAL YIELD DATA AMALYSES FOR CORE

ANALYSIS MATERIAL KEY NO. 6706-C-N

SPECIAL YIELD DATA ANALYSES FOR CORE

ANALYSIS MATERIAL KEY NO. 7134-H-E

MEAPONS PROCESS ENGINEERING TECHNOLOGY

REPORT - JUNE 1965

WEAPON MANUFACTURING CAPABILITY

RAW DATA AND CALCULATIONS FOR WEAPON

MANUFACTURING CAPABILITY

FABRICATION PROCESS TEST EVALUATION TEST

NO. 64-39 - 88-C GENERATING TOOLS

THORIUM - U-233 SEPARATIONS AT THE PUREX

PLANT CRITICAL MASS CONTROL

SPE.CIFICATIONS

TEMPLATE DATA

WEAPONS PROCESS ENGINEERING TECHNOLOGY

REPORT - JULY 1965
SMITH AE

SMITH AE

TOMLINSON RE.

TONLINSON RE, SWITH, AE SRD 4

$03 / 31 / 65$

TOMLINSON RE

SRD

42

$04 / 19 / 65$

TOMLINSON RE

SRD

11

$04 / 19 / 65$

BARBER AE

SRD

SRD

$04 / 26 / 65$

$04 / 29 / 65$

FIELD ST

SRD

8

05/06/65

SMITH AE

SINSLI RA

HALKER CM

SRD

CRD

SRD

118

11

$06 / 08 / 65$

$06 / 01 / 65$

$06 / 01 / 65$

SMITH AE

SRD

8

$06 / 15 / 65$

BRAYMEN HH, SMITH HE

SRD

SRD

11

$06 / 16 / 65$

BRAYMEN WH, SHITH HE

SRD

3

$06 / 16 / 65$

BRAYMEN WH, SHITH HE

SRD

SRD

$$
7
$$

PINKERTON JM

PIHKERTON JM

SRD

SRD

17

ARMSTROMG DA

SRD

22

$07 / 31 / 65$

KENDALL JB

SRD

24

$08 / 04 / 65$

SMITH AE 
Page No. 254

$04 / 28 / 93$

LIST OF HANFORD-GENERATED CLASSIFIED

DOCUMENTS DATED BETHEEN 1/1/61 AND 12/31/72

TITLE

AUTHOR

LEVE

PGS

DATE

RL-SEP-0669 PT1

DEVELOPMENT OF A POWER-ROLL-FORMIMG PROCESS: PART 1. FOUND DEVELOPMENT AND EXPERIENCE

RL-SEP-0669 PT3 DEVELOPMENT OF A POWER-ROLL-FORMIMG PROCESS, PART III. DETERMIMATION OF PONER-ROLL-FORMING PARAMETERS

RL-SEP-0669 PT4

RL-SEP-0670

RL-SEP-0672

RL-SEP-0697

RL-SEP-0705

RL-SEP-0709

RL-SEP-0715

RL-SEP-0716

RL-SEP-0720

RL-SEP-0720 1

RL-SEP-0724

RL-SEP-0724 1

RL-SEP-0745

RL-SEP-0746

RL-SEP-0750

RL-SEP-0762

RL-SEP-0765

RL-SEP-0778

RL-SEP-0807

RL-SEP-0831

RL-SEP-0840

RL-SEP-0844

RL-SEP-0848
DEVELOPMENT OF A POUER-ROLL-FORMING

PROCESS, PART IV. MMCHINING

PONER-ROLL-FORMED WEAPONS COMPONENTS

A-LINE PRCOUCT CHARACTERISTICS, JUNE -

JULY 1965

JONOG 22 PLUTONIUN MEETING

INSPECTION METHOOS FOR 104-C COMPONENTS WEAPONS PROCESS ENGINEERING TECHNOLOGY REPORT - AUCUST 1965

O3 PROGRAM SUPPORT PROPOSAL

TEMPLATE DATA

A-LINE PRODUCT CHARACTERISTICS, AUGUST 1965

EVALUATION OF FABRICATION PROCESS TEST 64-3, ISOSTATIC PRESS STABILIZATION OF UNALLOYED PLUTONIUN WEAPON COMPONENTS EVALUATION OF FPT-64-3, ISOSTATIC PRESS STABILIZATION OF UNALLOYED PLUTONIUN MEAPON COMPONENTS

ANALYSIS OF ISOSTATIC PRESSING RESULTS 600 TYPE PARTS

ANALYSIS OF ISOSTATIC PRESSING RESULTS 600 TYPE PARTS

104-C METHOOS APPROVAL

WEAPONS PROCESS ENGINEERING TECHNOLOGY REPORT - SEPTEMBER 1965

TRIP REPORT, VISIT TO SAVAMMAH RIVER

PLANT, SEPTEMBER 16-17, 1965

A-LINE PROOUCT CHARACTERISTICS, SEPTEMBER 1965

EVALUATION: FPT 64-21, 1807 SURFACE "V" FLATMESS

EVALUATION REPORT: FPT 64-22, ADDENDUN

1, 03 DEVELOPMENT RUNS RED-25-6-301 THRU 6-310

PUREX PROCESSING - MEASUREMENTS QUALITY CONTROL PROGRESS REPORT

WEAPONS PROCESS ENGINEERING TECHNOLOGY REPORT - OCTOBER 1965

A-LINE PROOUCT CHARACTERISTICS, OCTOBER 1965

PERMANENT SHAPE MOLD DEVELOPNEMT PROGRAM 104-C COMPONENT RADIUS AND POLAR HEIGHT INSPECTION barber ae

GRO

26

LAWTOW WL, OBERG GC

SRD

21

08/03/65

PINKERTON JM, VAN DER

COOK RE

SNITH AE

SRD

11

08/03/65

SWITH AE

MCGRECOR LL

SRD

SRD

SRD

94/65

$09 / 08 / 65$

$08 / 31 / 65$

SHITH AE

SMITH AE

SMITH, AE

SRD

SRD

SRD

09/03/65

$09 / 08 / 65$

WALKER CM

SRD

16

$09 / 10 / 65$

WALKER CH

SRD

16

$09 / 21 / 65$

TAYLOR IN

SRD

15

$09 / 15 / 65$

TAYLOR IN

SRD

14

$10 / 26 / 65$

SWITH AE

SRD

$09 / 28 / 65$

$09 / 30 / 65$

HILL OF

CRD

4

$09 / 24 / 65$

SHITH AE

SRD

11

$10 / 12 / 65$

VAN DER COOK RE

SRD

4

$10 / 08 / 65$

FIELD ST

SRD

25

$10 / 18 / 65$

KIMBLE FG

SRD

7

$10 / 21 / 65$

SRD

7

$11 / 01 / 65$

SNITH AE

SRD

10

$11 / 03 / 65$

WALKER CY

SMITH AE 
Page No. 255

$04 / 28 / 93$

LIST OF HANFORD-GENERATED CLASSIFIED

DOCUNENTS DATED BETWEEN 1/1/61 AND 12/31/72

DOC. NO.

TITLE

AUTHOR

LEVEL

PGS

DATE

RL-SEP-0866

RL-SEP-0877

RL-SEP-0879

RL-SEP-0880

RL-SEP-0881

RL-SEP-0896

RL-SEP-0911

RL-SEP-0912

RL-SEP-N-00001

RL-SEP-N-00005

RL-SEP-N-00006

RL-SEP-N-00007

RL-SEP-N-00010

RL-SEP-H-00011

RL-SEP-N-00017

RL-SEP-N-00019

RL -SEP-N-00021

RL-SEP-N-00022

USCM-4
THORIUM PROCESSING hUCLEAR SAFETY REVIEW

MEAPONS PROCESS EMGINEERING TECHNOLOGY REPORT - MOVEMBER 1965

EVALUATION: FPT-65-3, INCOTING OF UMALLOYED PLUTONIUN BUTTOWS SEMIANDULAL PROCRESS REPORT, OS RED, MAY 1. 1965 TO DECEMEER 1, 1965 SEMIANNUAL PROCRESS REPORT O3 PROCESS DEVELOPMENT MAY 1, 1965 TO DECEMAER 1. 1965

A-LINE PRCOUCT CHARACTERISTICS

MOVEMBER-DECEMBER 1965

PO-210 COSTS AT HANFORO

MEAPONS PROCESS EMGIMEERING TECHMOLOGY

REPORT - DECEMBER 1965

PERSONAL MOTES

PERSONAL NOTES

PERSONAL NOTES

OUALITY CONTROL NOTES

PROCESS DATA NOTES

PROCESS DATA COATROL

MEASUREMENT RELIABILITY QUALITY CONTROL NOTES

PERSONAL MOTES

MANAGERS MOTES URANIUM OXIDE

PERSONAL NOTES

FINAL REPORT, RESEARCH PROJECT, CONTRACT CA-264, CHEMICAL MILLING OF COUNTERBORE RECESSES IN THE URANILN WALL OF ZIRCALOY-2 CLAD URANILA TUBES

\begin{tabular}{|c|c|c|c|}
\hline \multirow{2}{*}{$\begin{array}{l}\text { CLAYTOW ED, GEIER RG, } \\
\text { LEVERETT MC }\end{array}$} & SRD & 17 & $12 / 01 / 65$ \\
\hline & SRD & 8 & $12 / 01 / 65$ \\
\hline MALKER CH & SRD & 27 & $11 / 30 / 65$ \\
\hline TONLIMSON RE & SRD & 14 & $12 / 01 / 65$ \\
\hline TOHLINSON RE & SRD & 56 & $12 / 01 / 65$ \\
\hline SWITH AE & SRD & 11 & $12 / 14 / 65$ \\
\hline HILL OF & $\begin{array}{l}\text { SRD } \\
\text { SRD }\end{array}$ & $\begin{array}{l}6 \\
4\end{array}$ & $\begin{array}{l}12 / 29 / 65 \\
12 / 30 / 65\end{array}$ \\
\hline MC JACOBS & SECR & & $10 / 14 / 64$ \\
\hline OA ARASTRONG & SECR & & $10 / 27 / 64$ \\
\hline RE FELT & SRD & & $11 / 05 / 64$ \\
\hline RR LANERT & SECR & & $11 / 13 / 64$ \\
\hline EF KRAUTTER & SECR & & $12 / 14 / 64$ \\
\hline RR LAMBERT & SECR & & $12 / 14 / 64$ \\
\hline DJ ROCHOW & SECR & & $02 / 11 / 65$ \\
\hline CE MILBER & SECR & & $02 / 12 / 65$ \\
\hline CW MLCOY & SECR & & $05 / 05 / 65$ \\
\hline RJ SORENSON & SECR & & $06 / 03 / 65$ \\
\hline ATKIMS DC & CRD & 44 & $03 / 28 / 69$ \\
\hline
\end{tabular}



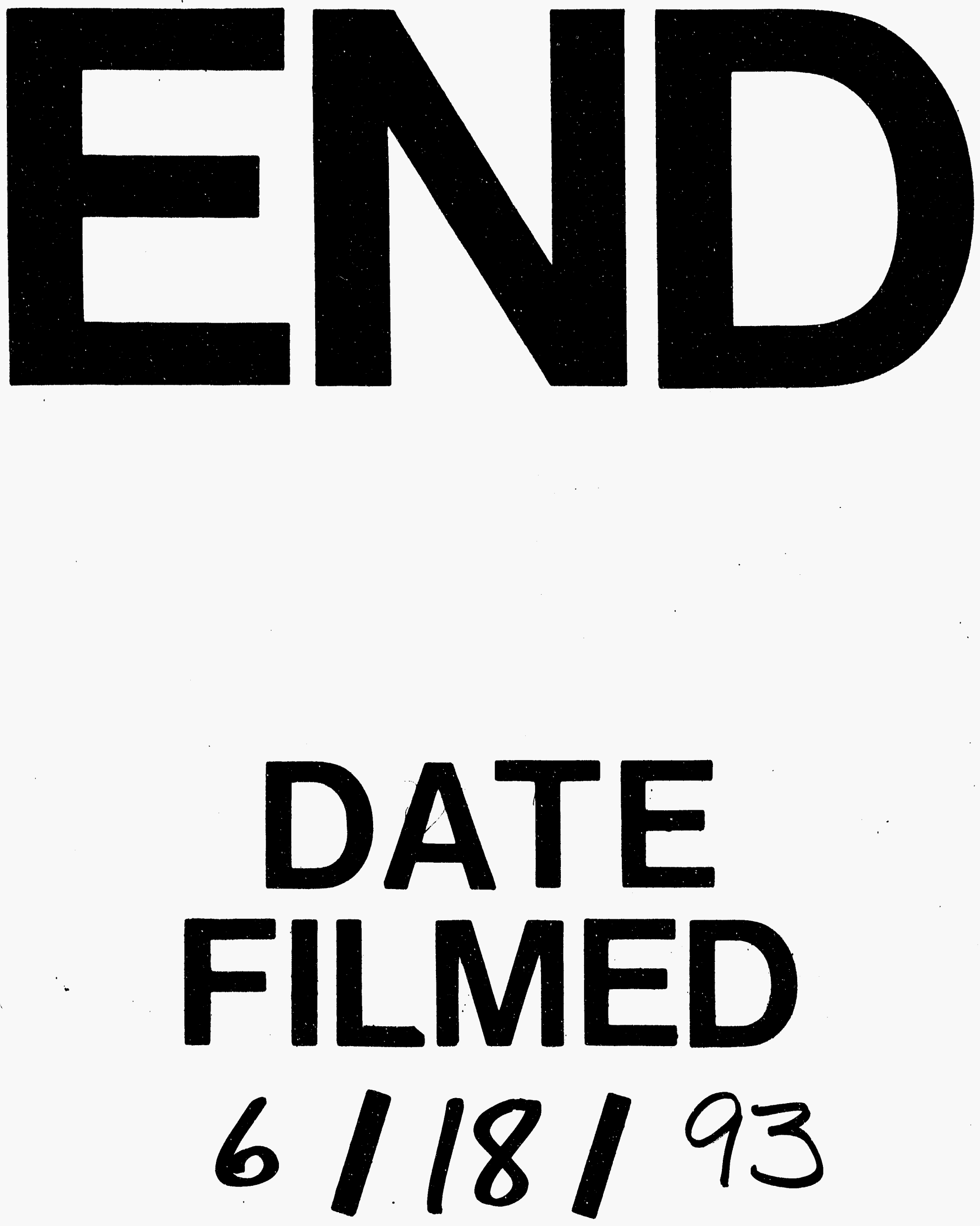


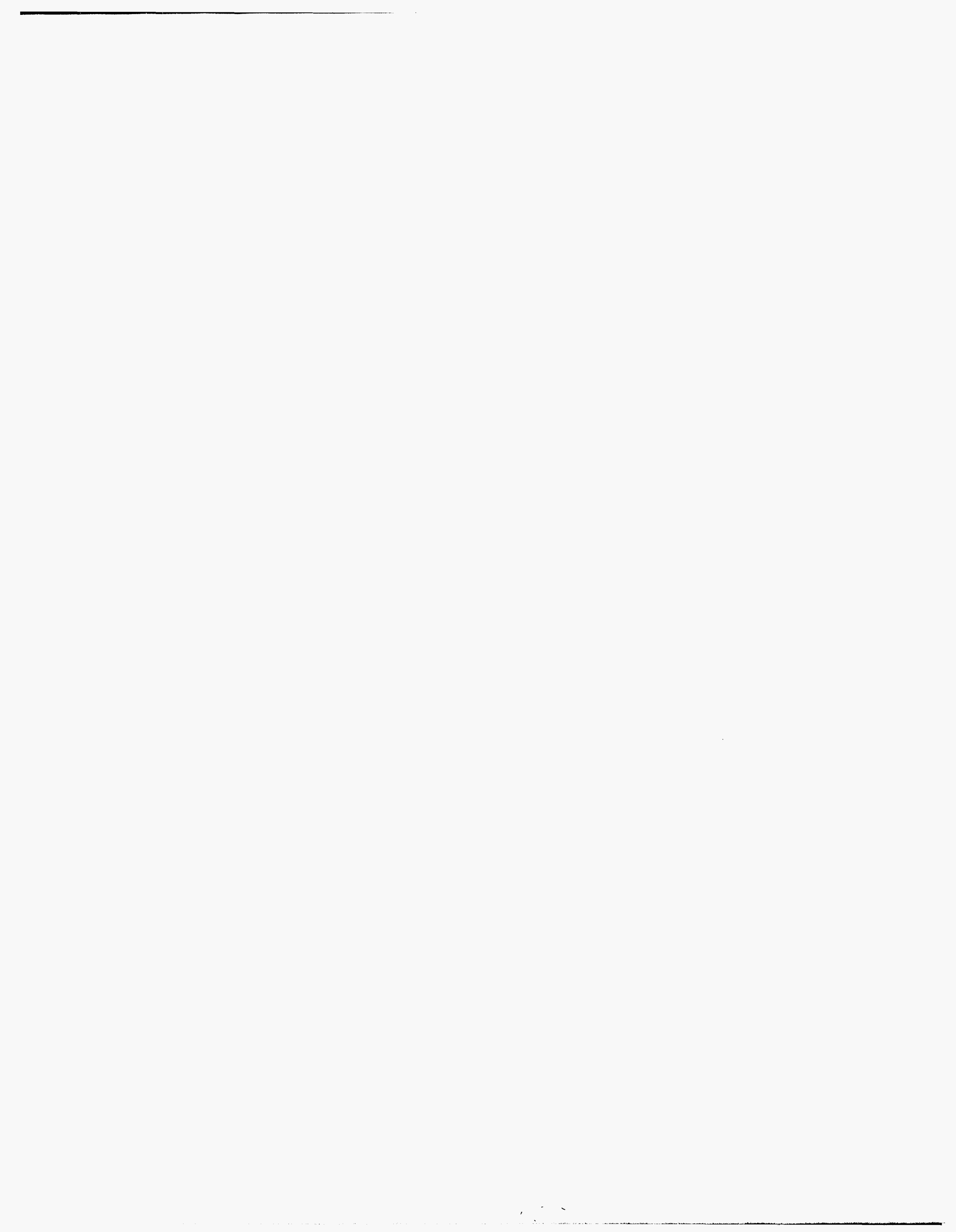

